\title{
A monograph of Hyaloscypha and allied genera
}

\author{
SEPPO HUHTINEN
}

HUHTINEN, S. 1989(1990): A monograph of Hyaloscypha and allied genera. Karstenia 29:45-252.

The genera Hyaloscypha Boud., Phialina Höhnel and Hamatocanthoscypha Svr. are monographed on a world basis. Hyaloscypha, with 27 recognized taxa, is circumscribed to contain whitish and mostly lignicolous species with tapering and aseptate hairs. Dextrinoid reactions, hair vesture and ascal development are shown to be important diagnostic characters. Two subgenera are recognized within Hyaloscypha. Seven new species are described: $H$. britannica Huhtinen, $H$. diabolica Huhtinen, $H$. epiporia Huhtinen, $H$. latispora Huhtinen, $H$. nipponica Huhtinen, $H$. strobilicola Huhtinen, and $H$. usitata Huhtinen. A new variety is published in Hyaloscypha secalina Velen. A new name, $H$. occulta Huhtinen, is proposed for $H$. parvula Arendh. \& R. Sharma. The following new combinations are made: Hyaloscypha albohyalina (P. Karst.) Boud. var. spiralis (Velen.) Huhtinen (Chrysothallus s.), H. albohyalina var. tigillaris (P. Karst.) Huhtinen (Peziza t.), Hyaloscypha fuckelii Nannf. var. alniseda (Velen.) Huhtinen (Hyaloscypha a.), Hyaloscypha leuconica (Cooke in Stevenson) Nannf. var. bulbopilosa (Feltg.) Huhtinen (Dasyscypha hamata var. b.), Hyaloscypha querciola (Velen.) Huhtinen (Uncinia q.) and Hyaloscypha aureliella (Nyl.) Huhtinen (Peziza a.). The genus Phialina, with eight recognized species, is circumscribed to contain foliicolous and herbicolous taxa with yellow pigment inside the paraphyses and the tapering and often septate hairs. Presence of croziers and lack of dextrinoid reaction are also stressed at the generic level. One new species, $P$. setiigera Huhtinen is described. The following new combinations are made: Phialina lachnobrachyoides (Raitv.) Huhtinen (Phialoscypha l.), Phialina foliicola (Graddon) Huhtinen (Uncinia f.). A new name, Phialina pusilla Huhtinen, is proposed for Hyaloscypha pusilla Arendh. \& R. Sharma. The genus Hamatocanthoscypha, with 10 recognized taxa, is delimited to comprise mostly white to coloured, stipitate inhabitants of litter. The hairs are typically cylindrical-tapering and uncinate. Three new species are described: Hamatocanthoscypha helicotricha Huhtinen, $H$. obsoleta Huhtinen and $H$. ocellata Huhtinen. The following new combinations are made: Hamatocanthoscypha melanobasis (Arendh. \& R. Sharma) Huhtinen (Debaryoscyphus m.), Hamatocanthoscypha laricionis (Velen.) Svr. var. dryopteridis (Bøhler) Huhtinen (Hyaloscypha d.), Hamatocanthoscypha uncipila (Le Gal) Huhtinen (Hyaloscypha u.), Hamatocanthoscypha uncinata (Phill.) Huhtinen (Peziza u.), Hamatocanthoscypha uncinata var. phaeotricha (K. Holm \& L. Holm) Huhtinen (Unciniella laricionis (Velen.) K. Holm \& L. Holm var. p.), and Hamatocanthoscypha laricionis var. minutissima (Velen.) Huhtinen (Uncinia m.). Of the 137 excluded taxa, Phialina montana Raitv. and Pezizella myriadea Mouton are combined with Calycellina Höhnel and Hyaloscypha sulfurina Dennis with Trichopeziza Mont. \& Dur. The pattern of variation in the taxa studied is illustrated in detail. Sporograms and frequency distribution curves are provided for most taxa. Ascal and spore dimensions and shape are shown to be dependent on the reagent used. The taxonomic status of 15 closely related genera is treated.

Cheiromycella microscopica (P. Karst.) Hughes is shown to be the anamorphous state of Hyaloscypha aureliella (H. stevensonii (Berk. \& Broome) Nannf.). A phialidic anamorph characterizes the type variety of Hamatocanthoscypha laricionis (Velen.) Svr. and H. uncipila. An immature, dematiaceous anamorph developed in cultures of $P$ hialina lachnobrachya (Desm.) Raitv.

Seppo Huhtinen, Department of Biology, University of Turku, SF-20500 Turku, Finland 


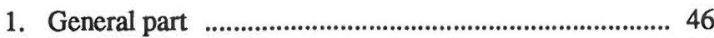

Introduction ................................................................. 46

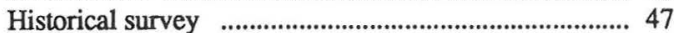

Hyaloscypha .................................................... 47

Hamatocanthoscypha ........................................... 49

Phialina ................................................................. 49

Generic relationships ................................................. 50

Delimitation of the monographed genera ................... 55

Morphology ............................................................. 58

Apothecia ............................................................. 58

Excipulum ......................................................... 58

Hairs ................................................................ 60

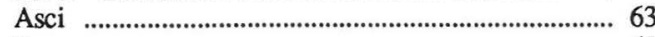

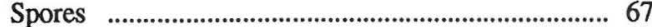

Paraphyses ........................................................... 71

Pigments, resinous exudates and histochemistry .... 71

Ecology ................................................................. 72

Hyaloscypha ..................................................... 72

Hamatocanthoscypha ............................................. 74

Phialina ................................................................ 74

Distribution ............................................................. 74

Phenology ................................................................... 77

Taxonomic approach ............................................... 77
2. Cultural studies $\quad$........................................................ 79

Mycelium ............................................................... 79

Anamorph and teleomorph ........................................... 79

Substrate preference ....................................................... 81

3. Material and methods ….............................................. 85

Morphological studies ................................................. 85

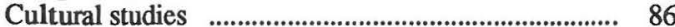

4. Taxonomic part .......................................................... 87

A synoptic key to the species and varities of Hyalo-

scypha, Hamatocanthoscypha and Phialina ............ 87

Hyaloscypha .............................................................. 90

Hamatocanthoscypha ............................................. 181

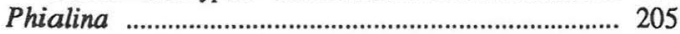

5. Excluded, dubious and imperfectly known taxa ........ 233

6. Acknowledgements .................................................. 244

7. References ..... 244

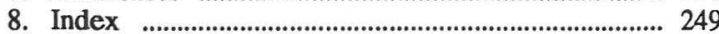

Dedicated to Serpula lacrymans,

who ate our house

but saved the manuscript.

\section{GENERAL PART}

\section{Introduction}

The ascomycete genus Hyaloscypha Boud. (Hyaloscyphaceae, Helotiales) possesses taxonomic characters that long passed unnoticed. For instance, it took nearly one hundred years before the colour change of the excipulum and hairs in Melzer's reagent was recognized as diagnostic (Svrček 1977c). The importance of the ascal development and hair vesture were not realized and there was hardly any agreement regarding the species delimitation. Over 1700 specimens were examined for the present study and detailed, annotated drawings were prepared from most of the collections. The variation and degree of stability could thus be thoroughly evaluated. Future genetic work on the ascus development may, nevertheless, change the classification proposed here. The weight to be attached to the character croziers versus simple septa is a serious problem in Hyaloscypha. Not even the mycogenetists I consulted could estimate the value of this difference. Although ascal development is constant within a given taxon, the taxonomic level at which the difference should be recognized remains unclear.

Authors of monographs tend to illustrate one, preferably "ideal" specimen of a species, giving a simplified picture of the situation in nature and excluding undesirable, but inevitable, variation. In this paper equal attention is paid to stability and variation. The depicted populations were selected to illustrate both constant and variable features, which could well be adopted as practice in future monographs.

The elements of a monograph are usually deposited in scores of herbaria all around the world, so that painstakingly collected information too often becomes fragmented or lost. The unpublished annotations filed with the specimens make up the geographically scattered pieces of a jigsaw puzzle, which require much effort to fit together. For this reason, a copy set of my annotations and drawings will be kept in herb. TUR, enabling a rapid survey of each taxa.

Since 1983 I have studied not only Hyaloscypha, but also the related genera. Of these, Hamatocanthoscypha Svr. and Phialina Höhnel are treated in detail. Dematioscypha Svr. has already been dealt with (Huhtinen 1987d). Some of the excluded elements have no genus to accommodate them and their placement must await further studies. Erection of monotypic genera for each case would be easy, but undesirable.

The ecological characteristics of each species and genus have been outlined. Besides conventional morphological studies, the histochemistry and cultural characters have also been examined. Special attention has been paid to methodological problems, which in the past have sometimes been responsible for masking the existing differences. 


\section{Historical survey}

Hyaloscypha

The genus Hyaloscypha was published by Boudier (1885) in a key, but the generic name had already appeared in the literature six years earlier, in the index of Gillet's (1879) flora. Under "Hyaloscypha Boudier" Gillet listed one species, Peziza dentata Pers., which he combined with Mollisia (Fr.) P. Karst. Apparently Boudier had released his new classification of Discomycetes well before publishing it, because Gillet's flora contains numerous genera that were established by Boudier six years later. Boudier (1885) placed Hyaloscypha in his "famille" Dasyscyphes "groupe" Subhirsutes. The diagnostic characters given in his key are 1) cylindrical to somewhat clavate, branched and septate paraphyses, which do not protrude above the hymenium, 2) somewhat fleshy and sessile apothecia, 3) apothecia less hairy and with broader and less clearly branched paraphyses (than in Arachnoscypha Boud.), 4) aseptate and guttulate ascospores. Boudier (1885) designated no type for the genus; he only mentioned $\mathrm{He}$ lotium vitreorum P. Karst. (in error for "vitreolum") and Peziza dentata as examples of the species included.

The circumscription of Hyaloscypha was later modified by Boudier (1907) and in all 34 species were referred to the genus. The noteworthy new diagnostic characters were the translucent apothecia, the tapering and septate hairs, and the lignicolous or corticolous growth habit. Many of the 34 species, however, disagree with Boudier's concept, either in their ecology (on herbaceous stems or leaves) or in being hairless. When including taxa he had not studied himself, Boudier was not always misled by insufficient descriptions in the early literature. For example, he synonymized the genus Allophylaria (P. Karst.) P. Karst. with Hyaloscypha and included all the five species listed by Saccardo (1889) in Hyaloscypha, although the genus was stated to be glabrous and the taxa caulicolous (Karsten 1869, Saccardo 1889). It is obvious that the expanded concept presented by Boudier in 1907 was burdened by uncertain circumscription.

When trying to reconstruct Boudier's (1885) concept of Hyaloscypha, we should consult the herbarium material he actually studied. In Paris there are three such collections: one of Hyaloscypha as understood at present, and two belonging to Cistella Quél. and Mollisina Höhnel. The specimen bearing the name Hyaloscypha hyalina belongs to $H$. aureliella (Nyl.) Huhtinen. It is not, however, the specimen which was depicted in "Icones Mycologicae" (Bou- dier 1905-1910) as H. hyalina. Further information on Boudier's concept can be extracted from the four species he illustrated in his Icones Mycologicae as Hyaloscypha, and had consequently studied. Three of these may represent species of Hyaloscypha as currently delimited. Their exact identity is, however, dubious. In the light of the 1907 publication, we can conclude that Boudier established a genus he only partly understood. But in the cases in which we know that he based his concept on close scrutiny of specimens, only two species are seriously misplaced.

For nomenclatural purposes, the two original species, Helotium vitreolum and Peziza dentata, are essential and problems arising from the lectotypification with $P$. dentata by Clements and Shear (1931) have been discussed by Huhtinen and Cannon (1987). In that paper conservation of Hyaloscypha was proposed with $H$. vitreola (P. Karst.) Boud. as the conserved type.

Hyaloscypha was largely ignored by Boudier's contemporaries. Phillips (1887), Saccardo (1889, 1913), Massee (1895), Schroeter (1908) or Höhnel (in his "Fragmente zur Mykologie") did not mention it at all, and Rehm (1896) referred to it only in a list of synonyms under Pezizella dentata (Pers.) Rehm. A single species, $H$. hyalina, was treated by Lagarde (1906). In 1913 the generic name appeared in Thome's Kryptogamen-Flora (Migula 1913), but only due to a slip of the pen. Under "Hyaloscypha Fuckel" Migula treated one species, "H. ciliata Fuckel". It seems that Migula copied the description from Höhnel (1902), who used the correct spelling "Hyalopeziza". A similar error occurred in Höhnel's (1926b) treatment of Pezizella Fuckel, where the two names occur together. The short discussion by Höhnel indicates that he accepted both clavate and tapered hairs in Hyaloscypha.

Clements and Shear (1931) took up Hyaloscypha when lectotypifying the genus, but placed it under the synonymy of Pezizella Fuckel sensu Rehm. The year 1932 saw the rebirth of Hyaloscypha in the valuable study by Nannfeldt. He (Nannfeldt 1932) was the first to reserve the genus for species with tapered, aseptate and thin-walled hairs, and his concept has prevailed ever since. As typical members of the genus, Nannfeldt cited Boudier's plates of $H$. vitreola and $H$. hyalina. Nannfeldt ignored the fact that the plates and descriptions of these two species indicate the occurrence of truly septate hairs in the genus. This does not prevent the use of Hyaloscypha in Nannfeldt's emended sense, since hair septation is present in the genus, although rare. Nannfeldt emphasized hair morphology in his delimitation. By synonymizing Phialina with Hyaloscypha he allowed variation in excipular wall thickness and in the gen- 
eral structure of the excipulum. He mentioned, however, that the outermost layer of excipulum is close to textura prismatica in Phialina as well. Some difficulty is encountered, however, in connecting the emended concept of Nannfeldt with Hyaloscypha sensu Boudier. Nannfeldt excluded the two original species of Boudier from his concept and thus created a later homonym Hyaloscypha Nannf. (Huhtinen \& Cannon 1987). This homonym largely covers Hyaloscypha as presently circumscribed and could be made available by conservation. But the species depicted and studied by Boudier indicate that he had a fairly clear idea of the genus. By conserving the typification with $P$. vitreola, citation of the original author of Hyaloscypha can be retained.

The next major treatment of Hyaloscypha was that of Velenovsky (1934). He expanded the genus markedly, especially as regards the hair morphology: the generic diagnosis stated "pilis rectis, brevibus". The genus was split into four groups of undefined rank, but largely matching Hyalopeziza and Cistella sensu Nannfeldt (1932), Hyaloscypha Nannf. and Incrupila Raitv. The division was based on the type of hairs: 1) hairs narrow at base, acute, septate; 2) hairs obtuse to clavate, septate; 3 ) hairs basally widened, tapering to a solid apex, aseptate to rarely sparsely septate; 4) hairs cylindrical, obtuse, septate and granulate. Svrček's (1985) and my studies on Velenovsky's material show the marked heterogeneity of Hyaloscypha sensu Velenovský.

In two type studies of British discomycetes, Nannfeldt $(1936,1939)$ transferred four species to Hyaloscypha. This notably widened his generic concept, especially the inclusion of Peziza dematiicola Berk. \& Broome. with a brown excipulum. This treatment was later followed by Dennis (1949). Seven new species were added to the genus in later papers of Velenovský $(1940,1947)$.

Dennis (1949) treated Hyaloscypha in detail and stressed both the nomenclatural problem caused by Nannfeldt and the problems of species delimitation. He largely followed Nannfeldt (1932, 1936, 1939), but admitted that the genus was artificial. His generic diagnosis was meagre, emphasizing mainly the aseptate, thin-walled and acutely pointed hairs. For the first time, the taxonomic difficulties caused by the whitish, lignicolous Hyaloscyphas were recognized. Dennis regarded them as forms of $H$. hyalina.

The genus was dealt with anew in Boudier's sense by Grelet (1951). Although the generic diagnosis contained no important new characters, two species with curved hairs or paraphyses were accepted. In a revision of the British Helotiaceae, Dennis (1956) treated some excluded species that Karsten had re- ferred to Helotium Pers. Some of these were found to be typical species of Hyaloscypha, but formal combinations were first made in 1970, in Raitviir's (1970a) study on the Hyaloscyphaceae. Raitviir had a narrow concept of Hyaloscypha, restricting the genus to whitish, lignicolous taxa (except in the case of $H$. paludosa). H. lachobrachya (Desm.) Nannf., with narrowly fusoid spores, was excluded and transferred to Phialina. Three years later Korf (1973) expanded the genus to embrace Microscypha $\mathrm{H}$. Sydow \& P. Sydow and Unguiculella Höhnel, i.e. taxa having almost cylindrical or clearly hooked hairs.

When describing Hyaloscypha dennisii, Bertault (1977) divided the genus into two sections. He considered that an excipulum composed of textura oblita was not an alien element in the genus. For such species, section Phialina (Höhnel) Bertault was established, with $H$. dennisii as the type. The other species included were $H$. deparcula (P. Karst.) Dennis and $H$. puberula (Lasch) Höhnel. Bertault's conclusions were, however, contradictory. The material examined by him was a sclerotiniaceous fungus (see Excluded taxa): the excipulum was not composed of textura oblita but was textura prismatica with slightly thickened walls, as already pointed out by Arendholz (1979). Furthermore, $H$. dennisii was not available for typification. Being based on Phialina, Bertault's sectional name must be typified from among the binomials included by Höhnel (Art. 7.12 and 49.1 of the Code; Greuter et al. 1988).

The next major treatment of the genus was that by Raschle (1978). He supported Nannfeldt's (1932) delimitation, but excluded Phialina as being congeneric with Calycellina Höhnel. In that and an earlier paper Raschle (1977) described the hairs as aseptate to septate. In the earlier paper he recognized the occasionally solid hair apices, already noted by Velenovský (1934). Starting from the fifties, the genus Hyaloscypha has received numerous new species (Grelet 1951, Le Gal 1954, Dennis 1955c, Nannfeldt 1958, Dennis 1960, 1962, Raitviir 1970a, Graddon 1972, Bøhler 1974, Sherwood 1974, Nag Raj \& Kendrick 1975, Descals \& Webster 1976, Svrček 1977a, Sherwood 1977, Ahmad 1978, Korf 1978, Raschle 1978, Svrček 1978, 1982, 1983, 1985, Dennis 1981, Abdullah \& Webster 1983, Arendholz \& Sharma 1983, Sharma et al. 1986, Spooner 1987, Svrček 1986, 1987a, 1988). The 80 taxa described by Velenovský were revised by Svrček (1985), who removed the discordant elements.

The addition of these new species obscured the delimitation. The characters accepted for Hyaloscypha include, for example, brown-walled and septate hairs, conspicuously warted hairs, multiseptate and 
tapering hairs totally covered by coloured resin, and branched hairs. Consequently, many other hyaloscyphaceous genera have become involved with the rather poorly circumscribed Hyaloscypha.

\section{Hamatocanthoscypha}

The genus Uncinia Velen. was established by Velenovský in 1934. It was taken up by Nannfeldt (1958), who transferred Uncinia laricionis Velen. to Hyaloscypha, and treated anew by Raitviir (1970a). By selecting $U$. laricionis as lectotype, the latter connected the genus with tiny hyaloscyphaceous species having cylindrical-tapering and curved hairs. The name is a later homonym of Uncinia Pers. and therefore the generic name Unciniella $\mathrm{K}$. Holm \& L. Holm was proposed (Holm \& Holm 1977). In the same year Svrček (1977a) established Hamatocanthoscypha. His study appeared five months earlier than that by the Holms and Unciniella thus falls into the synonymy of Hamatocanthoscypha.

Raitviir (1970a) placed the genus close to Cistella Quélet, whereas the generic diagnosis offered by Svrcek stressed the differences from Unguiculella. Velenovský (1934) gave the curved, septate and basally slender hairs, together with fleshy apothecia, as characters delimiting the genus from Hyaloscypha. Type studies on Uncinia showed that this heterogeneous genus includes species of Hyaloscypha, Mollisina and Unguiculella.

Svrček (1977a) circumscribed Hamatocanthoscypha to embrace small, sessile to shortly stipitate apothecia with short hairs. The hairs were characterized as being tapering to narrowly cylindrical, clearly curved to hooked, thin-walled, smooth, and aseptate to three-septate. The paraphyses were described as not protruding above the ascal tips and not hooked. Inclusion of new species in the genus has been limited, the only additions being those by Holm and Holm (1977), Svrček (1983) and Graddon (1986). The delimitation has changed, however, with these new taxa and the taxa discovered during the present study. Here, Hamatocanthoscypha is considered to be closely related to Hyaloscypha, and the monotypic genus Debaryoscyphus Arendh. \& R. Sharma is merged with it.

\section{Phialina}

The genus was established for Peziza deparcula P. Karst. and $P$. puberula Lasch by Höhnel (1926a: 61): "Ich stelle für die Pezizella deparcula die neue Gattung Phialina auf, die sich von Pezizella vornehmlich durch das parallelfaserige, nicht parenchymatische Excipulum unterscheidet." A few pages later he transferred Pezizella puberula (Lasch) Rehm in the genus, basing its inclusion on the study of two exsiccata under that name (Fuckel's Fungi rhenani 1150 and Rehm's Ascomyceten 1162). The similarity in general structure was stressed ("... ganz so gebaut wie Pezizella deparcula (Karst.) und muss daher auch zu Phialina gestellt werden. Das Gewebe ist knorperlig-faserig."). Due to Höhnel's words on page 61, $P$. deparcula has been regarded as the holotype of Phialina (Nannfeldt 1932, Raitviir 1970a, Arendholz 1979, Cannon et al. 1985), but actually Höhnel did not indicate which of the two species should be the type of the genus. $P$. deparcula may simply have happened to be the species treated first. The protologue of Phialina includes the discussion under $P$. puberula (cf. footnote to Art. 8.1).

The generic name is not, however, open to lectotypification. Nannfeldt (1932) cited Peziza deparcula as "Eutypus" of Phialina, thereby referring to the holotype of a given taxon (Nannfeldt 1932: 8). Unfortunately, the Code is vague about such errors in type citations. Nannfeldt actually lectotypified the genus by erroneously citing $P$. deparcula as holotype. In the absence of a relevant rule, the more or less established practice (Preamble 9) is to accept such lectotypifications. Were the generic name open to lectotypification, Peziza puberula Lasch could be selected, placing Phialina in the synonymy of Calycellina (see below). In that case, Setoscypha Velen. would be the correct name for the genus with the present circumscription. The type species is conspecific with $P$. lachnobrachya and the genus would be typified by a central element. At present Phialina unfortunately becomes typified by a somewhat marginal species.

It is evident that hair characteristics were not stressed by Höhnel (1926a) in the delimitation of $P$ hialina. When discussing the placement of $P$. deparcula suggested by Starbäck (1895), Höhnel excluded close relationship to Eupezizella candida. This view was based solely on the different excipular structure. From Starbäck's detailed illustrations the marked difference in hair structure between the two species is evident. The material that Höhnel cited under $P$. puberula was later shown to belong to Calycellina by Lowen and Dumont (1984) and was characterized by cylindrical and blunt hairs. Such hairs differ greatly from those of $P$. deparcula, but Höhnel emphasized the cartilaginous-fibrillous structure of the excipulum. Due to the somewhat poorly defined nature of the genus, it has received different delimitations. Nannfeldt (1932) treated Phialina as 
synonymous with Hyaloscypha, but refrained from making the combinations. Dennis (1949), who transferred the species, supported Nannfeldt's view and indicated some relationship to Hyaloscypha lachnobrachya. Later, Dennis (1960) accepted Phialina and stressed the cylindrical hairs. In his synopsis, Raitviir (1970a) included the original two species, together with $P$. lachnobrachya, in the genus. Both tapering and cylindrical hairs were still accepted in the genus when Raitviir (1977a) combined, for instance, Peziza flaveola Cooke with Phialina. In the same year, (Raitviir 1977b) the foliicolous $P$. lachnobrachya was made a type of a new genus, Phialoscypha Raitv. The closely related Peziza flaveola remained in Phialina, together with two other species with tapering hairs, namely Phialina pseudopuberula (Graddon) Raitv. and P. ulmariae.

Korf (1973) treated Phialina as synonymous with Calycellina and later (Korf 1982) transferred $P . u l$ mariae to that genus. This synonymy was accepted by Baral and Krieglsteiner (1985). Bertault (1977) had earlier reduced Phialina to sectional rank in $\mathrm{Hya}$ loscypha. This was criticized by Arendholz (1979), who accepted the genus for species with tapering hairs and a thick-walled excipulum. He correctly treated the type of excipulum characterizing some species of Phialina as a modification of textura prismatica. The term "textura prismatica incrassata" was introduced for a basically prismatic structure with thickened cell walls. One new species, $P$. plowrightii Arendh., was referred to the genus. The placement of Peziza lachnobrachya in Phialina, which had been proposed by Raitviir (1970a), was rejected due to differences in the excipulum.

The short history of Phialina is characterized by contradictory views of its delimitation. Few genera comprising only two species have been the subject of such controversy regarding their delimitation and validity. Unfortunately, new delimitation is still necessary and is given in the present study.

\section{Generic relationships}

Many genera have been reduced to partial or full synonymy with Hyaloscypha or are recent segregates. Some of the genera that have been connected with Hyaloscypha and Phialina are enumerated below (see also Historical survey).

\section{Allophylaria}

Allophylaria was established (Karsten 1869) with a diagnosis stating the apothecia to be glabrous. Sur- prisingly, the genus was later synonymized with $\mathrm{Hy}$ aloscypha by Boudier (1907) and this treatment was adopted by Grelet (1951). Boudier listed five species belonging to Hyaloscypha, apparently following Saccardo (1889), as they are listed in exactly the same order by him. The diagnoses by Saccardo and Karsten contain no indication that the species bear hairs. As they disagree with Boudier's (1907) diagnosis of $\mathrm{Hy}$ aloscypha in other respects too, the synonymy presented by Boudier remains a mystery. The genus Allophylaria was recently treated by Carpenter (1981) and found to be related to Crocicreas Fr. and Bisporella Sacc.

\section{Amicodisca}

Amicodisca Svr. is a recent, monotypic genus. The type species is Dasyscypha brdensis Velen. (Svrček 1987a). When establishing the genus Svrček wrote that it is closely related to Hyaloscypha. The distinguishing characters given are a coloured excipulum, fimbriate ascus pore and presence of yellow pigment. My experience of the genus rests on material identified and kindly loaned for study by Dr. Raitviir. These three collections originate from Siberia, but match well with Svrček's description.

Amicodisca differs from Hyaloscypha in fairly many respects. In two of the Siberian collections septate hairs prevail and these are common in the third collection also. The spores often have two or three septa. The olivaceous pigment is seen mainly inside and on the hairs, and to a lesser degree inside the paraphyses and spores. The excipulum is basally clearly brown. All the above characters are anomalous in Hyaloscypha. They may be considered to outweigh the similarity in hair shape and justify recognition at generic level.

\section{Betulina}

The genus was established by Velenovský (1947) for a single species, $B$. hirta Velen., differentiated from his concept of Hyaloscypha by stipitate apothecia. The species possessed pure white apothecia reaching $1.5 \mathrm{~mm}$ in diameter, narrow and up to $1 \mathrm{~mm}$ long, solid hairs and tiny, narrowly cylindrical spores. No type material exists, but the material in Velenovský's possession was probably Hyalopeziza ciliata Fuckel. Velenovsky described the same species under the names Hyaloscypha subtilis Velen. var. subtilis, $H$. mirabilis Velen. and Zoellneria acerum Velen. (Svrček 1985 and in litt.). From the original diagnosis it can be concluded that Betulina hirta was 
characterized by long cylindrical hairs $(2 \mu \mathrm{m}$ wide, $1000 \mu \mathrm{m}$ long). Such a species matched Velenovský's wide concept of Hyaloscypha and this explains his statement that Betulina is close to Hyaloscypha.

The genus was taken up again by Graddon (1974), who included in it his new phyllophilous species, $B$. fuscostipitata. Graddon stressed the stipitate habit but neglected the fact that Velenovský reported solid hairs and did not describe them as tapering. No generic name is available for Graddon's species. The hairs are tapering as in Hyaloscypha, but the pigmented excipulum, stipitate apothecia and the ecology prevent inclusion in that genus. The pigmentation of the in excipulum accords with Dematioscypha (Huhtinen 1987d). Graddon's species is closely linked with an unnamed, foliicolous specimen from Japan (CUP-JA 2695), which has rounded outer excipulum, but the inner layers close to textura oblita. With two such contradictory but connected taxa, establishment of a new genus should wait for further material.

\section{Calycellina}

In a recent paper Lowen and Dumont (1984) synonymized the other original species of Phialina, $P e$ ziza puberula Lasch, with the type of Calycellina. Peziza puberula was shown to have been inconsistently interpreted and misapplied. As is evident below, Phialina in its present circumscription differs from Calycellina in the hair characteristics; although related in its pigmentation, ecology and morphology, the latter genus has round-tipped and hyphoid hairs. This feature was not stressed by Lowen and Dumont (1984) or Baral and Krieglsteiner (1985), who accepted both hair types in Calycellina. The latter authors noted the yellow pigment present in the paraphyses and hairs of the constituent species. From the treatment by Lowen and Dumont it is not evident whether the yellow pigment is present in the numerous other species they accepted in Calycellina. The monotypic Scutoscypha Graddon was synonymized with the present genus by Baral and Krieglsteiner (1985).

\section{Chrysothallus}

Chrysothallus Velen. is one of the ill-defined genera established by Velenovský (1934). In later papers (Velenovský 1939, 1947) eight taxa were described, bringing the species number up to 16 . According to type studies, at least five of these belong to Hyaloscypha, one to Incrupila and one to Hamatocanthoscypha. The protologue stressed such partly illusory differences from Hyaloscypha as fleshy apothecia, presence of "hypothallus" and prosenchymatic tissue. The heterogeneous nature of the genus is unquestionable.

\section{Cistella}

The generic name Cistella was introduced by Quélet (1886) for nine species, the first in the list being $P e$ ziza dentata. The generic characters listed by Quélet were cupulate to scutellate apothecia with waxy and pruinose excipulum and an often elegantly crenulate margin. The spores were described as elongate-fusiform, the apothecial colours as "beautifully" hyaline. The species were stated to be lignicolous.

Nannfeldt (1932) pointed out the heterogeneity of the genus. He selected Cistella dentata (Pers.: Fr.) Quél. as the generic type calling it a "pseudotypus". As explained by Nannfeldt (1932: 8) himself, this term indicates the use of the "first species rule", which under the present Code is inadmissible (Art. 8.1). This typification was safeguarded, however, by acceptance of the proposal for conservation by Cash and Stevenson (1961). A year earlier, Clements and Shear (1931) had typified Hyaloscypha with the same species. As Hyaloscypha antedates Cistella it should be taken instead of Cistella, unless conservation is allowed. A conservation proposal, annulling the typification of Clements and Shear, was made by Huhtinen and Cannon (1987).

No material of Peziza dentata is preserved in Persoon's herbarium in Leiden. The illustration presented by Persoon (1798) shows a sessile, scutellate, lignicolous fungus with a clearly dentate margin. This, together with the short description, does not disagree with our present-day interpretation of Cistella. The taxon illustrated can either be a present-day Cistella or one of the few species which have hairs bearing resin and thus glued together to form "teeth", as seen in Protounguicularia barbata (Velen.) Huhtinen (Huhtinen 1987a). On the basis of a specimen sent from Quélet to Boudier (Herb. Boudier, in PC), we can conclude that their interpretation of Persoon's species matches Nannfeldt's (1932) circumscription of the genus. This concept has prevailed ever since, a major modification being the acceptance of herbicolous species as well (Matheis 1977, Raschle 1978). Nannfeldt's concept of Hyaloscypha has also been widely accepted since 1932 and we should not be upset by the dubious identity of Peziza dentata, but 
safeguard both generic names, as was proposed by Huhtinen and Cannon (1987).

The studied specimen of Cistella dentata sensu Quélet and Boudier belongs to the group of Cistellas bearing narrow, sharp and cyanophilous spines on the clavate hairs. The differences in hair vesture were pointed out by Matheis (1977), who established two subgenera. Subgenus Verrucotricha Math. is distinguishable from subgenus Cistella by hairs bearing cyanophilous spines. The other subgenus embraces species with hairs bearing nonstainable warts. To my mind this offers a partial solution for the delimitation of Cistella. The element that is clearly distinguishable from related genera is the subgenus Verrucotricha. At the generic level three segregates have been established. Discocistella Svr. was typified by Peziza fugiens Buckn. and at present comprises herbicolous species (Svrček 1962, 1987d). Clavidisculum Kirschst. was recently delimited as monotypic by Svrcek (1987d), embracing only Peziza acuum Alb. \& Schw. A study of the holotype specimen of Cistellina auricolor Raitv. revealed a hair vesture consisting of blunt acyanophilic spines. As stressed by Raitviir (1978), the excipulum is narrow-celled. Cistellina contains two species, the other being $C$. stereicola (Cooke) Raitv.

\section{Dematioscypha}

Dematioscypha was established by Svrček (1977c) as a monotypic genus to accomodate Peziza dematiicola Berk. \& Broome. According to Svrček, the main differences from Hyaloscypha were the dark-coloured excipulum, the occurrence amongst Haplographium Berk. \& Broome and the inamyloid asci. Recently (Huhtinen 1987d) I showed that the "P. dematiicola" of Svrček was in fact another, albeit congeneric species. The characters stressed by Svrcek appear to justify the separation of Dematioscypha. Furthermore, the genus possesses hairs that are either regularly or more rarely multiseptate. When combining $P$. dematiicola with Hyaloscypha, Nannfeldt (1936) mentioned the tapering hairs and the textura prismatica of the excipulum. On the other hand, he admitted that the brown excipulum is untypical of that genus. The other species now placed in Dematioscypha, Peziza richonis Boud., has also been combined with Hyaloscypha. The septa in the hairs were overlooked (Velenovský 1934 as Hyaloscypha olivacea, Dennis 1949). Dematioscypha has many characters that are either untypical of or absent from Hyaloscypha. Hence, Svrcek's generic division is justified.

\section{Eupezizella}

Starbäck (1895) divided Pezizella into two subgenera on the basis of the excipular structure and marginal hyphae. The subgenus Eupezizella Starb. (nom. inval., Art. 21.3) embraced species in which the whole excipulum consisted of textura prismatica and the margin bore specialized hairs. In the subgenus Ctenoscypha Starb., the excipulum had both textura intricata (at the base) and textura prismatica, and the margin was characterized by hyphal ends closely resembling the paraphyses. From his discussion (Starbäck 1895: 30), it is clear that Starbäck believed that the difference between these two types of margins was fundamental. In Eupezizella the margin was thought to be part of the excipulum, whereas in Ctenoscypha it was formed by the hymenium, i.e. by a thick layer of protruding paraphyses. Pezizella punctiformis (Grev.) Rehm was treated as an intermediate species. According to an authentic specimen in Stockholm, this is a species of Calycellina. Starbäck listed only species he had studied personally and this may be the main reason why he used Rehm's concept of Pezizella. In the original sense Pezizella contained only rarely collected species.

Eupezizella was accepted and published in generic rank by Höhnel (1926a: 61 and 79) with Pezizella candida Starb. as "Grundart". Nannfeldt (1932) listed Eupezizella as a synonym of Hyaloscypha, a solution which has been accepted ever since. Höhnel's use of the term "Grundart" was interpreted by Nannfeldt (1932: 8) as indicating explicit choice of the type. From Höhnel's own statements (1926: 54, 56), however, it is evident that the word "Grundart" was simply an indication that the species was the first mentioned in a list. Therefore, the typification of Eupezizella with Pezizella candida is not valid (Art. 8.1 ), but is validated here.

Starbäck did not stress hair shape in his delimitation of Eupezizella. This can be concluded from the material in his possession at the time that subg. Eupezizella was established and from the illustrations in his 1895 study. To him the fact that the hairs were clearly different in structure from the paraphyses was of great importance. The material contains species which were included in Hyaloscypha by Nannfeldt (1932), some of which are considered heterogeneous in the present study. But of the six taxa listed by Starbäck, four belong to Hyaloscypha as presently circumscribed. Pezizella hyalina (Pers.: Fr.) Rehm, P. granulosella (P. Karst.) Starb. and Pezizella atomaria Starb. all represent Hyaloscypha aureliella. This species is closely related to Pezizella candida. 
The blunt, aseptate, thin-walled, resinaceous hairs together with the lack of dextrinoid reactions delimit a group of species for which Eupezizella Höhnel is available as a valid name. Typified by Pezizella candida this group is recognized as the core of Hyaloscypha subgenus Eupezizella (Höhnel) Huhtinen.

\section{Fuscoscypha}

Fuscoscypha Svr. is a monotypic genus recently established for Lachnum aciculare Velen. (Svrček 1987b). A study of the holotype confirmed that there was no genus in the Hyaloscyphaceae to accommodate the species. The excipulum is composed of dark brown textura oblita. The narrowly conical hairs taper to a narrow apex and bear a lump of resin dissolving in heated lactic acid. Fuscoscypha may be related to Hamatocanthoscypha. The similarities in ecology, apothecia and hymenial characters are outweighed, however, by the dissimilar hairs and excipulum.

\section{Hyalopeziza}

The genus Hyalopeziza Fuckel belongs to the glassyhaired Hyaloscyphaceae. In recent treatments it has received various delimitations (Raschle 1977, Korf \& Kohn 1980, Huhtinen 1987c, Svrček 1987d), but the thick-walled hairs have remained the main character. In Hyaloscypha only one species shows marked similarity to species of Hyalopeziza. When typically developed, the hairs in H. leuconica (Cooke in Stevenson) Nannf. are firm-walled and in some populations they may have thin septa. The septa mostly occur in the basal parts, but they may occasionally occur above the middle level of the hairs. One such population was erroneously determined as Hyalopeziza sp. in an earlier study (Huhtinen 1984). Such septate hairs are a marginal character in $H$. leuconica. The range of variation also covers populations with thinwalled hairs. As most populations have aseptate hairs, affinity to Hyaloscypha is beyond doubt.

\section{Microscypha}

This genus was included in Hyaloscypha in the general key to discomycetes presented by Korf (1973), and the synonymy reappeared in a list of Eriksson and Hawksworth (1986). The type species, Microscypha grisella (Rehm) H. Sydow \& P. Sydow, is well known and widely distributed in Europe, being characterized by cylindrical and septate hairs and a brownish excipulum. The range of variation includes hairs that are slightly tapering and aseptate in their protruding part, but they are rare in the species. Rehm's type (Rehm, Ascom. 766) represents the same species beautifully illustrated by Boudier (1905-1910). Original material was studied by Sydow and Sydow (1919) when they established Microscypha, but they did not emphasize hair morphology in the diagnosis. They largely accepted the arguments of Höhnel (1909), stressing the textura prismatica structure of the excipulum.

The type specimen indicates, however, that the genus comprises small hyaloscyphaceous species on herbaceous litter characterized by cylindrical hairs and excipulum composed of brown to hyaline textura prismatica. This delimitation has been widely accepted (Nannfeldt 1932, Dennis 1949, 1971, Bøhler 1974, Raschle 1977). Svrček's concept is basically the same, but he has admitted a more rounded type of excipulum into the genus (Svrcek 1967, 1976, 1985). Microscypha cannot be closely related to $H y$ aloscypha, in view of the differences in the hairs, pigmentation, ecology and excipulum. The potential related genera are Hamatocanthoscypha and Psilocistella Svr.

\section{Phaeoscypha}

The genus was established for Hyaloscypha cladii Nag Raj \& Kendr. as the sole species (Kirk \& Spooner 1984). Phaeoscypha Spooner was stated to differ from Hyaloscypha in the brown and septate hairs, brown excipulum, eventually brownish spores and the association with Chalara (Corda) Rabenh. To my mind, this character combination justifies separation from Hyaloscypha. The genus is discussed further in section 5 .

\section{Phialoscypha}

The genus was described by Raitviir (1977a), with Peziza lachnobrachya Desm. as type species. It was considered to be closely related to Phialina on the basis of hymenial characters but to merit generic recognition as a small, natural, foliicolous genus. Characters differentiating it from Hyaloscypha were the long, filiform, solid hair apices. Three species were accepted in the genus, all of which are treated here under Phialina. The generic type of Phialoscypha is conspecific with that of the earlier Setoscypha, which would have offered an earlier name for the genus. 


\section{Psilocistella}

The species of Psilocistella are characterized by cylindrical, septate, straight and thin-walled hairs (Svrček 1977c, 1978, 1979, 1983, 1985). The genus is generally clearly delimited from Hamatocanthoscypha. However, the neotype of $P$. conincola (Velen.) Svr. is intermediate between the two genera. Its marginal hairs are straight and septate, whereas the hairs on the lower flanks are occasionally uncinate. In the excipulum and especially the cuneiform spores it matches well with Hamatocanthoscypha. In its ecology and morphology $P$. conincola is very close to the type variety of $H$. laricionis (Velen.) Svr. The straight and septate hairs at the margin, however, seem to indicate that Dasyscypha conincola Velen. belongs to Psilocistella. The rest of the species in the two genera are well differentiated from each other and delimitation is difficult in only this one species.

\section{Setoscypha}

The monotypic genus Setoscypha was taken up in a recent key to hyaloscyphaceous genera (Svrček 1987d). Velenovský (1934) stressed the close relationship to Hyaloscypha and spores with an "apical clavula". Other characters mentioned in the diagnosis were the basally dark brown parenchymatic excipulum and the lack of paraphyses. The generic name refers to the fact that the species bears up to $300 \mu \mathrm{m}$ long, septate hairs.

After studying the holotype, I can confirm synonymy with Peziza lachnobrachya (Svrček 1987d). The apothecia with well-developed hairs were not found, but smaller fruit bodies of the species were present. The "clavula" in the spores, stressed by Velenovský, is a frequent feature in spores of $P$. lachnobrachya that are situated at the ascus apex. They apparently become bent due to lack of space at the narrowing apex. In the present monograph Setoscypha is merged with Phialina.

\section{Truncicola}

The genus was established by Velenovský (1934) for a single species, $T$. parasitica Velen., differentiated from his concept of Hyaloscypha by furcate paraphyses and large apothecia. Later, Velenovský (1947) included another species in Truncicola and mentioned a possible connection with algae. Both species belong, however, to Hyaloscypha.

\section{Unguiculella}

The genus appeared in Höhnel's (1906b) revision of Feltgen's material. Under Pezizella hamulata Feltg. he concluded: "Hierdurch weicht der Pilz von Unguicularia ab und stellt vielleicht eine eigene Gattung dar, für die ich den Namen Unguiculella vorschlage". On the next page Höhnel writes (when treating $P$. aggregata Feltg.): "Der Pilz gehört mit Unguicularia falcipila v. H. (= Pezizella hamulata Feltg.), und Trichopeziza hamulata Sacc. in eine eigene Gattung, die ich Unguiculella nenne". Then follows the formal diagnosis of the genus and species are listed anew, $U$. hamulata (Feltg.) Höhnel being mentioned first.

As discussed below, typification of the genus is of major importance. The words of Höhnel have caused confusion in the same way as with Phialina. The protologue includes all the taxa treated by Höhnel on the two pages (cf. footnote to Art. 8.1). The words "... for which I propose the name Unguiculella" bear no major importance. Nannfeldt (1932) did not regard Pezizella hamulata as the type of Unguiculella. Using the term "Pseudotypus", he referred to the "first species rule". Consequently, his statement should not be taken as a lectotypification. Later, Müller (1967), Raitviir (1970a), Raschle (1977), and Korf and Kohn (1980) have taken the view that Pezizella hamulata is the holotype. None of these authors used the term "lectotype" for $P$. hamulata. But as such an error in type citation is not significant under the present Code, the earliest of these references, i.e. that of Müller (1967), is a valid lectotypification (Preamble 9). Müller referred to Pezizella hamulata as the type species, not to Unguicularia falcipila Höhnel, which was given as a synonym by Höhnel in the same study.

The isotype convolute of $P$. hamulata in Höhnel's herbarium ( $\mathrm{FH})$ is empty, except for a poorly preserved slide. This slide fails to elucidate many necessary morphological and histochemical details. In a sheet of annotations filed with the specimen, Korf writes that Feltgen's herbarium in Luxemburg may contain original collections by Feltgen. A search for more material should be made before the typification of Unguiculella is fixed. If no material exists in Feltgen 's herbarium, a new lectotype should be selected. Unfortunately, description of $P$. hamulata is vague and its identity with Unguicularia falcipila is not clear. Höhnel (1906b) noted a difference in the reaction of the ascal pores in Melzer's reagent and his observations on U. falcipila were confirmed by Korf (in herb., sheet of annotations). To some extent, Unguiculella parallels both Hyaloscypha and Unguiculariopsis Rehm. Its typification should thus be 
carefully considered in the light of the morphological and histochemical data on the commoner species (Nannfeldt 1936, Svrček 1978, Dennis 1950, Raitviir 1970a, Arendholz 1979, Korf \& Kohn 1980, Sharma et al. 1986, Huhtinen 1987c).

Species included in Unguiculella by the abovementioned authors do differ from the genus Hyaloscypha. Separating characters are, e.g., brown excipulum, with inner layers of textura intricata and outer layers of modified textura prismatica, hooked, hairlike paraphyses with firm walls, curved hairs with firm walls and in some species with dull or refractive contents, asci often with MLZ-pores, and herbicolous and foliicolous habit in many species. There is, however, only one character common to all species: the curved hairs. Besides the hairs and paraphyses, Höhnel's original diagnosis stressed the MLZ-ascal pores, the excipulum having a parallel hyphoid structure, at least towards the margin, and the rather clear parenchymatic medullary excipulum. From the description of Unguicularia falcipila (Höhnel 1906a) and that of connection to Mollisia hamulata Rehm (Höhnel 1918), it can be concluded that both solid and firm-walled hairs and paraphyses were accepted by Höhnel. He did not include species with thinwalled paraphyses. The later additions to Unguiculella (Nannfeldt 1936, Dennis 1950, 1955a, b, 1963, Müller 1967, Bøhler 1974, Raitviir 1977b, Svrček 1978) have widened the genus in this respect, but Höhnel's concept has largely been followed by these authors. Korf (1973), however, merged the genus with Hyaloscypha. Raschle (1977) pointed out that this synonymy is not correct for species which match Höhnel's generic diagnosis, mentioning $U$. rehmii $\mathrm{E}$. Müller. In the generic diagnosis, Höhnel used the term "Granne" in connection with hair and paraphysis apices. This term does not necessarily refer to solidified apices, as in U. rehmii. The original material in Höhnel's herbarium shows that the term also referred to paraphyses with protruding, hooked but not totally solidified apices.

Unguiculella was recognized as a subgenus of Hyalopeziza by Korf and Kohn (1980). Whatever its status, Unguiculella deserves recognition on the basis of two features, protruding paraphyses with hooked and/or glassy apices and the brown excipulum seen in many species. In a recent monograph Zhuang (1988) recognized the independent status of Unguiculariopsis. Differences from Unguiculella were found in excipular structure, excipular cell colour, hair colour, and shape of the paraphyses.

\section{Delimitation of the monographed genera}

Some of the species recognized here under Phialina or Hamatocanthoscypha have earlier been placed in Hyaloscypha. However, the three genera are clearly delimited from each other. The genus Phialina has many features that are rare or lacking in the other two. The distinction between Hyaloscypha and $\mathrm{Ha}$ matocanthoscypha is clear in the great majority of the species. In only two species of Hamatocanthoscypha is the difference less marked.

A vacuolar yellow pigment inside the hairs and paraphyses is diagnostic of the species of Phialina. The pigment is absent from Hyaloscypha and Hamatocanthoscypha, though a yellow pigment, apparently of different chemical composition, was seen in two species of Hamatocanthoscypha. Many species of Hyaloscypha have a yellowish resin, which does not occur in the other two genera. Brown pigmentation of the excipular walls is found only in some species of Hamatocanthoscypha. The pigments and exudates are discussed more thoroughly under "Pigments, resinous exudates and histochemistry".

The hair characters in the three genera show only partial overlap. In Phialina the basic shape is narrowly conical and the hairs taper to a narrow apex (Figs. 5, 6). In most of the species the apex is strongly curved, flexuose or cincinnate. Branched apices occur in two species. Whenever elongated, the hairs tend to be septate. In Hyaloscypha the hair shape is basically the same, but fairly wide variation is seen (Figs. 1, 2). Straight hairs prevail and septation is rare. The majority of the species exhibit a dextrinoid reaction, which is lacking in the other two genera. A further chemical difference is the clearly amyloid inclusions in the hairs of some Hyaloscypha species. Hamatocanthoscypha is characterized by short, blunt and uncinate hairs (Figs. 7, 8). Modifications of this basic type are rare.

The excipular structure is partly similar, but contributes to some extent to differentiate the genera. The prismatic structure characteristic of Hyaloscypha is modified in Hamatocanthoscypha. The cells on the middle flanks are clearly elongated, except in $H$. uncinata. In that species the excipular cells are almost isodiametric, a shape characteristic of Phialina. Differences in the middle flank cells are reflected in the mean Q values ( $H$. uncinata excluded from the calculations): 1.8 for Phialina, 2.2 for Hyaloscypha and 2.7 for Hamatocanthoscypha. The dextrinoid reaction in many species of Hyaloscypha is another major 
distinction. The excipular wall thickness is not a character of generic value.

The typical spores are different in each genera. In Hamatocanthoscypha small, often cuneate, spores prevail. The other extreme is seen in Phialina, which tends to have long and fusoid or voluminous spores. Hyaloscypha occupies an intermediate position with typically elliptic or oblong-elliptic spores (Fig. 13). A corresponding difference is not present in ascus size
(Fig. 72). Asci arising from simple septa are characteristic of many species of Hyaloscypha: in all 15 taxa out of 27 show this type of ascal development. Simple-septate asci are absent from Phialina and found in only one variety in Hamatocanthoscypha. Four-spored asci are seen only in Phialina. Notably broad paraphyses are typical of Phialina, while the other two genera differ less markedly from each other in paraphysis width. In Hamatocanthoscypha the ter-
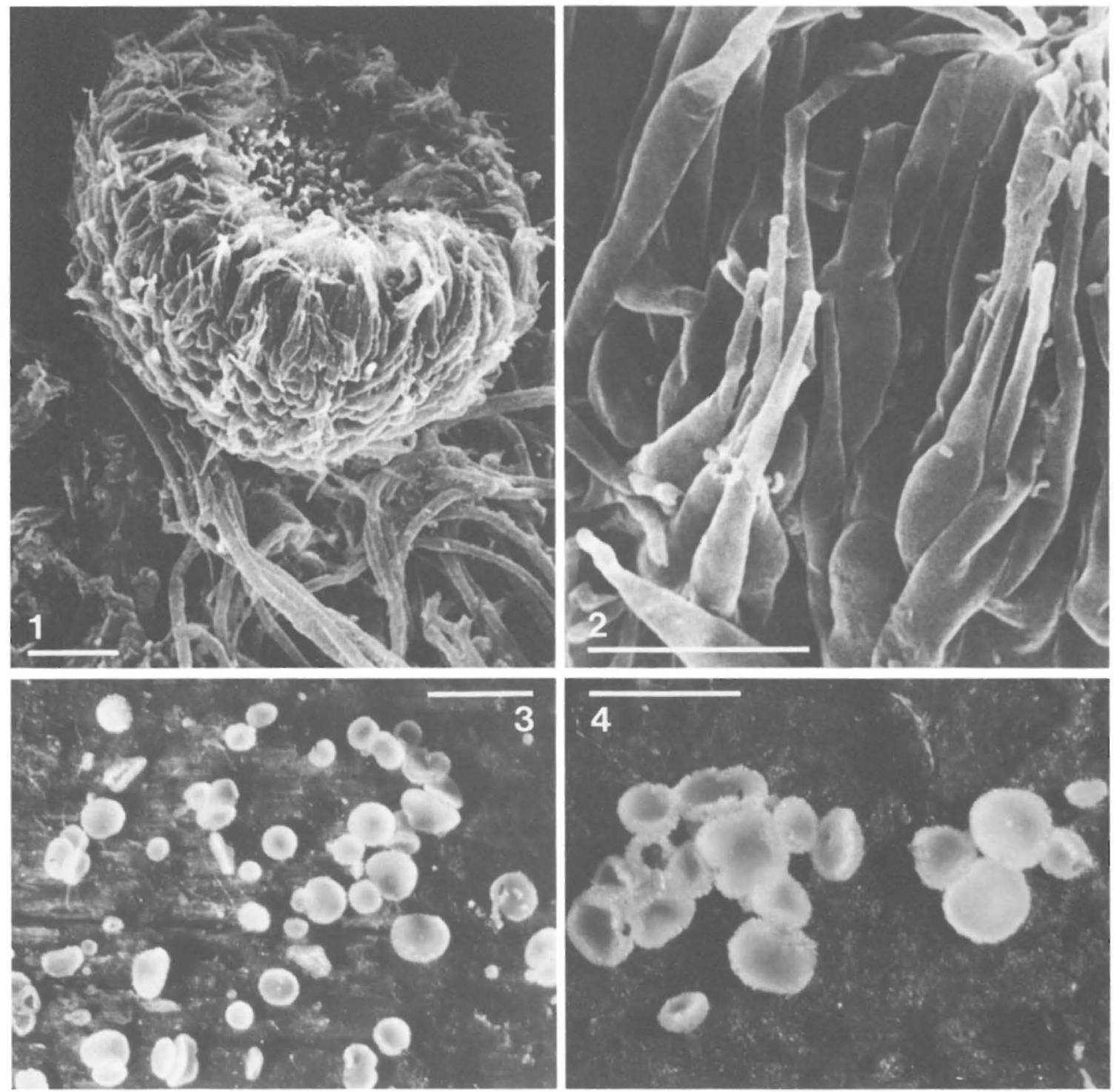

Figs. 1-4. Hyaloscypha. - 1: An apothecium of H. fuckelii var. alniseda (SH 88/103), scale $10 \mu \mathrm{m}$. - 2: Hairs close to the margin, scale $10 \mu \mathrm{m}$. - 3: Apothecia of $H$. aureliella (SH 88/108), scale $500 \mu \mathrm{m}$. - 4: Apothecia of $H$. aureliella (SH 87/206), scale $500 \mu \mathrm{m}$. - Photos: 1, 2, Kaarina Pihakaski; 3, 4, S. Huhtinen. 
minal cells are often clearly longer than in Hyaloscypha and the first septum often occurs at or below the level of the ascal bases.

The apothecia in Hamatocanthoscypha are clearly stipitate, while the other genera comprise mostly species with sessile apothecia. A convex hymenium with margins appressed to the substrate and with a spongy texture is characteristic of some species of Phialina. In other respects the appearance of the apothecia is fairly similar in all three genera (Figs. 1, 3, $4,6,7)$.
The genera differ in their ecology. Most species of Hyaloscypha occur on bulky wood substrates. Occurrence on leaves is most unusual and species fruiting on forest litter or herbaceous stems are clearly outnumbered by the lignicolous ones. The species of Hamatocanthoscypha are genuine litter inhabitants, mostly confined to forest debris. Occurrence on bulky wood substrates is rare. Most Phialina species are confined to leaves, only two species growing on ferns and Filipendula. Occurrence on forest litter is exceptional in Phialina.
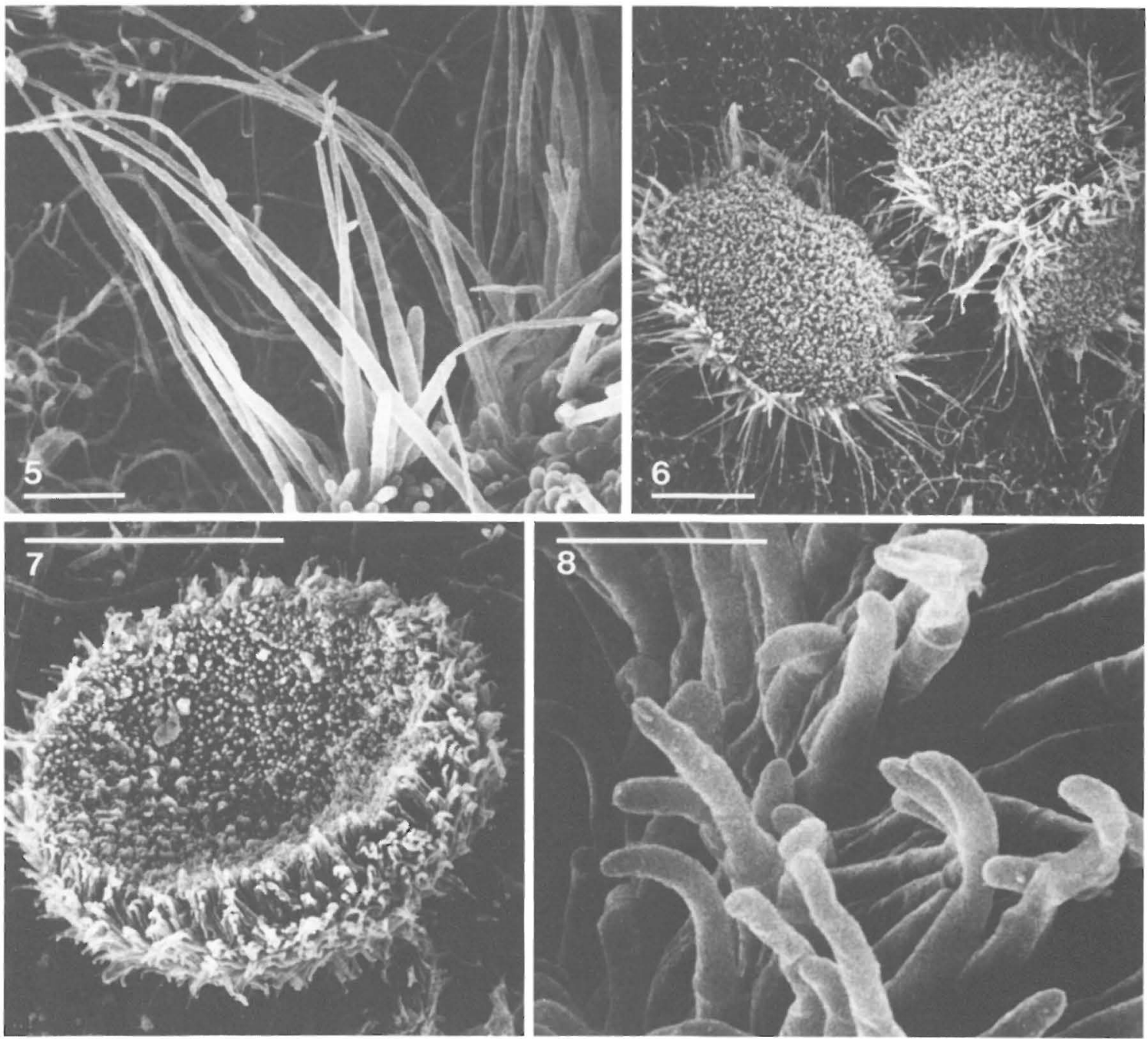

Figs. 5-8. Phialina and Hamatocanthoscypha. - 5: Marginal hairs of $P$. setiigera (SH 87/154), scale $10 \mu \mathrm{m}$. - 6: Cultured apothecia, scale $50 \mu \mathrm{m}$. - 7: An apothecium of Hamatocanthoscypha laricionis var. laricionis (SH 88/102), scale $50 \mu \mathrm{m}$. - 8: Hairs close to the margin, scale $10 \mu \mathrm{m}$. - Photos: Kaarina Pihakaski. 


\section{Morphology}

\section{Apothecia}

Most species of Hyaloscypha and Phialina have sessile to subsessile apothecia with a narrowing base. A short stipe is found in a few species only, and is often hidden when the apothecia expand upon maturity. The only clearly stipitate species are $H$. diabolica Huhtinen and $H$. epiporia Huhtinen. A distinct, somewhat slender stipe is typical of Hamatocanthoscypha. All the genera have cupulate apothecia, but in the juvenile stages the shape is occasionally turbinate or clavate. In foliicolous Phialina scutelloid apothecia and seemingly pulvinate apothecia are also common. These pulvinate apothecia are, however, centrally sessile and basically cupulate. With age, the margins bend down to the substrate, concealing the receptacle, so that the hymenium becomes convex.

The apothecia are roundish in all genera. Apothecia with crenulate or undulate margins are occasionally found in Hyaloscypha aureliella, $H$. daedaleae Velen. and Hamatocanthoscypha laricionis. The general appearance of the margin depends on the amount and length of marginal hairs. When the hairs are scarce the apothecia may be indistinguishable from those of hairless genera, as occasionally happens with Hyaloscypha vitreola in the field. The paraphyses may form a narrow inner fringe on the margin, but in general the margin is composed of hairs and hair-like hyphal ends. The excipulum shows no tendency to grow above the level of the hymenium. Rarely, irregular "teeth" may occur when abundant exudate glues the hairs together.

The disc is concave to plane, or in a few species rarely convex. Under the dissecting microscope the disc is seen to be homogeneous in texture in both Hyaloscypha and Hamatocanthoscypha. In foliicolous Phialina, however, it is often pronouncedly spongy. In herbarium material the discs are often hidden under the strongly incurved margin.

When dried, the apothecia may have darker colours than fresh material. Although there are no special pigments, the colour change is a relatively stable character in some species. For instance in the type variety of Hyaloscypha fuckelii Nannf. the dry apothecia of numerous populations are reddish brown. The same colour also characterizes $H$. herbarum Velen., but is rare or lacking in most other species of Hyaloscypha and the other two genera. Occasional variation in some populations diminishes the taxonomic value of such colour changes. A light, yellowish brown tone on the flanks is typical of dried apothecia of white species. In dried condition the flanks are never snow-white, but may appear to be so, due to a dense cover of hyaline hairs. When fresh apothecia of "white" species are placed on white paper they are mostly seen to be more grey in colour, due to water in the tissues. In many species fresh apothecia have greyish discs. This is not caused by pigmentation, but by the dark substrate apparent through the translucent, light tissue. Only Phialina and Hamatocanthoscypha have conspicuous, true pigments. The nature and taxonomic value of pigmentation are discussed below.

Dark colours may also be due to resinous exudates. In $H$. aureliella the apothecia are frequently brownish to reddish brown when dried. This is caused by the abundant resin characterizing over $90 \%$ of the collections. In fresh material the resin is much lighter and distributed over a wider surface and the resulting yellowish colour is less conspicuous. This colouring by resinous exudates is beautifully illustrated in a photograph by Breitenbach and Kränzlin (1981, as Hyaloscypha leuconica).

Crystals are exuded to the outer surface of apothecia in abundance only in Hamatocanthoscypha obsoleta Huhtinen and Hyaloscypha latispora Huhtinen. Both species are known from a single collection only and the stability of these crystalline exudates cannot be judged. If constant, they will be good taxonomic characters, absent from other species of the genera.

\section{Excipulum}

A clearly differentiated medullary excipulum is lacking in all the three genera (Figs. 9-11). Tissue resembling textura intricata is restricted to the hypothecium, i.e. to the ascogenous tissue. The outermost layers of excipulum are composed of textura prismatica. The basic type of textura prismatica, characterizing Hyaloscypha, is modified in the other two genera, the cells being more isodiametric in Phialina and more elongated in Hamatocanthoscypha. The basic prismatic shape may be modified in Hyaloscypha as well. In some populations of $H$. aureliella, the cells are more rounded in surface view. In the type specimen of Hyaloscypha cerberi Velen. also, the outermost cells are notably rounded, and their appearance was apparently responsible for placement of the species in Dennisiodiscus Svr. (Svrček 1978). These cells serve as basal hair cells, however, which are fairly often widened in Hyaloscypha. Below this layer typical textura prismatica prevails. In those species with sessile to subsessile apothecia, the excipulum grades towards the base into textura angularis or textura globulosa-angularis. In stipitate apothecia the excip- 

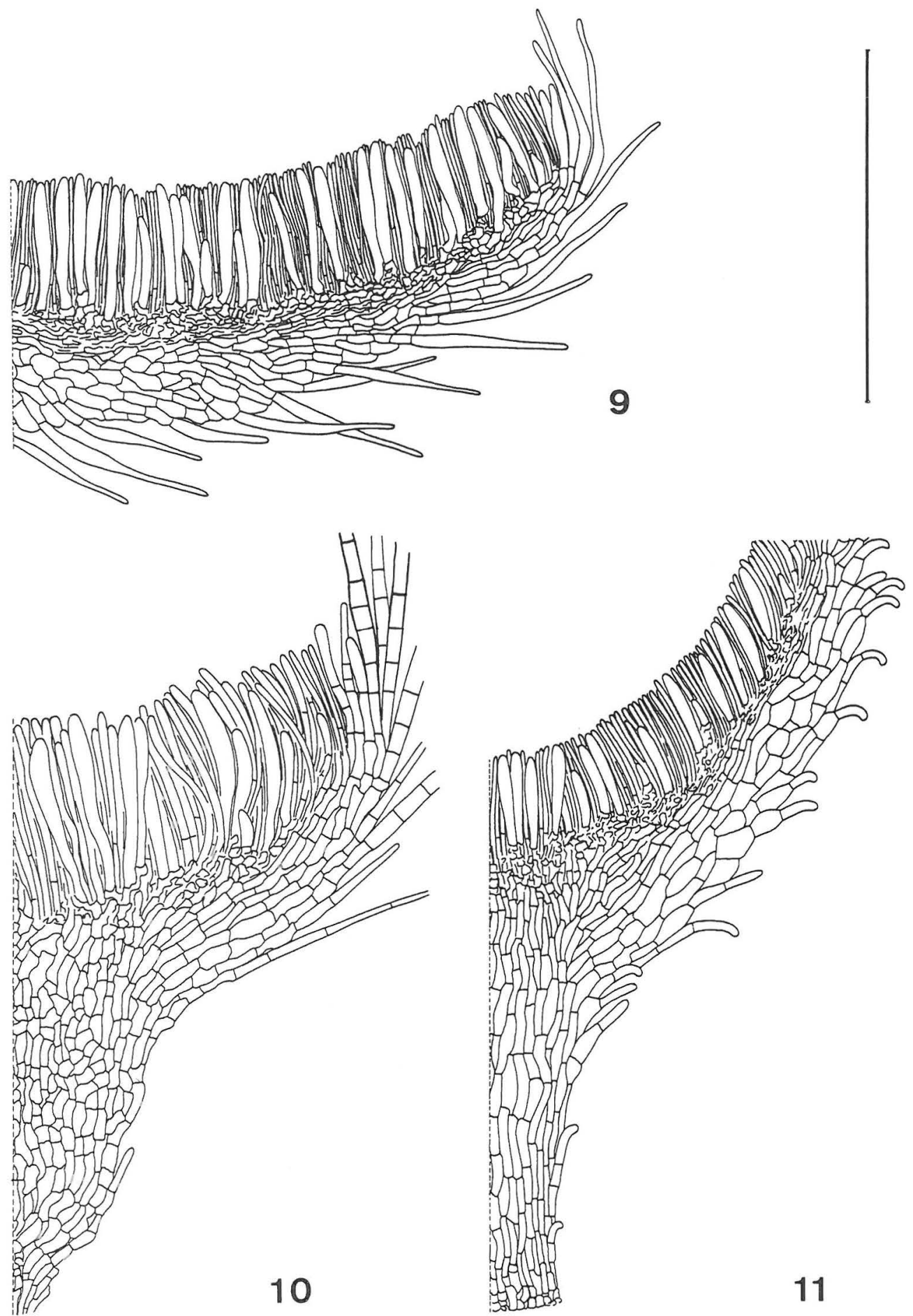

Figs. 9-11. Morphology of apothecia in Hyaloscypha, Phialina and Hamatocanthoscypha. - 9: Hyaloscypha aureliella (SH 88/ 105). - 10: P. setiigera (SH 87/154). - 11: Hamatocanthoscypha laricionis var. laricionis (SH 88/102). - Scale $100 \mu \mathrm{m}$. 
ulum cells may become narrowly prismatic on the stipe. Towards the margin, the prismatic shape prevailing on the middle flanks may change. In Hyaloscypha daedaleae, for instance, the excipulum cells close to the margin are almost hyphoid. The cells are often similar in shape to but smaller than the middle flank cells. This change in shape is of limited taxonomic value. The inner layers are usually composed of narrower textura prismatica, the change being gradual. In rare cases the narrowed cells form textura porrecta; this tissue typically forms an indistinct transitional layer adjacent to the hymenium. There is no covering layer on the excipulum.

The basic excipular tissue is textura prismatica with thin to moderately thickened cell walls. The cell shape is relatively constant, but in fairly many taxa both size and wall thickness may vary widely between the populations. Such variation is only rarely seen within a single population and possibly reflects differences in microhabitats. Relatively little attention has been paid to the variation in excipular wall thickness. More often the discussion has centred on the shape of the excipular cells and its taxonomic value at species and family level (e.g., Nannfeldt 1932, Raschle 1977).

In all three genera, the range of variation in wall thickness between populations of the same species was $0.5-0.8 \mu \mathrm{m}$. Variation exceeding $1 \mu \mathrm{m}$ is rare, but occasionally seen between the populations of $H$. aureliella and in some species of Phialina. Far-reaching conclusions from scanty material are taxonomically unsound and the excipular cell wall thickness may be assumed to show marked variation. In $P . u l$ mariae the variation is extreme, resulting in excipula of widely differing appearance: in some populations the cell walls are $2.5 \mu \mathrm{m}$ thick, in others only $0.3-$ $0.4 \mu \mathrm{m}$. In the majority of the populations the tissue is textura prismatica, in the others it has been modified, often becoming "textura prismatica incrassata", i.e. having the upper and side cell walls equally thickened (Fig. 258). More rarely, material has accumulated on the side walls only, giving the appearance of textura oblita (Fig. 261). In the light of the variation between populations, however, this tissue has probably originated from prismatic cells. I have touched on this phenomenon in a study of a species of Arachnopeziza Fuckel (Huhtinen 1987d). In classifying these two basically different types of excipular tissue, one can rely to some extent on the typically much more hyphoid nature of textura oblita.

The excipular cell size often varies considerably between populations of a given species. Two-fold differences are not exceptional. Nevertheless, excipular cell size has taxonomic value at the species level. Many variable taxa have relatively stable excipular tissue. The cells lie parallel to the surface of the receptacle and form more or less regular, parallel chains of prismatic cells. In $H$. aureliella the cells were noted to surface at a slight angle to the flanks (Fig. 9). Pronounced intercellular spaces are lacking in all taxa. Any space between the adjacent cell walls is visible only after strong staining in cotton blue. The rare intercellulary matrix is best seen in largecelled excipula. This was reported earlier by me for Calyptellopsis Svr. (as Hyaloscypha reticulata Vacek) and Dasyscyphus capitata (Peck) Le Gal (Huhtinen 1987c). The chemical reactions are discussed in detail in another section below.

\section{Hairs \\ Gross morphology}

Among the relatively few characters available for generic delimitation in the Hyaloscyphaceae, hairs are of major importance. In many cases they undoubtedly delimit natural entities, as is seen in, e.g., Dasyscyphella Tranzschel (cf. Raitviir 1977c, 1986) or Mollisina. The key taxonomic position of hairs at the generic level has not been seriously challenged. In this and earlier (Raschle 1977) studies, they have been stable in cultures. In the Hyaloscyphaceae the basis for generic delimitation is thus more rewarding than in the Leotiaceae, where the sterile portions of the ascocarps have received little attention (cf. Korf \& Dumont 1968) or are considered more variable than hymenial characters (Carpenter 1981). Hairs are defined here as morphologically and/or histochemically differentiated cells arising from the excipulum. The boundary between cells of the excipulum and hairs is not always clear cut, especially in the case of marginal hairs with narrow bases. Such hairs often have a few narrow basal cells which can be considered to be part of the excipulum or the basal part of a basally septate hair (Fig. 226). In such cases, a hair was measured, and its possible septation evaluated, on the part clearly protruding from the excipulum. When the margin was composed of a very dense layer of hairs, the boundary of the excipulum was taken as the level at which the cells started to be loosely arranged. This may not be a natural definition, but it is more exact than those so far in use. In the diagnoses presented by modern authors, the word "septate" unfortunately has two meanings: either the hairs have septa in the protruding part or they have a septate base (part of excipulum) and the protruding part is aseptate (such hairs are considered aseptate in the present study). 
Considering the taxonomic importance attached to hair morphology, we know surprisingly little about their functions and variation. A function of which we have direct proof is excretion and this has been noted by Raitviir (1986). The end products of this excretory process are seen in the resinous exudates, granulous and spiny surfaces, crystalline caps, chemically distinct saps and solidifying substances. It is noteworthy that these products are particularly characteristic of the hairs. If they were connected with the substrate, the hair exudates should vary with the chemical environment, for example, between foliicolous and lignicolous species of the same genus. In general, this is not the case; the many species of Lachnum, growing on a wide variety of substrates, produce similar granules on the hairs. Moreover, the glassy-haired species produce solidified hairs on such artificial substrate as malt extract (Raschle 1977). It is more likely that hair exudates in general are the by-products of certain chemical processes of the fungus, which are often characteristic of a certain genus. Variation arises at subgeneric level, where substrate specific exudates may occur, as seen, for instance, in the case of Protounguicularia barbata (Velen.) Huhtinen forma resinacea (Dennis) Huhtinen on Sorbus. The suggested excretory nature of hairs does not exclude the possible protective function of the hair cover against desiccation or herbivores.

The nature and causes of variation in hair morphology have not been thoroughly treated in the Hyaloscyphaceae. However, Raschle (1977) made an interesting cultural study. Although his material was not large, the results were intriguing. Raschle obtained apothecia of four glassy-haired species in culture and noted that while the dimensions of the hairs were variable the shape and chemistry were constant. My observations support these results as regards the gross morphology of the hairs. The same features which were noted to vary between natural populations showed variation in culture. The features examined were the amount of warts and apical solidifications and the dextrinoid reaction. In the species in which warts and solidifications occur, their abundance may partly be governed by the microhabitat. Variability of hair length was observed not only in herbarium specimens, but also in culture. The ontogenic variation in hair cover is meagre.

Hair septation has long been recognized as an important generic character in the Hyaloscyphaceae. It should be noted, however, that any genus with mainly septate hairs may include short-haired species in which septa are lacking. In my opinion, presence vs. absence of septa should always be judged in re- lation to hair length. In long hairs, septa possibly serve to strengthen the structure, being unnecessary in short hairs. Another possible explanation of their occurrence lies in the partial regularity of septal intervals generally seen in hyphoid structures. Hairs shorter than the "typical" septal intervals remain aseptate.

Both Phialina and Hamatocanthoscypha contain species with septate hairs. In the former genus, the tendency for longer hairs to be multiseptate in their apical parts is evident. But in such species as $P$. foliicola (Graddon) Huhtinen the dwarfish hairs are aseptate. In Hamatocanthoscypha the hairs tend to be septate in their basal parts. Hyaloscypha has been delimited both to include and to exclude septate hairs. The matter is not of fundamental importance, but is historically interesting as, Boudier (1907) in his emended and disputable diagnoses included hair septation in the genus. In his "Icones Mycologicae" (Boudier 1905-1910) he depicted the hairs of $H$. vitreola as being septate in their apical part as well, but in the description (Boudier 1911) they are said to be basally septate. My study of the holotype of Urceolella richonis Boud. showed that although his illustrations are of high quality, not all the taxonomic characters are correctly depicted. In the case of $U$. richonis, this is seen in the omitted septa and the exaggerated tapering of the hairs (cf. Huhtinen 1987d). Hence, we should interpret Boudier's "septate" with caution.

Septate hairs are not, however, anomalous in the genus, occurring in some populations of $H$. fuckelii, $H$. leuconica, $H$. vitreola and $H$. aureliella. But the septate hairs always form a small minority in an apothecium. In $H$. fuckelii, hair septation occurs more frequently than in any other species of the genus, whereas in, e.g., $H$. daedaleae septa are almost totally lacking. Consequently, hair septation serves as an additional character at the species level. The hair walls are thin in a majority of species in all the three genera. These walls are not refractive in cotton blue mounts. When the hairs are different from this prevailing type, the walls are seen as refractive in $\mathrm{CB}$ mounts or may have a stronger dextrinoid reaction. Four types of thickened walls are seen: 1 . The hairs may have a markedly thickened wall for most of their length, as in Hyaloscypha leuconica (Fig. 125). 2. Only the basal parts and apex may be thin-walled. Otherwise, thin-walled hairs may have local massive thickenings which usually occur at the constriction of lageniform hairs, but this is a relatively rare character in Hyaloscypha and is lacking in the other two genera. 3. The hairs may be thickened at the apex only, as seen in many species of Hyaloscypha. 4. The 
thickening is caused by a concentration of brown pigment, as is seen in the type variety of Hamatocanthoscypha uncinata (Phill.) Huhtinen. Wall thickness varies in importance from group to group. In some species, such as $H$.leuconica, thickened walls prevail, such hairs being intermixed with totally thinwalled hairs only in scattered populations. In $H$. vitreola both hair types are common. In this case the refractive walls offer a rapid means of recognition in some populations, but this does not warrant separation from the thin-walled populations at varietal or species level. In a few thin-walled species of Hyaloscypha untypical populations with slightly thickened walls occur. Such populations are rare and are here treated as firm-walled variants. In foliicolous Phiali$n a$, thickened hair walls characterize only one species and occur rarely in another. The accumulating material seems to be chemically identical to that of the wall proper. This can be concluded, at least in Hyaloscypha, from the overall dextrinoid reaction of the hair walls. To some extent, the overall thickenings stand out as being different from the apical solidifications, which may often be clearly dextrinoid in otherwise indextrinoid hairs.

The tendency to accumulate wall material at a basal constriction is seen in only a few populations and species of Hyaloscypha (Fig. 126). It has limited taxonomic value at the species or varietal level and is seldom necessary for determinations. The solidifications in hair apices also show marked variation between the populations. Solidified apices were included in the generic diagnoses by Velenovský (1934) and Raschle (1977). Although variable, the solid apices are diagnostic at the species level. They are concentrated in Hyaloscypha, being a marginal character in the other two genera. Apical solidifications and hair pigmentation are discussed further below.

Hair shape is fairly constant in a given species. The range of variation of hair length and width depends on the species. For example, $H$. fuckelii shows a wide range of variation in both features. At the other end of the scale is $H$. daedaleae, with minimal variation. Hair length has, quite correctly, received little taxonomic attention. Dennis (1949) recognized Peziza araneocincta Phill. as a variety of Hyaloscypha lachnobrachya, writing that it was "... probably no more than a long-haired variant of it." Owing to the observed gradation of hair length, this variety has been rejected in the present study. The basal width of hairs varies within narrower limits, but reaches the percentage variation of hair length.

The width of hair apices is a more reliable taxonomic character. For instance, blunt hairs characterize most species of subg. Eupezizella, whereas most species of subg. Hyaloscypha have hairs that taper to an acute point. The presence of apical knobs is of taxonomic value, although varying between the populations. $H$. fuckelii, in particular, is characterized by hairs with small, knob-like apices. Such hairs were depicted earlier by Le Gal (1939). The tendency to form such hair apices is seen in a few other species of Hyaloscypha. In Phialina, the hairs are drawn to an often long, filiform, flexuose apex. In Hamatocanthoscypha also, the blunt and uncinate hair apices serve as characters for generic delimitation. Cincinnate apices assist in species delimitation, but may vary between populations. Hyaloscypha cincinnata Sherwood is here synonymized with $H$. leuconica, whereas in H. epiporia such hair apices coincide with other morphological characters. Branched hair apices are seen in two species of Phialina.

\section{Hair vesture}

The different types of hair vesture have largely been neglected as taxonomic characters in Hyaloscypha and its closest allies. This is surprising, since the relatively prominent warts in, e.g., Hyaloscypha albohyalina (P. Karst.) Boud. can hardly have passed unnoticed. Warts play an important role in species delimitation in Hyaloscypha, but are less significant in the other two genera. For the recognition of $\mathrm{Hya}$ loscypha species, the presence of warts on hairs is taxonomically more important than their absence. In many species with warted hairs some populations may have totally smooth hairs. But if populations with prominently warted hairs have never been encountered in a given species, as in $H$. vitreola and $H$. leuconica, the absence of warts is a valid taxonomic character.

In the Hyaloscyphaceae, the two most common types of hair topography are: crystalline and more or less resinous. Crystalline warts are either firmly attached to the hairs, as in Lachnum, or occur loosely, as in Incrupila. They are persistent in most mountants. Furthermore, they often differ in their optical qualities from the wall proper and are thus visible as an overall vesture. Roughness caused by resinous exudates is most often seen as amorphous, not crystalline, bodies loosely covering the hairs. Such hair vesture is often easily washed off by ordinary reagents, e.g. in Albotricha Raitv. (Huhtinen 1985a).

In Hyaloscypha hairs may bear small warts throughout their length or only on the apical part. These are firmly fixed to the wall, but are often damaged by ordinary mountants, becoming deformed or totally dissolved. They are exuded on the wall and 


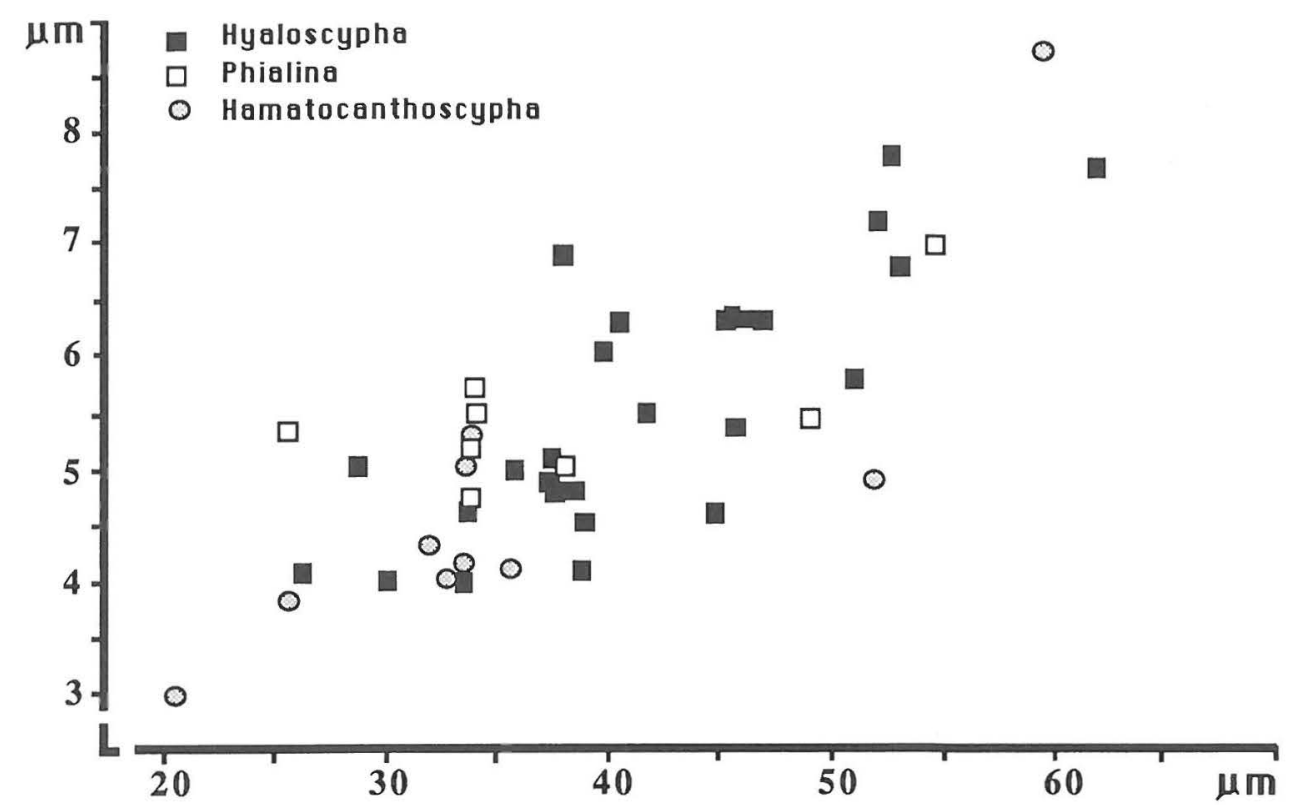

Fig. 12. Mean ascus length and width (in $\mathrm{CB}$ ) in the species and varieties of three hyaloscyphaceous genera.

are probably related to other resinous exudates. When the warts are deformed but not completely dissolved, hyaline globules are seen on the hair surface (Fig. 52). The warts in Hyaloscypha are not clearly different from the wall proper in their optical qualities. Hence, they are observed merely in optical section, where their arrangement often suggests a banded structure, rather than the presence of scattered warts. The tendency to deform in some species excludes their origin as protuberances of the wall proper, which remains intact in all reagents. The presence of "warts" of such a type was not observed in any of the genera studied.

In both $H$. fuckelii and $H$. daedaleae the hairs may be seen to bear prominent, loose encrustations when living material is studied in water mounts (Figs. 95, 102). These encrustations are lost with other ordinary reagents and never seen in dry herbarium material. Apparently, this type of roughness is caused by an excretory process. As it occurs in two species only, it assists determination when fresh material is available.

The occurrence of warts shows wide variation. In $H$. albohyalina, the whole range from populations with prominently warted to populations with perfectly smooth hairs is seen. In the apothecia of intermediate populations, warted and smooth hairs are equally common. In H. quercicola (Velen.) Huhtinen and $H$. herbarum also, variation is seen between populations and within a single apothecium. The ratio of smooth to warted hairs is often highly variable. Such differences can also result from the methodology; hairs seen as totally smooth in cotton blue mounts may bear scattered warts in Melzer's reagent, the reason being that when a cotton blue mount is heated, the warts are more liable to be destroyed. When persistent in reagents, the warts are alike in fresh and dried material. The resinous warts of the Hyaloscyphaceae are rather unusual. In addition to Hyaloscypha, they occur in some species of Phialina. In Phaeoscypha they were a marginal character. The warts in Hamatocanthoscypha are mostly pigmented, rarely hyaline. The hyaline warts, at least, are related to those of Hyaloscypha. I could not make out whether the brown warts originated from conglomerated pigment or a resinous exudate.

\section{Asci \\ Gross morphology}

The asci are eight-spored in all species of Hyaloscypha and Hamatocanthoscypha. Four-spored asci are found in only three species of Phialina. The asci are cylindrical-clavate and their shape varies little be- 
tween the species; the differences lie mainly in the size of the asci. The apex is somewhat conical in all but one species in Hyaloscypha.

The thickness of the apical wall in herbarium material has limited taxonomic value, as it is linked with two more useful characters. In larger asci and asci with inamyloid pores, the apical wall tends to be thicker than in smaller or amyloid asci. When fresh material is studied in water, the apical wall in mature asci is thin. The dramatic change taking place in apical wall thickness when the asci are killed was discussed by Baral (1987a, b). As the ascal apices were not studied with the precision outlined by Baral (1987a), closer scrutiny may result in further taxonomic characters.

The size of asci is a traditional morphological criterion. The length and width show a positive correlation (Fig. 12), greatest deviations being seen in, for example, Hyaloscypha latispora and Hamatocanthoscypha melanobasis (Arendh. \& R. Sharma) Huhtinen. In all the studied genera, ascus length varied more than width. An example of the average variation in Hyaloscypha is given in Table 1. Pronounced differences in ascus length were frequent even within a single apothecium. A similar trend is evident in the spore measurements (Table 4). Greater stability of ascus width was also reported by Hein (1976) for some dermateaceous genera. Raschle (1978) reported notable variation in ascus length in Cistella grevillei (Berk.) Raschle. An explanation of this, probably widespread, phenomenon is offered by the arrangement of the hymenium. Any element increasing in volume meets greater pressure in the horizontal direction, due to the densely packed asci and paraphyses. Hence, the more voluminous elements tend to grow in a vertical direction.

The dimensions and shape of asci were found to depend on the reagent. From Table 2 it is evident that the shrinkage in width taking place when a living ascus is treated with common reagents is ca. $15 \%$. The osmotic pressure inside the asci has a greater impact on the side walls of asci than in a vertical direction. Hence, when the pressure ceases the shrinkage in width is more pronounced than that in length. The shape of asci thus differs between living and killed material. The magnitude of this difference is indicated in Table 2. A difference in shrinkage of ca. 9\% was also noted between cotton blue and Melzer's reagent. Osmotic effects have seldom been stressed in mycological taxonomy. Scattered observations have been made by Luard (1983), Döbbeler (1984), Huhtinen (1985a) and Baral (1987a), and a detailed study is in preparation (Baral, pers. comm.). The reaction of the ascal pore in Melzer's reagent has traditionally re- ceived much attention. In the past it was reported only as either "I-" or "I+". In the papers by Kohn and Korf (1975) and Nannfeldt (1976), KOH pretreatment was introduced. Hein (1976) commented on the taxonomic value of the intensity of a positive reaction. More recently, Baral (1987a, b) published many interesting and important details concerning the chemical reactions and asci.

The intensity of the staining reaction has some diagnostic value. In all but one species of Phialina, the apical pore stains intensely. In $H$. aureliella and its closest relatives, the reaction was also intense, the resulting colour being deep purplish. Most species showed variation between the populations, the proportions for faint and intense reactions being different. The variation within a population was slight. The ontogenic variation was not, however, thoroughly scanned. These differences are masked by $\mathrm{KOH}$ pretreatment.

In Hyaloscypha usitata Huhtinen and H. nipponi$\mathrm{ca}$ Huhtinen the ascal pores are stained only after $\mathrm{KOH}$ pretreatment. The third known collection of the latter species from North America shows, however, the variable nature of this feature. Similar difficulties in weighing the character were met in Hamatocanthoscypha uncipila (Le Gal) Huhtinen. The feature inamyloid versus amyloid ascal pores contrasted clearly with the overall homogeneity of all other morphological characters. As an interim solution, these variants were not accorded any formal status.

In four taxa of Hyaloscypha and two of Hamatocanthoscypha the asci are inamyloid even after $\mathrm{KOH}$ pretreatment. In $H$. intacta Svr. and $H$. quercicola this character is linked with the clearly thickened apical wall. In $H$. latispora ascus apices are otherwise untypical in being thin-walled and clearly rounded. In all the three cases inamyloidity is combined with other differences, justifying recognition at species level. The same applies to two species of Hamatocanthoscypha, whereas a deviating collection from Svalbard, allied to Hyaloscypha britannica Huhtinen but with inamyloid asci, was recognized at varietal level only. Taxa with amyloid asci may rarely have populations with inamyloid asci, as seen in the type variety of $H$. albohyalina. If these asci are not coupled with other marked differences, recognition at the species level may not be justified. The taxonomic value of the ascus pore reaction should always be judged critically.

Interesting variation in the amyloid reaction of asci was noted in two specimens of Hyaloscypha. In the type specimen of Hyaloscypha sarothamni Velen. $(H$. herbarum), some asci in a given apothecium produced conidia. Although the asci with normal as- 
Table 1. The coefficient of variation (V,\%) for ascus width and length in 12 common taxa of Hyaloscypha. $\mathrm{CB}=$ cotton blue, $\mathrm{MLZ}=$ Melzer's reagent, $\mathrm{w}=$ width, $1=$ length (both in $\mu \mathrm{m})$.

\begin{tabular}{|c|c|c|c|c|c|c|}
\hline & $\begin{array}{l}\text { CB } \\
\text { w }\end{array}$ & $\begin{array}{l}\text { CB } \\
1\end{array}$ & $\begin{array}{l}\text { MLZ } \\
\text { w }\end{array}$ & $\begin{array}{l}\text { MLZ } \\
1\end{array}$ & $\begin{array}{l}\mathrm{CB} \\
\mathrm{n}\end{array}$ & $\begin{array}{l}\text { MLZ } \\
\text { n }\end{array}$ \\
\hline H. albohyalina var. albohyalina & 4.4 & 16.1 & 10.5 & 16.7 & 157 & 90 \\
\hline H. albohyalina var. spiralis & 12.9 & 17.0 & 14.6 & 14.8 & 285 & 132 \\
\hline H. aureliella & 13.2 & 13.3 & 8.0 & 17.1 & 83 & 60 \\
\hline H. britannica var. britannica & 8.9 & 10.3 & 13.0 & 9.3 & 52 & 63 \\
\hline H. daedaleae & 10.3 & 17.8 & 11.1 & 24.7 & 81 & 90 \\
\hline H. fuckelii var. fuckelii & 13.6 & 17.1 & 12.6 & 21.2 & 185 & 86 \\
\hline H. fuckelii var. alniseda & 15.3 & 20.0 & 12.0 & 19.8 & 127 & 113 \\
\hline H. leuconica var. leuconica & 17.0 & 24.6 & 17.9 & 23.2 & 39 & 84 \\
\hline H. leuconica var. bulbopilosa & 8.8 & 15.0 & 9.7 & 16.9 & 76 & 44 \\
\hline H. priapi & 10.2 & 13.6 & 11.9 & 12.4 & 52 & 59 \\
\hline H. quercicola & 12.1 & 15.7 & 11.9 & 12.1 & 80 & 43 \\
\hline H. vitreola & 11.2 & 14.6 & 16.3 & 14.0 & 159 & 59 \\
\hline Mean & 11.5 & 16.3 & 12.5 & 16.9 & $\mathrm{n}=1378$ & 923 \\
\hline
\end{tabular}

Table 2. Mean ascus width and mean Q-values in intact and killed asci of ten common taxa of Hyaloscypha. CB = cotton blue, $\mathrm{MLZ}=$ Melzer's reagent, $\mathrm{H}_{2} \mathrm{O}=$ fresh in water, $\mathrm{w}=$ width (both in $\mu \mathrm{m}$ ).

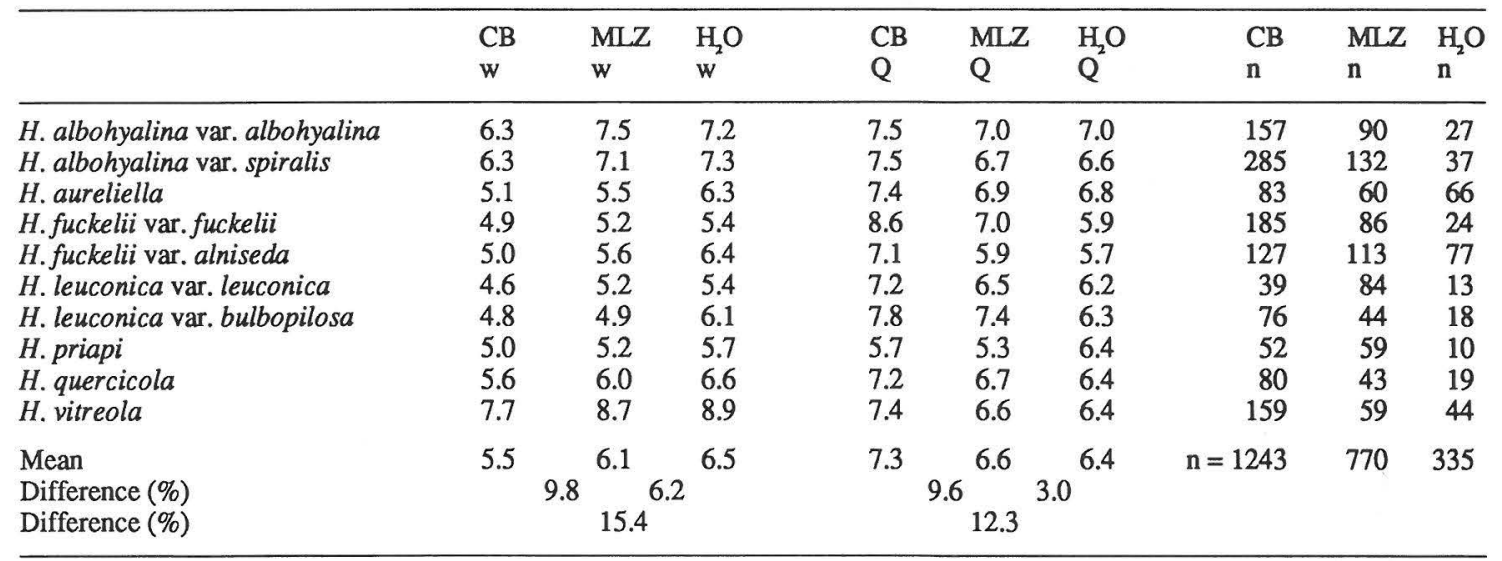

cospores were amyloid, the deviating asci were permanently inamyloid. The second collection (Herb. Korf 53-46) with similar features belonged to $H$. albohyalina var. albohyalina.

A hemiamyloid reaction in ascus pores, as defined by Baral (1987b: 434), was observed in only two species. In Hyaloscypha usitata a red and blue reaction was observed within the same mount. Apparent- ly, the difference is due to slight differences in iodine concentration between the different parts of the squash mount. In $H$. nipponica a red reaction characterized the two populations in which $\mathrm{KOH}$ pretreatment was necessary to evoke an amyloid reaction. In the third known population, with an amyloid reaction independent of $\mathrm{KOH}$ treatment, the reaction in Lugol's solution was blue. Unfortunately, in 
the three genera studied, the hemiamyloid reaction has little taxonomic value.

Ascal walls are not stained with any of the stains or reagents used. The staining reactions of the contents varied somewhat with the reagent. For instance, in $H$. aureliella the basal plasma frequently stained deep orange in Melzer's reagent, but deep reddish in Lugol's solution. It was noted that in many species the reaction is masked in Melzer's reagent, whereas a deep red colouring results when material is placed in Lugol's solution. The variation in these staining reactions diminishes their taxonomic value. In some species, such as $H$. vitreola, the ascal contents very occasionally stain orange in Melzer's reagent.

\section{Development of asci}

Although quite frequently included in species descriptions, especially in North American studies, ascal development has seldom received serious attention as a taxonomic character. Early reports of related taxa differing in their ascal bases were given by, e.g., Emmons (1932), Greis (1938) and Cain (1948). Later, the presence or absence of croziers has occasionally been stressed at the species level in the operculate discomycetes, e.g., by Rifai (1968), Pfister (1976), and Korf and Zhuang (1984). Weber (1972), Harmaja (1977) and Häffner (1987) recognized ascal development as diagnostic at both the species and the sectional level in Helvella Fr. Absence of croziers was even treated as a generic character in Microcalicium Vainio by Tibell $(1978,1987)$.

White $(1943,1944)$ and Korf (1951) published observations on the ascal bases in inoperculate discomycetes. White (1943) used them to differentiate some closely related foliicolous Helotium species. More recent North American studies reporting this character are those of Julien (1958), Haines (1974, 1980), Haines and McKnight (1977), Kohn (1979), Haines and Dumont (1984), Korf and Zhuang (1985), Zhuang and Korf (1986), and Zhuang (1988). In the recent European literature, ascal development has largely been neglected, especially in the inoperculate discomycetes. Arendholz (1979: 14), however, used ascal bases to distinguish between closely allied species. Baral (1984, 1987a) also recognized ascal bases as a valid character at the species level and in his 1984 paper he described differences in croziers between Lachnellula P. Karst. and other hyaloscyphaceous genera.

In the three genera treated here, the presence of croziers has largely remained unreported. To my knowledge, Arendholz (1979) was the first to mention this character. In Hyaloscypha the two different types of ascal bases play an important taxonomic role. In a given apothecium and population this character is stable. The taxonomic level at which the difference should be recognized is not, however, as obvious as postulated in many studies. Automatic recognition at the species level would result in unbalanced taxonomy, as this difference is coupled with a varying amount of other dissimilarities. In some cases the ascus base is the sole differentiating character, being backed only by weak diverging trends in morphology. Such a case is seen in $H$. fuckelii, whereas in $H$. leuconica the other morphological differences are more obvious. In $\mathrm{H}$. britannica and between $\boldsymbol{H}$. aureliella and $\boldsymbol{H}$. candida (Starb.) Boud. more clear-cut morphological or chemical differences are involved. And finally, in allied species the ascal bases may be only one of many differentiating characters. Hence, this character cannot be recognized at only a single taxonomic level.

Berthet (1964) reviewed the earlier literature on ascal development, a corner-stone in which was Chadefaud's (1943) study. Berthet identified the pleurorhyncous and aporhyncous species (largely equivalent to presence and absence of croziers) in many ascomycete genera. He found that the majority of species fall clearly into one of these two categories, although occasionally the two types of ascus development are seen in a single apothecium, e.g. in Pyrenopeziza mercurialis (Fuckel) Boud. In the taxonomically difficult Hymenoscyphus scutula (Pers.) Phill. complex, such variation was reported between two populations, which may, however, have belonged to different taxa. For four other species Berthet reported coexistence of simple septa and croziers, which apparently correspond to the "downward protuberances" discussed below. Many later observations on ascal bases confirm the stable nature of this feature. In view of its potential importance in Hyaloscypha, reports of variation within an apothecium or population deserve careful attention.

Variation within a single fruit body has been reported by Berthet (1964), Rogers (1972), Jones (1981) and Huhtinen (1987a). Less definite reports, some possibly due to taxonomic confusion, are found in White (1943) and Haines and McKnight (1977). In these it is not clear whether the variation was observed within a single fruit body or between different populations. Such observations as "often with croziers" may result from the fact that croziers are clearly visible in side view only. A substantial portion of ascogenous hyphae may appear to be simple-septate when lying in another position. There seems to be only one report of variation in the prevailing simple-septate state (Huhtinen 1987a). When 
the variation is seen between populations, two different but closely related taxa can be involved.

The rarity of variation in ascal development within a single fruit body and population is illustrated by observations of conidiogenous asci seen on two occasions. In the type variety of $H$. albohyalina, one population showed both normal amyloid asci and conidiogenous/inamyloid asci within a single fruit body. These abnormal asci were also devoid of croziers, which always characterize the normal asci. This particular population was the only one in which variation in ascus development could be confirmed and even in this case it was coupled with other abnormalities in the asci. The literature suggests clearly that in the majority of the infrageneric taxa studied in this respect, the ascal base characters are stable and reliable characters. The exceptions in which a clearly defined taxon has been critically observed are few. In the present study the stability of this feature was ascertained. Two main types of ascus base morphology were noted. The asci arise either from croziers (Figs. 36,42 ) or from simple septa (Fig. 117). A deviation from the former type is seen in the type variety of Hyaloscypha strobilicola Huhtinen, which, besides normal croziers and ascus bases, has a different type of crozier resulting in a more forked base (Fig. 161). The downward protuberance fuses with the lower stalk cell. A similar crozier was illustrated in $\mathrm{Hy}$ menoscyphus herbarum (Pers.) Dennis by Berthet (1964). Protuberances with free ends are rare. Somewhat similar free ascus bases are seen in some populations of $H$. herbarum, $H$. leuconica var. leuconica and $H$. quercicola. These taxa have asci arising from simple septa and the modification occurs in scattered populations only. These lateral to downward protuberances affect the shape of the ascus bases, but do not fuse with the stalk cell. This can be ascertained from their free, rounded ends and lack of the pore typical of true septa. Similar protuberances are very rarely seen in other taxa and assist in recognition of the species.

In Hyaloscypha, taxa with and without croziers are almost equally common. In Phialina, all the species have croziers and in Hamatocanthoscypha all but one taxon. This difference is intriguing, because developmental sequences of asci as taxonomic or phylogenetic criteria at higher levels have been compiled by, e.g., Jones (1981), Jones and McGawley (1984) and Jones and Clay (1987). In basidiomycetous fungi much discussion has arisen on the taxonomic and phylogenetic value of clamps (e.g. Savile 1955, Furtado 1966, Boidin 1971). The application of these ideas to ascomycetous fungi greatly depends on whether croziers are accepted as homologous to clamps (Boidin 1971).

In Hyaloscypha the difference in ascal bases could be one of the first morphological signs of divergence between the populations. This would explain the observation that many "taxon pairs" with small to more marked differences exist. During further speciation, the ascal bases, being conservative or genetically fundamental characters, would remain stable, while further differences in the less conservative features developed. The first step in evolution, according to this scheme, could be examplified by the two varieties of $H$. fuckelii with few morphological differences. Divergence appears to have gone slightly further in $H$. leuconica, and still further in $H$. albohyalina, in which the variational patterns are overlapping but different. The final stage, here recognized at the species level, shows marked differences and only slight or no overlap in variation ranges. The almost total lack of taxa with asci developing from simple septa in Hamatocanthoscypha and Phialina possibly indicates a longer evolutionary history, during which the capacity for divergence has decreased. Hyaloscypha, in which both types of ascal development are equally common, could be in an active evolutionary stage.

\section{Spores}

\section{Variability}

In Hamatocanthoscypha small cuneiform spores prevail and in Phialina the spores tend to be large or fusoid. The genus Hyaloscypha, with mostly ellipsoid medium-sized spores, is intermediate between the other two genera (Fig. 13). In each genus, however, single species have deviating spore types.

The spore shape is more useful than the ascus shape in species delimitation. The more diversified shape is evident from Fig. 13 where many taxa deviate from the correlation characterizing asci (Fig. 12). Spore shape and size are relatively stable characters in all the genera. But the range of variation differs from species to species. Foliicolous Phialina lachnobrachya and $P$. pseudopuberula are variable, with sporograms rarely encountered in Hyaloscypha (Figs. 236,252 ). The same amount of variation in spore width is seen in $H$. intacta, whereas most Hyaloscyphas with medium-sized spores have quite different, mutually similar sporograms. In infraspecific taxa, as in the varieties of $H$. leuconica and $H$. fuckelii, the spores are often alike. Similarity of variational 


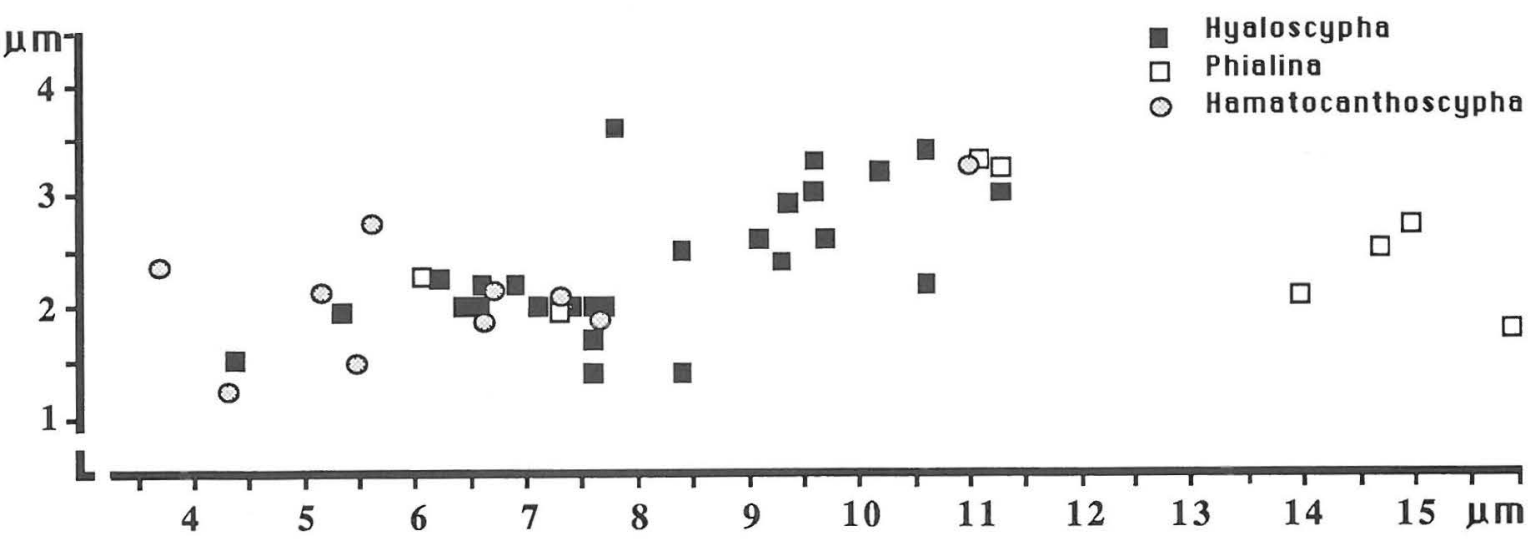

Fig. 13. Mean spore length and width (in CB, MLZ) in the species and varieties of three hyaloscyphaceous genera.

pattern can imply closeness of taxa, but there are exceptions. It could, however, be postulated that a very narrow species concept should not be applied to an unknown foliicolous Phialina.

The shrinkage of living spores in common reagents shows the same pattern as in the asci. The smaller shrinkage in length (Table 3) has the same explanation as in the asci and is partly masked by the larger variability of the length. No difference in shrinkage between cotton blue and Melzer's reagent could be detected. Only two different spore measurements are thus needed. Any taxonomic consideration of these genera should take account of the slight difference in spore shape between fresh and killed material ( $Q$ values in Table 3).

Parmasto and Parmasto (1987) concluded that in the Hymenomycetes the variation in spore length and width is almost identical. In the present study it was noted that in many species the spores tend to vary more in length than in width. The tendency is clearly seen at the specimen level. At the species level it is evident in the frequency distributions for spore quotient $\mathrm{Q}$, which are frequently skewed to the right. Atchley et al. (1976) warned, however, about the use of ratios, showing leptocurtotic and right-skewed distribution patterns, as did also Phillips (1983). Although the ratio $Q$ is used here to plot the shape, instead of constructing two frequency distribution graphs, spore elongation is also evident when spore length alone is plotted. In the sporograms, too, elongated spores markedly extend the frequency distribution pattern (see e.g. Fig. 115). The greater range of spore length is also evident from the mean coefficients of variation for spore length and width (Table 4).
The overall variation in length has a number of different components. In a single ascus the lowermost spore may be larger and more elongated than other spores. In a given apothecium the total variation between mature spores is often notable. The frequency of elongated spores is different from $1 / 8$, which suggests differences between asci or a tendency to elongate after discharge. Another possible explanation lies in the closeness of apothecia in a given population (possibly different individuals). After release, spores land on all the neighbouring apothecia and a mount from a single apothecium may actually contain spores from numerous individuals. The sporal dimorphism reported in the family by Raitviir (1970b, 1986) was not observed. Due to the variation of shape within populations, variation between populations is generally evident in spore volume rather than in spore shape.

Parmasto and Parmasto (1987) concluded that in the majority of Hymenomycetes spore width increases with spore length. This is true of the ellipsoid-spored taxa in the genera studied here, but is partly masked by the tendency to elongate. In species with typically narrow spores, the frequency distribution of the $Q$ values is clearly more platykurtotic than in ellipsoid-spored taxa. This is exemplified by Phialina ulmariae (Lasch in Rabenh.) Dennis and $P$. lachnobrachyoides (Raitv.) Huhtinen. The character under stronger stabilizing pressure in these taxa is possibly spore width, or the platykurtotic shape of the graph can be attributed to the use of a ratio.

The general horizontal orientation of the sporograms (variation in length) could be due to natural selection against an increase in spore width. In an inoperculate ascus, with only a narrow pore for spore 
Table 3. Mean spore measurements and mean Q values in living and killed spores of 10 common taxa of Hyaloscypha. $\mathrm{CB}=$ cotton blue, $\mathrm{MLZ}=$ Melzer's reagent, $\mathrm{H}_{2} \mathrm{O}=$ fresh in water, $\mathrm{w}=$ width, $\mathrm{l}=$ length (both in $\mu \mathrm{m}$ ).

\begin{tabular}{|c|c|c|c|c|c|c|c|c|}
\hline & $\begin{array}{c}\mathrm{MLZ} \\
\mathrm{CB} \\
1\end{array}$ & $\begin{array}{c}\mathrm{H}_{2} \mathrm{O} \\
1\end{array}$ & $\begin{array}{c}\mathrm{MLZ} \\
\mathrm{CB} \\
\mathrm{w}\end{array}$ & $\begin{array}{c}\mathrm{H}_{2} \mathrm{O} \\
w\end{array}$ & $\begin{array}{c}\mathrm{MLZ} \\
\mathrm{CB} \\
\mathrm{Q}\end{array}$ & $\begin{array}{c}\mathrm{H}_{2} \mathrm{O} \\
\mathrm{Q}\end{array}$ & $\begin{array}{c}\mathrm{MLZ} \\
\mathrm{CB} \\
\mathrm{n}\end{array}$ & $\mathrm{H}_{2} \mathrm{O}$ \\
\hline H. albohyalina var. albohyalina & 9.7 & 11.3 & 2.6 & 3.2 & 3.9 & 3.6 & 632 & 87 \\
\hline H. albohyalina var. spiralis & 9.1 & 9.6 & 2.6 & 2.8 & 3.6 & 3.4 & 1112 & 73 \\
\hline $\begin{array}{l}\text { H. aureliella } \\
\text { Pa }\end{array}$ & 6.9 & 8.1 & 2.2 & 2.5 & 3.3 & 3.3 & 356 & 89 \\
\hline H. fuckelii var. fuckelii & 7.4 & 7.0 & 2.0 & 2.1 & 4.0 & 3.4 & 604 & 30 \\
\hline H. fuckelii var. alniseda & 7.3 & 7.9 & 2.0 & 2.4 & 3.7 & 3.3 & 313 & 143 \\
\hline H. leuconica var. leuconica & 7.1 & 7.5 & 2.0 & 2.3 & 3.7 & 3.1 & 198 & 19 \\
\hline H. leuconica var. bulbopilosa & 7.6 & 8.9 & 2.0 & 2.4 & 4.1 & 3.6 & 304 & 41 \\
\hline H. priapi & 7.6 & 7.4 & 1.7 & 2.1 & 4.5 & 3.3 & 165 & 16 \\
\hline H. quercicola & 7.7 & 9.1 & 2.0 & 2.5 & 3.9 & 3.7 & 272 & 41 \\
\hline H. vitreola & 11.3 & 12.1 & 3.0 & 3.5 & 3.8 & 3.5 & 577 & 103 \\
\hline Mean & 8.2 & 8.9 & 2.2 & 2.6 & 3.9 & 3.4 & $\mathrm{n}=4533$ & 642 \\
\hline Difference & \multicolumn{2}{|c|}{$7.9 \%^{8.9}$} & \multicolumn{2}{|c|}{$15.4 \%$} & \multicolumn{2}{|c|}{$12.8 \%$} & & \\
\hline
\end{tabular}

Table 4. The coefficient of variation $(\mathrm{V}, \%)$ for spore width and length in 12 common taxa of Hyaloscypha. CB = cotton blue, $\mathrm{MLZ}=$ Melzer's reagent, $\mathrm{H}_{2} \mathrm{O}=$ fresh in water, $\mathrm{w}=$ width, $\mathrm{l}=$ length (both in $\mu \mathrm{m}$ ).

\begin{tabular}{|c|c|c|c|c|c|c|}
\hline & $\begin{array}{c}\mathrm{MLZ} \\
\mathrm{CB} \\
\mathrm{w}\end{array}$ & $\begin{array}{c}\mathrm{MLZ} \\
\mathrm{CB} \\
1\end{array}$ & $\begin{array}{c}\mathrm{H}_{2} \mathrm{O} \\
\mathrm{w}\end{array}$ & $\begin{array}{c}\mathrm{H}_{2} \mathrm{O} \\
1\end{array}$ & $\begin{array}{c}\mathrm{MLZ} Z \\
\mathrm{CB} \\
\mathbf{n}\end{array}$ & $\begin{array}{c}\mathrm{H}_{2} \mathrm{O} \\
n\end{array}$ \\
\hline H. albohyalina var. albohyalina & 14.5 & 16.4 & 13.5 & 15.8 & 632 & 87 \\
\hline H. albohyalina var. spiralis & 13.3 & 14.9 & 10.7 & 15.7 & 1112 & 73 \\
\hline H. aureliella & 13.0 & 15.3 & 10.8 & 13.4 & 356 & 89 \\
\hline H. britannica var. britannica & 11.4 & 14.4 & - & - & 295 & - \\
\hline H. daedaleae & 7.8 & 14.4 & - & - & 241 & - \\
\hline H. fuckelii var. fuckelii & 10.6 & 16.4 & 7.0 & 12.9 & 604 & 30 \\
\hline H. fuckelii var. alniseda & 11.7 & 18.2 & 15.1 & 13.7 & 313 & 143 \\
\hline H. leuconica var. leuconica & 20.4 & 18.9 & - & - & 198 & - \\
\hline H. leuconica var. bulbopilosa & 14.4 & 16.0 & 10.3 & 13.8 & 304 & 41 \\
\hline H.priapi & 16.7 & 17.2 & - & - & 165 & - \\
\hline H. quercicola & 13.6 & 16.2 & 12.8 & 14.5 & 271 & 41 \\
\hline H. vitreola & 9.4 & 10.7 & 7.7 & 12.8 & 577 & 103 \\
\hline Mean & 13.1 & 15.8 & 11.0 & 14.1 & $\mathrm{n}=5030$ & 607 \\
\hline
\end{tabular}

liberation, any increase in spore volume can be expected to be achieved through elongation. In the three genera studied here, $4.5 \mu \mathrm{m}$ seems to be a "critical" limit for spore width. In operculate discomycetes, lacking the narrow pore, the spores are frequently very wide. In inoperculate discomycetes, the spore length may reach, or even be many times as great as the length in operculate taxa, while the same tendency is not seen in width. The view of the significance of ascus dehiscence for spore width does not agree, however, with the observations of Beckett (1981) and Baral $(1987 \mathrm{a}, \mathrm{b})$, which suggest that the ascus pore is highly elastic.

Infraspecific variation clearly connected with the substrate was observed in one taxon only. In $H$. albohyalina var. spiralis (Velen.) Huhtinen the fre- 


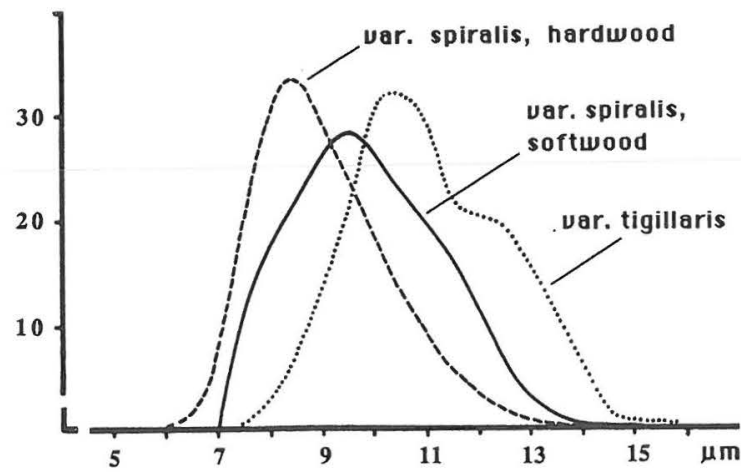

Fig. 14. The distribution of spore length in two varieties of Hyaloscypha albohyalina.

quency of elongated spores in populations on softwood was higher than in populations on hardwood (Fig. 14). A parallel but weaker tendency for spores to be wider in softwood populations was also observed. As discussed in the taxonomic part, this was taken as an indication of current divergence. Peziza tigillaris P. Karst., confined to softwood and with spores of even greater volume, was recognized as a variety. In this particular case a major hiatus in spore characteristics was recognized at varietal level only.

The coefficient of variation in the present material is higher than reported earlier in Hymenomycetes, e.g., by Parmasto and Parmasto (1987). Their study dealt with variability of mean spore width and length, but the calculations for Table 4 were made from individual spore measurements. In 100 hymenomycetous species the variability of spore length and width was mostly below $10 \%$, the means being $6.5 \%$ and $6.7 \%$. The geographical coverage in the Parmastos' material was, by their own admission, restricted, which has resulted in somewhat smaller variability than would be found in material from a larger part of the total distribution area. The difference in the mean values given in Table 4 is, however, intriguing. Methodological inaccuracy in measuring the often small in operculate spores may have added to the variability. The use of individual spore measurements instead of specimen means probably enhanced the difference. But observations suggest that the spore shape in inoperculate taxa is in fact often more variable than in hymenomycetous fungi, which rarely attain the present variation percentages (Parmasto \& Parmasto 1987: table 36).

\section{Gross morphology}

In addition to dimensions and shape, the spores have some other characters of taxonomic value, though this is reduced by their variability or dependence on other characteristics. One such character is the spore contents. In interpreting guttulation, it is important to distinguish between observations on living and killed material (Baral, pers. comm.). Ontogeny has a notable influence on the guttulae. In species with voluminous spores the (apparently oldest) spores lacking cyanophilous contents often show two regular globose guttulae with oily contents (Figs. 50, 58). The younger spores show conspicuous, less regular guttulae. In the genera studied, two main types of spore guttulation can be found: guttulae with light contents and small bodies appearing dark under the microscope. The size and prominence of guttulation is often related to spore size.

Spore septation, a character frequently reported by taxonomists, has diagnostic value at the species level in the present genera as well. The value is diminished by the dependence of this character on spore age. Upon germination many typically aseptate spores ultimately develop one cross septum. In such cases the septate spores seen in a squash mount are few and the mere words "spores aseptate to septate", without reference to frequencies or infraspecific variation, are of little taxonomic value. In the present study, spore septation was evaluated both on spores still inside asci and on discharged spores. Although spores seldom germinate on the disc, septation is partly linked with the time a spore has lain on the disc and very old spores should be ignored. Spores inside the asci may be septate because the asci are senescent and have failed to release the spores or may have become septate well before germination. In the latter case, septate spores are seen repeatedly. Large spores tend to become septate more frequently than smaller spores, and deviations from this tendency are of taxonomic value. Septation shows notable variation, however, between the populations. The situation is analogous to that of hair septation; an increase in volume is evidently accompanied by the presence of septa, which give a firmer structure. In few species of Hyaloscypha the septum was occasionally found to be dextrinoid. After prolonged exposure on the receptacle, the spores in some species may show brownish pigment in the walls.

The spores of Phialina pseudopuberula are exceptional in the three studied genera. In most populations many spores bear an apical cilium. Populations with "normal" spores also occur and thus the character is not significant above the species level. Such seemingly anomalous spore appendages have also been 
reported in Arachnopeziza (Korf 1951, Huhtinen 1987d), in which the character is diagnostic at the infrageneric levels only.

\section{Paraphyses}

In Phialina the paraphyses are clearly wider than in the other two genera. The subclavate apices are often 3-4 $\mu \mathrm{m}$ wide. Close septation below the terminal cell is also somewhat characteristic of the genus. The yellow pigment, which turns golden yellow in Melzer's reagent, is a clearly distinguishing character. The paraphyses are closely similar to those of Calycellina. In Hyaloscypha most species have paraphyses with a mean width of $1-2 \mu \mathrm{m}$, in only one, H. latispora, are the paraphyses as wide as in Phialina. In Hamatocanthoscypha the paraphyses are like those in Hyaloscypha, but tend to have long terminal cells with a septum occurring below the ascal bases.

Paraphysis width is a valuable, relatively stable character at the species level. It is not correlated with ascus width. The length of the terminal cell varies even within an apothecium and is of little diagnostic value. Paraphyses do not clearly protrude above the ascal apices. Branching is usually dichotomous and only Hyaloscypha priapi Velen. clearly has more intense branching. In species of Hyaloscypha with a clear dextrinoid reaction in the hair and excipular walls, the paraphyses closest to the margin may also be dextrinoid. As this character is correlated with the overall reaction, it is not taxonomically significant.

\section{Pigments, resinous exudates}

\section{and histochemistry}

In studies on the discomycetes the words "pigment" and "resin" have not been clearly defined. In the present study, a pigment is considered to be a coloured substance occurring inside the paraphyses and hairs or incorporated in the hair or excipular walls. Pigments are typically persistent in most reagents. A resin is here defined as an excretory product, which in many species is vulnerable to common mountants. In the Hyaloscyphaceae the excretory function is concentrated in the hairs, but is also seen in subicular hyphae (Huhtinen 1987c). The resin frequently occurring on the excipulum may be excreted by the hairs or the excipulum itself. In the present genera, the paraphyses have no obvious excretory function, though excretion by the paraphyses is a valuable taxonomic character in the Dermateaceae (Hein 1981). The accumulation of glassy substances inside the hairs or paraphyses should be recognized as fundamentally different from these excretory processes (Korf \& Kohn 1980, Huhtinen 1987c).

Most of the species here placed under Phialina were earlier included in Hyaloscypha, but the generic delimitation of the genera poses no problem. The yellow pigment that turns golden yellow in Melzer's reagent was recognized as diagnostic of Phialina early in the present study. It is absent from the two other genera. Later, it proved to be coupled with other morphological, chemical and ecological features typical of Phialina. An identical, persistent, cyanophilous pigment that turns golden in Melzer's reagent occurs in foliicolous Calycellina and Scutoscypha and was observed in one species of Mollisina. Its wide distribution among herbicolous and foliicolous genera is interesting from the phylogenetic point of view.

Although usually occurring on the hair walls, a resinous exudate may, in species, occur inside the hairs as well, but is in such cases lacking from the paraphyses. The resinous exudates in Hyaloscypha usually dissolve rapidly in Melzer's reagent and cotton blue. Their value as a generic character is diminished by the occurrence of identical resin in a wide variety of genera: Arachnopeziza, Betulina sensu Graddon, Dasyscyphella, Dematioscypha, Hyaloscypha, Lachnum, Protounguicularia Raitv. \& Galán and Psilocistella (Korf 1951, Graddon 1972, 1974, Raitviir 1977c, Svrček 1977c, 1978, Haines 1980, Haines \& Dumont 1984, Spooner \& Dennis 1985, Huhtinen 1987a, d, Spooner 1987). This resin is not found in combination with any particular type of substrate. At species level it is relatively stable and taxonomically more significant. A faint yellowish pigment, present in both paraphyses and hairs, is characteristic of Hamatocanthoscypha uncipila. This pigment is seen clearly only when fresh material is studied in water. In water-mounted herbarium material it is not observed after the mount has been squashed. Unlike the pigment in Phialina, it dissolves in cotton blue and Melzer's reagent. Traces of it were also seen in a few populations of $H$. laricionis (Velen.) Svr. var. laricionis.

Brown pigments incorporated in excipular walls are taxonomically useful at the generic level when the excipulum is dark brown. Faint or occasional brown pigmentation is less valuable in generic delimitation. In a few species of Hyaloscypha a faint brown tinge was observed in some populations. In Phialina pseudopuberula the excipular walls are brownish in all populations, and the colouring is thus valuable as a species character. Three species of Hamatocanthoscypha are characterized by brown pigmentation in 
the excipular walls and occasionally also hair walls. In $H$. uncinata one variety is characterized by stable pigmentation, while the other shows the whole scale of variation from hyaline to dark brown apothecia. The tendency to develop brown pigmentation is valuable, however, in delimitating Hamatocanthoscypha.

An important observation on the chemistry of Hyaloscypha was made by Dennis (1956), who noted that the excipulum and hairs of Peziza tigillaris stained reddish brown in Melzer's reagent. The reaction was later observed by Svrček (1977c), who emphasized its significance, referring to it somewhat misleadingly as "amyloid". Later (Svrček 1985) he described of the resulting colour as "... more or less violet or greyish violet or sometimes greenish...". The purplish red colour in Melzer's reagent (Cailleux $\mathrm{N} 13, \mathrm{~N} 15$ ) is only slightly more violet than the gill trama in Mycena (Pers.: Fr.) Roussel and this reaction is here termed "dextrinoid" in accordance with the terminology in hymenomycetous studies (Hora 1960). The reaction in Hyaloscypha is the same as that observed in the hairs of, for example, Unguicularia Höhnel. Inaccuracy in the terminology is also seen in the study by Raschle (1977), in which "amyloid" in the key is changed to "dextrinoid" in the descriptions. As clear amyloid reactions also occur in the hairs of Hyaloscyphaceae (see below), the term dextrinoid shoud be used in the sense suggested by Hora (1960). A dextrinoid reaction in the excipulum has so far been reported in only a few genera, namely Pezizella (Svrček 1982), Cudoniella Sacc. (Huhtinen 1985b) and Phaeohelotium Kan. (Svrček 1987a). In a recent revision of Velenovsky's material of Helotium Svrček (1984) reported dextrinoid excipula in several species.

The dextrinoid reaction is valuable in delimiting the genera studied here, being present only in Hyaloscypha. But the slight variability of this chemical reaction can be misleading. The hairs are more stable in their reaction than the excipulum. In nine of the 17 taxa with dextrinoid hairs the reaction is constant; in the rest of these taxa occasional populations may have indextrinoid hairs, while in nine species the reaction is totally absent. The subgenus Eupezizella lacks dextrinoid reactions, whereas these are common in subg. Hyaloscypha. When delayed, the reaction is often first seen in the solidified hair apices or in the excipular cells starting hair formation.

An amyloid reaction is rarer in the hairs or excipulum than a dextrinoid and never occurs as an overall reaction. The substances responsible for the blackish violet colour in Melzer's reagent occur in relatively small nodules inside the excipular cells.
They are closely attached to the wall but apparently not part of it. More rarely, the same nodules are seen inside the hairs. This reaction is characteristic of the subgenus Eupezizella and is most common in $H$. aureliella. In Hamatocanthoscypha it is seen in one taxon. A similar reaction was described by Svrček (1983) for Hyaloscypha amyloideopilosa Svr. (i.e. H. aureliella). It was also characteristic of two of the original species of Protounguicularia (Huhtinen 1987d). Recently, Spooner and Dennis (1985) described a violet reaction in the excipulum of a new species of Hymenoscyphus Gray. Svrček (1984) reported amyloid reactions in many species of Helotium sensu Velenovský.

In $H$. aureliella similar nodules were very occasionally dextrinoid. This exceptional reaction was observed only a few times, but it may indicate some relationship between the amyloid and dextrinoid reactions in Hyaloscypha. Both reactions were reported to occur in the same apothecia in Phaeohelotium imberbe (Bull.: Fr.) Svr. by Svrček (1984).

The water-based dyes were of little taxonomic value at the specific level. Quite reliable differences between Hyaloscypha and the two other genera were seen, however; in Hyaloscypha, the excipulum mostly stained deeply in basic fuchsin, cresyl blue, neutral red and toluidine blue. Possibly the colouring is due to an intercellular matrix which is seldom stained in cotton blue. In the two other genera, a strong staining reaction in these dyes was exceptional and was totally lacking in most taxa.

\section{Ecology}

The genera differ clearly from each other in their ecology, which strengthens their delimitation. Hyaloscypha is predominantly lignicolous with a tendency to fruit on bulky wood substrates. Less often, it fruits on herbaceous or arboreal litter. Species of Hamatocanthoscypha occur mainly on forest debris: twigs, needles, pieces of bark or cones. Fruiting on large branches or trunks is much rarer. The genus Phialina fruits mostly on leaves but also on herbaceous stems, and occurrence on wood is exceptional.

\section{Hyaloscypha}

The species of Hyaloscypha are saprophytes, attacking wood which is already modified by other fungi, mites and bacteria. There are no collections from intact wood. In some collections the wood may have a firm inner core but the surface is always softened and degraded by other organisms. Although Hyaloscypha 
occurs relatively late in the lignicolous succession, the abundance and wide distribution of its populations, at least in the temperate zone, gives it a dominant place among the lignicolous Helotiaceae. With the possible exception of Mollisia, Hyaloscypha is the most common genus on bulky wood substrates, clearly outnumbering such genera as Arachnopeziza, Cistella, Dasyscyphella, Hyalopeziza, Lachnum, Unguicularia, Perrotia Boud. and Psilocistella. The information from the tropics is too limited to allow conclusions in this respect.

Nannfeldt (1932) believed Hyaloscypha to be rather strictly substrate-specific. He doubtless based his opinion on two species, $H$. aureliella and $H$. daedaleae (H. quercus Nannf.), which are restricted to one type of wood. He described a new species, $H$. fuckelii, thought to be limited to Salix. However, $H$. fuckelii has a much wider ecological amplitude. Velenovský $(1934,1947)$ carried the idea of substrate specificity to an extreme, which resulted in taxonomic chaos. Dennis (1949) pointed out that populations occurring on a certain type of wood should not be taken uncritically as separate species of Hyaloscypha. Since 1950, however, the idea of rather narrow habitat requirements has led to the separation of many new species in the genus. The members of $\mathrm{Hy}$ aloscypha are not limited to a certain tree genus. In Hyaloscypha six major ecological groups can be distinguished: 1) species restricted to softwood 2) species restricted to hardwood 3) species restricted to oak wood (one only) 4) species occurring on both softwood and hardwood 5) species inhabiting all types of litter 6) species confined to herbaceous litter. The strict distinction between softwood and hardwood is well known among the inoperculate genera. Deviations, e.g., in $H$. aureliella, were indicated by herbarium labels, but an analysis of wood substrates showed that there were no exceptions among the nearly 350 collections. Three other species of subg. Eupezizella are confined to softwood. Five species of subg. Hyaloscypha, with more than occasional collections, are known to be confined to hardwood. Many other lignicolous species in this subgenus prefer to inhabit hardwood. Thorough decomposition of wood may explain those cases in which a species is exceptionally found on softwood. The chemical properties of the wood may have changed to such an extent that recognition is no longer possible.

Only one species, Hyaloscypha daedaleae, is confined to oak wood. In Europe it is easily distinguished from its closest relatives growing on other hardwoods. But, as discussed under $H$. daedaleae, there are no clear gaps in morphological characters in North American material. In Europe oak seems to be a relatively inaccessible substrate for many species of Hyaloscypha. Besides $H$. daedaleae, only $H$. quercicola fruits often on oak wood. Most of the other hardwood-inhabiting species avoid Quercus completely or almost so. The total substrate selection of these fungi is very diversified, and covers nearly all the common hardwood genera. The only unfavourable substrates were found to be poplars and oak. It could be postulated that only Quercus has played a major role in the speciation of Hyaloscypha. Such a trend is in contrast to what we know about higher fungi, where specialization on a single host genus is common. However, these fungi often colonize living trees or arrive soon after their death (Cooke \& Rayner 1984). Such pioneers face substrates with a different structure and chemistry than those of the partly decomposed remnants, favoured by Hyaloscypha The basic differences between softwood and hardwood in composition and wood extractives, or other properties significant for fungal growth, are likely to persist longer.

Hyaloscypha has few species with populations fruiting on both hardwood and softwood. In the type variety of $H$. fuckelii, only four collections originate from softwood. In the other variety and also in $H$. herbarum, a single collection was made from a coniferous tree. These observations suggest that softwoods are not easily accessible to these species. They can possibly be colonized only after degradation by pioneer organisms or when competition is diminished. $H$. albohyalina and $H$. leuconica can decompose both wood types, occurring on conifers more than just occasionally. However, there are no species preferring softwood and occurring occasionally on hardwood.

Two species of Hyaloscypha are frequently encountered on arboreal and herbaceous litter. Only $H$. secalina is confined to the latter substrate; $H$. herbarum is often found on herbaceous litter, but can fruit on various other substrates, too. Such a wide ecological amplitude, ranging from dead trunks and branches to cupules of Quercus or stems of Epilobium, is exceptional in the genus. One collection even originates from oak leaves. This particular species was found to grow rapidly in culture, which may reflect specialization to decomposition of litter rather than bulky wood. For example, the difference in growth rate from $H$. vitreola, which fruits on large trunks in a late stage of succession, was pronounced.

Two species of the genus have specialized substrate requirements. Hyaloscypha epiporia fruits on the pore surface of annual polypores growing on conifers. $H$. strobilicola fruits on conifer cone scales and seems to be confined to warm and dry climates. 
No species of Hyaloscypha is corticolous, and occurrence on the inner or outer surface of bark is only occasional. Fruit bodies were only exceptionally found on ephemeral substrates such as old sacking, decaying leaves or old newspaper. When old pyrenomycetes are present in abundance on the substrate, many Hyaloscypha species tend to form apothecia close to or on their stromata or perithecia. This may indicate that the mycelium extracts nutrition from pyrenomycetous tissues. In many such cases apothecia also occur on wood far from the pyrenomycetes, so there is no firm connection such as mycoparasitism.

\section{Hamatocanthoscypha}

The species of Hamatocanthoscypha are mostly litter decomposers. Only $H$. uncipila favours bulky wood, but it occurs on cone scales as well. Four of the 10 known taxa fruit solely or predominantly on coniferous litter, cones and needles being the main substrate. Small twigs and bark of conifers are also utilized by Hamatocanthoscypha. These substrates and conifer needles are not particularly favoured by the species of Hyaloscypha. The generic difference in preference is evident in $H$. laricionis, which grows also on Lycopodium and ferns. Hamatocanthoscypha helicotricha Huhtinen occurs on ferns as well.

Ecology plays an important role in the specific and subspecific delimitation of Hamatocanthoscypha. Its species seem to be ecologically more exacting than the Hyaloscypha species, contrasting markedly, for example, with the litter-inhabiting Hyaloscypha herbarum, which has a wide ecological amplitude. The material of many taxa of Hamatocanthoscypha is limited, however, and larger material of the type variety of $H$. laricionis revealed that in addition to coniferous litter, it grows on Lycopodium. It is intriguing that the genus clearly tends to concentrate on systematically primitive hosts.

\section{Phialina}

Six species of Phialina are foliicolous. The other two are more or less confined to Filipendula and Pteridium. In the foliicolous taxa, the host genus seems to play a minor role. Deviations from the typical type of substrate are few: in the caulicolous $P$. ulmariae two foliicolous collections are known and the foliicolous $P$. lachnobrachya has been found twice on capsules of Aesculus. The ecology of the genus suggests closer relationship to Calycellina than to either of the two lignicolous genera treated in the present study.

\section{Distribution}

The species of Hyaloscypha are distributed in the temperate zone, where they have been extensively collected, except in arctic and alpine habitats, and the distribution of the genus appears to be continuous (Fig. 15). The distribution pattern mirrors the availability of substrates for the fungi. The ability to grow on nearly all kinds of softwoods and hardwoods allows occurrence throughout the forested temperate zone. In every place I visited, I could find a population of Hyaloscypha within a few minutes, even in the middle arctic parts of Svalbard, so that the genus appears to be truly widespread and abundant.

Hyaloscypha aureliella roughly mirrors the total range of the genus, being its commonest species and apparently occupying the whole temperate zone. It is also present further south, as seen from the collections from Jamaica and the Philippines, and extends northward to Svalbard. Other widespread species are $H$. albohyalina, $H$. fuckelii, $H$. vitreola and $H$. leuconica, which have been collected in most temperate areas visited by discomycetologists. The very disjunct finds of some rarely collected species $(H$. epiporia, H. occulta Huhtinen) suggest that many may be expected to have a wide geographical range. Collections from more southern areas are too few and scattered to show the distribution. As many Hyaloscyphas seem to fruit in a late stage of succession, occurrence in the tropics may be limited by the rapid mineralization of fallen timber.

The collections of Phialina and Hamatocanthoscypha are rather scanty. In general, the distribution matches that of Hyaloscypha but strikingly few specimens derive from North America. Again, disjunct populations of the rare $H$. ocellata and $P$. foliicola suggest a wider range than is apparent from the collections.

The discomycetous floras of Eurasia and North America are very similar, especially in the northern boreal zone and the Arctic (e.g. Raitviir 1985, Huhtinen 1985b, 1987b, 1988, Huhtinen \& Niemelä 1985, Schumacher \& Sivertsen 1987). In the present material, only a few species, with more than occasional collections, showed a restricted distribution. Three Hyaloscypha species are known only from Europe: $H$. daedaleae, $H$. intacta and $H$. quercicola. In Phialina, the most restricted is $P$. lachnobrachyoides. Two common species of the genus seem to be lacking from North America, though further material may change the picture. Only a few collections of Hamatocanthoscypha derive from North America or Siberia, although material of Hyaloscypha has been collected there. 


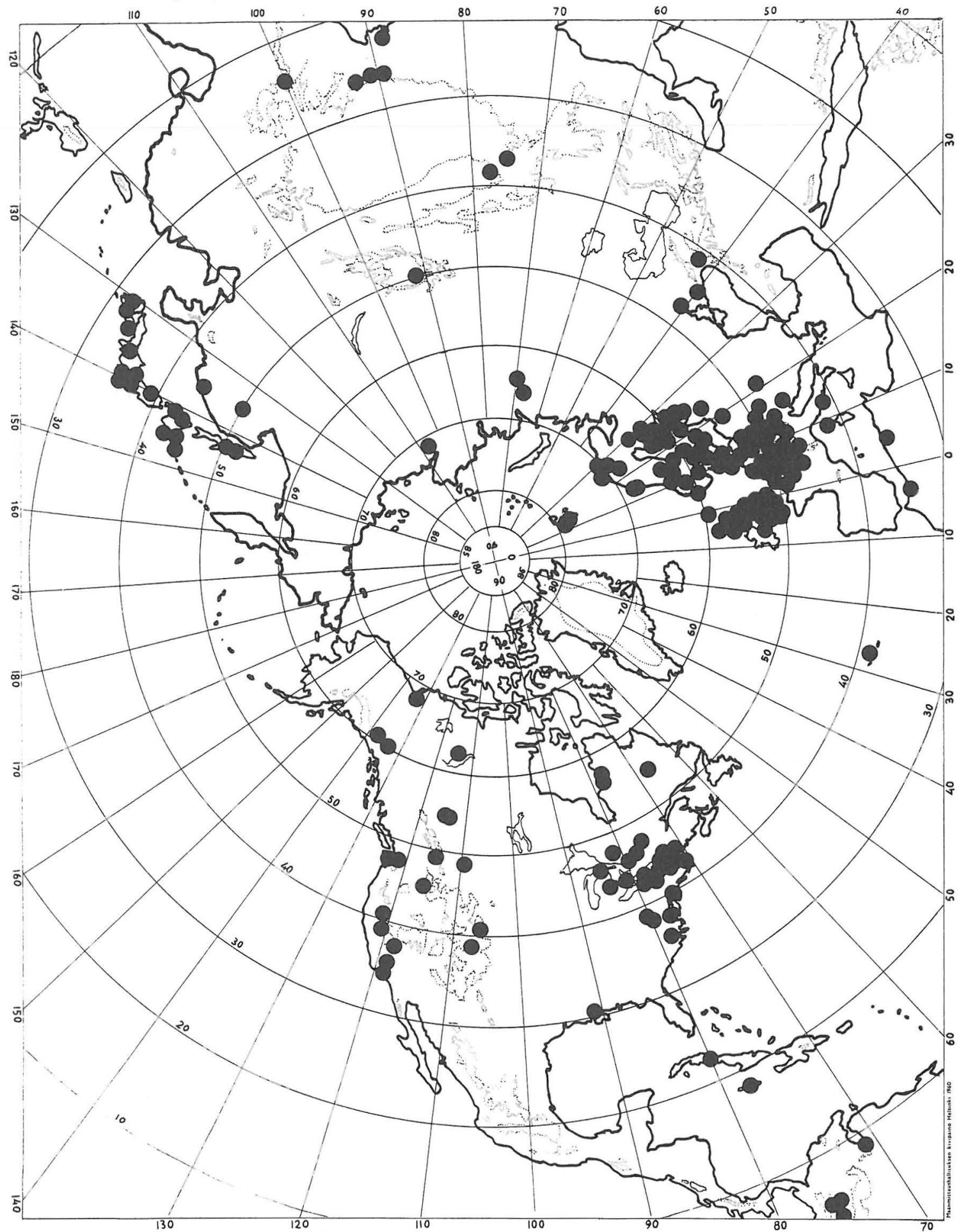

Fig. 15. Collection sites of Hyaloscypha, Phialina and Hamatocanthoscypha. Specimens of Hyaloscypha were collected from almost every site; the two other genera are less widely distributed. Sites not shown in the map: Chile (Valdivia), Tierra del Fuego, Tristan da Cunha, Philippines (Mindanao). 

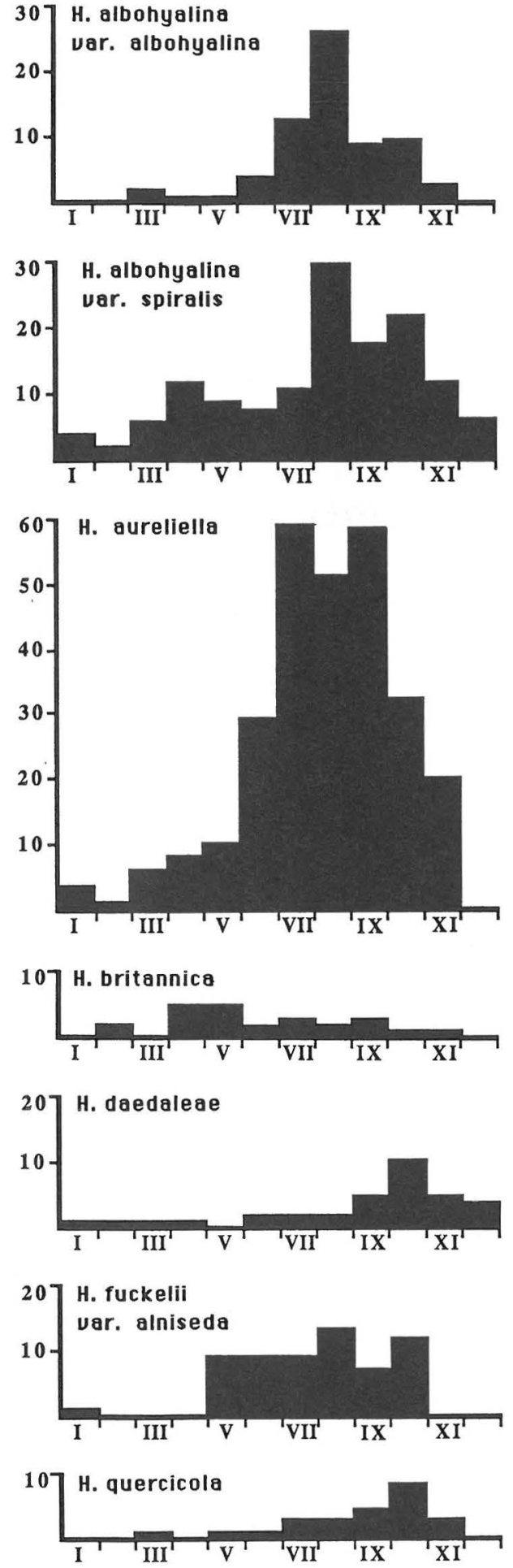
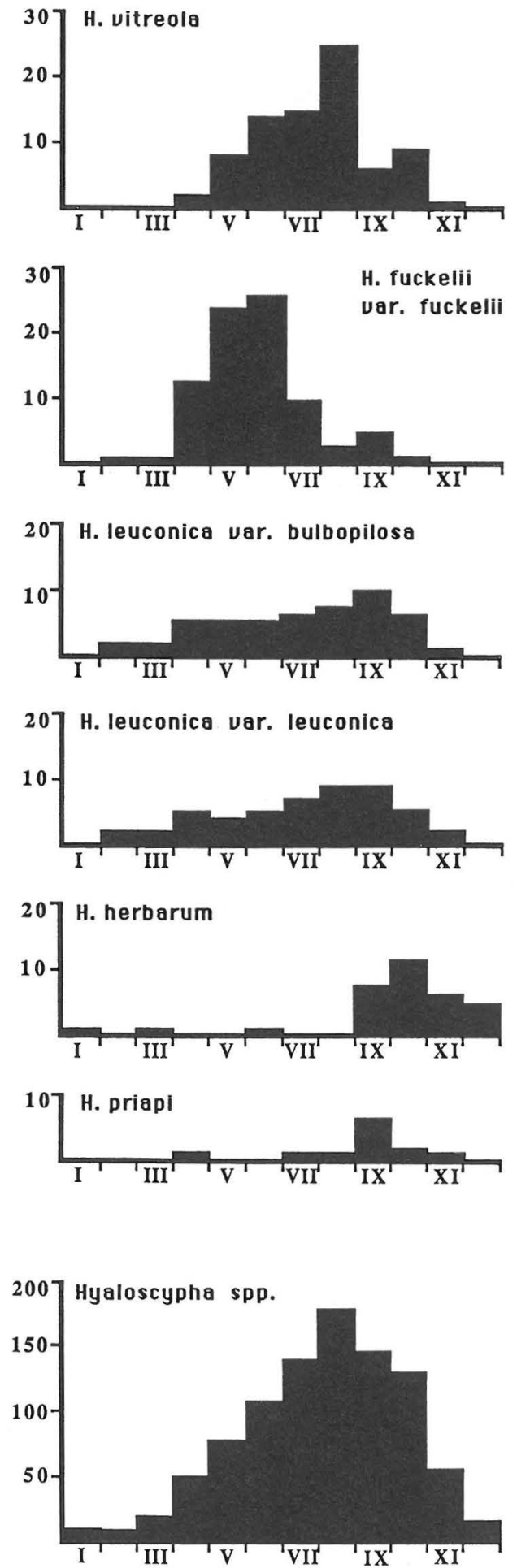

Fig. 16. Phenology of Hyaloscypha in the temperate and boreal zones. 

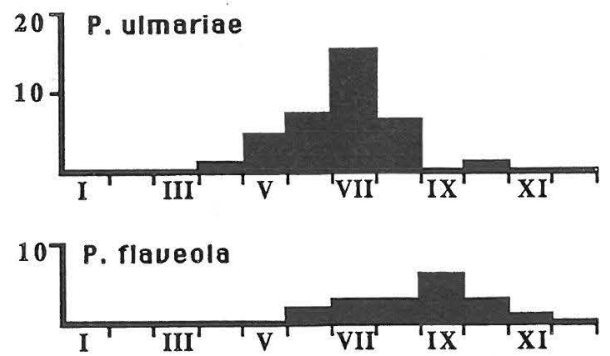

107 P. pseudopuberula
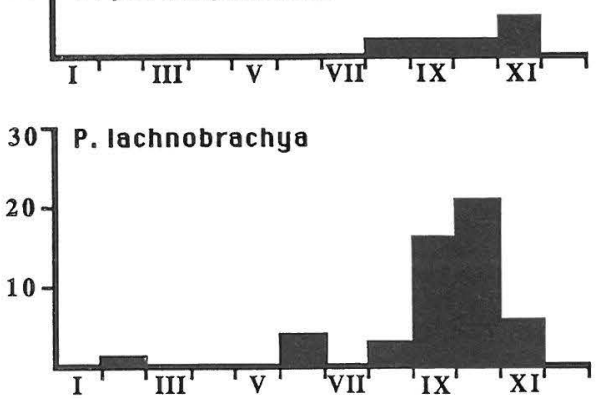
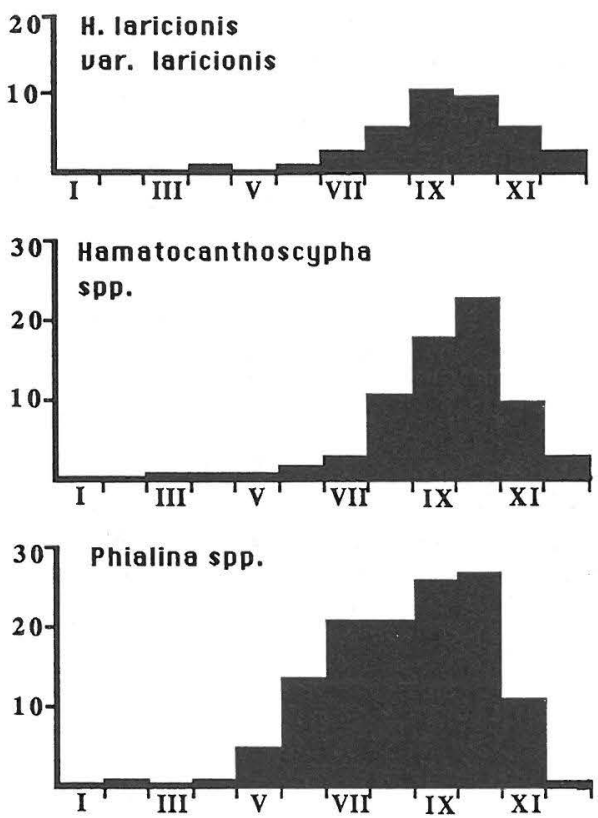

Fig. 17. Phenology of Phialina and Hamatocanthoscypha in the temperate and boreal zones.

\section{Phenology}

Species of the three genera can be observed during the whole frost-free season. The differences in phenology between the genera are slight (Figs. 16,17). In Hyaloscypha the amount of fruiting populations diminishes from September on, whereas in the other two genera the trend is the opposite. Most species in these genera have long fruiting periods, but differences are seen in their optima. The taxa fruiting mainly during the summer months are $P$. ulmariae and the type variety of $H$. fuckelii. $P$. pseudopuberula and $H$. herbarum are rarely found in summer. $H$. daedaleae, $H$. herbarum, $P$. lachnobrachya and $H$. laricionis tend to fruit late in the season. Many of the populations collected during the winter and spring originate from Great Britain and represent late fruiting. No vernal species are known in the three genera.

\section{Taxonomic approach}

The wide variability in these small discomycetes made it inadvisable to use single characters in separating the species. At the generic and species level character combinations were used. The diagnostic value of a given character was found to vary with the group. For example, the yellow pigmentation in
Phialina is common to all the species, whereas in Hamatocanthoscypha it may be characteristic of a species or population. Only the ascal development was considered to be a fundamental character in every genus, always separating a taxon of some rank.

Taxa were recognized as varieties if they differed in at least one character known or likely to be constant and well defined. The admittedly unfortunate word "likely" is due to insufficient collections of some taxa. In such cases the variation patterns, the factors controlling variation and the supposed mechanism of isolation in closely allied taxa were taken into consideration. The key character, which was often ascal development, was usually accompanied by other dissimilarities. When even a large material revealed only slight diverging trends, the varietal level was maintained. These trends were taken as a sign of ongoing divergence. Additional differences, especially when clear-cut, justified recognition at species level. Consequently, the species differ from each other in at least two diagnostic characters (cf. Kuyper 1985 , 1988), morphological, histochemical or ecological.

Although the above principles seem clear, numerous unanswered questions hamper their application. For example, the ecological isolation of Phialina 
lachobrachya, $P$. flaveola and $P$. ulmariae may have resulted in genetic isolation. The hiatus between the three taxa is, however, smaller than that between other species of Phialina. As cultural studies could be of critical value in the delimitation, a conservative decision was made pending such studies. In some cases the question whether the differentiating characters were independent remained unanswered. Are the longer spores in Hamatocanthoscypha laricionis var. dryopteridis Bøhler (Huhtinen) linked with the occurrence on ferns? In $P$. lachnobrachyoides the narrowness of the spores, which is the other main character thought to justify the species status, might be attributed to the fact that the asci are eight-spored. As the variational patterns in $P$. lachnobrachya and $P$. lachnobrachyoides are somewhat similar, the status of the latter is not self-evident. Spore guttulation offers a third difference from $P$. lachnobrachya, but even then intuition is needed in deciding its status. Tentative and intuitive decisions are an inevitable ingredient.

The taxonomical delimitations varied between the genera. In Phialina a wider variation in spore width had to be accepted. Ontogenic variation and the continuum between the populations suggested that the variational pattern in the foliicolous and herbicolous Phialinas may differ critically from that in the two other genera. In Hyaloscypha morphological and chemical discontinuities outnumbered the ecological criteria. In Hamatocanthoscypha and Phialina ecological characters play a more important role.

In view of the wide variation and differences in its pattern between the genera, the use of taxonomic forms was not attractive. The cases in which nonheritable, partially intergrating characteristics might allow separation were many. Hair wall thickness, shape of hairs and dextrinoid reactions showed discontinuities in many taxa of Hyaloscypha, but intergraded in the genus as a whole. Recognition of forms would have complicated the nomenclature, already burdened with numerous varieties. Many potential forms are discussed as variants in the text.

Many of the taxa showed surprisingly wide distribution. My earlier observations on inoperculate discomycetes of Canada and Svalbard also support the view that geographical isolation does not play a major role in their speciation (Huhtinen 1985b, 1987b, 1988). The mycoflora of a given area has often proved less original than expected (e.g., Raitviir \& Galán 1986), and the likelihood of finding subspecies with clearly defined ranges is not great (cf. Davis \& Heywood 1963). Two such cases were noted. Phialina lachnobrachyoides is known from seven collections in the Soviet Far East and Japan, whereas the related $P$. lachnobrachya has an extensive range. They are sympatric, however, and the diagnostic value of the dissimilarities is somewhat vague. A conservative solution was to withhold species status. In the other case species status was accorded. Hyaloscypha strobilicola Huhtinen differs both ecologically and geographically from the widely distributed and closely related $H$. aureliella. Occurrence in warm and arid areas and on conifer cone scales is atypical in $H$. aureliella. The morphological differences were considered sufficiently great for recognition at specific level (cf. Kuyper 1988). 


\section{CULTURAL STUDIES}

\section{Mycelium}

The morphology of the mycelium in culture proved to be a valuable tool in the early stage of this study. Stability in culture contributed to identification of the diagnostic characters and to correction of many narrow concepts. It also became evident that in some taxa the cultural morphology could vary drastically and that stability of the cultural morphology was not always linked with stability of the apothecial morphology. Linkage was seen in, e.g., $H$. quercicola and $H$. priapi. The morphology of the asci, spores and hairs in $H$. albohyalina showed wide variation, but strains of this species originating from a geographically wide area were identical in culture. In $H$. aureliella the situation was the opposite. Self-fertility is apparently not connected with variability of apothecial characters, because taxa with and without production of apothecia in single-spore strains did not differ in their overall variability. The cultural morphology is a valuable taxonomic tool, but must be used with caution Figs. 18-29 illustrate the mycelium of some of the cultivated taxa.

The diagnostic characters of the mycelium (on MA) were: 1) dark versus light overall colour, 2) presence vs. absence and type of aerial mycelium, 3) growth rate, 4) bright colours, 5) presence vs. absence of yeast-like growth, 6) presence vs. absence of mycelial strands, and 7) presence vs. absence of zonation. The differences present on MA disappeared on wood agars, on which all taxa showed a spaced pattern of hyphae. Formation of aerial mycelium was mostly lacking and, when present, was slow.

In many taxa the most constant character in the mycelium was its basic colour on MA. Taxa without any dark colours are rare and thus easily recognized. The amount of aerial hyphae was found to be more variable. The extremes, however, show stability and species normally lacking aerial mycelium develop it only exceptionally, as was seen in a three year old storage tube of $H$. vitreola. Another extreme was $H$. herbarum, always with a densely woolly appearance. Average growth rates are listed in Table 5. All the taxa grew slowly in culture. Two-fold differences between the extremes do, however, occur. And even a difference of a few millimetres in monthly radial growth becomes diagnostic after a few months of cultivation. Yeast-like growth was common in the two species of Hamatocanthoscypha and rare in other taxa. A bright green colour characterized the otherwise snow-white mycelium of $H$. intacta. In $H$. britannica var. roseoguttata Huhtinen the mycelium was clearly tinted red at the outer margin and under a thin blackish cortical layer. Prominent zonation characterized nearly all strains of $H$. daedaleae and was often seen in H. fuckelii var. alniseda (Velen.) Huhtinen, as well.

\section{Anamorph and teleomorph}

Production of teleomorphs and anamorphs was lacking in eight of the 19 taxa cultured. The results are summarized in Table 5. Had the selection of substrates been wider, conidia and teleomorphs would probably have occurred in more taxa. Due to the slow growth rate, the cultures were maintained for long periods. Although they were partly sealed and rarely opened, the risk of contamination was considered high. Hence, in fairly many cases scanty occurrence of a hyphomycete was not treated as a potential anamorph, even if it occurred more than once. These potential anamorphs are not reported here. No farreaching conclusions on relationship can be drawn from the anamorphs. Cheiromycella, Pseudaegerita and Phialophora-type anamorphs were observed in Hyaloscypha (Figs. 30-32). Clathrosphaerina Beverw. has also been connected with the genus (Descals \& Webster 1976). The two cultured species of Hamatocanthoscypha had fairly similar phialidic anamorphs (Figs. 33, 34) and in Phialina an immature, dematiaceous anamorph was seen. But within a certain species or variety the production of an anamorph was highly significant. For instance in $H$. albohyalina var. spiralis the Pseudaegerita anamorph together with stability in the vegetative morphology connected a major part of the variation range. In $H$. aureliella different excipular types were connected by the anamorph.

As also observed by Raschle (1977), the gross apothecial morphology often differed in the cultures. A caespitose habit or restricted hymenium was among the aberrations. But in general the results justified the conclusions drawn from herbarium material. The ascal development was found to be a constant character, as was also the typical pigment of Phialina. The overall morphology of the hairs remained unchanged, e.g., the typical hair shape and their somewhat firm walls in $H$. vitreola and $H$. leuconica var. bulbopilosa (Feltg.) Huhtinen were unaltered on MA and BA. Absence of hair vesture was constant in all but $H$. intacta. In Hyaloscypha, where the hymenial characters are more stable than in Phialina, their conservative nature was confirmed. The general size and shape of the spores remained unchanged. Ascus size was also usually fairly constant. The thickness of the excipular cell walls was mostly 

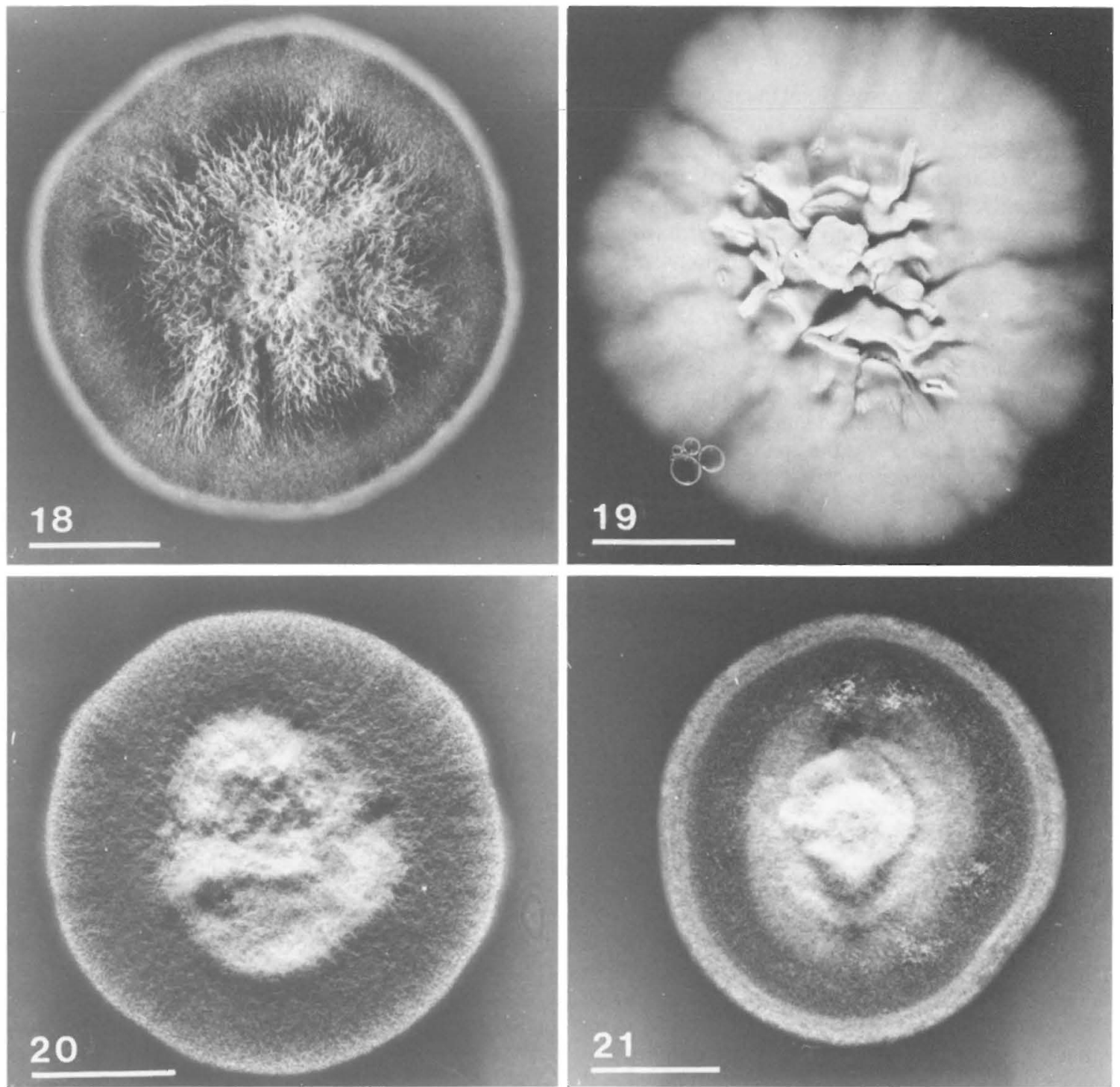

Figs. 18-21. Mycelia of single-spore strains of Hyaloscypha on malt extract agar. - 18: H. fuckelii var. alniseda (SH 86/96). 19: H. vitreola (87/49). - 20: H. fuckelii var. fuckelii (87/47). - 21: H. leuconica var. leuconica (87/91). — Scales $1 \mathrm{~cm}$. - Photos: Matti Tipuri.

stable, but in one strain of $H$. vitreola notably thicker walls were observed in culture. The hair excretory function was seen to be somewhat labile in $H$. intacta (discussed under that species) and $H$. vitreola. One strain of the latter produced abundant resin on the hairs - a feature not observed in nature. This resin was like that seen in $H$. aureliella. Observations on hairs in culture suggest that the often wide variation in hair vesture noted, for instance, in H. albohyalina occurs in many taxa.

Raschle (1977) concluded that the ascus and hair dimensions vary widely in culture, whereas the spore dimensions are stable, even on different substrates. Notable variation in ascus size was found only in $H$. 

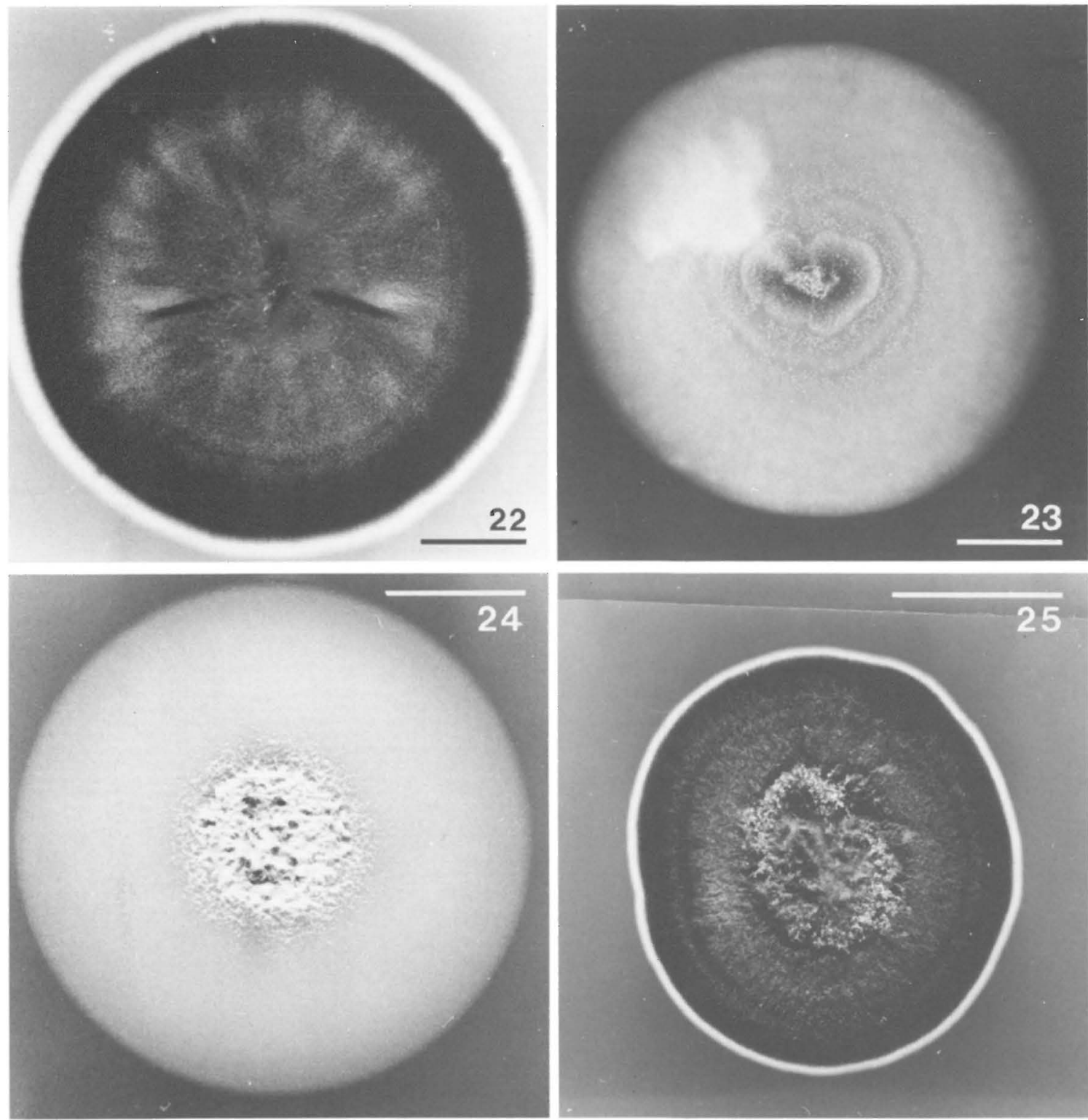

Figs. 22-25. Mycelia of Hyaloscypha and Hamatocanthoscypha on malt extract agar. - 22: Hamatocanthoscypha uncipila (SH 87/179, single-spore). - 23: Hyaloscypha epiporia (isotype, multispore). - 24: Hamatocanthoscypha laricionis var. laricionis (87/ 207, single-spore). - 25: Hyaloscypha daedaleae (86/173, single-spore). — Scales $1 \mathrm{~cm}$. — Photos: Matti Tipuri.

intacta, where the asci in culture were $20 \%$ smaller than in the original population. Variation noted in the hair dimensions in nature was not evident in culture. The prismatic nature of the excipulum showed stability, as also noted by Raschle (1977).

\section{Substrate preference}

Although clearly defined in many taxa in nature, the ecological preferences were less evident in the laboratory. $H$. daedaleae, which is restricted to Quercus 

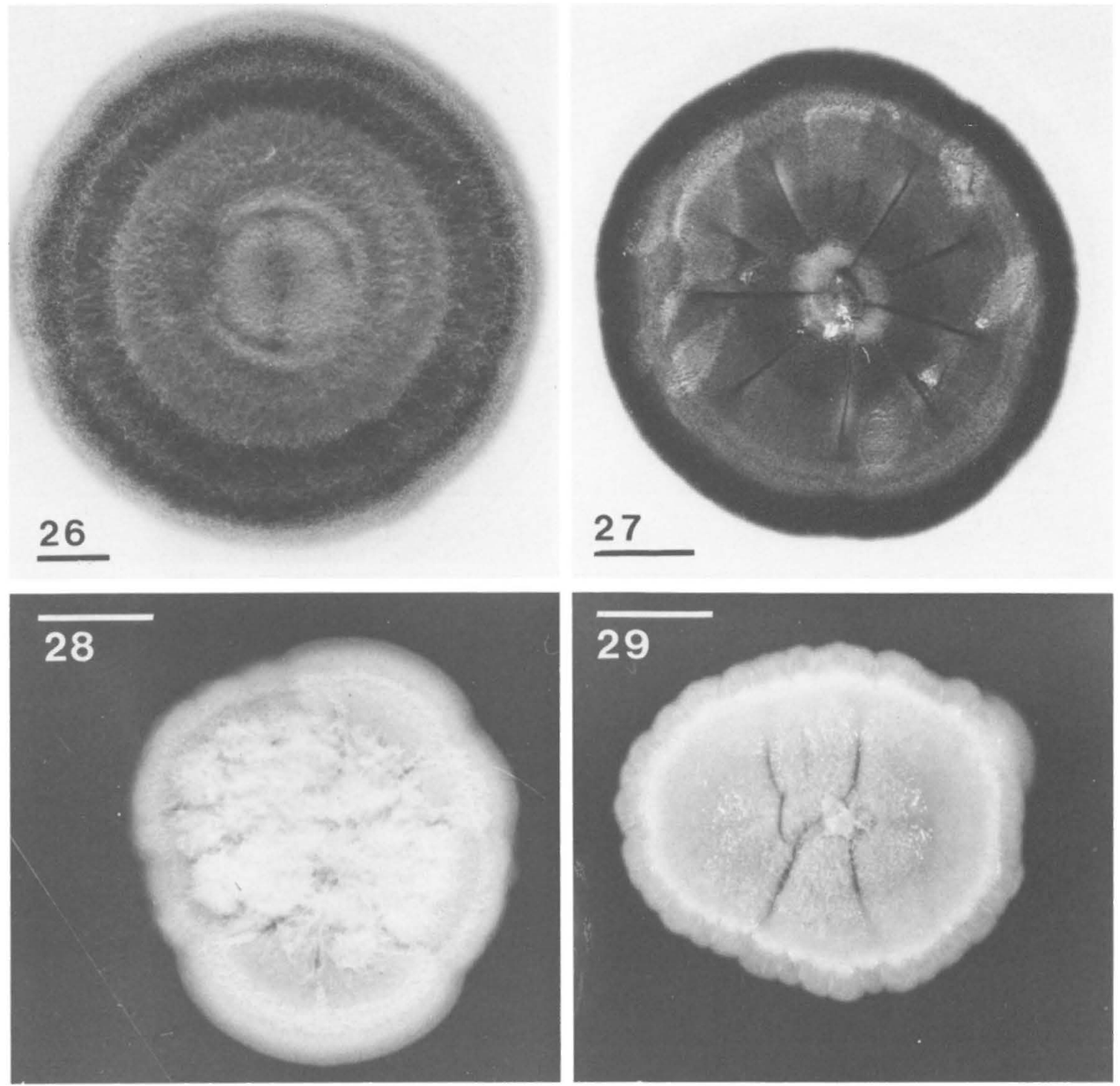

Figs. 26-29. Mycelia of single-spore strains of Hyaloscypha on malt extract agar. - 26: H. herbarum (SH 87/200). - 27: H. daedaleae (86/174). - 28: H. intacta (87/103). - 29: H. quercicola (86/169). — Scales $1 \mathrm{~cm}$. — Photos: Matti Tipuri.

grew well on Pinus agar. H. aureliella, which has never been found on softwood produced, the Cheiromycella anamorph on all the substrates tested and grew equally well on Betula agar, Quercus agar and leaf agar. Production of conidia on the "wrong" type of wood was not, however, observed in any other taxa. Apothecia mostly developed on malt agar or agar mixed with the wood preferred by the species, but the foliicolous Phialina setiigera Huhtinen produced scanty fruit bodies on Betula agar.

Alteration of the substrate by addition of agar, which changes the $\mathrm{pH}$ reaction of the wood, and by autoclaving may prevent substrate recognition. Another possible explanation is the lack of competition from other fungi in laboratory conditions. The ecological preferences seen in nature may be due to 

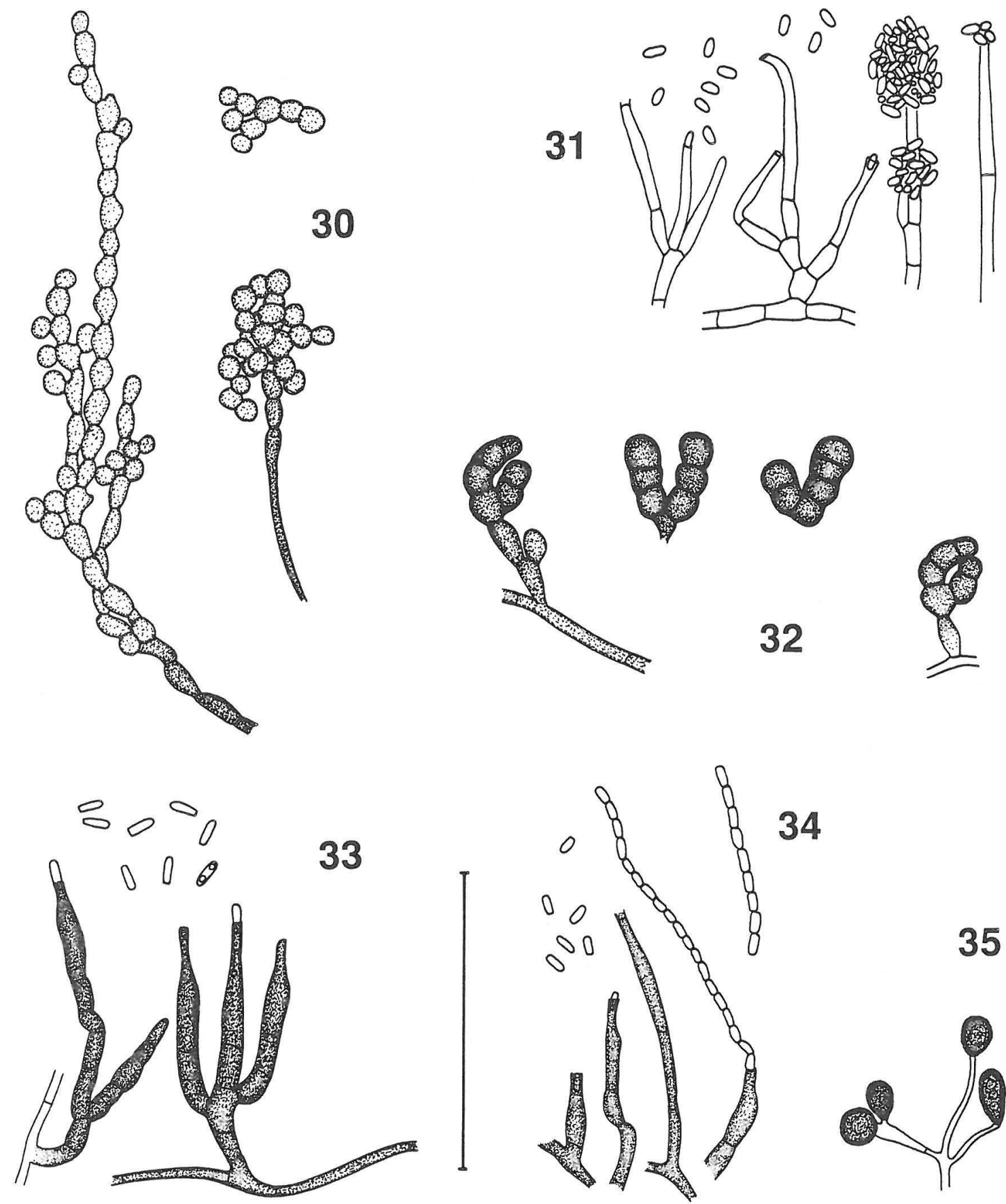

32
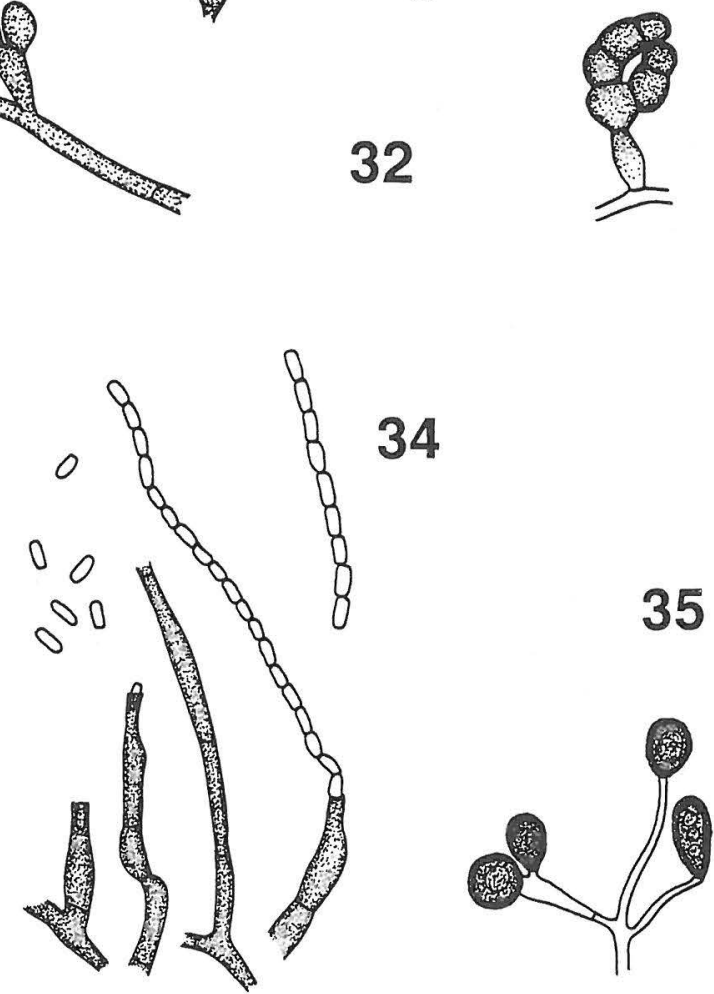

Figs. 30-35. Anamorphs of Hyaloscypha and Hamatocanthoscypha. - 30: Pseudaegerita sp. - 31: Phialophora-type (from Hyaloscypha fuckelii var. fuckelii). - 32: Cheiromycella microscopica. - 33: Phialidic anamorph of Hamatocanthoscypha laricionis var. laricionis. - 34: Phialidic anamorph of Hamatocanthoscypha uncipila. - 35: Chlamydospores produced in old cultures of Hyaloscypha intacta. - Scale $50 \mu \mathrm{m}$. 
Table 5. Cultural characters in Hyaloscypha, Hamatocanthoscypha and Phialina, observed on single-spore strains on malt and wood agars (asterisk = multispore strain). Symbol in parenthesis indicates sterile status; for abbreviations of substrates see "Materials and Methods".

\begin{tabular}{|c|c|c|c|c|c|c|c|}
\hline & $\begin{array}{l}\text { Radial } \\
\text { growth } \\
\mathrm{mm} / 30 \mathrm{~d}\end{array}$ & Anamorph & Months & Subs. & Apoth. & Months & Subs. \\
\hline H. albohyalina var. albohyalina & 13.7 & - & - & - & - & - & - \\
\hline H. albohyalina var. spiralis & 11.7 & + & 4 & all & _- & - & - \\
\hline H. aureliella & 7.0 & + & 1 & all & - & - & - \\
\hline H. britannica var. roseoguttata & 7.2 & - & - & - & - & - & - \\
\hline H. daedaleae & 10.7 & - & - & - & - & - & _- \\
\hline H. epiporia* & 10.4 & - & - & - & + & 18 & MA \\
\hline H. fuckelii var. fuckelii & 9.1 & + & 1 & MA & _- & - & - \\
\hline H. fuckelii var. alniseda & 7.8 & - & - & - & _- & _- & - \\
\hline H. herbarum & 13.8 & _- & _- & _- & _- & _- & - \\
\hline H. intacta & 11.6 & - & - & - & + & 19 & BA \\
\hline H. leuconica var. leuconica & 7.4 & - & - & - & - & - & - \\
\hline H. leuconica var. bulbopilosa & 8.3 & - & - & - & + & 18 & $\begin{array}{c}\text { MA } \\
\text { BA }\end{array}$ \\
\hline H. priapi & 8.3 & _- & _- & _- & _- & _- & DA \\
\hline H. quercicola & 11.8 & - & - & - & - & - & - \\
\hline H. vitreola & 7.6 & - & - & - & + & $\begin{array}{c}8 / 11 \\
16 \\
\text { BA }\end{array}$ & $\begin{array}{l}\text { BA } \\
\text { MA }\end{array}$ \\
\hline H. laricionis var. laricionis & 10.9 & + & 9 & all & $(+)$ & 9 & MA \\
\hline H. uncipila & 15.1 & + & 8 & $\begin{array}{l}\text { PA } \\
\text { QA }\end{array}$ & - & - & - \\
\hline P. lachnobrachya & 10.3 & $(+)$ & 9 & MA & _- & - & - \\
\hline$P$. setiigera & 10.7 & - & - & - & + & 10 & $\begin{array}{l}\text { MA } \\
\text { BA }\end{array}$ \\
\hline
\end{tabular}

competition rather than to the physical or chemical properties of the wood. Moreover, although fruiting on hardwoods has not been observed in, e.g., $H$. aureliella, this is not definite proof that mycelia never develop on hardwoods. Interestingly, Raschle (1977) concluded that apothecial production in the glassyhaired Hyaloscyphaceae occurred on an "unnatural" substrate under laboratory conditions. His observation seems to be based on a single species, Unguicularia millepunctata (Lib.) Dennis. Raschle used fern stems as substrate, which are ecologically not very different from the natural substrates of this herbicolous species. 


\section{MATERIALS AND METHODS}

\section{Morphological studies}

Material was received from the following herbaria (abbreviations after Holmgren et al. 1981): BPI, B, BHU, BISH, BR, C, CUP, DAOM, E, F, EXR, G, $\mathrm{H}, \mathrm{HO}, \mathrm{IMI}, \mathrm{JE}, \mathrm{K}, \mathrm{L}, \mathrm{M}$, MICH, MPU, NY, NYS, O, PC, PAD, PRM, RO, S, TAA, TUR, UPS, W. Material was also received from the following private herbaria: Herb. Arendholz, Herb. Galan, Herb. Graddon, Herb. Korf, Herb. Matheis. Collections were also received from Dr. H.O. Baral, Dr. P.F. Cannon, Mr. L. Kosonen, Mr. T. Lassøe, Mr. S. Olsen,-Dr. B. Spooner, Dr. Å. Strid, Mr. U. Söderholm, Mr. R. Toft and Mr. J. Vauras. At a rough estimate, the collections studied represent 70 $80 \%$ of the available herbarium material of Hyaloscypha, Hamatocanthoscypha and Phialina.

The material was studied with a Wild 20 research microscope with bright field and phase contrast optics. A magnification of ca. x1500 was used. Observations of apothecial colours under the microscope and the intensity of the chemical reactions were made with $\times 150$ magnification. The great majority of the over 1700 collections were depicted in detail. They were drawn with a drawing tube to the scale of $50 \mu \mathrm{m}=68 \mathrm{~mm}$. In a number of the drawings, the scale is 3\% too long. A set of copies of all drawings will be deposited in TUR. Copies will also be attached to the herbarium specimens.

Fresh material was available for $50 \%$ of the taxa. These collections were first studied in water to retain the structure and dimensions of living specimens. The wording "when fresh in water" indicates the characters of living material. Water or water-based dyes were also used to check the presence of resin in herbarium specimens. Most of the observations were made on material in CB and MLZ (for abbreviations, see below). Squash mounts were used except for preparing Figs. 9-11, for which the apothecia were fixed in 3\% glutaraldehyde, embedded in epoxy resin and cut on an ultramicrotome (ca. $1 \mu \mathrm{m}$ sections). The number of apothecia studied from each population was mostly 2-10.

MLZ was used without heating. For well-known taxa, the apothecium was placed straight into a drop of MLZ, allowed to soak for a minute and then squashed. For critical specimens, MLZ was added to the margin of the coverslip of a water mount and allowed to saturate the mount, while the chemical reactions were observed. This procedure occasionally gave only a faint dextrinoid reaction. In these cases another mount was made by placing material straight into MLZ. If the MLZ reaction was very faint or fragmentary, the apothecium was allowed to soak in MLZ for several minutes before adding new reagent, applying the coverslip and checking the reaction anew. $\mathrm{KOH}$ pretreatment was used to check the stability of a lacking MLZ reaction. The apothecium was placed in a large drop of $10 \% \mathrm{KOH}$, which was gently heated until it had clearly diminished (the concentration of $\mathrm{KOH}$ increasing). The excess $\mathrm{KOH}$ was then removed, the apothecium was rinsed twice with water, MLZ was added, and a coverslip applied. The CB mounts were heated twice until smoke developed. The excess reagent was removed, and a drop of lactophenol was added before putting on the coverslip. The slide was gently heated and squashed. This procedure ensures a strong contrast between the deeply stained tissue and light background. The CR mounts were heated gently, as were also mounts made in water-based dyes. The excess dye was removed to obtain a neutral background. Mounts in LUG were made by the methods of Baral (1987b), the reagent being allowed to saturate the water mount.

When not otherwise stated, "MLZ++" or "MLZ+" indicate strong and moderate/faint reactions without $\mathrm{KOH}$ pretreatment. For ascal plugs they signify an amyloid reaction, for other structures a dextrinoid reaction. For staining reactions in CR the intensity is indicated as CR++ or CR+. As regards the dyes, the reaction was strong and more or less uniform unless otherwise stated. "LUG+" indicates a hemiamyloid reaction in ascal plugs or contents (Baral 1987b). All the reactions reported are for dry material, unless otherwise stated. The formulas for the reagents are:

1. Melzer's reagent (MLZ): $0.5 \mathrm{~g}$ iodine, $1.5 \mathrm{~g} \mathrm{KI}, 20 \mathrm{~g}$ chloral hydrate, $20 \mathrm{ml}$ distilled water.

2. IKI solution (LUG): A solution with $1 \%$ of iodine (Baral 1987b: 409).

3. Cotton blue (CB): $0.5 \mathrm{~g}$ of cotton blue (Hollborn, Leipzig), dissolved in $99.5 \mathrm{ml}$ of lactophenol (equal proportions of phenol, glycerine, lactic acid and distilled water).

4. Congo red (CR): A saturated solution in concentrated (25\%) ammonium hydroxide, $\mathrm{pH} 12$.

5. Basic fuchsin (BF), cresyl blue (CRB), neutral red (NR), andtoluidine blue (TB) were used as strong aqueous solutions.

The depicted spores and asci were selected at random. Measurements were made from the drawings. Only discharged spores and asci with mature ascospores were measured. An "illusory" accuracy of $0.1 \mu \mathrm{m}$ was used in measuring the spores and ascus width. Such measurements were made only in calculating the individual $Q$ values (for each spore and ascus) and the mean population values. For statistical calculations and preparation of sporograms, a classification with $0.5-\mu \mathrm{m}$ class intervals was used. Ascus 
length was measured to the nearest $1 \mu \mathrm{m}$ and the class interval in statistical calculations was $5 \mu \mathrm{m}$. The measurements and means cover a major part of the collections studied, though not always based on measurements of exactly the same specimens. The number of observations per specimen also varied. The danger of over-representation of critical specimens, which needed a closer scrutiny, was avoided by studying a large number of populations in most taxa. The values for fresh material mostly originate from my own collections, the geographical and ecological coverage being smaller than for measurements of material in CB and MLZ. The excipulum was depicted and measured in surface view and the wall thickness measured on the joined wall of adjacent middle flank cells. For paraphyses the width was measured in roughly the lower third of the asci, unless otherwise stated. Hair length was measured on the protruding part (see section Morphology). Sporograms and distribution graphs for spore $Q$ values are based on measurements of material in CB and MLZ and the pooled data of individual spores of all the collections studied. Mean Q values for asci and spores were calculated from the individual $Q$ values of the whole material, whereas for excipular cells they were calculated from the overall mean values for length and width.

Spore and ascal measurements are given with the $90 \%$ confidence limits. These were not determined with the formula recommended by Parmasto and Parmasto (1987), because in most cases the distribution patterns were skewed. The limits were set by excluding $5 \%$ of the variational range from each end. The calculations were made from the tabulations for standard deviation. The extreme value, lying outside the tolerance limit, is given in parenthesis.

The descriptions are based on personal observations, unless otherwise stated. For lignicolous specimens the substrate was assigned, on the basis of a transverse section, to one of three categories: hardwood, softwood, Quercus wood. When the substrate falls into the correct category, the tree genus given by the collector is accepted and listed in the section Ecology for each species.

For scanning electron micrographs apothecia were fixed in 3\% glutaraldehyde, dehydrated in an ethanol series and dried with a critical point dryer. Samples were coated with gold and examined in a JEOL JEM 100 CX scanning electron microscope.

The following abbreviations were used (see also formulas above): AR = Raitviir, JAN = Nannfeldt, $\mathrm{JV}=$ Velenovský, $\mathrm{SH}=$ Huhtinen. Colours are given according to the code of Cailleux (1981) and rarely that of Küppers (1978).
When not otherwise indicated, the figures were drawn from $\mathrm{CB}$ mounts. "CB, MLZ" = drawing composed of elements in both reagents.

\section{Cultural studies}

The populations from which strains were isolated are marked with an asterisk in the lists of studied material. Mostly, four single-spore isolates were taken from a population. An apothecium was placed on a Petri dish and spores were teased apart at $\times 150$ magnification using a micromanipulator. In view of the closeness of the apothecia, the strains do not necessarily originate from a single apothecium.

Strains were transferred to storage tubes after 1-2 weeks, which were kept in the dark at $+5^{\circ} \mathrm{C}$. For storage and observations of growth rate and mycelial morphology, 2\% Difco Bacto Agar with 1.5\% malt extract was used (abbreviation MA). An inoculum of $2 \times 2 \mathrm{~mm}$ was placed in a $90 \mathrm{~mm}$ Petri dish for cultural studies. The strains were grown at $+15^{\circ} \mathrm{C}$ with a combination of normal and blue light and a $12 / 12 \mathrm{~h}$ : light/dark cycle. The diameter was measured at monthly intervals.

In the present monograph the term anamorph refers solely to those states of fungi where conidia are produced. Testing for teleomorph or anamorph production was carried out under similar conditions on malt agar and on the following substrates:

1. Betula agar (BA): $2 \%$ agar with $0.6 \%$ of decayed birch wood from various sources, $\mathrm{pH}$ of the wood $4.5, \mathrm{pH}$ of $\mathrm{BA}$ after autoclaving 4.4 .

2. Pinus-Picea agar (PA): $6 \%$ agar with $0.4 \%$ of decayed softwood from various sources, $\mathrm{pH}$ of the wood $3.8, \mathrm{pH}$ of PA after autoclaving 4.5.

3. Quercus agar (QA): $6 \%$ agar with $0.4 \%$ of brown-rotted wood (probably decomposed by Laetiporus sulphureus (Bull.: Fr.) Murr., pH of wood 3.4, pH of QA 4.0.

4. Leaf agar (LA): $2 \%$ agar with $0.2 \%$ of mixed deciduous leaf litter (Populus tremula, Betula, Salix).

Due to the long time required for anamorph and teleomorph production, the Petri dishes were partially sealed with plastic tape or placed in a half-open plastic bag. When apothecia developed, the spores were isolated and their vitality ascertained. Dried cultures were placed with the herbarium specimens.

To check the ecological requirements of the species, a few strains were isolated and/or grown on wood and leaf agars. The ability to grow on a particular substrate was evaluated from the abundance/ scantiness of hyphae on the agar surface, from the depth to which the hyphae grew in the agar and from the overall growth rate. In cases in which spores were not directly isolated on wood agars, the observations were made bearing in mind the nutrition in the inoculum with MA. 


\section{TAXONOMIC PART}

\section{A synoptic key to the species and varieties of Hyaloscypha, Hamatocantho- scypha and Phialina}

The characters are presented roughly according to their diagnostic value. Cultural characters, although often diagnostic, are listed at the end of the key because of their infrequent application in routine work. Enter the key in the first section. If your specimen is not keyed out after going through the first section the other sections need to be checked. Parentheses indicate a marginal character in the taxon concerned. When the same taxon is listed under two character states without parentheses, both characters are equally characteristic. Spore dimensions refer to $\mathrm{CB}$ and MLZ mounts, ascus dimensions to MLZ mounts.

\section{SECTION 1}

\section{Ascus development}

Asci arise from croziers: $1,4,5,6,8,11,15,17,22,24,26$, $27,28,29,30,32,33,34,35,36,37,38,39,40,41,42,43$, 44,45

Asci arise from simple septa: $2,3,7,9,10,12,13,14,16$, $18,19,20,21,23,25,31$

\section{Ascus reaction}

Ascal pores MLZt: 1, 2, 3, 4, 5, 7, 8, 9, 10, 11, 12, 13, 16, $17,19,20,22,23,24,25,26,27,29,30,31,33,34,35,36$, $37,38,39,40,41,42,43,44,45$

Ascal pores MLZ+ only after KOH pretreatment: (1), (2), (11), 18,26

Ascal pores MLZ-: 6, 14, 15, 21, 28, 32, 37

\section{Number of spores}

Asci four-spored: $38,40,(42), 45$

Asci eight-spored: 1-37, 39, 41, 42, 43, 44

\section{Mean spore width ( $90 \%$ confidence limits)}

$<2 \mu \mathrm{m}: 4,7,8,9,10,11,12,13,16,17,20,21,22,23$, $24,25,29,30,31,34,35,36,37,38,39,41,43,45$

$2.0<2.5 \mu \mathrm{m}: 1,2,4,5,7,8,9,10,11,12,13,(14), 16,17$, $19,(20), 21,(22), 24,26,(30), 32,33,34,(35), 36,37,38$, $39,40,(41), 42,43,45$

$2.5<3.0 \mu \mathrm{m}: 1,2,3,5,6,10,13,14,(16), 19,(21), 26,27$, $28,32,(33),(36), 37,38,40,42,43,44,(45)$
$3.0<3.5 \mu \mathrm{m}: 1,2,3,6,14,15,18,26,27,28,32,38,40$, 42,44

$>3.5 \mu \mathrm{m}: 3,14,15,18,28,42$

\section{Mean spore length ( $90 \%$ confidence limits)}

$<7 \mu \mathrm{m}: 4,7,8,9,10,11,12,(15), 16,17,19,20,21,23$, $24,25,29,30,31,32,33,34,35,36,37,39,43$

$7<10 \mu \mathrm{m}: 1,2,3,4,5,6,7,8$, (9), 10,11, 12, 13,14, 15, $16,17,18,19,20,21,22,23,26,27,(28),(29), 30,35,36$, $37,39,42,(43), 44$

$10<12 \mu \mathrm{m}: 1,2,3,5,6,13,14,18,(22),(23), 26,27,28$, $36,38,40,41,42,44,45$

$>12 \mu \mathrm{m}: 1,3,13,14,27,(28), 38,40,41,42,45$

\section{Hair reaction (without $\mathrm{KOH}$ pretreatment)}

Hair walls dextrinoid: $1,2,8,9,10,11,12,13,14,16,17$, $19,(20), 21,22,23,26,27$

Hair walls not dextrinoid: $1,2,3,4,5,6,7,8,10,(13), 15$, $18,20,(21), 23,24,25,(26), 28,29,30,31,32,33,34,35$, $36,37,38,39,40,41,42,43,44,45$

Hairs may show amyloid areas: 4, 5, 18

\section{Hair shape}

Hairs conical, apex tapering to a narrow point: $1,2,3,8,11$, $12,13,14,15,16,17,(20), 22,23,24,25,26,27,33,34$, $38,40,41,42,43,44,45$

Hairs conical, apex broad, blunt: 2, 3, 4, 5, 6, 7, 15, 18

Hairs conical, apex cincinnate-irregularly bent: $3,10,19,26$, $38,39,40,41,45$

Hair uncinate: $9,19,29,30,31,32,(33), 34,35,36,37,39$, 42

Hairs cylindrical-conical: 20

Hairs helicoid: 28

Hair apices may be knob-like: 11, 12, (13)

Hair apices may be branched: 42,43

\section{Resinous exudates (in water mounts)}

Hairs bearing prominent lumps of resin: 4, 5, 6, 7, 18, 24, 25

Hairs may have traces of resin or an apical lump: $1,2,3,8$, $13,(16),(17), 21,22 ?, 23,26,42$ 
Hairs not bearing resinous exudates: (1), (2), 3, (8), 9, 10, 11, $12,13,14,15,16,17,19,20,26,27,28,29,30,31,32,33$, $34,35,36,37,38,39,40,41,42,43,44,45$

\section{Hair wall thickness}

Hairs may show apical solidification: 1, 2, 3, (8), 9, 10, 11, 12 , (13), 14, (17), (19), 20, 21, 22, 23, 26, (27), (29), (34), (40), 42

Hairs may show localized thickenings: $1,(2),(11), 12,16,17$, $27,(44)$

Hairs may be firm-walled: (1), (10), (11), 15, 16, 17, 19, 27, 40,44

Only the outer curve of uncinate hairs firm: 35

\section{Hair vesture (in $C B$ and $M L Z$ )}

Warts lacking: (1), (2), 4, 5, 6, 7, 8,9, 10, 11, 12, 13, 14, $15,16,17,18,19,20,(21), 22,23,24,25,26,27,28,29$, $30,31,32,33,34,35,36,37,38,39,40,41,42,43,44,45$

Hairs warted: $1,2,3$, (4), (6), (7), 8, (9), (11), (12), (13), (14), $18,20,21,(22),(23), 24,25,(27),(29),(32),(34),(35),(36)$, (37), 38, 40, 41, (45)

\section{Ecology}

On bulky hardwood (excluding oak wood): 1, 2, 11, 12,13, $14,16,17,18,19,20,21,26 ?, 27,37$

On bulky oak wood: (1), 2, 8, 16, (17), 20, 21, 37

On bulky softwood: $1,2,3,4,5,6,7,9$, (11), (12), (13), 16, 17,37

On softwood bark: 1, (2), (4), 5, 19, 13, 26?, (27), 34, 35

On hardwood bark: 1, (2), 11, 12, 13, (14), 16, 21, 32

On arboreal litter: (2), 13, 15, 16, 17, 24, 25, 29, 31, 36, 37, (40)

On herbaceous litter: $11,13,(16),(17), 22,23,28,29,30$, $38,43,45$

On leaves: (13), 39, 40, 41, 42, 43, 44, (45)

On polypores: $10,(11)$

\section{SECTION 2}

\section{Yellow pigment inside hairs and paraphyses}

Pigment lacking: $1,2,3,4,5,6,7,8,9,10,11,12,13,14$, $15,16,17,18,19,20,21,22,23,24,25,26,27,28,29,30$, $31,32,33,34,35,36,37$
Amorphous pigment, turning golden yellow in MLZ, frequently present: $38,39,40,41,42,43,44,45$

Diffuse pigment, dissolving in MLZ, present: (29), 37

\section{Mean ascus width ( $90 \%$ confidence limits):}

Up to $5 \mu \mathrm{m}: 7,9,22,23,24,25,29,31,33,34,35,43$

$5-6 \mu \mathrm{m}: 4,8,10,11,12,16,17,20,30,32,37,39,41,44$, 45

$>6-8 \mu \mathrm{m}: 1,2$, (4), 5, 6, (12), 13, 15, 19, 21, 26, 36, 38, (39), 40, 42, (44), (45)

$>8 \mu \mathrm{m}:(1), 3,(6), 14,(15), 18,27,28$

\section{Ascus length ( $90 \%$ confidence limits)}

Up to $35 \mu \mathrm{m}: 20,23,29,31,33,39,43$

$>35-50 \mu \mathrm{m}: 4,7,8,9,11,12,15,16,17,19,(21), 22,24$, $25,26,30,(33), 34,35,36,37,38,40,41,45$

$>50-60 \mu \mathrm{m}: 1,2,5,6,10,13,21,32,44$

$>60 \mu \mathrm{m}: 3,14,18,27,28,42$

\section{Pigmentation of excipular walls (under the microscope)}

Walls hyaline: $1,2,3,4,5,6,7,8,9,10,11,12,13,14,15$, $16,17,18,19,20,21,22,23,24,25,26,27,28,29,30,31$, (36), $37,38,39,40,41,44,45$

Walls on middle flanks brownish or yellowish: (3), (4), (13), $32,33,34,35,36,(37), 42,43$

\section{Excipular wall thickness}

Up to $0.6 \mu \mathrm{m}$ : all taxa except $4,8,9,29$ which are thicker

Up to $1.0 \mu \mathrm{m}: 1,2,4,6,8,9,10,11,12,13,14,15,16,17$, $29,33,36,38,40,41,42,45$

Up to $1.5 \mu \mathrm{m}: 4,9,10,11,14,15,41,42,45$

Up to $2.5 \mu \mathrm{m}: 15,41,45$

\section{Mean length of middle flank cells}

Up to $10 \mu \mathrm{m}: 1,2,3,4,(5), 8,11,12,(13), 14,18,19,20$, $21,23,24,25,26,35,36,38,39,40,41,42,43$

$>10 \mu \mathrm{m}: 1,2,3,4,5,6,7,9,10,11,12,13,15,16,17$, $18,22,23,26,27,28,29,30,31,32,33,34,37,38,(40)$, 44,45 


\section{Length of marginal hairs}

Reaching $30 \mu \mathrm{m}: 8,13,14,20,21,23,25,28,29,30,31$, $32,33,34,35,36,37,38,39,40,42,43,45$

Reaching $50 \mu \mathrm{m}: 1,2,3,4,5,6,8,9,10,11,12,13$, (14), $15,18,19,(21), 22,(23), 24,(25), 26,27,(28), 32$, (34), 35, (37), $38,40,41,45$

Reaching $100 \mu \mathrm{m}: 4,5,6,7,11,12,15,16,17,18,27,40,41$

Reaching $150 \mu \mathrm{m}: 16,17,40,41,44$

Reaching $300 \mu \mathrm{m}: 16,17,44$

\section{Hair septation (protruding part)}

Hairs aseptate: all but $40,41,44$

Hairs septate: (4), (8), (11), (12), (16), (17), (18), (19), (21), (27), 28, 29, 30, 31, 32, 33, 34, 35, (36), 37, 38, 40, 41, 44, 45

\section{Spore septation}

Spores aseptate in all populations: $4,7,8,9,15,22,24,25$, $29,31,32,33,34,35,39,41,43,44,45$

Spores may be septate in some populations: $1,2,3,5,6$, (10), (11), (12), 13, 14, (16), (17), (18), 19, 20, 21, 23, 26, $27,28,(30),(36), 37,38,40,42$

\section{Stipe}

Apothecia sessile-shortly stipitate: $1,2,3,4,5,6,7,8,11$, $12,13,14,15,18,19,20,21,22,23,24,25,26,27$, (35), 38 , $39,40,41,42,43,44,45$

Apothecia prominently stipitate: 9, 10, (15), 16, 17, 28, 29, $30,31,32,33,34,35,36,37$, (44), (45)

\section{Size of apothecia (when dry)}

Up to $200 \mu \mathrm{m}$ in diam: $1,2,3,6,9,10,11,12,14,19,21,24$, $25,26,28,30,31,32,34,36,37,39,43$

$200-400 \mu \mathrm{m}: 4,5,7,(11),(12), 13,16,20,22,23,27,29,35$, $38,40,42$

$>400 \mu \mathrm{m}: 4,8,15,17,18,33,41,44,45$

\section{Mean width of paraphyses (excluding the widened apex)}

Up to $1.2 \mu \mathrm{m}: 4,5,6,7,8,20,24,25$

$1.3-1.5 \mu \mathrm{m}: 1,9,14,17,19,21,31,33,35$

$1.6-1.8 \mu \mathrm{m}: 2,3,10,11,12,13,16,18,22,23,26,29,30,32$, $34,36,37$
$1.9-2.0 \mu \mathrm{m}: 27,28,39$

$>2.1 \mu \mathrm{m}: 15,38,40,41,42,43,44,45$

SECTION 3. CULTURAL CHARACTERS (on malt agar).

\section{Anamorph}

Cheiromycella: 4

Pseudaegerita: 2

Phialidic: $11,29,37$

Dematiaceous: 40

\section{Basic colour of mycelium (excluding aerial hyphae)}

Mycelium predominantly light coloured: 4, 10, 11, 12, 14, (17), 20, 21, 27, 29, 40

Mycelium predominantly dark coloured: $1,2,4,6,8,11$, (12), 13, 16, 17, 37, 44

\section{Aerial mycelium}

Aerial mycelium lacking: 4, 8, 10, (11), 12, 14, (16), 17, 20, $21,27,29,37,40$

Aerial mycelium scanty-moderate: $4,6,(8),(12),(14), 17$, $29,(40)$

Aerial mycelium abundant: $1,2,4,11,13,16,17,(29), 44$

\section{Growth rate (radial growth in $\mathrm{mm} / 30$ days)}

7-9 mm: 4, 6, 11, 12, 16, 17, 20, 27

>9-12 mm: 2, 8, 10, 14, 21, 29, 40, 44

$>12 \mathrm{~mm}: 1,13,37$

\section{Bright colours on mycelium}

Producing bright green, yellow, orange or red colours: 6, 14, 29

No bright colours: $1,2,4,8,10,11,12,13,16,17,20,21$, $27,37,40,44$

\section{Zonation}

Mycelium may show colour bands and zones: (4), 8, 12, 14, (27), 29, (40)

Zonation lacking: $1,2,4,6,8,10,11,(12), 13,14,16,17,20$, $21,27,29,37,40,44$ 


\section{Mycelial strands (excluding erect strands on inoculum)}

Mycelial strands frequent: (1), (8), 10, 12, 14, 16, (17), 29, 37

Mycelial strands lacking: $1,2,4,6,8,11,12,13,16,17,20$, $21,27,(29), 40,44$

\section{Yeast-like growth}

Yeast-like growth frequent: (4), 21, 29, 37

Yeast-like growth lacking: $1,2,4,6,8,10,11,12,13,14,16$, $17,20,21,27,40,44$

\section{Hyaloscypha}

Hyaloscypha Boud., Bull. Soc. Mycol. France. 1:118. 1885, nom. cons. prop. - Lectotype: Peziza vitreola P. Karst., Not. Sällsk. Fauna Flora Fenn. Förh. 10:180. 1869, typ. cons. prop., Huhtinen \& Cannon (1987).

Eupezizella Höhnel, Mitt. Bot. Inst. Techn. Hochsch. Wien 3:61. 1926. - Lectotype (selected here): Pezizella candida Starb., Bih. Svensk Vet.-Akad. Handl. 21:30; Pl. 1: 16a-c. 1895.

Truncicola Velen., Monogr. Discom. Bohem.: 289. 1934. - Holotype (the only binomial mentioned): Truncicola parasitica Velen., Monogr. Discom. Bohem.: 289. 1934.

?Hyaloscypha Nannf., Nova Acta Soc. Sci. Upsal. Ser. 4., 8:266. 1932, homon. illeg. (Art. 48.1). For the controversy over the publication of this homonym, see Huhtinen \& Cannon (1987). - Not typified.

Non Hyaloscypha Fuckel sensu Migula, Thome's Kryptogamen-Flora 3(3, 2): 1092. 1913, lapsus calami pro Hyalopeziza Fuckel.

Apothecia gregarious, more rarely confluent, superficial, 50-800(-1 500) $\mu \mathrm{m}$ in diam when fresh, $50-500(-700) \mu \mathrm{m}$ in diam when dry, cupulate, mostly sessile to very shortly stipitate, rarely clearly stipitate. Disc shallow to plano-convex, margin consisting of a thin to prominent layer of hairs, not incurved when fresh, often clearly incurved when dry. Hair cover dense and prominent, more rarely sparse or inconspicuous. Colour pure white to whitish and greyish, more rarely yellowish or yellowish brown when fresh, in one species with rosy red colour at the margin. Dry apothecia yellowish to brownish, more rarely Apricot or reddish brown, these colours modified by the whitish hair cover or by a brownish to Amber resin. Ectal excipulum of textura prismatica, cells lying parallel to the receptacle surface, rarely at a slight angle, on middle flanks with mean length ranging between 7 and $16 \mu \mathrm{m}$, mean width between 2.7 and $7.0 \mu \mathrm{m}$, mean $\mathrm{Q}$ value 2.2 , becoming narrower inwards, more roundish towards the base, towards margin alike or becoming smaller or narrower. Inner excipulum lacking or poorly developed, show- ing weak delimitation from the thin subhymenium. Walls in ectal parts thin to $1 \mu \mathrm{m}$ thick, rarely 1.0 $2.5 \mu \mathrm{m}$ thick, hyaline to very pale brownish or yellowish, MLZ++ (vinaceous) to MLZ-, rarely with amyloid nodules. Hairs narrowly conical-conicallageniform, more rarely almost cylindrical or filiform, straight, rarely apically strongly curved or cincinnate, aseptate, rarely sparsely septate. Apex blunt to tapering, more rarely with a knob, thin-walled to solidified, with or without an apical globule in MLZ. Wall thin to clearly thickened, hyaline, smooth to warted, MLZ++ (vinaceous) to MLZ-, in few taxa with amyloid inclusions. Warts stable to deforming in CB and MLZ. Hairs with or without resinous exudates, these appearing as a small apical lump or as massive deposits, pigment lacking. Asci cylindricalclavate, with a conical apex, in one species apex clearly rounded, eight-spored, mean length ranging between ca. 26 and $62 \mu \mathrm{m}$, mean width between 3.8 and $7.7 \mu \mathrm{m}$ pore $\mathrm{MLZ+}$, more rarely $\mathrm{MLZ}$-, arising from croziers or simple septa. Spores elliptic to oblong-elliptic, rarely cuneiform, cylindrical or broadly elliptic, mean length ranging between 4.4 and $11.3 \mu \mathrm{m}$, mean width between 1.4 and $3.6 \mu \mathrm{m}$, aseptate to septate, septum MLZ- to MLZ+, aguttulate to clearly guttulate. Paraphyses filiform-cylindrical to apically slightly widened, mean width of lower parts ranging between 1 and $2 \mu \mathrm{m}$, in one species $2.7 \mu \mathrm{m}$, not protruding clearly above the level of the asci, unpigmented, sparsely dichotomously branched, rarely with numerous branches close to apex, sparsely septate. Inhabiting bulky wood substrates, more rarely on arboreal or herbaceous litter, only exceptionally on leaves.

\section{Division of Hyaloscypha}

In the genus Hyaloscypha few taxa are restricted to softwood. Three of these, $H$. aureliella, $H$. britannica and $H$. candida, have similar morphological and chemical properties, sharing blunt hairs, occurrence of resin and lack of dextrinoid reactions. H. nipponica, which grows on hardwood, resembles them morphologically and the amyloid reactions link it with $H$. aureliella and $H$. britannica. The fifth species to be included in the same subgenus is $H$. strobilicola, which has slightly different hair characteristics, but in other respects is close to the four other species.

The new subgenus Eupezizella (Höhnel) Huhtinen is here typified with Pezizella candida. When Eupezizella was raised to generic rank by Höhnel (1926), Pezizella candida was cited as "Grundart". As discussed in the section Generic relationships, the later unintentional lectotypification by Nannfeldt (1932) is 
not valid. Although originally recognized under $P e$ zizella (Starbäck 1895), subg. Eupezizella was largely delimited in the same way as here. $H$. aureliella comprised most of the material listed by Starbäck (1895) for his new subgenus. The earlier division of Hyaloscypha into two sections by Bertault (1977) was based on a misconception of the genus. Velenovskýs (1934) subdivisions of the genus have no taxonomic status.

The subgenus Eupezizella differs from the subgenus Hyaloscypha in the following characters: 1) resinous exudates are abundant, 2) the hairs are predominantly blunt, 3 ) dextrinoid reactions are lacking, 4) most species are confined to softwood, and 5) some species and populations show an amyloid reaction in the hairs and/or excipula. The subgenus occupies an intermediary position between subg. Hyaloscypha and the species with long, blunt and septate hairs encountered during the study. Two such species were originally described under Protounguicularia and were later transferred to Arachnopeziza
(Huhtinen 1987d). A study of all such species is needed. At present their multiseptate hairs justify their exclusion from Hyaloscypha.

Hyaloscypha Boud. subg. Eupezizella (Höhnel) Huhtinen, comb. et stat. nov.

Eupezizella Höhnel, Mitt. Bot. Inst. Techn. Hochsch. Wien 3: 61. 1926. - Pezizella Fuckel subg. Eupezizella Starb., Bih. Svensk Vet.-Akad. Handl. 21:29. 1895, nom. inval. (Arts. $21.3+32.1$ (b)). - Lectotype (designated here): Pezizella candida Starb., Bih. Svensk Vet.-Akad. Handl. 21:30; P1. 1:16a-c. 1895.

\section{Hyaloscypha Boud. subg. Hyaloscypha}

Hyaloscypha Boud., Bull. Soc. Mycol. France. 1:118. 1885, nom. cons. prop. - Lectotype: Peziza vitreola P. Karst., Not. Săllsk. Fauna Flora Fenn. Förh. 10:180. 1869, typ. cons. prop. (Huhtinen \& Cannon 1987).

\section{Key to species and varieties of Hyalloscypha}

The spore measurements were made on material mounted in $\mathrm{CB}$ or $\mathrm{MLZ}$, the ascus measurements on material in MLZ.

1 With or without dextrinoid reactions, hairs pointed, rarely blunt, smooth to warted, not bearing abundant resin in water mounts (but may show relatively inconspicuous lumps at hair apices, hairs then not markedly blunt)

- Without overall dextrinoid reactions, hairs blunt, with yellowish, brownish, reddish or hyaline resin usually dissolving in $\mathrm{CB}, \mathrm{MLZ}$...................................................................................................... 2

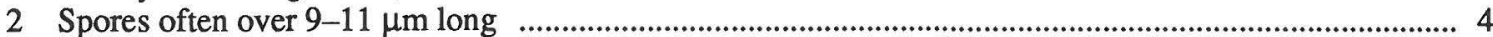

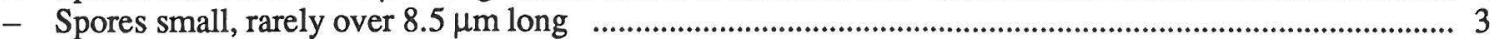

3 . With yellowish brown to brown resin, asci arise from croziers, excipulum and hairs often with amyloid nodules, on softwood .......................................................................................................... $4 \mathrm{H}$. aureliella

- With hyaline resin, asci arise from simple septa on softwood $7 \mathrm{H}$. candida

4 Asci arise from simple septa, spores 3-4 $\mu \mathrm{m}$ wide, rarely septate, excipulum with amyloid nodules, on hardwood 18 H. nipponica

- Asci arise from croziers, spores 2-3 $\mu \mathrm{m}$ wide, often septate, on softwood

5 Resin yellowish brown to brown, ascal plug MLZ+ 5 H. britannica var. britannica

- Resin rosy red under hand lens in fresh condition, ascal plug MLZ - .. 6 H. britannica var. roseoguttata

6 Spore mean length $4.5-8.5 \mu \mathrm{m}$, spores rarely over $10 \mu \mathrm{m}$ long

- Sporemean length over $9.5 \mu \mathrm{m}$, spores mostly over $10 \mu \mathrm{m}$ long

7 Spores over $2 \mu \mathrm{m}$ wide

- Spores almost cylindrical, up to $2 \mu \mathrm{m}$ wide, mean spore width $1.4 \mu \mathrm{m}$

8 Asci $39-47 \mu \mathrm{m}$ long, arising from croziers, hairs narrowly conical $22 H$ secalina var secalina

- Asci 23-36 $\mu \mathrm{m}$ long, arising from simple septa, hairs lageniform 23 H. secalina var. paludicola

9 Asci permanently MLZ-, arising from simple septa, hairs short, mostly smooth, often with apical solidifications, MLZ+ $14 \mathrm{H}$. intacta 
10 Hairs minutely to clearly rough in $\mathrm{MLZ}$, rarely smooth 13

- Hairs predominantly smooth in MLZ (rarely apically sparsely warted) .......................................... 11

11 Asci arise from simple septa, spores clearly elongated, hairs relatively short, $18-40 \mu \mathrm{m}$ long, fruiting on bulky wood, arboreal litter and herbaceous stems $13 \mathrm{H}$. herbarum

- Asci arise from croziers 12

12 Spores oblong-elliptic to phaseoliform, hairs with long, narrow apices of equal width and uniformly thickened wall, no apical solidifications, ascal plugs MLZ+ without $\mathrm{KOH}$ pretreatment, on bulky hardwood

27 H. vitreola

- Hairs narrowly conical to lageniform, walls thin, ascal plugs MLZ - without $\mathrm{KOH}$ pretreatment

26 H. usitata

13 Asci arise from croziers, spores elliptic to oblong-elliptic, often septate, guttulate, dextrinoid reaction frequent in hairs and excipulum, rarely lacking

- Asci arise from simple septa 1 H. albohyalina var. albohyalina

14 Hairs warted to smooth mostly MIZt. oblong-elliptic

2 H. albohyalina var. spiralis

- Hairs with low, platelike crust, apically cincinnate, MLZ-, asci 53-70 $\mu \mathrm{m}$ long, spores 3-4 $\mu \mathrm{m}$ wide, phaseoliform to broadly elliptic to oblong-elliptic, on softwood 3 H. albohyalina var. tigillaris

15 Ascal plugs MLZ+ 17

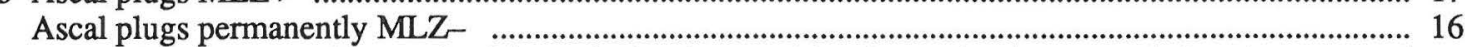

16 Spores $1.5-2.5 \mu \mathrm{m}$ wide, asci with conical apices, arising from simple septa, hairs short, smooth to clearly warted, apical solidifications frequent, excipulum MLZ-, hairs MLZ- or MLZ+

- Spores 3-4 $\mu \mathrm{m}$ wide, asci arise from croziers, apices rounded, hairs 40-60 $\mu \mathrm{m}$ long, smooth, not solidified, excipulum MLZ+, hairs MLZ-

15 H. latispora

17 Hairs straight, slightly bent or undulating to apically cincinnate, asci arise from croziers or simple septa .

- Hairs predominantly uncinate or apically clearly hooked or forked, asci arise from simple septa .......... 18

18 Restricted to pore surface of annual polypores, hair apices frequently solidified, excipulum with thickened walls 10 H. epiporia

- Fruiting on ordinary wood substrates

19 Hairs with apically uniformly thickened, refractive wall, apices tapering to $0.3-1.0 \mu \mathrm{m}$, hairs deep violet in LUG, smooth, mean spore size $8.4 \times 2.5 \mu \mathrm{m}$, spores often over $10 \mu \mathrm{m}$ long, frequently septate

- Hairs thin-walled, smooth to warted, apices blunt, tapering to $1-2 \mu \mathrm{m}$, wall LUG-, mean spore size $6.2 \times$ $2.1 \mu \mathrm{m}$, spores shorter than $7.5 \mu \mathrm{m}$, aseptate 9 H. diabolica

20 Spore mean width over $1.6 \mu \mathrm{m}$ and mean length over $6 \mu \mathrm{m}$, fruiting on various substrates 24

- Spores narrowly cylindrical, mean width $1.4 \mu \mathrm{m}$ or elliptic with mean length $<6 \mu \mathrm{m}$, fruiting on herba-

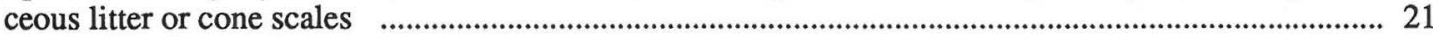

21 On cone scales

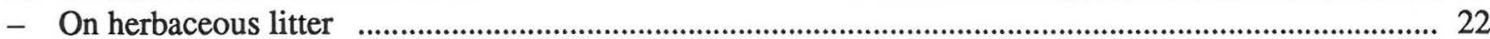

22 Asci 39-47 $\mu \mathrm{m}$ long, arising from croziers, hairs narrowly conical ............... $22 \mathrm{H}$. secalina var. secalina

- Asci 23-36 $\mu \mathrm{m}$ long, arising from simple septa, hairs lageniform ............ $23 \mathrm{H}$. secalina var. paludicola

23 Asci arise from croziers, $28-44 \mu \mathrm{m}$ long, mean spore size $5.4 \times 1.9 \mu \mathrm{m}$, spores often over $6 \mu \mathrm{m}$ long ...... 24 H. strobilicola var. strobilicola

- Asci arise from simple septa, 19-34 $\mu \mathrm{m}$ long, mean spore size $4.4 \times 1.5 \mu \mathrm{m}$, spores rarely over $6 \mu \mathrm{m}$ long 25 H. strobilicola var. parvispora

24 Hairs often almost cylindrical to cylindrical, intermixed with conical hairs, prominently warted, often apically cincinnate, MLZ- or fragmentarily MLZ+, mean spore width $1.7 \mu \mathrm{m}$..................... $20 \mathrm{H}$. priapi

- Hairs lageniform to narrowly conical, relatively clearly tapering towards the apex, mostly MLZ+ for whole length 
25 Spores predominantly cuneiform, short, rarely over $8 \mu \mathrm{m}$ long, hairs narrowly conical to slightly lageniform, predominantly smooth, apothecia occasionally yellowish to naked eye, on Quercus

Majority of spores elliptic, oblong-elliptic or clearly elongated, rarely subfusoid

26 Hairs up to $90 \mu \mathrm{m}$ long, without uniformly thickened and refractive wall, smooth to warted, apices widened or not, solidified or not

- Hairs up to $200 \mu \mathrm{m}$ long, even when shorter with smooth, uniformly thickened, refractive wall, apices not widened or solidified, occasionally cincinnate

27 Asci arise from simple septa, hairs rarely with constrictions

16 H. leuconica var. leuconica

- Asci arise from croziers, hairs often irregularly constricted below 17 H. leuconica var. bulbopilosa

28 Hairs 30-60(-90) $\mu \mathrm{m}$ long, smooth or bearing loose encrustation when fresh in water, smooth in CB, MLZ, tapering to a fine point, mean spore size $7-8 \times 2 \mu \mathrm{m}$, spores rarely over $2.5 \mu \mathrm{m}$ wide or over $10 \mu \mathrm{m}$ long, septation exceptional

- Hairs 30-50 $\mu \mathrm{m}$ long, warted to smooth in CB, MLZ, tapering to a fine point or apically $1-2 \mu \mathrm{m}$ wide, mean spore length $7-13 \mu \mathrm{m}$, mean spore width $2-3 \mu \mathrm{m}$, spores often over $2.5 \mu \mathrm{m}$ wide or over $10 \mu \mathrm{m}$ long, rarely to frequently septate

29 Asci arise from croziers, hairs smooth to conspicuously warted in CB, MLZ

1 H. albohyalina var. albohyalina

Asci arise from simple septa

30 Spores elliptic to oblong-elliptic, hairs with tapering to broad and blunt apices, smooth to conspicuously warted in CB, MLZ, on bulky wood, rarely on litter 2 H. albohyalina var. spiralis

- Spores oblong to oblong-elliptic, hairs smooth to minutely warted in CB, MLZ, often on herbaceous or arboreal litter $13 \mathrm{H}$. herbarum

31 Asci arise from croziers

$11 \mathrm{H}$. fuckelii var. fuckelii

- Asci arise from simple septa $12 H$. fuckelii var. alniseda

1. Hyaloscypha albohyalina var. albohyalina

Hyaloscypha albohyalina (P. Karst.) Boud., Hist. classific. discom. Europe: 127. 1907. - Peziza albohyalina P. Karst., Not. Sällsk. Fauna Flora Fenn. Förh. 10:189. 1869. - Helotium albohyalinum (P. Karst.) P. Karst., Not. Sällsk. Fauna Flora Fenn. Förh. 11:241. 1870. - Pezizella albohyalina (P. Karst.) Rehm ex Sacc., Syll. Fung. 18:57. 1906. — Lectotype (selected here): [Finland,] Tavastia australis, Tammela, Mustiala, ad lign. Alni, 8.X.1868 Karsten (H, Herb. Karsten 579, examined). The specimen was earlier vaguely selected? as lectotype (Dennis 1950: 184) by the wording "may be selected as lectotypus".

Peziza lectissima P. Karst., Not. Sällsk. Fauna Flora Fenn. Förh. 10:184. 1869. - Helotium lectissimum (P. Karst.) P. Karst., Not. Sällsk. Fauna Flora Fenn. Förh. 11:242. 1870. - Phialea lectissima (P. Karst.) Sacc., Syll. Fung. 8:272. 1889. - Hymenoscyphus lectissimus (P. Karst.) O. Kuntze, Revis. gen. pl. 3:485. 1898. - Micropodia lectissima (P. Karst.) Boud., Hist. classific. discom. Europe: 128. 1907. Hyaloscypha lectissima (P. Karst.) Raitv., Scripta Mycol. 1:29. 1970. - Holotype (the only specimen mentioned): [Finland,] Tavastia australis, Tammela, Mustiala, ad Salic. phyl., 29.IX.1867 Karsten (H, Herb. Karst. 589, examined). The specimen has been indicated as lectotype by various authors (in herb.), but is the sole collection, as stated by Karsten (1869). It was cited as holotype by Carpenter (1981).

Chrysothallus pezizellaeformis Velen., Monogr. Discom. Bohem.: 269; P1. 6, Fig. 25. 1934. - Holotype (the only specimen mentioned): [Czechoslovakia,] Bohemia, Mnichovice, collis Plechác, Corylus avellana, XI.1928 Velenovský (PRM 153073, examined).

Hyaloscypha protonematosa Velen., Novitates mycol. noviss.: 138. 1947. - Holotype (indicated as such on the label by Svř̌ek): [Czechoslovakia,] Bohemia, Mnichovice, Mirošvice (viaductus), in fossa aquosa silv. ad lignum carpineum, 18.V.1941 Velenovsky (PRM 151097, examined). In contrast to the original diagnosis, the substrate was found to be coniferous.

Apothecia gregarious, rarely confluent, superficial, up to $800 \mu \mathrm{m}$ in diam when fresh, mostly 50 $200(-300) \mu \mathrm{m}$ in diam when dry, cyathiform to cupulate when juvenile, cupulate when mature, narrowly to broadly sessile or with a gradually tapering base. Disc shallow to plano-convex when fresh, bordered by a thin zone of marginal hairs, which rise only slightly above the hymenium; margin fleshy and incurved when dry, typically obscuring the hymenium. Hair cover dense at margin, sparse to dense below, snow-white. Colour pure white to watery greyish when fresh; dry apothecia ranging from very light yellowish brown to K77 to Straw (K79) to L80, in three populations reddish brown (R15).

Ectal excipulum of textura prismatica, cells on middle flanks regularly prismatic, $8-13(-16) \times 3.0$ 

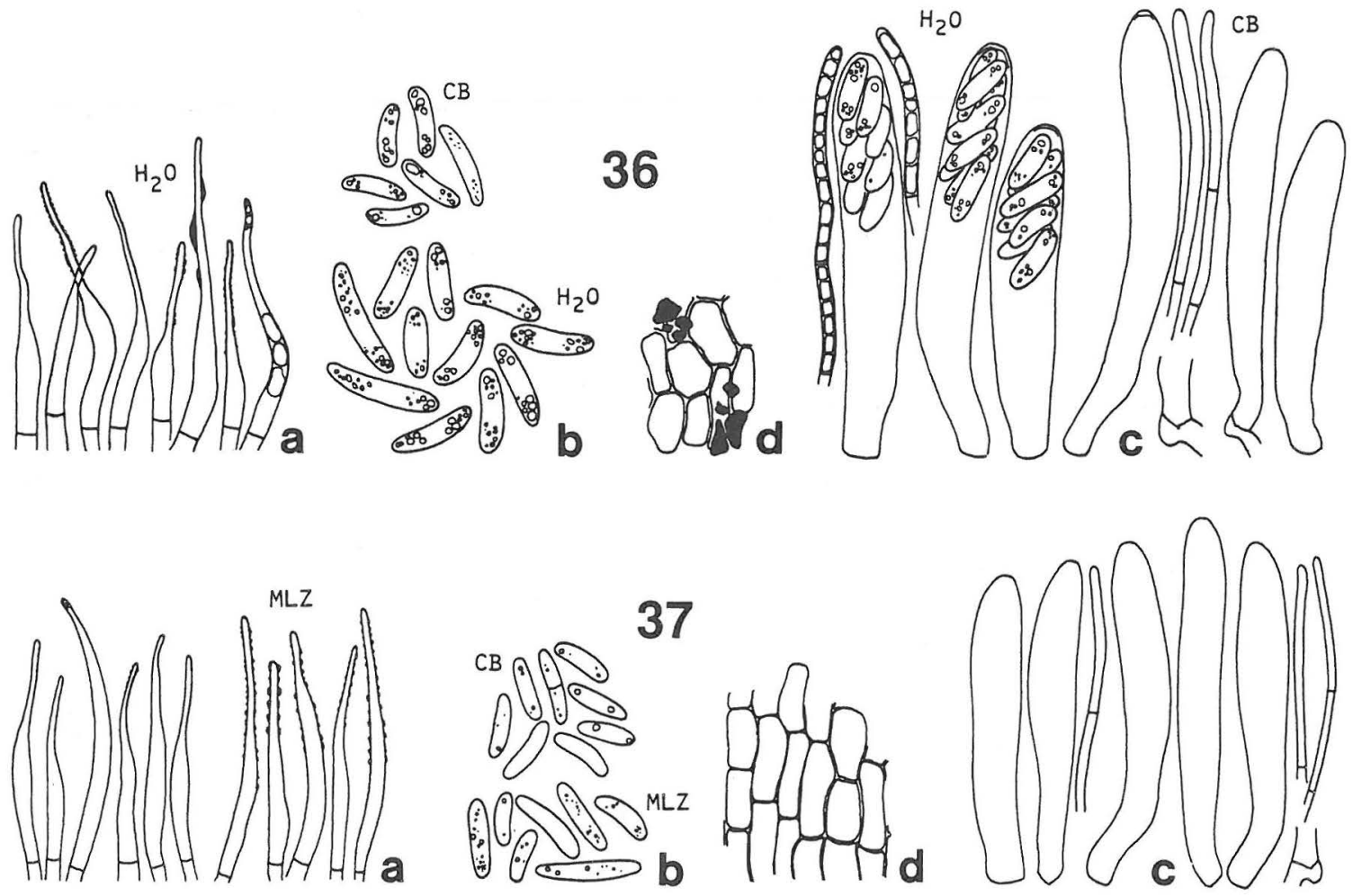

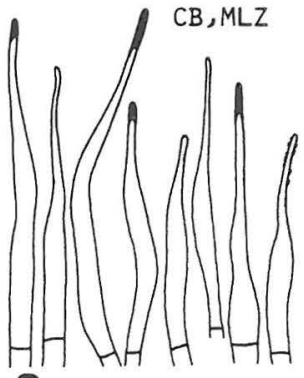

a

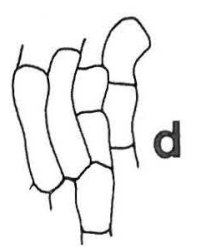

38

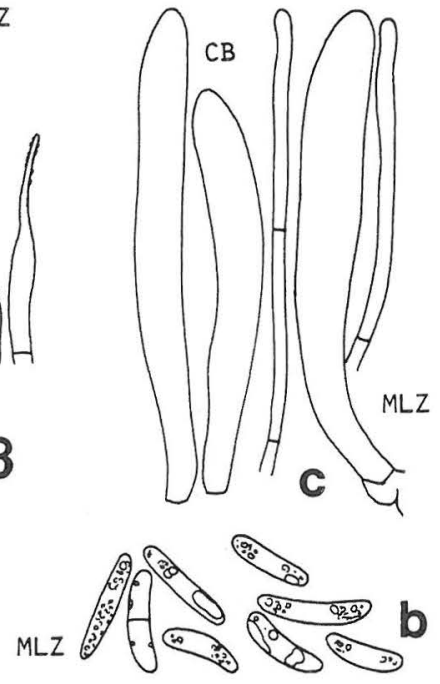

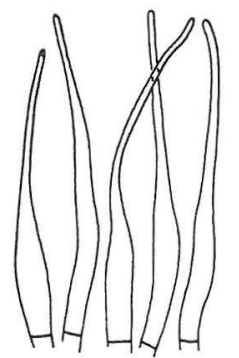

a

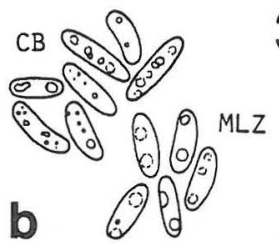

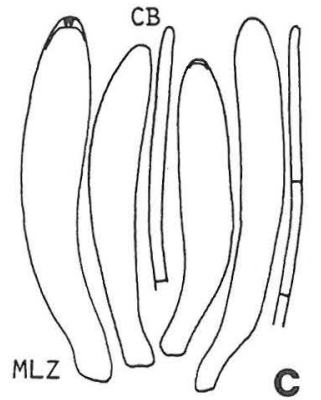

39

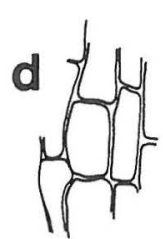

Figs. 36-43. Hyaloscypha albohyalina var. albohyalina. a) hairs, b) spores, c) asci and paraphyses, d) excipulum. Scale $50 \mu \mathrm{m}$. 36: SH 87/181. - 37: Holotype of Hyaloscypha protonematosa. - 38: Jamalo-Neneckij, 1969 AR. - 39: Korf \& Tachibana 3449. -40: Boroni, 1967 AR. - 41: 1982 Spooner. - 42: Holotype of Peziza lectissima. - 43: Cain 15870. 

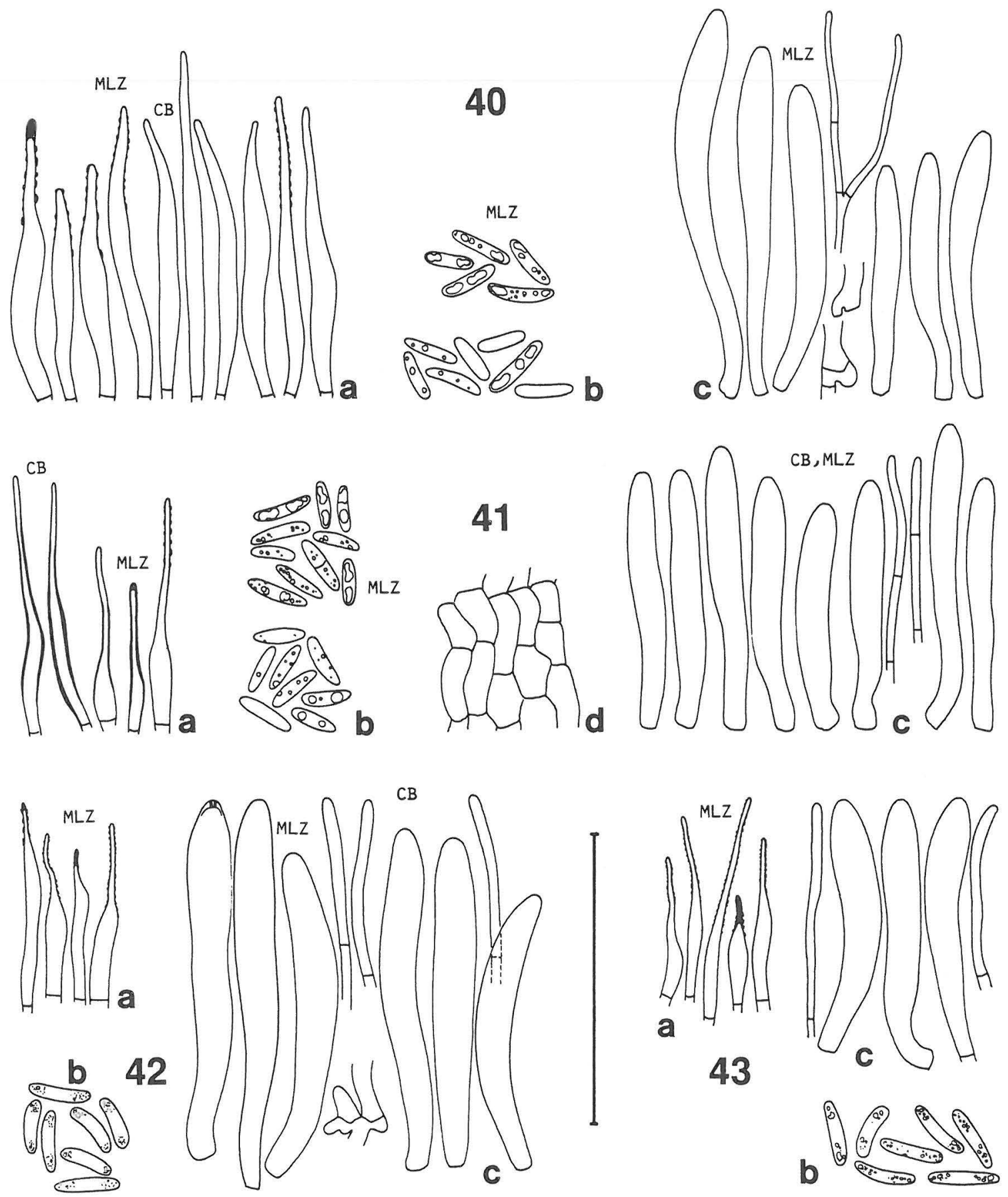
$5.5 \mu \mathrm{m}, \overline{\mathrm{Q}}=2.7(\mathrm{n}=40)$ in $94 \%$ of the populations; broadly prismatic, $10-18 \times 6.5-9.0 \mu \mathrm{m}, \mathrm{Q}=2.0(\mathrm{n}=$ 15 ) in others, not notably changing shape towards margin, at the base more roundish. Inner excipulum of narrower textura prismatica, cells ca. 9-13 $\times 2.5-$ $4.8 \mu \mathrm{m}, \mathrm{Q}=2.8-4.8(\mathrm{n}=15)$. Walls in ectal parts $0.4-0.6 \mu \mathrm{m}$ thick in $95 \%$ of populations, up to $1.0 \mu \mathrm{m}$ thick in others, becoming thinner inwards, hyaline, MLZ+ to MLZ++ when fresh and dry in $90 \%$ of the populations, MLZ- or fragmentarily $\mathrm{MLZ}+$ in others after $\mathrm{KOH}$ pretreatment, $\mathrm{BF}+$, $\mathrm{CRB}+, \mathrm{NR}+, \mathrm{TB}+$, fragmentarily $\mathrm{CR}+$, occasionally $\mathrm{CB}+$, LUG-.

Hairs $30-50 \times 1.8-5.0(-8.0)$, varying between the populations from almost filiform or narrowly conical to slightly lageniform, straight to bent, undulating or not but never apically cincinnate, aseptate. Apex tapering to $0.5-1.0 \mu \mathrm{m}$, in a few populations to $1.5-2.0 \mu \mathrm{m}$, rarely swollen, in $75 \%$ of the populations without solidifications, frequently prominently solidified in others, often bearing a resinous, hyaline lump, deforming in $\mathrm{MLZ} ; \mathrm{BF}+, \mathrm{CB}+, \mathrm{CR}+, \mathrm{CRB}+$, LUG-, MLZ-, NR-, TB-; solidifying matter CR++, MLZ++, rarely only after $\mathrm{KOH}$ pretreatment. Wall thin (in three populations uniformly $0.5 \mu \mathrm{m}$ thick), occasionally locally thickened (up to $0.5 \mu \mathrm{m}$ ) at the constriction, hyaline, dull or refractive, in $83 \%$ of the populations $M L Z+$ to $M L Z++$ when fresh and dry, MLZ - or fragmentarily MLZ+ after $\mathrm{KOH}$ pretreatment in others, with sparse to dense, prominent warts, rarely with a low, platelike crust, in $25 \%$ of the populations smooth or inconspicuously warted; warts deforming or not in $\mathrm{CB}$ and $\mathrm{MLZ}, \mathrm{BF}+$, rarely $\mathrm{CB}+, \mathrm{CR}+, \mathrm{CRB}+$. Marginal hairs intermixed with abundant, cylindrical-clavate, thin-walled cells, 10 $30 \times 3-5 \mu \mathrm{m}$.
Asci 34-63(-74) x 5.8-9.1(-10.5) $\mu \mathrm{m}, \overline{\mathrm{x}}=48.8$ $\times 7.2 \mu \mathrm{m}(\mathrm{n}=27), \bar{Q}=7.0$ when fresh in water, 36$62(-69) \times 5.3-10.5(-12.4) \mu \mathrm{m}, \overline{\mathrm{x}}=47.6 \times 7.5 \mu \mathrm{m}$ $(\mathrm{n}=90), \overline{\mathrm{Q}}=7.0$ in MLZ; $35-60(-63) \times 4.5-$ 8.2(-10.0) $\mu \mathrm{m}, \overline{\mathrm{x}}=47.1 \times 6.3 \mu \mathrm{m}(\mathrm{n}=157), \bar{Q}=$ 7.5 in $\mathrm{CB}$, eight-spored, cylindrical-clavate, with slightly conical apex. Apical pore MLZ++ in $60 \%$ of the populations when fresh and dry, MLZ+ in others (in one population permanently MLZ-, in one MLZ+ after $\mathrm{KOH}$ pretreatment), LUG-. Wall in mature asci apically $1.0-2.0 \mu \mathrm{m}$ thick (in CB, MLZ), negative in all reagents; contents often LUG+, rarely MLZ+ (orange). Asci arising from croziers.

Spores 8.4-14.6(-15.4) x 2.5-4.0(-4.2) $\mu \mathrm{m}, \overline{\mathrm{x}}=$ $11.3 \times 3.2 \mu \mathrm{m}(\mathrm{n}=87), \overline{\mathrm{Q}}=3.6$ when fresh in water; 7.2-12.3(-16.3) x 2.0-3.3(-3.8) $\mu \mathrm{m}, \overline{\mathrm{x}}=9.7$ $\times 2.6 \mu \mathrm{m}(\mathrm{n}=632), \bar{Q}=3.9$ in CB and MLZ, elliptic to oblong-elliptic, with rounded to slightly tapered apices, frequently one-septate after being discharged, more rarely while still inside the asci; septum thin, central, rarely MLZ+. Wall hyaline, occasionally brownish in aged discharged spores. Guttulae prominent and light when fresh in water, appearing either as one larger globule at each end or as abundant, smaller, apically grouped globules; in dry material prominent, light, separate to coalescent, rarely lacking; the spores with $\mathrm{CB}$ - contents with one regular, globose, light guttula at each end.

Paraphyses filiform or cylindrical to slightly widened at the apex, unpigmented, first dichotomous branches occurring at the level of lowermost $1 / 3$. Terminal cells $10-43(-49) \times 1.0-2.0(-2.5) \mu \mathrm{m}, \overline{\mathrm{x}}=$ $27.1 \times 1.5 \mu \mathrm{m}(\mathrm{n}=60)$.

Cultural characters (12 populations, 35 strains). Radial growth slow on MA, $13.7 \mathrm{~mm} /$ month $(\mathrm{n}=$
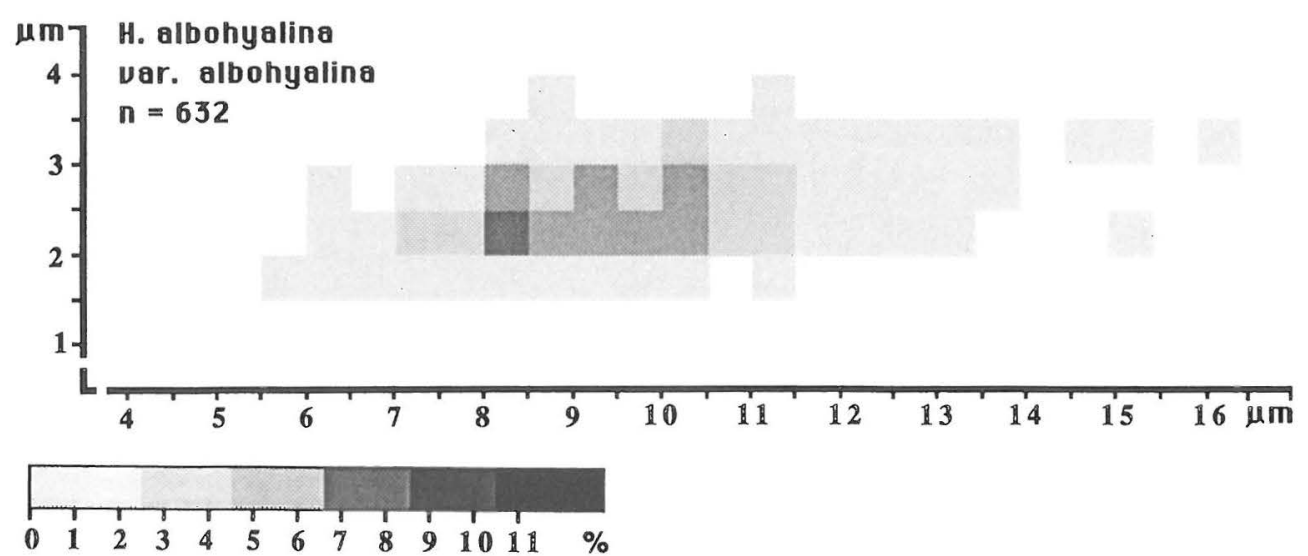

Fig. 44. Percentage sporogram of Hyaloscypha albohyalina var. albohyalina. 
87, from 5 popul., 14 strains). Mats with black to blackish brown basic colour, in centre $\mathrm{T} 71$, at the margin dark brown (P75, R75), lacking greenish tinges, extreme margin rarely with a 1-2 $\mathrm{mm}$ wide yellowish brown (K89, K90) band. Aerial mycelium densely woolly, rarely appressed, extending to 2$5 \mathrm{~mm}$ from the margin, greyish white (L92) to brownish (M75, N70, N71), in one population more reddish brown (N55). Margin indistinct, fimbriate, submerged. Hyphal strands present only in one population, these free but prostrate, occurring within $1 \mathrm{~cm}$ of the inoculum. Zonation lacking, sector formation rare, no colour change in surrounding agar, no yeast-like growth. Context somewhat tough at centre, odour not specific.

Spores germinating readily on wood agars, growth moderate and equal on BA, PA, QA, retarded on LA. Aerial mycelium formed moderately on QA, lacking in other wood agars. Anamorph and teleomorph production lacking in single and multispore trials.

The stability of the cultural morphology among the strains is outstanding. In culture, the taxon is fairly easily distinguished by the blackish basic colour combined with abundant aerial mycelium and lack of zonation, and by the growth rate. Identical morphology is seen in var. spiralis, which, however, frequently produces a Pseudaegerita anamorph in culture. $H$. herbarum is somewhat similar in culture, but the growth rate is markedly faster. Many populations of $H$. aureliella resemble those of the present taxon, but differ in their anamorph and notably slower growth on MA.

The cultured populations originate from both softwood and hardwood, and one population also from Quercus. Some populations have smooth hairs; the others have minutely to clearly warted hairs. Populations differing from each other in narrow versus

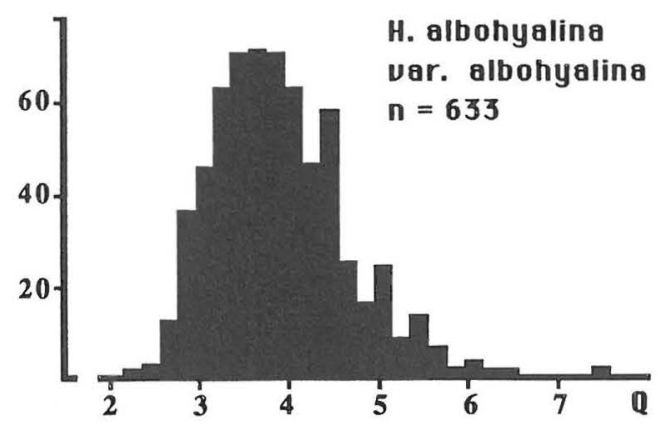

Fig. 45. The distribution of spore quotient Q in Hyaloscypha albohyalina var. albohyalina. blunt hairs and solidified versus unsolidified hairs are linked by similar cultural morphology. Two of the cultured populations lack dextrinoid reactions in the hairs and excipulum or show only a few scattered MLZ+ areas, whereas the others show an overall MLZ+ reaction. The mean spore length in cultured populations varied from 8.4 to $11 \mu \mathrm{m}$. The observed variability among the populations embraced in var. albohyalina is mostly linked by the cultural characters.

Distribution and phenology. The present variety does not seem to be as widely distributed or as common as var. spiralis. The scattered collections show, however, that var. albohyalina also fruits throughout North America and Europe and extends through Siberia and the Caucasus to the Soviet Far East and Japan. Populations exploiting building wood were also found in two middle arctic areas of Svalbard. The fruiting period is long, consisting of the whole frost-free season. There is, however, a peak in fruiting populations between July and October (Fig 16). In general, the fruiting period shows no marked differences from that of var. spiralis.

Ecology. According to the present material, var. albohyalina prefers to fruit on hardwoods. In all 45 collections originate from hardwoods, and 22 from softwoods. The same trend is seen in the larger material of var. spiralis, which has an almost identical ratio between the two types of substrates. Of the above-mentioned collections, 10 are corticolous: $\mathrm{Be}$ tula (2), Crataegus (2), Pinus (3), unidentified hardwood bark (4). The ecological amplitude is apparently narrower than that of var. spiralis, because all the collections are on strictly ligneous substrates, as follows (corticolous populations excluded): Alnus (8), Salix (5), Betula (4), Pinus (3), Fagus (2), Tilia (2), Cryptomeria (1), Corylus (1), Larix (1), Populus tremula (1), Prunus spinosa (1), Quercus (1).

Discussion. Hyaloscypha albohyalina var. albohyalina is a relatively variable taxon. Without observations of the ascal bases, it can be confused with a variety of closely related taxa. But after the presence of croziers has been confirmed, a collection is relatively easily recognized. The closely related taxa with similar ascal bases are $H$. fuckelii var. fuckelii, $H$. vitreola, $H$. daedaleae, and to some extent also $H$. aureliella.

From the first taxon var. albohyalina is best distinguished by the more voluminous spores and asci and the typically rough hairs. The overlap in the hymenial characters is narrow, however, and the popu- 
lation mean of each parameter is often enough for a correct determination. The typical hairs of $H$. fuckelii and $H$. albohyalina differ widely and again the overlap is small. From $H$. vitreola the present variety can be distinguished by the widely different, firm-walled, smooth hairs and the larger spores and asci of $H$. vitreola. Var. albohyalina has been found only once on Quercus. In addition to this difference in ecology, the cuneiform spores of $H$. daedaleae are a reliable and rapid means of recognition if a population growing on oak wood is suspected to belong to var. albohyalina. As some populations of var. albohyalina on softwoods tend to have small spores, narrow paraphyses and no or fragmentary dextrinoid reactions, they can be confused with those of $H$. aureliella, especially when an apothecium is mounted directly in $\mathrm{CB}$ or MLZ and the yellowish resin of $H$. aureliella is thus lost. In these cases re-examination of material mounted in water is needed to check whether resin is present on the hairs. Other characters of $H$. aureliella are also diagnostic, viz. the blunt and thin-walled hairs, amyloid reactions, especially in the excipulum, sparse septation and indistinct guttulation.

From the other two varieties of the species, the present taxon is distinguished by the presence of croziers. This stable character is coupled with other, more variable features. The clearly lageniform type of hairs with broad apices and prominent warts is almost lacking in var. albohyalina, whereas the other two varieties have populations with such hairs. Excipulum with broadly prismatic cells on the middle flanks is seen in clearly fewer populations of var. albohyalina than in var. spiralis.

Though the hair roughness and the dextrinoid reaction of the hairs vary widely in var. albohyalina, the hair shape is less variable than in var. spiralis. Hair roughness shows a gradual decline from populations characterized by totally and permanently warted hairs in every apothecium to populations showing sparsely granulated and smooth hairs in certain apothecia. The populations with consistently smooth hairs are in a clear minority. The rare variants with uniformly thickened, smooth hair walls are, however, clearly linked with other populations. Solidification of hair apices also varies between the populations, but when present is much more pronounced than that seen in $H$. fuckelii. The present variety does not show the difference in spore shape between populations on softwood and hardwood evident in var. spiralis (Fig. 14). The dextrinoid reaction of the hair and excipular walls shows a smooth gradation from negative to fragmentary to strong reactions in MLZ, the variation being of the same magnitude as in $H$. fuckelii.
In a few populations the euamyloid reaction of the apical pore is retarded or lacking in MLZ. As in var. spiralis, $\mathrm{KOH}$ pretreatment resulted in a normal reaction, except in one population with permanently MLZ - asci. This variation must be accepted within the present taxa, as the populations are linked by so many other characters. Of special interest is one population from the Adirondack Mts. (Herb. Korf 53-46), in which some apothecia have asci filled with small ascoconidia. This feature is coupled with constantly MLZ-apical pores and lack of croziers. In the same population most apothecia show asci that are normal for var. albohyalina. Similar variation in MLZ reaction is seen only in the type of $H$. sarothamni. It thus seems that these genetically fairly strictly controlled characters can occasionally show marked variation.

An interesting collection from Alberta (Edmonton, Wagner bog, SH 87/181) extends the variation of the present taxon. This single collection probably represents a chemically distinct variant, characterized by abundant, bright yellow resin. When fresh, this resin gives a bright lemon yellow colour to the whole fungus. When dry, the resin is seen loose in the hymenium and on the excipulum (in water) and even filling many spores. Like the brownish resin of $H$. aureliel$l a$, this resin is immediately dissolved when $\mathrm{CB}$ or MLZ is added to a water mount. Such a bright yellow resin is unknown in the other species of Hyaloscypha. As it does not consistently occur inside the paraphyses and hairs, it cannot be closely related to the golden yellow pigment characterizing the genus Phialina. Further collections from the area are needed to clarify the taxonomic value of this character.

Material studied: Canada. Alberta: Edmonton, $1987 \mathrm{SH}$ 87/181* (TUR). Québec: Poste-de-la-Baleine, $1982 \mathrm{SH}$ 82/134, 82/142a, 82/146, 82/199 (TUR). Schefferville, 1967 Măkinen 67-1386b, 67-1402 (TUR). Ontario: Algonquin Nat. Park, 1939 Cain 15870 (MICH). Yukon: Kluane Lake, 1987 SH 87/113 (TUR). Czechoslovakia. Bohemia: Mnichovice, 1926, 1928 (type of Chrysothallus pezizellaeformis), 1933, 1934, 1941 (type of Hyaloscypha protonematosa) JV (PRM). Slovakia: Bratislava area, 1986 SH 86/63, 86/75* (TUR). Finland. Varsinais-Suomi: Nauvo, 1986 SH 86/155* (TUR). Parainen, 1984, 1985 SH 84/82, 85/36 (TUR). Turku 1984, 1985 SH 84/55, 85/171* (TUR). Uusimaa: Tammisaari, 1986 SH 86/143* (TUR). Tenhola, 1986 SH 86/150* (TUR). Satakunta: Punkalaidun, 1986 SH 86/174* (TUR). Etelä-Häme: Korpilahti, 1986 SH 86/93*, 86/101* (TUR). Lammi, 1985 SH 85/117* (TUR). Tammela, 1867, 1868 PAK 579 (type of Peziza albohyalina), 580, 589 (type of Peziza lectissima, isotype also in UPS), 590, 594 (H). Tampere, 1985 Söderholm 1225 (TUR). Pohjois-Savo: Siilinjärvi, 1985 SH 85/93* (TUR). Koillismaa: Juuma, 1978 Korf et al. (CUP). France. Corsica: Chisoni, 1972 Demoulin \& Korf 72-113 (Herb. Korf). Col de Vizzavona, 1972 Demoulin \& Korf 72-23 (Herb. Korf). Great Britain. Hampshire: Matley Bog, 1982 
Spooner (K). Surrey: Ham Common, 1948 Hughes (IMI, two collections). Yorkshire: Halifax (CUP - D 160). Mull: Loch Buic, 1976 Clark (E). Japan. Kochi Pref.: Ochi-machi, 1959 Korf et al. 1435 (CUP). Kanagawa Pref.: Fuji-Hakone Nat. Park, 1963 Korf \& Korf 2757 (CUP). Yokohama, 1958 Korf et al. 982 (CUP). Gumma Pref.: Lake Marunuma, 1957 Korf 134 (CUP). Honshu, Nagano-shi, 1969 Tachibana \& Korf 3449 (CUP). Norway. Østfold: Fredrikstad, 1985 SH 85/41* (TUR). Oppland: Dovre, 1985 SH 85/58, 85/65, 85/71 (TUR). Nordland: Træna, 1988 Olsen (TUR). Finnmark: Karasjok, 1978 Korf et al. (CUP) Talvik, $1978 \mathrm{Korf}$ et al. (CUP, two collections). Svalbard: Ny Ålesund, 1988 Knudsen, SH 88/14 (TUR); Longyearbyen, 1988 SH 88/52 (TUR). Sweden. Uppland: Bondkyrka, 1931 Lundell \& JAN 4195 (UPS). Jämtland: Åre, 1951 JAN 11794 (UPS). Torne Lappmark: Jukkasjärvi, 1928 JAN 1522 (UPS). Switzerland. Graubünden: Val Tavru, 1984 SH 84/149 (TUR). Tübingen: Schönbuch, 1977 Matheis 776 (Herb. Matheis). Unlocalized, 1970 Dennis \& Müller (K). U.S.A. Colorado: Tolland, 1910 Seaver \& Bethel (NY). Rocky Mts. Nat. Park, 1948 Korf \& Korf 1405, 1447 (CUP). New York: Marcy Dam, 1953 Schaffer \& Korf 53-46 (Herb Korf). Michigan: Emmet Co., 1969 Korf 3766 (Herb. Korf). U.S.S.R. Estonian S.S.R.: Distr. Tartu, Voore, 1971 Kullman (TAA). Boroni Nature Res., 1967 AR (TAA) Vooremaa, 1969 Kalamees (TAA). Krasnodar Region: Caucasian Res., 1974 Waasma (TAA). Tuva S.S.R.: Tannu-Ola Mts., 1972 AR (TAA). Tjumen Region: Jamalo-Neneckij, Krasnyi Kamenj 1969 AR (TAA, two collections). Khabarovsk Region: Sikhote Alin Mts., 1973 Kullman (TAA).

\section{Hyaloscypha albohyalina var. spiralis}

Hyaloscypha albohyalina (P. Karst.) Boud. var. spiralis (Velen.) Huhtinen, comb. et stat. nov. - Chrysothallus spiralis Velen., Monogr. Discom. Bohem.: 269. 1934. - Lectotype (indicated on the label by Svrcek, formally designated here): [Czechoslovakia,] Bohemia, Mnichovice, Hubačkov, Corylus avellana, 29.XII.1928 Velenovský (PRM 150477, examined).

Truncicola parasitica Velen., Monogr. Discom. Bohem.: 289 ; Pl. 25:30, 31. 1934. Holotype (the only specimen mentioned): [Czechoslovakia,] Bohemia, Radotín, IX.1925 leg. Fechtner, det. Velenovský (PRM 148816, examined).

Hyaloscypha lignicola Abd. \& Webster, Trans. Brit. Mycol. Soc. 80:253. 1983. - Holotype (original designation): [Great Britain,] Devon, Minehead, Salix wood, stagnant water, Sept. 1978 S.K. Abdullah (stated to be in HME, not extant; isotype in IMI 266914, examined).

Hyaloscypha hyalina (Pers.: Fr.) Boud. "typical form" sensu Dennis, Mycol. Pap. 32:70. 1949, p.p. minore.

Hyaloscypha hyalina (Pers.: Fr.) Boud. "form 3" sensu Dennis, Mycol. Pap. 32:69. 1949, p.p. majore.

Hyaloscypha hyalina (Pers.: Fr.) Boud. "form 4" sensu Dennis, Mycol. Pap. 32:70. 1949, p.p. minore.

Ectal excipulum of textura prismatica, cells on middle flanks regularly prismatic, $7.5-11.0 \times 3-$ $5 \mu \mathrm{m}, \bar{Q}=2.4(\mathrm{n}=30)$ in $80 \%$ of the populations broadly prismatic, $10-17 \times 5-8 \mu \mathrm{m}, \bar{Q}=2.0(\mathrm{n}=$ 50 ) in others, not notably changing shape towards margin, at the base more roundish. Walls in ectal parts $0.4-0.6 \mu \mathrm{m}$ thick in $80 \%$ of populations, up to $1.0 \mu \mathrm{m}$ thick in others, becoming thinner inwards, hyaline, MLZ+ to MLZ++ when fresh and dry in $87 \%$ of the populations, MLZ - or fragmentarily $\mathrm{MLZ}+$ in others after $\mathrm{KOH}$ pretreatment, $\mathrm{BF}+$, $\mathrm{CRB}+, \mathrm{NR}+, \mathrm{TB}+$, fragmentarily $\mathrm{CR}+, \mathrm{CB}-$, $\mathrm{LUG}-$.

Hairs $30-50 \times 1.8-5.0(-8.0)$, varying between the populations from almost filiform or narrowly conical to clearly lageniform, straight to bent, undulating or not but never apically cincinnate, aseptate. Apex in narrow-haired populations tapering to 0.5 $1.0 \mu \mathrm{m}$, in others to ca. $1.2-2.0 \mu \mathrm{m}$, rarely swollen, in $25 \%$ of the populations clearly solidified, typically bearing a lump of hyaline exudate, deforming in $\mathrm{MLZ}, \mathrm{BF}+, \mathrm{CB}+, \mathrm{CR}+, \mathrm{CRB}+, \mathrm{LUG}-, \mathrm{MLZ}-$, NR-, TB-; solidifying matter CR++, MLZ++, rarely only after $\mathrm{KOH}$ pretreatment. Wall thin, ca. $0.4 \mu \mathrm{m}$ thick throughout in $3 \%$ of the populations, seldom locally thickened but even then without massive thickenings, hyaline, dull or refractive, in $84 \%$ of the populations MLZ+ to $\mathrm{MLZ++}$ when fresh and dry, MLZ - or MLZ+ after $\mathrm{KOH}$ pretreatment in others, with sparse to dense, prominent warts, rarely with a low, platelike crust, warted in $97 \%$ of the populations, smooth or inconspicuously warted in others; warts deforming or not in $\mathrm{CB}$ and $\mathrm{MLZ}, \mathrm{BF}+\mathrm{CB}+$, $\mathrm{CR}+$, rarely $\mathrm{CRB}+$.

Asci 37-55(-59) x 5.6-9.1 $\mu \mathrm{m}, \overline{\mathrm{x}}=46.7 \times$ $7.3 \mu \mathrm{m}(\mathrm{n}=37), \bar{Q}=6.6$ when fresh in water; $31-$ $59(-63) \times 5.6-9.2(-10.0) \mu \mathrm{m}, \overline{\mathrm{x}}=46.7 \times 7.1 \mu \mathrm{m}(\mathrm{n}$ $=132), \bar{Q}=6.7$ in MLZ; $34-60(-70) \times 5.1-7.8$ $(-9.5) \mu \mathrm{m}, \overline{\mathrm{x}}=46.4 \times 6.3 \mu \mathrm{m}(\mathrm{n}=285), \bar{Q}=7.5 \mathrm{in}$ CB. Apical pore MLZ+ in $50 \%$ of the populations, MLZ++ in others (in two populations MLZ-, after $\mathrm{KOH}$ pretreatment $\mathrm{MLZ+}$ ), LUG-. Wall in mature asci apically $1.0-2.0 \mu \mathrm{m}$ thick (in CB, MLZ), negative in all reagents; contents often LUG+, rarely $\mathrm{MLZ+}$ (orange). Asci arising from simple septa.

Spores 7.6-12.5(-12.8) × 2.0-3.4 $\mu \mathrm{m}, \overline{\mathrm{x}}=9.6 \times$ $2.8 \mu \mathrm{m}(\mathrm{n}=73), \overline{\mathrm{Q}}=3.4$ when fresh in water; $6.6-$ $11.5(-15.3) \times 2.0-3.4(-4.0) \mu \mathrm{m}, \overline{\mathrm{x}}=9.1 \times 2.6 \mu \mathrm{m}$ $(\mathrm{n}=1112), \bar{Q}=3.6$ in $\mathrm{CB}$ and MLZ.

Paraphyses cylindrical or slightly widened at the apex, asci, unpigmented, first dichotomous branches occurring at the level of lowermost $1 / 3$. Terminal cells $10-38(-45) \times 1.0-2.0(-2.5) \mu \mathrm{m}, \overline{\mathrm{x}}=22.0 \times$ $1.7 \mu \mathrm{m}(\mathrm{n}=80)$

Cultural characters (13 populations, 32 strains). Radial growth slow on MA, $11.7 \mathrm{~mm} /$ month ( $\mathrm{n}=$ 72, from 3 popul., 10 strains). Cultural morphology largely identical to that of var. albohyalina, but lacking hyphal strands, producing reddish brown (P 17) 

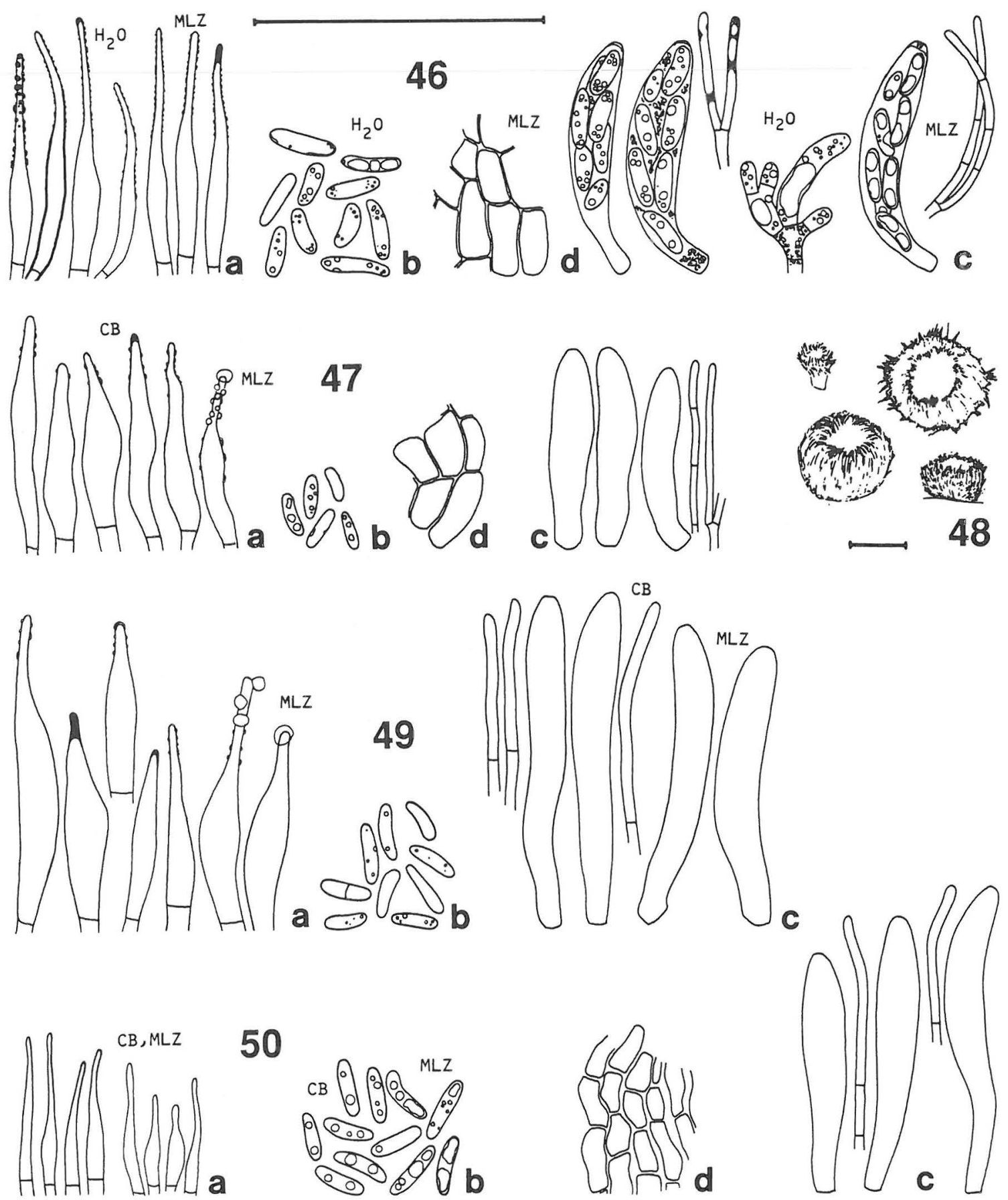

50

Figs. 46-54. Hyaloscypha albohyalina var. spiralis. a) hairs, b) spores, c) asci and paraphyses, d) excipulum. Scale $50 \mu \mathrm{m}$, for apothecia $100 \mu \mathrm{m}$. - 46: SH 87/43. - 47: Isotype of Hyaloscypha lignicola. - 48: Dry apothecia from SH 86/65. - 49: Vrábsko, 1968 Svrčk. - 50: 1978 Hindley. — 51: 1950 Dennis. — 52: 1960 Moravec. — 53: Palmer 64052. — 54: JAN 19119. 
KARSTENIA 29 1989(1990)

HUHTINEN: HYALOSCYPHA AND ALLIED GENERA

101
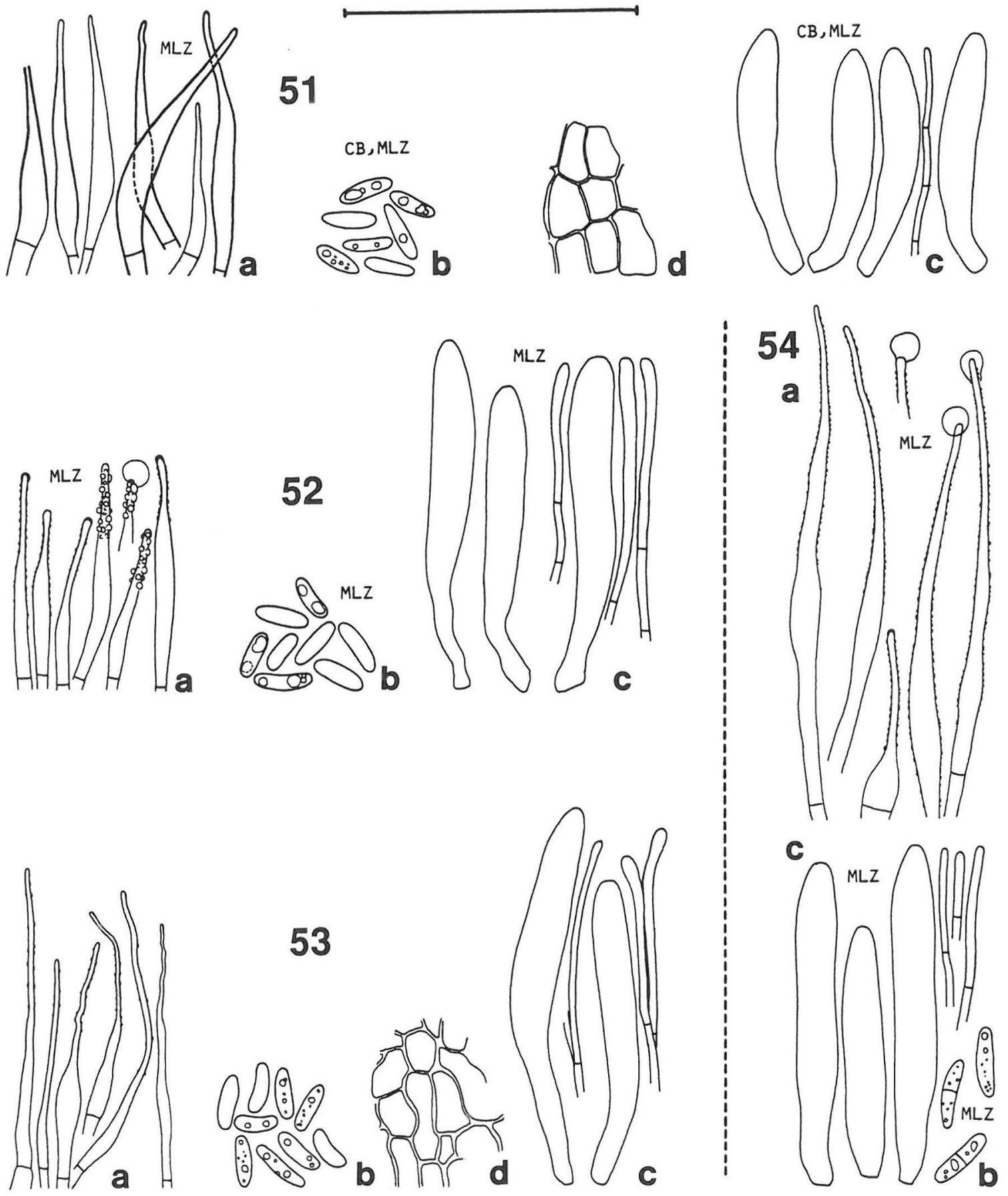
guttulae on aerial mycelium in one strain, agar changing to brownish (M70, N77) in one strain.

Spores germinating readily on wood agars, growth moderate on BA, PA, QA, LA. Aerial mycelium lacking on wood agars but in one strain producing scattered, small tufts of erect, moniliform, brown hyphae. Producing Pseudaegerita readily in culture on all substrates, teleomorph not produced in single or multispore trials.

As in the type variety, the morphology of the cultures is remarkably stable, although the strains originate from such widely distant areas as Czechoslovakia, southern Norway, Finland and Svalbard. Pseudaegerita is produced by populations originating from both softwood and hardwood. Only three of the eleven populations tested did not produce the anamorph. Populations differing in hair breadth and spore length are linked by similar cultural characters, as are also populations with sparsely warted hairs, but data on cultures of smooth-haired populations are still lacking.

When describing Hyaloscypha lignicola, Abdullah and Webster (1983) reported the connection with Pseudaegerita sp., which they suspected to be conspecific with $P$. corticalis (Peck) Crane \& Schokn. The large variation in the colour of the conidia reported by Abdullah and Webster (1983) and Abdullah et al. (1986) was also observed in the present studies. The conidia are mostly brown (R51, T71), but also olivaceous (N91, P91), yellow or whitish. According to the above authors, Pseudaegerita is aero-aquatic and the anamorph is often collected on decaying wood in water. It seems, however, to survive on land, as well, as seen in some of the present collections, in which the apothecia were accompanied by the anamorph. The high water content of decayed wood and the high relative humidity of the immediate surroundings possibly contribute to its survival. For many aero-aquatic hyphomycetes the ecological range is limited (Fisher 1978, Sanders \& Webster 1978).

Distribution and phenology. $H$. albohyalina var. spiralis is apparently more widely distributed than the type variety, being especially common in western and central Europe, and known also from northern Europe, the Caucasus, Tian Shan Mts., northern Ural Mts., Soviet Far East, Japan and disjunct areas in North America. The collections from Chile, Tierra del Fuego and from Svalbard notably extend the range.

Fruit bodies are produced fairly steadily during the frost-free season, with a possible peak in autumn (Fig. 16). This peak may also be caused by greater collection activity. In Great Britain the variety has mostly been collected during late autumn and early spring, and fruiting is apparently diminished during the summer months.

Ecology. Hyaloscypha albohyalina var. spiralis occurs on both hardwoods and softwoods, but prefers the former substrate: in the present material 35 collections originate from softwoods and 102 from hardwoods. The ratio between the two types of substrate is the same as in the type variety. A corticolous habit is less characteristic of the present variety, as only five collections originate from bark (two from Betula, one each from Fagus and Larix, one from unidentified coniferous bark). The ecological amplitude of var. spiralis is wide. It occurs on both sapwood and heartwood, on stroma of old pyrenomycetes and there are also single collections from Rubus stems, from cupules of Castanea and Aesculus, from a Picea cone, from an unidentified deciduous leaf and from a stem of Sasa paniculata. Furthermore, var. spiralis is capable of fruiting on Quercus. The abovementioned collections are not included in the following list of data from the collectors: Fagus (10), Alnus (9), Picea (7), Quercus (8), Betula (5), Salix (4), Pi-

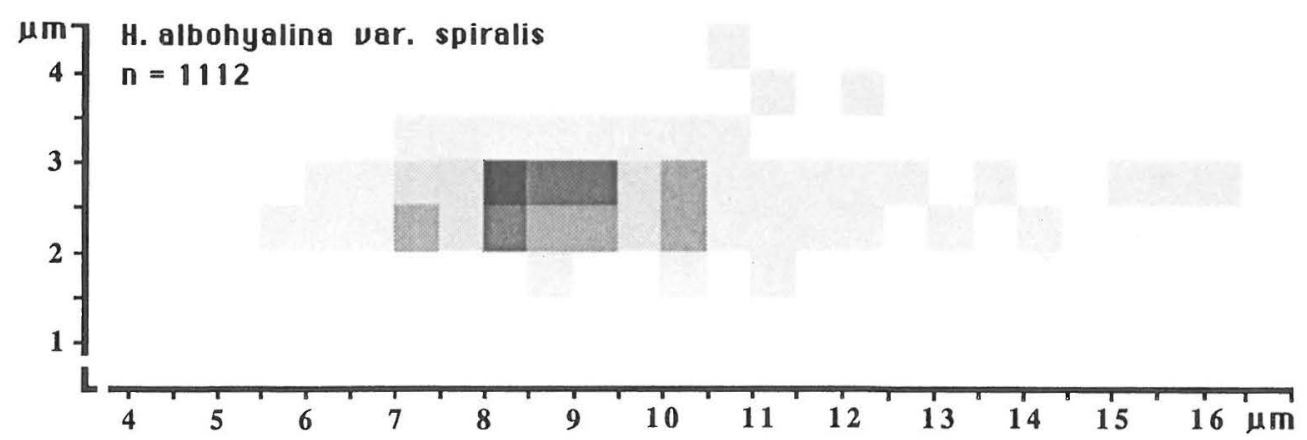

Fig. 55. Percentage sporogram of Hyaloscypha albohyalina var. spiralis. 
nus (3), Corylus (2), Carpinus (1) Crataegus (1), Fraxinus (1), Larix (1), Nothofagus (1), Populus tremula (1), Prunus avium (1), Sambucus (1), Ulex (1).

Discussion. Hyaloscypha albohyalina var. spiralis is variable, showing affinities to fairly many of the taxa discussed below. It is perhaps most difficult to differentiate from $H$. herbarum, which is another variable species. The overlap between these two taxa, both with asci arising from simple septa, causes difficulties in some populations. Their cultural characters differ, however, and support the distinction made here. All the cultured strains of $H$. herbarum grew rapidly, the cultures on malt agar differ markedly in morphology and no anamorph is known in $H$. herbarum. But when the cultural characters are not known, each population needs to be studied in detail before the distinction can be made. In general, $H$. herbarum has longer, more elongated spores, with tardily developing or no septa and without clear, light guttulae. These characters are coupled with smooth, lageniform hairs, showing warts in only a few populations As these populations are also those with the shortest and least elongated spores, they come close to var. spiralis, but, as seen from Figs. 55 and 115, the spore size and shape clearly differ between the two taxa and even in such problematic cases the spores of $H$. herbarum are narrower and more elongated.

Hyaloscypha albohyalina var. spiralis is easily distinguished from the type variety by the lack of

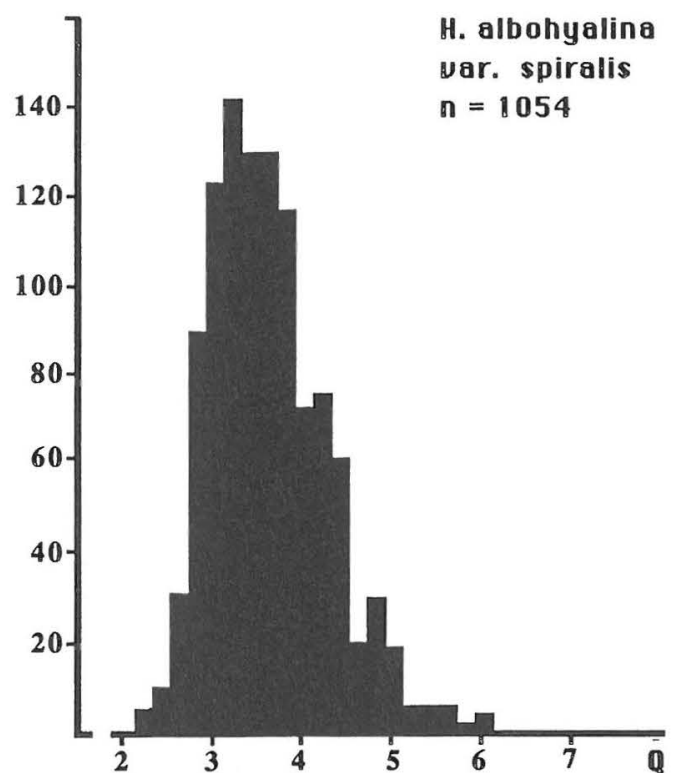

Fig. 56. The distribution of spore quotient Q in Hyaloscypha albohyalina var. spiralis. croziers at the ascal bases. The other differences are less well defined and show considerable overlap. These differences, here considered to indicate ongoing divergence of the varieties (see discussion under var. tigillaris), are mostly seen in the hair characters. In var. spiralis the hairs are more often lageniform with relatively broad and blunt apices, whereas in the type variety populations with hairs of this type are in the minority. In var. spiralis local hair wall thickenings and uniformly thickened walls are rarer than in the type variety. The cultural characters are identical, but so far the anamorph, Pseudaegerita sp., has been obtained only from var. spiralis. The ecological range of the type variety is more restricted, which suggests that var. spiralis is diverging from the main gene pool and invading new types of substrates.

When the hairs bear prominent warts, the present taxon is easily recognized; warted hairs are not present in $H$. fuckelii or $H$. vitreola. The broad hair apices with distinct solidifications are also a rapid means of recognition. The absence of croziers differentiates var. spiralis from both $H$. vitreola and $H$. aureliella. The latter species may be confused with the small-spored, indextrinoid populations when the typical resin on $H$. aureliella is lost in $\mathrm{CB}$ and $\mathrm{MLZ}$ mounts.

In general, $H$. albohyalina has more robust asci and spores than $H$. fuckelii and even a smooth-haired population is fairly easily recognized by these characters alone. Even the small spores in var. spiralis are typically more broadly ellipsoid with broader ends. Additional characters that can be used to separate $H$. albohyalina are the more frequent septation and guttulation of the spores, together with the more frequent apical exudates on the hairs and the often larger-celled excipulum of var. spiralis. The cultures of the two taxa are different, and $H$. albohyalina can be recognized by the morphology of vegetative mycelium on agar.

Hyaloscypha quercicola is another species with asci arising from simple septa and hairs showing prominent warts. But this species also has smaller asci and spores than those of var. spiralis. Furthermore, $H$. quercicola is differentiated by consistently MLZ-ascal pores, small-celled excipulum, relatively narrow hairs and distinctive cultural characters. Thus, confusion between the two taxa is hardly possible. $H$. priapi can also be distinguished by the dimensions of its asci and spores, and its broad short hairs. The size ranges of the spores and asci in $H$. intacta overlap with those of var. spiralis, but $H$. intacta has constantly MLZ- asci and its short hairs are smooth or bear only a few tiny warts.

In var. spiralis variability occurs mainly in the hair characteristics. There are two main hair types, which 
do not coexist within a population. Clearly lageniform hairs with broad apical parts usually have prominent warts and are often apically solidified. Hairs of the other type are narrow with filiform apices, mostly having smaller warts and the apices only minutely solidified. This hair type can coexist with perfectly smooth hairs even in the same apothecium. In four populations the hairs have mostly smooth and firm walls, this feature being coupled with a very weak or no dextrinoid reaction. These populations originate from both softwood and hardwood and are geographically widely distributed. Thus the total variation in hair shape and vesture is large. Part of the variation seen in spore length is explained by the obvious tendency for spores to become elongated in populations growing on softwoods (Fig. 14). An increasing trend on softwood is not seen in spore width, or in ascus length or width. The excipular variation and variation in chemical reactions match those of other closely related taxa.

Stability is seen mainly in the ascus and spore characteristics. The asci are more robust than in, for example, $H$. fuckelii and they always arise from simple septa. The spores retain their typical oblongelliptic shape in different environments and septal formation and clear guttulae are fairly typical. The dextrinoid reaction in the hair and excipular walls is evident in ca. $90 \%$ of the populations.

As the holotype is apparently lost, the lectotypification suggested by Svrcek on the label is here validated. The specimen fits well with the original diagnosis. Svrček (1985) listed Chrysothallus spiralis among the synonyms of Hyaloscypha perpusilla Velen., a species here synonymized with $H$. fuckelii. The interpretation offered by Dennis (1975) is erroneous and refers to Hyaloscypha uncipila, which belongs to Hamatocanthoscypha.

The spore length and width in the type specimen of var. spiralis are close to the peak frequency for both parameters. The populations producing $\mathrm{Pseu}$ daegerita in culture have a mean spore length ranging from 7 to $11 \mu \mathrm{m}$ (in CB, MLZ). Although the collections with the longest spores have not been connected to the lower end of the spore length range variation by cultural studies, they are unquestionably linked by a smooth gradation in a variety of morphological characters. This gradation shows one hiatus, which is discussed under var. tigillaris. The effect of softwood substrates on spore length is apparently a manifestation of ongoing divergent evolution.

Exsiccatae studied: Sydow, P., Mycotheca Marchica 671, $\mathrm{S}, \mathrm{NY}$.
Material studied: Argentina. Tierra del Fuego: Ushuaia, 1989 Cannon et al. 516c (TUR). Austria. East-Tirol: Dolsach, 1922 Keissler (W). Sonntagsberg, 1909 Strasser (F). Schneeberggebiet, 1902 Höhnel (F). Belgium. Namen: Malonne, 1882 Thiry (BR). Liège, Mouton (no number) and 401 (BR). Not localized, Mouton (BR). Canada. Ontario: York Co., Nashville, 1955 Cain (BPI). Québec: Schefferville, 1967 Mäkinen 67-1351 (TUR). Czechoslovakia. Bohemia: Jimy, 1922 JV (PRM). Mnichovice, 1924, 1926, 1928 (type of Chrysothallus spiralis), 1929, 1941 JV (PRM). Praha, 1960 Wichansky (PRM). Radotín, 1925 Fechtner (PRM, type of Truncicola parasitica) Vrabsko, 1968 Svrček (PRM). Slovakia: Bratislava area, 1986 SH 86/55*, 86/57*, 86/65, 86/69*, 86/70, 86/71, 86/76*, 86/78, 86/79*, 86/80 (TUR). Srdiecko, 1960 Moravec (PRM). Chile. Région de los Lagos: Valdivia, 1985 Cannon (IMI). Denmark. Jylland: Als, 1988 Lassøe (TUR). Sjalland: Hvalso skov, 1952 JAN (UPS). Federal Republic of Germany. Baden-Württemberg: Weingartnerer Moor, 1987 Winterhoff (TUR). Rheinland-Pfalz: Boppard, 1937 Sponheimer (B). Unlocalized, 1894 Fuckel (G), 1973 Engel 285 (K). Finland. Varsinais-Suomi: Kalanti, 1987 SH 87/209 (TUR). Lohja, 1986 SH 86/3* (TUR). Parainen, 1984 SH 84/112 (TUR). Turku, 1984, 1985 SH 84/12, 84/51, 84/252, 84/253, 85/ 19, 85/161* (TUR). Etelä-Häme: Asikkala, 1986 SH 86/20* (TUR). Kangasala, 1987 Kosonen (TUR) SH 87/43 (TUR). Tottijärvi, 1986 SH 86/178* (TUR). Inarin Lappi: Utsjoki, 1965 JAN (UPS). France. Doubs: Mont Morond, 1954 JAN (UPS). Unlocalized, 1884 (P). Great Britain.Devon: Stoke Wood, 1947 Wakefield (K, UPS). Not localizable, 1981 Webster (IMI). Somerset: Minehead, 1978 Abdullah (IMI, type of Hyaloscypha lignicola). Sussex: Ardingley, 1968 Dennis (K, two collections). Unlocalized, 1941 Reid (K). Surrey: Esher Common, 1980 (K). Horsley, 1949, 1952 Dennis (K). Ockley, Reid (K). Oxshott, 1948 Hughes (K). Ranmore, 1947 Dennis (K, IMI), 1974 Dennis $(\mathrm{K})$. Walton-on-Thames, 1978 Spooner (K). Essex: Hockley Woods, 1976 Spooner (K). Gloucestershire: Thombury, 1950 Dennis (K). Carmarthenshire: Llandovery, 1948 Dennis (K). Suffolk: Blythburg, 1978 Ellis \& Ellis (IMI). Norman Gwatkin Res., 1978 Ellis \& Ellis (IMI). Southwold, 1978 Ellis \& Ellis (IMI). Huntingdonshire: Holme Fen, 1976 Watling 11693 (E). Derbyshire: Bakewell, 1964 Apinis (K). Yorkshire: Bradford, Soppitt (NY). Dalby, 1976 Bramley K/77/8 (K). Hackness, 1965 Bramley 20/65 (K). Limb Valley, 1948 Hughes (IMI). Pickering, 1963, 1972, 1981, 1982 Bramley K/63/91, K/72/17, K/81/19, K/82/2 (K). Roche Abbey, 1948 Hughes (IMI, K). Sheffield, 1948 Hughes (IMI). Sunwood, 1892 Crossland (K). Wadsworth, 1894 (K, Herb. Crossland). Peebles: Glentress Forest, 1974 Coppins (E). Perthshire: Craiglush, 1954 (E). Rannoch, Watling \& Coppins 1563 (E), 1983 SH 83/369 (TUR). Inner Hebrides: Isle of Ulva, 1969 Dennis (K). Skye, 1980 Bennell (E). Wester Ross: Ardnamurchan, 1950 Dennis (K). Inverness-shire: Head of Glen More, 1949 Dennis (K). Shetland: Kergord, 1987 Dennis (K, TUR). Not localizable, 1964 Palmer 64052 (K), 1872 (K, Herb. Broome); Grovely Wood, 1978 Hindley (K). Hungary. Matra Mts.: Kekes, 1978 Korf et al. (CUP). Japan. Nagasaki Pref.: Unzen Nat. Park, 1957 Korf et al. 312 (CUP). Kochi Pref.: Ochi-machi, 1959 Korf et al. 1422 (CUP). Gumma Pref.: Lake Marunuma, 1957 Korf 132 (CUP). Ishikari Prov.: Sapporo, 1958 Korf et al. 980 (CUP). Kushiro Prov: Akan Nat. Park, 1958, 1963 Korf et al. 828, 2560 (CUP). Norway. Oppland: Dovre, 1985 SH 85/46* (TUR). Svalbard: Ny Ålesund, 1988 SH 88/9* (TUR). Sweden. Småland: Kärda, 
1944 Lundell (UPS). Öland: Föra, 1953 JAN 13138c (UPS). Västmanland: Sala, 1945 Molander 18 (UPS). Uppland: Bondkyrka, 1929 Lundell (UPS). Danmark, 1930 Lundell (UPS). Uppsala, 1944 JAN 7544 (UPS). Gästrikland: Gävle, 1952 JAN 12632 (UPS). Switzerland. Graubünden: S-Charl, 1984 SH 84/130, 84/135, 84/137, 84/138 (TUR). Val Tavru, 1984 SH 84/ 152, 84/157 (TUR). U.S.A. California: 1888 (K, Herb. Grove). New York: Danby, 1958 Korf 58-59 (Herb. Korf). Genesee Co., Bergen Swamp, 1949 Rogerson (NY). Ithaca, 1901 Durand (S). 1978 Carpenter \& Rogerson (NY). 1947, 1960 Korf 627, 3084 (Herb. Korf) Orleans Co., Lyndonville, 1905, 1908 Fairman (CUP - D 444, 445). Newfield-Nina road, 1947 Perkins \& Korf 547 (Herb. Korf). Bowness, Atkinson (K, Herb. Phillips). U.S.S.R. Estonian S.S.R.: Boroni Nature Res., 1967 AR (TAA, two collections). Poelendmaa, 1966 AR (TAA). Krasnodar $R e-$ gion: Caucasian Res., 1975 Pallo (TAA). Karachayevo-Cherkess: Teberda, 1968 AR (TAA). Kirghizia: Terskei Alatau Mts., 1965 AR (TAA). Sary-Tshelek Res., 1967 AR (TAA). Tjumen Region: Jamalo-Neneckij, Suryskary, 1976 Murdvee (TAA). Primorsk Region: Kavalerovo, 1977 Saar (TAA). Khabarovsk Region: Korfovsk, 1966 Remm (TAA). Sakhalin Region: Nabili Mts., 1970 Kullman \& AR (TAA). Ozersk, 1970 Denini (TAA).

\section{Hyaloscypha albohyalina var. tigillaris}

Hyaloscypha albohyalina (P. Karst.) Boud. var. tigillaris (P. Karst.) Huhtinen., comb. et stat. nov. - Peziza tigillaris P. Karst., Not. Sällsk. Fauna Flora Fenn. Förh. 10:184. 1869. - Helotium tigillare (P. Karst.) P. Karst., Not. Sällsk. Fauna Flora Fenn. Förh. 11:240. 1870. — Phialea tigillaris (P. Karst.) Sacc., Syll. Fung. 8:270. 1889. - Hymenoscyphus tigillaris (P. Karst.) O. Kuntze, Revis. gen. pl. 3:486. 1898. - Micropodia tigillaris (P. Karst.) Boud., Hist. classific. discom. Europe: 128. 1907. - Hyaloscypha tigillaris (P. Karst.) Raitv., Scripta Mycol. 1:30. 1970. - Lectotype (selection made here among discordant elements of the holotype, Art. 9.2): [Finland,] Tavastia australis, Tammela, Teurois [Teuro] ...pini, 11.IX.1868 Karsten (H, Herb. Karsten 594, examined). The isotype (Karst. 593) has erroneously been indicated as lectotype by Raitviir (in herb.) and as holotype by Carpenter (1981), with the erroneous "Aug." and "592" citations. The heterogeneous elements in the type specimen are discussed below.

Apothecia scattered, superficial, up to $500 \mu \mathrm{m}$ in diam when fresh (according to Karsten), up to $150 \mu \mathrm{m}$ in diam when dry, cyathiform to cylindrical when juvenile, cupulate when mature, with a gradually tapering base to shortly stipitate. Margin incurved when dry. Hair cover dense to sparse at margin, sparse below, snow-white when dry. Colour whitish when fresh, dry apothecia somewhat reddish brown, (either N45, N80 or M5), the hair cover occasionally obscuring these colours.

Ectal excipulum of textura prismatica, cells on middle flanks regularly prismatic, $8-14 \times 4-7 \mu \mathrm{m}, \bar{Q}$ $=2.3(n=10)$, not changing shape towards margin, at the base more roundish. Walls in ectal parts 0.4 $0.6 \mu \mathrm{m}$ thick, hyaline to fragmentarily pale brown- ish, MLZ-; $\mathrm{BF}+, \mathrm{CRB}+, \mathrm{NR}+, \mathrm{TB}+$, fragmentarily $\mathrm{CR}+, \mathrm{CB}-$, LUG-.

Hairs $25-40(-45) \times 2.2-4.0 \mu \mathrm{m}$, narrowly conical to lageniform, straight to apically strongly cincinnate and curved, often tardily reviving and remaining collapsed and entangled, aseptate. Apex tapering to 0.8$1.0(-2.0) \mu \mathrm{m}$, rarely solidified, with or without apical exudate and an apical globule in MLZ. Wall thin, never locally thickened, hyaline, dull, negative in all reagents, with dense and prominent warts or platelike crust, rarely smooth or inconspicuously warted, this variation occurring within a single apothecium; warts deforming or not in $\mathrm{CB}$ and $\mathrm{MLZ}$; $\mathrm{BF}+$, $\mathrm{CRB}+, \mathrm{NR}+, \mathrm{TB}+$, occasionally $\mathrm{CB}+$, faintly $\mathrm{CR}+$.

Asci $53.5-70(-88) \times 7.0-8.9(-10.0) \mu \mathrm{m}, \overline{\mathrm{x}}=$ $62.1 \times 8.2 \mu \mathrm{m}(\mathrm{n}=13), \bar{Q}=7.6$ in $\mathrm{MLZ}$, eightspored, cylindrical-clavate, with slightly conical apex. Apical pore $\mathrm{MLZ}+$. Wall in mature asci apically $1.0-1.8 \mu \mathrm{m}$ thick (in MLZ), negative in all reagents-; contents MLZ-. Asci arising from simple septa.

Spores 8.9-13.5(-15.0) x 2.9-4.0(-4.3) $\mu \mathrm{m}, \overline{\mathrm{x}}=$ $10.7 \times 3.3 \mu \mathrm{m}(\mathrm{n}=164), \bar{Q}=3.3$ in $C B$, phaseoliform or broadly elliptic to oblong-elliptic, with rounded to blunt apices, mostly aseptate after being discharged ( $1 \%$ being one-septate), not seen to become septate while still inside the asci; septum thin, central, MLZ-. Wall hyaline, CR-. Guttulae prominent and light in dry material, rarely lacking; spores with CB- contents with two, rarely one, large, light guttula.

Paraphyses cylindrical to slightly widened at the apex, unpigmented. Terminal cells $13-37 \times 1.3-$ $2.1 \mu \mathrm{m}, \overline{\mathrm{x}}=25.5 \times 1.8 \mu \mathrm{m}(\mathrm{n}=12)$.

\section{Cultural characters. Not known.}

Distribution and phenology. This variety has been collected three times from widely separated areas. The type comes from Karsten's collecting localities in South Finland, and the other two from the Rocky Mountains (U.S.A.). All the collections were made in early autumn.

Ecology. All the three collections originate from decorticated coniferous wood. Karsten (1869) gave the substrate as building wood of Pinus, i.e. Pinus sylvestris

Discussion. Hyaloscypha albohyalina var. tigillaris is differentiated from the other two varieties of the species by the larger spores and asci, lacking dextrinoid reactions, and the heavily plated, apically cincinnate and tardily reviving hairs. The coniferous substrate also seems to be characteristic of this varie- 
57
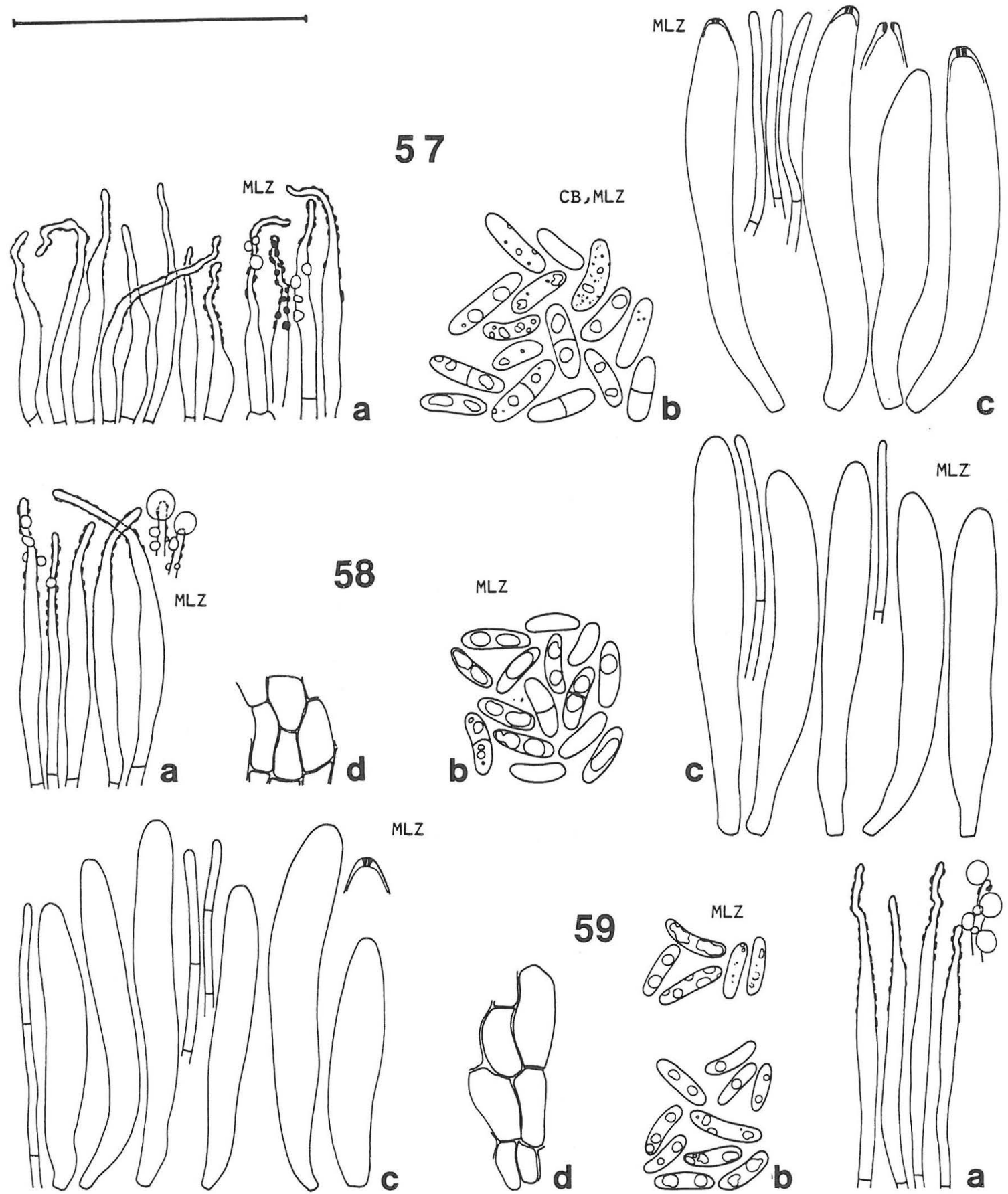

Figs. 57-59. Hyaloscypha albohyalina var. tigillaris. a) hairs, b) spores, c) asci and paraphyses, d) excipulum. Scale $50 \mu \mathrm{m}$. -57 : Lectotype of Peziza tigillaris. - 58: CUP 37702. - 59: CUP 37737. 
ty. The spore characteristics distinguish it from populations of var. spiralis, in which spores tend to be longer in softwood populations. Spore length and especially spore width clearly separate the present three collections from the rest of the softwood populations. In var. spiralis spore width differs less obviously between populations from softwoods and hardwoods. Hence, var. tigillaris is clearly separated by the wider spores.

The hairs of var. tigillaris have strongly cincinnate/curved apical parts, with a low but prominent plate-like crust. Such a hair type is not seen in the other two varieties. The hairs also lack wall thickenings, apical solidifications are rare, the apices are typically blunt and the asci are more voluminous all indicating a divergent line of evolution. The main reason for keeping the present taxon under $H$. albohyalina is the link with the other two varieties provided by softwood populations of var. spiralis. Without the difference in spore length between hardwood and softwood populations of var. spiralis, var. tigillaris could be taken for an independent species. Karsten's original material consists of two specimens, both mixed collections. The original collection (Karst. 594) contains pieces of coniferous wood, which are of different origin. Only one weathered piece was found to bear apothecia of the present tax- on, the others bearing $H$. albohyalina var. albohyalina. Karsten's original notes and description show that only the apothecia from lectotype were studied for the description of Peziza tigillaris. The other species apparently passed unnoticed. The isotype (Karst. 593), separated by Karsten in 1883 , contains the same kind of weathered coniferous chips as the selected lectotype bearing identical apothecia. In addition, the package contains one chip of hardwood, again with $H$. albohyalina. Accordingly, within the isotype an isolectotype has been selected.

Type material of Peziza tigillaris was later studied by Dennis (1956), who apparently examined only $H$. albohyalina, since he reported a dextrinoid excipular reaction and smaller clearly narrower spores. This seems to be the first report of the dextrinoid reaction in Hyaloscypha. P. tigillaris was transferred to Hyaloscypha by Raitviir (1970a), who had also studied the type. From his short description in the key, giving the spore width as $2.5-4.5 \mu \mathrm{m}$, it is obvious that his concept of $H$. tigillaris also partly embraces $H$. albohyalina var. albohyalina.

Material studied: Finland: Etelä-Häme: Tammela, Teuro, 11.IX.1868 PAK (H, type). - U.S.A. Colorado: Rocky Mts. Nat. Park, Bear Lake, 29.VIII.1948 Korf \& Korf (CUP 37737). Montana: Glacier Nat. Park, Josephine Lake, 17.VIII.1948 Korf \& Korf 1405 (CUP 37702).

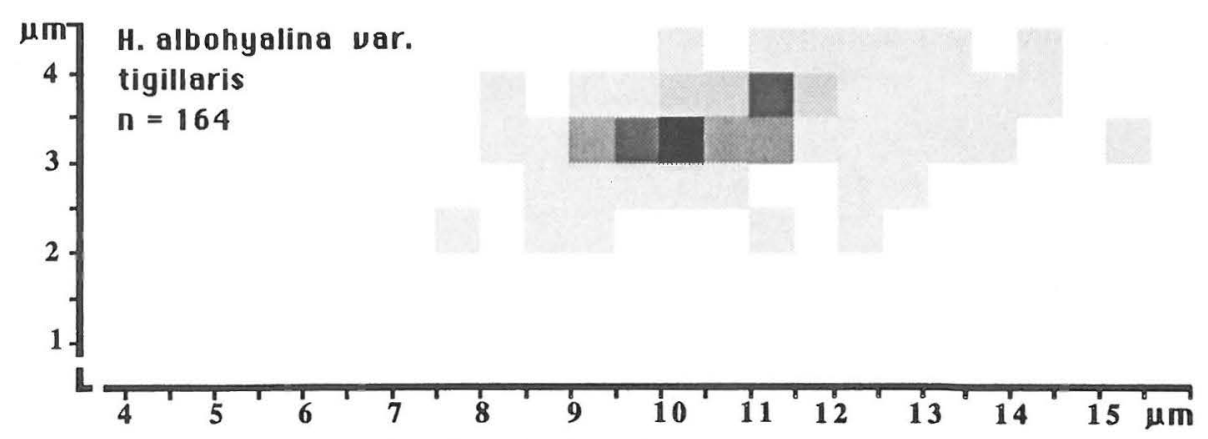

Fig. 60. Percentage sporogram of Hyaloscypha albohyalina var. tigillaris.

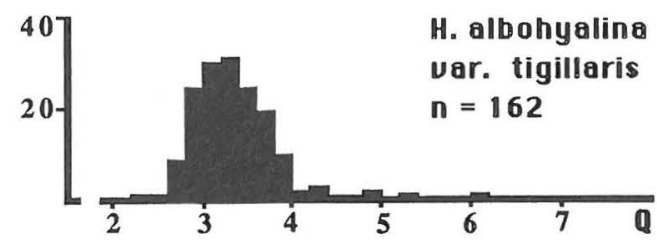

Fig. 61. The distribution of spore quotient Q in Hyaloscypha albohyalina var. tigillaris.

\section{Hyaloscypha aureliella}

Hyaloscypha aureliella (Nyl.) Huhtinen, comb. nov. - Peziza aureliella Nyl., Not. Sällsk. Fauna Flora Fenn. Förh. 10:49. 1869. - Mollisia aureliella (Nyl.) P. Karst., Bidr. Känned. Finlands Natur Folk 19:206. 1871. - Tapesia aureliella (Nyl.) P. Karst., Acta Soc. Fauna Flora Fenn. 2:137. 1885. - Eriopeziza aureliella (Nyl.) Rehm, Rabenh. Krypt.-Fl. ed 2., 1(3): 695. 1896. - Holotype (the number of collections from Helsinki not clearly stated in the protologue, but only one speci- 

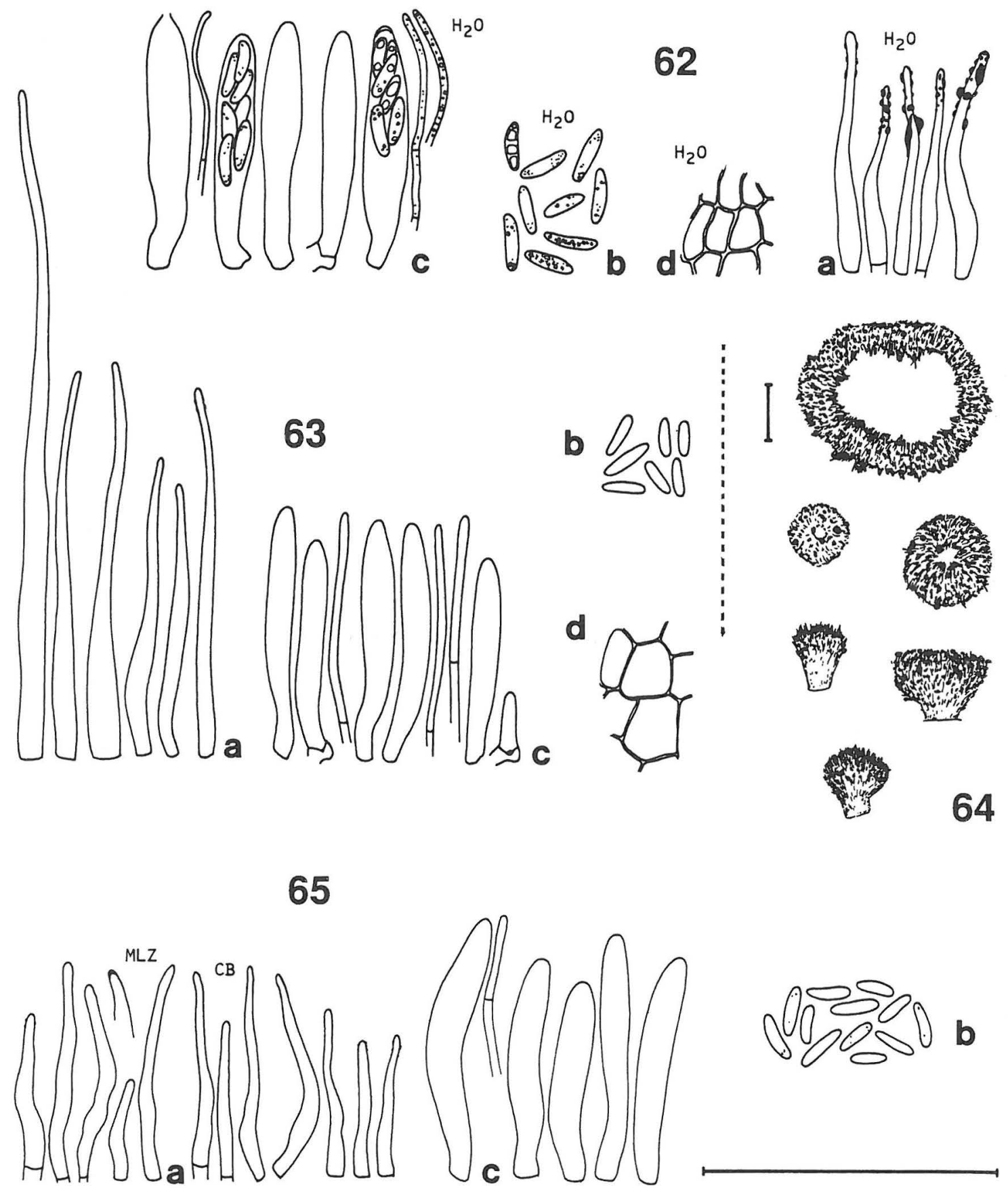

Figs. 62-65. Hyaloscypha aureliella. a) hairs, b) spores, c) asci and paraphyses, d) excipulum. Scale $50 \mu \mathrm{m}$, for apothecia $100 \mu \mathrm{m}$. -62: SH 87/202. - 63: JAN 15090a. - 64: Dry apothecia from various collections. - 65: Holotype of Peziza stevensonii. 
men with two separate fragments exists): [Finland,] Nylandia [Uusimaa,] Helsingfors [Helsinki,] autumno 1858 W. Nylander ( $\mathrm{H}$, examined).

Peziza stevensonii Berk. \& Broome, Ann. \& Mag. Nat. Hist. Ser. 4, 15:38. 1875. - Lachnella stevensonii (Berk. \& Broome) Phill., Man. Brit. Discomyc.: 235. 1887. - Dasyscypha stevensonii (Berk. \& Broome) Sacc., Syll. Fung. 8: 454. 1889. - Urceolella stevensonii (Berk. \& Broome) Boud., Hist. classific. discom. Europe: 130. 1907. - Hyaloscypha stevensonii (Berk. \& Broome) Nannf., Trans. Brit. Mycol. Soc. 20:206. 1936. - Holotype (the only specimen mentioned): [Great Britain,] Glamis, in herb. M.J.B., 52 Rev. J. Anderson (K, Herb. Broome, sub Peziza anderssoni Berk. \& Broome; examined). The two isotypes in $\mathrm{K}$ contain the following additional data: "1874" and "ex Stevenson". The former matches the collection data "Glamis, Hunter's Hill, 1874 J.S." given by Stevenson (1879). The latter isotype confirms Nannfeldt's (1936) suspicion that the specimen was erroneously annotated. The holotype was apparently illustrated by Spooner \& Dennis (1985), whereas the collection depicted as "type" by Dennis (1949, Fig. 78) is apparently based on another isotype.

Pezizella atomaria Starb., Bih. Svensk. Vet.-Akad. Handl. 21:31. 1895. - Urceolella atomaria (Starb.) Boud., Hist. classific. discom. Europe: 130. 1907. - Hyaloscypha atomaria (Starb.) Nannf., Nova Acta Soc. Sci. Upsal. Ser. 4., 8:273. 1932. - Holotype (the only specimen mentioned): [Sweden,] vid Glottern, 2.VIII. (S, apparently Haglund's original label; examined). The isotype in $\mathbf{S}$ contains the following additional data (in Starbäck's handwriting): "Suecia, Ostragothia, par. Qvillinge, ad lacuum Glottern, ad lignum pineum, 2.VIII.91 leg. Haglund".

Pezizella granulosella (P. Karst.) Rehm var. sydowii Rehm, Rabenh. Krypt.-Fl. ed 2., 1(3): 655. 1892. - Pseudohelotium granulosellum (Karst.) Sacc. var. sydowii (Rehm) Sacc., Syll. Fung. 14: 773. 1899. - Mollisiella granulosella (P. Karst.) Boud. var. sydowii (Rehm) Boud., Hist. classific. discom. Europe: 142. 1907. - Lectotype (selected here): [German Democratic Republic,] Neumark, Marienspring bei Cladow, auf einem faulenden Baumstemme, VII.1888 P. Sydow (S, Herb. Rehm; examined).

Dasyscypha resinifera Höhnel, Ann. Mycol. 1:396. 1903. - Hyaloscypha resinifera (Höhnel) Boud., Hist. classific. discom. Europe: 127. 1907. - Lectotype (selected here): [Austria,] Nördliche Wienerwald, Rekawinkel-Kronstein, 29.VI.1902 Höhnel (F 8612, examined).

Chrysothallus pinaceus Velen., Monogr. Discom. Bohem.: 270; P1. 6: 22. 1934. - Holotype (the only specimen mentioned): [Czechoslovakia,] Bohemia, Mnichovice, ad truncum pini (loco insolato), 24.VI.1929 Velenovsky (PRM 150472, examined).

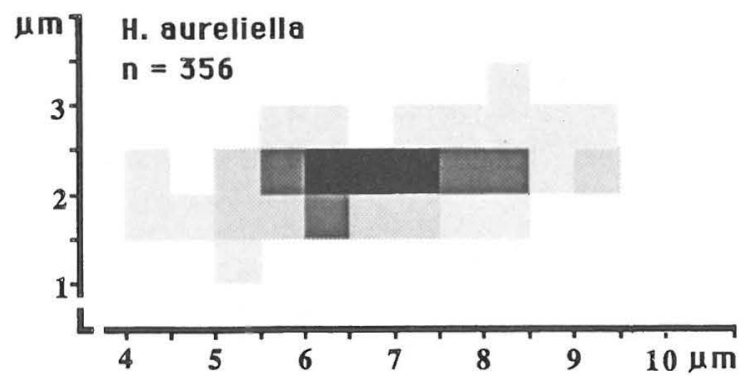

Fig. 66. Percentage sporogram of Hyaloscypha aureliella.
Hyaloscypha albolutea (Pers.) Velen. var. obscura Velen., Monogr. Discom. Bohem.: 273. 1934. - Holotype (the only specimen mentioned): [Czechoslovakia,] Bohemia, Mnichovice, Populus tremula, 24.VIII.1925 Velenovsky (PRM 824924 , examined). In contrast to the protologue, the substrate was found to be coniferous.

?Hyaloscypha falcata Velen., Monogr. Discom. Bohem.: 278; PI. 14: 8. 1934. - Holotype (the only specimen mentioned): [Czechoslovakia,] Bohemia, Mnichovice, supra Hrusice, Juniperus communis, X.1928 Velenovský (PRM 151125 , examined). One fragment of apothecium studied, collection now empty. The long spores depicted by Velenovsky not found.

Truncicola copiosa Velen., Novitates mycol. noviss.: 142. 1947. - Holotype (the only specimen mentioned): [Czechoslovakia,] Bohemia, Mnichovice, Bỉá Skala pr. Božkov, ad truncum pini silv., 6.V.1941 Velenovsky (PRM 150757, examined).

Hyaloscypha velenovskii Graddon, Trans. Brit. Mycol. Soc. 58:152, Fig. 5a. 1972. - Holotype (original designation): [Great Britain,] Cheshire, Wildboardclough, on Larix, 1.VII.1961 Graddon (K, examined). The isotype in TAA contains the additional data "no. 1538".

?Hyaloscypha amyloideopilosa Svr., Ceská Mykol. 37:65. 1983. - Holotype (original designation): [Czechoslovakia,] Bohemia, Pořî́ko apud Čřenice, in valle rivi Křešický potok, ad lignum durum rami deiecti Carpini betuli, 18.X.1968 Svrček (PRM 829226, not examined).

Peziza hyalina (Pers.: Fr.) sensu Nylander, Not. Sällsk. Fauna Flora Fenn. Förh. 10:53. 1869, p.p.

Peziza hyalina (Pers.: Fr.) sensu Karsten, Not. Sällsk. Fauna Flora Fenn. Förh. 10:180. 1869, p.p. majore.

Peziza hyalina (Pers.: Fr.) sensu Karsten, Bidr. Känned. Finlands Natur Folk 19:144. 1871, p.p. majore?

Lachnella hyalina (Pers.: Fr.) Phill., Man. Brit. Discomyc.: 267; Pl. 8: 48. 1887, p.p.

Pezizella hyalina (Pers.: Fr.) Rehm, Rabenh. Krypt.-Fl. ed. 2, 1(3): 653. 1892, p.p.

Pezizella granulosella (P. Karst.) Rehm, Rabenh. Krypt.Fl. ed. 2, 1(3): 654. 1892, p.p.

Pezizella hyalina (Pers.: Fr.) Rehm, sensu Starb., Bih. Svensk. Vet.-Akad. Handl. 21:29; P1. 1:18. 1895, p.p. majore.

Dasyscypha hyalina (Pers.: Fr.) Massee, Brit. fung.-fl.: 338. 1895.

Hyaloscypha hyalina (Pers.: Fr.) Boud. var. hyalina sensu Velenovský, Monogr. Discom. Bohem.: 284; PI. 15:49. 1934, p.p. (the partial synonymy indicated by Svrček 1978c: 202).

Hyaloscypha albolutea (Pers.) Velen. var. albolutea, Monogr. Discom. Bohem.: 272; Pl. 14:3, 22. 1934, p.p. (the partial synonymy indicated by Svrček 1985: 205).

Hyaloscypha hyalina (Pers.: Fr.) Boud. sensu Raitviir, Scripta Mycol. 1:30. 1970.

Apothecia gregarious to confluent, superficial, up to $1.5 \mathrm{~mm}$ in diam when fresh, mostly $200-500 \mu \mathrm{m}$ in diam when dry, cupulate throughout development, often clearly undulate-crenulate when large, narrowly to broadly sessile or with a gradually tapering base, rarely with a $20-30 \mu \mathrm{m}$ long, stout stipe. Disc shallow to plano-convex when fresh, bordered by a copious zone of hairs which rise more or less clearly above the hymenium; fleshy and incurved when dry, 
obscuring much of the hymenium. Hair cover dense, seldom sparse below, white to yellowish. Colour when fresh varying within a single population from whitish to light yellowish brown to Straw (K79) to Corn (K80), the yellowish colour due to resinous exudate most prominent on marginal hairs; dry apothecia mostly varying between the populations from very light yellowish brown to yellowish brown (L80) to orange brown (N40) to deep reddish brown (R13); apothecia frequently covered by lumps of yellowish to yellowish brown resin when fresh, resin becoming amber to reddish brown upon drying.

Ectal excipulum of textura prismatica, cells on middle flanks varying between the populations from regularly prismatic, 7.5-12.0 × 3.5-6.0 $\mu \mathrm{m}$, to more roundish, $12-15 \times 7-10 \mu \mathrm{m}, \bar{Q}=1.8(\mathrm{n}=50)$, rarely becoming smaller and narrower towards margin, at the base more roundish, often lying at a slight angle to the surface. Inner excipulum of narrower textura prismatica. Walls in ectal parts $0.8-1.5 \mu \mathrm{m}$ thick in $70 \%$ of the populations, $0.4-0.8 \mu \mathrm{m}$ in others, becoming thinner inwards, hyaline to rarely pale brownish, MLZ-, except for the sparse to dense, blackish violaceous nodules occurring in $29 \%$ of the populations and similar but dextrinoid nodules present in $3 \%$ of the populations, fragmentarily $\mathrm{BF}+$, $\mathrm{CRB}+, \mathrm{NR}+, \mathrm{TB}+, \mathrm{CR}-, \mathrm{LUG}-, \mathrm{CB}-$.

Hairs 25-60(-110) x 2.5-5.2(-7.8) $\mu \mathrm{m}$, narrowly conical to slightly lageniform, straight to slightly flexuose, aseptate (in $2 \%$ of the populations basally one-septate hairs rarely seen), in $6 \%$ of the populations with scattered, blackish violet inclusions in MLZ. Apex blunt, gradually tapering to $1.0-2.0 \mu \mathrm{m}$, not solidified, lacking an apical globule in MLZ. Wall thin, never locally prominently thickened, dull, hyaline, smooth to apically inconspicuously encrusted in all mountants, negative in all reagents, frequently bearing lumps of amorphous, resinous matter. Resin persistent in water based stains and $\mathrm{KOH}$, forming loose, oily, brown globules to totally dissolving in $\mathrm{MLZ}, \mathrm{CB} ; \mathrm{BF}+, \mathrm{CRB}+, \mathrm{NR}+$, TB+, LUG-.

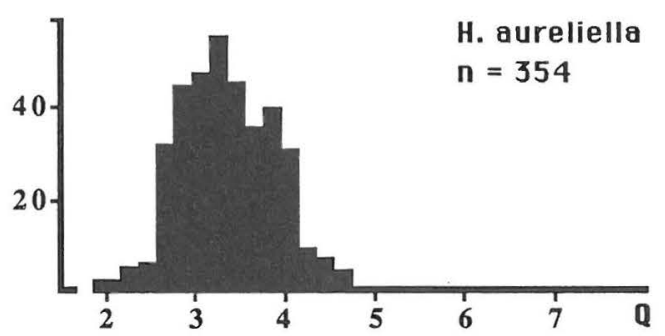

Fig. 67. The distribution of spore quotient $\mathrm{Q}$ in Hyaloscypha aureliella.
Asci $35-54(-63) \times 5-8(-8.4) \mu \mathrm{m}, \overline{\mathrm{x}}=44.0 \times$ $6.3 \mu \mathrm{m}(\mathrm{n}=66), \bar{Q}=6.8$ when fresh in water; $32-$ $48(-65) \times 5.0-6.4(-6.8) \mu \mathrm{m}, \overline{\mathrm{x}}=39.1 \times 5.5 \mu \mathrm{m}(\mathrm{n}=$ $60), \bar{Q}=6.9$ in $\mathrm{MLZ} ; 29-44(-49) \times 4.0-6.3$ $(-6.6) \mu \mathrm{m}, \overline{\mathrm{x}}=37.5 \times 5.1 \mu \mathrm{m}(\mathrm{n}=83), \bar{Q}=7.4$ in $\mathrm{CB}$; eight-spored, cylindrical-clavate with a slightly conical apex. Apical pore MLZ++ when fresh and dry, LUG+ (blue). Wall in mature asci apically 0.4 $0.8 \mu \mathrm{m}$ thick (in CB, MLZ), negative in all reagents. Contents often LUG+, MLZ+ (orange). Asci arising from croziers.

Spores $6.5-10.0 \times 2-3(-3.2) \mu \mathrm{m}, \overline{\mathrm{x}}=8.1 \times$ $2.5 \mu \mathrm{m}(\mathrm{n}=89), \bar{Q}=3.3$ when fresh in water; 5.3 $8.8 \times 1.8-2.4(-3.0) \mu \mathrm{m}, \overline{\mathrm{x}}=6.9 \times 2.2 \mu \mathrm{m}(\mathrm{n}=$ 356), $\bar{Q}=3.3$ in $\mathrm{CB}$ and MLZ; elliptic to oblong-elliptic, with rounded to slightly tapered apices, aseptate, irregularly biseriate and situated in upper portion of asci. Wall hyaline, not seen to become brownish in aged spores. Guttulae lacking or inconspicuous in fresh and dry material, when present grouped at spore ends, light.

Paraphyses filiform, unpigmented, first dichotomous branches occurring at the level of the lowermost $1 / 3$. Terminal cells $10-36 \times 1.0-1.6(-1.8) \mu \mathrm{m}$, $\overline{\mathrm{x}}=21.8 \times 1.2 \mu \mathrm{m}(\mathrm{n}=50)$.

Cultural characters (26 populations, 65 strains). Radial growth slow on MA, $7.0 \mathrm{~mm} /$ month $(\mathrm{n}=95$, from 6 popul., 21 strains). Mats variable in general appearance, variation mostly between the populations, notable variation within a population seen in four collections. Mats in ca. $40 \%$ of the populations with black to blackish brown basic colour, in centre black to T71, at the margin dark brown (P75), lacking greenish tinges. Aerial mycelium densely woolly, greyish (P92, S92) to brownish (N70). Margin indistinct, fimbriate. Mats in ca. $36 \%$ of the populations with creamy whitish basic colour and a doughlike surface, with or without blackish grey (S92) or brown (P75) bands developing ca. 5-10 mm away from the inoculum. Aerial mycelium lacking to abundant, whitish to grey (M92, N92). Margin indistinct, fimbriate, diffuse. Mats in rest of the populations showing the two basic types within the population.

Hyphal strands lacking or confined to the inoculum, scattered, erect, giving the inoculum a shaggy appearance. Zonation rare, sector formation occasional, agar changing to reddish brown (R17, R39) in one population, this particular population with prominent yeast-like growth, with abundant deep red (S17) conglomerations on agar surface. Context somewhat tough at surface, odour not specific.

Spores germinating readily on wood agars, growth moderate and equal on BA, PA, QA, LA. 
Aerial mycelium mostly lacking, rarely sparsely formed on QA. Producing Cheiromycella microscopica (P. Karst.) Hughes in 54\% of the populations on all substrates, teleomorph not produced in single spore or multispore trials.

The cultures derived from morphologically identical populations of $H$. aureliella are surprisingly variable. The variation between single spore strains from the same populations, which in most species of $\mathrm{Hya}$ loscypha is minimal, proved to be large in many populations. The situation is the reverse of that seen in, e.g., H. albohyalina, in which the apothecia vary, while the mycelia in culture are uniform. The cultures are easily recognized by production of Cheiromycella which, however, diminishes with prolonged storage. Had all the populations been tested on different substrates at an early stage, the percentage would most likely have exceeded the above 54 . When the anamorph is not produced, $H$. aureliella resembles fairly many species in its cultural morphology.

The conidial stage produced in culture is identical to that abundant in the type specimen of Dicoccum microscopicum P. Karst (holotype in H studied), and combination with Cheiromycella Höhnel was proposed by Hughes (1958). Like the teleomorph, the anamorph may be confined to softwood (Karsten 1887, Höhnel 1903, Ellis 1971, Ellis \& Ellis 1985). Production of conidia was observed, however, on all the substrates studied, though this may be due to the semi-natural nature of the media.

Interesting variation was observed in three Finnish populations which produced brown conidia on the surface and inside the agar in storage tubes after one and a half years. These strains did not produce $C h e i-$ romycella. The apothecia were identical to $H$. aureliella in occurrence of resin, size of hymenial elements, nature of the hairs, and chemical characteristics. In one population, however, the excipulum had dextrinoid nodules, which are a rare character in $H$. aureliella, whereas another collection showed the typical amyloid nodules. Unfortunately, the collections are too scanty for close scrutiny and the significance of the possibly different anamorph remains to be assessed.

Distribution and phenology. Hyaloscypha aureliella is undoubtedly the most widespread and common taxon of the genus. The present text is based on 347 studied populations. In Europe, it ranges from the northern parts of the Nordic countries to Spain in the south (R. Galán, pers. comm.) and a further extension is seen in collections from Morocco and the Azores. In the U.S.S.R. the taxon has been collected from the Estonian S.S.R., Ural Region, Caucasus,
Altai Mts., Kirghizia, Yakutsk, and from the Primorsk Region and Sakhalin in the Soviet Far East. It is also known from Japan. In Canada $H$. aureliella has been collected from the Canadian timberline in Québec, the Northwest Territories (Inuvik) and Yukon, and is also known from Alberta. In the U.S.A. it is known from California, Louisiana, Colorado, Montana, Ohio, North Carolina, New Jersey, Connecticut, New York and Michigan. Interesting extensions to the south are represented by collections from Jamaica and the Philippines. It is possibly also known from India (Sharma 1986). The range extends as far north as Svalbard.

In the temperate and boreal zone $H$. aureliella is an autumnal taxon, the peak in the number of fruiting populations being reached between July and September (Fig. 16). Most of the collections dating from the winter months and early spring have been made in Great Britain, where the species has a more or less continuous fruiting period. In Finland $H$. aureliella is very common and abundant, and can be found in a few minutes in any site with fallen coniferous branches or trunks. My collections from Scotland, Norway and Switzerland indicate that it is equally common in large parts of Europe. In northern Europe at least, the hyaloscyphaceous flora on softwood shows marked ecological specialization. Smaller dry twigs which are still attached to an erect tree are the domain of Lachnellula spp. Twigs, pieces of fallen cortex and cone scales are attacked by Hamatocanthoscypha laricionis and Hyaloscypha leuconica, while the more bulky substrates are occupied by $H$. aureliella. At the Canadian timberline, H. laricionis is replaced by Ciliolarina ligniseda (Velen.) Svr., which seems to occur with $H$. aureliella on decorticated wood as well (Huhtinen 1990). The present taxon appears to be less abundant in the Canadian north than in the boreal zone in Finland.

Ecology. Hyaloscypha aureliella is restricted to softwoods, and shows a marked preference for decorticated fallen trunks and larger branches. Only a single collection was made from coniferous bark, and only one specimen has been collected from cone scales of Picea. Decorticated, only slightly decomposed wood is the most typical substrate for $H$. aureliella. Often only a thin superficial layer has been partly degraded and softened by microorganisms, while the inner layers are still fairly hard and seemingly intact. The following list is compiled from the data provided with the specimens: Pinus (91), Picea (39), Larix (16), Abies (8), Juniperus (2), Pseudotsuga (1), Taxus (1). Seven collections originate from different types of building wood, and single 
collections originate from an old fence post, from roofing shingles, and from an old broom handle. The remainder of the collections originates from undetermined softwood.

Discussion. Hyaloscypha aureliella has many distinguishing features. The hairs are typically blunt and almost always bear at least traces of brownish resin, which is easily observed in water mounts but dissolves rapidly in CB and MLZ. The substrate is always coniferous, the asci always arising from croziers and nearly always with a very deep euamyloid reaction at their apices. The spores are aseptate and small and the paraphyses filiform. Dextrinoid reactions are lacking, or in a very few populations small dextrinoid areas are visible in the excipulum. Much more often the excipulum shows intensively amyloid nodules, the presence of which assists in distinguishing juvenile apothecia, which may occasionally bear hyaline resin only. The hairs are never apically inflated and never show prominently thickened areas. After the resin has dissolved, the hairs are perfectly smooth or bear a few minute warts at their apices. In such mounts the species may be confused with coniferous populations of, e.g., $H$. fuckelii var.fuckelii.

Variability in $H$. aureliella is seen in the excipulum and the chemical reactions, and to a lesser degree in the resinous exudate. The outer excipulum varies between the populations from small-celled to largecelled and from regularly prismatic to more roundish. The wall thickness also varies between the populations, from thin to $1.5 \mu \mathrm{m}$ thick, the majority of the populations being characterized by more or less firm walls. The amyloid reaction, visible in the excipulum and more rarely inside the hairs, varies between the populations, one third of which show this feature either in the excipulum or in the hairs. The density of amyloid excipular nodules may also vary within a population. Two different colours are seen in MLZ. At the amyloid end of the scale, the colour is always blackish violet and a deviating reaction is seen as wine red colouring. No gradation exists between the two reactions. The dextrinoid and amyloid nodules in the excipulum are morphologically alike, but the former are never conspicuous or abundant. When abundant in the hairs and excipulum, the amyloid substance is an eye-catching feature. Apparently such a population was described by Svrček as a new species, $H$. amyloideopilosa, because all the morphological and chemical characteristics given in the protologue match perfectly with the present material (Svrček 1983). The resinous exudate varies in colour. In a few populations, the resin may be hyaline or lacking, but this feature is due to lack of mature apothecia and characterizes juvenile apothecia only.

In one collection (Morocco, 14.I.1957 Bertault, MPU 6352), the same amyloid nodules occurred abundantly in the wood close to apothecia. They were not noted further away from apothecia. This amyloid substance may be exuded from the fungus, although in the other collections it occurs as restricted inclusions within the hairs and excipular cells.

This species has many fairly constant characters: the hair morphology, all ascal characters and the small, aseptate spores. The spores distinguish it from $H$. britannica, which has notably larger and often septate spores. The great majority of populations have fairly blunt hairs and when present, the narrower hairs make up a minority only. The ascal size shows smaller variation than in other species of the genus and the spores also vary little between the populations. The euamyloid reaction, whose intensity varies between the populations of many species is a very constant character in $H$. aureliella

The frequent presence of abundant resin has mostly been neglected as a taxonomic feature, as was noted by Spooner and Dennis (1985). No doubt this is due to the solubility of the resin in the mountants commonly in use. The resin was recognized by Höhnel (1903) and Svrček (1978c). Nannfeldt $(1932,1936)$ did not mention it, although he frequently studied and collected the taxon. This exudate was used to separate the taxonomically superfluous $H$. velenovskii Graddon, due to the assumption that $H$. hyalina sensu Velenovský (1934) is a non-resinous species. Peziza aureliella Nyl. was synonymized with Arachnopeziza eriobasis (Berk.) Korf by Korf (1951). This was done without studying the type collection, and was apparently due to Nylander's (1869) mention of "subiculum" in the original treatment of the species. As in numerous other collections of $H$. aureliella, this "subiculum" is only a wood-inhabiting, resupinate basidiomycetous fungus. Korf's solution condemned Peziza aureliella to become a "forgotten" epithet.

Exsiccatae studied: Ellis, J.B. \& Everhart, B.M., North American Fungi 2810 (K, L, M, NY; fragments of the same collection also in BPI, NY, CUP). - Lundell, S. \& Nannfeldt, J., Fungi Exsiccati Suecici 987a (K, S) and 987b (K). Sydow, H. \& Sydow, P., Mycotheca Germanica 607 (F). Sydow, P., Mycotheca Marchica 580 (K, S), 2442 (K), 2851 (K), 3377 (K), 4763 (NY). - Rabenhorst, G., Fungi Europaei 1615 (G, K, L, M).

Selected specimens (347 collections studied): Canada. Alberta: Edmonton, 1987 SH 87/180* (TUR). Yukon: Whitehorse, 1987 SH 87/178 (TUR). Kluane Lake, 1978 SH 87/162 (TUR). Denmark. Sjalland: Bromme Lilles $\varnothing, 1978$ Korf et 
al. (CUP). Finland. Varsinais-Suomi: Turku, 1984, 1986 SH 84/45, 86/123 (TUR). Satakunta: Punkalaidun, 1984, 1985, SH 84/35, 85/123*, 85/167* (TUR). Etelä-Häme: Tampere, 1987 SH 87/28* (TUR). Pohjois-Häme: Laukaa, 1986 SH 86/89* (TUR). France. Corsica: Corte, 1972 Demoulin \& Korf 72-80 (Herb. Korf). Great Britain. Surrey: Boxhill, 1948 Hughes (IMI, K). Suffolk: Dunwich Forest, 1978 Ellis \& Ellis (IMI). Warwickshire: Tapster Valley, 1978 Clark (IMI). Jamaica. Cinchona Bot. gard., 1971 Korf et al. (CUP). Japan. Miyazaki Pref.: Miyazaki, 1957 Korf et al. 369 (CUP). Norway. Svalbard: Ny Ålesund, 1988 SH 88/34 (TUR). Morocco. Tanger, 1957 Bertault 6352 (MPU). Philippines. Mindanao: Cortobato, 1966 Dumont SA2212 (NYS). Portugal. Azores: Terceira, 1975 Dennis (K). Sweden. Östergötland: Gryt, 1957 JAN 15090a (UPS). Switzerland. Graubünden: S-Charl, 1984 SH 84/136 (TUR). U.S.A. California:; Siskiyou Co., Mt. Shasta, 1965 (Herb. Korf). Colorado: Jackson Co., Cameron Pass, 1954 Rogerson (Herb. Korf). North Carolina: Lake Ravenel, 1952 Rosinski \& Korf 2591 (Herb. Korf). Connecticut: Castlewood Lake, 1947 Korf \& Korf 877 (Herb. Korf). U.S.S.R. Lithuanian S.S.R.: Varna, 1966 AR (TAA 44173, 44204). Sakhalin Region: Nabili Mts., 1970 Kullman (TAA).

\section{Hyaloscypha britannica sp. nov.}

Dasyscypha resinifera Höhnel, Ann. Mycol. 1:396. 1903, p.p. minore.

Cistella tapesioides Starb. in Vestergren, sensu Dennis, Mycol. Pap. 32:60. 1949.

Apothecia cupulata, sessilia vel breviter stipitata, pilosa; specimina exsiccata usque ad $350 \mu \mathrm{m}$ lata, straminea vel aurantiobrunnea. Excipulum externum textura prismatica, cel-

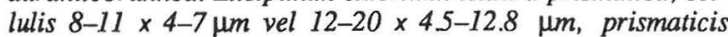
vel aliquantum rotundatis, aliquantum crassotunicatis, hyalinis, in solutione Melzeri raro sparsim vinosis. Pili 35-100(-130) x $2-4(-5) \mu m$, anguste conici vel anguste lageniformes, in apice 1-2 $\mathrm{m}$ lati, in solutione Melzeri raro sparsim amyloidei, leves vel minuter incrustati, aseptati, tenuiter tunicati, exudato resinoso, persistenti vel diffluenti. Asci 41-59 x 5.0-7.5 $\mathrm{m}$, amyloidei, in basi uncinati. Sporae 7.0-11.6 (-12.5) $\times 2.0$ 2.8(-3.2) $\mu \mathrm{m}$, ellipsoideae vel oblongo-ellipsoideae, saepe septatae. Paraphyses filiformae, 0.8-1.4 um latae, cellulis terminalibus 16-44 $\mathrm{um}$ longis.

Holotype: Great Britain. Berkshire: Reading Foray, on very rotten wood, 11.V.1947 Marsh (K).

Apothecia gregarious to confluent, superficial, up to $350 \mu \mathrm{m}$ in diam when dry, cupulate, narrowly to broadly sessile to stipitate on a $20-30 \mu \mathrm{m}$ long, stout stipe. Margin prominent, incurved, obscuring most of the hymenium when dry. Hair cover dense, white to yellowish when dry. Colour varying between the populations from light yellowish brown to Straw (K79) to orange brown (N39); frequently covered by lumps of amber to reddish brown resin when dry.
Ectal excipulum of textura prismatica, cells on middle flanks varying between the populations from regularly prismatic, $8-11 \times 4-7 \mu \mathrm{m}$ to similar or more roundish, $12-20 \times 4.5-12.8 \mu \mathrm{m}, \overline{\mathrm{Q}}=2.1 \mathrm{(n}=$ $70)$. Walls in ectal parts varying between the populations from 0.4 to $0.8 \mu \mathrm{m}$ thick, hyaline, MLZ- except for the scanty dextrinoid nodules present in two populations; $\mathrm{BF}+, \mathrm{CRB}+, \mathrm{TB}+$, fragmentarily $\mathrm{NR}+$, CB-, CR-, LUG-.

Hairs 35-100(-130) x 2-4(-5) $\mu \mathrm{m}$, narrowly conical to slightly lageniform, straight to slightly flexuose, aseptate, in one population infrequently with blackish violet inclusions in MLZ. Apex blunt, tapering to $1-2 \mu \mathrm{m}$, not solidified. Wall thin, never locally prominently thickened, dull, hyaline, frequently bearing lumps of amorphous, yellowish brown resinous matter. Resin persistent in waterbased stains, $\mathrm{KOH}$ and $\mathrm{CR}$, somewhat resistant to $\mathrm{CB}$ and $\mathrm{MLZ}$, often forming globose bodies on the wall in $\mathrm{MLZ}$; $\mathrm{BF}+, \mathrm{CRB}+, \mathrm{NR}+, \mathrm{TB}+, \mathrm{CB}-, \mathrm{CR}-$, LUG-.

Asci $42-56(-58) \times 5.0-7.5(-8.8) \mu \mathrm{m}, \overline{\mathrm{x}}=48.9 \times$ $6.1 \mu \mathrm{m}(\mathrm{n}=63), \bar{Q}=8.0$ in $\mathrm{MLZ} ; 41-59(-62) \times$ $5.0-6.8 \mu \mathrm{m}, \overline{\mathrm{x}}=50.7 \times 5.8 \mu \mathrm{m}(\mathrm{n}=52), \bar{Q}=8.7$ in MLZ; eight-spored, cylindrical-clavate, with a slightly conical apex. Apical pore MLZ++ when dry. Wall in mature asci apically $0.4-0.8 \mu \mathrm{m}$ thick (in $\mathrm{CB}$, MLZ). Contents often LUG+, MLZ+ (orange). Asci arising from croziers.

Spores 7.0-11.6(-12.5) × 2.0-2.8(-3.2) $\mu \mathrm{m}, \overline{\mathbf{x}}=$ $9.3 \times 2.4 \mu \mathrm{m}(\mathrm{n}=295), \bar{Q}=4.0$ in CB and MLZ, elliptic to oblong-elliptic, with rounded to slightly tapered apices, frequently becoming septate (ca. 4 $23 \%$ of the discharged spores), in a few populations often while still inside the asci; septum indextrinoid to dextrinoid in aged spores. Wall hyaline, becoming brownish in aged spores. Guttulae conspicuous in dry material, light.

Paraphyses filiform, unpigmented, first dichotomous branches occurring at the level of the lowermost $1 / 3$. Terminal cells $16-44 \times 0.8-1.4 \mu \mathrm{m}, \overline{\mathrm{x}}=$ $27.6 \times 1.1 \mu \mathrm{m}(\mathrm{n}=30)$.

\section{Cultural characters. Not known.}

Distribution and phenology. The type variety of this species has mostly been collected from Great Britain; 78\% of the collections originate from England and Scotland. The other collections originate from Austria, central Finland, Norway and Sweden and from the timberline area in Québec. With the exception of three collections (from Austria, Finland and Sweden), the material originates from areas with a more or less maritime climate. 

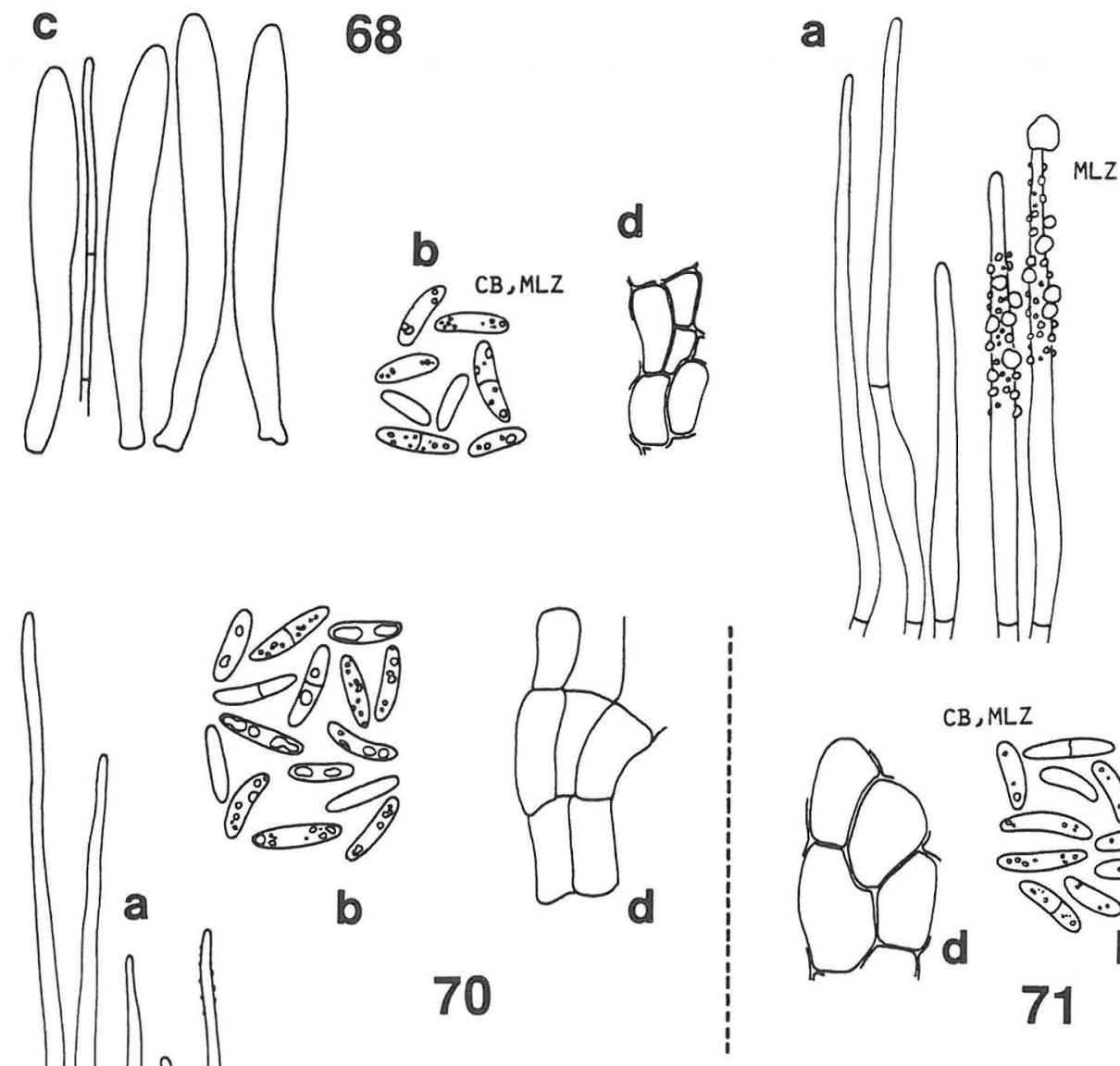

MLZ
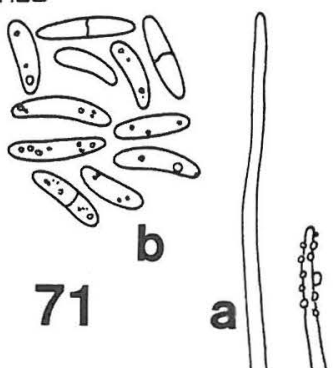

69
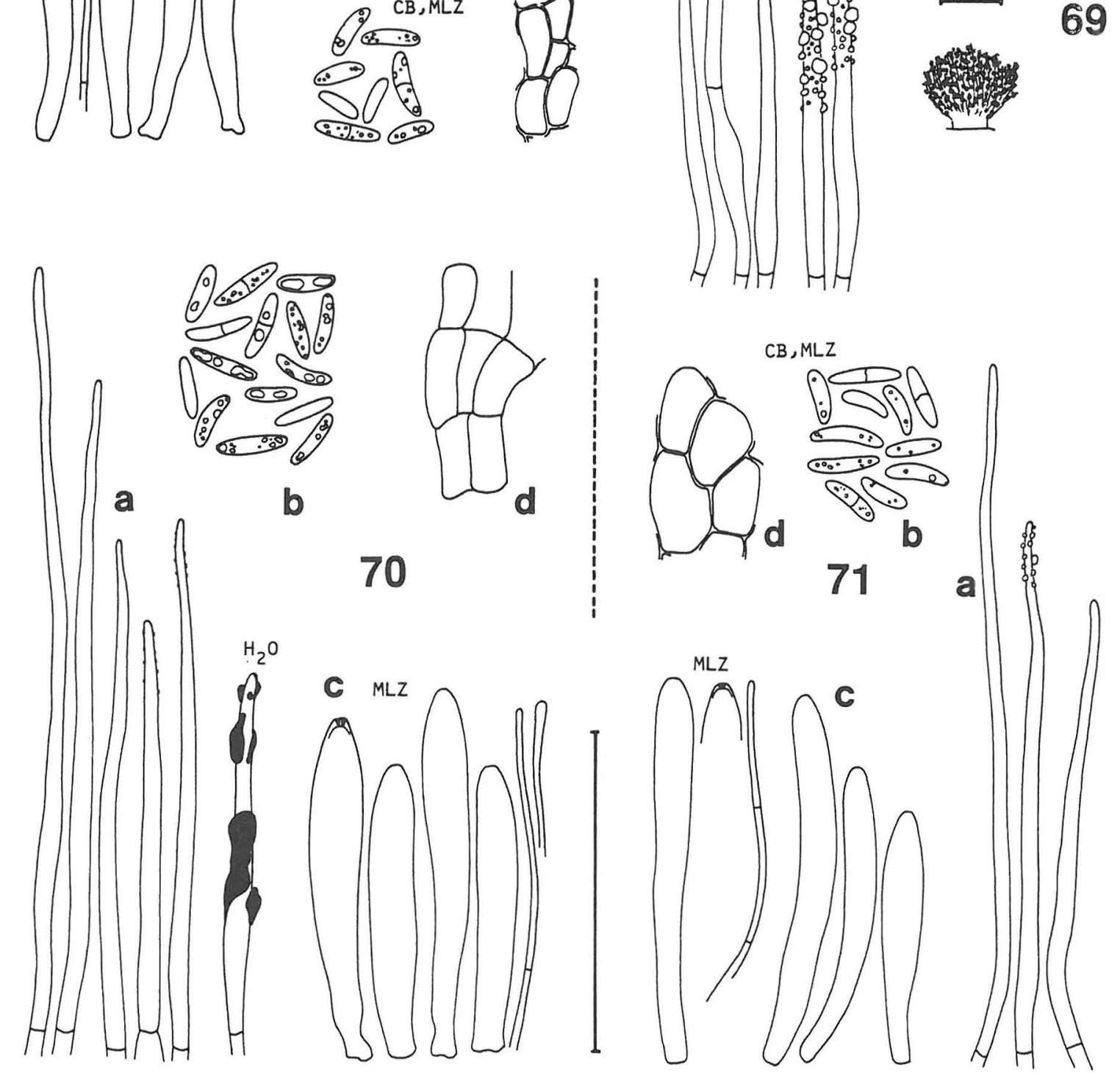

Figs. 68-71. Hyaloscypha britannica var. britannica. a) hairs, b) spores, c) asci and paraphyses, d) excipulum. Scale 50 $\mu \mathrm{m}$, for apothecia $100 \mu \mathrm{m}$. - 68: 1978 Spooner. — 69: Dry apothecia from SH 82/107. — 70: SH 83/382. — 71: Holotype. 
The fruiting period for var. britannica is almost continuous. As in the closely related $H$. aureliella, the collections made during the winter and spring originate from Great Britain.

Ecology. The type variety of $H$. britannica is ecologically similar to $H$. aureliella and $H$. candida. All the collections originate from softwoods, as basically also the collection from Norway, which was made from old newspaper. Sixteen populations fruited on decorticated wood, five originate from bark of conifers and one from building wood. A difference from $H$. aureliella is seen in the proportion of populations fruiting on bark. The exact substrate was indicated by the collectors as follows: Larix (5), Picea (4).

Discussion. This taxon is best distinguished from $H$. aureliella by its clearly larger, often prominently guttulate and septate spores. In $H$. aureliella septate spores are very rare, whereas in some populations of $H$. britannica the spores are even one-septate while still inside the asci. The frequency of spore septation in discharged spores varies between the populations, usually from 4 to $13 \%$, though nearly one quarter of the discharged spores may be one-septate.
A further difference which appears to have taxonomic value lies in the resinous exudate of $H$. britannica. In many populations the resin is not totally dissolved in MLZ or CB, as it is in H. aureliella. In untapped MLZ mounts the hairs are often covered by globose bodies, consisting of partly deformed resin or warts. Some resin is left on the hairs even when MLZ is repeatedly added to the mount. Hence, saturation of MLZ is not the reason for residual undissolved resin, as is occasionally the case in $H$. aureliella. In general the hairs in $H$. britannica tend to be longer than in $H$. aureliella, which is a useful albeit overlapping diagnostic character. The differences from var. roseoguttata are discussed below. The present taxon shows affinities with $H$. aureliella and $H$. candida in the ecology, morphology, phenology and chemical characters. Hence, it clearly belongs to the subgenus Eupezizella. The sparse occurrence of dextrinoid nodules in two populations parallels their rare occurrence in $H$. aureliella. Though apparently responsible for a deep amyloid reaction, these nodules may rarely show a deviating reaction. As holotype, I have selected the specimen treated by Dennis (1949) under the name Cistella tapesioides The substrate is coniferous wood, not Quercus as stated by

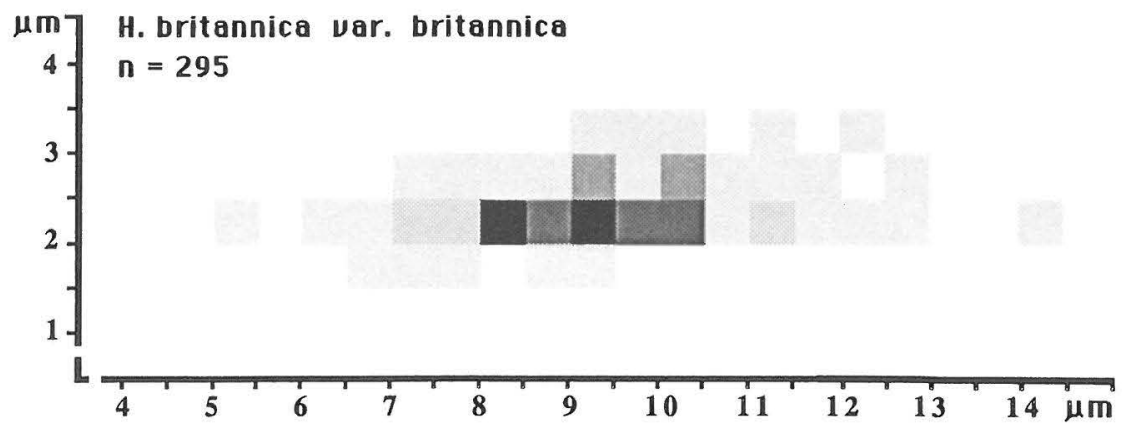

Fig. 72. Percentage sporogram of Hyaloscypha britannica var. britannica.

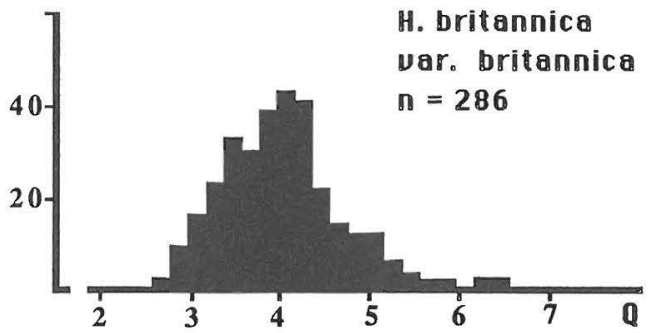

Fig. 73. The distribution of spore quotient Q in Hyaloscypha britannica var. britannica.
Dennis. The discrepancy in naming the specimen was discussed by Korf (1951: 159), who also mentioned an isotype in herb. F, which I have not studied. Two years later, Dennis (1953) acknowledged Korf's correction and referred the collection to Hyaloscypha quercina Velen. var. barbata Velen., basing his view on the description and figures by Velenovsky (1934). As shown by me (Huhtinen 1987a), Velenovský's taxon belongs to Protounguicularia.

Material studied: Austria. Schneeberggebiet, 1902 Höhnel (F, syntype of Dasyscypha resinifera). Canada. Québec: Manitounuk Islands, 1982 SH 82/107 (TUR). Finland. Poh- 
jois-Häme: Laukaa, 1986 SH 86/87 (TUR). Great Britain. Devon: Bellever Forest, 1967 Hawksworth 4881 (IMI). Exeter, 1978 Spooner (K). Berkshire: Reading, 1947 Marsh (K, type). Gloucestershire: Haywood, 1975 Graddon 2497 (Herb. Graddon). Stafford: Rudyard Lake, 1949 Graddon 363 (K). Yorkshire: Hackness, 1955 Watling 151/1 (E). Howe Dale Clough, 1957 Watling 151/2 (E). Wathe, 1959 Watling 151/5 (E). Pickering, 1958 Bramley (K). Galloway: Glen Trool, 1986 Cannon (IMI). Bennan Forest, 1986 Lowen (IMI). Peebles: Glentress Forest, 1954 Henderson 1554 (E). Perthshire: Inver, 1983 SH 83/382 (TUR). Mull: Glen Aros, 1970, 1974 (collector not indicated, E). Inverness-shire: Clachnaharry, 1951 Korf 2179 (Herb. Korf). St. Kilda, 1978 Gilbert (E). Norway. Hordaland: Bergen, 1987 (TUR), 1988 Olsen (BG). Sweden. Uppland: Bondkyrka, 1944 Lundell (UPS).

\section{Hyaloscypha britannica var. roseogut- tata var. nov.}

A varietate typica valde similis, sed ascis in solutione Melzeri non coloratis et resina roseotincta differt.

Holotype: Norway. Svalbard: Longyearbyen, $200 \mathrm{~m} \mathrm{SE}$ of the Svalbard Museum, on a wooden box, 17.VIII.1988 SH 88/62 (TUR, isotypi in CUP, $\mathrm{K}$, cultures in ATCC, CBS).

Apothecia gregarious, superficial, up to $250 \mu \mathrm{m}$ in diam when dry, cupulate throughout development, narrowly to broadly sessile. Disc shallow when fresh, bordered by a prominent zone of marginal hairs, which rise above the level of the hymenium; margin fleshy and incurved when dry, totally concealing the disc. Hair cover dense, rosy to white when fresh, white to brownish when dry. Colour white below, at the margin modified with rosy (M17) exudate, more rarely white throughout; dry apothecia whitish to brownish.

Ectal excipulum of textura prismatica, cells on middle flanks at a slight angle to the surface, prismatic to more rounded, 7.6-13.5(-16.0) $\times 4.8-7.0 \mu \mathrm{m}$, $\overline{\mathrm{x}}=11.7 \times 5.8 \mu \mathrm{m}(\mathrm{n}=25), \overline{\mathrm{Q}}=2.0$, becoming smaller and prismatic towards margin, at the base more roundish. Inner excipulum of narrower textura prismatica. Walls in ectal parts $0.5-1.0 \mu \mathrm{m}$ thick, becoming thinner inwards, hyaline, $\mathrm{MLZ}-; \mathrm{BF}+$, $\mathrm{CRB}+, \mathrm{NR}+, \mathrm{TB}+, \mathrm{CB}-, \mathrm{CR}-$, LUG-.

Hairs $40-90 \times 2.5-4.0 \mu \mathrm{m}$, narrowly conical, straight to slightly flexuose, aseptate. Apex blunt, gradually tapering to $1.3-2.0 \mu \mathrm{m}$, not solidified, without an apical globule in MLZ. Wall thin, never locally thickened, dull, hyaline, smooth to apically encrusted in MLZ, negative in all reagents, frequently bearing lumps of amorphous resinous matter, which are rosy under a hand lens but yellowish brown under the microscope. Resin persistent in water-based stains and CR, dissolving in $\mathrm{CB}$ and $\mathrm{MLZ} ; \mathrm{BF}+$, $\mathrm{CRB}+, \mathrm{NR}+, \mathrm{TB}+, \mathrm{CR}-$, LUG-, MLZ-.
Asci $46-60(-64) \times 6.0-8.2 \mu \mathrm{m}, \overline{\mathrm{x}}=52.3 \times$ $7.2 \mu \mathrm{m}(\mathrm{n}=20), \bar{Q}=7.4$ in $C B$, eight-spored, cylindrical-clavate with a slightly conical apex. Apical pore $\mathrm{MLZ}$ - when fresh and dry, even after $\mathrm{KOH}$ pretreatment, LUG-. Wall in mature asci apically $0.5-1.0 \mu \mathrm{m}$ thick in CB, MLZ, negative in all reagents. Contents MLZ-, frequently LUG+. Asci arising from croziers.

Spores 8.0-11.4(-13.8) x 2.8-3.4(-3.6) $\mu \mathrm{m}, \overline{\mathbf{x}}=$ $9.6 \times 3.0 \mu \mathrm{m}(\mathrm{n}=48), \bar{Q}=3.2$ in $\mathrm{CB}$ and $\mathrm{MLZ}$, elliptic to oblong-elliptic, more rarely subfusoid, with rounded to slightly tapered apices, $26 \%$ of the discharged spores one-septate, septum MLZ-, not seen to be septate while still inside the asci, not seen to become brownish in aged spores. Guttulae prominent, light, with oily contents when fresh in water, similar in dry material.

Paraphyses filiform, unpigmented, first dichotomous branches occurring at the level of ascal bases. Terminal cells $14-39 \times 1.0-1.5 \mu \mathrm{m}, \overline{\mathrm{x}}=23.4 \times$ $1.2 \mu m(n=20)$.

Cultural characters (1 population, 6 strains). Radial growth slow on MA, $7.2 \mathrm{~mm} /$ month $(\mathrm{n}=9$, from 1 popul., 6 strains). Mats with blackish basic colour masked by the dense overall aerial mycelium, below a dark cortex the mycelium deep reddish orange, at the margin with a $0.5-1.5 \mathrm{~mm}$ wide zone of similar mycelium. The aerial mycelium velvety, greyish (M92, P92). Zonation lacking, sector formation lacking, mycelial strands lacking, no colour change in surrounding agar, no yeast-like growth. Context tough, odour not specific. Not producing anamorphs or teleomorphs in single-spore trials on MA.

The prominent reddish orange colouring distinguishes the variety from all the other cultured taxa. Although the colouring apparently mirrors the unique resin of this variety, no traces of resin were seen in the mycelium.

Distribution and phenology. Surprisingly, the sole known collection originates from Svalbard, where suitable habitats on coniferous wood are offered only by man. The variety may have a northern distribution because it has not been detected in the extensive boreal and hemiboreal European material. The collection was made in mid-August.

Ecology. The type was found on the underside of an old wooden box lying in arctic heath. The wood was still relatively hard and seemingly unattacked by any other fungi. In Svalbard coniferous wood occurs solely as building timber or driftwood, the latter be- 


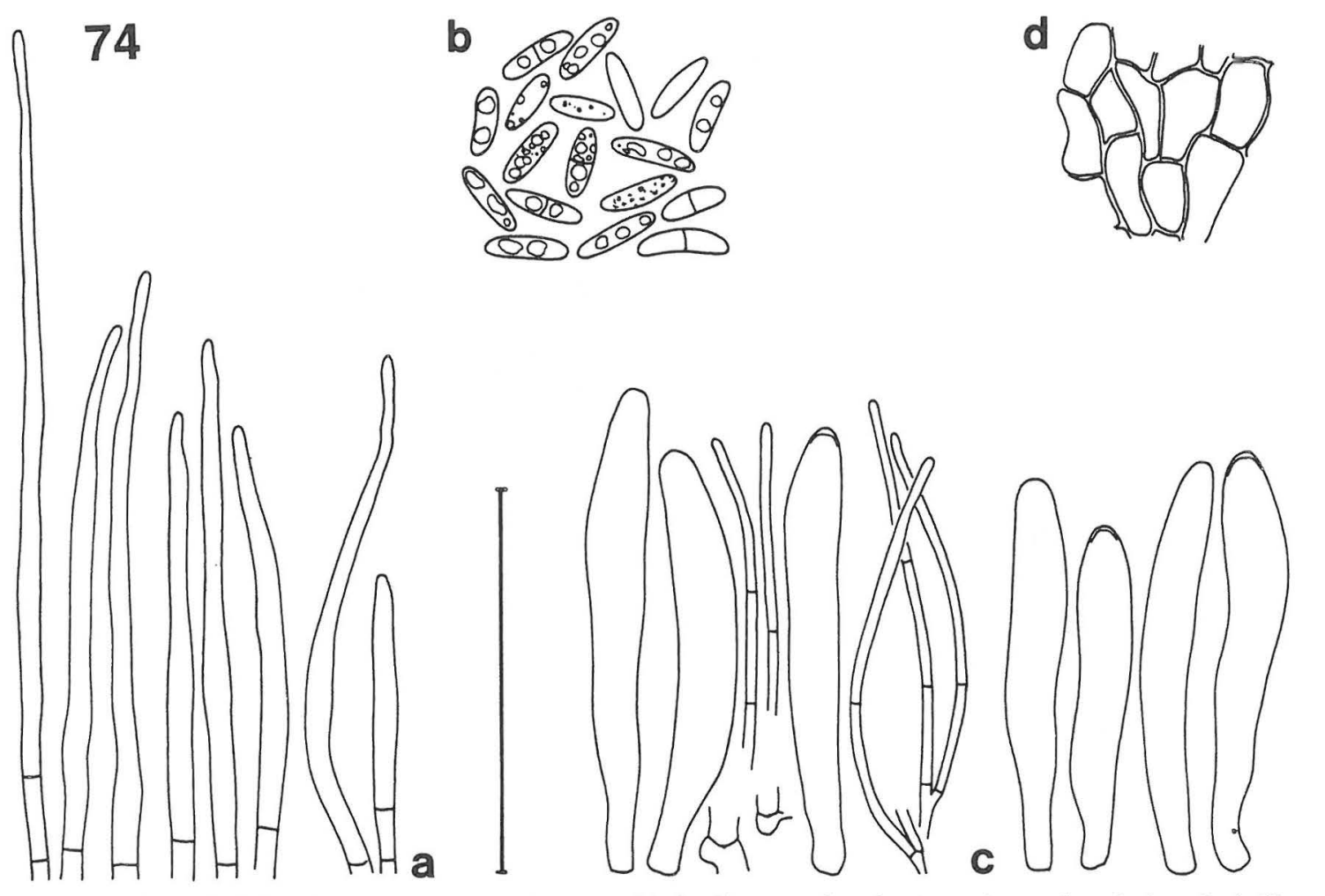

Fig. 74. Hyaloscypha britannica var. roseoguttata, holotype. a) hairs, b) spores, c) asci and paraphyses, d) excipulum. Scale $50 \mu \mathrm{m}$.

ing relatively inaccessible to fungi due to the harsh habitat on the seashore. The combined differences in the resin and asci seem to exclude the possibility that the material arose through environmental modification.

Discussion. H. britannica var. roseoguttata is easily recognized in the field by the beautiful, rosy exudate concentrated at the margin. The rosy tinge prevails but intermixed one can find totally hyaline apothecia, apparently lacking resin. The large, guttulate and frequently septate spores, the large asci arising

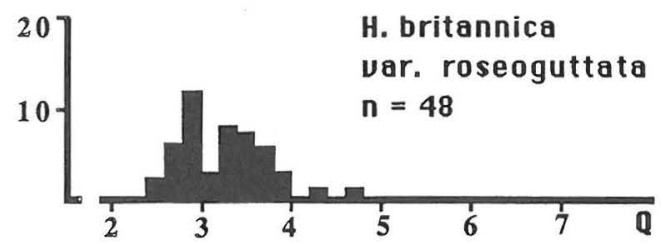

Fig. 75. The distribution of spore quotient $\mathrm{Q}$ in Hyaloscypha britannica var. roseoguttata. from croziers, the narrow paraphyses, the lack of dextrinoid reactions and the ecology link the collection with $H$. britannica. Clear differences are seen in the colour of the exudate and in the MLZ-ascal pores in var. roseoguttata. The value of these characters requires further assessment.

Though the MLZ reaction of the ascal pores shows variation between the populations in $H$. albohyalina var. spiralis, this is very exceptional in Hyaloscypha. Under the microscope and in dry material, the resin loses its rosy hue and appears identical to the resin of var. britannica and H. aureliella, which diminishes its value in taxonomy. The two distinguishing characters appear to be independent and the taxonomic strategy outlined previously could justify its recognition as a species. However, the similarities to var. britannica seem to be taxonomically more important than the differences, and consequently the Svalbard collections should be recognized at varietal level pending cultural studies of the type variety.

Material studied: Norway. Svalbard: Longyearbyen, 1988 SH 88/62* (TUR, type). 


\section{Hyaloscypha candida}

Hyaloscypha candida (Starb.) Boud., Hist. classific. discom. Europe: 127. 1907. - Pezizella candida Starb., Bih. Svensk. Vet.-Akad. Handl. 21:30; P1. 1: 16a-c. 1895. -Eupezizella candida (Starb.) Höhnel, Mitt. Bot. Inst. Techn. Hochsch. Wien 3:61. 1926. - Holotype (the only specimen mentioned): [Sweden,] near Eriksberg, 30.VIII (S). The isotype (in S) contains the following additional data: Sweden, Östergötland, Kolmården Forest, Betula, 1891 Haglund 3.

Apothecia gregarious, superficial, up to $350 \mu \mathrm{m}$ in diam when dry, cupulate, broadly sessile. Margin prominent, incurved, obscuring most of the hymenium when dry. Hair cover dense at the margin, sparse below, snow-white. Colour Straw (K79) to more orange brown (L60) when dry.

Ectal excipulum of textura prismatica, cells on middle flanks prismatic to broadly prismatic, 10.6$20.0 \times 4.8-11.4 \mu \mathrm{m}, \overline{\mathrm{x}}=14.7 \times 6.9 \mu \mathrm{m}, \overline{\mathrm{Q}}=2.1(\mathrm{n}$ $=25$ ), not notably changing size or shape towards the margin, at the base more roundish. Inner excipulum of narrower textura prismatica, cells ca. 8-11 $\times 2.5-4.2 \mu \mathrm{m}$. Walls in ectal parts $0.4-0.6 \mu \mathrm{m}$ thick, hyaline, MLZ- even after $\mathrm{KOH}$ pretreatment.

Hairs $55-125 \times 2.5-3.8 \mu \mathrm{m}$, narrowly conical to almost cylindrical, straight to slightly flexuose, aseptate. Apex blunt, tapering to $1.2-2.0 \mu \mathrm{m}$, not solidified. Wall thin, never locally thickened, dull, hyaline, MLZ-, CB-, smooth to apically encrusted and bearing hyaline, resinous matter. Resin deforming in $\mathrm{MLZ}$ and forming globose bodies on the wall, partly persistent in MLZ and CB.

Asci $36-47(-48) \times 4.2-5.0(-5.2) \mu \mathrm{m}, \overline{\mathrm{x}}=41.4 \times$ $4.7 \mu \mathrm{m}(\mathrm{n}=21), \bar{Q}=9.0$ in MLZ; 34 $45(-47) \times$ 3.3-5.1(-5.3) $\mu \mathrm{m}, \overline{\mathrm{x}}=38.8 \times 4.1 \mu \mathrm{m}(\mathrm{n}=32), \bar{Q}=$ 9.6 in $\mathrm{CB}$; eight-spored, cylindrical-clavate to cylindrical, with slightly conical apex. Apical pore MLZ++, LUG+. Wall in mature asci apically 0.6$0.8 \mu \mathrm{m}$ thick (in CB, MLZ). Contents MLZ-. Asci arising from simple septa.

Spores 5.6-7.6(-8.0) x 1.8-2.2 $\mu \mathrm{m}, \overline{\mathrm{x}}=6.5 \times$ $2.0 \mu \mathrm{m}(\mathrm{n}=107), \bar{Q}=3.3$ in CB and MLZ, elliptic to oblong-elliptic, with rounded to slightly tapered apices, aseptate. Wall hyaline, not seen to become brownish in aged spores. Guttulae prominent and light in CB and MLZ.

Paraphyses filiform to cylindrical, rarely apically slightly widened, unpigmented, first dichotomous branches occurring at the level of the lowermost $1 / 3$. Terminal cells $14-34 \times 1.0-1.2(-2.0) \mu \mathrm{m}, \overline{\mathrm{x}}=21 \mathrm{x}$ $1.2 \mu \mathrm{m}(\mathrm{n}=15)$.

Cultural characters. Not known.
Distribution and phenology. Known only from the type locality in southern Sweden. The collection dates from late August.

Ecology. The type derives from decorticated softwood, although the original diagnosis gives the substrate as wood of Betula.

Discussion. $H$. candida is distinguished from all the other taxa in the genus by the long (stated to reach $200 \mu \mathrm{m}$ in length in the original diagnosis), blunt, aseptate and MLZ- hairs coupled with the small spores and asci arising from simple septa. It resembles $H$. aureliella in morphology, histochemistry and ecology. The hairs are like those of $H$. aureliella but clearly longer. The asci arise from simple septa, which offers a rapid means of recognition in the case of a long-haired population of $H$. aureliella. $H$. candida is also distinguished from $H$. aureliella by the guttulate spores, the narrower asci, hyaline resin, and lack of amyloid excipular reaction. It can be separated from $H$. britannica by the spore size and septation, together with differences in ascus development and resin. $H$. aureliella and $H$. candida are here recognized as independent species. Their delimitation poses no problems, but the taxonomic level at which it should be carried out is not clear. The difference in ascal bases, together with the other dissimilarities, could justify varietal level. Additional differences are found, however, in the resinous exudates. H. aureliella has brownish resin dissolving completely in MLZ and CB, whereas the present taxon has a hyaline exudate, which is partly persistent in these mountants. Especially in MLZ, many hairs are covered by globose bodies. As seen in $\mathrm{H}$. britannica var. roseoguttata, the resinous exudates may be specific to a given taxon. The resin in $H$. candida probably has taxonomic value, adding to the differences from $H$. aureliella. It also differs from the resin of $H$. britannica in being completely hyaline. The amyloid excipular nodules present in nearly $1 / 3$ of the populations of $H$. aureliella were not observed in $H$. candida, but their absence allows no definite conclusions.

Erection of two varieties under $H$. aureliella would group the same hair type and ecological requirements under one species. But, as seen in $H$. nipponica, this hair type also exists in a hardwood species, and is not necessarily indicative of conspecificity. Moreover, such a grouping would unite dissimilar resinous exudates. Unfortunately, the cultural features are known only in $H$. aureliella, and only a provisional solution can be proposed until the variability of 

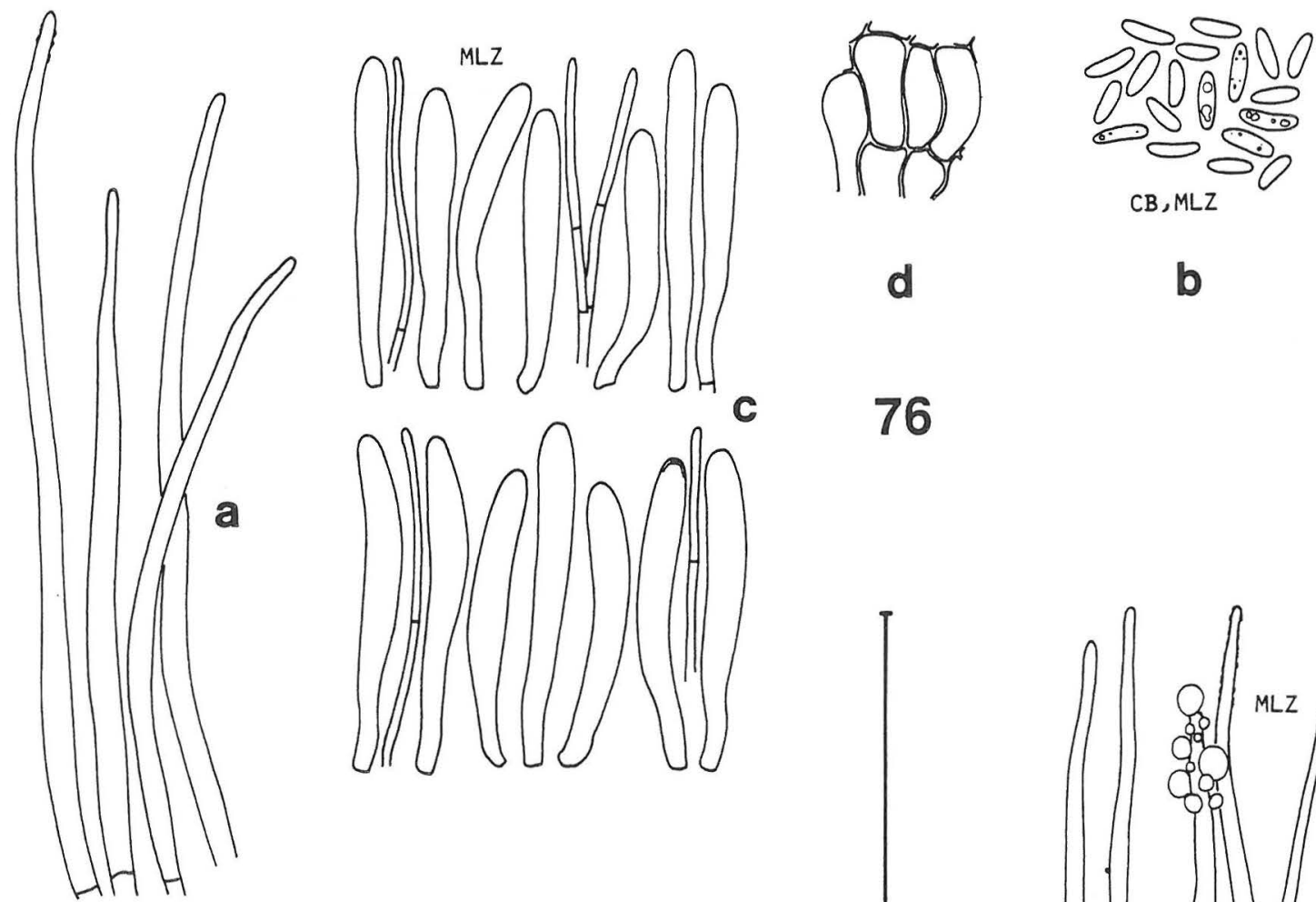

d

b
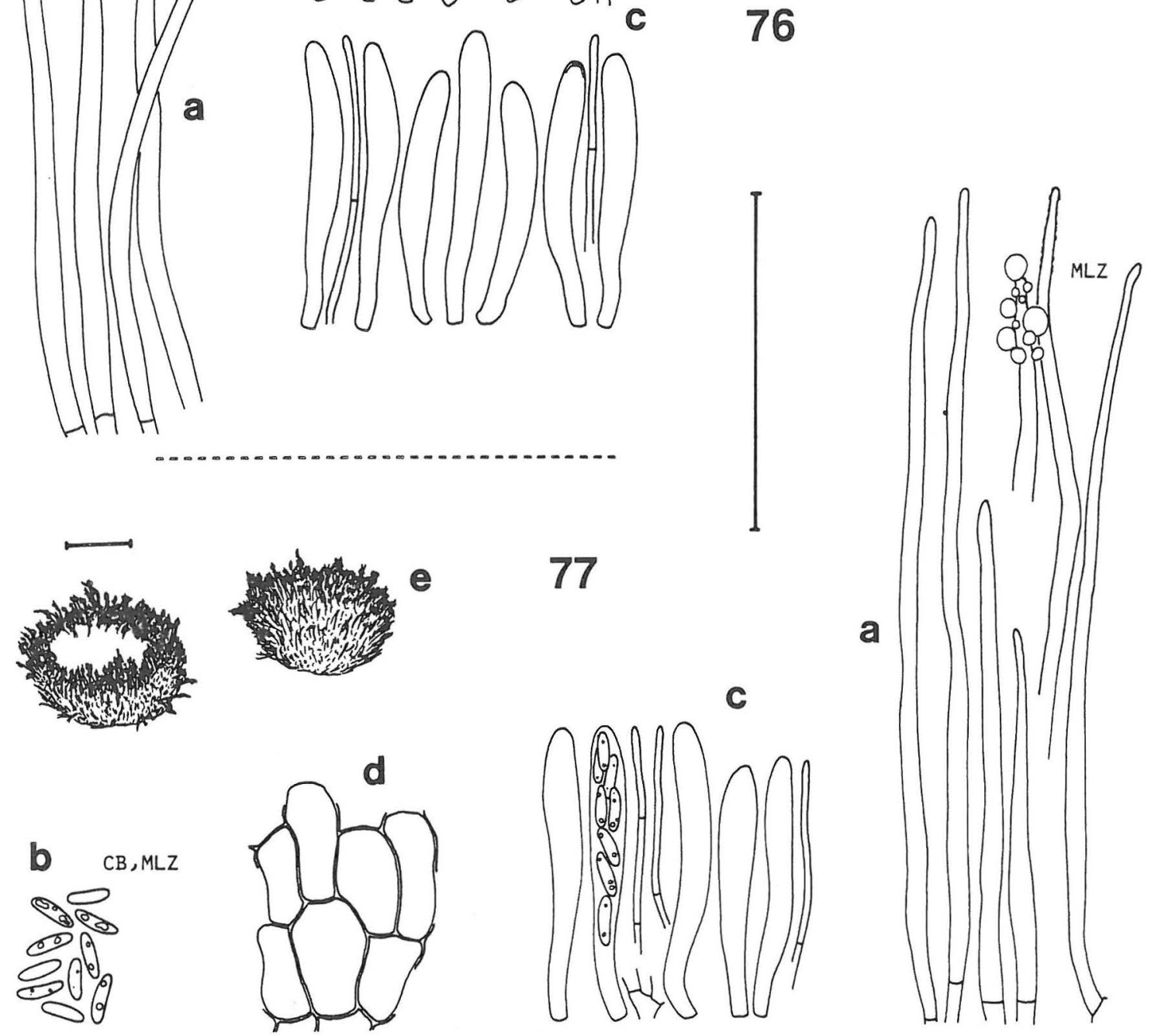

Figs. 76-77. Hyaloscypha candida. a) hairs, b) spores, c) asci and paraphyses, d) excipulum, e) dry apothecia. Scale $50 \mu \mathrm{m}$, for apothecia $100 \mu \mathrm{m} .-76:$ Holotype. - 77: Isotype. 
$H$. candida and its cultural characters can be evaluated. If the possible anamorph proves to be a species of Cheiromycella, the taxonomic solution offered here may have to be reconsidered.

$H$. candida belongs to the subgenus Eupezizella. Due to the scantiness of the type material, the excipular staining reactions were not checked thoroughly. A mount in TB showed, however, that the excipulum is only fragmentarily stained in waterbased stains, as is the case in many other members of the subgenus. In subg. Hyaloscypha the staining is strong.

Material studied: Sweden. Östergötland:, Kolmården, 1891 Haglund (S, type).

\section{Hyaloscypha daedaleae}

Hyaloscypha daedaleae Velen., Monogr. Discom. Bohem.: 285; P1. 15:56. 1934. - Holotype (the only specimen mentioned): [Czechoslovakia,] Bohemia, Mnichovice, Hubackov, Daedalea quercina, 14.IX.1931 Velenovsky (PRM 151201, examined).

?Hyaloscypha quercus Nannf., Nova Acta Soc. Sci. Upsal. Ser. 4., 8:274; Fig. 44c. 1932, nomen dubium, see discussion below. - Lectotype (selected here): Plate 525 in "Icones Mycologicae" (Boudier 1910, as Hyaloscypha hyalina (Pers.) Boud.).

Hyaloscypha luteopallida Svr., Ceská Mykol. 42:79; Fig. 5. 1988. - Holotype (original designation): [Czechoslovakia,] Bohemia, Praha-Liboc, in arboreto Hvězda, in codice Quercus, 24.X.1982 Svrček (PRM 855437, examined).

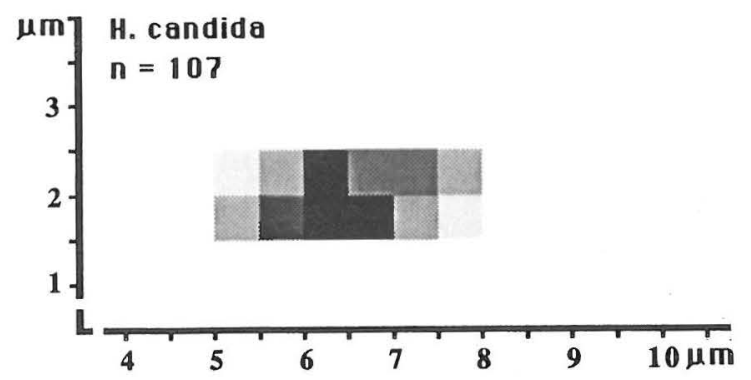

Fig. 78. Percentage sporogram of Hyaloscypha candida.

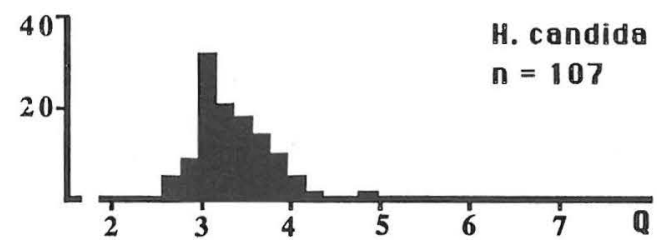

Fig. 79. The distribution of spore quotient Q in Hyaloscypha candida.
Hyaloscypha trapezispora Svr., Ceská Mykol. 42:79; Figs. 6, 7. 1988. - Holotype (original designation): [Czechoslovakia,] Bohemia, montes Brdské Hřebeny, in cacumine montis Hradec $(628 \mathrm{~m})$, ad radicem putr. Quercus, 1.V.1984 Svrčk (PRM 855438, examined).

Hyaloscypha hyalina, "typical form" sensu Dennis, Mycol. Pap. 32:70. 1949.

Apothecia gregarious to confluent, superficial, up to $1 \mathrm{~mm}$ in diam when fresh, typically $200-400$ $(-700) \mu \mathrm{m}$ when dry, cupulate throughout development, often clearly undulate-crenulate when large, narrowly sessile to shortly stipitate on a slender to stout stipe up to $100 \mu \mathrm{m}$, more rarely broadly sessile. Disc shallow to plano-convex when fresh, bordered by a copious zone of short hairs which rise only slightly above the hymenium; margin fleshy and incurved when dry, often obscuring much of the hymenium. Hair cover the same on flanks and at margin, extremely dense, snow-white. Colour watery greyish to yellowish brown (max. M79) when fresh; greyish to light yellowish brown (K77) to Straw (K79) to M79 when dry, in a few populations reddish brown (R15, R17), the dense overall hair cover obscuring these colours.

Ectal excipulum of textura prismatica, cells on middle flanks regularly prismatic, ranging between populations from 5-7 $\times 2.5-4.0 \mu \mathrm{m}$ to $10-13 \times 3$ $5 \mu \mathrm{m}, \overline{\mathrm{Q}}=2.4(\mathrm{n}=50)$, becoming clearly narrower towards margin, at the base more roundish. Inner excipulum of narrower textura prismatica to textura porrecta, cells ca. 8-15 x 2-3 $\mu \mathrm{m}, \mathrm{Q}=3.1-5.0$. Walls $0.8-1.0 \mu \mathrm{m}$ thick in ectal parts, more rarely $0.4 \mu \mathrm{m}$, becoming thinner inwards, hyaline, MLZto MLZ+ when fresh, MLZ+ to MLZ++ when dry, $\mathrm{BF}+, \mathrm{CRB}+, \mathrm{NR}+, \mathrm{TB}+$, fragmentarily $\mathrm{CR}+, \mathrm{CB}-$, LUG-.

Hairs 25-35(-46) x 2-3(-4) $\mu \mathrm{m}$, narrowly conical to conical to slightly lageniform, such variation occurring within a single apothecium, straight to undulating, aseptate (one-septate hairs very rare). Apex tapering to $0.5-0.8(-1.0) \mu \mathrm{m}$, often with a clearly delimited, up to $1.0(-1.5) \mu \mathrm{m}$ wide knob, rarely minutely solidified, often bearing minute, hyaline, apical exudate, which is deformed in MLZ. Wall thin, never locally prominently thickened, hyaline, dull, smooth to loosely granulated on central and apical parts when fresh in water, this granulation removed by all reagents and not visible in dry material, smooth or with persistent, scattered, tiny warts on apical parts when dry, warts deformed or not in $\mathrm{CB}$ and MLZ; wall MLZ - or MLZ+ when fresh and dry, basally $\mathrm{CR}+$, apically $\mathrm{CR}-$, negative in other reagents. Marginal hairs intermixed with abundant cylindrical-clavate, thin-walled cells, $8-20 \times 3-4 \mu \mathrm{m}$. 

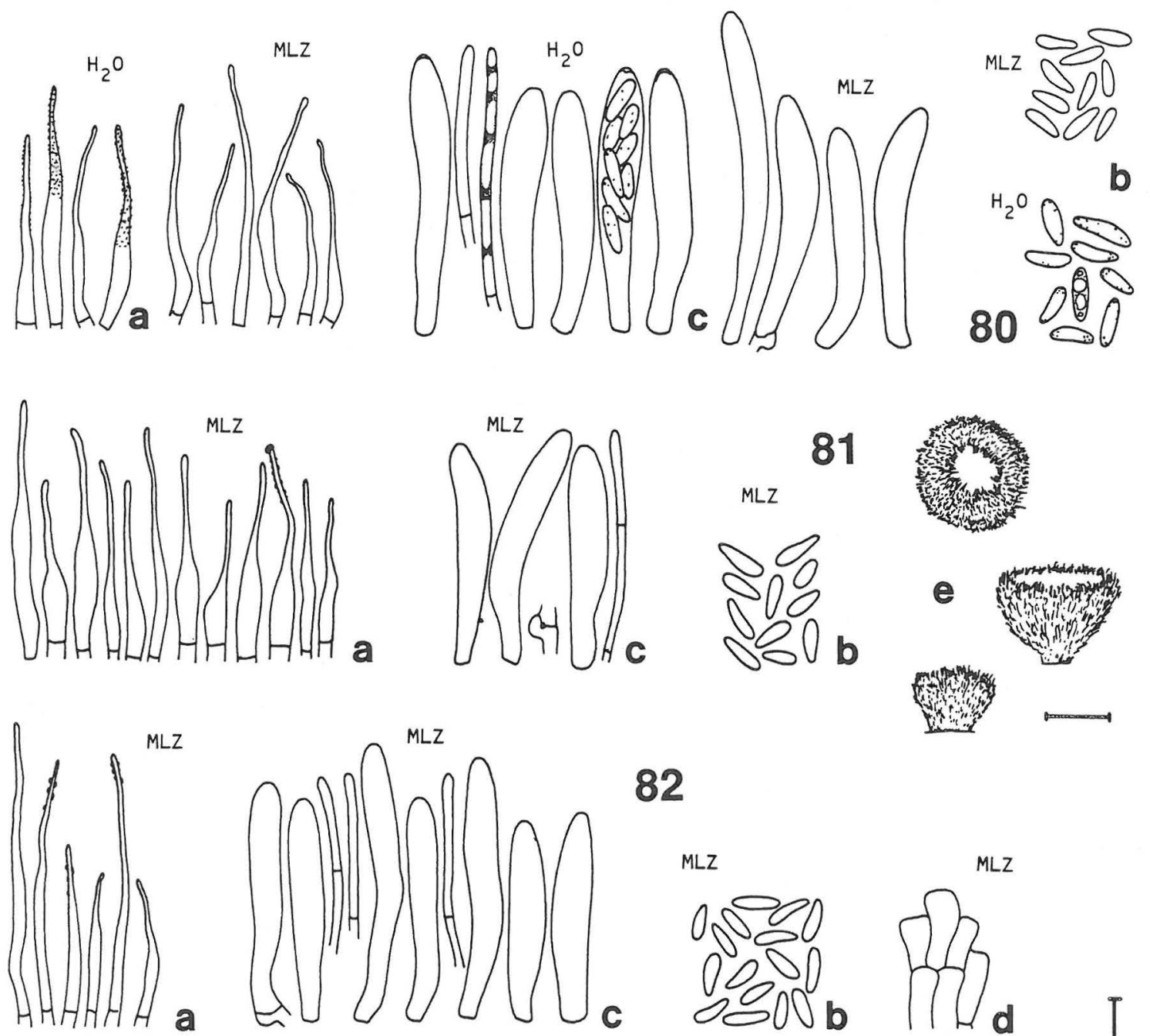

82
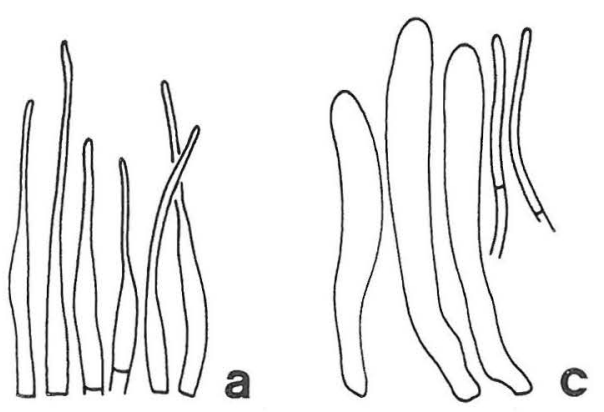

83

Figs. 80-83. Hyaloscypha daedaleae. a) hairs, b) spores, c) asci and paraphyses, d) excipulum, e) dry apothecia. Scale $50 \mu \mathrm{m}$, for apothecia $100 \mu \mathrm{m}$. - 80: SH 87/99. - 81: 1964 Mäkinen et al. - 82: Holotype of Hyaloscypha trapezispora. - 83: Holotype of Hyaloscypha daedaleae. 
Asci 34-53(-64) x 4.8-6.5 $\mu \mathrm{m}, \overline{\mathrm{x}}=42.6 \times$ $6.1 \mu \mathrm{m}(\mathrm{n}=20), \overline{\mathrm{Q}}=3.1$ when fresh in water; 29 $49(-53) \times 4.3-6.3(-6.8) \mu \mathrm{m}, \overline{\mathrm{x}}=37.1 \times 5.1 \mu \mathrm{m}(\mathrm{n}=$ 90), $\bar{Q}=7.2$ in $\mathrm{MLZ} ; 28-50(-56) \times 4.0-5.8$ $(-6.0) \mu \mathrm{m}, \overline{\mathrm{x}}=38.4 \times 4.8 \mu \mathrm{m}(\mathrm{n}=81), \bar{Q}=8.0$ in $\mathrm{CB}$, eight-spored, cylindrical-clavate, with slightly conical apex. Apical pore MLZ++ in $80 \%$ of the populations, MLZ+ in others, LUG-. Wall thin, in mature asci apically $0.2-0.4 \mu \mathrm{m}$ thick (in CB, MLZ), negative in all reagents; contents frequently LUG+, rarely $\mathrm{MLZ++}$ (orange). Asci arising from croziers.

Spores $5-9(-10) \times 1.8-3.0(-3.8) \mu \mathrm{m}, \overline{\mathrm{x}}=7.0 \times$ $2.3 \mu \mathrm{m}(\mathrm{n}=43), \bar{Q}=3.1$ when fresh in water; 5.0 $7.8(-10) \times 1.8-2.2(-2.6) \mu \mathrm{m}, \overline{\mathrm{x}}=6.5 \times 2.0 \mu \mathrm{m}(\mathrm{n}=$ 241), $\bar{Q}=3.2$ in CB and MLZ, ca. $90 \%$ are cuneiform-subfusiform within a population, ca. $10 \%$ oblong-ellipsoid to slightly subfusiform, not seen to become septate, biseriate and situated obliquely inside the asci. Wall hyaline, CR-. Guttulae mostly lacking when fresh in water, only scattered, tiny, dark bodies present; in dry material the same, or a few light guttulae present in MLZ.

Paraphyses cylindrical to slightly clavate, unpigmented, first dichotomous branches occurring typically below the level of ascal bases. Terminal cells $14-40 \times 1-2 \mu \mathrm{m}, \overline{\mathrm{x}}=35.5 \times 1.5 \mu \mathrm{m}(\mathrm{n}=90)$, septa $\mathrm{CR}++$.

Cultural characters (5 populations, 13 strains). Radial growth slow on MA, $10.7 \mathrm{~mm} /$ month $(\mathrm{n}=$ 45, from 3 popul., 10 strains). Mats with overall black basic colour or with black to dark brown (R75) centre, outwards prominently banded, with whitish and brown (P75, R75, N75), rarely olivaceous (P91) bands, extreme margin black to olivaceous in totally black mats, whitish in others. Aerial mycelium lacking or confined to $1 \mathrm{~cm}$ from the inoculum, woolly, thin, greyish (P92) to dark brown (R75), in one population densely woolly to the margin, grey (P92, S92). Margin distinct, even, undulating. Hyphal

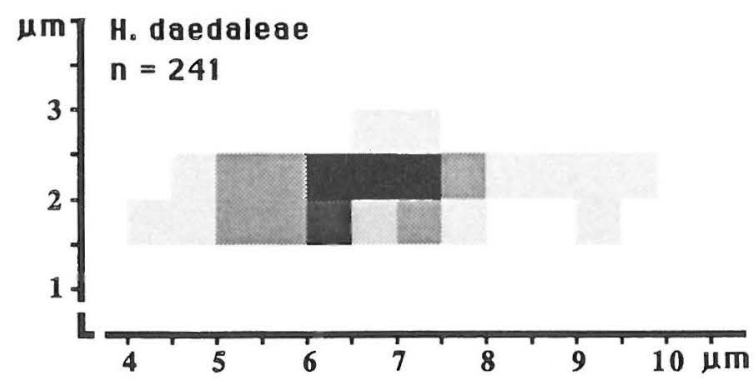

Fig. 84. Percentage sporogram of Hyaloscypha daedaleae. strands present in one population, running in the agar, extending to the margin. Sector formation occasional, no colour change in surrounding agar, no yeast-like growth. Context tough at surface, odour disagreeable in one population tested.

Spores germinating readily on wood agars, growth good on PA, QA, retarded on BA, LA. Aerial mycelium lacking, zonation evident in PA, QA. Anamorph lacking, producing juvenile apothecia on $\mathrm{QA}$ in single spore trials.

The banded appearance of cultures or the overall blackish colour combined with sparse or no aerial mycelium characterize $H$. daedaleae. Though their amount is easily modified by the environment, the dark pigments distinguish $H$. daedaleae from $H$. fuckelii, which often shows zonation in culture, but lacks blackish pigments. Strong mycelial strands are rarely seen in the present species, whereas they often characterize $H$. fuckelii.

Distribution and phenology. Hyaloscypha daedaleae has so far been collected only in Europe. The possible occurrence in North America is discussed below. The fairly small number of collections implies that the peak of fruiting in $H$. daedaleae is later than in the closely related H. fuckelii (Fig. 16). In North Europe most collections were made in late autumn and in central and western Europe $H$. daedaleae also fruits through the winter. By contrast, $H$. fuckelii var. fuckelii has a clear peak during the summer months.

Ecology. Hyaloscypha daedaleae is confined to Quercus. I have not seen collections from other substrates and the earlier reports (cf. Dennis 1949) are erroneous, mostly representing $H$. albohyalina. Like the other species of the genus, $H$. daedaleae prefers decorticated wood; in the present material only one collection originates from oak bark. The type collection originates from an old fruit body of Daedalea quercina L.: Fr. In the ecological sense, such fruiting is not very different from fruiting on oak wood. In the present material apothecia were also found on Hymenochaete rubiginosa (Dicks.: Fr.) Lév.

Discussion. $H$. daedaleae is closely related to $H$. fuckelii, and can be confused with var. fuckelii, since both taxa have asci arising from croziers. The best morphological character distinguishing the two taxa is undoubtedly the cuneiform spore shape predominating in $H$. daedaleae. Exceptional occurrences of $H$. fuckelii var. fuckelii on oak wood can be identified by the oblong-ellipsoid spores. As seen in Figs. 84 and $100, H$. daedaleae has somewhat shorter 
spores than var. fuckelii. Other differences are the short and thin-walled hairs of $H$. daedaleae with very few septa. Due to their shortness, the hairs never become multiseptate, as is occasionally seen in var. fuckelii. Furthermore, many apothecia of $\mathrm{H}$. daedaleae are shortly stipitate and the largest show the typical crenulate-undulating habit even in dry material. When fresh, the apothecia are often slightly yellowish and clearly larger than var. fuckelii. The very dense and equal hair cover of $H$. daedaleae makes dry specimens strikingly different and easily recognized under the dissecting microscope. Among the less obvious differences, revealed in larger material of $H$. daedaleae, are the intensity of the ascal euamyloid reaction, the firm-walled and small-celled excipulum and the commoner occurrence of deformed hair exudates and warts. As $H$. fuckelii occurs only exceptionally on Quercus, the substrate alone is also a fairly good clue to the species.

There is, however, another species growing predominantly on oak: Hyaloscypha quercicola. It is easily recognized under the microscope by its rough hairs, mosty with solidified apices, the different spore shape and asci lacking an euamyloid reaction. In Europe, the difference between $H$. daedaleae and $H$. fuckelii is clear-cut, but in a number of collections of $H$. fuckelii var. fuckelii from the northeastern states of the U.S.A., I found that the difference was less pronounced. Some collections showed a ring-porous structure possibly indicative of Quercus wood and some of these specimens had slightly cuneiform, short spores coupled with short hairs and a firm excipulum. The differences from populations with the character combination of var. fuckelii are, however, not clearly defined. It therefore seems that in North America these taxa are still in the process of speciation, or the ecological barrier blocking gene exchange in Europe is lacking and the taxa undergo introgression. As $H$. fuckelii var. fuckelii undoubtedly occurs in North America, these difficult specimens are referred to that taxon.

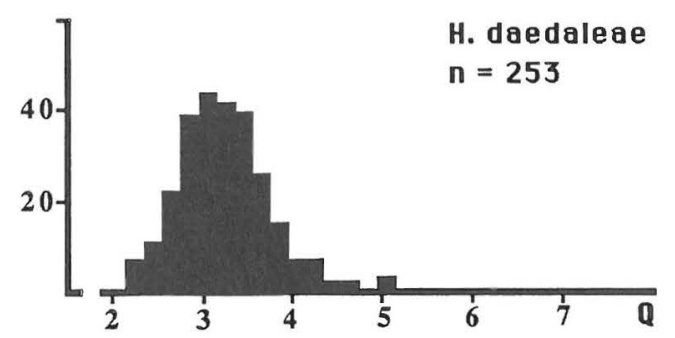

Fig. 85. The distribution of spore quotient Q in Hyaloscypha daedaleae.
Variation in $H$. daedaleae is mainly seen in the ascal length, size of excipular cells, hair roughness and exudates. As in the related species, the ascal length varies with the population. Although the asci are clearly longer in some populations, their width is more constant. The majority of the populations have fairly small-celled excipulum, and only a few have larger cells approaching the lower limit of the excipular cells in var. fuckelii. In some populations the hairs frequently show a hyaline, apical, roundish lump of resinous matter swelling somewhat in MLZ. This substance is different from the occasional, larger, apical globule of var. fuckelii.

Constant characters in the present species are the typical spores, lack of clear guttulae and septa in spores, hair size and shape, lack of prominent solidifications in hairs, ascus bases, ascal width and to a lesser degree the intensity of the euamyloid reaction. The species is clearly delimited from its closest relatives in Europe. The yellowish colour and cuneiform spores recently led Svrcek (1988) to describe two new species from Quercus wood, but study of the type specimens revealed that they both match perfectly with the large material studied here.

The typification of $H$.quercus Nannf. is somewhat complicated. Nannfeldt (1932) published the name $H$. quercus, together with $H$. fuckelii, as a "new name" to clarify the misapplications of Persoon's Peziza hyalina in the earlier literature. In doing so, he published a new species, not a nomen novum (Art. 33.3 Note 1). Although no description was given, the name is validly published, due to Nannfeldt's direct reference to the earlier description by Boudier (1911, as Hyaloscypha hyalina). The words "only on oakwood" on page 268 can hardly be taken as a diagnosis in the sense of the Code (Art. 32.2). Hence, the type of the name H.quercus must be traced back to Boudier's concept of $H$. hyalina rather than to the material in Nannfeldt's possession (Art. 7.16). Of the many synonyms cited, $H$.quercus was unquestionably linked with $H$. hyalina sensu Boudier, as seen also from the list of new combinations and names (Nannfeldt 1932: 336). Although nomenclaturally correct, the link with Boudier's concept is taxonomically unfortunate, since Nannfeldt had studied, many rich collections of the present taxon, suitable for lectotypification.

The sole collection in Boudier's herbarium under the name Hyaloscypha hyalina contains a species confined to coniferous wood, $H$. aureliella. The label bears the text "ex Quélet", indicating that Boudier received the specimen from Quélet, but the material depicted in "Icones Mycol." was collected by Boudier himself from Quercus wood (Boudier 1911: 308) and 
definitely represents another species. Hence, the only element available for typification of $H$. quercus is Boudier's (1910) plate 525 (Art. 9.3).

This plate is accurate and represents a species characterized by the cuneiform-subclavate spores diagnostic of $H$. daedaleae. But the hairs are shown and specifically mentioned to be cylindrical-obtuse and septate. This, together with the small size given for the apothecia, makes conspecificity of the plate and the present species improbable. In conclusion, Nannfeldt's nomenclatural treatment unfortunately makes $H$. quercus a dubious name.

Two years later, Velenovský (1934) published Hyaloscypha daedaleae, for which an apparent holotype exists. The type is unfortunately scanty, but study of one apothecium showed that the specimen undoubtedly represents the present taxon. In the original diagnosis Velenovský (1934) stressed the cuneiform shape of the spores and their small size. The small-celled excipulum is also indicated in the illustration presented by Velenovský. Dennis (1949), following the taxonomic reasoning of Nannfeldt (1932), suggested that the present species should be assigned to Peziza hyalina Pers. This view was based on the fact that Fries (1823) cited Nees's (1817) illustration, i.e. a species stated to grow on Quercus by Nees, in his protologue of Peziza hyalina. As discussed under $P$. hyalina (see Excluded taxa), there is not enough evidence for such a solution, because the illustration by Nees is far too poor in critical taxonomic characters.

Material studied: Czechoslovakia. Bohemia: Mnichovice, $1931 \mathrm{JV}$ (PRM, type of Hyaloscypha daedaleae), $1940 \mathrm{JV}$ (PRM). Praha-Liboc, 1982 Svrček (PRM, type of Hyaloscypha luteopallida). Brdské Hřebeny Mts., 1984 Svrček (PRM, type of Hyaloscypha trapezispora). Slovakia: Zvolen, 1965 Reid et al. (K). Denmark. Jylland: Honssnap Skov,

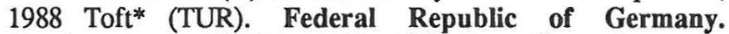
Hamburg area: Sachsenwald, 1903 Jaap (S, Herb Rehm). Bayern: Günzburg, 1963 Doppelbaur (M). Not localized, 1974 Engel 441 (K). Finland. Varsinais-Suomi: Kaarina, 1986 SH 86/173* (TUR). Turku, 1964 Mäkinen et al. (TUR), 1983, $1984,1985,1987 \mathrm{SH} 83 / 32,84 / 248,84 / 254,85 / 142,85 / 143$, $85 / 144 *$, 86/160*, 87/99* (TUR). German Democratic Republic. Brandenburg: Tamsel, 1906 Vogel (S). Great Britain. Devon: Slapton Ley, 1974 Clark (IMI). Surrey: Ranmore, 1947 Dennis (K, IMI. TAA). Sutton, 1888 (K, Herb. Grove). Huntingdonshire: Monks Wood, 1960 Dennis (K). Derbyshire: 1960 Palmer (K). Suffolk: Eastham Wood, 1912 (K, Herb. Ellis). Scotland: Loch Lomond, 1980 Spooner (K). Middleton, New Park, 1885 (K, Herb. Grove). Sweden. Västergötland: Långared, 1943 Hasselrot (UPS). Östergötland: Gryt, 1957 JAN 15305 (UPS). Uppland: Bondkyrka, 1927 JAN 3617 (UPS); 1928, 1930, 1932 Lundell (UPS). U.S.S.R. Lithuanian S.S.R.: Distr. Alytus, Punia For. Res. 1966 AR (TAA).

\section{Hyaloscypha diabolica sp. nov.}

Apothecia cupulata, stipitata, pilosa; specimina exsiccata straminea vel pallide aurantio brunnea, pili nivea. Excipulum externum textura prismatica, cellulis 12-24 x 4-10 $\mu \mathrm{m}$, crassotunicatis, hyalinis, in solutione Melzeri coloratis. Pili 29-50 x 2.8-5.0 $\mu \mathrm{m}$, anguste conici, uncinati, leves vel minuter incrustati, tenuiter tunicati, in apice interdum solidi, aseptati, in solutione Melzeri colorati. Asci 29-48 x 4.3-5.5 $\mu \mathrm{m}$, octospori, amyloidei, non uncinati. Sporae 5.3-7.2 x 1.8-2.3 $(-2.7) \mu \mathrm{m}$, ellipsoideae vel oblongo-ellipsoideae vel subfusoideae, aseptati, minuter guttulati. Paraphyses cylindraceae, 1.2$1.6 \mu \mathrm{m}$ latae, cellulis terminalibus $13-37 \mu \mathrm{m}$ longis.

Holotype: U.S.A. Oregon: Marion Co., Silver Creek Falls campground, on log end, 2.III.1968 Haines 815 (NYS).

Apothecia gregarious to confluent, superficial, up to $200 \mu \mathrm{m}$ in diam when dry, cylindrical to cupulate when juvenile, cupulate when mature, stipitate on a stout, $50-80 \mu \mathrm{m}$ long, $30-50 \mu \mathrm{m}$ wide stipe, more rarely sessile on a gradually tapering base. Margin fleshy and incurved when dry, obscuring most of the hymenium. Hair cover dense at margin, dense to sparse below, snow-white. Colour Straw (K79) to orange brown (M59) when dry, modified by the hair cover.

Ectal excipulum of textura prismatica, cells on middle flanks prismatic to rounded or somewhat elongated, $12-24 \times 4-10 \mu \mathrm{m}, \overline{\mathbf{x}}=15.9 \times 6.0 \mu \mathrm{m}(\mathrm{n}=$ 25 ), $Q=2.7$, becoming smaller towards margin, on stipe clearly elongated. Inner excipulum of narrower textura prismatica, cells elongated, ca. $12-18 \times 2.8-$ $3.2 \mu \mathrm{m}$. Walls in ectal parts $0.8-1.5 \mu \mathrm{m}$ thick, hyaline, MLZ++; $\mathrm{BF}+, \mathrm{CRB}+, \mathrm{NR}+, \mathrm{TB}+, \mathrm{CB}-, \mathrm{CR}-$, LUG-.

Hairs $29-50 \times 2.8-5.0 \mu \mathrm{m}$, narrowly conical to slightly lageniform, uncinate to bent, rarely straight, aseptate. Apex tapering to $1-2 \mu \mathrm{m}$, fairly often solidified, especially in hairs from lower flanks, often showing an apical globule in MLZ; solidifying matter $\mathrm{MLZ}++, \mathrm{CR}++, \mathrm{CB}-$. Wall thin, not locally thickened (except for apex), hyaline, dull, MLZ+, negative in other reagents, smooth, more rarely prominently warted, warts deformed or not in CB, MLZ.

Asci $29-48 \times 4.3-5.5 \mu \mathrm{m}, \overline{\mathrm{x}}=38.8 \times 4.9 \mu \mathrm{m}(\mathrm{n}=$ 15), $\bar{Q}=8.0$ in MLZ; $30-46 \times 4-5 \mu \mathrm{m}, \overline{\mathrm{x}}=38.7 \times$ $4.4 \mu \mathrm{m}(\mathrm{n}=15), \bar{Q}=8.8$ in $\mathrm{CB}$, eight-spored, cylindrical to cylindrical-clavate, with slightly conical apex. Apical pore MLZ++, LUG+ (blue). Contents MLZ-, LUG+. Wall in mature asci apically $0.5-$ $0.8 \mu \mathrm{m}$ thick (in $\mathrm{CB}, \mathrm{MLZ}$ ), negative in all reagents. Asci arising from simple septa.

Spores $5.3-7.2 \times 1.8-2.3(-2.7) \mu \mathrm{m}, \overline{\mathrm{x}}=6.2 \times$ $2.1 \mu \mathrm{m}(\mathrm{n}=54), \bar{Q}=3.0$ in CB and MLZ, elliptic to 


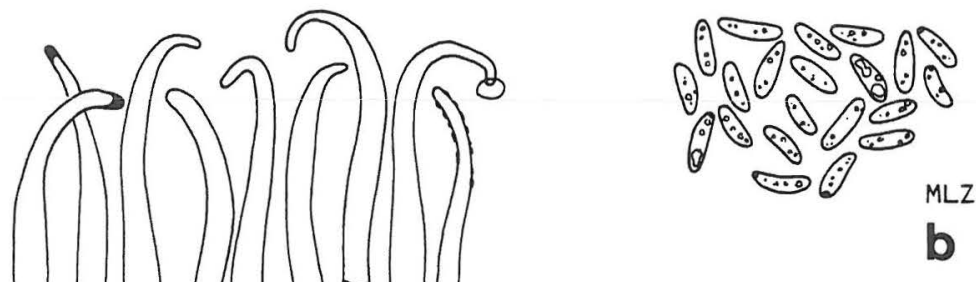

H H H H H H H

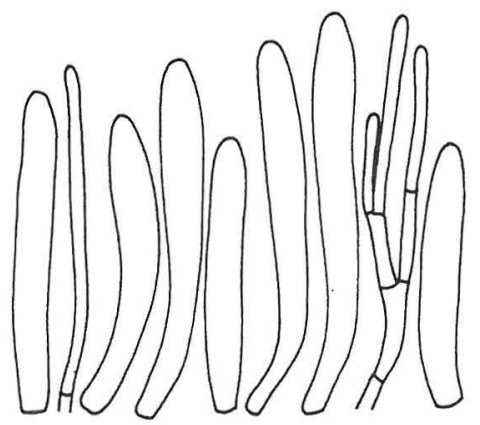

a

$C B, M L Z$

86

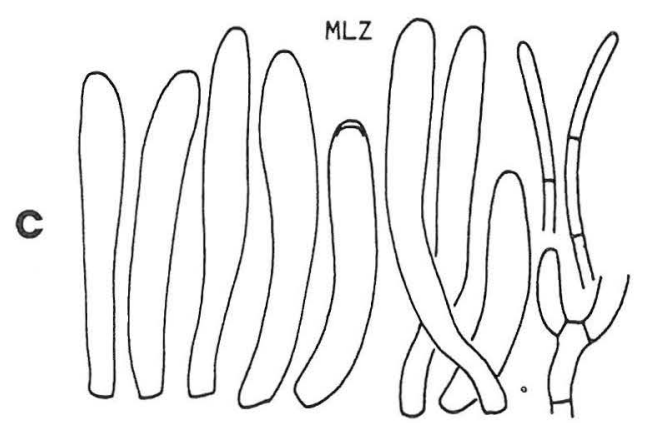

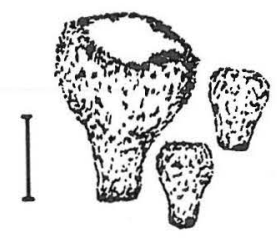

e
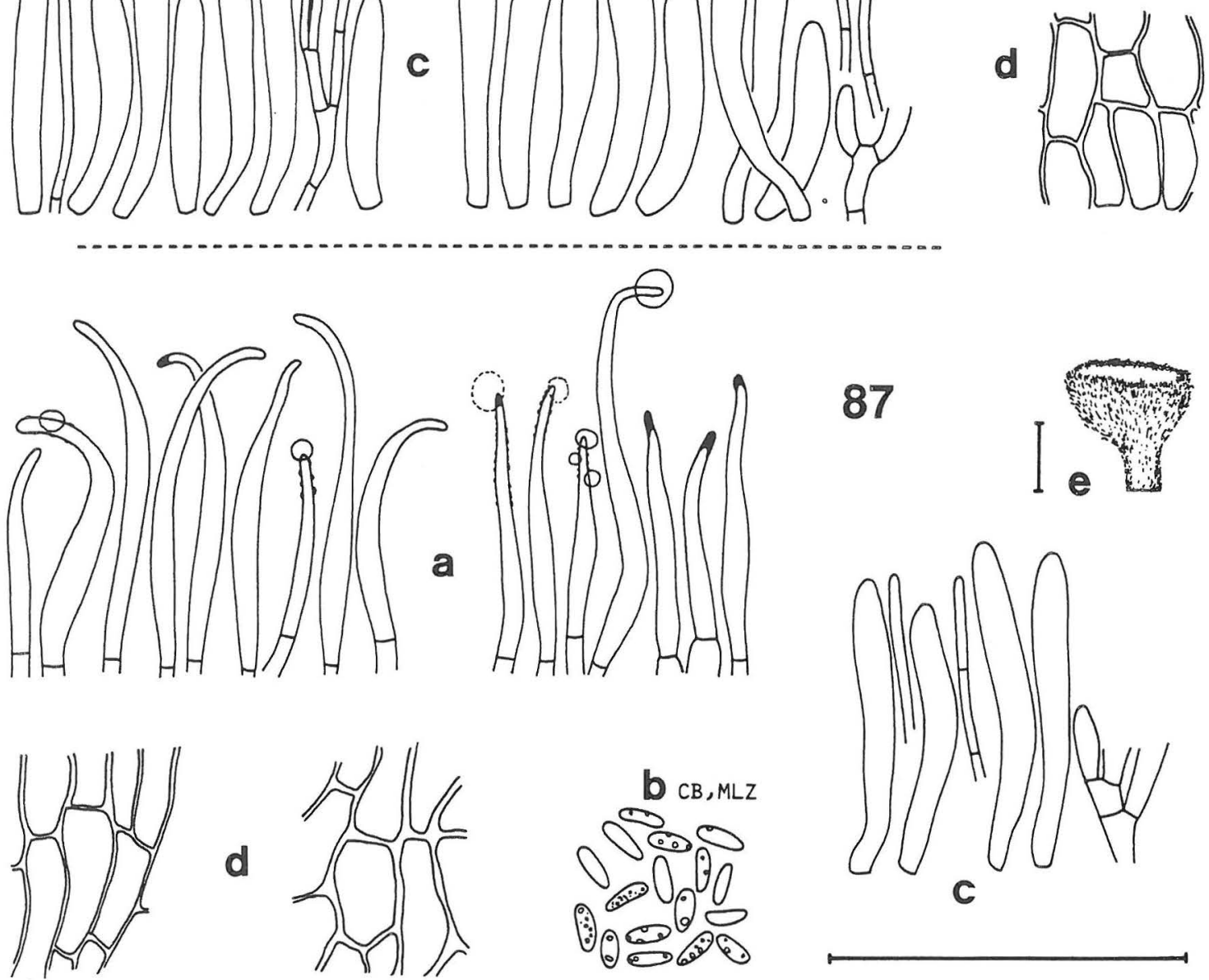

Figs. 86-87. Hyaloscypha diabolica. a) hairs, b) spores, c) asci and paraphyses, d) excipulum, e) dry apothecia. Scale $50 \mu \mathrm{m}$, for apothecia $100 \mu \mathrm{m}$. - 86: Holotype. - 87: Rogerson 3985. 
oblong-elliptic to subfusoid, aseptate. Wall hyaline, not seen to become brownish in aged spores. Guttulae prominent and light in $\mathrm{CB}$ and $\mathrm{MLZ}$, spores with CB-contents have two regular, yellowish, oily globules in water-based stains.

Paraphyses cylindrical, unpigmented, first dichotomous branches occurring at the level of lowermost 1/3. Terminal cells $13-37 \times 1.2-1.6 \mu \mathrm{m}, \overline{\mathrm{x}}=20 \times$ $1.4 \mu \mathrm{m}(\mathrm{n}=10)$.

\section{Cultural characters. Not known.}

Distribution and phenology. Hyaloscypha diabolica is known from only two areas in Oregon and was collected in March and August. As this conspicuous species has not been encountered in the extensive European collections, it may be truly North American.

Ecology. Both collections originate from bulky softwood. In the type collection the substrate species was not indicated, in the other it was Picea sitchensis.

Discussion. Hyaloscypha diabolica is an intermediate species which at first sight might be included in Hamatocanthoscypha, due to the uncinate hairs, but which shows many characters of Hyaloscypha. The hairs and the stipitate apothecia would support placement in Hamatocanthoscypha, but the dextrinoid re-

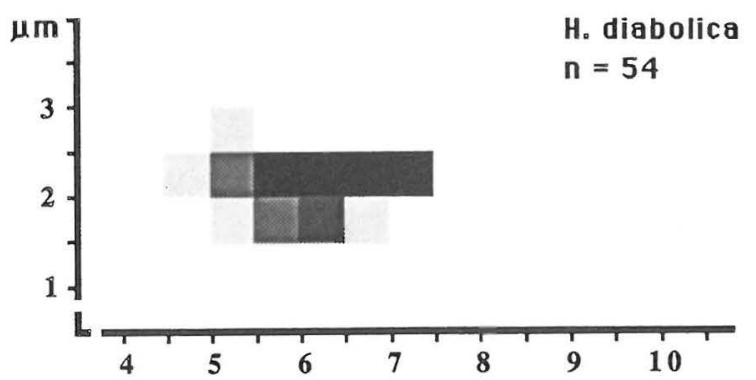

Fig. 88. Percentage sporogram of Hyaloscypha diabolica.

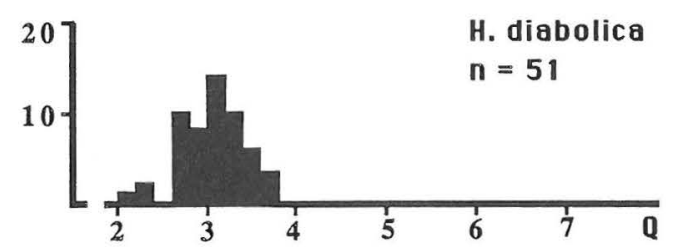

Fig. 89. The distribution of spore quotient Q in Hyaloscypha diabolica. action in the hair and excipular walls is a feature lacking in Hamatocanthoscypha and characteristic of many species of Hyaloscypha. Also characteristic of Hyaloscypha are the hyaline globule at the hair apex in MLZ, the frequently solidified hair apices, the deforming warts, the aseptate hairs and the ecology. Furthermore, asci arising from simple septa are seen in many species of Hyaloscypha, but are known in only two populations in Hamatocanthoscypha minutissima. The distinctive character combination allows rapid recognition under the microscope.

$H$. diabolica seems to be related to $H$. albohyalina, most resembling var. spiralis, but the massively thickened excipulum, the small spores and asci and the uncinate hairs lie outside the range of variation of var. spiralis. The variation between the two known collections is negligible. They are identical in all the critical characteristics.

Material studied: U.S.A. Oregon: Lincoln-Lane Co., Cape Perpetua State Park, 1962 Rogerson 3985 (NY). Marion Co., Silver Creek Falls campground, 1968 Haines 815 (NYS, type).

\section{Hyaloscypha epiporia sp. nov.}

Apothecia cupulata, breviter stipitata vel basaliter constricta, minuter pilosa, alba; specimina exsiccata 50-250 $\mu \mathrm{m}$ lata, ochracea vel armeniaca, pili nivei. Excipulum externum textura prismatica, cellulis 10-15 $\times 2-3 \mu$ m prope marginem, cellulis deorsum 7-16 x 3.4-5.0(-6.5) $\mu \mathrm{m}$, aliquantum crassotunicatis vel crassotunicatis, hyalinis, in solutione Melzeri vinosis. Pili $25-40(-50) \times 2.5-3.5(-4.0) \mu m$, anguste conici, in apice cincinnati, leves, tenuiter tunicati vel in apice solidi, in solutione Melzeri hyalini vel vinosi. Asci 35-55 x 4.5$6.0 \mu \mathrm{m}$, amyloidei, in basi non uncinati. Sporae 5.6-8.2 $x$ $1.8-2.8 \mu \mathrm{m}$, ellipsoideae vel subfusoideae, raro septatae, guttulatae. Paraphyses cylindraceae, 1-2 $4 \mathrm{~m}$ latae, cellulis terminalibus 14-30 um longis.

Holotype: Finland. Pohjois-Häme: Saarijărvi, Pyhä-Häkki National Park, on pore surface of Amylocystis lapponica growing on a fallen Picea trunk, 21.VIII.1986 Strid 17761 (S, isotype in TUR; cultures in ATCC, CBS).

Apothecia densely gregarious, often confluent, superficial, up to $350 \mu \mathrm{m}$ in diam when fresh, $50-$ $200(-250) \mu \mathrm{m}$ when dry, cupulate throughout development, shortly stipitate or with a gradually tapering base; stipe short and stout, ca. 50-70 x 50 $\mu \mathrm{m}$. Margin narrow, inconspicuous and hardly incurved to fleshy and obscuring much of the hymenium (in one population) when dry. Hair cover concentrated in the margin (uniform in one population), snow-white. Colour whitish when fresh; dry apothecia varying from light yellowish brown (K77) to Apricot (L65) and M65. 

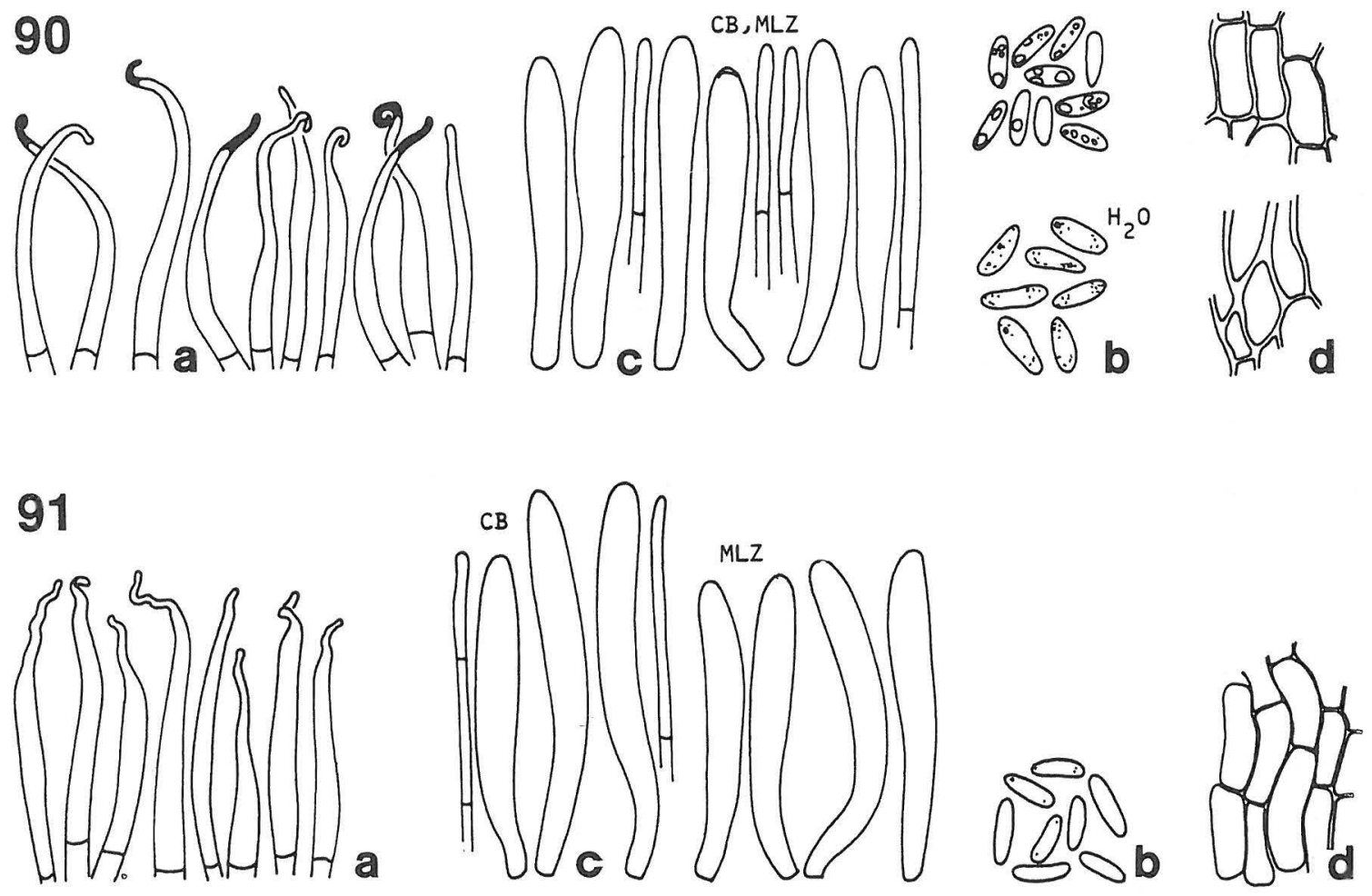

\section{2}
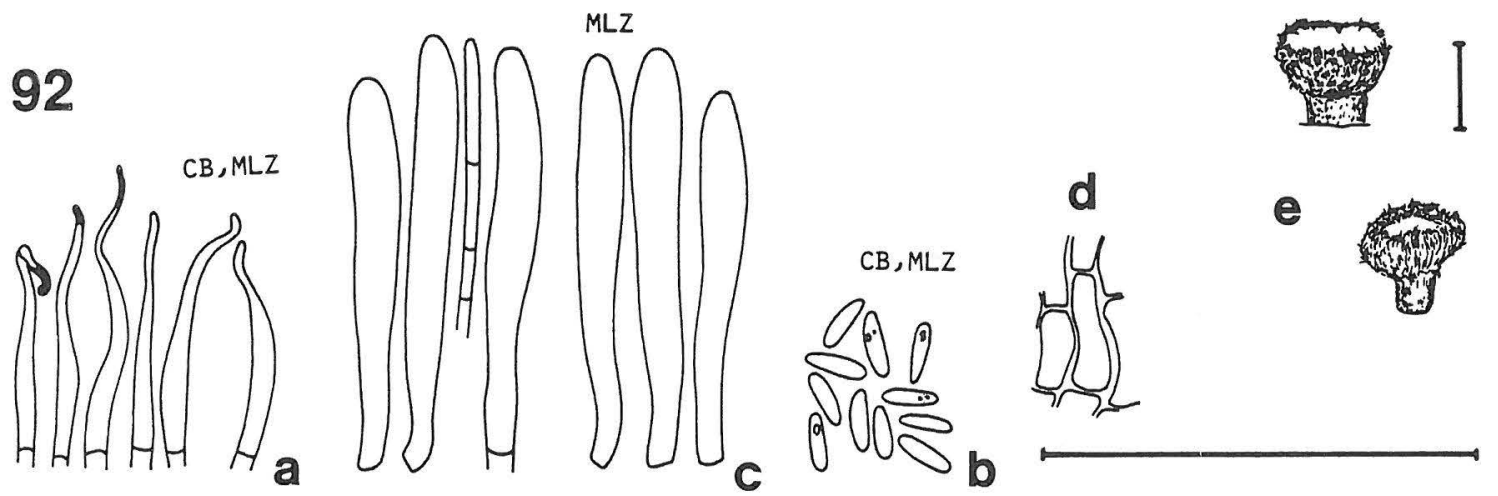

Figs. 90-92. Hyaloscypha epiporia. a) hairs, b) spores, c) asci and paraphyses, d) excipulum, e) dry apothecia. Scale $50 \mu \mathrm{m}$, for apothecia $100 \mu \mathrm{m}$. - 90: Isotype. — 91: 1957 Dennis. — 92: Jackson 12384.

Ectal excipulum of textura prismatica, cells on middle flanks regularly prismatic to somewhat roundish, 7-16 x 3.4-5.0(-6.5) $\mu \mathrm{m}, \overline{\mathrm{x}}=10.6 \times$ $4.2 \mu \mathrm{m}(\mathrm{n}=40), \bar{Q}=2.5$, becoming more elongate towards margin, $10-15 \times 2-3 \mu \mathrm{m}$, at the base more roundish. Inner excipulum of more elongate cells, ca.
14-18 $\times 3.0-3.5 \mu \mathrm{m}$. Walls in ectal parts $0.6-$ $1.5 \mu \mathrm{m}$ thick, hyaline, MLZ+ when dry, $\mathrm{CRB}+$, $\mathrm{NR}+, \mathrm{TB}+$, fragmentarily $\mathrm{CR}+, \mathrm{BF}-, \mathrm{CB}-, \mathrm{LUG}-$.

Hairs $25-40(-50) \times 2.5-3.5(-4.0) \mu \mathrm{m}$, narrowly conical, apically strongly curved and cincinnate, rarely straight, aseptate. Apex tapering to 0.8- 
$1.0 \mu \mathrm{m}$, prominently solidified, rarely thin-walled, without an apical globule in MLZ, the solidifying matter MLZ++, CR++. Wall thin to basally $0.5 \mu \mathrm{m}$ thick, never locally prominently thickened (except for apices), hyaline, dull to refractive, smooth in all reagents when fresh and dry, varying between the populations from MLZ- to MLZ+, negative in other reagents.

Asci $35-48(-55) \times 4.5-5.5(-6.4) \mu \mathrm{m}, \overline{\mathrm{x}}=40.9 \times$ $5.3 \mu \mathrm{m}(\mathrm{n}=51), \bar{Q}=7.5$ in MLZ; 35-55 x 4.5$6.0(-6.4) \mu \mathrm{m}, \overline{\mathrm{x}}=45.8 \times 5.3 \mu \mathrm{m}(\mathrm{n}=18), \bar{Q}=8.5$ in CB; eight-spored, cylindrical-clavate, with slightly conical apex. Apical pore MLZ+, LUG+ (blue). Wall in mature asci apically $0.5-1.0 \mu \mathrm{m}$ thick (in CB, $M L Z)$, negative in all reagents, contents frequently LUG+, MLZ+ (orange). Asci arising from simple septa.

Spores $5.6-8.2 \times 1.8-2.8 \mu \mathrm{m}, \overline{\mathrm{x}}=6.6 \times 2.2 \mu \mathrm{m}$ $(\mathrm{n}=125), \bar{Q}=3.2$ in $\mathrm{CB}$ and $\mathrm{MLZ}$, elliptic to elliptic-subfusoid to narrowly elliptic, with rounded to tapered apices, straight to slightly curved, becoming septate very rarely (ca. $1 \%$ one-septate after being discharged, not seen to become septate while still inside the asci). Wall hyaline. Guttulae prominent, light in dry material, spores with $\mathrm{CB}-$ contents with usually one regular, dull guttula.

Paraphyses cylindrical, unpigmented, first dichotomous branches appearing at the level of lowermost 1/3. Terminal cells $14-30 \times 1-2 \mu \mathrm{m}, \overline{\mathrm{x}}=22.3 \times$ $1.6 \mu \mathrm{m}(\mathrm{n}=25)$.

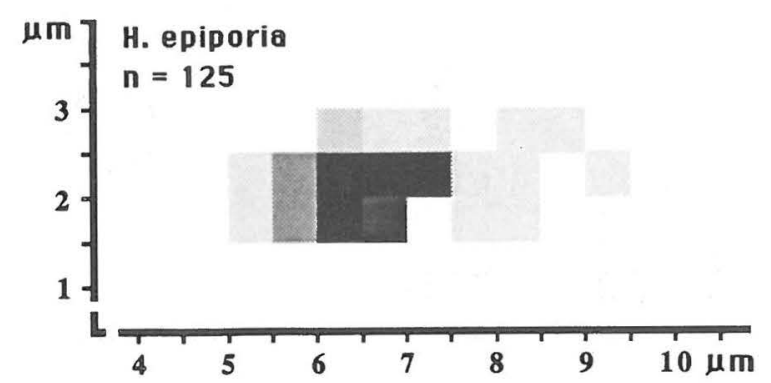

Fig. 93. Percentage sporogram of Hyaloscypha epiporia.

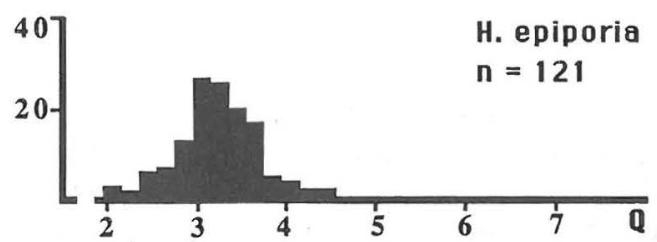

Fig. 94. The distribution of spore quotient Q in Hyaloscypha epiporia.
Cultural characters (1 populations, 2 multispore strains). Radial growth slow on MA, $10.4 \mathrm{~mm} /$ month $(n=5)$. Mats with light basic colours, in centre brown (N75), fading outwards to yellowish brown (K90) to Cream (K75) to whitish. Aerial mycelium lacking. Margin distinct, even, submerged. Hyphal strands inconspicuous but dense to $2 \mathrm{~cm}$ from the inoculum, running in the agar, at the inoculum erect. Sector formation occasional, no colour change in surrounding agar, no yeast-like growth. Context somewhat tough at surface, odour sweet. Anamorph not produced on MA, apothecia produced in abundance on MA after 18 months.

Distribution and phenology. The few collections of $H$. epiporia originate from central and northern Finland, Scotland and Ontario, Canada. The collections have been made between late July and late $\mathrm{Au}$ gust.

Ecology. Three of the collections derive from old, already partly decayed fruit bodies of Amylocystis lapponica (Rom.) Singer, a species restricted to softwoods (Ryvarden 1976). In every case the fruit bodies occur only on the pore surface. The fourth collection (from Scotland) contained a small fragment of polypore hymenium originating from pine logs. I owe the determination of this fragment as cf. Postia lowei (Pilát) Jülich to Dr. Tuomo Niemelä. Again, the apothecia were crowded on the pore surface. The main ecological requirement for $H$. epiporia is possibly the annual nature of the polypore and its occurrence on softwood. In the type specimen at least, the fruit bodies of Amylocystis were those of the current year.

The strict ecological demands make Hyaloscypha epiporia unique in the genus. The species was, however, one of the few Hyaloscyphas to fruit in the laboratory. Several apothecia arose from a multispore isolate on MA after 18 months. The limits of its ecological tolerance are probably not set by the nature of the substrate but by the competition in less specialized habitats.

Discussion. Hyaloscypha epiporia is a rare species that is easily recognized by the substrate and the apically curiously cincinnate and partly solidified hairs. Also typical of the species are the densely gregarious growth habit and the regularly shortly stipitate apothecia.

Variation is seen between the populations in one main character. The collection made from the rarer polypore species (Scotland) lacks the apical solidifications which characterize the great majority of the 
hairs in the apothecia of the three other collections. As all the other diagnostic characters are found in the material from Scotland, the lack of solidified hair apices must be considered to lie within the variational range of the species. This character was relatively constant in culture, only the percentage of the apically solid hairs diminished slightly. Whether the solidifications are influenced by the substrate can be decided when further collections are available.

With the exception of solidified hair apices, the morphological characters of $H$. epiporia are relatively stable. The excipulum is always at least fairly firmwalled, walls up to $1.0 \mu \mathrm{m}$ thick being typical. The chemical characters show limited variation. A dextrinoid reaction is always seen in the excipulum and in only one population were the hair walls MLZ- (not the solidifications). In contrast to those of many Hyaloscyphas, the asci frequently show an orange reaction in MLZ. However, the apothecia obtained from culture were totally indextrinoid. As in many species, the dextrinoid reaction is lacking in fresh and dry material of some populations. In each case such variation was accepted within the species on account of numerous other diagnostic characters. The variation in the apothecia of the present species in vitro supports the view that this chemical character may be altered by external factors.

The reference of the species to Hyaloscypha is based on the general morphology of the hairs and excipulum and on the dextrinoid reaction. The curved to cincinnate hair apices may be interpreted as evidence of relationship to Hamatocanthoscypha, but in that genus dextrinoid reactions and apically solidified hairs are not present. Furthermore, the hair apices almost totally ceased to be cincinnate in cultured apothecia, which may indicate that this character changes with external factors.

Material studied: Canada. Ontario: Lake Timagami, 1935 Jackson 12384 (BPI). Finland. Pohjois-Häme: Pyhä-Häkki Nat. Park, 1986 Strid* $^{*} 15547$ (S, holotype; isotype in TUR). Koillismaa: Valtavaara, 1978 Strid (CUP). Great Britain. Perthshire: Loch Rannoch, 1957 Dennis (K).

\section{Hyaloscypha fuckelii var. fuckelii}

Hyaloscypha fuckelii Nannf., Nova Acta Soc. Sci. Upsal. Ser. 4., 8:273. 1932. No specimens mentioned. - Lectotype (selected here): [Federal Republic of Germany,] am Rheinufer bei Oestrich, Salix, 1894 (S, Herb. Barbey-Boissier 1222, Herb. Fuckel 1894, ex Herb. Rehm; isolectotype in G, examined).

?Hyaloscypha capreolaria Velen., Mon. Discom. Bohem.: 411. 1934. - Holotype (the only specimen mentioned):
[Czechoslovakia,] Bohemia, Mnichovice, Trémblaty, ad truncum carpineum, 28.V.1934 Velenovský (PRM 151246, examined). In contrast to the data on the label, the substrate is coniferous.

Chrysothallus copiosus Velen., Monogr. Discom. Bohem.: 270; PI. 7:31. 1934. - Holotype (the only specimen mentioned): [Czechoslovakia,] Bohemia, Mnichovice, Quercus, 25.V.1931 Velenovský (PRM 150470, examined).

Hyaloscypha hyalina (Pers.: Fr.) Boud. var. laricina Velen., Mon. Discom. Bohem.: 284. 1934. - Lectotype (Svrček 1985: 210): [Czechoslovakia,] Bohemia, Mnichovice, Hrusice, Larix decidua, 19.VII.1926 Velenovsky (PRM 151187, examined).

Hyaloscypha perpusilla Velen., Monogr. Discom. Bohem.: 282; P1. 15:46. 1934. - Lectotype (Svrcek 1985: 214): [Czechoslovakia,] Bohemia, Mnichovice, Carpinus, V.1934 Velenovsky (PRM 151248, examined).

Hyaloscypha spinosae Velen., Monogr. Discom. Bohem.: 411. 1934. - Holotype (the only specimen mentioned): [Czechoslovakia,] Bohemia, Mnichovice, Hrusice, Prunus spinosa, V.1934 Velenovsky (PRM 151260, examined).

Hyaloscypha tiliae Velen., Monogr. Discom. Bohem.: 272; Pl. 14:24. 1934. — Lectotype (Svrčk 1985: 218): [Czechoslovakia,] Bohemia, Ondřejov, Tilia, 27.V.1925 Velenovský (PRM 150875, examined).

Hyaloscypha mali Velen., Novitates mycol.: 193. 1939. Holotype (the only specimen mentioned): [Czechoslovakia,] Bohemia, Mnichovice, in horto nostro, ad lignum mali, VII.1939 Velenovský (PRM 151117, examined).

Hyaloscypha australis Dennis, Results Norweg. Exped. Tristan da Cunha 36:10, Fig. 1. 1955. - Holotype (original designation): Tristan da Cunha, Phylica arborea, 8.I.1938 Mejland 995 (K, examined).

Apothecia gregarious, rarely confluent, superficial, up to $700 \mu \mathrm{m}$ in diam when fresh, $50-150$ $(-600) \mu \mathrm{m}$ when dry, cyathiform to cupulate-stipitate when juvenile, cupulate when mature, narrowly sessile or with a gradually tapering base, rarely with a 30-50 $\mu \mathrm{m}$ long, slender stipe, the exceptionally large apothecia broadly sessile. Disc shallow to planoconvex when fresh, bordered by a conspicuous zone of hairs, which rise above the hymenium; fleshy and incurved when dry, obscuring much of the hymenium. Hair cover dense, snow-white. Colour when fresh pure snow-white to watery greyish to clearly grey (due to the dark substrate shining through); dry apothecia ranging between populations from reddish brown (L25, P15, R17; prevailing) to yellowish brown (K71, K77, K89) to orange brown (M59).

Ectal excipulum of textura prismatica, cells on middle flanks regularly prismatic, ranging between populations from 5-12 $\times 3-5 \mu \mathrm{m}$ to $13-17 \times 3.8-$ $7.0 \mu \mathrm{m}, \bar{Q}=2.4(\mathrm{n}=50)$, not changing size or shape towards margin, at the base more roundish. Inner excipulum of narrower textura prismatica, cells ca. $8-12 \times 2.5-4.0 \mu \mathrm{m}, \bar{Q}=3.3-4.5$, rarely narrowing to the extent of forming textura porrecta. Walls in ectal parts $0.4 \mu \mathrm{m}$ thick (in ca. $20 \%$ of populations $0.8-1.5 \mu \mathrm{m})$, becoming thinner inwards, 

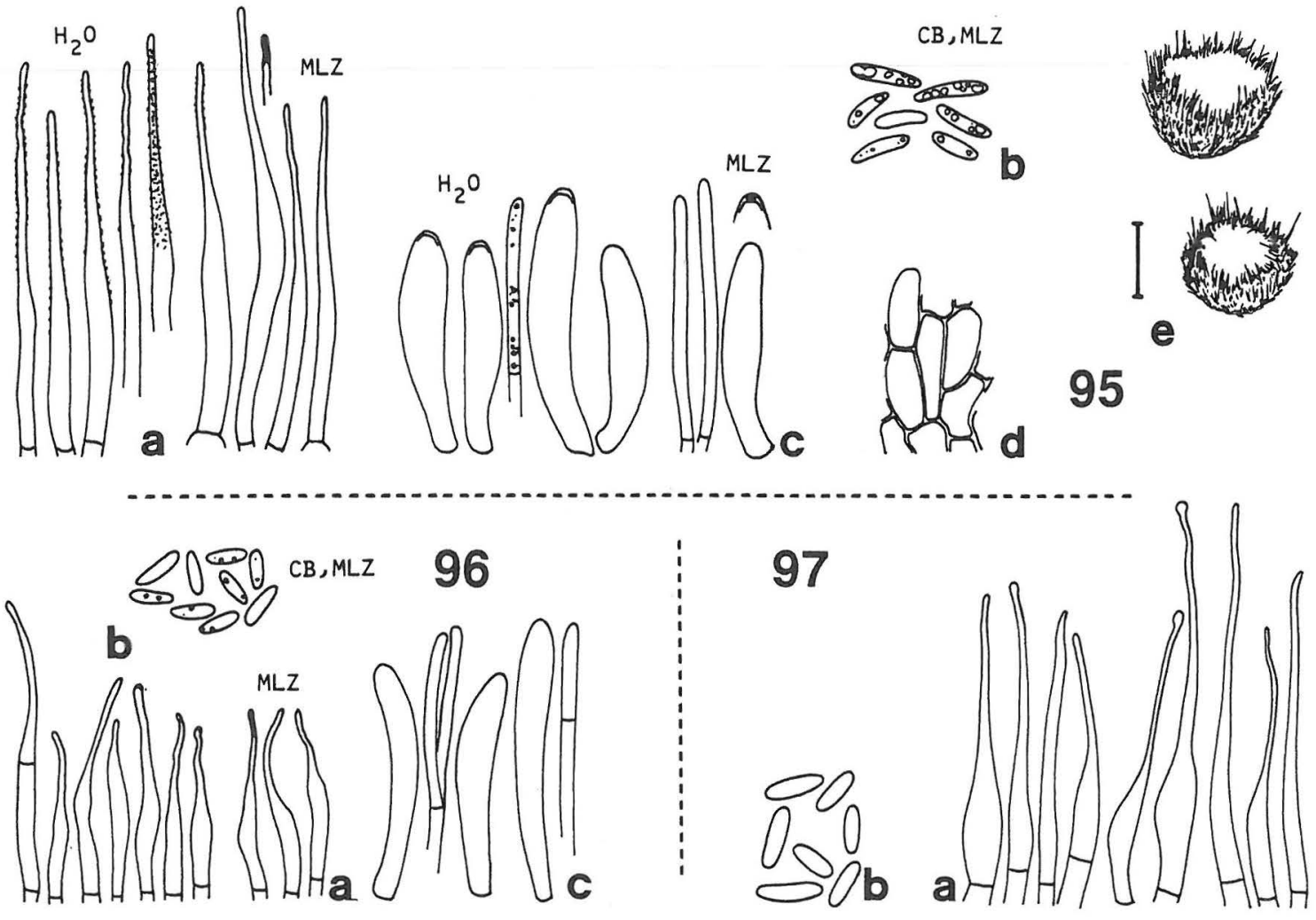

97
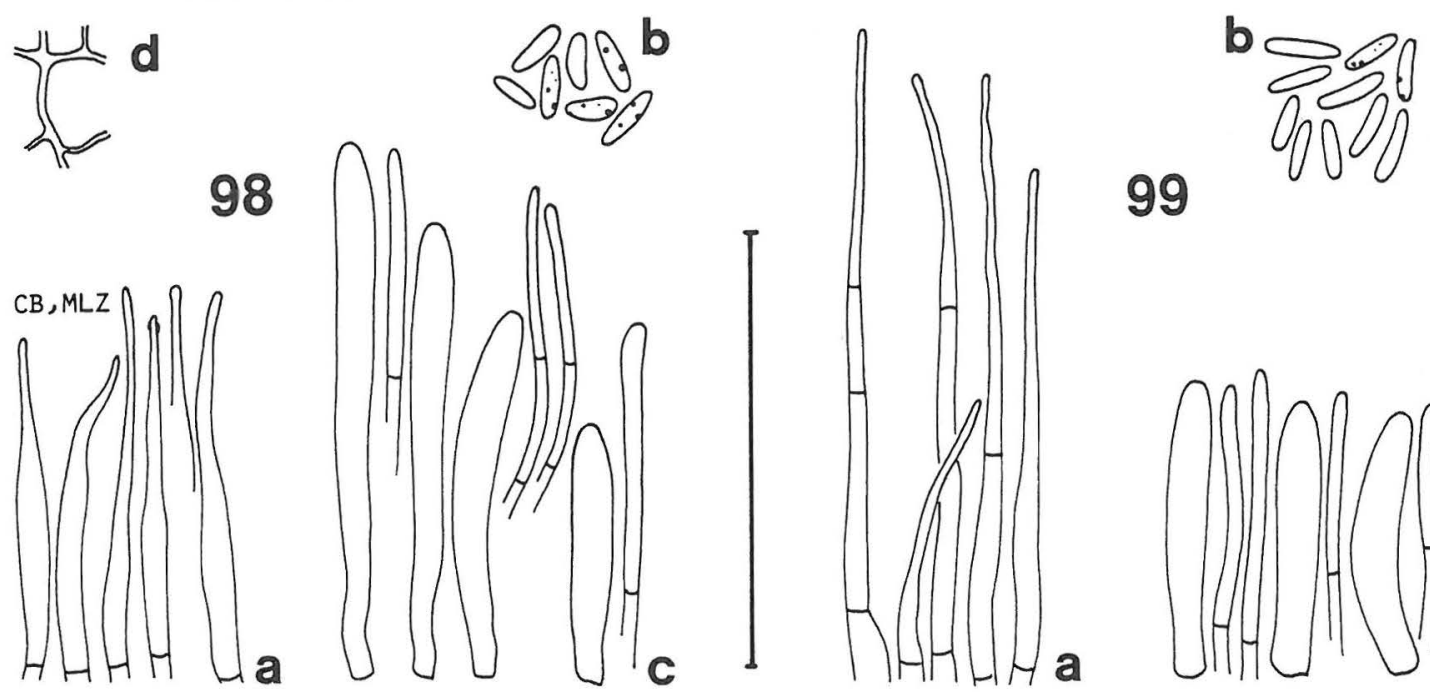

Figs. 95-99. Hyaloscypha fuckelii var. fuckelii. a) hairs, b) spores, c) asci and paraphyses, d) excipulum, e) dry apothecia. Scale $50 \mu \mathrm{m}$, for apothecia $100 \mu \mathrm{m}$. — 95: SH 87/46. — 96: Korf \& Korf 921. — 97: Smith 29259. — 98: Svrcek 89/66. — 99: JAN 10309. 
hyaline, MLZ+ to MLZ++ when fresh and dry (in ca. 7\% of populations MLZ- or fragmentarily MLZ+ even after $\mathrm{KOH}$ pretreatment), $\mathrm{CRB}+, \mathrm{NR}+, \mathrm{TB}+$, fragmentarily $\mathrm{BF}+, \mathrm{CR}+, \mathrm{CB}-$.

Hairs $30-60(-87) \times 2-6 \mu \mathrm{m}$, rarely $20-30 \times 2-$ $4 \mu \mathrm{m}$, narrowly conical to conical to lageniform, such variation occurring mostly between the populations, straight to apically cincinnate, aseptate or with one basal or central septum (ca. 15\% of the populations showing some tendency to become multiseptate). Apex tapering to $0.8-1.2 \mu \mathrm{m}$, often terminating in a clearly delimited, up to $2.0 \mu \mathrm{m}$ wide, partly solidified knob, rarely with an apical globule in MLZ. Wall thin, rarely somewhat thickened, seldom locally thickened but even then without massive thickenings, hyaline, dull or refractive, loosely granulated when fresh in water, this granulation removed by all reagents and not visible in dry material, smooth (rarely bearing a few tiny apical warts, not deformed in MLZ or $\mathrm{CB}), \mathrm{MLZ}+$ to $\mathrm{MLZ++}$ when fresh and dry, basally and in thickened areas $\mathrm{CR}++$, towards apex negative in all other reagents. Marginal hairs intermixed with abundant cylindrical-clavate, thin-walled cells, 8-20 x 4-7 $\mu \mathrm{m}$.

Asci $25-44(-47) \times 4.6-6.5(-7.0) \mu \mathrm{m}, \overline{\mathrm{x}}=31.7 \mathrm{x}$ $5.4 \mu \mathrm{m}(\mathrm{n}=24), \bar{Q}=5.9$ when fresh in water; 25 $45(-61) \times 4.2-6.7(-7.0) \mu \mathrm{m}, \overline{\mathrm{x}}=34.6 \times 5.4 \mu \mathrm{m}(\mathrm{n}=$ 86), $\mathrm{Q}=7.0$ in MLZ; $30-50(-69) \times 4-6(-7) \mu \mathrm{m}, \overline{\mathrm{x}}=$ $37.2 \times 4.9 \mu \mathrm{m}(\mathrm{n}=185), \bar{Q}=8.6$ in $\mathrm{CB}$, eightspored, cylindrical-clavate, with slightly conical apex. Apical pore MLZ+ to MLZ++, rarely MLZ-, in those cases $\mathrm{MLZ}+$ to $\mathrm{MLZ++}$ after $\mathrm{KOH}$ pretreatment, LUG+ (blue). Wall thin, in mature asci apically $0.8-1.0 \mu \mathrm{m}$ thick (in CB, MLZ), negative in all reagents; contents frequently $L U G+$, rarely $\mathrm{MLZ}++$ (orange). Asci arising from croziers.

Spores $5.6-8.5 \times 1.9-2.3 \mu \mathrm{m}, \overline{\mathrm{x}}=7.0 \times 2.1 \mu \mathrm{m}$ $(\mathrm{n}=30), \bar{Q}=3.4$, when fresh in water; $5.5-9.5$ $(-11.2) \times 1.8-2.4(-2.9) \mu \mathrm{m}, \overline{\mathrm{x}}=7.4 \times 2.0 \mu \mathrm{m}(\mathrm{n}=$ 604), $\bar{Q}=4.0$ in CB and MLZ, elliptic to oblong-el-

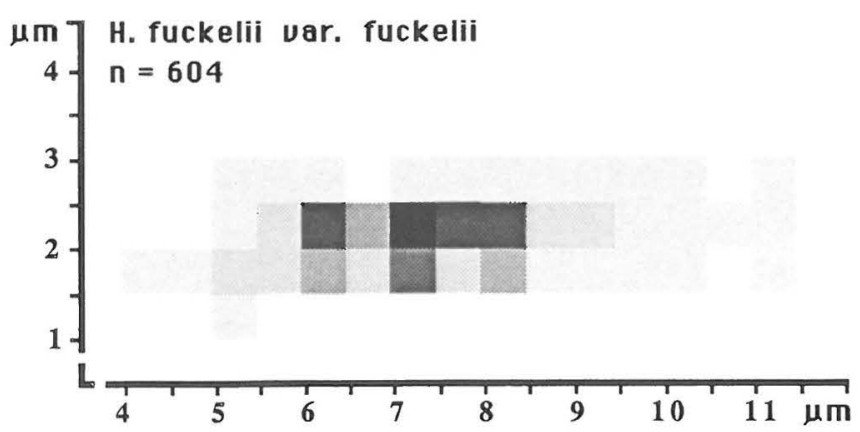

Fig. 100. Percentage sporogram of Hyaloscypha fuckelii var. fuckelii. liptic, rarely fusiform-elliptic, becoming septate very rarely (1.8\% of the discharged spores), once seen to be septate while still in the asci. Wall hyaline, rarely brownish in aged discharged spores, CR-. Guttulae mostly lacking when fresh in water, only scattered, tiny, dark, mostly apical bodies present, rarely with two light apical globules, or multiguttulate; in dry material alike.

Paraphyses cylindrical to slightly clavate (lobate in one collection), unpigmented, first dichotomous branches occurring at the middle level of the asci. Terminal cells $16-37 \times 1.2-2.2 \mu \mathrm{m}, \overline{\mathrm{x}}=24.7 \times$ $1.7 \mu \mathrm{m}(\mathrm{n}=60)$, septa $\mathrm{CR}++$.

Cultural characters (4 populations, 11 strains). Radial growth slow on MA, $9.1 \mathrm{~mm} /$ month $(\mathrm{n}=47$, from 3 popul., 8 strains). Mats in Finnish strains either with creamy white basic colour, dough-like surface without aerial mycelium and strong mycelial strands running in agar (i.e. like some strains of var. alniseda) or with black basic colour, at the margin dark brown (S71), aerial mycelium densely woolly, greyish (P92) to brownish (N71), lacking mycelial strands. The Canadian strain snow-white, surface mealy, no aerial mycelium, no mycelial strands. Margin indistinct, fimbriate, submerged in all types. Zonation lacking, sector formation rare, no colour change in surrounding agar, no yeast-like growth. Context somewhat tough at surface, odour not specific.

Spores germinating readily on wood agars, growth moderate and equal on BA, PA, QA, LA. Producing a $P$ hialophora-type anamorph on MA in all the four Canadian single-spore strains, in Finnish strains anamorph not produced. Not producing apothecia in single spore or multispore trials.

Only a few strains could be studied and the conclusions are consequently tentative. The cultural morphology partly parallels that of var. alniseda but its characteristic zonation is lacking. 
Distribution and phenology. Hyaloscypha fuckelii var. fuckelii is common and widely distributed. It is known from most of Europe, from the Soviet Far East and the Caucasus, from Japan, from Canada and the northeastern U.S.A., and even from the South Atlantic. In Europe no records derive from the Mediterranean area, but this variety reaches the timberline in the north. From the temperate to the boreal zone var. fuckelii fruits from February to October. A clear peak is reached between March and July, $87 \%$ of the present material being collected in that period.

Ecology. Hyaloscypha fuckelii var. fuckelii is mostly confined to hardwoods. In the present material 79 collections originate from hardwoods and only five from softwoods. Most collections originate from sapwood or subcortical or cortical layers, and only a few from soft, water-soaked heartwood, which is the domain of $H$. vitreola. Five populations originate from inner or outer bark: Betula (1), Fraxinus (1), Prunus spinosa (1), unidentified (3). Occurrence on stroma and perithecia of pyrenomycetes is also characteristic of the species; I have seen five such collections. In three further cases the apothecia grew on other wood-rotting fungi, Hymenochaete sp., Peniophora sp. and Phellinus sp., also occurring on naked wood beside these fungi.

The few collections from herbaceous substrates are of special interest. Although its ecological amplitude on ligneous substrates is wide, var. fuckelii is not very successful in occupying herbaceous substrates. It seems to prefer monocotyledonous litter, but the variety has even been collected from a cultivated iridaceous Chilean plant, Libertia formosa, in

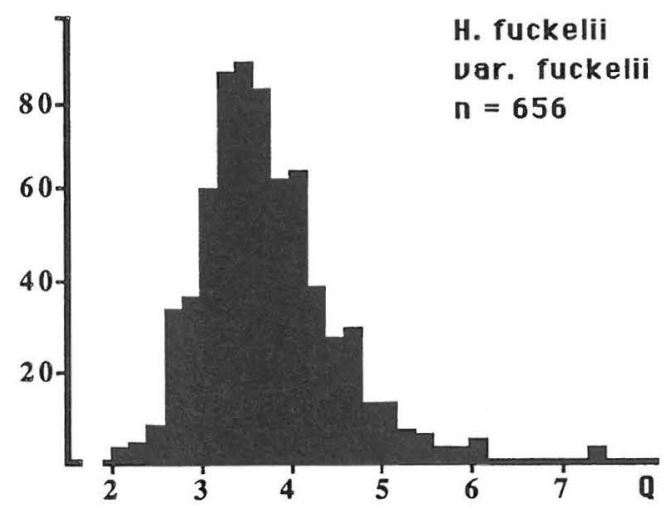

Fig. 101. The distribution of spore quotient Q in Hyaloscypha fuckelii var. fuckelii.
England. Other collections originate from stems of Juncus (1), and leaves of Juncus (1), Typha (1), and Molinia (1).

The substrates in the lignicolous collections are described as follows (populations on bark excluded): Betula (8), Salix (6), Quercus (5), Alnus (4), Carpinus (3), Populus tremula (3), Rubus (2), Abies (1), Corylus (1), Fagus (1), Larix (1), Malus (1), Phylica (1), Picea (2), Populus tremuloides (1), Prunus spinosa (1), Tilia (1), Viburnum (1)

Discussion. Hyaloscypha fuckelii is recognized mainly by the small spores and asci combined with thin-walled hairs. The hairs have loose incrustations when fresh in water, but appear smooth when dry material is mounted. The hair characteristics distinguish it clearly from the rough-haired species $H$. albohyalina, because $H$. fuckelii rarely shows an apically exuded globule or permanent warts on the hairs. Abundant and prominent warts, which often deform markedly in MLZ, characterize $H$. albohyalina, the small-spored populations of which could perhaps be confused with the present taxon.

In many populations of $H$. fuckelii the hairs show two additional characters: a tendency to become septate in their exceeding part and a tendency to form an apical knob. Such populations are easily recognized. The long-haired populations never show such uniformly thick-walled hairs as in $H$. leuconica. Hair wall thickenings are rare in var. alniseda and are hardly ever seen in the type variety. The asci and spores are similar in size to those of $H$. leuconica, and the ecology partly overlaps, but the hair characteristics show only minimal overlap. In such cases strong dextrinoidity of the hairs, a negative reaction in the excipulum, and lack of loose roughness on the hairs in fresh material are indicative of $\mathrm{H}$. leuconica. In Europe the type variety of $H$. fuckelii can be distinguished fairly easily from the related $H$. daedaleae, which also has asci arising from croziers and whose hairs overlap the lower end of the range of hair variation in $H$. fuckelii. The distinguishing features are discussed in detail under that species, which is mostly recognized by its cuneiform spores and faint to lacking dextrinoid reactions. In the great majority of $H$. fuckelii specimens, the dextrinoid reactions are clear to moderate in both the excipulum and hairs, and even the few populations on Quercus lack cuneiform spores. In North America, however, the characters of the species overlap (see $H$. daedaleae).

The two varieties of $H$. fuckelii are distinguished by the difference in the ascal bases. The other, less important differences are discussed under var. alniseda. 
The few herbaceous collections are, with the exception of the specimen from Libertia, characterized by variable spores, many of which are elongated and rather narrow. Such spores are found in $H$. herbarum and $H$. secalina, which may at first be difficult to differentiate. The former species has asci arising from simple septa and wide variation in the spore length. The latter is characterized by narrower spores, smaller asci, and tiny apothecia. Although all the herbicolous collections are linked by their monocotyledonous substrates, they resemble the wood-inhabiting specimens so closely that no taxonomic separation can be made. Merging of these collections in $H$. secalina is not acceptable, because even the most elongated spores differ from the narrowly cylindrical spores of $H$. secalina.

Variation in H. fuckelii is mainly seen in hair and excipular characters. Throughout the range of the species, populations occur with capitate and/or often septate hairs, with small and narrowly conical hairs, with thin-walled or thick-walled excipulum and with large-celled or small-celled excipulum. There seem to be no geographical, ecological or phenological reasons for this variation. Although fresh material shows little variation in colouring, when dry the apothecia are reddish brown in many populations and yellowish brown in others. The curious reddish tone is also present in, e.g., $H$. vitreola and $H$. herbarum, but to a lesser extent.

$H$. fuckelii has stable hymenial characters. The average spore size varies within narrow limits between the populations. Septa are rare and guttulae are almost totally absent. The ascal length varies more widely, but the width is fairly constant.

Nannfeldt (1932), treating Peziza hyalina Pers. as a nomen confusum, published two new names for species in this complex believed to be substrate-specific. His delimitation of "H. quercus" (H. daedaleae) was correct, because the species is confined to Quercus and differs morphologically from the related species. But $H$. fuckelii, proposed to embrace Fuckel's (1869-1870) concept of Pseudohelotium hyalinum (Pers.: Fr.) Fuckel, was based on the erroneous assumption that the species was confined to Salix. When proposing $H$. fuckelii as a "new name" for $P$. hyalinum sensu Fuckel, Nannfeldt in fact published a new name of a new species (Art. 33.3 Note 1). He did not give a formal description, but referred to Fuckel's (1869-1870) description. Fuckel's concept of $P$. hyalinum was based on specimens from his herbarium distributed in Herb. Barbey-Boissier 1222 , bearing data identical to those published by Fuckel. This exsiccata is also preserved in $S$ and represents the present species. It bears no annotations by Nannfeldt, but this is irrelevant from the nomenclatural standpoint. This particular specimen is here selected as lectotype.

Two years later, Velenovsky (1934) described the species as $H$. perpusilla. Although the numerous collections in PRM show that Velenovsky did not have a sound concept of the species complex, the majority of the material belongs to $H$. fuckelii as presently circumscribed. Svrček (1985) correctly lectotypified $H$. perpusilla with the smooth-haired, small-spored group showing dextrinoid reactions in both the hairs and excipulum (reported as amyloid by him), the choice falling on the taxon with asci arising from croziers. As concluded by Svrček (1985), the present species has often been reported and collected as Hyaloscypha hyalina, as may be seen in, e.g., Le Gal's (1939) study.

The type specimen of $H$. capreolaria contains apothecia only from coniferous substrates, although Velenovsky's diagnosis gives Carpinus as the host. The scanty specimen with juvenile apothecia is here referred to $H$. fuckelii, although it also has some features pointing to $H$. aureliella. The spore and ascus size and the lack of dextrinoid reactions fit either taxon. No resin was noted, but whether this is an artifact is not clear. The hairs with relatively narrow apices indicate $H$. fuckelii.

Exsiccatae studied: Phillips, W., Elvellacei britannici 24 (K, E, M, PAD). - Roumeguere, C., Fungi Sel. Exs. 7169 $(\mathrm{G}, \mathrm{NY})$.

Material studied: Canada. Yukon: Kluane Lake, 1987 SH 87/136* Czechoslovakia. Bohemia: Chrustenice, 1946 Vacek (PRM). Klánovice, 1964 Svrček 716/64 (PRM). Krč, 1925 JV (PRM). Malá Chuchle, 1966 Svř̌ek 89/66 (PRM). Mnichovice, 1924, 1926, 1926 (type of Hyaloscypha hyalina var. laricina), 1930, 1931 (type of Chrysothallus copiosus), 1934 (five collections; type of Hyaloscypha spinosae, type of Hyaloscypha perpusilla, type of Hyaloscypha capreolaria) JV (PRM), 1939 (two collections; type of Hyaloscypha mali) JV (PRM). Novohradské Mts., 1966 Svræek \& Kubicka (PRM) Ondrejov, $1925 \mathrm{JV}$ (PRM, type of Hyaloscypha tiliae). Radotín, 1924 JV (PRM). Rěvvice, 1947 Donbova (PRM). Roblín, 1923 JV (PRM). Vyslovka, 1927 JV (PRM). Slovakia: Bratislava area, 1986 SH 86/73 (TUR). Denmark. Sjælland: Ørholm, 1977 Hauerslev (C). Federal Republic of Germany. Nordrhein-Westfalen: Oestrich (S, G; type of Hyaloscypha fuckelii). Not localizable, 1973 Engel 388 (K). Finland. Varsinais-Suomi: Turku, 1984, 1986, 1987 SH 84/48, 86/14, 87/77 (TUR). Etelä-Häme: Kangasala, 1982, 1987 Söderholm 822 (TAA), 1366 (TUR). Tampere, 1987 Söderholm 1369, SH 87/42* (TUR). Satakunta: Parkano, 1987 SH 87/47* (TUR). Pomarkku, 1987 SH 87/46* (TUR). Great Britain. Surrey: Oxshott, 1948 Dennis (K). Rockhampton, 1948 Modelin (IMI). Salop: Almond Park, 1887 (K, Herb. Phillips). Warwickshire: Coleshill Pool, 1979 Clark (IMI). Knavenhill Wood, 1973 Evans 1180 (K). Suffolk: Walberswick Common, 1979 Ellis \& Ellis (IMI, two collections). 
Norfolk: Swanton, 1946 Evans (K). Yorkshire: Austwich Moors, 1963 Bramley (K). Rosper plantation, 1954 Watling 113 (E). Howe Dale Clough, 1957 Watling 114-3 (E). Buckinghamshire: not localizable, 1953 Reid (K). Mull: Not localizable, 1968 Henderson 8079 (E). Strathclyde: Brodick Castle, 1982 Dennis (K). Rosshire: Shielding, 1947 Dennis (UPS). Delamere forest, 1910 Ellis (K). Not localizable, 1977 Spooner (K). Japan. Hokkaido: Nopporo, 1958 Korf e t al. 730 (CUP). Lake Akan, 1958 Korf et al. 857 (CUP). Honshu: Kyoto, 1958 Korf et al. 636 (CUP). Sweden. Östergötland: Gryt, 1949 JAN 10309 (UPS). Uppland: Bondkyrka, 1931 Lundell \& JAN 4190, 4203 (UPS). Ärentuna, 1933 Lundell (UPS). Gästrikland: Gävle, 1953, 1957 JAN 12822, 15317 (UPS). Torne Lappmark: Jukkasjärvi, 1928 JAN 1247 (UPS). Tristan da Cunha (GB). 1938 Mejland (K, type of Hyaloscypha australis). U.S.A. North Carolina: Macon Co., 1947 Korf 811 (Herb. Korf). Dry Falls, 1952 Rosinski \& Korf 2554 (Herb. Korf). Connecticut: Candlewood Lake, 1947 Korf \& Korf 921, 1059, 1065, 1090 (Herb. Korf). New Hampshire: Petersborough, 1956 Stevenson (BPI). New York: Danby, 1947 Rogerson \& Korf 661, 981 (Herb. Korf). Ithaca, 1947, 1949, 1954 Korf et al. 54-4, 54-6, 675, 904, 959, 1605 (Herb. Korf). Essex Co., 1959 Korf et al. 3805 (Herb. Korf). Ulster Co., 1959 Smith 29259 (NY). Unlocalized, Bachman (S, Herb. Bresadola). Michigan: Ann Arbor, 1948 Korf et al. 1291 (Herb. Korf). Halfmoon Lake, 1945 Smith 20287 (MICH). U.S.S.R. Georgian S.S.R.: Not localizable, 1967 AR (TAA). Lithuanian S.S.R.: Distr. Alytus, Punia For. Res., 1966 AR (TAA). Krasnodar Region: Caucasian Res., 1976 Pallo (TAA). Kuril Islands: Kunashir, 1970 Remm (TAA, two collections).

\section{Hyaloscypha fuckelii var. alniseda}

Hyaloscypha fuckelii Nannf. var. alniseda (Velen.) Huhtinen stat. nov. - Hyaloscypha alniseda Velen., Monogr. Discom. Bohem.: 283; P1. 15:47. 1934. - Lectotype (selection made among discordant elements of the holotype): [Czechoslovakia,] Bohemia, Stránčice, St. Anna, Alnus V.1928 Velenovský (PRM 150831, examined).

Apothecia ranging from watery grey to K71 to Cream to K75 and K77 when dry within the same population, rarely clearly reddish brown (L25, P15).

Ectal excipulum of textura prismatica, cells on middle flanks regularly prismatic, ranging between populations from $8-12 \times 3.8-5.0 \mu \mathrm{m}$ to $13-17 \times$ 3.8-7.0 $\mu \mathrm{m}, \bar{Q}=2.4(\mathrm{n}=50)$, in two populations more isodiametric, $5.5-8.8 \times 4.0-6.5 \mu \mathrm{m}, \mathrm{Q}=1.4$ 1.8. Walls in ectal parts $0.4 \mu \mathrm{m}$ thick, ca. $8 \%$ of populations with $0.8-1.0 \mu \mathrm{m}$ thick walls.

Hairs 30-60(-90) × 2-6(-8) $\mu \mathrm{m}$, rarely $20-30 \times$ $2-4 \mu \mathrm{m}$, varying between populations from narrowly conical to conical to clearly lageniform, aseptate to occasionally basally or centrally one-septate, ca. $8 \%$ of populations with fairly many multiseptate hairs in an apothecium; septum thin. Wall thin, rarely prominently thickened at the constriction. Apex tapering to
$0.8-1.2 \mu \mathrm{m}$, typically with minutely thickened apical wall.

Asci $26-54(-61) \times 5.1-7.7(-7.8) \mu \mathrm{m}, \overline{\mathrm{x}}=36.8 \times$ $6.4 \mu \mathrm{m}(\mathrm{n}=77), \bar{Q}=5.7$ when fresh in water; 25 $44(-64) \times 4.6-6.8(-7.0) \mu \mathrm{m}, \overline{\mathrm{x}}=33.3 \times 5.6 \mu \mathrm{m}(\mathrm{n}=$ 113), $\bar{Q}=5.9$ in $\mathrm{MLZ} ; 25-48 \times 4.0-6.3(-7.0) \mu \mathrm{m}, \overline{\mathrm{x}}=$ $35.7 \times 5.0 \mu \mathrm{m}(\mathrm{n}=127), \overline{\mathrm{Q}}=7.1 \mathrm{in} \mathrm{CB}$; arising from simple septa.

Spores $6.1-10.0(-12.0) \times 2.0-2.9(-3.2) \mu \mathrm{m}, \overline{\mathrm{x}}=$ $7.9 \times 2.4 \mu \mathrm{m}(\mathrm{n}=143), \bar{Q}=3.3$ when fresh in water; $5.4-9.9(-12.2) \times 1.6-2.3(-2.8) \mu \mathrm{m}, \overline{\mathrm{x}}=7.3 \times$ $2.0 \mu \mathrm{m}(\mathrm{n}=313), \overline{\mathrm{Q}}=3.7$ in CB and MLZ, elliptic to oblong-elliptic, very rarely one-septate.

Cultural characters (11 populations, 23 strains). Radial growth slow on MA, $7.8 \mathrm{~mm} /$ month $(\mathrm{n}=$ 129 , from 6 popul., 18 strains). Mats variable in colouring, usually creamy white at centre, outwards with prominent brown (M70, P69, P71, S51) or Beige (L91) zonation, the brown colours rarely prevailing, surface dough-like especially at centre. Aerial mycelium absent or inconspicuous, cottony, restricted to $2-5 \mathrm{~mm}$ from the inoculum, in two strains moderate, tomentose, extending $5-10 \mathrm{~mm}$ from the margin, greyish (N92, R92). Margin distinct or indistinct, fimbriate to even, submerged. Prominent submerged hyphal strands present in five strains, lacking in others; all with erect strands arising from the inoculum. Small, white hyphal conglomerations frequent in and on the agar. Sector formation rare, no colour change in adjacent agar, no yeast-like growth. Context somewhat tough at surface, odour not specific.

Spores germinating readily on wood agars, growing well on BA, PA, QA. Aerial mycelium lacking. Anamorphs and teleomorphs not seen in single spore or multispore trials.

Although heterogenous in colouring, var. alniseda can mostly be recognized in culture by the dough-like surface, prominent mycelial strands running in the agar and strong creamy white to brownish zonation.

Distribution and phenology. Hyaloscypha fuckelii var. alniseda is mostly sympatric with the type variety. The present material indicates, however, that var. alniseda is more northerly in its occurrence. This is seen most clearly in large material originating from the same country, e.g. Great Britain, Czechoslovakia and Finland. In Finland var. alniseda is much commoner than var. fuckelii, but in the other two countries the situation is the opposite. Unlike the type variety, var. alniseda fruits from late spring to late autumn, without any obvious peaks during the season. 

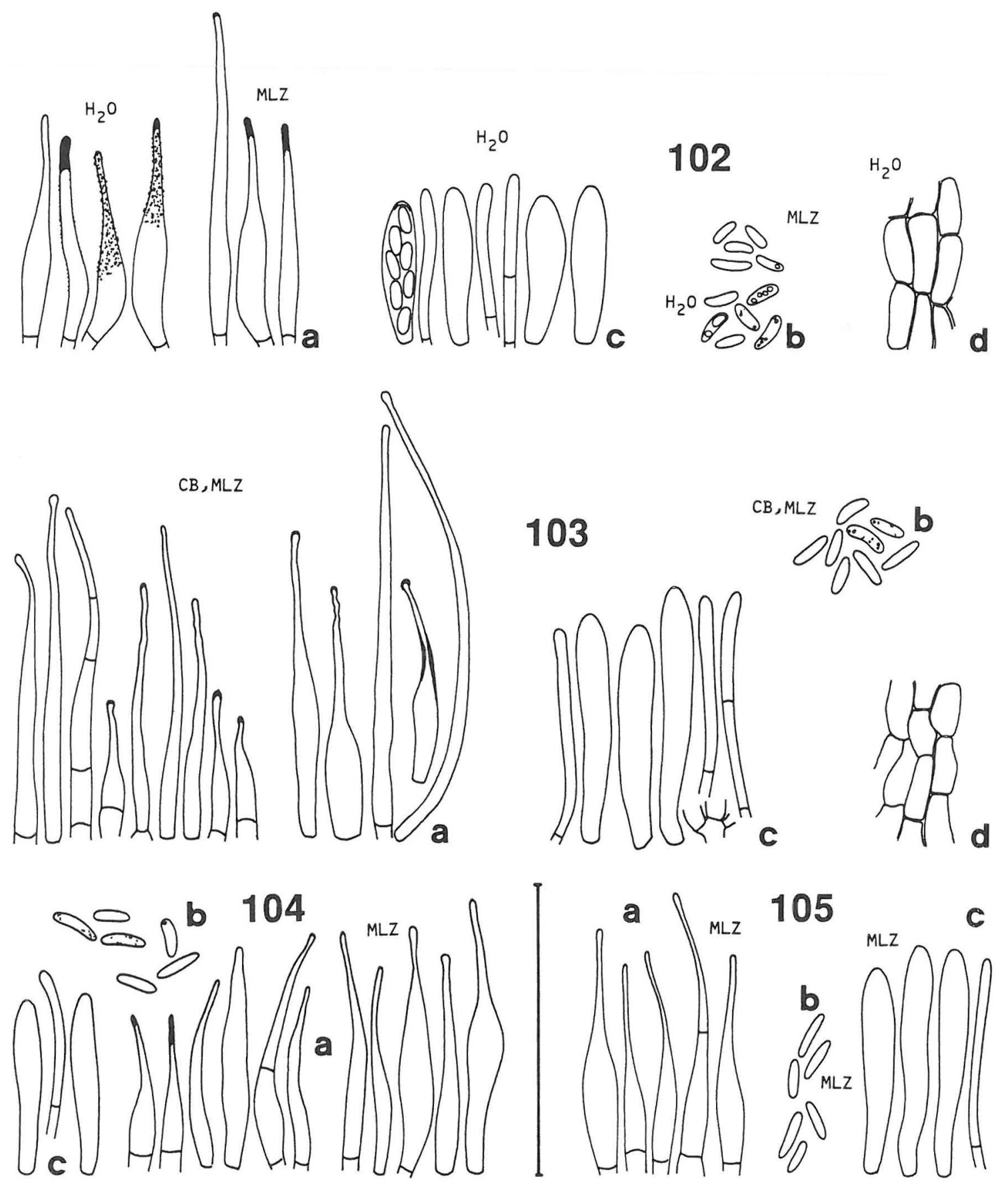

Figs. 102-105. Hyaloscypha fuckelii var. alniseda. a) hairs, b) spores, c) asci and paraphyses, d) excipulum. Scale $50 \mu \mathrm{m} .-102$ : Söderholm 1374. - 103: JAN 20611. - 104: Smith 13432. - 105: 1976 Murdvee. 
Ecology. Var. alniseda does not differ markedly from the type variety. It also prefers to fruit on hardwoods; the present material contains 65 collections from hardwoods and only two from softwoods. Similarly most collections originate from sapwood, or subcortical and cortical layers, and very few from soft or water-soaked heartwood. Eleven populations originate from the inner or outer surface of bark: $B e$ tula (8), Fagus (1), Prunus spinosa (1), Sorbus (1). Occurrence on stroma or perithecia of pyrenomycetous fungi is also typical of var. alniseda; in seven populations the preference for fruiting directly on a pyrenomycete is clear. One collection from old sacking (England) most likely belongs to present variety. Like the type variety, this taxon is rarely found on herbaceous substrates. One collection originates from dead leaves of grass. The following hosts have been listed: Alnus (13), Betula (5), Prunus padus (2), Sorbus (2), Salix (1), Symphoricarpus (1), Lonicera (1), Fagus (1), Populus tremula (1), P.trichocarpa (1), Picea schrenkiana (1). No collection from Quercus could be detected.

Discussion. Var. alniseda is differentiated from the type variety by the asci lacking a basal crozier. Other diagnostic characters are few and the sporograms are almost identical. When present, the hair wall thickenings are more prominent in var. alniseda, especially at the constriction of the lageniform hairs, but such thickenings occur in only a few populations.

The present variety may be confused with smallspored populations of $H$. albohyalina var. spiralis. When the hairs of the latter are warted or broadly lageniform, they differ clearly from those of var. alniseda. Furthermore, although the spores of var. spiralis are at the lower end of the size range, they tend to be more broadly elliptic and to have blunter ends than those of var. alniseda.
In material fruiting on herbaceous substrates, the spore variation is wider. As in the type variety, elongated or very narrow spores are then commoner than in lignicolous populations. The spores resemble those of $H$. secalina, though not as narrow. In the herbaceous population of var. alniseda the average $\mathrm{Q}$ value was $4.3(\mathrm{n}=20)$ and in $H$. secalina var. paludicola it was 5.6. The partial overlap in ecology and spore size may be confusing in identifying a herbicolous collection.

The type collection of $H$. alniseda is unfortunately a mixture of three taxa, the additional ones being $H$. vitreola and $H$. fuckelii var. fuckelii $(=H$. perpusilla). Although Velenovsky's (1934) diagnoses of $H$. perpusilla and $H$. alniseda indicate differences in spore size between the two species, these do not exist. Velenovský treated material now placed under $H$. herbarum as conspecific with $H$. perpusilla, which explains why he gave the spore length as 8-18 $\mu \mathrm{m}$ for $H$. perpusilla. It is obvious that Velenovský mixed the two related taxa in the type collection. Hyaloscypha perpusilla was lectotypified by Svrček (1985) to embrace the taxon with asci arising from croziers and the other taxon is here selected as a lectotype of $H$. fuckelii var. alniseda (Art. 9.2., T.4.(e)). The host is not Quercus as suggested by Svrcek (1985), because its vessels lie in bundles (Grosser 1977).

Earlier reports of this taxon are few. It was recorded from the U.S.A. by Kanouse (see Seaver 1961: 274) and my study of the specimen confirmed this determination. Svrček (1985) treated H. alniseda as a synonym of $H$. perpusilla.

Material studied: Canada. Québec: Poste-de-la-Baleine, 1982 SH 82/132 82/157, 82/199 (TUR). Northwest Territories: Yellowknife, 1984 SH 84/239 (TUR). Czechoslovakia. Bohemia: Stránčice, $1928 \mathrm{JV}$ (PRM, type) Slépiči Hory, 1962 Svrček (PRM). Mnichovice, 1986 SH 86/38*

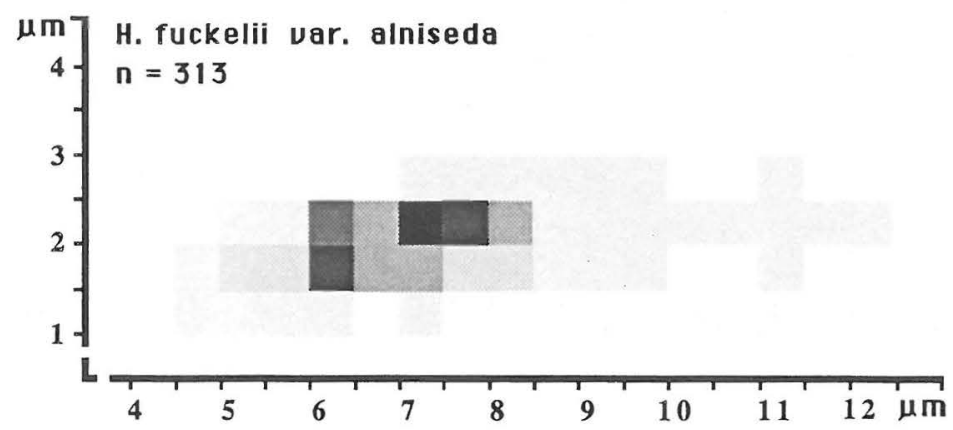

Fig. 106. Percentage sporogram of Hyaloscypha fuckelii var. alniseda. 
(TUR). Slovakia: Tatra Mts., 1924, 1926 Pilát (PRM). Finland. Varsinais-Suomi: Kaarina, 1986 SH 86/167* (TUR) Marttila, 1984 Yli-Mattila (TUR). Piikkiö, 1987 SH 87/22* (TUR). Rymättylä, 1984 SH 84/30 (TUR). Turku, 1984, 1985 , 1986 SH 84/46 84/98 85/133, 85/135*, 85/140*, 86/122, 86/139* (TUR). Uusimaa: Hanko, 1986 SH 86/148 (TUR). Etelä-Häme: Kangasala, 1985, 1986, 1988 Söderholm $1169,1270,1484$ (TUR). Korpilahti, 1986 SH 86/96* (TUR). Lammi, 1985 SH 85/108, 85/111, 85/112 (TUR). Nokia, 1987 Söderholm 1374 (TUR). Tammela, 1868 PAK 2636 (H). Tampere, 1985 Söderholm 1220 (TUR), 1987 SH 87/23. Satakunta: Parkano, 1987 SH 87/4 (TUR) Pori, 1987 SH 87/45* (TUR). Punkalaidun, 1984, 1985 SH 84/260, 84/261, 85/166*, 85/168 (TUR). Vammala, 1986, 1987 SH 86/177, 87/196* (TUR). Inarin Lappi: Utsjoki, 1965 JAN 19119 (UPS). Great Britain. Wiltshire: Batheaston, 1872 (K, Herb. Broome). Bedfordshire: Potton Wood, 1960 James (K). Suffolk: Henham Park Estate, 1982 Ellis \& Ellis (IMI). Yorkshire: Hebden Bridge, 1956 Watling 185 (E). Pickering, 1958 Bramley 59/14a (K). Argyll: Isle of Mull, 1968 Henderson 8052 (E). France. Unlocalized (P, Herb. Montagne). German Democratic Republic. Potsdam, 1971 Benkert (BHU). Norway. Akershus: Oslo, 1985 Gams (TUR), SH 85/45 (TUR). Oppland: Dovre, 1985 SH 85/50, 85/65, 85/72 (TUR). Finnmark: Levajok, 1978 Korf. et al. (CUP). Sweden. Småland: Femsjö, 1929 JAN 3262 (UPS). Gästrikland: Gävle, 1952, 1969, 1971 JAN 12414, 20611, 21836 (UPS). Uppland: Börje, 1947 JAN 9170 (UPS). Jämtland: Åre, 1951 JAN 11571 (UPS). U.S.A. Idaho: Valley Co., 1977 Wells (TAA). New York: Genesee Co., 1948 Korf et al. (CUP). Washington: Olympic Nat. Park, 1934, 1939 Smith 13074, 13432, 13542 (MICH). Vermont: Grafton, 1961 Korf 3058 (Herb. Korf). U.S.S.R. Tjumen Region: Jamalo-Neneckij, Suryskary, 1976 Murdvee (TAA). Kirghizia: Terskei Alatau Mts., 1968 AR (TAA).

\section{Hyaloscypha herbarum}

Hyaloscypha herbarum Velen., Monogr. Discom. Bohem.: 284; Pl. 14:21. 1934. - Lectotype (Svrček 1985): [Czechoslovakia,] Bohemia, Mnichovice, Myšlín, Verbascum lychnitis, 3.IX.1924 Velenovský (PRM 150018, examined).

Hyaloscypha cerberi Velen., Monogr. Discom. Bohem.: 411. 1934. - Dennisiodiscus cerberi (Velen.) Svr., Ceská Mykol. 32:204. 1978. - Holotype (the only specimen mentioned): [Czechoslovakia,] Bohemia, Mnichovice, Božkov, Phragmites, 7.VI.1934 Velenovsky' (PRM 151262, examined).

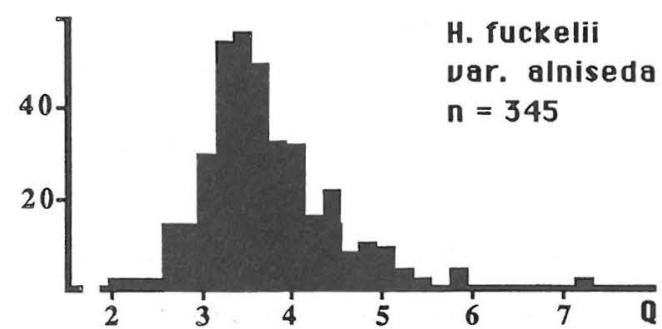

Fig. 107. The distribution of spore quotient Q in Hyaloscypha fuckelii var. alniseda.
Hyaloscypha sarothamni Velen., Monogr. Discom. Bohem.: 274. 1934. - Holotype (the only specimen mentioned): [Czechoslovakia,] Bohemia, Mnichovice, Menčice, Sarothamnus, X.1933 Velenovský (PRM 151263, examined). Concerning the discrepant reference to the name under $H$. herbarum, see discussion in the text.

Uncinia ruborum Velen., Monogr. Discom. Bohem.: 296. 1934. - Holotype (the only specimen mentioned): [Czechoslovakia,] Bohemia, Mnichovice, Rubus frut., XI.1931 Velenovsky (PRM 153251, examined). The year written by Velenovsky has later been corrected to "1933" by Svrcek, on the basis of Velenovsky's notes.

Hyaloscypha acerina Velen., Novitates mycol. noviss.: 138. 1947, nom. illeg. (Art. 64.1., non Hyaloscypha acerina Velen. 1934, see Phialina pseudopuberula.). - Holotype (the only specimen mentioned): [Czechoslovakia,] Bohemia, Mnichovice, collis Plechác, Acer pseudoplatanus, 19.IX.1940 Velenovský (PRM 151107, examined).

Hyaloscypha rubi-fruticosi Svr., Česká Mykol. 32:203. 1978. - Holotype (original designation): [Czechoslovakia,] Bohemia, Praha, Divoká Šárka, ad sarmenta Rubi fruticosi, 24.X.1972 Svrček (PRM 770993, examined).

Hyaloscypha cupularum Svr., Ceská Mykol. 41:22; P1. 2:3. 1987. - Holotype (original designation): [Czechoslovakia,] Bohemia, Mnichovice, Hubackov, ad cupulas Quercus, 4.XII.1926 Velenovský (PRM 147458, examined).

Apothecia gregarious to confluent, superficial, up to $1 \mathrm{~mm}$ in diam when fresh, typically $100-300$ $(-500) \mu \mathrm{m}$ in diam when dry, cupulate throughout development, broadly sessile or with a gradually tapering base. Disc shallow to plano-convex when fresh, bordered by an inconspicuous zone of hairs, which rise only slightly above the hymenium; margin thick when dry, fleshy and incurved, obscuring part of the hymenium. Hair cover sparse below, dense at the margin, snow-white. Colour greyish white to yellowish brown when fresh; dry apothecia ranging from faint yellowish to faint brownish $(\mathrm{K} 90, \mathrm{~L} 77)$ to Apricot (L65) to reddish brown (R15, R17, S50).

Ectal excipulum of textura prismatica, cells on middle flanks prismatic to somewhat rounded, 7$14(-15) \times 4.0-7.3(-8.0) \mu \mathrm{m}, \overline{\mathrm{x}}=10.6 \times 5.3 \mu \mathrm{m}(\mathrm{n}=$ 80 ), $Q=2.0$, not changing size or shape towards margin, at the base more roundish; in one population the outermost layer serving as hair bases with clearly rounded, loosely arranged cells. Inner excipulum of similar to slightly narrower cells. Walls in ectal parts $0.4-0.6 \mu \mathrm{m}$ thick in $50 \%$ of the populations, 0.8 $1.2 \mu \mathrm{m}$ thick in others, hyaline to somewhat brownish, varying between the populations from fragmentarily MLZ+ to MLZ++ when fresh and dry (in one population only after $\mathrm{KOH}$ pretreatment), $\mathrm{BF}+$, $\mathrm{CR}+, \mathrm{CRB}+, \mathrm{NR}+, \mathrm{TB}+, \mathrm{CB}-$ to fragmentarily $\mathrm{CB}+$, LUG-.

Hairs $18-40(-52) \times 2.0-4.5(-6.0) \mu \mathrm{m}$, narrowly conical to lageniform with or without a narrow base, straight to bent, aseptate. Apex tapering to $0.8-1.3$ $(-1.8) \mu \mathrm{m}$, rarely clavate or subcapitate (up to 

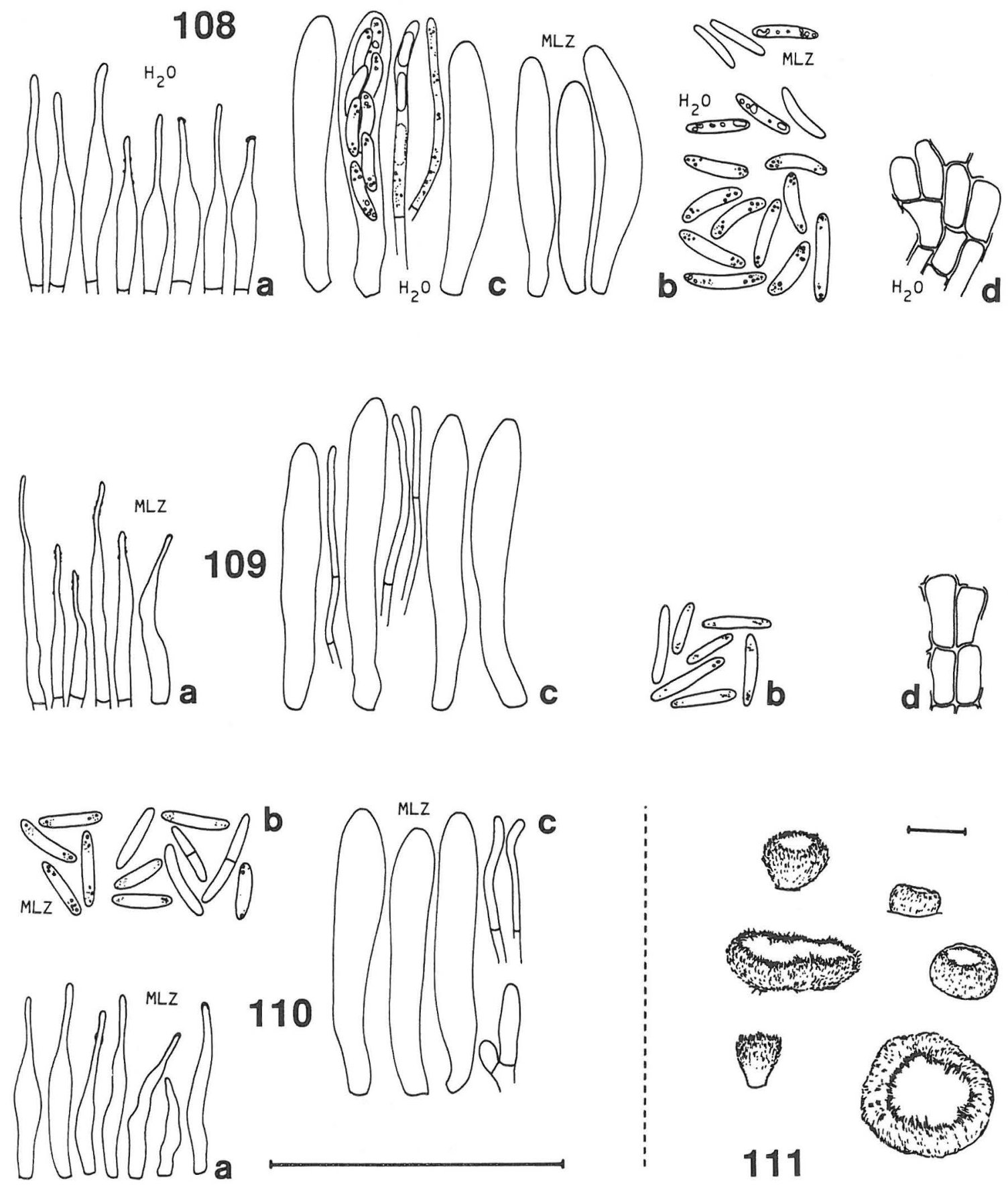

Figs. 108-114. Hyaloscypha herbarum. a) hairs, b) spores, c) asci and paraphyses, d) excipulum. Scale $50 \mu \mathrm{m}$, for apothecia $100 \mu \mathrm{m}$. - 108: SH 87/200. - 109: Holotype of Hyaloscypha cupularum. - 110: Holotype of Hyaloscypha acerina. - 111: Dry apothecia from various collections. - 112: 1968 AR. - 113: Holotype of Hyaloscypha sarothamni. - 114: Holotype of Hyaloscypha cerberi. 

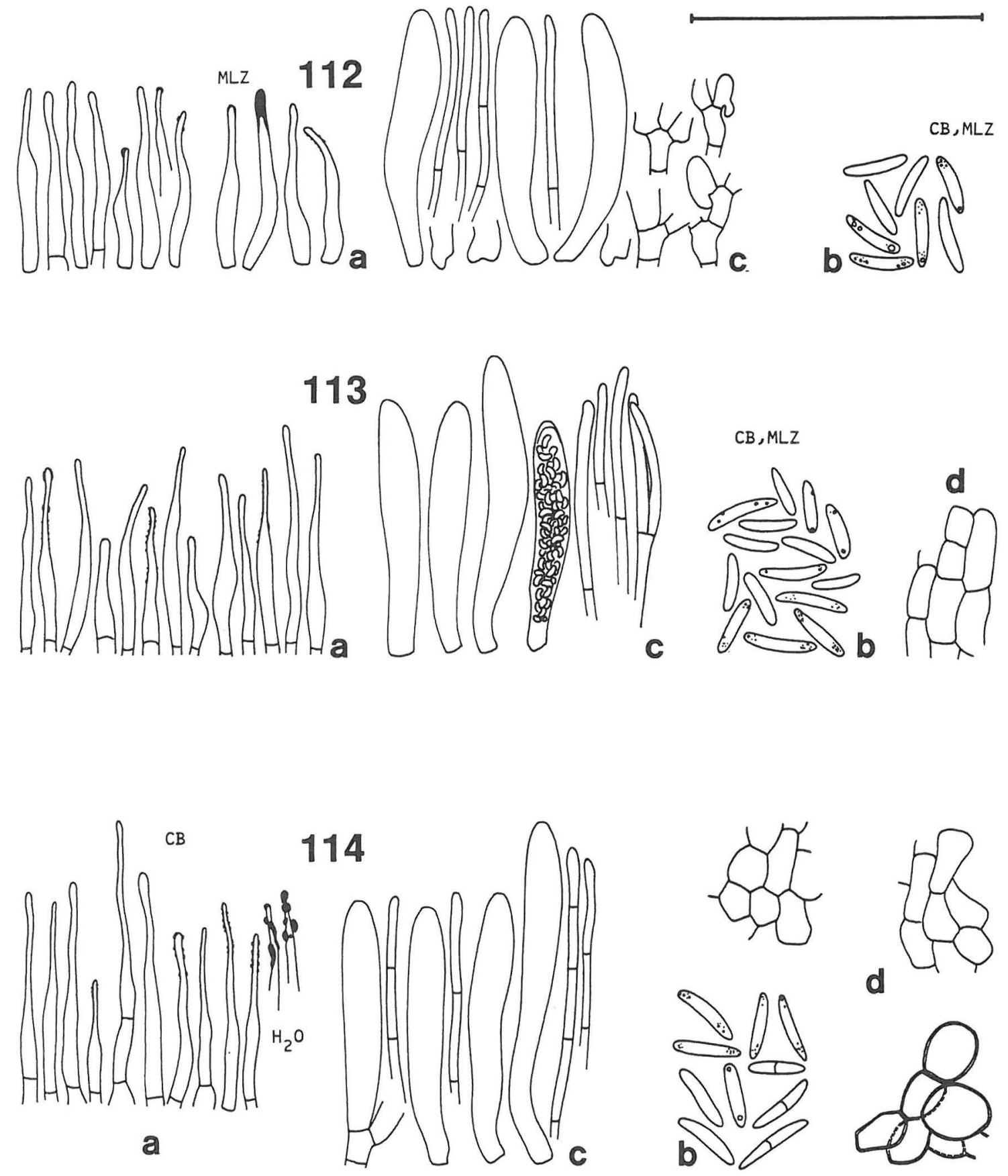
$1.8 \mu \mathrm{m}$ wide), very rarely prominently solidified, occasionally bearing a lump of hyaline to slightly yellowish exudate, almost totally dissolved in MLZ, totally dissolved in $\mathrm{CB}$ or showing a few $\mathrm{CB}+$ remnants, mainly at the apex. Wall thin, not locally clearly thickened (except for the apices), hyaline, dull, MLZ+ to MLZ++ in $93 \%$ of the populations, MLZ - to MLZ+ only after $\mathrm{KOH}$ pretreatment in others, smooth to inconspicuously apically warted when fresh and dry, such variation occurring within the same apothecium; warts rarely deformed, $\mathrm{BF}+$, $\mathrm{NR}+, \mathrm{CB}-, \mathrm{CR}-$. Marginal hairs intermixed with abundant, cylindrical-clavate, thin-walled cells, 10$20 \times 3.5-4.5 \mu \mathrm{m}$.

Asci 39-55(-56) $\times$ 6.0-8.4(-8.6) $\mu \mathrm{m}, \overline{\mathrm{x}}=46.2 \times$ $7.3 \mu \mathrm{m}(\mathrm{n}=31), \mathrm{Q}=6.5$ in water; $37-51(-53) \times$ $5.1-8.2 \mu \mathrm{m}, \overline{\mathrm{x}}=44.5 \times 6.8 \mu \mathrm{m}(\mathrm{n}=30), \bar{Q}=6.8 \mathrm{in}$ MLZ; 34-58(-64) $\times 5.0-7.5(-8.0) \mu \mathrm{m}, \overline{\mathrm{x}}=45.5 \times$ $6.3 \mu \mathrm{m}(\mathrm{n}=85), \bar{Q}=7.4$ in $\mathrm{CB}$, eight-spored, cylindrical-clavate, with slightly conical apex. Apical pore MLZ++ in $64 \%$ of the populations, MLZ+ in others, LUG+. Wall in mature asci apically 0.8$1.3 \mu \mathrm{m}$ thick (in $\mathrm{CB}, \mathrm{MLZ}$ ), negative in all reagents; contents regularly LUG+, very rarely MLZ+ (orange). Asci arise from simple septa with occasional downward hooks.

Spores $9.5-17.5(-19.0) \times 2.1-3.1(-3.2) \mu \mathrm{m}, \overline{\mathrm{x}}=$ $12.5 \times 2.8 \mu \mathrm{m}(\mathrm{n}=97), \bar{Q}=4.6$ in water; 8.5 $13.4(-19.0) \times 1.8-2.8(-3.4) \mu \mathrm{m}, \overline{\mathrm{x}}=10.6 \times 2.2 \mu \mathrm{m}$ $(\mathrm{n}=290), \bar{Q}=5.1$ in $\mathrm{CB}$ and $\mathrm{MLZ}$, oblong to oblong-elliptic to elliptic-fusiform, with rounded to tapered apices, rarely elliptic, up to $10 \%$ becoming one-septate after being discharged, more rarely while still inside the asci; septum thin, central, MLZ-. Wall hyaline, CR-. Guttulae small, dark, apically grouped when fresh in water, more rarely larger and light; in dry material alike or coalescent.

Paraphyses cylindrical to slightly widened at the apex, unpigmented, first dichotomous branches oc- curring at the middle level of the asci. Terminal cells $10-33 \times 1.2-2.0 \mu \mathrm{m}, \overline{\mathrm{x}}=22.6 \times 1.6 \mu \mathrm{m}(\mathrm{n}=50)$.

Cultural characters ( 6 populations, 16 strains). Radial growth slow to moderate on MA, $13.8 \mathrm{~mm} /$ month ( $\mathrm{n}=105$, from 6 popul., 12 strains). Mats with blackish to blackish brown basic colour, in centre blackish (S92) to dark olivaceous (S91), at margin olivaceous (P91), extreme margin occasionally whitish for $0.5 \mathrm{~mm}$. Aerial mycelium prominent, densely woolly, greyish (P92, L73) to greyish brown (N71), extending almost to margin. Margin indistinct, fimbriate, submerged. Hyphal strands lacking, zonation lacking, sector formation lacking, no colour change in surrounding agar, no yeast-like growth. Context somewhat tough at surface, odour not distinct.

Spores germinating readily on wood agars, some hardwood-originating strains growing tardily on PA and the single softwood strain growing tardily on BA, the others showing good growth on all wood agars, growth retarded on LA. Anamorph and teleomorph lacking in single spore and multispore trials.

$H$. herbarum is relatively easily recognized in culture due to its rapid growth combined with olivaceous basic colour, lack of zonation and hyphal strands and due to the extensive aerial mycelium.

Distribution and phenology. The present material originates mainly from central, western and northern Europe. The only exception is a geographically isolated find from northern Caucasus in the U.S.S.R. $H$. herbarum is clearly an autumnal species: most collections have been made between September and December. The remaining collections date from January, March and June (Fig. 16).

Ecology. In contrast to many other Hyaloscyphas, $H$. herbarum occurs more often on thin ligneous

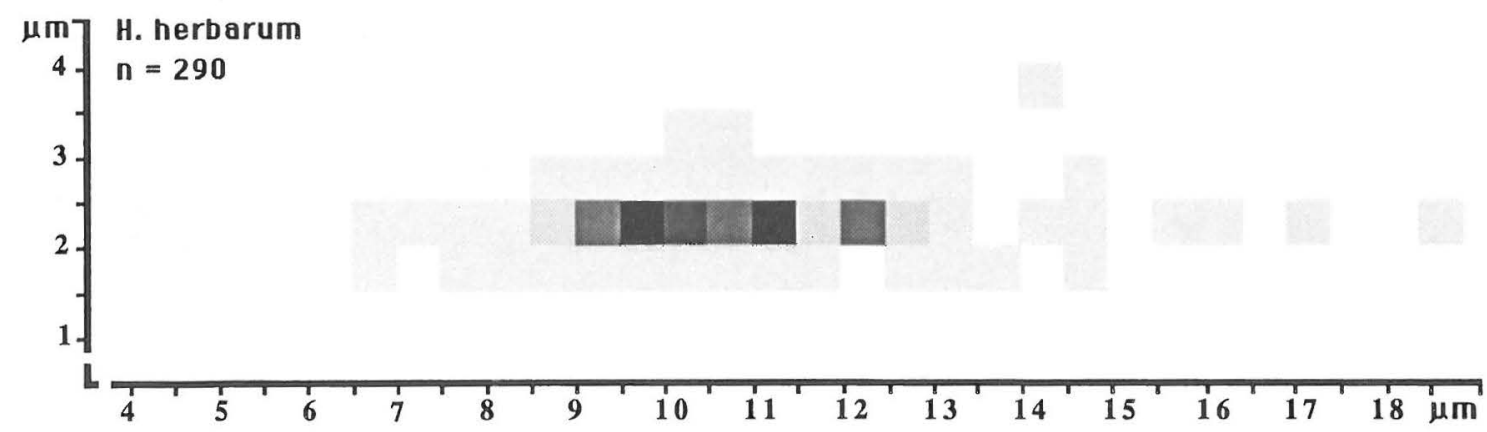

Fig. 115. Percentage sporogram of Hyaloscypha herbarum. 
substrates, amongst litter, and on herbaceous stems. The ecological range is wide and includes decayed trunks and branches, but the amount of collections from other types of substrate indicates that $H$. herbarum is well able to grow outside the major domain of the genus. This adaptation is possibly mirrored in the more vigorous growth rate of the species, which enables utilization of fairly rapidly degraded substrates.

Eight collections originate from bark, often being in close association with pyrenomycetes, but also fruiting without an obvious connection: Crataegus (2), Alnus (1), Betula (1), Pinus (1), Populus tremula (1), Quercus (1), unidentified (1). An uncommon kind of substrate was cupules of Quercus and Fagus ( 2 from each). One collection even originates from Quercus leaves. The rest of the material had the following substrates: Betula (2), Rosa (2), Rubus (2), Acer (1), Alnus (1), Corylus (1), Epilobium (1), Fagus (1), Melampyrum (1), Phragmites (1), Populus tremula (1), Sarothamnus (1), Symphoricarpus (1), Verbascum (1), hardwood (4). With the exception of one collection, $H$. herbarum occurred on hardwood and herbaceous substrates. Svrček (1985) cited a collection on Rumex.

Discussion. Hyaloscypha herbarum is best recognized by the elongated spores, asci arising from simple septa and short, predominantly smooth hairs. It is distinguished from the rough-haired taxa by the hair and spore characteristics. The ascal bases and presence of an euamyloid pore differentiate it from $H$. vitreola, $H$. intacta and $H$. daedaleae, but distinction from $H$. fuckelii var. alniseda may be difficult, because of the large variation in the spores of $H$. herbarum. At the lower end of the range, a population may be confused with var. alniseda, which also has simple-septate asci. The spores in the present species are narrower, however, and more often septate than in var. alniseda. The smaller asci in var.alnised $a$ and its characteristic hairs (apical knob, thickened wall at the constriction, roughened surface when fresh in water) also distinguish the taxa. When cultural characters are available, the morphology and growth rate of the mycelium are diagnostic. The over- lap in the natural populations indicates some relationship.

Following the general trend in the genus, the spores within a given apothecium in $H$. herbarum vary little in width but may vary markedly in length. The maximal spore length in $H$. herbarum is the greatest in the genus, though the average values of the populations are smaller than in, e.g., $H$. vitreola. As in many species, the thickness of the excipular cell walls varies greatly between the populations. The tendency for the spores to become one-septate also varies, the proportion of septate spores in a population ranging from one to ten percent. The hair characters differ little between the populations. The walls lack massive thickenings except for the rare solidified apices. In all populations the size and shape are the same and warts are scanty or lacking. In a few populations the hairs have a small apical lump of hyaline to yellowish exudate.

In the type collection of $H$. sarothamni s ome of the asci are filled with small ascoconidia, budding from the primary spores. Such conidia are very rare in Hyaloscypha and were noted in one other population only (see H. albohyalina var. albohyalina). The production of conidia is coupled with the ascal MLZpore and even within the same apothecia normal MLZ+ asci may be intermixed with MLZ- asci that produce conidia.

When describing $H$. herbarum, Velenovský (1934) cited the synonym "H. galiorum Velen. exs.". In the explanatory text of the plate illustrating the new species (Pl. 14:21) he also mentions Hyaloscypha sarothamni and $H$. galiorum (Figs. 19, 20). The original diagnosis of the former species (Velenovsky 1934) contained no reference to an illustration, and the latter name was never published by Velenovský. Although there is a slight possibility that $H$. sarothamni was indirectly used as a synonym of $H$. herbarum, Art. 34.1 of the Code should not be used in this context. It is not clear whether these illustrations were cited to indicate conspecificity or whether they were mentioned accidentally or even perhaps for the sake of comparison. These two illustrations are at first sight different from that of $H$. herbarum. Fur-

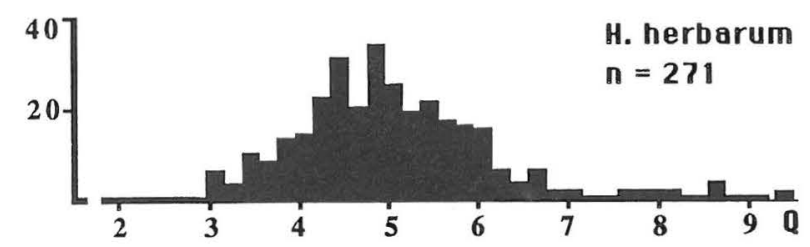

Fig. 116. The distribution of spore quotient Q in Hyaloscypha herbarum. 
thermore, it seems that Velenovský made a mistake in the generic name because the illustrations fit well with Uncinia sarothamni Velen. and U. galiorum Velen., both of which were described without reference to any illustration. Type studies of these species showed that they represent the fungus now known as Unguiculella eurotioides (P. Karst.) Nannf. The falcate hairs and short, elliptic and biguttulate spores of U. eurotioides are as depicted by Velenovský (1934; P1. 14: 19, 20). In view of the possible errors or comparative intention of Velenovskýs citation, the epithets of $H$. sarothamni, $U$. sarothamni and $U$. galiorum should be considered as not validly published under Art. 34.1 (a).

In a recent treatment of Velenovský's species of Hyaloscypha, Svrček (1985) accepted the present taxon as a good species and considered that $H$. sarothamni may also be a good species. He noted the close relationship to $H$.perpusilla and $H$.alniseda ( $H$. fuckelii var.alniseda), stressing it anew in the description of $H$. cupularum and mentioning the long ascospores and asci.

Hyaloscypha cerberi Velen. was placed in Dennisiodiscus Svr. by Svrček (1978). Though no explanation was given, the decision was apparently based on the roundish and slightly brownish cells in the outermost ectal excipulum, which I also noted when studying the holotype. Under the microscope, however, the brownish tone is very faint and does not represent a wide difference from the other species of Hyaloscypha. Moreover, the excipular cells tend to be somewhat roundish in $H$. herbarum. In the type of $H$. cerberi the rounded cells form one or two layers on normal textura prismatica, serving as hair bases, which may explain their somewhat loose arrangement. The remainder of the hairs is aseptate and the hairs are thus not typical of Dennisiodiscus (Svrček 1978). Although the dry apothecia have a curious greenish brown tone (R91, P90), Velenovský (1934) describes them as pure white when fresh. These characters are marginal in $H$. herbarum, but the asci, spores, hairs, hair exudate and chemical reactions all suggest conspecificity. Until further material from the full range of variation in this species becomes available, $H$. cerberi is best synonymized with $H$. herbarum. In any case, placement in Hyaloscypha is more natural than transfer to Dennisiodiscus.

Material studied: Czechoslovakia. Bohemia: Jevany, 1924 JV (PRM). Libochovičhy, 1926 Fechtner (PRM). Mnichovice, 1924 (type of Hyaloscypha herbarum), 1926 (type of H. cupularum), 1928, 1929, 1930, 1933 (type of $H$. sarothamni, type of Uncinia ruborum), 1934 (type of Hyaloscypha cerberi), 1940 (type of $H$. acerina) JV (PRM). Praha, 1972 Svrček (PRM, type of Hyaloscypha rubi-fruticosi). Federal Republic of Germany. Rheinland-Pfalz: Hunsruck, 1935
Sponheimer (B). Finland. Varsinais-Suomi: Turku, 1985, 1986,1987 SH 85/131*, 85/136*, 85/140, 86/132, 86/145*, $86 / 147,87 / 200^{*}$ (TUR). Satakunta: Punkalaidun, $1985 \mathrm{SH}$ $85 / 159 *, 85 / 160,85 / 164 *$. Great Britain. Surrey: Leatherhead, 1944 Dennis (K). Effingham, 1949 Dennis (K). Suffolk: Dunwich Forest, 1981 Ellis \& Ellis (IMI). Reydon Pits, 1978 Ellis \& Ellis (IMI, two collections). Holmwood Common, 1979 Dennis (K). U.S.S.R. Karachayvo-Cherkess: Teberda, 1968 AR (TAA).

\section{Hyaloscypha intacta}

Hyaloscypha intacta Svrček, Česká Mykol. 40:209; PI. 3:1. 1986. - Holotype (original designation): [Czechoslovakia,] Bohemia centralis, montes Brdské Hřebeny, in silvestria Červená Hlína dicta supra pagum Dobrichovice, ca 450 m.s.m., 28.VIII.1985 Svrček (PRM, not seen).

Apothecia gregarious, superficial, up to $500 \mu \mathrm{m}$ in diam when fresh, $100-250 \mu \mathrm{m}$ when dry, cupulate throughout development, narrowly to broadly sessile. Disc shallow to plano-convex when fresh, bordered by an inconspicuous zone of hairs, which rise only slightly above the hymenium; margin only slightly thicker when dry, not clearly incurved. Hair cover sparse to dense, concentrated in the margin, snow-white. Colour whitish when fresh; dry apothecia ranging from light greyish to faintly brownish (M69, M75) to Apricot (L65) to more orange (N59) between the populations.

Ectal excipulum of textura prismatica, cells on middle flanks regularly to irregularly prismatic, 6 $11(-13) \times 3.5-5.5(-5.8) \mu \mathrm{m}, \overline{\mathrm{x}}=9.3 \times 4.4 \mu \mathrm{m}(\mathrm{n}=$ 40 ), $\bar{Q}=2.1$, not changing size or shape towards margin, at the base more roundish. Inner excipulum of more elongate cells, ca. 9.0-11.5 x 2.5-3.2 $\mu \mathrm{m}$. Walls in ectal parts $0.6-1.6 \mu \mathrm{m}$ thick, hyaline, $\mathrm{MLZ}+$ to $\mathrm{MLZ}++$ when dry, $\mathrm{CRB}+, \mathrm{NR}+, \mathrm{TB}+$, fragmentarily $\mathrm{BF}+, \mathrm{CR}+, \mathrm{CB}-$, LUG-.

Hairs $18-38 \times 2.0-3.0(-3.5) \mu \mathrm{m}$, narrowly conical to almost filiform, straight to slightly bent, aseptate. Apex tapering to $0.6-1.3(-1.6) \mu \mathrm{m}$, thin-walled to often prominently solidified, this variation occurring in the same apothecium or between populations, not exuding an apical globule in MLZ, nor terminating in a clearly delimited knob. Wall thin, never locally thickened (except for apices), hyaline, dull, smooth when fresh and dry (rarely bearing a few, tiny, apical warts, not deformed in $M L Z$ and $C B$ ), $M L Z+$ to $M L Z++$ when fresh and dry, negative in other reagents. Marginal hairs intermixed with abundant cylindrical-clavate, thin-walled cells, $8-17 \times$ 2.2-4.5 $\mu \mathrm{m}$.

Asci 42-83(-93) x 7.2-9.8(-10.8) $\mu \mathrm{m}, \overline{\mathrm{x}}=61.3$ x $8.2 \mu \mathrm{m}(\mathrm{n}=25), \overline{\mathrm{Q}}=7.4$ in MLZ; $38-82(-90) \times$ 5.9-8.6(-9.0) $\mu \mathrm{m}, \overline{\mathrm{x}}=57.1 \times 7.0 \mu \mathrm{m}(\mathrm{n}=45), \bar{Q}=$ 

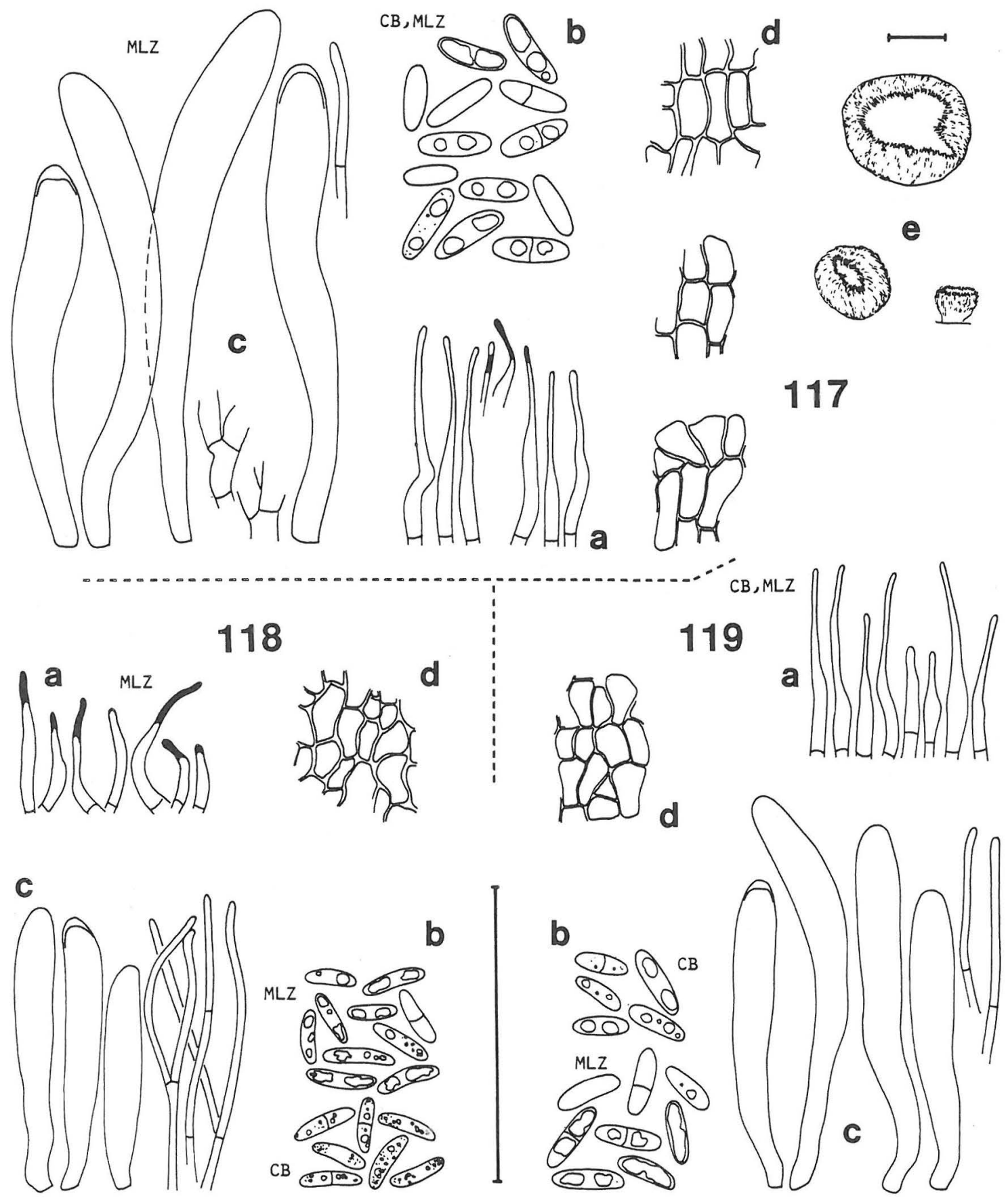

Figs. 117-119. Hyaloscypha intacta. a) hairs, b) spores, c) asci and paraphyses, d) excipulum, e) dry apothecia. Scale $50 \mu \mathrm{m}$, for apothecia $100 \mu \mathrm{m}$. - 117: 1930 Lundell. — 118: Söderholm 1334. - 119: Bramley K.72/16. 
8.1 in $\mathrm{CB}$, eight-spored, cylindrical-clavate, with rounded to slightly conical apex. Apical pore MLZeven after $\mathrm{KOH}$ pretreatment, LUG-. Wall in mature asci apically $1.5-2.5 \mu \mathrm{m}$ thick (in CB, MLZ), negative in all reagents; contents frequently LUG + , rarely MLZ++ (orange). Asci arising from simple septa.

Spores 7.9-12.5(-14.7) x 2.5-4.3(-4.9) $\mu \mathrm{m}, \overline{\mathrm{x}}=$ $10.2 \times 3.2 \mu \mathrm{m}(\mathrm{n}=214), \bar{Q}=3.3$ in $\mathrm{CB}$ and MLZ, broadly elliptical to oblong elliptical, straight to slightly bent, frequently becoming one-septate (5$20 \%$ of discharged spores, in the different populations), often septate while still inside the asci, septum central to eccentric, $\mathrm{CR}-(\mathrm{CR}++$ in old spores). Wall hyaline, rarely brownish in aged discharged spores, $\mathrm{CR}-(\mathrm{CR}++$ in old spores). Guttulae prominent in fresh and dry material, light.

Paraphyses cylindrical or apically slightly widened to apically branched/lobed, unpigmented, first dichotomous branches occurring at the level of the lowermost $1 / 3$. Terminal cells $10-40 \times 1.0-2.2 \mu \mathrm{m}, \overline{\mathrm{x}}=$ $24.1 \times 1.4 \mu \mathrm{m}(\mathrm{n}=30)$.

Cultural characters (1 population, 3 strains). Radial growth slow on MA, $11.6 \mathrm{~mm} /$ month $(\mathrm{n}=28)$. Mats snow-white but frequently with brilliant green colour (Küppers Y70-M00-C60) in most of the central part or in zones. Surface dough-like, aerial mycelium lacking, when present sparse and confined to a few millimetres around the inoculum. Margin distinct, even, undulating, submerged. Hyphal strands frequent in central parts, running in the agar, erect and prominent on the inoculum. Zonation occasional, sector formation lacking, no colour changes in surrounding agar, no yeast-like growth. Context somewhat tough at surface, odour not distinct.

Growth moderate on BA, retarded on PA. Single spore strains producing apothecia on BA after 19 months, the 24 strains extracted from cultivated apo- thecia all mutually identical. Apothecia not produced on PA or MA, anamorph lacking on all substrates. Producing abundant chlamydospores on BA after 22 months (Fig. 35).

Distribution and phenology. Although relatively few collections of $H$. intacta are known, the species seems to be widespread. So far it is known from southern Finland, central Sweden, Estonia, England, Scotland, Czechoslovakia and southern Canada. The collections date from January (1), June (1), August (1), September (2), October (3) and November (1).

Ecology. All the studied collections originate from hardwoods. Apparently the species is not able to fruit on softwoods, because even the mycelial growth is much retarded when cultured on softwood agar. A slight extension of the ecological range in seen in two collections, from the inner side of the bark of Sorbus aucuparia and from pyrenomycetous stroma on the inner bark of Betula. The host is indicated in only three additional collections: Carpinus (2), Salix (1).

Discussion. Hyaloscypha intacta is a well-delimited species, possessing many characteristic features, such as the large, permanently $\mathrm{MLZ}$ - asci arising from simple septa, the short and smooth hairs with frequently solidified apices, the large and frequently septate spores, the reliably dextrinoid reaction of the hairs and excipulum, and the distinctive cultural properties. Another species of Hyaloscypha with consistently MLZ- asci is $H$. quercicola, but it is distinguished by its smaller asci and spores, typically warted hairs and the different morphology of the vegetative mycelium in culture.

The hymenial variability of $H$. intacta is fairly wide, however. In six populations the ranges of the mean measurements for spores and asci are, re-

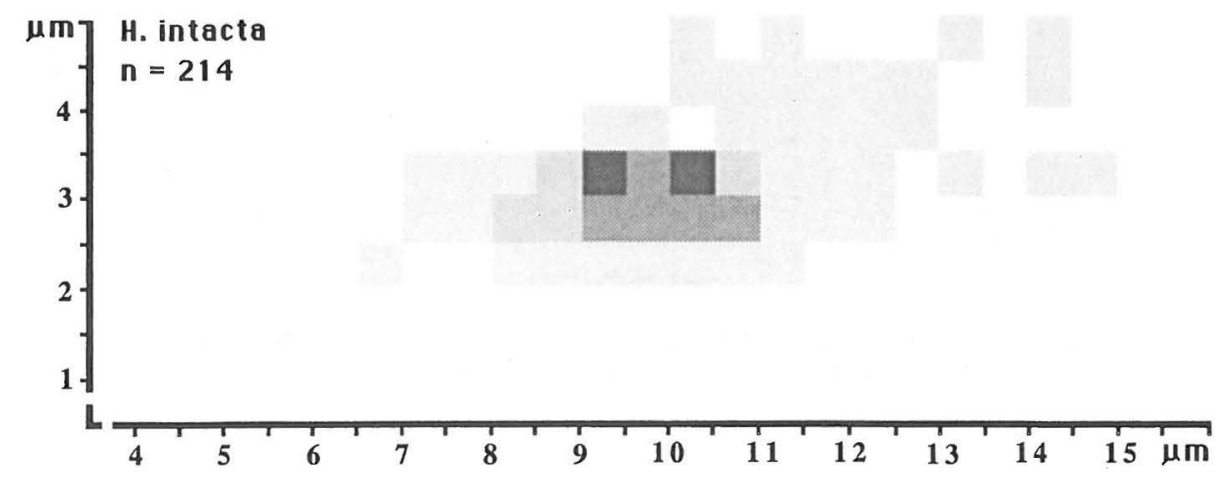

Fig. 120. Percentage sporogram of Hyaloscypha intacta. 
spectively, $8.9-10.3 \times 2.7-3.1 \mu \mathrm{m}$ and $40-58 \times 6.2-$ $7.2 \mu \mathrm{m}(\mathrm{CB})$. The collections from Sweden and Scotland add to the variation: $11.3-11.5 \times 3.1-$ $3.9 \mu \mathrm{m}$ and $66.5-77.6 \times 6.3-8.3 \mu \mathrm{m}$. Discontinuity is apparent in the ascal measurements, whereas the spore dimensions show a smooth gradation. The discontinuity may be due to insufficiently representative material and does not exceed that noted in other species of the genus. As the other diagnostic characters link the Swedish and Scottish collections with the populations with smaller asci and spores, there is no reason to separate them taxonomically until the total variation has been elucidated.

$H$. intacta was one of the few species to fruit in culture. A single-spore isolate from one collection formed apothecia in two trials after 19 months on BA. In both of these two cases marked variation was seen in one character only. These cultivated apothecia bore both smooth and densely warted hairs. As discussed in the general part, the hairs in some species have an excretory function whose extent depends on the physical and chemical properties of the microhabitat. The effect of the environment is especially clear in $H$. albohyalina var. spiralis, in which the hairs vary clearly between populations in this respect. In $H$. intacta, which has smooth to inconspicuously warted hairs in nature, the hair vesture is apparently also modified by the environment, prolonged cultivation with limited aeration possibly changing the rate of excretion. Of the other characters of these cultivated apothecia, only ascal size varied notably: the asci were $20 \%$ smaller than in the original material. No change was observed in vitro in the dextrinoid reaction, excipular characters, hair shape and size, spore characters, ascal bases or MLZ-reaction.

Many characters were stable and did not vary between the populations. The hairs and excipulum were always dextrinoid and the small-celled excipulum had more or less thickened cell walls. The hair shape and smoothness are also good characters in the present

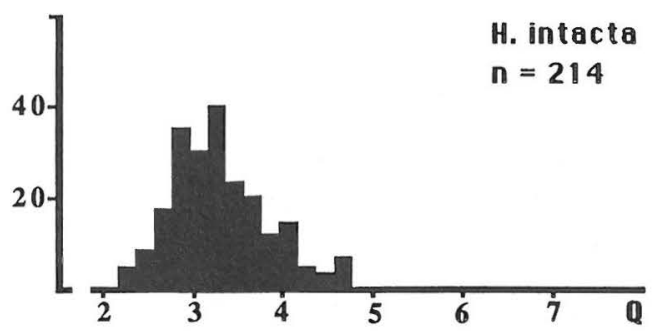

Fig. 121. The distribution of spore quotient Q in Hyaloscypha intacta. material, but though frequent, solid apices were more rare in two populations. The spores were septate in the whole material, septation even occurring rather frequently inside the asci. Although variable, the asci are always large and lack both croziers and MLZ+ reaction. The spores are always more or less elliptical, clearly guttulate and with broad ends.

I did not study the type of $H$. intacta, but there is little doubt of the identity of the present material. Only two characters in Svrček's (1986) original description deviate from my material: the thin-walled excipulum and the variation in hair shape in the type collection, in which Svrcek noted two kinds of hairs. The more lageniform kind is absent from the present material. However, this variation accords with that in, e.g., H. albohyalina var. spiralis. Svrcek (1986) did not mention the dextrinoid reactions in $H$. intacta.

Material studied: Canada. Ontario: Lake Opinicon, 1958 Korf (Herb. Korf 3885). Czechoslovakia. Bohemia: Mnichovice, 1926 JV (PRM). Finland. Varsinais-Suomi: Turku, 1985 SH 85/103* (TUR). Etelä-Häme: Tampere, 1986 Söderholm 1334 (TUR). Great Britain. Y orkshire: Pickering, 1972 Bramley K.72/16 (K). Midlothian: Dalmahoy, 1977 Coppins 3030 (E). Sweden. Uppland: Bondkyrka, 1930 Lundell (UPS). U.S.S.R. Estonian S.S.R.: Boroni Nat. Res., 1967 AR (TAA).

\section{Hyaloscypha latispora sp. nov.}

Apothecia cupulata, sessilia vel stipitata, pilosa; specimina exsiccata rubiginosa, pili nivei. Excipulum externum affinis textura prismatica, cellulis 13-18 $\times 5-9 \mu \mathrm{m}$, irregulariter crassotunicatis, hyalinis, in solutione Melzeri vinosis, crystallis hyalinis vel brunneis. Pili 40-60(-85) $\times 2.4-4.8 \mu \mathrm{m}$, anguste conici, leves, tenuiter tunicati, aseptati, in solutione Melzeri non colorati. Asci octospori, 41-49 x 6.8-8.2 (-9.8) $\mu \mathrm{m}$, non amyloidei, in apice rotundati, in basi uncinati, crystallis hyalinis vel brunneis. Sporae 6.9-9.2 (-10.0) x 3.1-4.2(-4.3) $\mathrm{um}$, late ellipsoideae, non septatae, perspicue guttulatae, crystallis similis. Paraphyses cylindraceae vel in apice minuter dilatatae, 2-3 um latae, cellulis terminalibus 16-31 $\mu \mathrm{m}$ longis, crystallis similis.

Holotype: Morocco. "Moyen Atlas. Face Nord du Bou Ighitten, au dessus de l' Aguelmam n' Sidi Ali, 2000 m env., sur tiges mortes de Rosa sp., 12.IV.1958 Malençon 5417" (MPU).

Apothecia gregarious, superficial, $150-600 \mu \mathrm{m}$ in diam when dry, cupulate, sessile to stipitate on a stout stipe, ca. $60 \times 60 \mu \mathrm{m}$. Disc shallow, bordered by a copiously incurved margin. Hair cover dense, concentrated in the margin, snow-white. Colour orange brown (M59) when dry, modified by the hair cover.

Ectal excipulum of modified textura prismatica, cells on middle flanks basically prismatic, but some- 

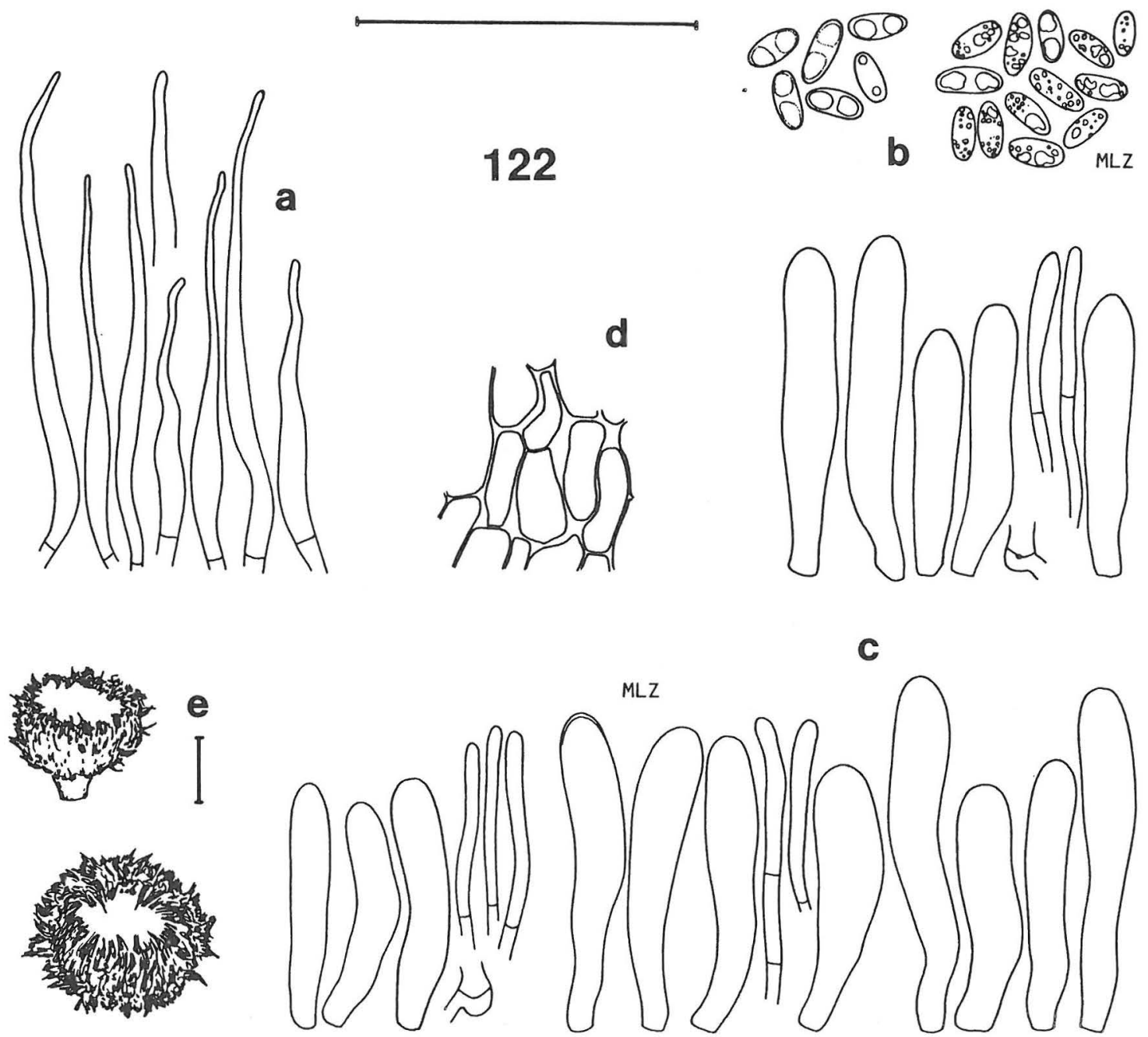

Fig. 122. Hyaloscypha latispora, holotype. a) hairs, b) spores, c) asci and paraphyses, d) excipulum, e) dry apothecia. Scale $50 \mu \mathrm{m}$, for apothecia $100 \mu \mathrm{m}$.

what irregular due to the unevenly thickened walls, $13-18 \times 5-9 \mu \mathrm{m}, \overline{\mathbf{x}}=15.4 \times 5.8 \mu \mathrm{m}(\mathrm{n}=10), \bar{Q}=$ 2.7 , not notably changing size or shape towards margin. Inner excipulum of similar tissue. Walls in ectal parts very variable in thickness, $0.4-2.5 \mu \mathrm{m}$ thick, hyaline, $\mathrm{MLZ}++$; $\mathrm{BF}+, \mathrm{CB}+, \mathrm{CR}+, \mathrm{CRB}+$, NR+, TB+, LUG-. Excipulum covered by abundant, hyaline to brownish crystals, dissolving in MLZ and LUG, for the most part dissolved in CB, persistent in $\mathrm{KOH}, \mathrm{CR}$ and water-based stains.

Hairs $40-60(-85) \times 2.4-4.8 \mu \mathrm{m}$, narrowly coni$\mathrm{cal}$, straight to somewhat undulating, aseptate. Apex tapering gradually to $0.5-1.8 \mu \mathrm{m}$, not solidified, without an apical globule in MLZ. Wall thin to slightly thickened for their whole length, hyaline, dull, smooth, negative in all reagents.

Asci $41-49 \times 6.8-8.2(-9.8) \mu \mathrm{m}, \overline{\mathrm{x}}=43.3 \times$ $7.6 \mu \mathrm{m}(\mathrm{n}=15), \bar{Q}=5.8$ in MLZ; $34-50 \times 6.2-$ $8.2 \mu \mathrm{m}, \overline{\mathrm{x}}=38.0 \times 6.6 \mu \mathrm{m}(\mathrm{n}=20), \bar{Q}=5.6 \mathrm{in} \mathrm{CB}$; eight-spored, cylindrical to cylindrical-clavate, with rounded apex. Apical pore not observed, MLZ-, LUG-. Walls in mature asci of equal thickness, 0.2$0.6 \mu \mathrm{m}$ thick (in $\mathrm{CB}$ ). Contents not turning bright orange in $\mathrm{MLZ}$, frequently LUG+, many asci with 
hyaline to brownish, crystalline basal inclusions, dissolving in MLZ, LUG, at least partly persistent in other reagents. Asci arising from croziers.

Spores 6.9-9.2(-10.0) $\times$ 3.1-4.2(-4.3) $\mu \mathrm{m}, \overline{\mathrm{x}}=$ $7.8 \times 3.6 \mu \mathrm{m}(\mathrm{n}=78), \bar{Q}=2.2$ in CB and MLZ, broadly elliptical, almost symmetrical, with rounded apices, aseptate. Wall hyaline. Guttulae prominent in all reagents, light, in $\mathrm{CRB}$ and $\mathrm{TB}$ spores with central, metachromatic (red) area; many spores with crystalline inclusions similar to those in asci and paraphyses.

Paraphyses cylindrical to apically slightly widened, unpigmented, with crystalline inclusions similar to those in asci and spores. Terminal cells $16-31 x$ $2-3 \mu \mathrm{m}, \overline{\mathrm{x}}=24 \times 2.7 \mu \mathrm{m}(\mathrm{n}=10)$.

\section{Cultural characters. Not known.}

Distribution and phenology. The sole collection was made in April from Moyen Atlas in Morocco.

Ecology. The apothecia occur on a piece of woody stem of Rosa sp. They are concentrated on old apothecia of an unknown discomycete occurring abundantly on the stem, but also occur on naked wood. A similar fungicolous habit is seen in other species of the genus, but $H$. latispora is the only one whose apothecia were seen fruiting on a discomycete.

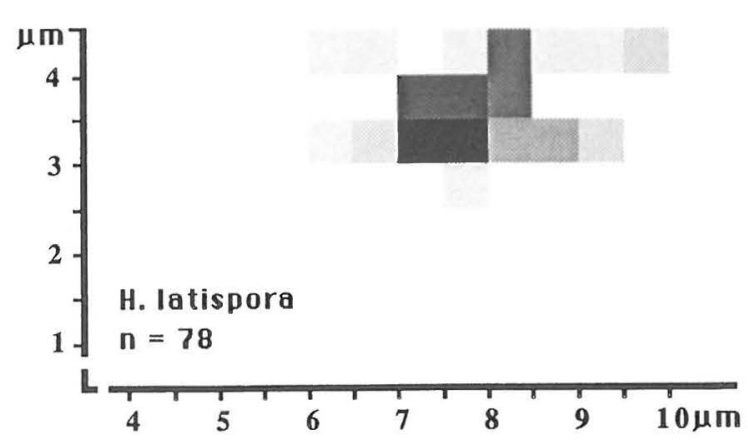

Fig. 123. Percentage sporogram of Hyaloscypha latispora.

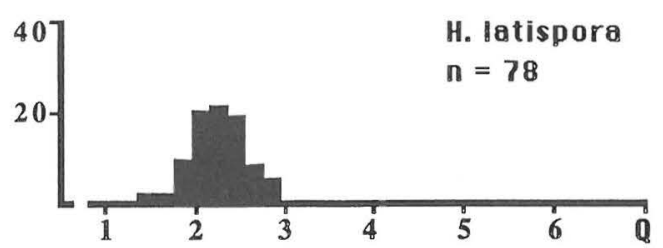

Fig. 124. The distribution of spore quotient Q in Hyaloscypha latispora.
Discussion. Hyaloscypha latispora is a distinctive species, easily recognized by the MLZ- asci with rounded apices and the almost symmetrical, broadly elliptic spores. The curious type of excipulum, with unevenly thickened walls, and the consequent irregularity in cell shape also differentiate it from the other species of Hyaloscypha. The MLZ- hairs are smooth and may show a slightly thickened wall. Crystalline inclusions characterize all structures. In $\mathrm{CB}$ they are usually seen in the spores only and they dissolve in MLZ. In water-based stains and in CR all structures except the hairs contain these crystals.

$H$. latispora is a somewhat alien element in any modern genus of the Hyaloscyphaceae. It is here accepted in Hyaloscypha mainly because of the similar hairs. The dextrinoid reaction of the excipulum is another character typical of the genus. No apical pore was observed and the asci do not show apically thickened walls, as is the case in all other species of Hyaloscypha. The strong variation in the thickness of the excipular cell walls, which masks the textura prismatica structure, is unmatched in the genus.

The evidence for placing the present species in Hyaloscypha is rather slender. The species can hardly be related to $H$. vitreola, as suggested by the collector, and it is not easy to pinpoint any other closely related species. However, in the classification of the Hyaloscyphaceae, the hair morphology has frequently proved to be a valuable character, being superior to, e.g., ascus morphology or excipular wall thickness.

Material studied: Morocco. Moyen Atlas: Bou Ighitten, 1958 Malençon (MPU, type).

\section{Hyaloscypha leuconica var. leuconica}

Hyaloscypha leuconica (Cooke in Stevenson) Nannf., Trans. Brit. Mycol. Soc. 20:206. 1936. - Peziza leuconica Cooke in Stevenson, Mycol. scot:: 317. 1879. - Lachnella leuconica (Cooke in Stevenson) Phill., Man. Brit. Discomyc.: 267. 1887. - Trichopeziza leuconica (Cooke in Stevenson) Sacc., Syll. Fung. 8:414. 1889. - Dasyscypha leuconica (Cooke in Stevenson) Massee, Brit. fung.-fl.: 334. 1895. — Urceolella leuconica (Cooke in Stevenson) Boud., Hist. classific. discom. Europe: 130 . 1907. - Holotype (the only specimen mentioned): [Great Britain,] Angus Clova, on fir wood of old sheep fence, $2900 \mathrm{ft}$, VII.1878 Stevenson (K, Herb. Cooke; a slide examined).

Hyaloscypha cincinnata Sherw., Phytologia 28:15, Fig. 1. 1974. - Holotype (original designation): [U.S.A.,] New York, Schuyler County, Lost Gorge, Hendershot Gulf near Swan Hill road, station 28, on a dead stick, 13.IX.1973 Sherwood 1316 (CUP, examined). 


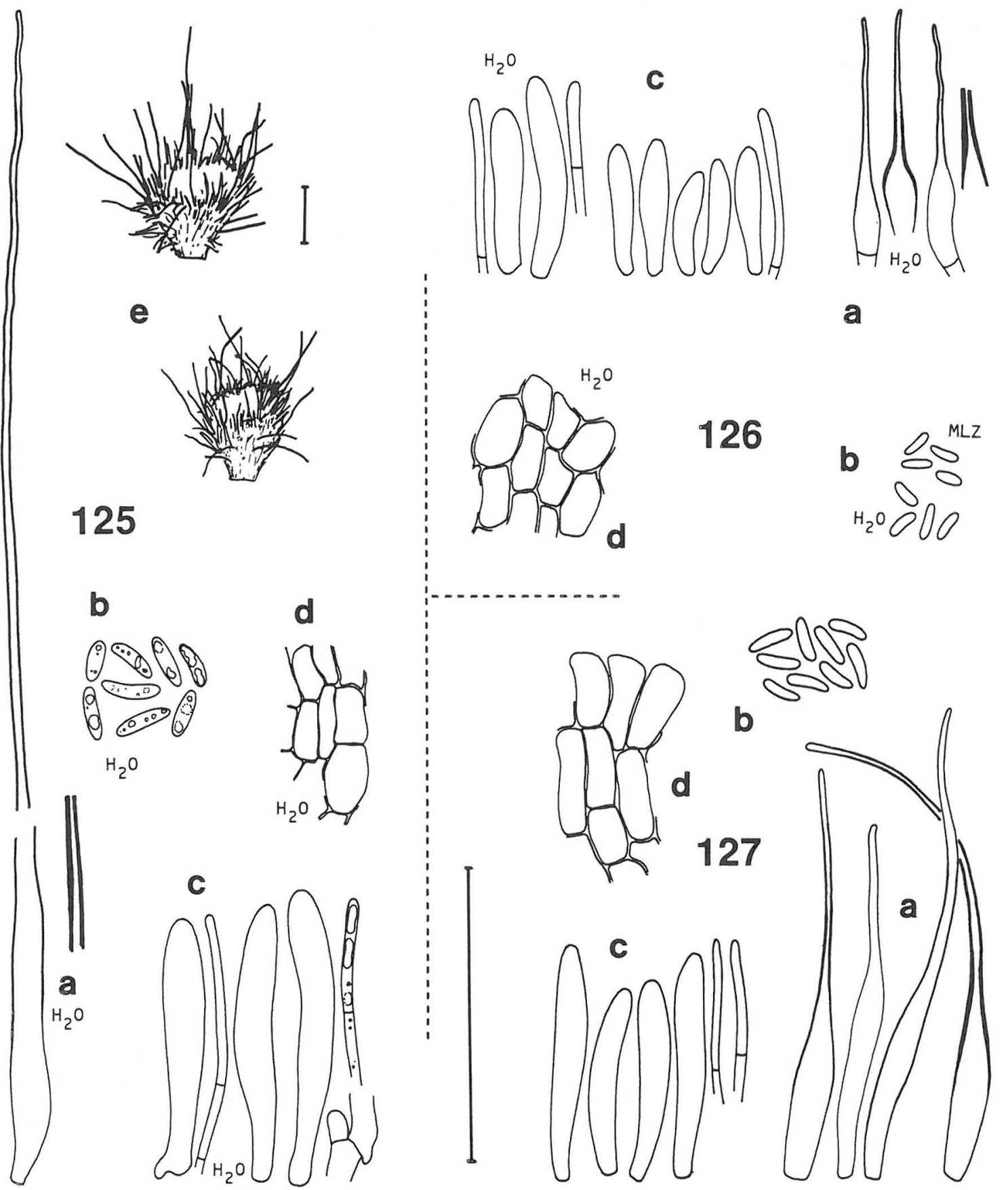

Figs. 125-127. Hyaloscypha leuconica var. leuconica. a) hairs, b) spores, c) asci and paraphyses, d) excipulum, e) dry apothecia. Scale $50 \mu \mathrm{m}$, for apothecia $100 \mu \mathrm{m}$. — 125: SH 87/91. — 126: SH 87/73. — 127: JAN 3303. 
Apothecia scattered, superficial, up to $500 \mu \mathrm{m}$ in diam when fresh, $100-400 \mu \mathrm{m}$ in diam when dry, cyathiform when juvenile, then cupulate on a stout stipe, up to $100 \mu \mathrm{m}$ long and $70 \mu \mathrm{m}$ wide. Disc bordered by a copious zone of hairs, which rise above the hymenium, margin strongly incurved and obscuring the disc when dry. Hair cover dense, snowwhite, longest hairs concentrated in the margin. Colour white to slightly yellowish brown (K77) when fresh; dry apothecia yellowish brown (L80) to Straw (K79), colour modified by the hair cover.

Ectal excipulum of textura prismatica, cells on middle flanks regularly prismatic, $8-17 \times 3.2-$ $7.0 \mu \mathrm{m}, \overline{\mathrm{x}}=11.3 \times 4.7 \mu \mathrm{m}(\mathrm{n}=70), \bar{Q}=2.4$, similar or becoming slightly smaller towards margin, in stipe narrower. Inner excipulum of narrower textura prismatica or forming an inconspicuous layer of textura porrecta, cells ca. $10-12 \times 1.5-3.0 \mu \mathrm{m}$. Walls in ectal parts varying between the populations from 0.4 to $1.0 \mu \mathrm{m}$ thick, hyaline, MLZ+ or MLZ++ in $75 \%$ of the populations, MLZ- in others, these MLZ+ or $\mathrm{MLZ}$ - after $\mathrm{KOH}$ pretreatment, $\mathrm{BF}+, \mathrm{CRB}+, \mathrm{NR}+$, $\mathrm{TB}+$, fragmentarily $\mathrm{CR}+, \mathrm{CB}-$, LUG-.

Hairs $50-250 \times 3.0-9.5 \mu \mathrm{m}$ in $74 \%$ of the populations, $30-80 \times 3-7 \mu \mathrm{m}$ in others, narrowly conical to basally clearly widened, straight to apically prominently cincinnate, mostly aseptate, basally sparsely septate in $18 \%$ of the populations, rarely septate at or above the middle level, septa thin. Apex gradually tapering to $0.5-1.6(-3.0) \mu \mathrm{m}$, not solidified, without an apical globule in MLZ. Wall at the base typically thin, rarely thickened, becoming thickened and refractive (mostly $0.5-1.0 \mu \mathrm{m}$ thick) upwards, occasionally obscuring the lumen, equal or becoming thinner at the extreme apex, in some populations such hairs intermixed with scattered, totally thin-walled hairs, wall hyaline, MLZ++, CR++, LUG+ (blue), negative in other reagents, structurally stable in $\mathrm{KOH}$, smooth when fresh and dry, in a single apothecium seen to bear hyaline resin (in LUG). Mar- ginal hairs intermixed with cylindrical-clavate, thinwalled cells, $15-25 \times 3-5 \mu \mathrm{m}$.

Asci $22-50 \times 4.5-6.8 \mu \mathrm{m}, \overline{\mathrm{x}}=34.4 \times 5.4 \mu \mathrm{m}(\mathrm{n}=$ $13), \bar{Q}=6.2$ when fresh in water; $22-46(-53) \times 3.8-$ 6.8(-7.0) $\mu \mathrm{m}, \overline{\mathrm{x}}=34.0 \times 5.2 \mu \mathrm{m}(\mathrm{n}=84), \overline{\mathrm{Q}}=6.5$ in MLZ; $21-48(-52) \times 3.0-5.8 \mu \mathrm{m}, \overline{\mathrm{x}}=33.8 \times$ $4.6 \mu \mathrm{m}(\mathrm{n}=39), \bar{Q}=7.2$ in CB; eight-spored, cylindrical-clavate, with slightly conical apex. Apical pore $\mathrm{MLZ}+$ in $70 \%$ of the populations, MLZ++ in others, LUG+ (blue). Wall in mature asci apically 0.5-1.0 $\mu \mathrm{m}$ thick (in CB, MLZ), negative in all reagents; contents irregularly $\mathrm{LUG}+$, very rarely MLZ+ (orange). Asci arising from simple septa, in $21 \%$ of the populations the basal septum frequently with a downward hook.

Spores $5.5-10.0 \times 1.7-2.8(-3.0) \mu \mathrm{m}, \overline{\mathrm{x}}=7.5 \mathrm{x}$ $2.3 \mu \mathrm{m}(\mathrm{n}=19), \overline{\mathrm{Q}}=3.1$ when fresh in water; 5.0 9.5(-11.0) x 1.3-2.6(-3.0) $\mu \mathrm{m}, \overline{\mathrm{x}}=7.1 \times 2.0 \mu \mathrm{m}(\mathrm{n}$ $=198$ ), $\bar{Q}=3.7$ in CB and MLZ; elliptic to oblongelliptic, with rounded apices, rarely becoming septate (ca. $3 \%$ of the discharged spores), septum thin, central, MLZ+. Wall hyaline, turning brownish in aged, discharged spores. Guttulae prominent, light, often apical when fresh in water, in dry material alike but smaller or lacking.

Paraphyses cylindrical to slightly widened at the apex, unpigmented, first dichotomous branches occurring at or below the middle level of the asci. Terminal cells $12-28 \times 1.0-2.5 \mu \mathrm{m}, \overline{\mathrm{x}}=17.9 \times 1.6 \mu \mathrm{m}$ $(n=70)$.

Cultural characters (5 populations, 14 strains). Radial growth slow on MA, $7.4 \mathrm{~mm} /$ month $(n=41$, from 4 popul., 8 strains). Mats with black basic colour, in centre black, at margin olivaceous (P91) or dark brown (S70), rarely extreme margin whitish for 1-2 mm. Aerial mycelium densely woolly, greyish (P92) to greyish white, occasionally with brown (P77) areas, extending nearly to the margin. One strain from Quercus with medium brown (M75) basic

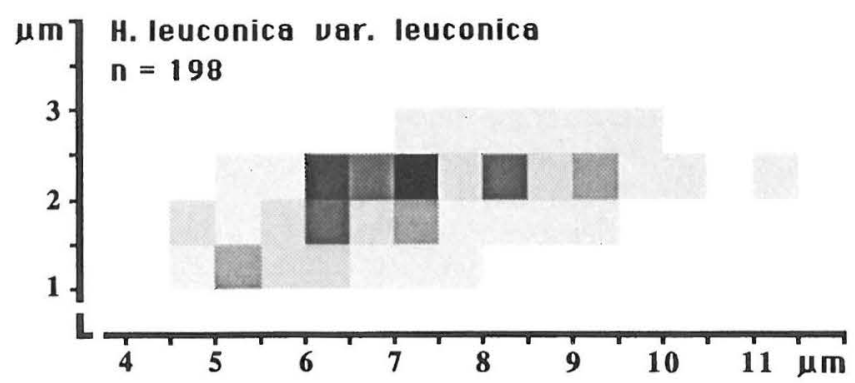

Fig. 128. Percentage sporogram of Hyaloscypha leuconica var. leuconica. 
colour, and wide creamy white margin, aerial mycelium lacking, duplicate trial with overall dark brown (T71) colour. Margin distinct, even, submerged. Hyphal strands distinct to none, running in the agar, inoculum occasionally with erect strands, no zonation, no sector formation, no colour change in surrounding agar, no yeast-like growth. Context tough at surface, odour not specific.

Spores germinating readily on wood agars, growth moderate on $\mathrm{BA}, \mathrm{PA}, \mathrm{QA}$, producing juvenile apothecia on MA after 6 months (single-spore strain), these not developed further. Anamorph not produced in single-spore or multispore trials. The cultures from stems of Calluna, catkins of Betula and a hardwood branch were fairly alike. Only a single strain originating from Quercus was somewhat exceptional. Compared with that in var. bulbopilosa, the variation is notably smaller. Identical strains can, however, be found in the two taxa.

Distribution and phenology. In Europe the taxon is distributed from the Nordic countries to central and western Europe. The few collections from the Canadian timberline (Québec and McKenzie District in N.W.T.), from West Virginia and New York in the U.S.A. and from the Altai Mts. in the U.S.S.R. suggest that the distribution may be wider than can be concluded from the present material.

Most collections have been made between June and November. In England var. leuconica, like many other members of the genus, fruits in winter as well.

Ecology. The present taxon shows a wide ecological range. Fruiting populations have been found both on bulky wood substrates and on pieces of wood in litter. The ecology parallels that of var. bulbopilosa, and is somewhat overlapping with that of $H$. herbarum, but the present taxon is apparently not capable of fruiting on herbaceous substrates.

Two collections have been made from bark (Betula, Quercus), four from litter (Betula catkin, Larix cone, Fagus cupule, small twig of Pinus), one from Rubus stems and the rest from wood: Betula (2),

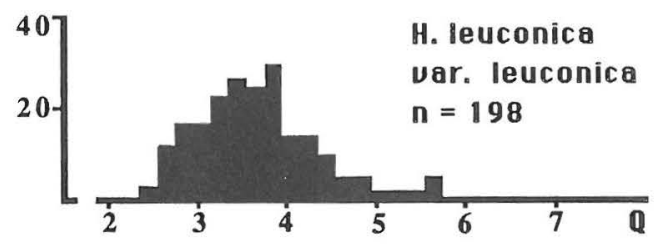

Fig. 129. The distribution of spore quotient Q in Hyaloscypha leuconica var. leuconica.
Calluna (3), Quercus (2), Alnus (1), Fagus (1), Pinus (1), Taxus (1), unidentified hardwood (7), unidentified softwood (2).

Discussion. Hyaloscypha leuconica var. leuconica is recognized by its long, thick-walled hairs, which have an immediate, clear dextrinoid reaction. $H$. albohyalina var. spiralis occasionally has fairly firmwalled hairs coupled with asci that arise without croziers, but its hairs are short and often somewhat warted, and it has larger asci and spores. Firm hairs also occur in $H$. vitreola, but it is differentiated by larger spores and ascal bases.

The long-haired populations of the present variety are easily distinguished from $H$. fuckelii var. alniseda. In the short-haired populations, the hymenial characters overlap, and the hair morphology and cultural characters can be used. A clearly thickened wall in the whole apical part of the hair is characteristic of var. leuconica, whereas in var. alniseda the rare wall thickenings are restricted to the constriction of the lageniform hairs. Solidified apices are lacking in var. leuconica and also apical knobs, both present in var. alniseda. Var. leuconica also lacks the loose granulation on intact hairs in water, which is typical of var. alniseda. The cincinnate hair apex and the clearly widened and relatively abruptly delimited hair bases are also useful, though less reliable, characters of var. leuconica. The distinction between the two varieties of the present species is discussed under var. bulbopilosa. The most variable character of this taxon is hair size, but due to the even gradation of the other characters a distinction based on the hairs would serve no useful taxonomical purpose. Although less marked, variation in hair length is seen in many species of the genus. The hair bases also vary from narrow to almost bulbous, the extremes mostly occurring in separate populations. With the exception of one population, discussed below, firm-walled hairs predominate in the apothecium, although the wall thickness may vary from thin to $1 \mu \mathrm{m}$ thick. The variability of the hymenial and excipular characters is concordant with the general trends in the genus. Small- and large-spored populations occur irrespectively of hair size.

In a few populations of the present variety simpleseptate ascal bases coexist with modified ascal bases. These cases are marked by a downward process, which does not fuse with the lower cells. Free asci are often characterized by a basal process and are morphologically clearly different from those arising from croziers. This modification is the same as discussed by Berthet (1964) for Lachnellula calycina Sacc. In Hyaloscypha it is seen in three taxa and is 
always coupled with aporhyncous ascus development. Such modified ascus bases are dominant in a few populations only.

The type specimen of $H$. cincinnata, agreeing in ascus development with other collections, is from the extreme of the range of variation in respect to its hair characteristics. In the type the hair wall is often thin throughout. This "marginal" type is linked with the other populations by the firmness of many hairs, the clearly swollen hair bases, the cincinnate and nonsolidified apices and the small spores, which exclude it from $H$. fuckelii var. alniseda. The spore measurements overlap, but the present variety has the smallest-spored populations. None of the morphological or chemical characters of the very scanty type of $H$. cincinnata link it unequivocally with var. alniseda.

The hairs of var. leuconica are smooth both when fresh in water and in dry material. In one apothecium (Schefferville, on softwood) hyaline resin, dissolving in MLZ and CB, was observed on the hairs. A few populations have septate hairs, the septa being mostly restricted to the basal hair parts. An interesting collection in this respect was treated and depicted by me under Hyalopeziza sp. (Huhtinen 1984). This population is characterized by very thick hair walls, which occasionally obscure the lumen. The presence of sparse thin septa in the upper part of some hairs as well misled me into placing the collection in Hyalopeziza. In $H$. leuconica there is a smooth gradation among the populations from aseptate hairs to occasional septation; the collection from Schefferville representing an extreme. The hair characteristics of $H$. leuconica may, however, indicate relationship between the two genera.

Three collections in the present material, including the type, were treated and depicted by Dennis (1949) under H. leuconica and Hyaloscypha sp. For the latter conspecificity with Hyalopeziza nectrioidea (Rehm) Raschle was suggested. Although I could not study the type specimen of Peziza leuconica, Dr. Brian Spooner kindly let me check a recent slide made from that collection. This slide showed that the type agrees well with the studied material.

Exsiccatae studied: Rehm, H., Ascomyceten 1360 (BR, $\mathrm{MICH}, \mathrm{PAD}, \mathrm{S})$.

Material studied: Canada. Québec: Schefferville, 1967 Heikkilä (TUR). Northwest Territories: Inuvik, 1984 SH

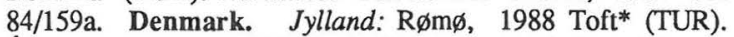
Åbenrå, 1988 SH 88/80* (TUR). Federal Republic of Germany. Westfalen: Siegen, 1948 Ludwig (B). Hannover area: Hildesheim, 1943 (B). Finland. Varsinais-Suomi: Nousiainen, $1987 \mathrm{SH} 87 / 91^{*}$ (TUR). Sauvo, $1987 \mathrm{SH} \mathrm{87/73*}$ (TUR). Etelä-Häme: Kangasala, 1982, 1985 Söderholm 821, 1169a. Pohjois-Häme: Konnevesi, 1986 SH 86/90 (TUR). Pohjois-Savo: Kuopio, 1985 SH 85/90 (TUR). German
Democratic Republic. Potsdam, 1969 Benkert (BHU). Great Britain. Kent: Plaxtol, 1975 Coppins 1491 (E). Yorkshire: Halifax, 1897 (UPS). Masham, 1950 Hughes (IMI). Banstead Woods, 1947 Hughes (IMI). Perthshire: Blackcraig Forest, 1975 Coppins 1147 (E) Calvine, 1983 Coppins 10089 (E). Craiglush, 1954 anonymous 1644 (E). Inverness-shire: Isle of Skye, 1980 Bennell (E). Norway. Hordaland: Bergen, 1987 Olsen* (TUR). Sweden. Småland: Femsjo, 1929 JAN 3303 (UPS). U.S.A. New York: Lost Gorge, 1973 Sherwood (CUP, type of Hyaloscypha cincinnata). Adirondack Mts., Marcy Dam, 1953 Schaffer \& Korf 53-46 (Herb. Korf). West Virginia: Randolph Co., Monongahela Nat. For., 1985 Haines 3721 (NYS). U.S.S.R. Kazakhstan: Altai Mts., Leninogorsk, 1965 AR (TAA).

\section{Hyaloscypha leuconica var. bulbopilosa}

Hyaloscypha leuconica (Cooke in Stevenson) Nannf. var. bulbopilosa (Feltg.) Huhtinen, comb. nov. - Dasyscypha hamata (Sacc.) Feltg. var. bulbopilosa Feltg., Rec. Mem. Trav. Soc. Bot. Luxemb. 16:68. 1903. - Dasyscypha bulbopilosa (Feltg.) Höhnel, Sitzungsber. Akad. Wiss. Wien, Math. Nat. Cl. 115: 1289. 1906. - Trichopeziza hamata Sacc. var. bulbopilosa (Feltg.) Sacc. \& D. Sacc., Syll. Fung. 18:80. 1906. - Holotype (the only specimen mentioned): [Luxemburg,] Leudelingen, an Salix Holz, IX.1902 Feltgen (F 8620, examined).

?Hyaloscypha degenerans Velen., Monogr. Discom. Bohem.: 274. 1934. - Lectotype (Svrček 1985: 208): [Czechoslovakia,] Bohemia, Mnichovice, Struharov, in conis pinis, VII.1933 Velenovský (PRM 151202, seen but found to be empty).

Hyaloscypha hyalina (Pers.: Fr.) Boud. var. setosa Velen., Monogr. Discom. Bohem.: 284. 1934. - Hyaloscypha setosa (Velen.) Svr., Česká Mykol. 16:96. 1962. — Holotype (indicated as such by Svrček 1985: 210): [Czechoslovakia,] Bohemia, Senohraby, Picea exc., 30.VIII.1926 Velenovský (PRM 150877 , examined). The label gives only one of the three types of substrate mentioned by Velenovsky (1934). Hence, a case of lectotypification may be involved.

Hyaloscypha longevestita Velen., Monogr. Discom. Bohem.: 274; P1. 15:58. 1934. — Lectotype (Svrcek 1985: 211): [Czechoslovakia,] Bohemia, Mnichovice, Hubačkov, Larix, 11.VII.1929 Velenovský (PRM 151136, examined).

Apothecia scattered, superficial, up to $800 \mu \mathrm{m}$ in diam when fresh, $100-500 \mu \mathrm{m}$ in diam when dry, disc bordered by a copious zone of hairs, which rise clearly above the hymenium, margin strongly incurved and often obscuring the disc when dry. Hair cover dense, snow-white, longest hairs concentrated in the margin. Colour white to slightly yellowish brown (K77) when fresh; dry apothecia yellowish brown (L80) to Straw (K79) to orange brown (L80), rarely reddish brown (R17), colour modified by the hair cover.

Ectal excipulum of textura prismatica, cells on middle flanks regularly prismatic, $8-15 \times 3.6-$ $6.0 \mu \mathrm{m}, \overline{\mathrm{x}}=11.0 \times 4.9 \mu \mathrm{m}(\mathrm{n}=80), \bar{Q}=2.2$, similar or becoming slightly smaller towards margin, in 


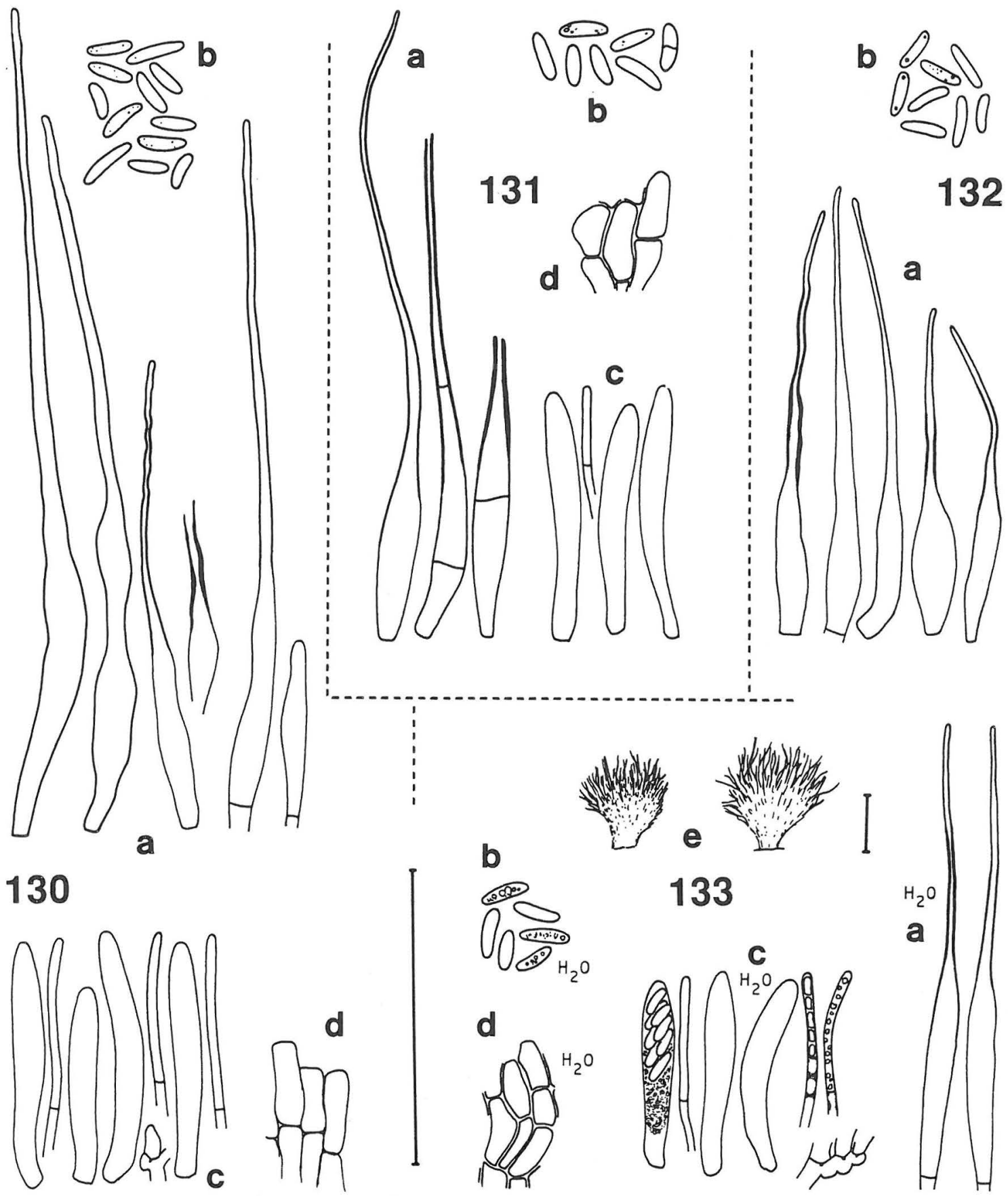

Figs. 130-133. Hyaloscypha leuconica var. bulbopilosa. a) hairs, b) spores, c) asci and paraphyses, d) excipulum, e) dry apothecia. Scale $50 \mu \mathrm{m}$, for apothecia $100 \mu \mathrm{m}$. - 130: Holotype of Hyaloscypha hyalina var. setosa. - 131: Coppins 545. - 132: 1944 Lundell. - 133: Vauras 2604. 
stipe narrower. Walls in ectal parts varying between the populations from 0.4 to $1.0 \mu \mathrm{m}$ thick, hyaline, MLZ+ or MLZ ++ in $57 \%$ of the populations, MLZin others, these MLZ+ or MLZ- after $\mathrm{KOH}$ pretreatment, $\mathrm{BF}+, \mathrm{CRB}+, \mathrm{NR}+, \mathrm{TB}+$, fragmentarily $\mathrm{CR}+$, CB-, LUG-.

Hairs (30-) $45-250 \times 2.2-11.0 \mu \mathrm{m}$ in $72 \%$ of the populations, $35-90 \times 2.5-8.2 \mu \mathrm{m}$ in others, narrowly conical to basally clearly widened, straight to apically prominently cincinnate, aseptate to basally sparsely septate (in $22 \%$ of the populations), once seen to be septate at or above the middle level, septa thin. Apex gradually tapering to $0.5-1.5(-1.8) \mu \mathrm{m}$, rarely solidified, without an apical globule in MLZ. Wall at the base typically thin, rarely thickened, becoming thickened and refractive (typically 0.5$1.0 \mu \mathrm{m}$ thick) upwards, occasionally obscuring the lumen, wall equal or becoming thinner at the extreme apex, in some populations such hairs intermixed with scattered, totally thin-walled hairs, wall hyaline, MLZ++ (in one population MLZ-, fragmentarily $\mathrm{MLZ}+$ after $\mathrm{KOH}$ pretreatment), CR++, LUG+ (blue), negative in other reagents, structurally stable in $\mathrm{KOH}$, smooth when fresh and dry, in a few populations seen to bear hyaline to yellowish resin in water, dissolving in MLZ. Marginal hairs intermixed with cylindrical-clavate, thin-walled cells, $15-25 \times 3-$ $5 \mu \mathrm{m}$.

Asci 34-51 x 5.3-6.7(-7.2) $\mu \mathrm{m}, \overline{\mathrm{x}}=40.0 \times$ $6.1 \mu \mathrm{m}(\mathrm{n}=18), \mathrm{Q}=6.3$ when fresh in water; 32 $48(-61) \times 4.0-6.3 \mu \mathrm{m}, \overline{\mathrm{x}}=37.4 \times 4.9 \mu \mathrm{m}(\mathrm{n}=44)$, $\mathrm{Q}=7.4$ in $\mathrm{MLZ} ; 28-47(-54) \times 4.0-5.8 \mu \mathrm{m}, \overline{\mathrm{x}}=$ $37.6 \times 4.8 \mu \mathrm{m}(\mathrm{n}=76) ; \bar{Q}=7.8$ in $\mathrm{CB}$; eightspored, cylindrical-clavate, with slightly conical apex. Apical pore MLZ+ in $73 \%$ of the populations, MLZ++ in others, LUG+ (blue). Wall in mature asci apically $0.5-1.0 \mu \mathrm{m}$ thick (in CB, MLZ), negative in all reagents; contents irregularly LUG+, very rarely $\mathrm{MLZ+}$ (orange). Asci arising from croziers.
Spores $7.0-11.3 \times 2.0-2.8(-3.2) \mu \mathrm{m}, \overline{\mathrm{x}}=8.9 \times$ $2.4 \mu \mathrm{m}(\mathrm{n}=41), \bar{Q}=3.6$ when fresh in water; 6.0 9.6(-11.6) $\times 1.5-2.2(-2.8) \mu \mathrm{m}, \overline{\mathrm{x}}=7.6 \times 2.0 \mu \mathrm{m}(\mathrm{n}$ $=304), \bar{Q}=4.1$ in $\mathrm{CB}$ and MLZ; elliptic to oblongelliptic, with rounded apices, becoming septate rarely (ca. $1.8 \%$ of the discharged spores), septum thin, central, MLZ+. Wall hyaline, becoming brownish in aged, discharged spores. Guttulae lacking or inconspicuous when fresh in water; in dry material lacking or small and light.

Paraphyses cylindrical to slightly widened at the apex. Terminal cells $10-39 \times 1.0-2.5 \mu \mathrm{m}, \overline{\mathrm{x}}=21.6 \mathrm{x}$ $1.5 \mu \mathrm{m}(\mathrm{n}=70)$.

Cultural characters ( 9 populations, 26 strains). Radial growth slow on MA, $8.3 \mathrm{~mm} /$ month $(\mathrm{n}=19$, from 4 popul., 6 strains). Mats variable, either with black basic colour and like those of var. leuconica or with basic colour creamy white, grey (S92) to dark brown (R75, T71) to olivaceous (S91). Aerial mycelium densely woolly, whitish to greyish, or none. Margin whitish for $1.2 \mathrm{~mm}$, even to fimbriate. Hyphal strands present in one population, strong, submerged. Zonation lacking, sector formation rare, two strains turning the agar yellowish (close to K85), no yeast-like growth. Context tough at surface, odour not specific.

Spores germinating readily on wood agars, growth moderate on BA, PA, QA, retarded on LA, producing apothecial primordia on MA after 10 months, these not developed further, producing apothecia on BA after 18 months (single-spore strain from hardwood), the 12 strains extracted from cultivated apothecia all alike, anamorph not produced in single spore or multispore trials.

The variation in cultural morphology occurs mostly between the populations. The two strains which coloured the surrounding agar originated from Picea wood and from unidentified hardwood. The

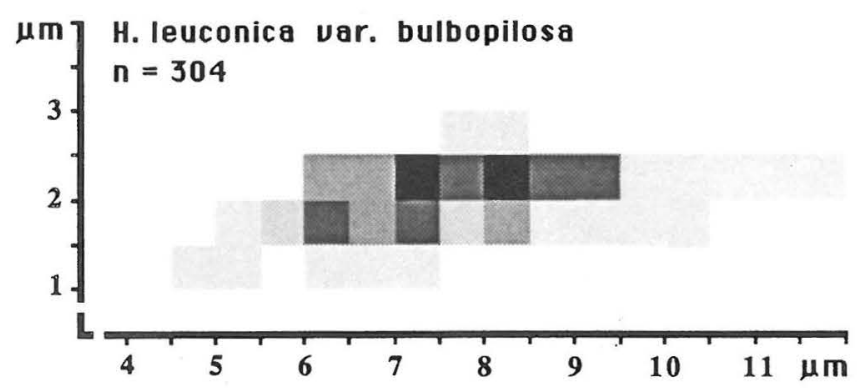

Fig. 134. Percentage sporogram of Hyaloscypha leuconica var. bulbopilosa. 
strains came from widely separate localities, in Czechoslovakia, Denmark and Finland, but the variation does not seem to be due to geographical distance. In fact, the variation between the Finnish populations is greater.

Distribution and phenology. In Europe H. leuconica var. bulbopilosa is distributed from the timberline areas of the Nordic countries to central and western Europe. One collection originates from northern Siberia and two from North America, from the timberline area in Québec and from New York State. The distribution pattern resembles that of var. leuconica.

Var. bulbopilosa fruits throughout the frost-free period. In northern Europe the collections date mainly from summer and autumn, whereas in Great Britain many collections have been made during the winter months. There is no major difference from the phenology of the type variety.

Ecology. The ecological amplitude of Hyaloscypha leuconica var. bulbopilosa is wide. It fruits on both hardwoods and softwoods and also on smaller ligneous substrates such as cones of Pinus (6) and Larix (4), small twigs of Larix (1) and Calluna stems (1), Vaccinium (1), Quercus (1). The collections from more bulky substrates originate from: Pinus (4), Alnus (4), Betula (2), Corylus (2), Larix (2), Picea (2), Populus (2), Fagus (1), Quercus (1), Salix (1), Sorbus (1), Symphoricarpus (1), unidentified hardwood (9), unidentified softwood (4), With the exception of the occurrence on Pinus cones, there is no major difference in ecology from var. leuconica.

Discussion. The basic difference between the two varieties lies in the ascal bases, which in var. leuconica have simple septa and in the present variety have clear croziers. In other respects the variational ranges in hair, spore and ascus characters of the two

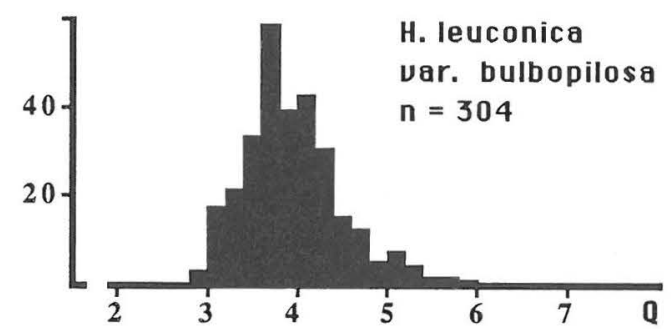

Fig. 135. The distribution of spore quotient Q in Hyaloscypha leuconica var. bulbopilosa. taxa form an almost uninterrupted gradation. However, the hair type of, for example, the type specimen of $H$. cincinnata seems to be lacking in var. bulbopilosa. A sign of possible divergence is seen in the hairs of var. bulbopilosa, which in many populations are characterized by moderate wall thickness and by frequent constrictions, which give the hairs a more irregular appearance than the thick-walled hairs of var. leuconica. Slight differences occur in spore guttulation also, but their taxonomic value is obscure. The ecological ranges of the two varieties are also alike and they both fruit on such rare (for a $\mathrm{Hya}$ loscypha) substrates as cones of Larix or Calluna stems. As in $H$. fuckelii, the present varieties seem to represent more recently diverged taxa than, e.g., the varieties in $H$. albohyalina, which have more pronounced morphological differences. The recognition of two varieties, rather than two forms, is due to the probable taxonomic significance of the ascal development.

The short-haired populations of the present variety are separated from the type varieties of $H$. fuckelii and $H$. albohyalina by the characters discussed under var. leuconica.

As in var. leuconica, the hairs are variable in size and shape. Populations with basally swollen hairs are linked by intermediate forms with populations with basally narrow and often irregularly constricted hairs. The wall thickenings are most marked in the basally swollen type with a relatively abrupt constriction. In the narrow hair type the walls tend to be regularly thickened in their central part, the base and extreme apex having thin walls. Although the wall thickness varies and uniformly thin-walled hairs are occasionally present, all the populations have firm and refractive hairs. In two populations the hairs bore yellowish to hyaline, dissolving resin. Basally sparsely septate hairs occur in many populations but are always in the minority. These hairs are otherwise indistinguishable from those that are aseptate. The variation in the size of the excipular cells between the populations is not linked with the variation in hair size (see discussion under var. leuconica). Although the hair walls were strongly dextrinoid, the excipular walls of fairly many populations remained negative. The presence or lack of the reaction was not connected with the nature of the substrate.

An interesting collection, originating from Austria (Herb. Matheis 965), showed short, firm-walled hairs with MLZ- walls. Even after $\mathrm{KOH}$ pretreatment the positive reaction was only fragmentary. Though chemically differentiated from the rest of the material, this collection is similar in morphological characters. It is provisionally placed in var. bulbopilosa because 
similar variation in the hair reactions was found in, e.g., H. albohyalina.

Stability is seen in the ascal bases and their size, spore size, sparse septation and spore guttulation, and in the lack of warts on the hair wall. This homogeneity is noteworthy in view of the wide ecological amplitude of the taxon.

The synonymy between $H$. leuconica and the two taxa of Velenovský was noted by Svrček (1985). I found no apothecia in the lectotype of $H$. degenerans Velen. Svrček (1985) synonymized this species with $H$. leuconica and my synonymization with the present variety is based on the ecology. So far var. leuconica has not been found on cones of Pinus (the original substrate for $H$. degenerans), whereas this seems to be a relatively usual substrate for the present variety. Due to its short, thin-walled, apically solidified and encrusted hairs, $H$. hyalina var. laricina is synonymized with $H$. fuckelii, rather than with $H$. leuconica as suggested by Svrček (1985).

Material studied: Austria. Tirol: Bieler Höhe, 1985, Matheis 965 (Herb. Matheis). Canada. Québec: Manitounuk Islands, 1982 SH 82/101 (TUR). Czechoslovakia. Bohemia: Duba, 1979 Minter (IMI). Mnichovice, 1929 JV (PRM, type of Hyaloscypha longevestita) $1986 \mathrm{SH} \mathrm{86/32*}$ (TUR). Senohraby, 1926 JV (PRM, type of Hyaloscypha hyalina var. setosa). Vsenory, 1926 JV (PRM). Denmark. Jylland: Kruså, 1978 Sivertsen \& Korf (CUP). Lovrup, 1988 Læssøe* (TUR). Sjolland: Copenhagen, 1987 Læssøe (TUR). Finland. Varsinais-Suomi: Kustavi, 1987 Vauras 2604* (TUR). Turku, 1985 SH 85/132*, 85/139* (TUR). Etelä-Häme: Lammi, $1985 \mathrm{SH}$ 85/110* (TUR). Tammela, 1987 SH 87/88* (TUR). Satakunta: Punkalaidun, 1985 SH 85/124* (TUR). Inarin Lappi: Utsjoki, 1986 Holm \& Holm (TUR). German Democratic Republic. Löbau, 1972 Benkert (BHU). Potsdam, 1969 Benkert (BHU, two collections). Suhl, 1981 Benkert (BHU). Great Britain. Sussex: Crawley, 1979 Minter (IMI). Surrey: Ockham Common, 1978 Minter (IMI). Peaslake, 1948 Dennis (F). Nottinghamshire: Sherwood Forest, 1979 Minter (IMI). Cheshire: Delamere Forest, 1910 (K). Yorkshire: Halifax, 1895 ?Crossland (CUP-D 162). Hebden Bridge, 1956 Watling 114-2 (E). Picking Gill Clough, 1959 Watling 114-4 (E). Ravenscarr Gill Wood, 1959 Watling 151-4 (E). Spa Gill Wood, 1954 Watling 114-1 (E). Northumberland: Stannington, 1979 Minter (IMI). Angus: Glen Isle, 1966 Watling 971 (E). Peebles: Blyth Bridge, 1979 Minter (E). Glentress Forest, 1974 Coppins 545 (E). Luxemburg. Leudelingen, 1902 Feltgen (F, type of Dasyscypha hamata var. bulbopilosa). Netherlands. Dwingelo Lhee, 1955 Maas Geesteranus (L). Wageningen, 1960 anonymous (L). Norway. Østfold: Fredrikstad, 1985 SH 85/40 (TUR). Sweden. Smaland: Kärda, 1929 JAN 2148, 1944 Lundell (UPS). Uppland: Bondkyrka, 1931 Lundell \& JAN 4197 (UPS). Börje, 1946 Melderis (K). Erentuna, 1928 Lundell (UPS). Läby, 1949 JAN 10696 (UPS). Jämtland: Åre, 1951 JAN 11794 (UPS). Torne Lappmark: Jukkasjärvi, 1927 JAN 6441948 Pilát \& JAN 9911 (UPS). U.S.A. New York: Ithaca, 1947 Korf 628 (Herb. Korf). U.S.S.R. Tjumen Region: JamaloNeneckij, Krasnosel Kup, 1964 Parmasto (TAA).

\section{Hyaloscypha nipponica, sp. nov.}

Apothecia cupulata, sessilia, minuter pilosa; specimina exsiccata aurantiaca vel rubiginosa, usque ad $700 \mu \mathrm{m}$ lata. Excipulum externum textura prismatica vel textura angularia, cellulis $6.2-12.5(-16.2) \times 3.4-7.2(-9.0) \mu \mathrm{m}$, aliquantum crassotunicatis, hyalinis, in solutione Melzeri sparsim amyloideis. Pili $30-85 \times 2.2-5.2 \mu \mathrm{m}$, anguste conici vel fere cylindracei, in solutione Melzeri raro sparsim amyloidei, raro exudato resinoso, aseptati, vel sparsim septati, tenuiter tunicati. Asci 58 $70(-76) \times 6.8-9.2(-9.3) \mu m$ in solutione Melzeri, non amyloidei vel. amyloidei sed post solutionem kalii amyloidei, in basi non uncinati. Sporae 8.3-10.9(-11.5) x 2.9-3.8 (-4.0) $\mu \mathrm{m}$, ellipsoideae vel oblongo-ellipsoideae, non septatae vel sparsim septatae. Paraphyses cylindraceae vel in apice minuter dilatatae, 1.2-2.2 4 m latae, cellulis terminalibus 19 $52 \mu \mathrm{m}$ longis.

Holotype: Japan. Hokkaido, Ibari Pref.: E end of Lake Shikotsu, on rotten wood, 20.V.1958 Imai, Kamei, Otani, Korf et al. (CUP-JA 755).

Apothecia gregarious to confluent, superficial, up to $700 \mu \mathrm{m}$ in diam when dry, cupulate, broadly sessile, firmly attached to the substrate. Margin prominent, incurved, obscuring most of the hymenium when dry. Hair cover dense at the margin, sparse below, snow-white. Colour orange brown (M59) or reddish brown (R17) when dry.

Ectal excipulum of textura prismatica-textura angularis, cells on middle flanks somewhat angular, 6.2-12.5(-16.2) x 3.4-7.2(-9.0) $\mu \mathrm{m}, \overline{\mathrm{x}}=9.4 \times$ $5.0 \mu \mathrm{m} ; \bar{Q}=1.9(\mathrm{n}=25)$, becoming more prismatic towards the margin, at the base more roundish. Walls in ectal parts $0.4-0.8 \mu \mathrm{m}$ thick, hyaline, becoming basally brownish with age MLZ- even after $\mathrm{KOH}$ pretreatment, except for the sparse to dense, blackish violaceous nodules, fragmentarily $\mathrm{BF}+, \mathrm{CRB}+$, $\mathrm{NR}+, \mathrm{TB}+, \mathrm{CB}-, \mathrm{CR}-$, LUG-.

Hairs $30-85 \times 2.2-5.2 \mu \mathrm{m}$, narrowly conical to almost cylindrical, straight to bent, aseptate to rarely basally or centrally one-septate, rarely with a few scattered, blackish violet inclusions in MLZ. Apex tapering to $1.2-2.0 \mu \mathrm{m}$, not solidified, without an apical globule in MLZ. Wall thin, never locally thickened, hyaline, dull, smooth to minutely encrusted in all mountants, negative in all reagents, bearing lumps of amorphous, hyaline to yellowish, resinous matter. Resin invariably persistent in all reagents.

Asci $58-70(-76) \times 6.8-9.2(-9.3) \mu \mathrm{m}, \overline{\mathrm{x}}=64.3 \times$ $7.8 \mu \mathrm{m},(\mathrm{n}=20), \bar{Q}=8.7$ in MLZ; $51-67(-72) \times$ 6.8-8.5(-88) $\mu \mathrm{m}, \overline{\mathrm{x}}=61.9 \times 7.7 \mu \mathrm{m}(\mathrm{n}=20), \overline{\mathrm{Q}}=$ 8.1 in $\mathrm{CB}$; eight-spored, cylindrical-clavate with a slightly conical apex. Apical pore MLZ-, MLZ++ after KOH pretreatment, LUG+ (red in two populations, blue in one). Wall in mature asci apically $0.5-$ 

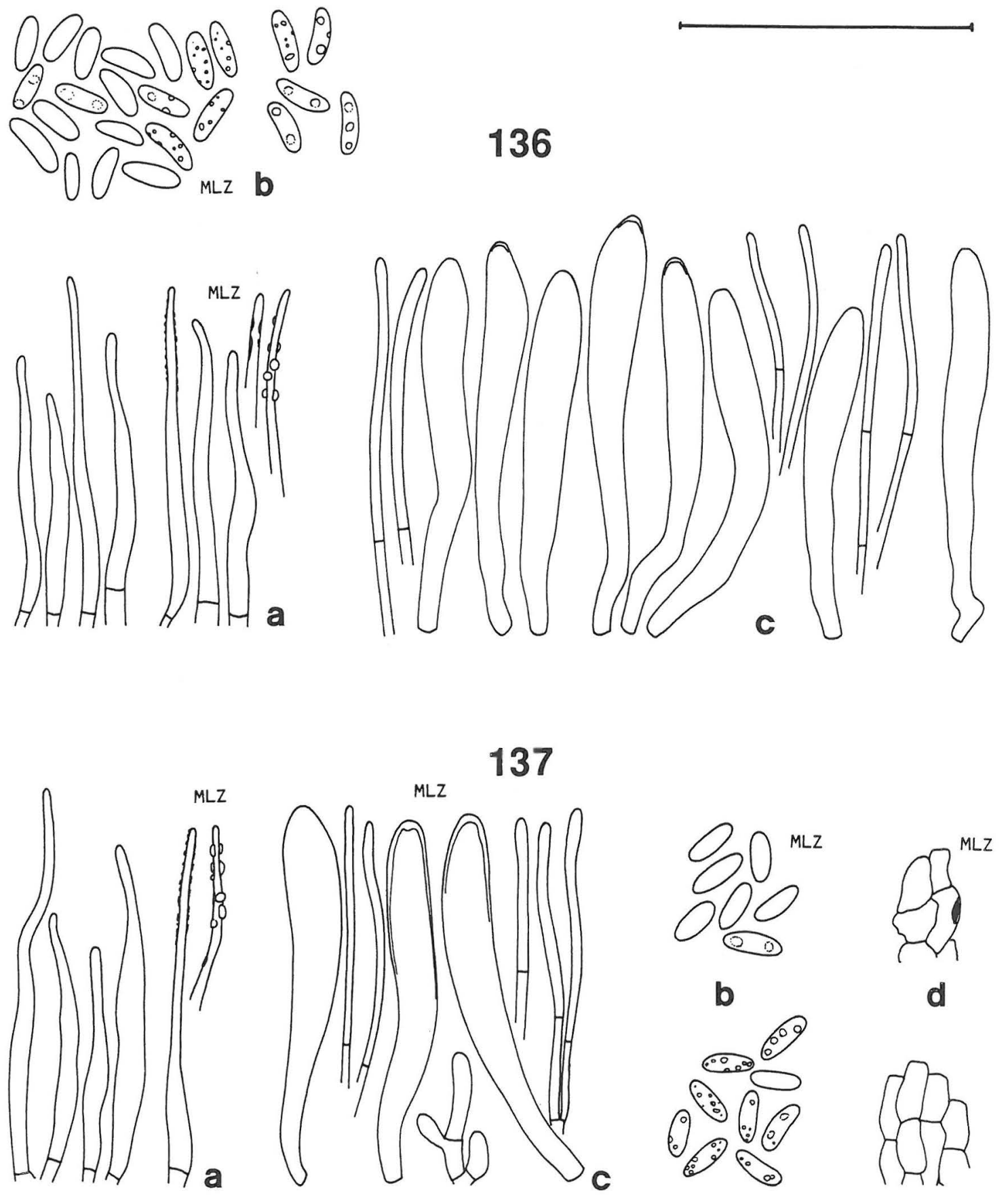

Figs. 136-137. Hyaloscypha nipponica. a) hairs, b) spores, c) asci and paraphyses, d) excipulum. Scale $50 \mu \mathrm{m}$. - 136: Holotype. - 137: Korf et al. 691. 
$1.0 \mu \mathrm{m}$ thick (in CB, MLZ), negative in all reagents. Contents frequently LUG+, infrequently MLZ+ (orange). Asci arising from simple septa.

Spores 8.3-10.9(-11.5) x 2.9-3.8(-4.0) $\mu \mathrm{m}, \overline{\mathbf{x}}=$ $9.6 \times 3.3 \mu \mathrm{m}(\mathrm{n}=96), \bar{Q}=3.0$ in $C B$ and MLZ, elliptic to oblong-elliptic, with rounded apices, aseptate, in one collection $2 \%$ of the discharged spores one-septate. Wall hyaline. Guttulae prominent to small, light in CB, smaller in MLZ.

Paraphyses cylindrical to apically slightly widened, unpigmented. Terminal cells 19-52 x 1.2$2.2 \mu \mathrm{m}, \overline{\mathrm{x}}=35.1 \times 1.8 \mu \mathrm{m}(\mathrm{n}=22)$.

\section{Cultural characters. Not known.}

Distribution and phenology. Two of the known collections originate from Hokkaido in northern Japan. The collections date from May. The third specimen comes from the northeastern U.S.A., New York State. This collection was made in October.

Ecology. The substrates in all collections are apparently hardwood. They differ structurally from each other, but are similar in softness and degree of decay. The ecology parallels that of $H$. vitreola, i.e. the species fruits on well decayed and soft, inner wood.

Discussion. Hyaloscypha nipponica is an easily recognizable species. The blunt, resinaceous, thinwalled and indextrinoid hairs place it in the subgenus

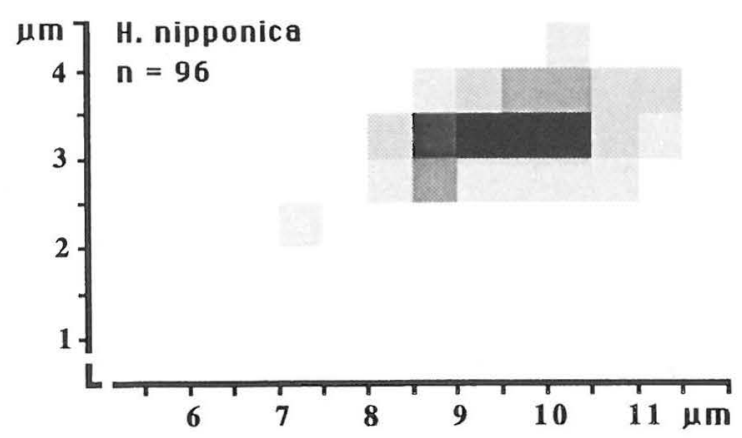

Fig. 138. Percentage sporogram of Hyaloscypha nipponica.

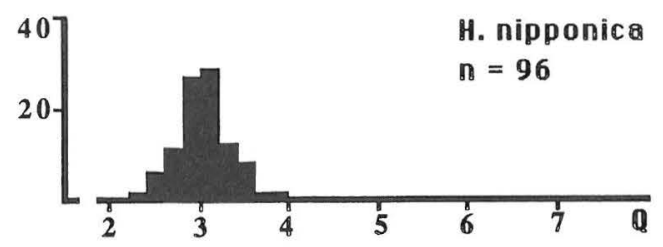

Fig. 139. The distribution of spore quotient $\mathrm{Q}$ in Hyaloscypha nipponica.
Eupezizella, close to H. aureliella. As in that species, the hairs and especially the excipulum show scattered, intensively amyloid areas, which allow rapid recognition. $H$. nipponica is distinguished from the other species in the subgenus by its hymenial characters. The ascal pore stains strongly in MLZ, but only after $\mathrm{KOH}$ pretreatment. The plug may become clearly red in LUG, which is rarely seen in Hyaloscypha. The asci arise from simple septa, and the large spores are characteristic.

Amyloid nodules were frequently observed in hyphae in the wood next to the apothecia. They are most abundant in these vegetative hyphae but occur to a lesser extent in the basal parts of the excipulum, too. From the base upwards, the nodules become increasingly infrequent. This feature was present, although rarely, in $H$. aureliella also.

In the mountants used most of the hairs lack resinous exudates. The exudate is somewhat different from that of $H$. aureliella in being partly resistant to $\mathrm{CB}$ and MLZ. In these mountants a few hairs have a platelike crust reminiscent of that of $H$. albohyalina var. tigillaris. As the spore and ascus sizes are alike, the taxa can be confused under the microscope, but they are differentiated by the chemical characters and the smooth hairs of $H$. nipponica. The difference in ecology, i.e. production of fruit bodies on either softwoods or hardwoods, may also be valuable.

The presence of sparsely septate, resinous hairs with amyloid inclusions indicates relationship to the marginal species of Arachnopeziza (Huhtinen $1987 d)$. Such hairs are equally rarely seen in the collection from Ithaca. The hair shape, chemistry and especially the predominantly aseptate hairs link the species with $H$. aureliella. Hence, $H$. nipponica is a typical member of the subgenus Eupezizella.

Notable hymenial variability is seen in the three collections. The MLZ- asci in the Japanese material contrast with the always MLZ+ asci in the third collection. This difference is repeated in LUG, in which the two former collections have red ascal plugs, the latter blue. Septate spores are seen only in the North American specimen, which also differs in the somewhat larger-celled textura prismatica.

The excipulum, although somewhat like textura angularis, has a structure similar to that in $H$. aureliella. The rounded and somewhat irregular appearance of the ectal cells in surface view is partly due to their lying at a narrow angle to the surface of the flanks. The same feature is seen in many populations of $H$. aureliella.

Material studied: Japan. Ibari Pref.: Lake Shikotsu, 1958 Korf et al. 755 (CUP, type). Ishikari Prov.: Toyohiracho, 1958 Korf et al. 691 (CUP). U.S.A. New York: Ithaca, 1901 Durand (CUP-D 1187). 


\section{Hyaloscypha occulta}

Hyaloscypha occulta Huhtinen, nom. nov. - Hyaloscypha parvula Arendh. \& R. Sharma, Mycotaxon 17:494; Pl. 8:1-6. 1983, nom. illeg. (Art. 64.1., non Hyaloscypha parvula (De Not.) Boud.). - Holotype (original designation): [India,] WBengal, Darjeeling, 6th mile, $2350 \mathrm{~m}$ a.s.l., angiospermous forest, 20.VIII.1980 R. Sharma (PAN 17640, not seen but an isotype examined).

Apothecia gregarious, superficial, up to $150 \mu \mathrm{m}$ in diam when dry, cupulate throughout development, sessile or with a gradually tapering base. Margin incurved and totally obscuring the disc when dry. Hair cover equal, dense, snow-white. Colour Straw (K79) when dry, the overall hair cover obscuring this colour.

Ectal excipulum of short-celled textura prismatica, cells on middle flanks regularly prismatic, 5.3-9.0(12.5) $\times 2.8-5.0 \mu \mathrm{m}, \overline{\mathrm{x}}=7.0-3.8 \mu \mathrm{m}(\mathrm{n}=30), \overline{\mathrm{Q}}=$ 1.8 , not changing size or shape towards margin, at the base small-celled and more roundish. Inner excipulum of only slightly narrrower cells. Walls thin to $0.4 \mu \mathrm{m}$ thick, hyaline, $\mathrm{MLZ}+$; $\mathrm{BF}+, \mathrm{CRB}+$, $\mathrm{NR}+, \mathrm{TB}+, \mathrm{CB}-, \mathrm{CR}-, \mathrm{LUG}-$.

Hairs $20-55 \times 2-4(-5) \mu \mathrm{m}$, narrowly conical, apically undulating to curved or hooked, more rarely straight, aseptate, rarely basally one-septate. Apex tapering to $0.3-1.0(-2.0) \mu \mathrm{m}$, not solidified (except for a few juvenile hairs), without an apical globule in MLZ. Wall basally thin, becoming uniformly or locally $0.4-0.7 \mu \mathrm{m}$ thick towards apex, hyaline, refractive, smooth in all reagents, MLZ++, LUG+ (violet), fragmentarily $\mathrm{CR}++$; negative in other reagents. Marginal hairs intermixed with abundant, cylindrical-clavate, thin-walled to apically massively thickened cells, 8-15 × 2.8-4.0 $\mu \mathrm{m}$.

Asci $35-43 \times 6-8(-8.2) \mu \mathrm{m}, \overline{\mathrm{x}}=40.2 \times 6.8 \mu \mathrm{m}$ $(\mathrm{n}=23), \overline{\mathrm{Q}}=6.0$ in $\mathrm{MLZ} ; 33-49(-53) \times 5.0-6.8(-$ 7.0) $\mu \mathrm{m}, \overline{\mathrm{x}}=39.3 \times 6.0 \mu \mathrm{m}(\mathrm{n}=22) ; \bar{Q}=6.6$ in $\mathrm{CB}$; eight-spored, cylindrical-clavate, with slightly conical apex. Apical pore MLZ+, LUG+ (blue). Wall in mature asci apically thin to $0.6 \mu \mathrm{m}$ thick (in CB, MLZ), negative in all reagents, contents frequently IKI+ (reddish orange), rarely $\mathrm{MLZ}+$ (faint orange). Asci arising from simple septa.

Spores 6.5-9.9(-10.6) $\times 2-3 \mu \mathrm{m}, \overline{\mathrm{x}}=8.4 \times 2.5 \mu \mathrm{m}$ ( $\mathrm{n}=71$ ), $\mathrm{Q}=3.5$ in CB and MLZ, elliptic to oblongelliptic to phaseoliform, with rounded to slightly tapered apices, 15 to $38 \%$ of them one-septate before and after discharge; septum thin, central, often MLZ+. Guttulae mostly inconspicuous in discharged spores, light; more prominent in spores maturing inside the asci.

Paraphyses cylindrical to slightly widened at the apex, unpigmented. Terminal cells $8-34 \times 1.2-$ $1.6 \mu \mathrm{m}, \overline{\mathrm{x}}=20 \times 1.4 \mu \mathrm{m}(\mathrm{n}=12)$.

\section{Cultural characters. Not known.}

Distribution and phenology. This interesting and easily recognizable species is known from only two, widely separated areas. Judging from the rather large amount of lignicolous Hyaloscyphas studied, it seems that the scantiness of collections reflects the true rarity of $H$. occulta. The collections were made in June and August.

Ecology. In the collection from India the apothecia occur on decorticated, unidentified wood. The other collection shows apothecia on the inner side of unidentified bark. These collections agree with the general lignicolous habit of Hyaloscypha.

Discussion. Hyaloscypha occulta is recognized by the firm-walled, intensively dextrinoid, smooth and curved hairs, which are unique in the genus. The small-celled excipulum is also distinctive. The firmwalled hairs may cause some confusion between $H$. occulta and $H$. leuconica. The latter species has smaller asci, but overlap exists, especially in the spore measurements. In these cases the type variety of $H$. leuconica is differentiated by its longer, more or less straight and basally widened hairs and largercelled excipulum. The ascal bases in $H$. occulta, which I found to lack croziers, though these are mentioned in the original diagnosis (Arendholz \& Sharma 1983), separate it from, e.g., such taxa as H. leuconica var. bulbopilosa and $H$. fuckelii var. fuckelii.

The two known collections vary only in spore and ascus characteristics. In the North American collection the spores are frequently one-septate even inside the asci, whereas in the type material the septation developed more tardily. The asci in the type occasionally show a uniformly orange inclusion in MLZ, whereas in the other collection the ascal contents are MLZ-.

This species was described by Arendholz and Sharma (1983) under the name $H$. parvula, which is a later homonym for $H$. parvula (De Not.) Boud. Being a species with clearly thickened, refractive hair walls, $H$. occulta is close to Hyalopeziza, as noted by Arendholz and Sharma (1983). However, the nonseptate and apically curved hairs agree with the character combination of Hyaloscypha and to a lesser degree with that of Hyalopeziza (see also discussion under "Generic relationships"). $H$. occulta would be a marginal species in Unguiculella also, due to the normal paraphyses and hyaline excipulum of textura prismatica. Hence, it is best placed in Hyaloscypha, together with its apparently closest relatives, such as H. leuconica var. bulbopilosa. 

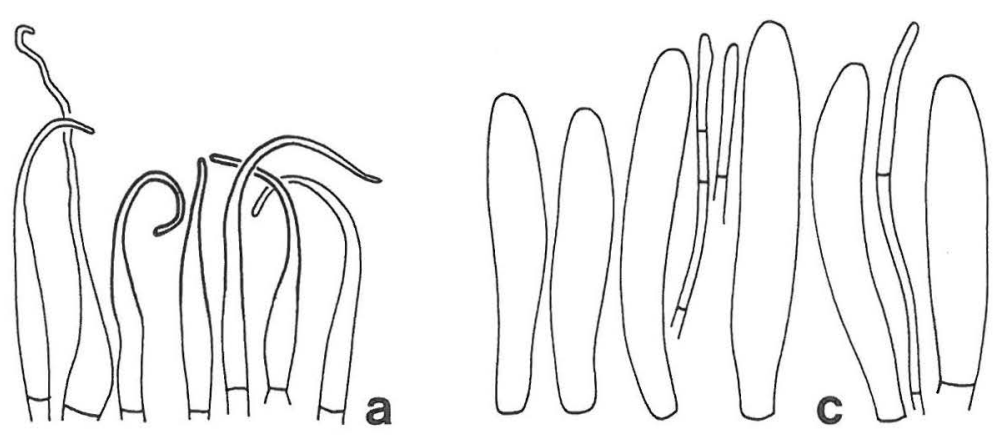

\section{0}
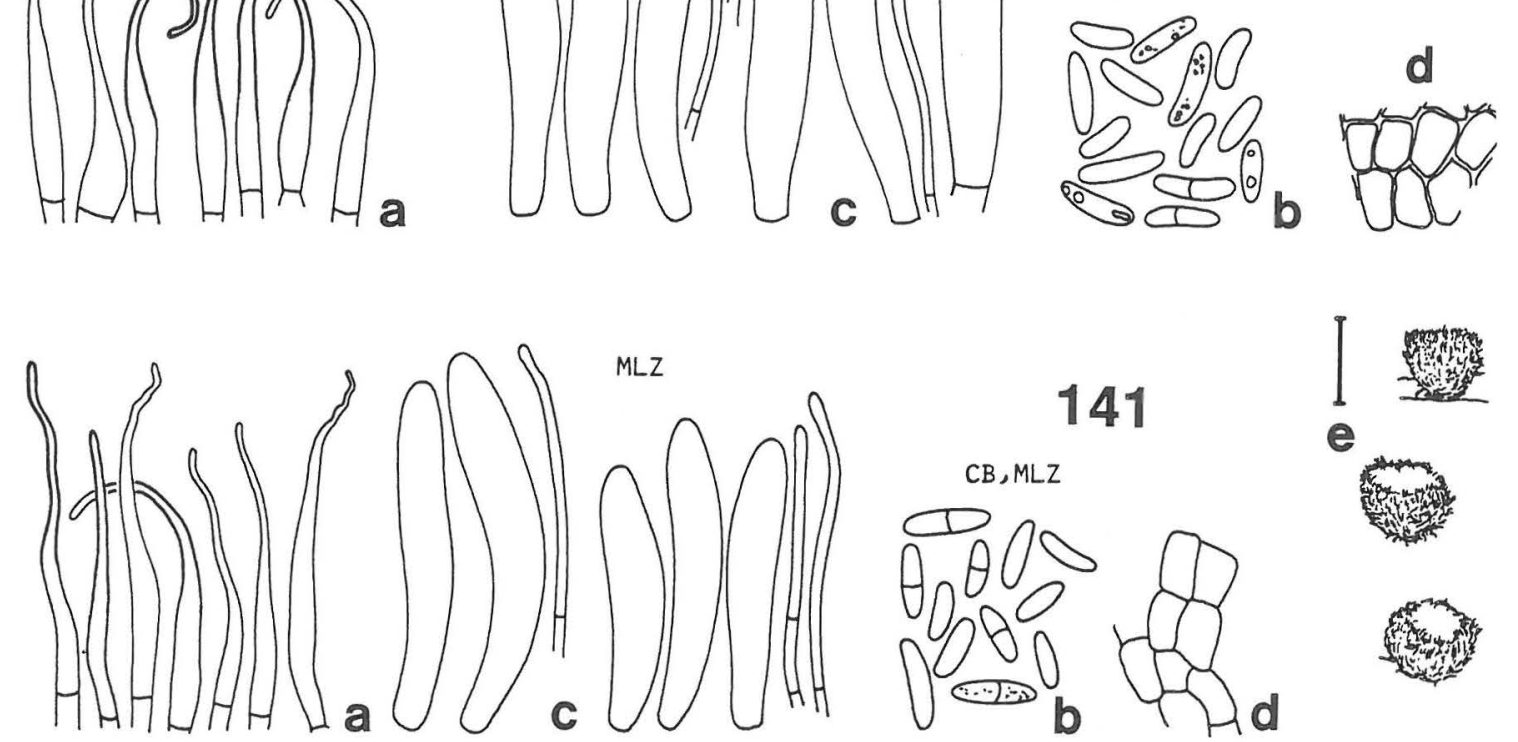

$M L Z$
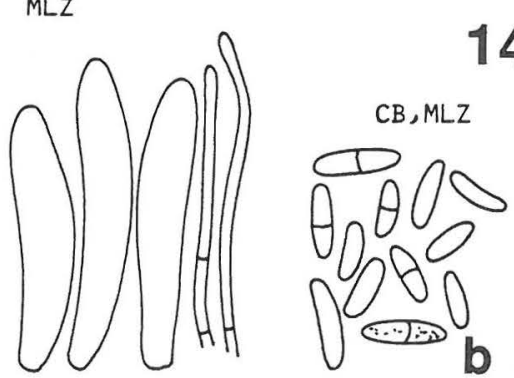

141

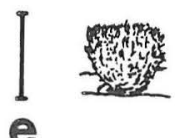

은
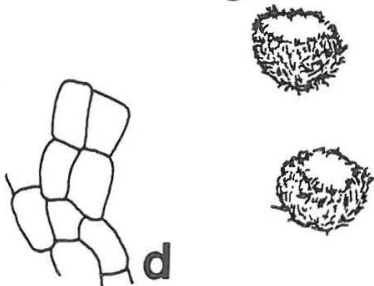

Figs. 140-141. Hyaloscypha occulta. a) hairs, b) spores, c) asci and paraphyses, d) excipulum, e) dry apothecia. Scale $50 \mu \mathrm{m}$, for apothecia $100 \mu \mathrm{m}$. - 140: Isotype. - 141: Korf et al. 3015.

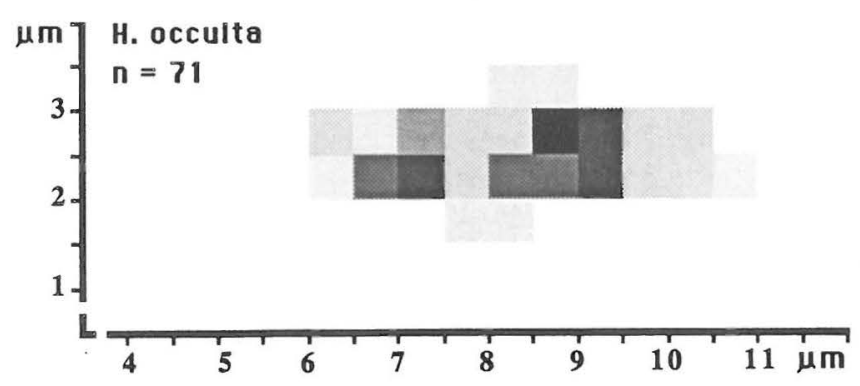

Fig. 142. Percentage sporogram of Hyaloscypha occulta.

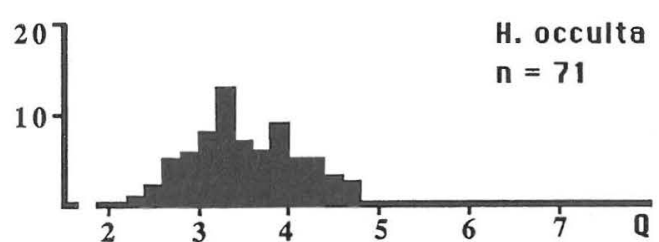

Fig. 143. The distribution of spore quotient $\mathrm{Q}$ in Hyaloscypha occulta.
The smooth and firm-walled hairs were beautifully illustrated by Arendholz and Sharma (1983). The original diagnosis accords with my results, except for the observation of ascal bases. The isotype, studied by me, had apothecia with asci arising from simple septa only. The chemical reactions of the hairs in $H$. occulta are intriguing. When LUG was added to a water mount, the hairs turned deep violet, the colour being bleached by addition of chloral hydrate. The 
reversible nature of this amyloid/dextrinoid reaction was confirmed by adding MLZ to the same mount, upon which the hairs became wine-red. Such a reaction is rare in Hyaloscypha, in which dextrinoid hairs are mostly negative in LUG.

Material studied: India. W-Bengal: Darjeeling, 1980 Sharma (Herb. Arendholz, isotype of Hyaloscypha parvula). U.S.A. New York: Alleghany State Park, 1961 Korf et al. 3015 (Herb. Korf).

\section{Hyaloscypha priapi}

Hyaloscypha priapi Velen., Monogr. Discom. Bohem.: 272; P1. 14:23 (See discussion). 1934. - Psilocistella priapi (Velen.) Svrček, Ceská Mykol. 39:215. 1985. — Lectotype (Svrcek 1977c: 215): [Czechoslovakia,] Bohemia, Jevany, [Fagus,] IX.1924 Velenovský (PRM 148814, examined).

Apothecia gregarious to often confluent, superficial, up to $300 \mu \mathrm{m}$ in diam when fresh, $50-250 \mu \mathrm{m}$ when dry, cupulate throughout development, varying during ontogeny and between populations from shortly stipitate to broadly sessile. Disc shallow to clearly convex when fresh, bordered by an inconspicuous zone of hairs, which rise only slightly above the hymenium; margin varying between populations from inconspicuous and hardly incurved to fleshy and strongly incurved. Hair cover varying between the populations from dense and equally distributed to almost lacking and marginal, snow-white. Colour whitish to yellowish when fresh; dry apothecia varying between the populations from light greyish to light greyish brown (K90) to light ochraceous to Straw (K79) and M79.

Ectal excipulum of textura prismatica, cells on middle flanks regularly prismatic, 7.0-11.5 $\times 3.2-$ $5.5(-7.8) \mu \mathrm{m}, \overline{\mathrm{x}}=8.8 \times 4.3 \mu \mathrm{m}(\mathrm{n}=50), \bar{Q}=2.0$, becoming smaller towards margin, at the base more roundish. Inner excipulum of more elongate cells, ca. $9-13 \times 2.0-3.2 \mu \mathrm{m}$. Walls in ectal parts $0.4-0.6 \mu \mathrm{m}$ thick, hyaline, MLZ- when dry except for a few, scattered $\mathrm{MLZ}++$ areas; $\mathrm{BF}+, \mathrm{CRB}+, \mathrm{NR}+, \mathrm{TB}+$, CB-, CR-, LUG-.

Hairs $20-30(-35) \times 2.0-3.5(-4.0) \mu \mathrm{m}$, varying in the same apothecium from narrowly conical to cylindrical, straight to often apically cincinnate, aseptate. Apex not tapering or tapering to $0.8-1.0 \mu \mathrm{m}$, rarely solidified, without an apical globule in MLZ; the solidifying matter MLZ++, CR++. Wall thin, never locally prominently thickened (except for apices), hyaline, dull, smooth to prominently warted when fresh and dry, such variation occurring in the same apothecium or between populations, very rarely locally $\mathrm{MLZ}+, \mathrm{CR}+$; warts deformed or not in all reagents,
$\mathrm{BF}+, \mathrm{CRB}+, \mathrm{NR}+, \mathrm{TB}+, \mathrm{CB}-, \mathrm{CR}-($ after $\mathrm{KOH}$ pretreatment $\mathrm{CR}+$ ).

Asci $29-37 \times 5.2-6.3 \mu \mathrm{m}, \overline{\mathrm{x}}=36.0 \times 5.7 \mu \mathrm{m}(\mathrm{n}=$ $10), \bar{Q}=6.4$ when fresh in water, $23-34 \times 4.3-6.8(-$ 7.2) $\mu \mathrm{m}, \overline{\mathrm{x}}=28.1 \times 5.2 \mu \mathrm{m}(\mathrm{n}=59), \bar{Q}=5.3 \mathrm{in}$ $\mathrm{MLZ} ; 24-34(-40) \times 4.0-5.8(-6.2) \mu \mathrm{m}, \overline{\mathrm{x}}=28.2 \times$ $5.0 \mu \mathrm{m}(\mathrm{n}=52), \bar{Q}=5.7$ in CB; eight-spored, cylindrical to cylindrical-clavate, with clearly to slightly conical apex. Apical pore MLZ++ to $M L Z+$ when fresh and dry, LUG+ (blue). Wall in mature asci apically $0.2-0.8 \mu \mathrm{m}$ thick (in CB, MLZ), negative in all reagents, contents without or with scattered LUG+ reaction, very rarely $\mathrm{MLZ+}$ (orange). Asci arising from simple septa.

Spores $5.8-10.0 \times 2.0-2.4 \mu \mathrm{m}, \overline{\mathrm{x}}=7.4 \times 2.1 \mu \mathrm{m}$ $(\mathrm{n}=16), \overline{\mathrm{Q}}=3.3$ when fresh in water; 5.5-9.5(11.0) $\times 1.3-2.0(-2.2) \mu \mathrm{m}, \overline{\mathrm{x}}=7.6 \times 1.7 \mu \mathrm{m}(\mathrm{n}=$ $165), \bar{Q}=4.5$ in $C B$ and MLZ; narrowly elliptic to elliptic-fusiform (in CB, MLZ), with rounded to tapered apices, straight to slightly bent, slow to rapidly becoming one-septate after discharge, such variation occurring between the populations, more rarely septate while still inside the asci; septum thin, central, MLZ-. Wall hyaline. Guttulae minute, light.

Paraphyses filiform to narrowly cylindrical, unpigmented, in most populations with numerous repetitive branches, dichotomously branched in others. Terminal cells $10-28 \times 0.8-1.2(-1.4) \mu \mathrm{m}, \overline{\mathrm{x}}=17.6 \mathrm{x}$ $1.1 \mu \mathrm{m}(\mathrm{n}=40)$.

Cultural characters ( 3 populations, 14 strains). Radial growth slow on MA, $8.3 \mathrm{~mm} /$ month $(\mathrm{n}=47$, from 3 popul., 9 strains). Mats Cream (K75) to creamy white throughout, surface dough-like to pruinose. Aerial mycelium lacking or restricted to the inoculum, snow-white, minutely cottony, sparse. Margin distinct, even to minutely fimbriate, superficial. No hyphal strands, zonation or sector formation, no colour change in surrounding agar, no yeastlike growth. Context tough at surface, odour not distinct.

Growth good on BA (strain from Betula), moderate on PA, QA, retarded on LA. Anamorph and teleomorph production lacking in single spore and multispore trials.

In general appearance and growth rate the mycelium matches that of $H$. vitreola and $H$. quercicola. Lack of dark colours and aerial mycelium distinguishes $H$. priapi easily from many other species of Hyaloscypha in culture.

Distribution and phenology. The few collections of $H$. priapi originate from widely separate areas. Judging from the ease with which the species was 

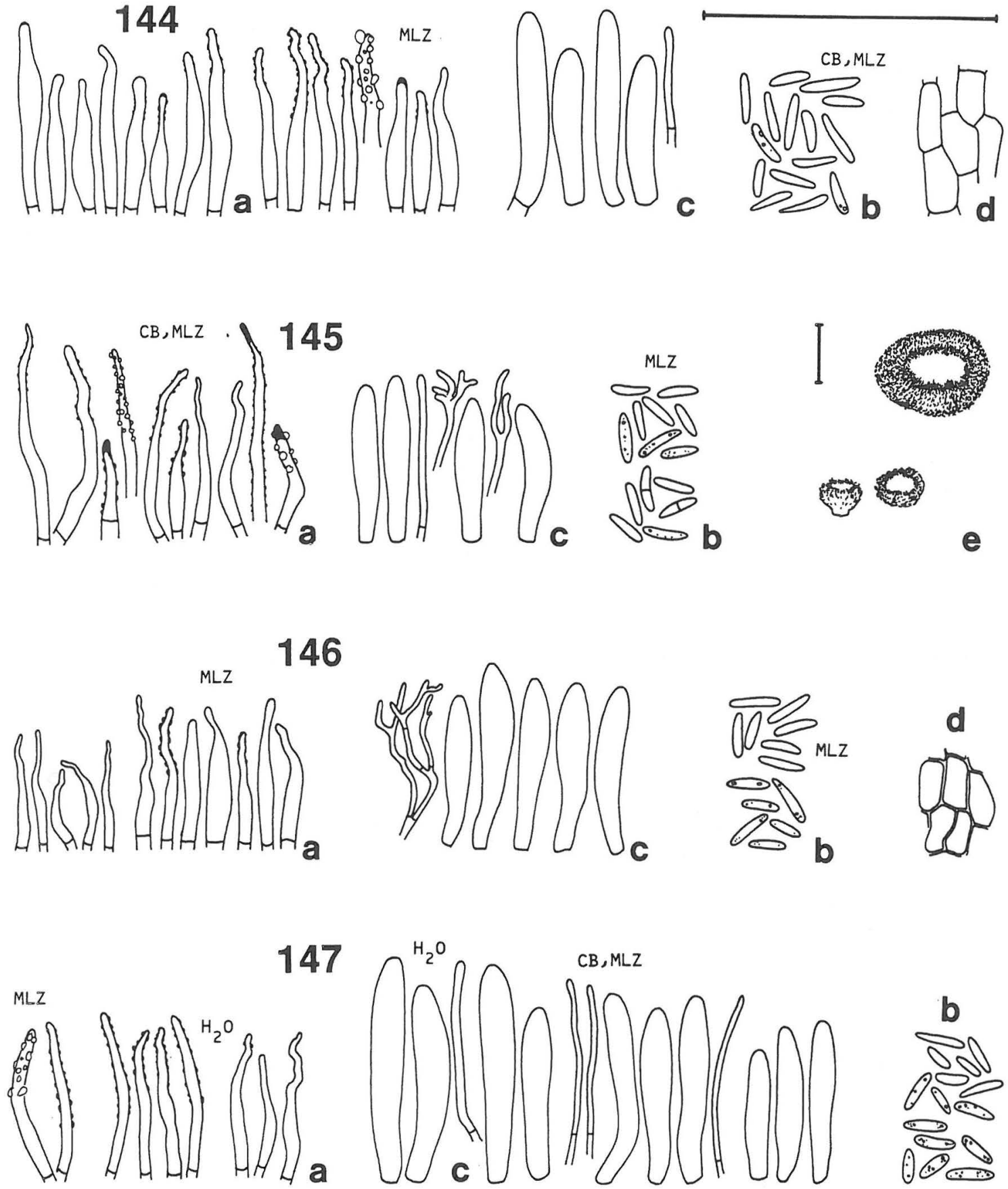

147
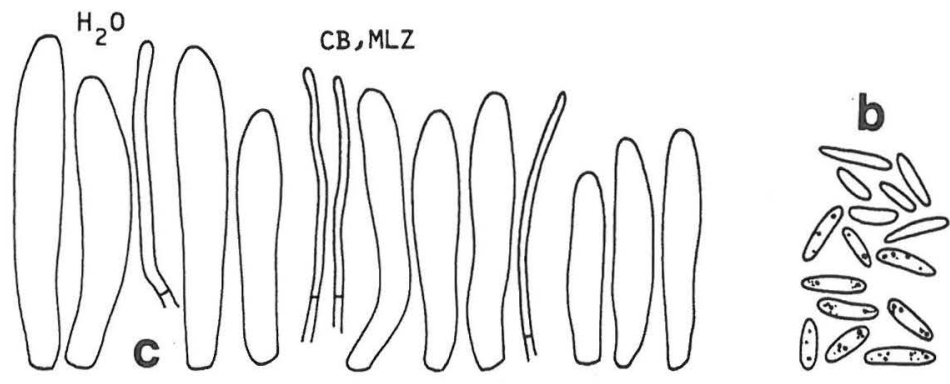

Figs. 144-147. Hyaloscypha priapi. a) hairs, b) spores, c) asci and paraphyses, d) excipulum, e) dry apothecia. Scale $50 \mu \mathrm{m}$, for apothecia $100 \mu \mathrm{m}$. - 144: SH 84/237. - 145: 1931 JV. - 146: SH 86/141. - 147: SH 86/137. 
found during a short stay in central Norway or Inuvik (N.W.T., Canada), it must be commoner than the small number of collections suggests. In SW Finland $H$. priapi was found in all my major collection areas. Further collections derive from the Lithuanian S.S.R., Scotland and from Czechoslovakia. The collections have been made between August and November. One collection from Finland, made in March, most probably, consisted of overwintered apothecia.

Ecology. The present species seems to be confined solely to hardwoods. Four collections originate from wood of Betula, two from Quercus, two from Fagus, and four from unidentified hardwood.

Discussion. Hyaloscypha priapi is distinguished from the other species of the genus by its warted, broad and apically cincinnate hairs. The closely related $H$. quercicola often has clearly warted hairs, but their shape does not vary from cylindrical to tapering, nor are cincinnate hair apices frequent in $\mathrm{H}$. quercicola. Furthermore, that species is differentiated by the common dextrinoid reactions, longer and MLZasci and broader spores. Small and narrow spores together with the often cylindrical hairs also distinguish $H$. priapi from $H$. albohyalina var. spiralis. The strongly branched paraphyses are also distinctive.

The present material varies mostly in the hairs and paraphyses. Variation in the hair morphology within an apothecium and between the populations is important in this genus. In $H$. quercicola the hairs ranged within the same apothecium from almost filiform to narrowly conical and from sparsely to densely warted. In the present species the variation extends from narrowly conical to cylindrical within an individual, but the hair granulation mostly differs between the populations. In most collections the paraphyses are highly branched in their apices, but a few populations have sparsely dichotomous paraphyses. As in other species of the genus, the spore septation varies between the populations.

The asci are short, often almost cylindrical, and fairly constant in shape. Lack of a crozier and MLZ+ apical pores are also reliable characters. The patchy dextrinoid staining is restricted to a few solidified hair apices and to a few areas of the excipulum, and a mounted apothecium often appears to be totally indextrinoid. The spore size and shape do not vary much; the great majority of the spores are characteristically narrow.

Svrček's (1977c) illustration of the lectotype shows the broad granulous hairs. A problem is posed by the contradiction between the type and some parts of Velenovský's (1934) diagnosis. Velenovský described and depicted the hairs as long, septate and tapering to an acute apex. However, the diagnosis indicated the small asci and small narrow spores characterizing the present material. Svrček (1977c) showed that the diagnosis was in fact a mixture of characters from two species. Apparently the illustration is also at least partly based on Pilát's collection from bark of Fagus (PRM 150884), which is placed under $H$. fuckelii var. alniseda in the present study. But the densely septate hairs described and illustrated by Velenovský are inexplicable (Svrček 1977c). Besides the lectotype and syntype, one further collection is linked with $H$. priapi by the identical herbarium name used by Velenovský. As this collection is also conspecific, it seems that Velenovský had a relatively sound concept of $H$. priapi and that the selected lectotype accords with his concept.

The generic placement of the species is difficult. Svrček listed it under Psilocistella, a genus with cylindrical, smooth and thin-walled hairs (Svrček 1977c, 1985). In the latter study Svrček also transferred Hyaloscypha priapi var. vernalis Velen. in Psilocistella and, consequently, the genus now includes elements with dextrinoid hairs and excipulum.

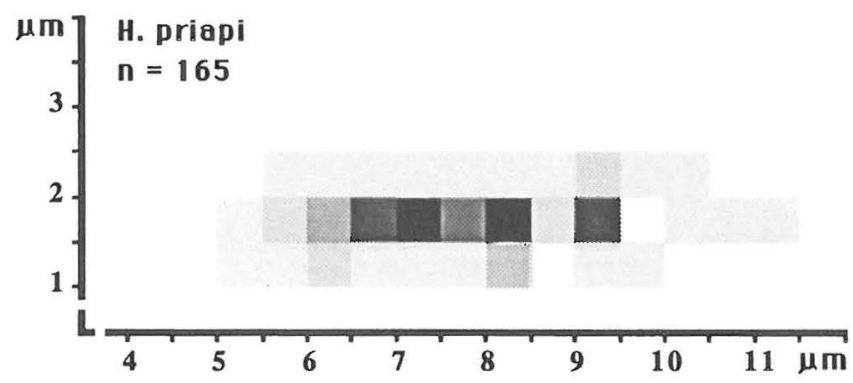

Fig. 148. Percentage sporogram of Hyaloscypha priapi. 
The dextrinoid reaction of Hyaloscypha is not sufficient on its own to determine the generic placement of $H$. priapi. Psilocistella vernalis (Velen.) Svrček has hairs typical of Psilocistella and the dextrinoid reaction is a rare and marginal character in that genus. During the present study I have come across many undescribed species which should be placed in Psilocistella. These, together with the types of $P$. lignatilis (Velen.) Svr., $P$. obsoleta (Velen.) Svr. and $P$. vernalis, which I have also studied, indicate that the genus should embrace only species with cylindrical and septate or aseptate hairs. With the exception of $P$. vernalis, all the species lack dextrinoid reactions. Furthermore, none of the species I have seen show hairs that vary from cylindrical to tapering, as in $H$. priapi. The deforming warts are also typical of $\mathrm{Hya}$ loscypha, and H. priapi is an alien element in Psilocistella in this respect. To my mind, H. priapi should not be placed in the genus Psilocistella.

H. priapi may perhaps be related to the genus Cistella. But as discussed earlier, a clear entity in Cistella is the subgenus Verrucotricha Matheis, with hairs bearing narrow, cyanophilous spines. These spines and the often clavate hairs clearly exclude a close relationship between $H$. priapi and such species of Cistella. Cistella also embraces species with warted hairs like those of the genus Hyaloscypha, but in many species the hairs are clavate or cylindricalclavate (cf. Nannfeldt 1932, Dennis 1949) and thus clearly different. A further difference is that the warts in Cistella do not seem to be resinous.

The new Cistella lagenipilus Spooner, however, is fairly close to $H$.priapi in the solid dextrinoid apices of its lower hairs and in its hair shape and granulation (Kirk \& Spooner 1984). A study of the type showed that the hairs are always coarsely granulous. Their lageniform shape is essentially the same as in Hyaloscypha, but they are much more robust and blunt. The present species and Cistella lagenipilus are best kept in separate genera. If Spooner's species turns out to be exclusively herbicolous, it will also be differentiated by its ecology.

The hair characteristics of $H$. priapi put it at one end of the range of variation in the genus. Although

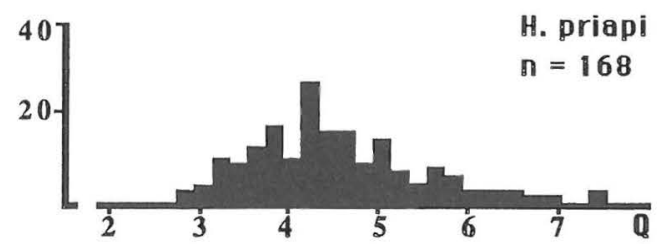

Fig. 149. The distribution of spore quotient $\mathrm{Q}$ in Hyaloscypha priapi. the hairs are often cylindrical, however, the apothecia also have more typical Hyaloscypha hairs and the warts deform in most mountants, which is a feature of Hyaloscypha. Hence, its placement in the genus seems to be justified. Possible difficulties in differentiating it from certain species of Cistella should be dealt with a wider study of that genus.

Material studied: Canada. Northwest Territories: Inuvik, 1984 SH 84/237 (TUR). Czechoslovakia. Bohemia: Jevany, 1924 (type). Mnichovice, 1918, 1931 JV (PRM). Finland. Varsinais-Suomi: Turku, 1984, 1986 SH 84/16, 86/136*, 86/141*, 86/161* (TUR). Great Britain. Perthshire: Calvine, 1983 SH 83/338 (TUR). Norway: Oppland: Dovre, 1985 SH 85/52 (TUR). U.S.S.R. Lithuanian S.S.R.: Distr. Alytus, Punia For. Res. 1966 AR (TAA).

\section{Hyaloscypha quercicola}

Hyaloscypha quercicola (Velen.) Huhtinen, comb. nov. Uncinia quercicola Velen., Monogr. Discom. Bohem.: 294. 1934. - Lectotype (indicated on the label by Svrcek, formally designated here): [Czechoslovakia,] Bohemia, Mnichovice supra Hrusice, in truncos Quercus, VIII.1928 Velenovský (PRM 147371, examined).

Hyaloscypha quercina Velen., Monogr. Discom. Bohem.: 275; P1. 14:30. 1934, p.p.? - Psilocistella quercina (Velen.) Svr., Česká Mykol. 31:197. 1977. — Lectotype (selection within the specimen indicated by Svrcek, followed here): [Czechoslovakia,] Bohemia, Jevany, 14.X.1922 Velenovsky (PRM 149148, sub Mollisia faginea Velen., examined).

Apothecia gregarious, superficial, up to $400 \mu \mathrm{m}$ in diam when fresh, 50-200(-300) $\mu \mathrm{m}$ when dry, cupulate throughout development, sessile or with gradually tapering base, rarely shortly stipitate on a stout, $20-40 \mu \mathrm{m}$ high stipe. Disc shallow to plano-convex when fresh, bordered by an inconspicuous zone of hairs, which rise only slightly above the hymenium; margin thicker when dry, fleshy and incurved, slightly to totally obscuring the hymenium. Hair cover sparse to dense, snow-white. Colour greyish white to yellowish brown (L87) to greyish brown (N90) when fresh; dry apothecia ranging between the populations from greyish to ochraceous to yellowish brown (L79, L87), the hair cover obscuring these colours.

Ectal excipulum of textura prismatica, cells on middle flanks regularly prismatic, $5.5-10.0(-12) \times$ $2.8-4.3(-4.9) \mu \mathrm{m}, \overline{\mathrm{x}}=8.1 \times 3.5 \mu \mathrm{m}(\mathrm{n}=50), \overline{\mathrm{Q}}=$ 2.3 , not changing size or shape towards margin, at the base more roundish. Inner excipulum of more elongate cells, ca. $10-15 \times 2.5-3.0 \mu \mathrm{m}$. Walls in ectal parts $0.4-0.8 \mu \mathrm{m}$ thick, hyaline, MLZ- (even after $\mathrm{KOH}$ pretreatment) to fragmentarily $\mathrm{MLZ}+$ to $\mathrm{MLZ}++$ when dry; $\mathrm{BF}+, \mathrm{CRB}+, \mathrm{NR}+, \mathrm{TB}+, \mathrm{CB}-$, CR-, LUG-. 
164

HUHTINEN: HYALOSCYPHA AND ALLIED GENERA

KARSTENIA 29 1989(1990)

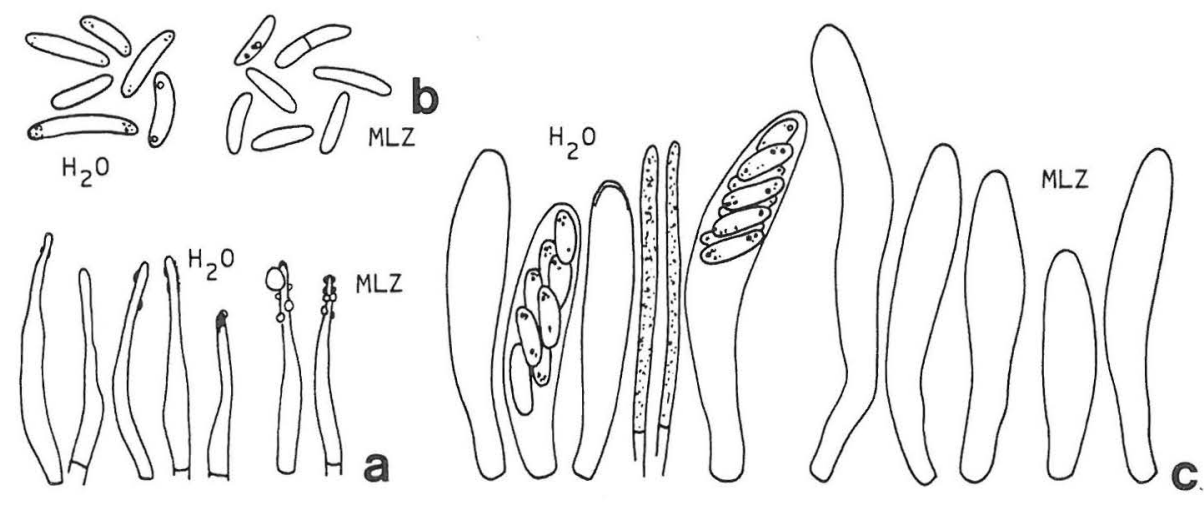

150
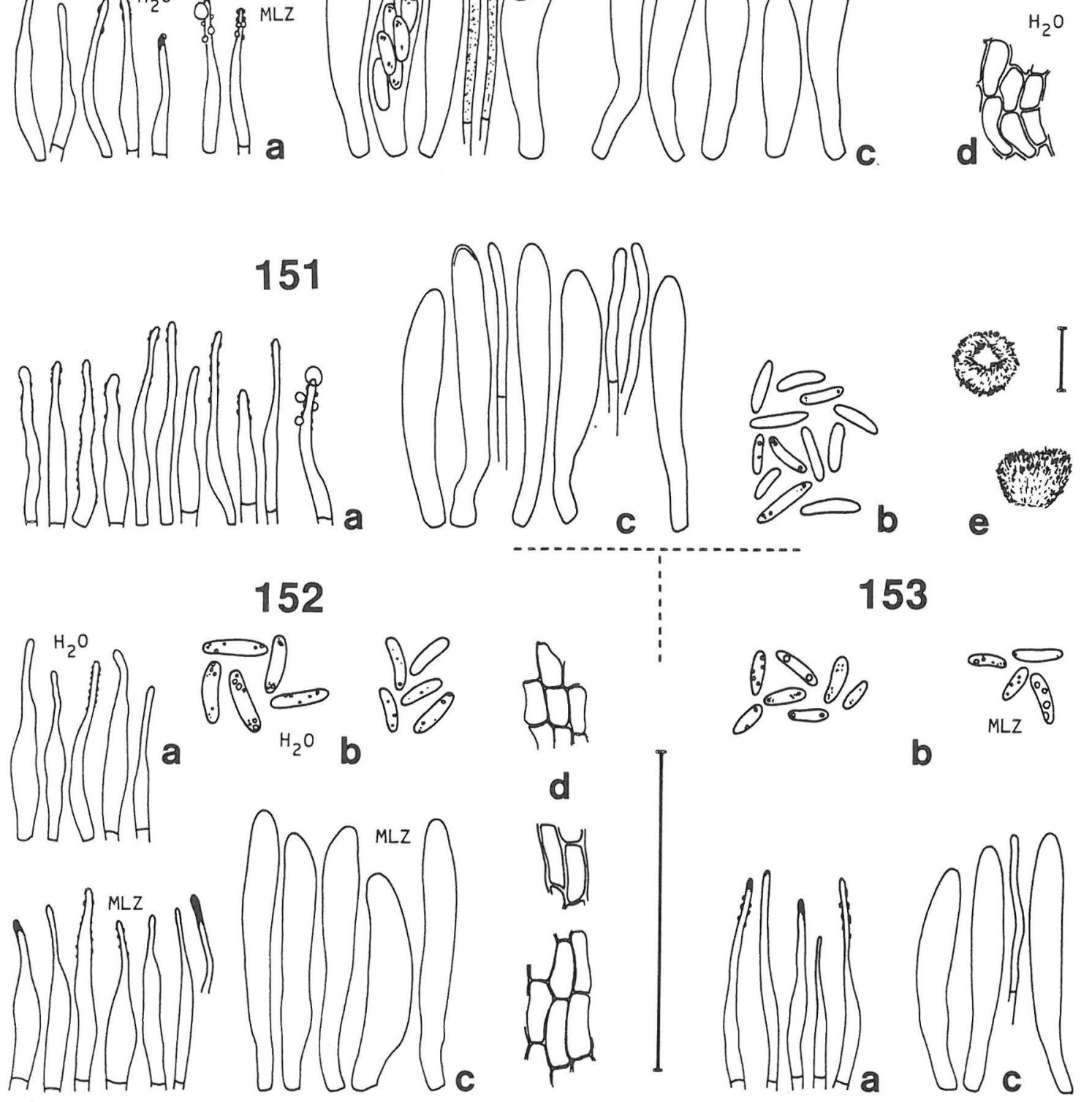

Figs. 150-153. Hyaloscypha quercicola. a) hairs, b) spores, c) asci and paraphyses, d) excipulum, e) dry apothecia. Scale $50 \mu \mathrm{m}$, for apothecia $100 \mu \mathrm{m}$. - 150: SH 87/203. - 151: SH 83/35. — 152: SH 86/121. — 153: $1986 \mathrm{AR}$. 
Hairs $20-35(-43) \times 1.8-3.5(-4.5) \mu \mathrm{m}$, narrowly conical to almost filiform, straight to bent, aseptate to rarely one-septate in lower half. Apex tapering to $0.8-1.5 \mu \mathrm{m}$, rarely swollen, in $85 \%$ of the populations often prominently solidified, frequently bearing a lump of hyaline to pale yellowish exudate, deforming in $M L Z$ and $C R$, not evident in $C B, C R+$; solidifying matter CR++, MLZ++. Wall thin, never locally thickened (except for apices) hyaline, dull, MLZ+ to MLZ++ when fresh and dry (in three populations MLZ-), with sparse to dense, prominent warts in a majority of hairs (in two populations hairs predominantly smooth) in water, MLZ and CR, becoming less clearly warted to smooth in $\mathrm{CB}$; warts deforming in MLZ and $\mathrm{CR} ; \mathrm{BF}+, \mathrm{CRB}+, \mathrm{NR}+$, rarely $\mathrm{CB}+$, $\mathrm{CR}+$. Marginal hairs intermixed with abundant, cylindrical-clavate, thin-walled or apically thickened cells, 8-15 × 2.0-3.8 $\mu \mathrm{m}$.

Asci $31-55(-57) \times 5.6-7.5(-8.5) \mu \mathrm{m}, \overline{\mathrm{x}}=44.6 \times$ $6.6 \mu \mathrm{m}(\mathrm{n}=19), \overline{\mathrm{Q}}=6.4$ when fresh in water; 33$50(-54) \times 4.3-7.2(-7.4) \mu \mathrm{m}, \overline{\mathrm{x}}=41 \times 6 \mu \mathrm{m}(\mathrm{n}=$ $43), \bar{Q}=6.7$ in $\mathrm{MLZ} ; 33-53(-65) \times 4.7-7.0$ $(-7.5) \mu \mathrm{m}, \overline{\mathrm{x}}=41.7 \times 5.6 \mu \mathrm{m}(\mathrm{n}=41), \bar{Q}=7.2$ in $\mathrm{CB}$, eight-spored, cylindrical-clavate, with slightly conical apex. Apical pore MLZ- even after $\mathrm{KOH}$ pretreatment, LUG-. Wall in mature asci apically $1.0-1.8 \mu \mathrm{m}$ thick (in CB, MLZ), negative in all reagents; contents rarely LUG+ and MLZ+ (orange). Asci arising from simple septa, in a few populations the basal septum frequently with a downward hook.

Spores 7.0-11.2(-12.5) x 2.0-2.9(-3.0) $\mu \mathrm{m}, \overline{\mathrm{x}}=$ $9.1 \times 2.5 \mu \mathrm{m}(\mathrm{n}=41), \bar{Q}=3.7$ when fresh in water; $6.0-9.9(-11.2) \times 1.5-2.5(-3.0) \mu \mathrm{m}, \overline{\mathrm{x}}=7.7 \times 2.0 \mu \mathrm{m}$ $(\mathrm{n}=271)$ in CB and MLZ, $\bar{Q}=3.9$, elliptic to oblongelliptic to elliptic-fusiform, with rounded to tapered apices, infrequently one-septate after being discharged, not seen to become septate while still inside the asci; septum thin, central, MLZ-. Wall hyaline, CR-. Guttulae mostly inconspicuous when fresh in water, light, intermixed with more frequent tiny, dark bodies; in dry material alike or lacking.

Paraphyses cylindrical to slightly widened at the apex, rarely apically branched or lobed, unpigmented, first dichotomous branches occurring at or above the middle of the asci. Terminal cells $13-31 \times 1.0$ $1.8 \mu \mathrm{m}, \overline{\mathrm{x}}=21.1 \times 1.3 \mu \mathrm{m}(\mathrm{n}=40)$.

Cultural characters (6 populations, 15 strains). Radial growth slow on MA, $11.8 \mathrm{~mm} /$ month ( $\mathrm{n}=$ 35 , from 3 popul., 8 strains). Mats Cream (K77) to creamy whitish, with a dough-like surface, rarely Buff (N80). Aerial mycelium lacking, or minutely cottony and restricted to $5 \mathrm{~mm}$ from the inoculum, whitish. Margin distinct, superficial, even to distinctly cloudy and submerged. Hyphal strands lacking. No zonation, sector formation or colour change in surrounding agar, yeast-like growth occasional. Context somewhat tough at surface, odour not distinct.

Spores germinating readily on wood agars, strain from Quercus growing well on $\mathrm{QA}$, growth retarded on BA, PA, strain from Corylus growing well on QA, moderately well on BA, PA. Apothecial primordia formed on QA (strain from Quercus), but not developed further. Anamorph not produced in single spore or multispore trials.

In general appearance $H$. quercicola resembles $H$. vitreola and $H$. priapi in culture. It differs greatly in growth rate from the former species, but the difference from $H$. priapi is smaller, though still recognizable. A smooth-haired population was also obtained in culture and it showed the typical morphology of rough-haired populations on MA. Such variation between the populations can be accepted in H. quercicola.

Distribution and phenology. The species seems to be restricted to central, western and northern Europe:

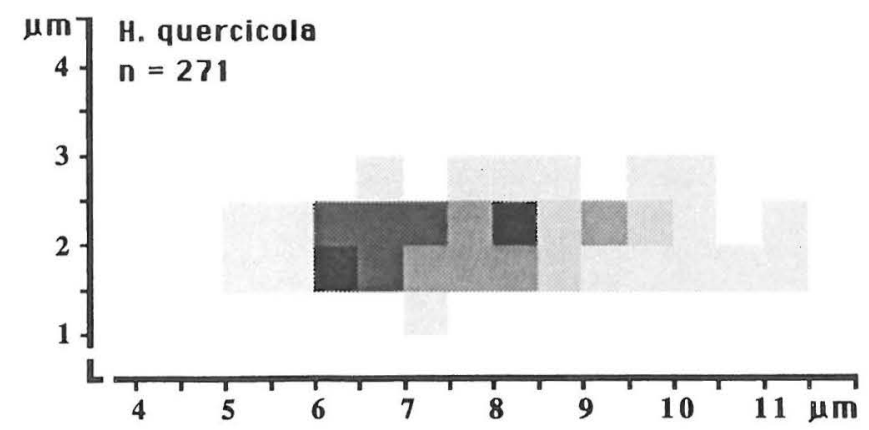

Fig. 154. Percentage sporogram of Hyaloscypha quercicola. 
the material seen ranged from Czechoslovakia northwards to southern Finland. Whether H. quercicola is truly southern and is fruiting at the limit of its range in Finland remains to be determined by further search in the north.

The fruiting period in Sweden and Finland starts in July and the number of fruiting populations apparently increases towards September-October. The two collections from England were made in March and May. H. quercicola is a relatively late-fruiting species (Fig. 16).

Ecology. Hyaloscypha quercicola is a hardwood species. The tendency to fruit on inner or outer bark of hardwood species is evident in $1 / 3$ of the present material: Quercus(2), Betula (2), Fagus (1), Populus (1), Prunus avium (1), unidentified (2). The rest of the material shows the following hosts: Quercus (9), Corylus (2), Betula (1), Crataegus (1), unidentified (4). It seems that the species fruits most often on oak, and has a corticolous habit more often than the other members of Hyaloscypha.

Discussion. Hyaloscypha quercicola is recognized under the microscope by the consistently MLZasci. $H$. intacta shows the same character, but has larger spores and asci and typically smooth hairs. In $H$. albohyalina and $H$. fuckelii var. fuckelii, populations with consistently MLZ- asci or asci MLZ+ only after $\mathrm{KOH}$ pretreatment are rare. Such rare populations can be distinguished by the clearly warted, short hairs, the small spores and asci of H. quercicola, and by the lack of croziers in that species. Hence, recognition of $H$. quercicola seldom causes serious problems.

The populations of the present species vary very little. In all but two, the hairs are strongly warted and in most of them the hairs have a tendency to apical solidification. The hairs are often markedly different when mounted in CB and MLZ: in CB they are pre-

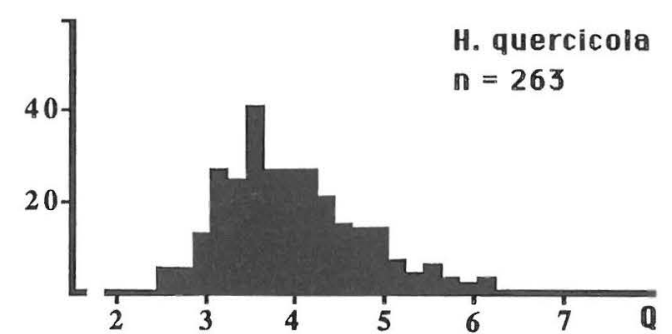

Fig. 155. The distribution of spore quotient Q in Hyaloscypha quercicola. dominantly smooth, because the warts are lost during heating; in $\mathrm{MLZ}$ the warts are visible and only slightly deformed. Many characters do not vary within or between the populations: ascal and spore size, ascal bases, MLZ- apical pores, spore shape, and the small-celled excipulum are constant characters in $H$. quercicola.

Due to the heterogeneous type specimen, $H$. quercina var. quercina is linked with the present species. The type is a mixture of three taxa: $H$. aureliella, the present species and a species on which the protologue was based. Uncertainty arose from the transfer of $H$. quercina to Psilocistella and its lectotypification (Svrček 1977c, 1985). Svrček gave no description of $H$. quercina, which according to the original diagnosis (Velenovský 1934) has cylindrical and septate hairs, much resembling the paraphyses. The cylindrical shape of the hairs in the lectotype fragment is evident from Svrček's (1977c) discussion under H. priapi Velen. var. vernalis Velen. But in a more recent discussion of var. vernalis (Svrček 1985), asci characterized by MLZ- apices are indirectly cited as typical of $H$. quercina. This rare character indicates that the lectotype also contained H. quercicola.

To clarify the typification, Dr. Svrček kindly reexamined the type material and separated the lectotypic element, after which it was studied again by me. The apothecia matching the original diagnosis are senescent and totally devoid of asci. The spore size matches $H$. quercicola, which suggests that the difference in the hair shape may have been overlooked by Velenovsky and that the part of the diagnosis referring to the hymenial characters may be based on $H$. quercicola. The indirect reference to MLZ-asci by Svrček (1985) is probably the result of mounting one of the apothecia of $H$. quercicola, present in abundance and in good condition on other wooden chips in the collection. Likewise, the proposed exclusion of Psilocistella quercina from that genus (Huhtinen 1987d) is based on a species not belonging to the lectotype.

Of the two taxa in the specimen which possibly affected the original diagnosis of $H$.quercina, the one with hairs matching the illustration seems a reasonable choice. It is possible that the senescent apothecia included a few younger apothecia, from which Velenovský extracted the ascus size for the diagnosis. Hence, the provisional selection by Dr. Svrček is supported here. This places $H$. quercina in Psilocistella, for which a combination already exists, though the species remains of uncertain identity.

Fortunately another name with a more uniform type collection exists for naming the present taxon, 
viz. Uncinia quercicola Velen. A lectotypification was indicated by Svrček on the label and my studies confirmed that the specimen fits the description fairly well. The spore length was given by Velenovský as 4-6 $\mu \mathrm{m}$, but a more comprehensive range is $6-9 \mu \mathrm{m}$. In all other respects also the specimen matches the bulk of the present material.

Exsiccatae studied: Sydow, P., Mycotheca Marchica 2846 (K, NY).

Material studied: Czechoslovakia. Bohemia: Mnichovice, 1922 (type of Hyaloscypha quercina), 1928 (type of Uncinia quercicola) JV (PRM) 1986 SH 86/43 (TUR). Slovakia: Bratislava, 1986 SH 86/62 (TUR). Denmark. Sjolland: Amager, 1978 Korf et al. (CUP). Federal Republic of Germany. Westfalen: Siegen, 1940 Ludwig (B, two collections). Finland. Varsinais-Suomi: Kaarina, $1986 \mathrm{SH}$ 86/168*, 86/169* (TUR). Turku, 1983, 1984, 1985, 1986, 1987 SH 83/35, 83/37, 84/255, 85/39, 86/121, 86/140*, 87/189*, 87/203*. Satakunta: Vammala, 1987 SH 87/194* (TUR). Great Britain. Worcester: NR Crowle, 1980 Clark (K). Derbyshire: Bakewell, 1964 (K). Sweden. Östergötland: Gryt, 1957, 1976 JAN 15063, 15066, 24417 (UPS). Uppland: Stockholm, 1889 Romell 16886 (S). U.S.S.R. Estonian S.S.R.: Valga Distr., Koiva Nat. Res., 1986 AR (TAA).

\section{Hyaloscypha secalina var. secalina}

Hyaloscypha secalina Velen., Monogr. Discom. Bohem.: 285; P1. 14:34. 1934. — Lectotype (Svrček 1985: 216): [Czechoslovakia,] Bohemia, Mnichovice, Habr, ad stramina, 3.VIII.1925 Velenovský (PRM 150133, examined).

Apothecia gregarious, superficial, up to $400 \mu \mathrm{m}$ in diam when dry, cupulate, sessile or with a short and stout stipe. Disc shallow when dry, bordered by an incurved margin. Hair cover sparse to dense, white. Colour varying from yellowish brown to deep reddish brown (S15), modified by the hair cover.

Ectal excipulum of textura prismatica, cells on middle flanks regularly prismatic, $7.4-14.0 \times 3-$ $5 \mu \mathrm{m}, \overline{\mathrm{x}}=11.0 \times 3.9 \mu \mathrm{m}(\mathrm{n}=15), \bar{Q}=2.8$, not notably changing shape or size towards margin, at the base more roundish. Inner excipulum a prominent layer of textura porrecta, cells 9.6-16.6 x 1.4 $2.6 \mu \mathrm{m}, \overline{\mathrm{x}}=13.2 \times 2.0 \mu \mathrm{m}(\mathrm{n}=10)$. Walls in ectal parts thin to $0.5 \mu \mathrm{m}$ thick, becoming thinner inwards, basally up to $0.8 \mu \mathrm{m}$ thick, MLZ+.

Hairs $20-55 \times 2.0-3.2 \mu \mathrm{m}$, narrowly conical, straight to apically slightly cincinnate, aseptate. Apex tapering gradually to $0.5-0.8 \mu \mathrm{m}$, occasionally inconspicuously solidified, without an apical globule in MLZ. Wall thin, never locally prominently thickened, hyaline, dull, MLZ+, smooth or rarely bearing a few tiny, apical warts not deformed in MLZ or CB. Mar- ginal hairs intermixed with abundant, cylindricalclavate, thin-walled cells, $12-19 \times 2.5-3.0 \mu \mathrm{m}$.

Asci $39-47 \times 4.44 .8 \mu \mathrm{m}, \overline{\mathrm{x}}=44.8 \times 4.6 \mu \mathrm{m}(\mathrm{n}=$ 5), $\bar{Q}=9.8$ in $\mathrm{CB}$, eight-spored, cylindrical-clavate, with slightly conical apex. Apical pore MLZ++. Contents not turning bright orange in MLZ. Asci arising from croziers.

Spores $7.0-10.3 \times 1-2 \mu \mathrm{m}, \overline{\mathrm{x}}=8.4 \times 1.4 \mu \mathrm{m}(\mathrm{n}=$ 16 ), $\bar{Q}=6.1$ in $C B$, cylindrical to oblong-elliptic, rarely fusiform-elliptic, aseptate. Wall hyaline. Guttulae mostly lacking, only minute, dark bodies present.

Paraphyses cylindrical, unpigmented. Terminal cells $17-31 \times 1.3-2.1 \mu \mathrm{m}, \overline{\mathrm{x}}=23.2 \times 1.8 \mu \mathrm{m}(\mathrm{n}=4)$.

\section{Cultural characters. Not known.}

Distribution and phenology. The sole collection originates from central Bohemia in Czechoslovakia and dates from early August.

Ecology. According to Velenovský's notes, the collection which Svrček selected as lectotype was made from dead culms of Secale cereale. H. secalina, with its two varieties, belongs to the ecologically deviating species of Hyaloscypha, apparently not capable of fruiting on ligneous substrates. The type variety of $H$. fuckelii also grows on herbaceous substrates, although the main substrate is wood. Another species adapted to growing on herbaceous litter, $H$. herbarum, has not been collected on monocotyledons.

Discussion. The delimitation of $H$. secalina is somewhat problematic. I know 11 herb-derived collections of Hyaloscypha species, with relatively small and elongated spores. One of these, from Libertia, is a perfect match with the core of $H$. fuckelii var. fuckelii. Four other collections, all on monocotyledons and with a large proportion of clearly elongated spores, are included in that variety although their spores lie at the limit of the range of variation in var. fuckelii. They do differ, however, from the almost cylindrical spores of $H$. secalina. Being linked with var. fuckelii by the collection from Libertia, they are best included in that wood-preferring species. One collection on gramineous leaves is filed under $H$. fuckelii var. alniseda.

The remaining five collections can be separated into two well-defined taxa on the basis of the ascus formation. In the type variety, the asci arise from croziers and in var. paludicola from simple septa. The other differences between the two varieties are discussed under var. paludicola. All these collections 

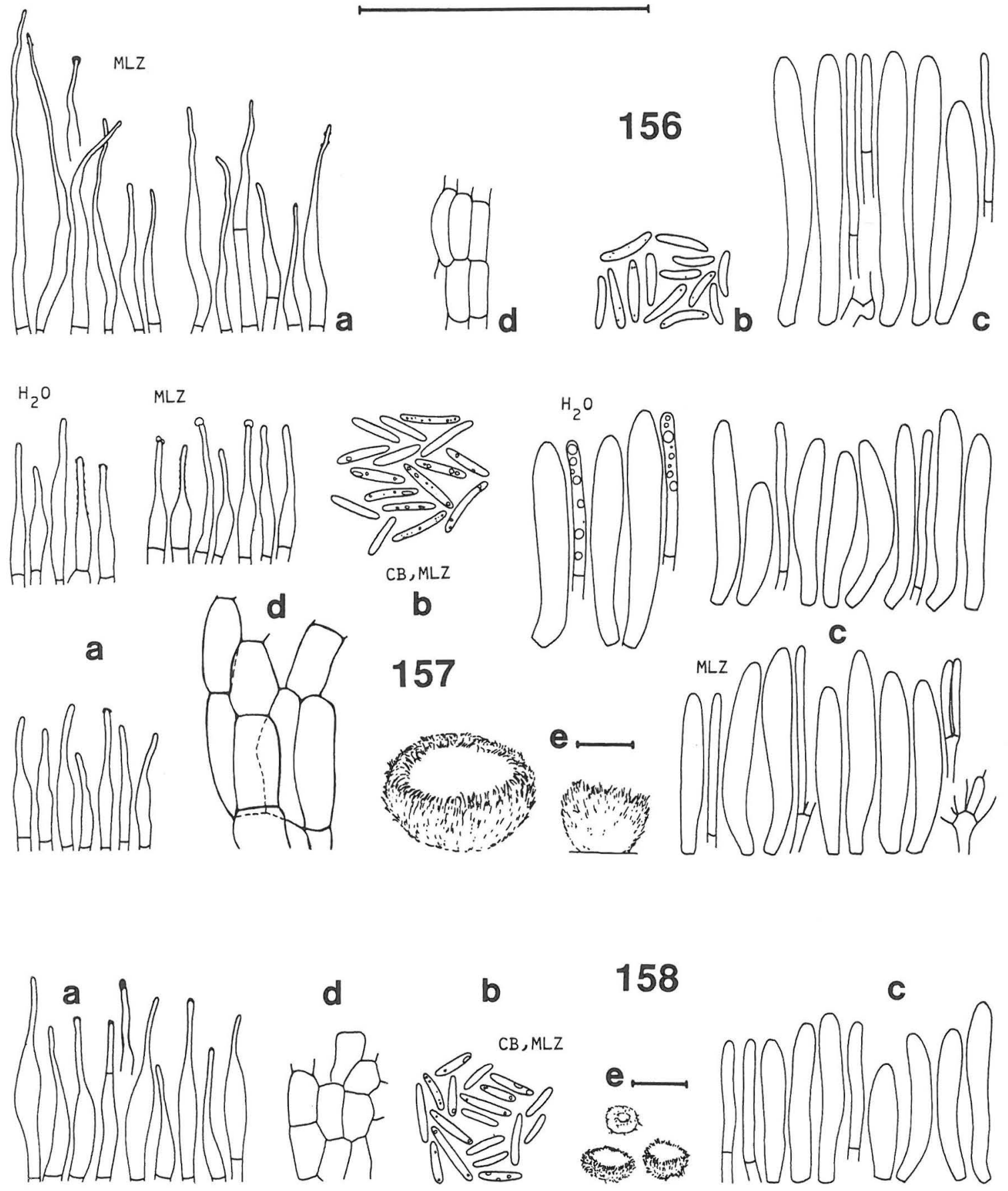

b

158

C

Figs. 156-158. Hyaloscypha secalina. a) hairs, b) spores, c) asci and paraphyses, d) excipulum, e) dry apothecia. Scale $50 \mu \mathrm{m}$, for apothecia $100 \mu \mathrm{m}$. - 156: Lectotype of var. secalina. - 157: Holotype of var. paludicola. - 158: Var. paludicola from Eriophorum, Söderholm 880. 
are characterized by almost cylindrical, very narrow spores, which are uncommon in the genus. As these collections are similar in ecology and morphology, and separated from related species by the spore morphology, they are here accepted as a species. Due to the scanty data, its delimitation will need to be confirmed by further material.

Svrček (1985) correctly stressed the closeness to the type variety of $H$. fuckelii (as $H$. perpusilla). It is possible that Velenovský's authentic material also contained elements here placed under $H$. fuckelii var. fuckelii. $H$. secalina was reported from England by Clark (1980), but I have not seen the collection.

In addition to the spores, the distinct layer of textura porrecta in the inner excipulum may characterize the present taxon. In var. fuckelii the inner layers are formed by narrowing textura intricata. Typical textura porrecta is seen only rarely, and in such specimens it is relatively inconspicuous.

The present taxon is easily differentiated from $H$. herbarum by the narrow spores and croziers. The asci in $H$. herbarum are also wider.

Material studied: Czechoslovakia. Bohemia: Mnichovice, 1925 JV (PRM, type).

23. Hyaloscypha secalina var. paludicola, var. nov.

A varietate typica valde similis, sed ascis parvis et non uncinatis et pilis lageniformis et resinosis differt.

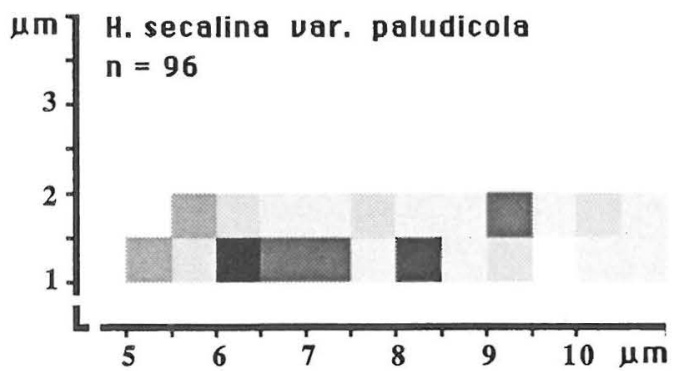

Fig. 159. Percentage sporogram of Hyaloscypha secalina var. paludicola.

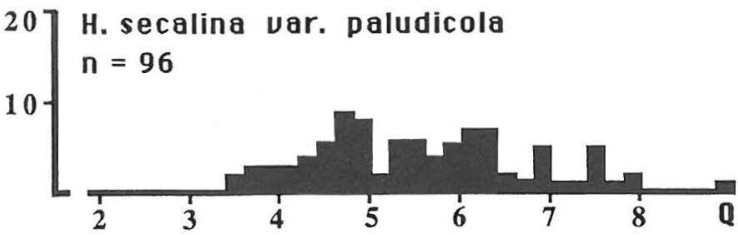

Fig. 160. The distribution of spore quotient Q in Hyaloscypha secalina var. paludicola.
Holotype: Finland. Varsinais-Suomi: Turku, Ruissalo, grid $27^{\circ} \mathrm{E} 67124: 2360$, on blackened stems of Potentilla palustris, 5.VII.1985 Huhtinen 85/12 (TUR).

Apothecia gregarious, superficial, up to $300 \mu \mathrm{m}$ in diam when dry, cupulate, sessile or with a gradually narrowing base when younger. Disc shallow when dry, bordered by a copiously incurved margin. Hair cover equal, dense, white. Colour white when fresh; dry apothecia very pale greyish to very pale yellowish.

Ectal excipulum of textura prismatica, cells on middle flanks regularly prismatic to somewhat roundish, $7.0-12.8 \times 3.2-6.3 \mu \mathrm{m}, \overline{\mathrm{x}}=9.5 \times 5.0 \mu \mathrm{m}$ $(\mathrm{n}=20), \bar{Q}=1.9$. Inner excipulum of narrower textura prismatica or an inconspicuous layer of textura porrecta, $9-12.8 \times 2.0-3.4 \mu \mathrm{m}, \overline{\mathrm{x}}=10.7 \times 2.7 \mu \mathrm{m}$ $(\mathrm{n}=10)$. Walls in ectal parts thin to $0.5 \mu \mathrm{m}$ thick, MLZ+ in two populations, MLZ- and only basally faintly $\mathrm{MLZ}+$ in the other; $\mathrm{TB}+$, fragmentarily $\mathrm{BF}+$, $\mathrm{CR}+, \mathrm{CRB}+, \mathrm{NR}+, \mathrm{CB}-, \mathrm{LUG}-$.

Hairs 18-35 x 2.8-4.3 $\mu \mathrm{m}$, lageniform, aseptate. Apex tapering gradually to $0.5-0.8 \mu \mathrm{m}$, occasionally inconspicuously thickened, frequently bearing an apical resinous lump, deformed in $\mathrm{CB}, \mathrm{MLZ}$ and $\mathrm{CR}$, stable in IKI, remnants $\mathrm{CB}+, \mathrm{CR}+$. Wall thin, never locally prominently thickened, dull, smooth, $\mathrm{MLZ}+$ in two populations, MLZ- in the other, negative in all other reagents.

Asci $23-35(-36) \times 3.3-4.9(-5.6) \mu \mathrm{m}, \overline{\mathrm{x}}=28.7 \times$ $4.1 \mu \mathrm{m}(\mathrm{n}=32), \bar{Q}=7.1$ in MLZ; $21-30(-34) \times$ 3.7-4.8(-5.0) $\mu \mathrm{m}, \overline{\mathrm{x}}=26.5 \times 4.1 \mu \mathrm{m}(\mathrm{n}=21), \overline{\mathrm{Q}}=$ 6.6 in $\mathrm{CB}$, eight-spored, cylindric-clavate, with slightly conical apex. Apical pore MLZ+, LUG+ (blue). Contents not turning bright orange in MLZ, infrequently and inconspicuously LUG+. Asci arising from simple septa.

Spores $5.5-10.4(-11.0) \times 1.1-1.7(-2.0) \mu \mathrm{m}, \overline{\mathrm{x}}=$ $7.6 \times 1.4 \mu \mathrm{m}(\mathrm{n}=96), \bar{Q}=5.6$ in $\mathrm{CB}$ and $\mathrm{MLZ}$, cylindrical to oblong-elliptic, rarely fusiform-elliptic, aseptate to frequently one-septate in one collection. Guttulae lacking to minute, light.

Paraphyses cylindrical to apically slightly widened, unpigmented. Terminal cells $14-29 \times 1.2-$ $2.2 \mu \mathrm{m}, \overline{\mathrm{x}}=20.4 \times 1.7 \mu \mathrm{m}(\mathrm{n}=16)$.

\section{Cultural characters. Not known.}

Distribution and phenology. This variety is known from four collections from southwestern and central Finland and northern Norway. These collections date to early July and August.

Ecology. As in the type variety, the collections originate from herbaceous litter, two of the Finnish collections and the Norwegian collection from $\mathrm{Po}_{\mathrm{O}}$ 
tentilla palustris, the fourth from Eriophorum vaginatum. The collections have been made in paludified sites, as was apparently the case with the type of var. secalina (Velenovsky 1934). The collections of $H$. fuckelii var. fuckelii from Juncus, Molinia and Typha are from the same type of habitat.

Discussion. Relatively numerous features distinguish var. paludicola from the type variety, but the scanty material makes the taxonomic value of the less clear-cut differences uncertain. Once again, the ascal development provides a reliable difference. Var. paludicola lacks croziers and has shorter asci than the type variety. The data available on the type variety are, however, scanty. Further differences are seen in the hair characteristics. In var. paludicola the hairs are clearly lageniform and capped with a lump of resin. The remnants of this apical lump are clearly stained in CB and CR. In LUG the exudate remains intact. It seems that the two varieties of $H$. secalina are closely linked in ecology and spore characteristics but differ in many other characters.

The present variety is distinguished from other species of Hyaloscypha by its ascal bases, exceptionally narrow spores and ecology. Rare herbaceous, long-spored collections of $H$. fuckelii var. alniseda are separated from var. paludicola by the latter's narrow cylindrical spores.

Material studied: Finland. Varsinais-Suomi: Turku, 1985 SH 85/12 (TUR, type). Etelä-Häme: Kangasala, 1982 Söderholm 880 (TAA). Pirkkala, 1989 Söderholm 1642 (TUR). Norway. Nordland: Træna, 1988 Olsen (TUR).

\section{Hyaloscypha strobilicola, sp. nov.}

Apothecia cupulata, sessilia, minuter pilosa; specimina exsiccata pallide ochracea vel aurantiaca. Excipulum externum textura prismatica, cellulis 5.2-11.0 × 3.2-7.0 $\mu \mathrm{m}$, prismaticis vel aliquantum rotundatis, aliquantum crassotunicatis, hyalinis, in solutione Melzeri non coloratis. Pili $25-40(-48) \times 1.8-$ $3.0 \mu \mathrm{m}$, anguste conici, in solutione Melzeri non colorati. leves vel minuter incrustati, in aqua exudato resinoso, aseptati, tenuiter tunicati. Asci 25-44 × 3.8-5.2 $\mu \mathrm{m}$, octospori, amyloidei, in basi uncinati. Sporae 4.2-6.6 x 1.6-2.0 $\mu \mathrm{m}$, ellipsoideae vel oblongo-ellipsoideae, non septatae. Paraphyses filiformae, 0.8-1.3 $\mathrm{m}$ latae, cellulis terminalibus 9-34 $\mu \mathrm{m}$ longis.

Holotype: U.S.A. California: Nevada County, 5 miles W of Grass Valley on Hy. 20, $2500 \mathrm{ft}$ [762 m] a.s.1., on old fallen needles [in error for cones] of Pinus sabiniana Dougl., open grassy slope with Pinus sabiniana, Arctostaphylos, 7.XI.1964 McLaughlin \& Tavares 1656; sub Hyaloscypha stevensonii, det. S. Carpenter 1973 (CUP 60975).
Apothecia gregarious to confluent, superficial, up to $250 \mu \mathrm{m}$ in diam when dry, cupulate throughout development, narrowly to broadly sessile. Disc concave, bordered by a fleshy, copiously incurved, somewhat raised margin. Hair cover dense, equal, white to yellowish. Colour varying from very light yellowish brown to orange brown (N40); apothecia covered by lumps of hyaline to brown resin when dry.

Ectal excipulum of textura prismatica, cells on middle flanks regularly prismatic to more roundish, $5.2-11.0 \times 3.2-7.0 \mu \mathrm{m}, \overline{\mathrm{x}}=8.5 \times 4.4 \mu \mathrm{m}(\mathrm{n}=30), \bar{Q}$ $=1.9$, becoming smaller and more regularly prismatic towards margin, at the base more roundish. Inner excipulum of only slightly narrower textura prismatica. Walls in ectal parts $0.4-0.8 \mu \mathrm{m}$ thick, hyaline, $\mathrm{MLZ}-; \mathrm{NR}+, \mathrm{TB}+$, fragmentarily $\mathrm{BF}+, \mathrm{CRB}+$, CB-, CR-, LUG-.

Hairs $25-40(-48) \times 1.8-3.0 \mu \mathrm{m}$, narrowly conical, straight to slightly flexuose, aseptate, not containing amyloid matter. Apex gradually tapering to $0.4-1.0 \mu \mathrm{m}$, never prominently solidified, without an apical globule in MLZ. Wall thin, never locally thickened, dull, hyaline, smooth to moderately encrusted in MLZ, more often perfectly smooth in CB, negative in all reagents, frequently bearing lumps of amorphous, resinous matter. Resin persistent in water based stains, $\mathrm{CR}$ and $\mathrm{KOH}$, dissolving in $\mathrm{MLZ}$ and $\mathrm{CB} ; \mathrm{BF}+, \mathrm{CRB}+, \mathrm{NR}+$, $\mathrm{TB}+$, LUG-.

Asci $28-44(-48) \times 3.8-5.2 \mu \mathrm{m}, \overline{\mathrm{x}}=33.5 \times$ $4.4 \mu \mathrm{m}(\mathrm{n}=35), \bar{Q}=9.6$ in MLZ; $25-41(-43) \times$ $3.2-4.3(-4.5) \mu \mathrm{m}, \overline{\mathrm{x}}=33.4 \times 4.0 \mu \mathrm{m}(\mathrm{n}=17), \overline{\mathrm{Q}}=$ 8.5 in $\mathrm{CB}$; eight-spored, cylindrical-clavate with a slightly conical apex, often with an elongated and slender basal part. Apical pore MLZ++ when dry, LUG+ (blue). Wall in mature asci apically 0.4 $0.6 \mu \mathrm{m}$ thick (in CB, MLZ), negative in all reagents. Contents not staining bright orange in $\mathrm{MLZ}$, rarely and inconspicuously LUG+. Asci arising from croziers, bases of free asci often conspicuously bifurcate.

Spores $4.2-6.6(-8.0) \times 1.6-2.0(-2.2) \mu \mathrm{m}, \overline{\mathrm{x}}=$ $5.4 \times 1.9 \mu \mathrm{m}(\mathrm{n}=126), \bar{Q}=2.9$ in CB and MLZ; elliptic to oblong elliptic, with rounded to slightly tapered apices, aseptate, often with resinous/oleaceous, $\mathrm{CR}-$ contents when mature. Wall hyaline, not seen to become brownish in aged spores. Guttulae lacking to inconspicuous in dry material.

Paraphyses filiform, unpigmented, first dichotomous branches occurring at the level of the uppermost $1 / 3$. Terminal cells 9-34 x 0.8-1.3 $\mu \mathrm{m}, \overline{\mathrm{x}}=$ $21.3 \times 1.1 \mu \mathrm{m}(\mathrm{n}=25)$.

Cultural characters. Not known. 


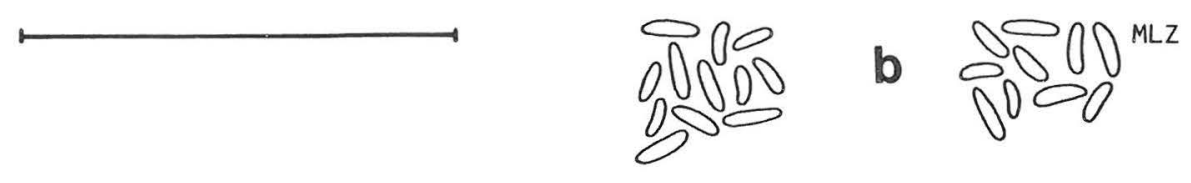

$C B, M L Z$
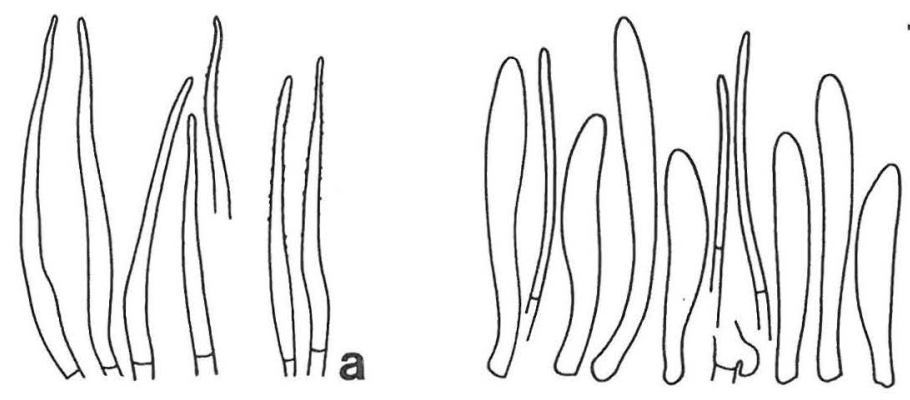

161
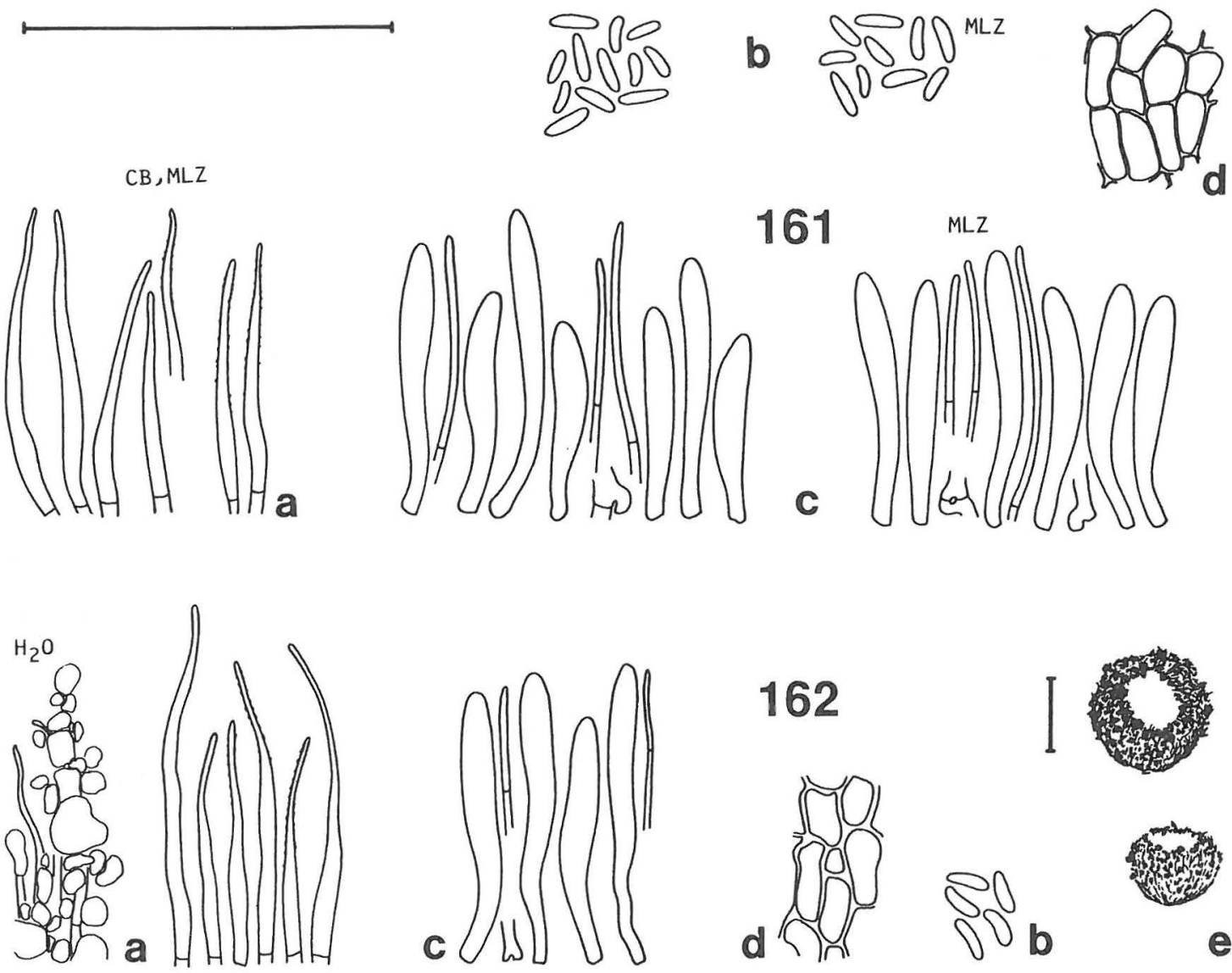

Figs. 161-162. Hyaloscypha strobilicola var. strobilicola. a) hairs, b) spores, c) asci and paraphyses, d) excipulum, e) dry apothecia. Scale $50 \mu \mathrm{m}$, for apothecia $100 \mu \mathrm{m}$. - 161: Holotype. - 162: 1981 Dennis.

Distribution and phenology. The three collections and the ample data on the distribution of the closely related $H$. aureliella suggest that this species is restricted to a Mediterranean climate. Due to the coniferous substrate and the resinous exudate, $H$. strobilicola var. strobilicola is likely to appear under $H$. aureliella $(H$. stevensonii) in herbaria. The present material of var. strobilicola originates from California and central Italy, and was collected in late October and November.

Ecology. The collections have been made from cone scales of Pinus sabiniana (California) and $P$. pinea (Italy). The Californian sites have been sunny and open to sparsely wooded. Unlike the other spe- cies of the genus, $H$. strobilicola may therefore be thermophilic. There also seems to be a marked ecological difference from $H$. aureliella, which has been found on cone scales only once.

Discussion. Hyaloscypha strobilicola var. strobilicola is closely related to $H$. aureliella, which it resembles in the resinous exudate, small spores, generally indextrinoid excipulum, filiform paraphyses and occurrence on softwoods. The distinguishing characters are found in the asci, hairs and excipulum. The asci arise from croziers in both, but many ascal bases in var. strobilicola show prominent bifurcation, a character not found in other species of Hyaloscypha. The ascal contents do not turn orange in 
MLZ, nor do they show the strong hemiamyloid reaction of many populations of $H$. aureliella. The hairs taper to a clearly narrower apex and both they and the excipulum lack the amyloid reaction of $H$. aureliella. The differences from the other variety of $H$. strobilicola are discussed under that taxon.

Mature spores are often resinous in both varieties of $H$. strobilicola. Almost the whole spore is evenly filled with resin, which remains unstained in CR. Such spores were not noted in the populations of $H$. aureliella and the feature may have diagnostic value.

$H$. aureliella has been found only once growing on cone scales. $H$. aureliella is mainly distributed outside the area of $H$. strobilicola. As they are clearly closely related, they could have been separated at subspecific level. In such small inoperculates geographical isolation is often illusory, but the clear-cut difference in ecology could be considered of subspecific value (cf. Kuyper 1988). However, clear discontinuities in other characters make species status justified. Although the hairs are notably tapering, $H$. strobilicola is best placed in subg. Eupezizella.

Material studied: Italy. Lazio: Sabaudia, 1981 Dennis (K). U.S.A. California: Colusa Co., S of Stonyford, 1964 Tavares et al. 1593 (G, H, UPS, TUR). Nevada Co., Grass Valley, 1964 McLaughlin \& Tavares 1656 (CUP, type).

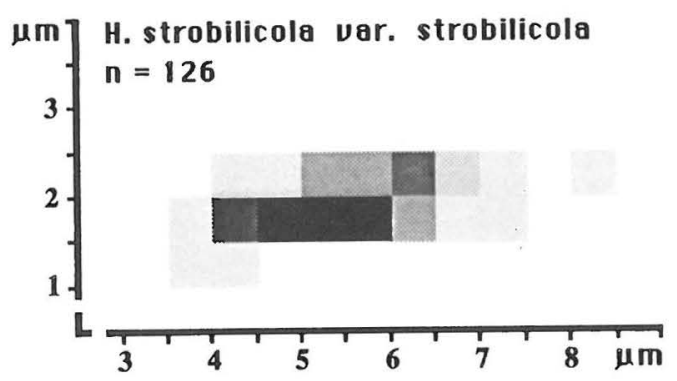

Fig. 163. Percentage sporogram of Hyaloscypha strobilicola var. strobilicola.

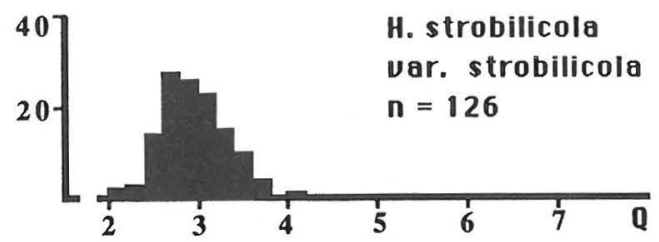

Fig. 164. The distribution of spore quotient Q in Hyaloscypha strobilicola var. strobilicola.
25. Hyaloscypha strobilicola var. parvispora, var. nov.

A varietate typica valde similis, sed sporis parvis et ascis non uncinatis differt.

Holotype: U.S.A. California: Western part of Yosemite National Park, roadside along highway 120, on decaying cone scales of Pinus lambertiana Dougl., 11.V.1962 Bonar (CUP 47333).

Ectal excipulum of textura prismatica, cells on middle flanks regularly prismatic to more roundish, 6.0-12.8 $\times 3.0-7.2 \mu \mathrm{m}, \overline{\mathrm{x}}=8.4 \times 4.7 \mu \mathrm{m}(\mathrm{n}=40), \bar{Q}=$ 1.8 , becoming smaller and more regularly prismatic towards margin, at the base more roundish. Inner excipulum of narrower textura prismatica to textura porrecta, $8-10 \times 2-3 \mu \mathrm{m}$. Walls in ectal parts MLZexcept for scattered dextrinoid areas in one collection.

Hairs $18-30(-43) \times 2.0-3.6 \mu \mathrm{m}$ narrowly conical to slightly lageniform. Wall thin, never locally thickened, smooth to clearly encrusted in MLZ, smooth to rarely inconspicuously encrusted in CB.

Asci $19-34 \times 3.7-5.0 \mu \mathrm{m}, \overline{\mathrm{x}}=25.3 \times 4.1 \mu \mathrm{m}(\mathrm{n}=$ 25), $\bar{Q}=7.5$ in MLZ; $20-42 \times 3.3-5.0 \mu \mathrm{m}, \overline{\mathrm{x}}=30.0$ $x 4.0 \mu \mathrm{m}(\mathrm{n}=12), \bar{Q}=6.4$ in $\mathrm{CB}$; arising from simple septa.

Spores $3.2-5.7(-6.0) \times 1.2-2.0 \mu \mathrm{m}, \overline{\mathrm{x}}=4.4 \times$ $1.5 \mu \mathrm{m}(\mathrm{n}=120), \overline{\mathrm{Q}}=2.9$ in CB and MLZ; elliptic to oblong-elliptic, often with resinaceous/oleaceous, $\mathrm{CR}$ - contents when mature.

Paraphyses filiform, terminal cells $12-20 \times 0.9-$ $1.2 \mu \mathrm{m}, \overline{\mathrm{x}}=15.8 \times 1.0 \mu \mathrm{m}(\mathrm{n}=10)$.

\section{Cultural characters. Not known.}

Distribution and phenology. Only Californian collections are known, and so the two varieties of $H$. strobilicola have somewhat similar distributions. The collections date from May and November.

Ecology. Both populations have fruited on cone scales of Pinus lambertiana and the ecology is similar to that of the type variety.

Discussion. Hyaloscypha strobilicola var. parvispora is very similar to the type variety. However, it can easily be distinguished by its asci arising from simple septa. The spore sizes partially overlap, but var. parvispora tends to have smaller spores. The ascal bases serve as a rapid means of differentiation from rare strobilicolous populations of $H$. aureliella. Many mature, discharged spores show the resinous/ oleaceous, MLZ- contents also characteristic of the type variety.

Material studied: U.S.A. California: Del Norte Co., Darlingtonia, 1938 Parks \& Parks 6174 (G, H, K, M, TAA, UPS). Yosemite Nat. Park, 1962 Bonar (CUP, type; isotype in BPI). 


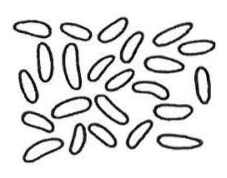

MLZ
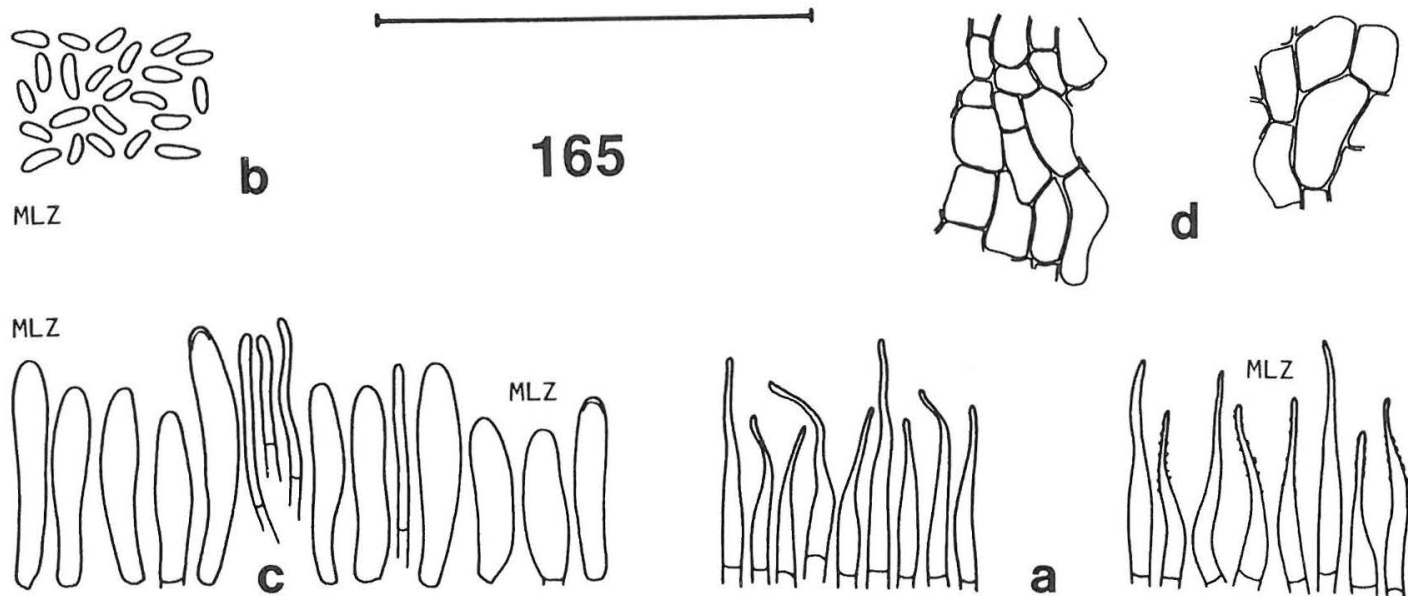

a

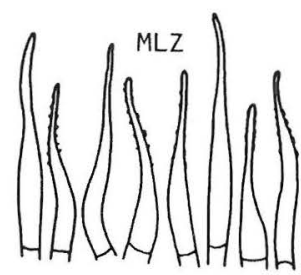

Fig. 165. Hyaloscypha strobilicola var. parvispora, holotype. a) hairs, b) spores, c) asci and paraphyses, d) excipulum. Scale $50 \mu \mathrm{m}$.

\section{Hyaloscypha usitata, sp. nov.}

Apothecia cupulata, sessilia vel basaliter constricta, minuter pilosa; specimina exsiccata armeniaca vel aurantiaca. Excipulum externum textura prismatica, cellulis 6.0-12.2 × 3.5$6.3 \mu \mathrm{m}$, aliquantum crassotunicatis, hyalinis, in solutione Melzeri vinosis. Pili 26-48 $\times 3.2-5.8(-6.2) \mu m$, lageniformes vel anguste conici, resinosi vel non resinosi, in solutione Melzeri vinosi, raro non colorati, aseptati, tenuiter tunicati, in collectio unico in apice cincinnati, saepe solidi. Asci 38-51(52) $\times$ 7.0-9.0(-9.2) $\mu \mathrm{m}$ in solutione Melzeri, non amyloidei

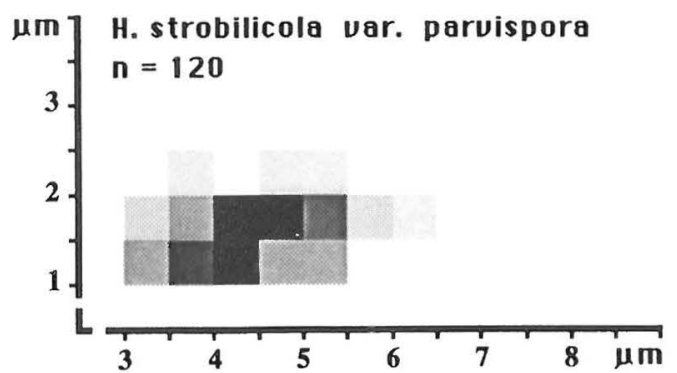

Fig. 166. Percentage sporogram of Hyaloscypha strobilicola var. parvispora.

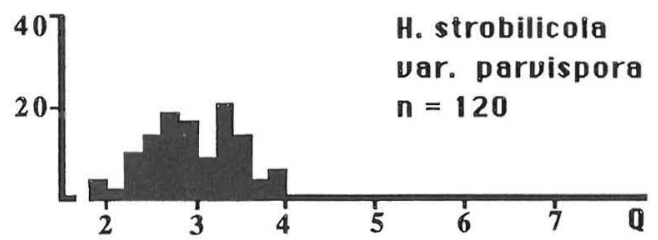

Fig. 167. The distribution of spore quotient Q in Hyaloscypha strobilicola var. parvispora. sed post solutione kalii amyloidei, in basi uncinati. Sporae 7.7-11.5(-12.1) x 2.2-3.2(-3.8) $\mu \mathrm{m}$, ellipsoideae vel oblongo-ellipsoideae, septatae vel non septatae. Paraphyses cylindraceae vel in apice minuter dilatatae, 1.3-2.0 um latae, cellulis terminalibus 17-35 $\mu \mathrm{m}$ longis.

Holotype: Colombia: Dpto. Antioquia: Medellin, Estacion Forestal Experimental Piedras Blancas, on unidentified wood, 19.VII.1974 Dumont, Haines \& Velasquez (NY, Dumont-Co 1591).

Apothecia gregarious, superficial, up to $250 \mu \mathrm{m}$ in diam when dry, cupulate, sessile or with a gradually tapering base. Margin copious when dry, incurved and typically obscuring most of the hymenium. Hair cover dense at the margin, sparse below, snowwhite. Colour varying within a given population from light greyish to Apricot (L65) to orange brown (M59).

Ectal excipulum of textura prismatica, cells on middle flanks regularly prismatic, 6.0-12.2 × 3.5$6.3 \mu \mathrm{m}, \overline{\mathrm{x}}=9.8 \times 4.7 \mu \mathrm{m} \quad(\mathrm{n}=23), \bar{Q}=2.1$, not changing size or shape towards margin, at the base more roundish. Inner excipulum of narrower textura prismatica. Walls in ectal parts $0.4-0.6 \mu \mathrm{m}$ thick, hyaline, MLZ++ to $\mathrm{MLZ}+; \mathrm{CRB}+, \mathrm{TB}+$, fragmentarily $\mathrm{BF}+, \mathrm{CR}+, \mathrm{NR}+, \mathrm{CB}-, \mathrm{LUG}-$.

Hairs $26-48 \times 3.2-5.8(-6.2) \mu \mathrm{m}$, lageniform to narrowly conical, straight to bent, in one population apically frequently cincinnate, aseptate. Apex tapering to $0.5-1.5 \mu \mathrm{m}$, in two populations not solidified, in one population frequently solidified, solidifying matter MLZ-, BF+, faintly $\mathrm{CR}+$; $\mathrm{CB}-, \mathrm{CRB}-$, NR-, LUG-, TB-. Wall thin, in the cincinnate apex locally 
$C B, M L Z$

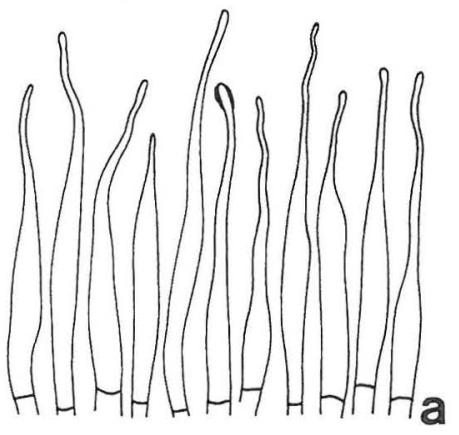

168

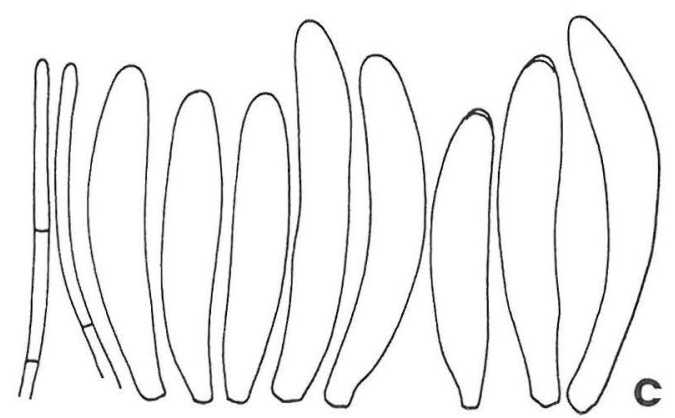

$C B, M L Z$
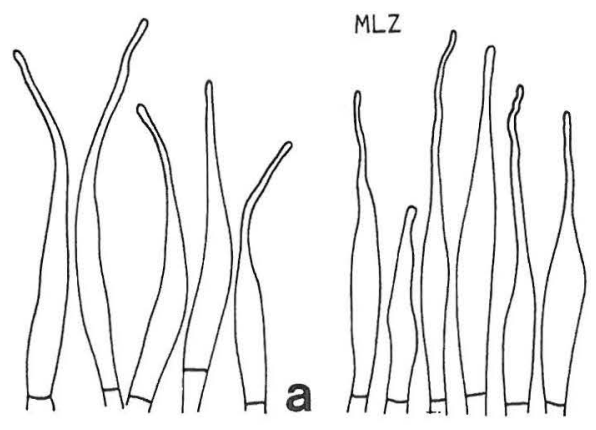

169
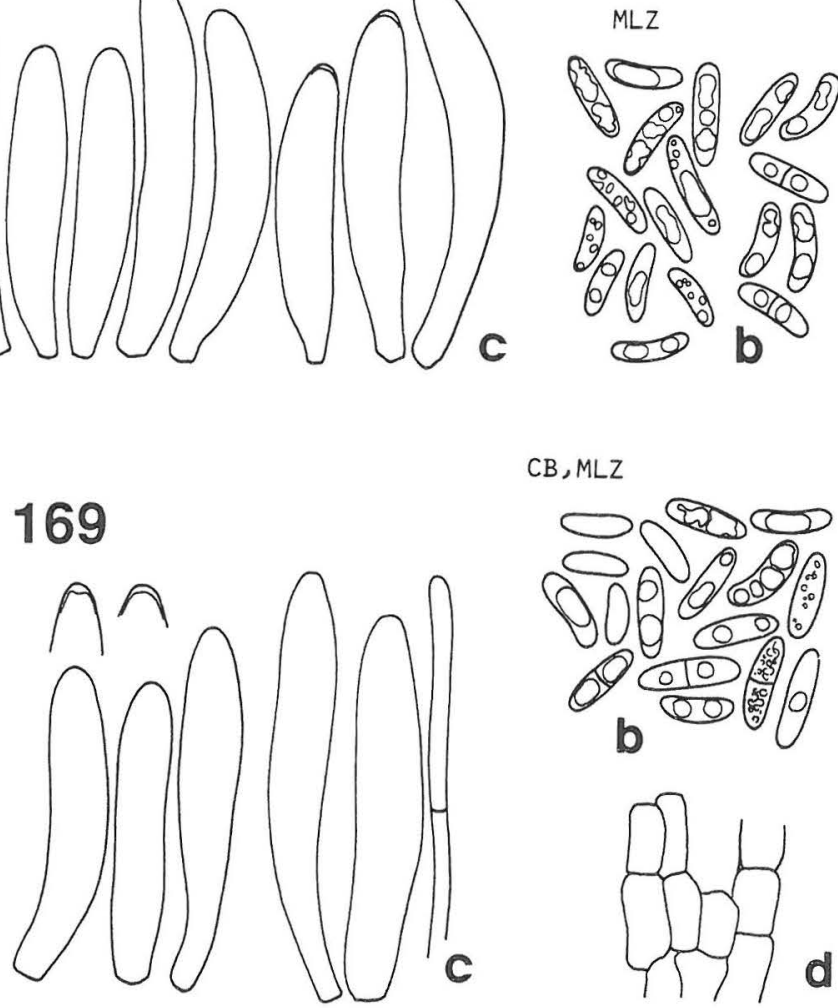

\section{a.m.}

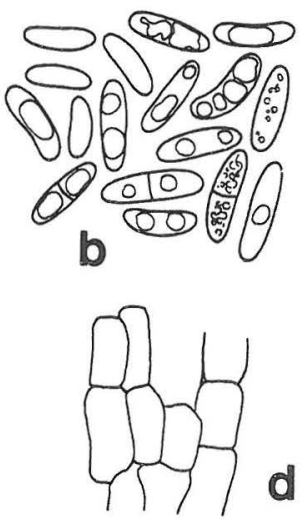

170
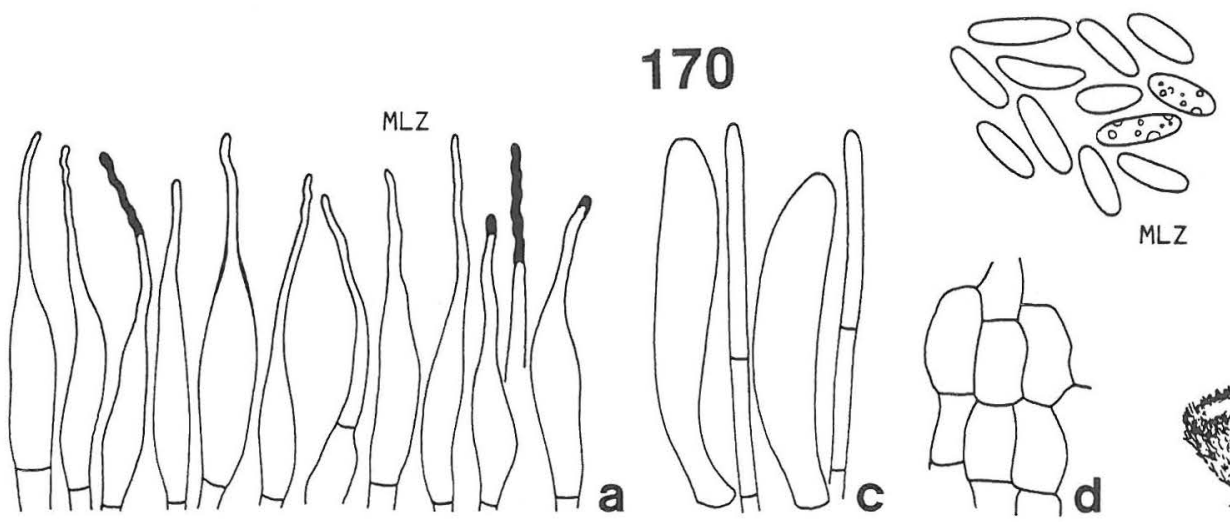

b : 9

MLZ
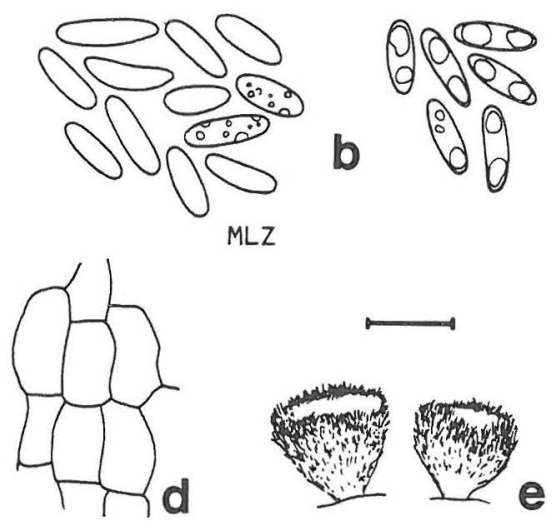

Figs. 168-170. Hyaloscypha usitata. a) hairs, b) spores, c) asci and paraphyses, d) excipulum, e) dry apothecia. Scale $50 \mu \mathrm{m}$, for apothecia $100 \mu \mathrm{m}$. - 168: Holotype. - 169: Dumont et al. 1600. — 170: Dumont et al. 2283. 
minutely thickened, which gives the wall a warted appearance, smooth, MLZ+, MLZ+ to MLZ- in one population, with or without scattered lumps of hyaline to yellowish, resinous matter, partly deforming in $\mathrm{CB}$ and $\mathrm{MLZ}$, resin faintly $\mathrm{CR}+\mathrm{NR}+, \mathrm{TB}+$, CRB-. Marginal hairs intermixed with abundant to scanty, clavate, thin-walled cells, $10-23 \times 3-6 \mu \mathrm{m}$.

Asci $38-51(-52) \times 7.0-9.0(-9.2) \mu \mathrm{m}, \overline{\mathrm{x}}=42.9 \times$ $7.8 \mu \mathrm{m}(\mathrm{n}=20), \mathrm{Q}=5.6$ in MLZ; $34-48(-50) \times$ $5.8-6.8(-7.0) \mu \mathrm{m}, \overline{\mathrm{x}}=40.6 \times 6.3 \mu \mathrm{m}(\mathrm{n}=29), \bar{Q}=$ 6.4 in $\mathrm{CB}$, eight-spored, cylindrical-clavate with a slightly conical apex. Apical pore MLZ-, MLZ++ after $\mathrm{KOH}$ pretreatment, LUG+ (red and blue). Wall in mature asci apically $0.4-1.0 \mu \mathrm{m}$ thick (in $\mathrm{CB}$, MLZ), negative in all reagents. Contents frequently LUG+ and MLZ+ (orange). Asci arising from croziers.

Spores 7.7-11.5(-12.1) x 2.2-3.2(-3.8) $\mu \mathrm{m}, \overline{\mathrm{x}}=$ $9.4 \times 2.9 \mu \mathrm{m}(\overline{\mathrm{x}}=124), \bar{Q}=3.3$ in CB and MLZ; elliptic to oblong-elliptic, with rounded apices, 4-6\% of the discharged spores one-septate in two populations, very rarely septate while still inside the asci, aseptate in one population. Wall hyaline, not turning brown in aged spores. Guttulae numerous, prominent, light, separate to coalescent in CB and MLZ; in spores with CB- contents one or two, oily, yellowish guttulae.

Paraphyses cylindrical to apically slightly widened, unpigmented. Terminal cells $17-35 \times 1.3-$ $2.0 \mu \mathrm{m}, \overline{\mathrm{x}}=24.7 \times 1.7 \mu \mathrm{m}(\mathrm{n}=24)$.

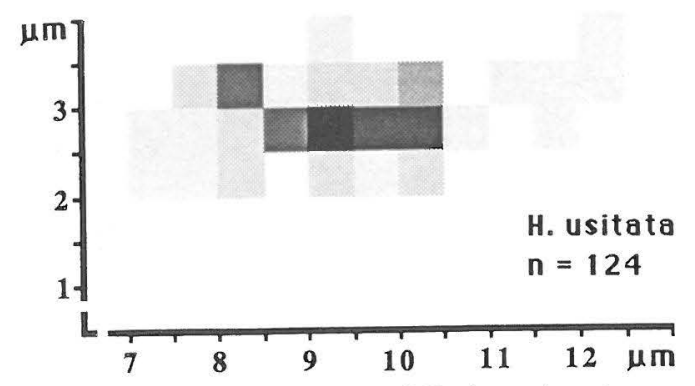

Fig. 171. Percentage sporogram of Hyaloscypha usitata.

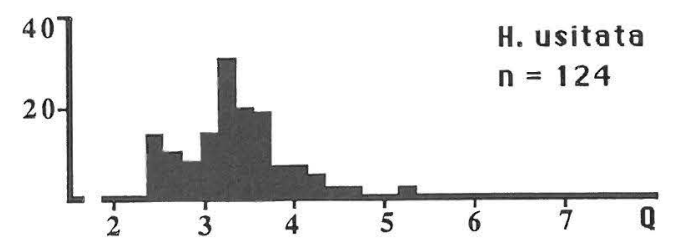

Fig. 172. The distribution of spore quotient Q in Hyaloscypha usitata.

\section{Cultural characters. Not known.}

Distribution and phenology. The material originates from the northern Andes, two collections were made in the same area in Colombia and one in Venezuela. They date from June and July.

Ecology. The collections differ ecologically from each other. The type grew on a small chip of unidentified wood and the apothecia occur on both naked wood and abundant pyrenomycetous fungi. The other Colombian collection is on unidentified bark. In the third collection, the apothecia occur on wood of Espeletia.

Discussion. As in $H$. nipponica, the asci have a plug, which is negative if put straight into MLZ, but shows deep staining after $\mathrm{KOH}$ pretreatment. In IKI the plug varies from red to blue within the same mount. The dextrinoid hairs are of the same type as in $H$. fuckelii, except in the collection from Venezuela (see below). The spore and ascus size distinguish $H$. usitata from $H$. fuckelii. Without the MLZ reaction the present species might be confused with the widespread $H$. albohyalina var. albohyalina. However, the hairs of the latter are warted and apically wider and show MLZ++ solidifications, although the variational range is partly overlapping. Resin and MLZ- asci are exceptional in var. albohyalina. At present the taxa seem clearly separate. The narrowly conical to filiform, often somewhat firm and refractive hairs of $H$. vitreola assist in distinguishing it from $H$. usitata, as do also the consistently MLZ+ asci.

The two collections from Colombia are identical, but variability in the hairs is seen between them and the collection from Venezuela. The other morphological features are alike. In the Venezuelan collection many hairs are apically solidified, and apices are often cincinnate. The clavate marginal cells have typical apical inclusions, which are not refractive in any mountant used. These characters are not present in the other two collections. Although the hairs in the Venezuelan material appear warted, this is not the case. The appearance is caused by minute wall thickenings situated at the cincinnate parts of the wall. The hairs in this material are also slightly more lageniform and lack the scanty resin present in the other two collections. The Venezuelan collection has aseptate spores.

From $H$. leuconica it seems clear that cincinnate apices alone do not justify separation at the species level. In many Hyaloscyphas the presence or absence of apical hair solidifications and spore septa varies 
between the populations. As the collections of $H$. usitata differ only in these respects, they are kept together for the time being.

Material studied: Colombia. Medellin, 1974, Dumont et al. 1591 (NY, type); Dumont et al. 1600 (NY). Venezuela. Merida: Sierra Nevada Nat. Park, 1971 Dumont et al. 2283 (NY).

\section{Hyaloscypha vitreola}

Hyaloscypha vitreola (P. Karst.) Boud., Bull. Soc. Mycol. France 1:118. 1885. - Peziza vitreola P. Karst., Not. Sällsk. Fauna Flora Fenn. Förh. 10:180. 1869. - Helotium v itreolum (P. Karst.) P. Karst., Not. Sällsk. Fauna Flora Fenn. Förh. 11:240. 1870. - Pseudohelotium vitreolum (P. Karst.) Sacc., Syll. Fung. 8:291. 1889. - Dasyscypha vitreola (P. Karst.) Massee, The Naturalist: 183. 1901. - Lectotype (Dennis 1956: 196): [Finland,] Tavastia australis. Tammela, Mustiala, ad lign. betul., 10.X.1868 P. Karsten (H, Herb. Karsten 2633, examined).

Pezizella incerta Allescher, Ber. Bot. Ver. Landshut 15:76. 1898. - Pseudohelotium incertum (Allescher) Sacc. \& P. Sydow, Syll. Fung. 16:729. 1902. - Hyaloscypha incerta (Allescher) Boud., Hist. classific. discomyc. Europe: 126. 1907. - Holotype (the only specimen mentioned): [Federal Republic of Germany, Bayern,] Benedictbeuern, monte "Zwiesel", Hab. ad lignum putridum Fagi, a Chlorosplenio aeruginoso vel aureginascente omnino colore aeruginoso tinctam, VIII.[18]85 Allescher (M, examined).

Uncinia maxima Velen., Novitates mycol. noviss.: 139. 1947. - Holotype (the only specimen mentioned): [Czechoslovakia,] Bohemia, Mnichovice, "Polvčiny", Alnus glut., 18.VII.1941 Velenovsky (PRM 153247, examined).

Uncinia spinosae Velen., Novitates mycol. noviss.: 139. 1947. - Holotype (the only specimen mentioned): [Czechoslovakia, Bohemia,] Mnichovice, Bila Skala-Božkov, Prunus spinosa, 30.VI.1941 Velenovsky (PRM 153255, examined).

Hyaloscypha hyalina (Pers.: Fr.) Boud. "form" 4, Mycol. Pap. 32:70. 1949, p.p. majore.

Apothecia gregarious to confluent, superficial, up to $600 \mu \mathrm{m}$ in diam when fresh, typically $200-300$ (500) $\mu \mathrm{m}$ when dry, cyathiform when juvenile, cupulate when mature, narrowly to broadly sessile or with a gradually tapering base, rarely with a $20-30 \mu \mathrm{m}$ long, stout stipe; prominent anchoring hyphae mostly lacking (present in two collections). Disc shallow to plano-convex when fresh, bordered by a thin zone of marginal hairs, which rise only slightly above the hymenium; margin fleshy and incurved when dry, obscuring much of the hymenium. Hair cover sparse, concentrated in the margin, seldom dense, such variation occurring within the same population, white. Colour pure white, brownish (K71), yellowish, or greyish when fresh, very old apothecia yellowish brown (L60, K80), dry apothecia ranging from light yellowish or Straw (K79) to more orange brown
(M59, M65), rarely reddish brown (P15, R17), such variation occurring within a given population.

Ectal excipulum of textura prismatica, cells on middle flanks regularly prismatic, ranging between populations from 8-13 $\times 3-6$ to $14-20 \times 5-9 \mu \mathrm{m}, \bar{Q}$ $=2.4(n=50)$, becoming narrower towards margin, at the base more roundish. Inner excipulum of narrower textura prismatica, cells $10-15 \times 2.5-3.2 \mu \mathrm{m}$, $\mathrm{Q}=3.3-5.5$. Walls in ectal parts $0.4 \mu \mathrm{m}$ (in ca. $10 \%$ of the populations up to $0.8 \mu \mathrm{m}$ ), becoming thinner inwards, hyaline, MLZ- to MLZ++ when fresh, $\mathrm{MLZ}+$ to $\mathrm{MLZ++}$ when dry; $\mathrm{CR}+$, fragmentarily $\mathrm{CRB}+, \mathrm{NR}+, \mathrm{TB}+, \mathrm{BF}-, \mathrm{CB}-\mathrm{LUG}-$.

Hairs $30-60(-85) \times 2-3(-6) \mu \mathrm{m}$, narrowly conical to almost filiform, straight to bent, of uneven width and mostly aseptate (ca. 5\% of the hairs in an apothecium with one thin basal or central septum). Apex tapering to $0.8 \mu \mathrm{m}$ or rarely swollen, very rarely partly solidified, not bearing apical exudates and without an apical globule in MLZ. Wall thin, or up to $0.4 \mu \mathrm{m}$ thick in upper half, seldom locally thickened but even then without massive thickenings, hyaline, dull to refractive, smooth, (very rarely bearing a few, tiny, hyaline apical warts, partly deformed in $\mathrm{MLZ}$ and $\mathrm{CR}), \mathrm{MLZ}+$ to $\mathrm{MLZ++}, \mathrm{CR}+$ to $\mathrm{CR}++, \mathrm{BF}-$, CB-, CRB-, LUG-, NR-, TB-. Marginal hairs intermixed with abundant, cylindrical-clavate, thinwalled cells, $16-30 \times 3-7 \mu \mathrm{m}$.

Asci $40-75(-84) \times 7.3-10.5(-12.8) \mu \mathrm{m}, \overline{\mathrm{x}}=56.8$ $\times 8.9 \mu \mathrm{m}(\mathrm{n}=44), \overline{\mathrm{Q}}=6.4$ when fresh in water, 44 $67(-72) \times 6.9-12.0(-12.8) \mu \mathrm{m}, \overline{\mathrm{x}}=55.0 \times 8.7 \mu \mathrm{m}$ $(\mathrm{n}=59), \bar{Q}=6.6$ in MLZ; 40-68(-74) x 6.3-9.5(11) $\mu \mathrm{m}, \overline{\mathrm{x}}=52.8 \times 7.7 \mu \mathrm{m}(\mathrm{n}=159), \bar{Q}=7.4 \mathrm{in}$ $\mathrm{CB}$; eight-spored, cylindrical-clavate, with slightly conical apex, rarely with large, refractive, basal bodies (seen in CB). Apical pore MLZ+ in 70\% of populations, MLZ++ in others, LUG+ (blue). Wall thin to $0.4 \mu \mathrm{m}$ thick, in mature asci apically $1.0-1.5 \mu \mathrm{m}$ thick (in CB, MLZ), negative in all reagents; contents often LUG+, rarely MLZ++ (orange), variation mostly between the populations. Asci arising from croziers.

Spores 8.5-15.0(-20) x 2.9-3.7(-4.2) $\mu \mathrm{m}, \overline{\mathrm{x}}=$ $12.1 \times 3.5 \mu \mathrm{m}(\mathrm{n}=103), \overline{\mathrm{Q}}=3.5$ when fresh in water; 9-14(-17) x 2.6-3.5(-4.0) $\mu \mathrm{m}, \overline{\mathrm{x}}=11.3 \times$ $3.0 \mu \mathrm{m}(\mathrm{n}=577), \bar{Q}=3.8$ in CB and MLZ, oblongelliptic to phaseoliform, with rounded to slightly tapered apex, frequently one-septate after being discharged, more rarely while still inside the asci (10\% of populations with tardily developing or no septa), septum thin, central, often MLZ+. Wall hyaline, brownish in aged discharged spores. Guttulae large and light when fresh in water; prominent, light, separate to coalescent in dry material; spores with $\mathrm{CB}-$ 

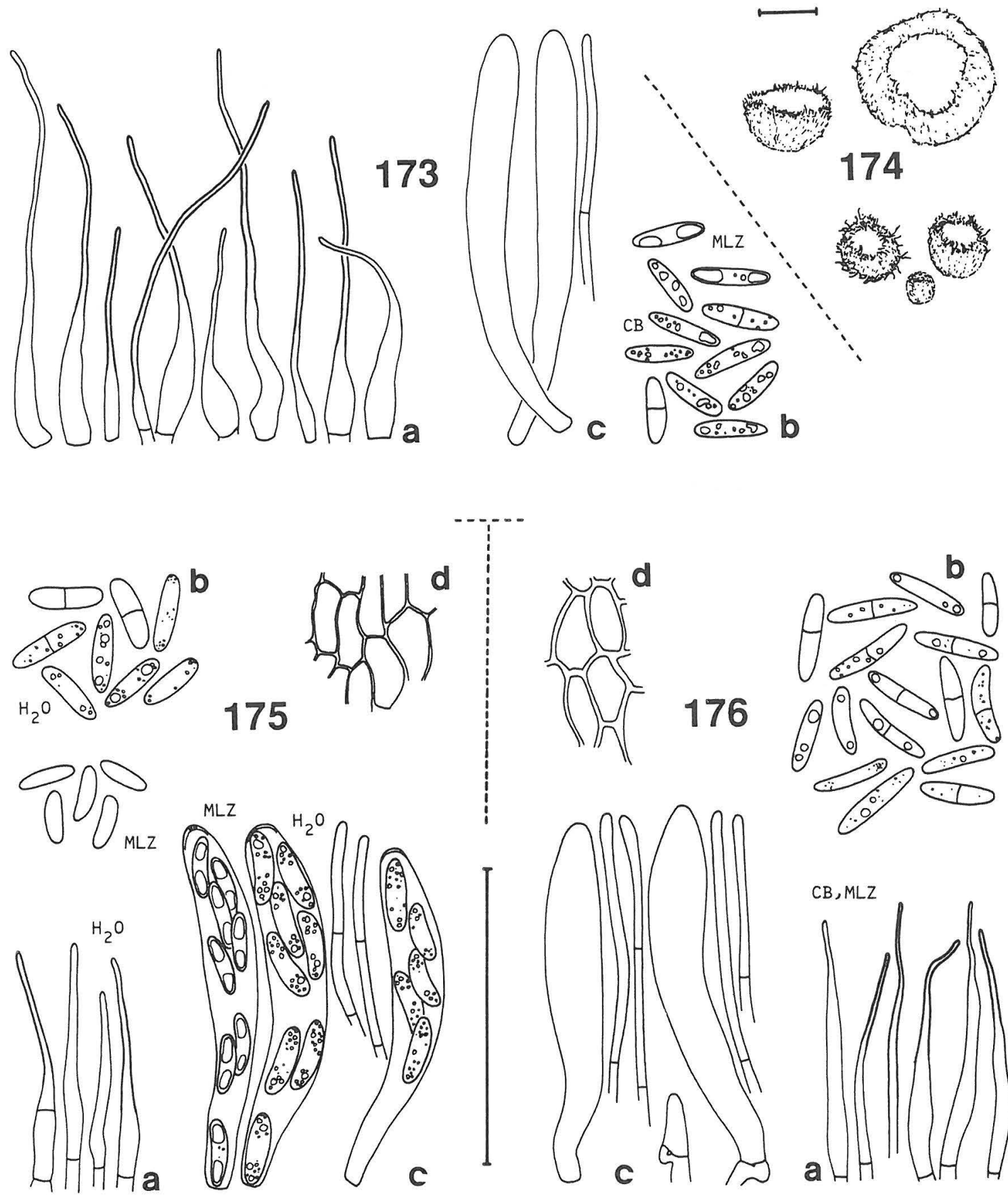

Figs. 173-176. Hyaloscypha vitreola. a) hairs, b) spores, c) asci and paraphyses, d) excipulum. Scale $50 \mu \mathrm{m}$, for apothecia $100 \mu \mathrm{m}$. - 173: JAN 20611. - 174: Dry apothecia from various collections. - 175: SH 87/49. - 176: Lectotype. 
contents with one regular, globose, light guttula at each end.

Paraphyses cylindrical or slightly widened at the apex, unpigmented, first dichotomous branches occurring at the level of lowermost $1 / 3$. Terminal cells $17-58 \times(1.3-) 1.5-2.5(-2.8) \mu \mathrm{m}, \overline{\mathrm{x}}=31.9 \times 1.9 \mu \mathrm{m}$ $(\mathrm{n}=148)$.

Cultural characters (9 populations, 25 strains). Radial growth slow on MA, $7.6 \mathrm{~mm} /$ month $(\mathrm{n}=91$, from 5 popul., 17 strains). Mats Cream (K75) to Straw (K79) to yellowish brown (M77) to Buff (N80) or whitish, raised, often strongly folded or convoluted, surface dough-like. Aerial mycelium lacking, but densely woolly, grey (N92) mycelium abundant after years in storage, occasionally covering the inoculum. Margin distinct, even, superficial. Hyphal strands lacking or inoculum with scattered, erect, white strands. Zonation rare, sector formation lacking, but rarely with dark brown (T71) to grey areas. No colour change in surrounding agar. Context tough at surface, odour not distinct.

Spores germinating readily on wood agars, growth moderate on BA, PA, QA, producing apothecia on MA after 16 months (single-spore strain), on BA after 8 months (single-spore and multispore), producing apothecia primordia on $\mathrm{PA}, \mathrm{QA}$, these not developed further. Anamorph not produced in single spore or multispore trials.

$H$. vitreola is easily recognized in culture due to the slow growth rate, light colouring and the often folded to convoluted surface. Occasionally the mats become up to $10 \mathrm{~mm}$ high, gyrose, and may be hollow inside. Isolations were made from populations with thin-walled and firm-walled hairs, septate and aseptate spores, and smaller and larger-celled excipula. All these populations were identical in culture.
Distribution and phenology. The distribution area of $H$. vitreola is large and so far the species is known from central, western and northern Europe, the U.S.A., the Caucasus and the Soviet Far East. The species is fairly common in North Europe. In the temperate and boreal zone $H$. vitreola fruits from March to November. A clear peak is reached during the summer months, and from September the fruiting populations diminish.

Ecology. In the present material the substrate of 72 collections could be determined as hardwood; in eight cases the collector reported a hardwood substrate and in four collections the wood could not be determined. $H$. vitreola prefers very soft, watersoaked inner wood which has been extensively degraded by other organisms. More rarely it is found growing on inner or outer bark (2 collections from Betula, one from Alnus, one unidentified). The substrates were indicated as follows: Alnus (22 collections), Betula (14), Fagus (3), Acer (1), Populus tremula (1), Prunus avium (1), P. padus (1), P. spinosa (2), Prunus sp. (1) and Ulmus (1). I have not seen any collection from Quercus.

Discussion. Hyaloscypha vitreola is a well-delimited species with relatively little variation. Diagnostic characters are the large asci showing croziers, large spores and smooth, narrow hairs. Even the smallest spores are usually larger than those of the allied $H$. leuconica, which is also a smooth-haired species, tending to have thickened hair walls. Populations with dwarfish narrow hairs are, however, not common in $\mathrm{H}$. leuconica and these two species are fairly easily separated. Dry material of Hyaloscypha fuckelii also has smooth hairs, but fresh hairs are often covered by loose, minute roughness, which is never

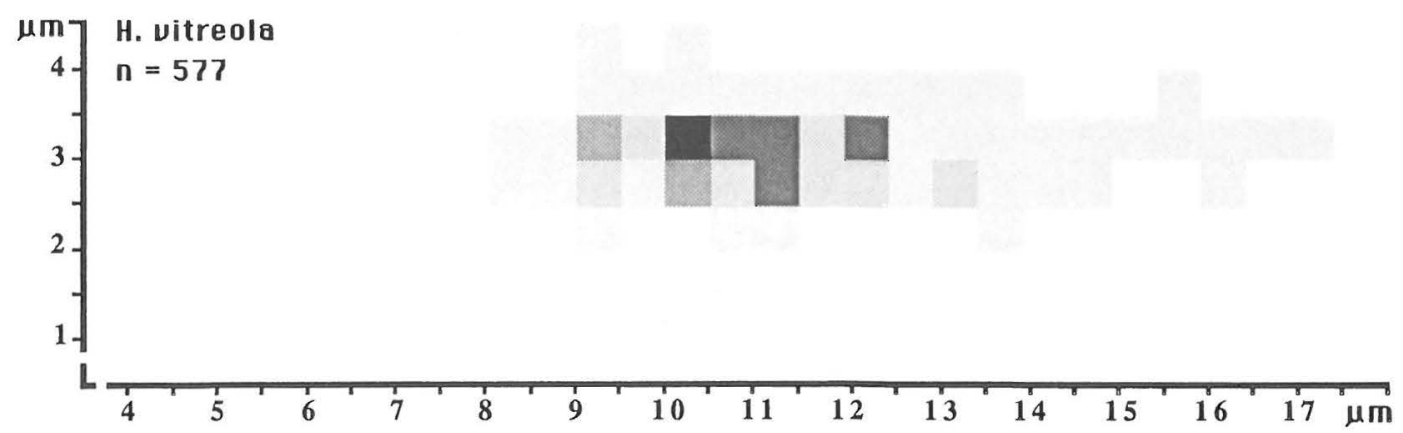

Fig. 177. Percentage sporogram of Hyaloscypha vitreola. 
present in $H$. vitreola, and the hairs often have thickened or globose apices or clearly widened bases. The overlap in ascal and spore size is slight and $H$. fuckelii var. alniseda does not have croziers.

The hair and spore characteristics separate Hyaloscypha vitreola from $H$. albohyalina var. albohyalina. Although the spore sizes overlap considerably, the shape is clearly more elongate in the latter. In $H$. vitreola, the spores are less variable than in $H$. albohyalina var. albohyalina, whose small-spored populations are thus easily recognized. But the spore characters should be matched with hair characters to ensure correct identification. In $H$. vitreola the hairs are smooth in all reagents, or very rarely and inconsistently bear one to four tiny apical warts. The hairs are very narrow in their elongated apical part, where the wall is thickened and refractive. They also lack prominent solidifications at the extreme apices. In the type variety of $H$. albohyalina, the hairs are shorter, thin-walled, roughened by abundant, deforming warts and often have solidified apices. The cultures of these species differ markedly. The other, sometimes smooth-haired variety of $H$. albohyalina is easily distinguished by the asci without croziers and by spores of the same type as in var. albohyalina.

The spores and asci in $H$. intacta have the same size range as in $H$. vitreola, but the species are not likely to be confused, because the asci of $\mathrm{H}$. intacta arise from simple septa and are consistently negative in MLZ and the short hairs have prominent apical solidifications.

Constant characters of $H$. vitreola are the hair shape, lack of hair roughness, lack of massive solidifications, spore size and shape, cultural characters, and to a lesser degree the ascal size. The ascal size varies mainly between populations. Spore septation

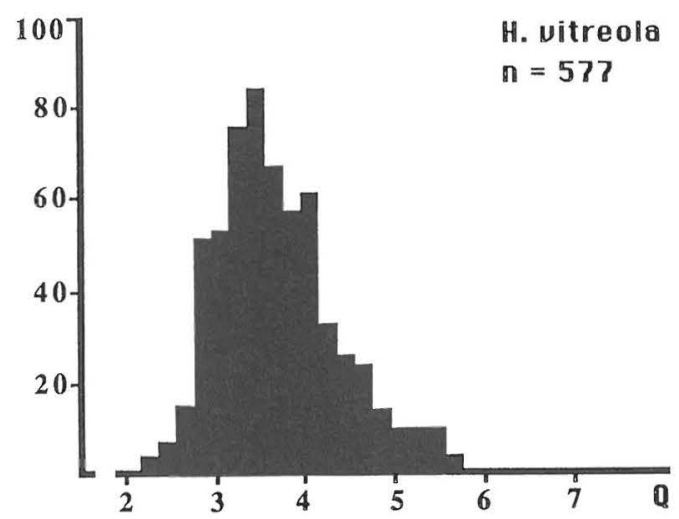

Fig. 178. The distribution of spore quotient Q in Hyaloscypha vitreola. and hair wall thickness are more variable. Populations with thin-walled hairs are in a clear minority and variation often occurs in the same apothecium. Spore septation tends to vary between the populations. Those with consistently aseptate spores are few and show no connection with the substrate, time of fruiting or geographical origin. As in most of the Hyaloscypha species, the excipulum varies between the populations, which can roughly be arranged in small- and large-celled types. The total variation between the populations of $H$. vitreola is relatively small and warrants no subdivisions.

Earlier treatments of $H$. vitreola are few. It was partly covered by Velenovský's concept of $H$. perpusilla and coincides with the greater part of $H$. hyalina "form 4". Dennis (1956) studied authentic material of Peziza vitreola and deduced the affinity to Hyaloscypha. But the unclarified status of the name (see below) apparently resulted in the species remaining "forgotten", in spite of Dennis's (1956) study and typification. Hyaloscypha vitreola was included in the key by Raitviir (1970a), who used the name in the original sense.

In a recent conservation proposal, typification of Hyaloscypha with $H$. vitreola was suggested (Huhtinen \& Cannon 1987), and the confused interpretation of the species was discussed. My own studies of the original material of Peziza vitreola in Karsten's herbarium, and also those by Dennis (1956), show that Karsten's concept of the species was clear. The discrepancy indicated by Nannfeldt (1932) and Raschle (1978) is due to unfortunate confusion in Fungi Exsiccati Fennici 731: the convolutes in UPS and $\mathrm{H}$ contain a helotiaceous species. Despite the slightly too pointed spores, the illustration and description of $H$. vitreola by Boudier $(1905-1910,1911)$ may represent Karsten's species. The narrow hairs with sparse septa and firm walls, the large asci, the size and biguttulate nature of the spores and occurrence on frondose wood all speak for this interpretation. In view of the abundant original material and Boudier's possibly correct interpretation, $H$. vitreola is a convenient choice for the generic type.

Exsiccatae studied: Karsten, P., Fungi Fenniae Exsiccati $731(\mathrm{~K})$.

Material studied: Czechoslovakia. Bohemia: Karlstejn, 1986 Svrčk \& SH 86/44* (TUR). Mnichovice, 1924, 1926, 1941 (type of Uncinia maxima, type of Uncinia spinosae) JV (PRM). Strancice, 1925, 1928 JV (PRM). Svojetice, 1924 JV (PRM). Treboň, 1966 Svrčk (PRM). Federal Republic of Germany. Bayern: Mt. Zwiesel, 1885 Allescher (M, type of Pezizella incerta). Regensburg, 1921 Killermann (M). Valley of river Tauber, 1938 Sponheimer (B). Finland. VarsinaisSuomi: Kaarina, 1986 SH 86/170* (TUR). Nauvo, 1986 SH 86/157* (TUR). Paimio, 1987 SH 87/49* (TUR). Parainen, 
1984 SH 84/82 (TUR). Turku, 1985, SH 85/38, 85/118, 85/134* (TUR). Uusimaa: Helsinki, 1984 SH 84/42 (TUR). Kirkkonummi, 1987 SH 87/84* (TUR). Vihti, 1986 SH 86/144* (TUR). Etelä-Häme: Kangasala, 1986 Söderholm 1269, 1270 (TUR). Korpilahti, 1986 SH 86/81, 86/84 (TUR). Lammi, 1985, SH 85/107, 85/113 (TUR). Tammela, 1865, 1868 PAK 2625, 2633 (type of Peziza vitreola), 2634, 2637, 2638 (H). Tampere, 1985 Söderholm 1172 (TUR). PohjoisHäme: Laukaa, 1986 SH 86/86* (TUR). Pohjois-Savo: Kuopio, 1985 SH 85/74, 85/77, 85/84 (TUR). Siilinjärvi, 1985 SH 85/83 (TUR). Vehmersalmi, 1985 SH 85/88 (TUR). Inarin Lappi: Utsjoki, 1965 JAN 19168 (UPS). Great Britain. Surrey: Esher Common, 1948 Dennis (K). Yorkshire: Roche Abbey, 1948 Hughes (IMI, K). Dumfries: Langholm, 1948 Dennis (K). Midlothian: Dalmahoy, 1954 Henderson 1394 (E). Unlocalized, 1899 Crossland (K). Weybridge, 1922 Rea (K). Norway. Oppland: Dovre, 1985 SH 85/49, 85/53 (TUR). Finnmark : Laevvajokka, 1965 Kankainen (TUR). Lyftjok, 1987 Svrček* (TUR). Sweden. Småland: Femsjö, 1929 JAN 3193 (UPS). Kärda, 1944 Lundell (UPS). Gästrikland: Gävle, 1950, 1956, 1969, 1972, 1973, 1974 JAN 10839, 14429, 14763b, 20611, 22358, 22976, 23988, 23090, 23557, 23560 (UPS). Hille, 1952 JAN 12503 (UPS). Hälsingland: Färila, 1956 JAN 14763b (UPS). Torne Lappmark: Jukkasjärvi, 1928 JAN 1064 (UPS). U.S.A. New York: Ithaca, 1894 Durand (CUP-D 680). Avalanche Lake, 1953 Schaffer \& Korf 53-13 (Herb. Korf). Marcy Brook (Adirondack Mts.), Schaffer \& Korf 53-30, 53-39 (Herb. Korf). Allegany State Park, 1961 Korf 3067, 3072 (Herb. Korf). Ringwood, Korf et. al. 633, 55-2 (Herb. Korf). Washington: Olympic Nat. Park, 1939 Smith 13391 (MICH). U.S.S.R. Lithuanian S.S.R.: Unlocalized, 1966 AR (TAA, two collections). Ukrainian S.S.R.: Sverdlovsk, 1973 Parmasto \& AR (TAA). Krasnodar Region: Caucasian Res., 1976 Pallo (TAA). Khabarovsk Region: Bolshekhekhchirsk Nat. Res., 1982 Parmasto (TAA). 


\section{HAMATOCANTHOSCYPHA}

Hamatocanthoscypha Svr., Ceská Mykol. 31:11. 1977. (February). - Holotype (original designation): Uncinia laricionis Velen., Monogr. Discom. Bohem.: 295; Pl. 16:42-44. 1934.

Uncinia Velen., Monogr. Discom. Bohem.: 293. 1934, nom. illeg. (Art. 64.1). - Lectotype (Raitviir 1970a: 73): Uncinia laricionis Velen., Monogr. Discom. Bohem.: 295; P1. 16:42-44. 1934 (see discussion in "Historical survey").

Unciniella K. Holm \& L. Holm, Symb. Bot. Upsal. 21:17. 1977 (August), nom. superfl. pro Hamatocanthoscypha Svr. (Art. 63.1). See discussion in "Historical survey".

Debaryoscyphus Arend. \& R. Sharma, Mycotaxon 25:134. 1986. - Holotype (original designation): Debaryoscyphus melanobasis Arendh. \& R. Sharma, Mycotaxon 25:134; Pl. 6:1-6. 1986.

Apothecia scattered to gregarious, superficial, 50$550 \mu \mathrm{m}$ in diam, cupulate, prominently stipitate, rarely sessile on a gradually tapering base. Disc shallow to plano-convex, margin consisting of a thin to prominent layer of hairs, not incurved when fresh, often clearly incurved when dry. Hair cover dense and minute, rarely sparse or prominent. Colour whitish to dark greyish when fresh, in one species bright yellow. Dry apothecia whitish to yellowish brown, more rarely blackish or bright yellow. Ectal excipulum of modified textura prismatica, cells lying parallel to the receptacle surface, on middle flanks with mean length ranging between 11.4 and $14.8 \mu \mathrm{m}$, mean width between 4.4 and $6.6 \mu \mathrm{m}$, mean $Q$ value 2.7, in one species consisting of textura prismatica-textura angularis, becoming narrower inwards, more roundish towards the base, towards margin similar or becoming smaller or narrower. Inner excipulum lacking or poorly developed, showing weak delimitation from the thin subhymenium. Walls in ectal parts thin to $1.2 \mu \mathrm{m}$ thick, hyaline to dark brown, MLZ-, MLZ+ (faint blue) in two species, in one taxon with amyloid nodules. Hairs almost cylindrical to narrowly conical, rarely lageniform, uncinate, more rarely straight or helicoid, aseptate to basally two-septate. Apex blunt, more rarely tapering to a fine apex, thin-walled, rarely inconspicuously solidified, without an apical globule in MLZ. Wall thin, hyaline to dark brown, smooth to warted, MLZ-. Warts stable to deforming in CB and MLZ. Hairs without resinous exudates or these very inconspicuous, in one species and one variant containing a faint yellow pigment dissolving in CB and MLZ. Asci cylindrical-clavate, eight-spored, mean length ranging between ca. 21 and $60 \mu \mathrm{m}$, mean width between 2.9 and $8.7 \mu \mathrm{m}$, pore MLZ+, more rarely MLZ-, asci arising from croziers, in one taxon from simple septa. Spores mostly small and cuneiformsubfusiform or elliptic, rarely larger and elliptic, mean length ranging between 3.7 and $11.0 \mu \mathrm{m}$, mean width between 1.3 and $3.2 \mu \mathrm{m}$, aseptate, more rarely septate, septum MLZ-, aguttulate to clearly guttulate. Paraphyses cylindrical to apically slightly widened, mean width of lower parts ranging between 1.2 and $2.0 \mu \mathrm{m}$, not protruding clearly above the level of the asci, unpigmented, in one species and one variant containing similar pigment to that seen inside the hairs, sparsely dichotomously branched, terminal cells often longer than the asci. Inhabiting wood litter and ferns, rarely on leaves (except needles) or bulky wood.

\section{Key to species and varieties of Hamatocanthoscypha}

The spore measurements were made on material mounted in CB or MLZ, the asci measurements on material in CB.

1 Apothecia whitish when fresh, light coloured when dry, lacking bright yellow or dark brown colours, excipular walls hyaline under the microscope except for stipe base

- Apothecia bright yellow, brown or greyish brown when fresh, alike or blackish when dry, excipular walls hyaline to clearly pigmented, brown colour not restricted to the very base of stipe

2 Apothecia bright yellow when fresh and dry, hairs and paraphyses with prominent yellow pigment when fresh in water 37 H. uncipila

- Apothecia with brown to blackish colours on flanks or stipe basally prominently blackish

3 Asci 46-57 $\mu \mathrm{m}$ long, plug MLZ-, spores elliptic to broadly elliptic, 2.2-3.2 $\mu \mathrm{m}$ wide $32 \mathrm{H}$. melanobasis

- Asci 29-45 $\mu \mathrm{m}$ long, plug MLZ+, spores cuneiform-subfusiform, 1.2-2.6 $\mu \mathrm{m}$ wide

4 Hairs showing one-sided thickenings, dry apothecia blackish 35 H. uncinata var. uncinata

- Hair walls of uniform thickness, dry apothecia with variable colouring, blackish to brown to creamcoloured 
5 Spores cuneiform-subfusiform, 1-2 $\mu$ m wide

- Spores elliptic, broadly elliptic or oblong-elliptic, $1.8-3.8 \mu \mathrm{m}$ wide

6 Spores ca. 10-12 × 3-4 $\mu \mathrm{m}$, often one-septate, hairs helicoid

28 H. helicotricha

- Spores not over $8 \mu \mathrm{m}$ long, aseptate, hairs straight to uncinate 7

7 Spores not over $5 \mu \mathrm{m}$ long, elliptic to broadly elliptic, excipulum covered by hyaline to brown crystals

- Spores frequently over $5 \mu \mathrm{m}$ long, elliptic, excipulum without covering crystals $33 \mathrm{H}$. obsoleta 34 H. ocellata

8 Asci arise from simple septa, $16-24 \mu \mathrm{m}$ long,spores not over $6 \mu \mathrm{m}$ long

- Asci arise from croziers, $23-41 \mu \mathrm{m}$ long, mean spore length $6.0-7.7 \mu \mathrm{m}$

31 H. laricionis var. minutissima

9 On ferns, spores $6-9 \mu \mathrm{m}$ long

$30 \mathrm{H}$. laricionis var. dryopteridis

- On litter of various conifers, spores 5-7 $\mathrm{m}$ long $29 \mathrm{H}$. laricionis var. laricionis

28. Hamatocanthoscypha helicotricha sp. nov.

Apothecia cupulata, stipitata, minuter pilosa; specimina exsiccata straminea, pili nivei. Excipulum externum textura prismatica sed cellulis variabilibus, elongatis, prismaticis vel isodiametricis, 11.0-19.5(-21.0) x 5.0-10.6(-11.8) $\mu \mathrm{m}$, aliquantum crassotunicatis, hyalinis, in solutione Melzeri non coloratis. Pili 15-38 x 2.3-4.3 $\mu \mathrm{m}$, anguste conici vel fere cylindrati, helicoidei vel uncinati, leves, tenuiter tunicati, aseptati vel basaliter biseptati, in solutione Melzeri non colorati. Asci 54-74 x 7.5-9.4 $\mu \mathrm{m}$, octospori, non amyloidei, uncinati. Sporae 9.8-12.2(-12.8) × 2.8-3.8(-4.0) $\mu \mathrm{m}$, ellipsoideae vel oblongo-ellipsoideae, saepe uniseptatae, manifeste guttulatae. Paraphyses cylindraceae, 1.8-2.0 $\mu$ m latae, cellulis terminalibus 31-48 $\mu \mathrm{m}$ longis.

Holotype: Colombia. Dpto. Huila: ca. $20 \mathrm{mi}[32 \mathrm{~km}]$ from Altamira on the Altamira-Florencia road, on undet. fern, 15.I.1976 Dumont, Buritica, Luteyn \& Molina (NYS, Co2694; isotype in NY).

Apothecia gregarious, superficial, $100-200 \mu \mathrm{m}$ in diam when dry, cupulate throughout development, clearly and stoutly stipitate, with up to $100 \mu \mathrm{m}$ high and $70 \mu \mathrm{m}$ wide stipe. Margin fleshy and incurved, partly obscuring the hymenium when dry. Hair cover dense, minute, white. Colour Straw (K79) when dry, modified by the overall hair cover.

Ectal excipulum of textura prismatica, cells on middle flanks prismatic to elongated or more isodiametric, $11.0-19.5(-21.0) \times 5.0-10.6(-11.8) \mu \mathrm{m}, \overline{\mathrm{x}}$ $=14.6 \times 6.6 \mu \mathrm{m}(\mathrm{n}=30), \mathrm{Q}=2.2$, becoming narrower and elongated towards margin, on stipe narrowly prismatic. Inner excipulum of narrower textura prismatica. Walls in ectal parts $0.4-0.8 \mu \mathrm{m}$ thick, hyaline, $\mathrm{CB}+, \mathrm{MLZ}-$.
Hairs $15-38 \times 2.3-4.3 \mu \mathrm{m}$, almost cylindrical to narrowly conical, apically coiled to uncinate, aseptate to basally two-septate. Apex blunt, tapering to 1.2$2.3 \mu \mathrm{m}$, not solidified, without an apical globule in MLZ. Wall thin, hyaline, dull, never locally prominently thickened, CB-, MLZ-, smooth.

Asci $54-74 \times 7.5-9.4 \mu \mathrm{m}, \overline{\mathrm{x}}=59.4 \times 8.7 \mu \mathrm{m}(\mathrm{n}=$ 5), $\bar{Q}=6.9$ in $\mathrm{CB}$, eight-spored, cylindrical-clavate with a slightly conical apex. Apical pore MLZ- even after $\mathrm{KOH}$ pretreatment. Walls in mature asci apically $0.8 \mu \mathrm{m}$ thick. Contents MLZ-. Asci arising from croziers.

Spores 9.8-12.2(-12.8) × 2.8-3.8(-4.0) $\mu \mathrm{m}, \overline{\mathrm{x}}=$ $11.0 \times 3.2 \mu \mathrm{m}(\mathrm{n}=24), \bar{Q}=3.5$ in CB and MLZ, elliptic to oblong-elliptic, frequently one-septate, septum MLZ-, wall not seen to turn brownish in aged spores. Guttulae conspicuous, numerous, light, spores with $\mathrm{CB}$ - contents typically containing two regular, large, yellowish guttulae.

Paraphyses cylindrical, unpigmented, first dichotomous branches occurring below the level of the asci. Terminal cells $31-48 \times 1.8-2.0 \mu \mathrm{m}, \overline{\mathrm{x}}=41 \times$ $2 \mu \mathrm{m}(\mathrm{n}=6)$.

Cultural characters. Not known.

Distribution and phenology. Known only from the type collection.

Ecology. The type grew on a rather woody fern rachis.

Discussion. The large spores and asci, and the helicoid hair apices distinguish $H$. helicotricha from 

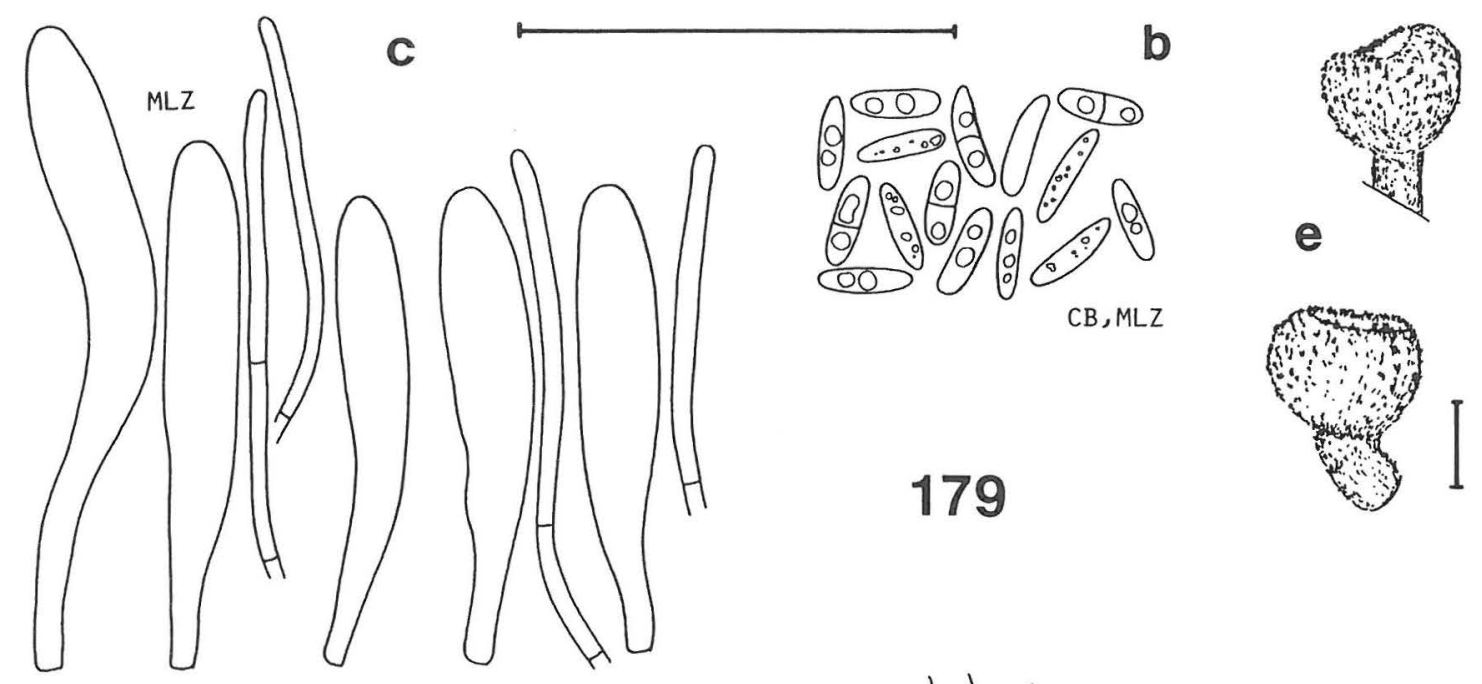

$C B, M L Z$
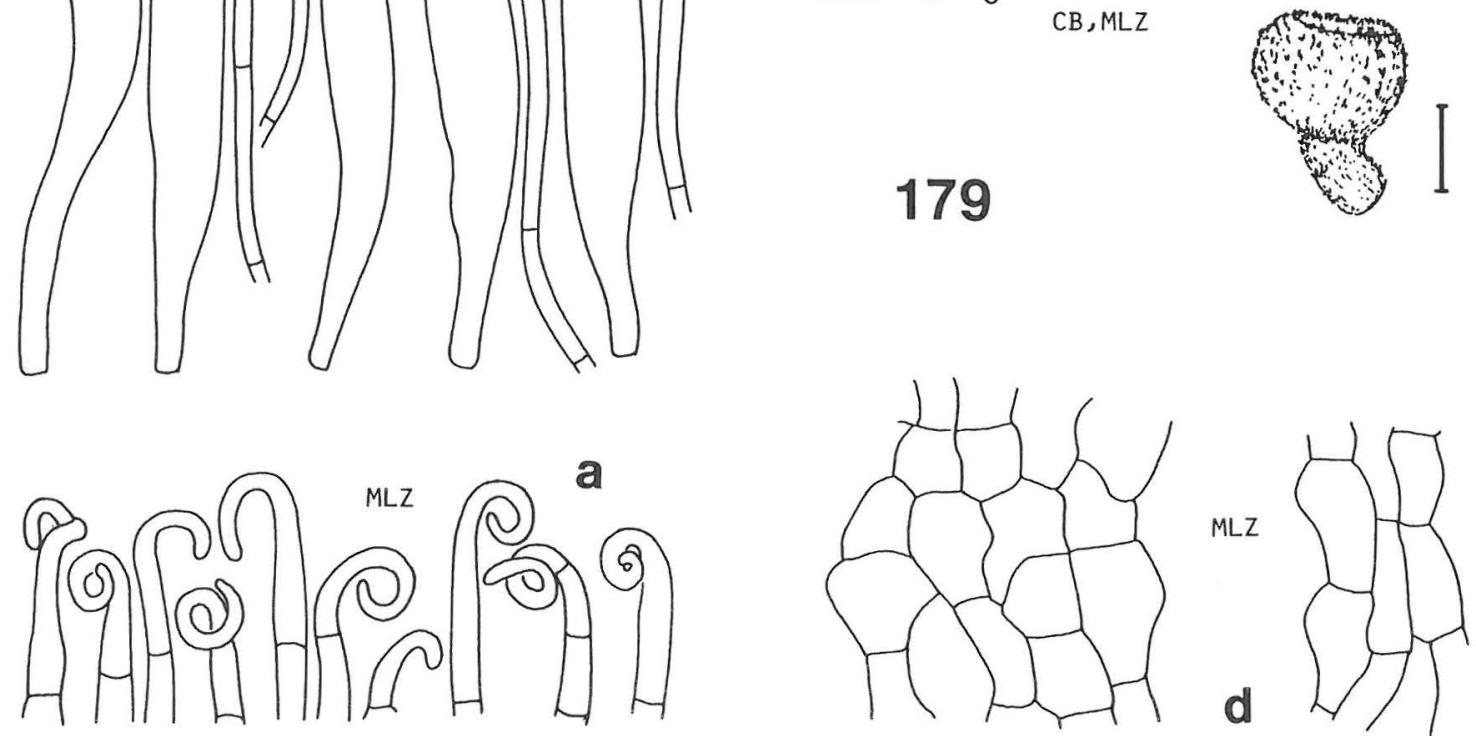

Fig. 179. Hamatocanthoscypha helicotricha, holotype. a) hairs, b) spores, c) asci and paraphyses, d) excipulum, e) dry apothecia. Scale $50 \mu \mathrm{m}$, for apothecia $100 \mu \mathrm{m}$.

all other species in the genus. The spores have the normal cuneiform shape of Hamatocanthoscypha only when juvenile; upon maturation they become less typical of the genus. Placement in Hamatocanthoscypha is supported by the typical hairs and apothecia, and by the lack of dextrinoid reactions. The excipular cells are variable in shape, but they often tend to become elongated, offering a further character diagnostic of the genus. The growth on litter, rather than on bulky wood or leaves, is also characteristic of Hamatocanthoscypha.

Although the apothecia were relatively large, the asci had matured surprisingly slowly, allowing only a small number of measurements. The specimen was also too scanty for checking all the staining reactions. The yellowish tinge in dried apothecia is not caused by the presence of a yellow pigment.

Material studied: Colombia. Dpto. Huila: Altamira, 1976 Dumont et al. 2694 (NYS, type).

\section{Hamatocanthoscypha laricionis var. laricionis}

Hamatocanthoscypha laricionis (Velen.) Svr., Česká Mykol. 31:11. 1977. - Uncinia laricionis Velen., Monogr. Discom. Bohem. 295; Pl. 16:42-44. 1934. - Hyaloscypha laricionis (Velen.) Nannf., Fungi Exsiccati Suecici Fasc. 51-52:39. 1958. - Unciniella laricionis (Velen.) K. Holm \& L. Holm var. laricionis, Symb. Bot. Upsal. 21:17. 1977. - Lectotype (selected here): [Czechoslovakia, Bohemia,] Mnichovice, Kozěny Vrch, Abies [in acubus,] 8.XII.1927 Velenovsky (PRM 151069 , examined). The only syntype which contains apothecia, one apothecium left.

Peziza acuum Alb. \& Schwein.: Fr. var. (Art. 35.3) tenuissima P. Karst., Not. Sällsk. Fauna Flora Fenn. Förh. 10:181. 1869. - Helotium acuum (Alb. \& Schwein.: Fr.) Fr. var. tenuissimum (P. Karst.) P. Karst., Not. Sällsk. Fauna Flora Fenn. Förh. 11:240. 1870. — Lectotype (selected here): [Finland,] Tavastia australis. Tammela, Mustiala, ad virgulta Abietis, 15.X.1868 Karsten (H, Herb. Karsten 2694, examined); cited also at subspecific level by Holm and Holm (1977).

Hyaloscypha cuneata Velen., Monogr. Discom. Bohem.: 274; Pl. 15:57. 1934. - Hamatocanthoscypha cuneata (Velen.) 

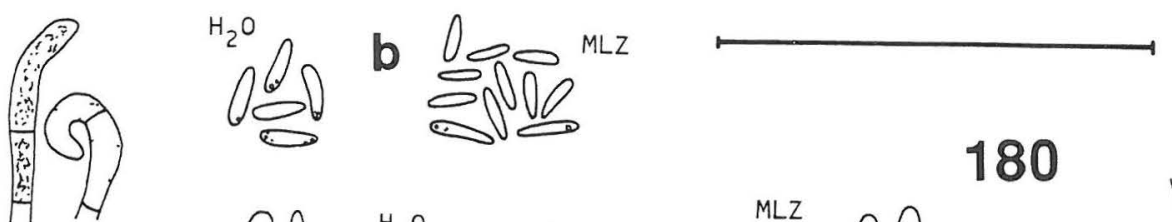

$\mathrm{H}_{2} \mathrm{O}$
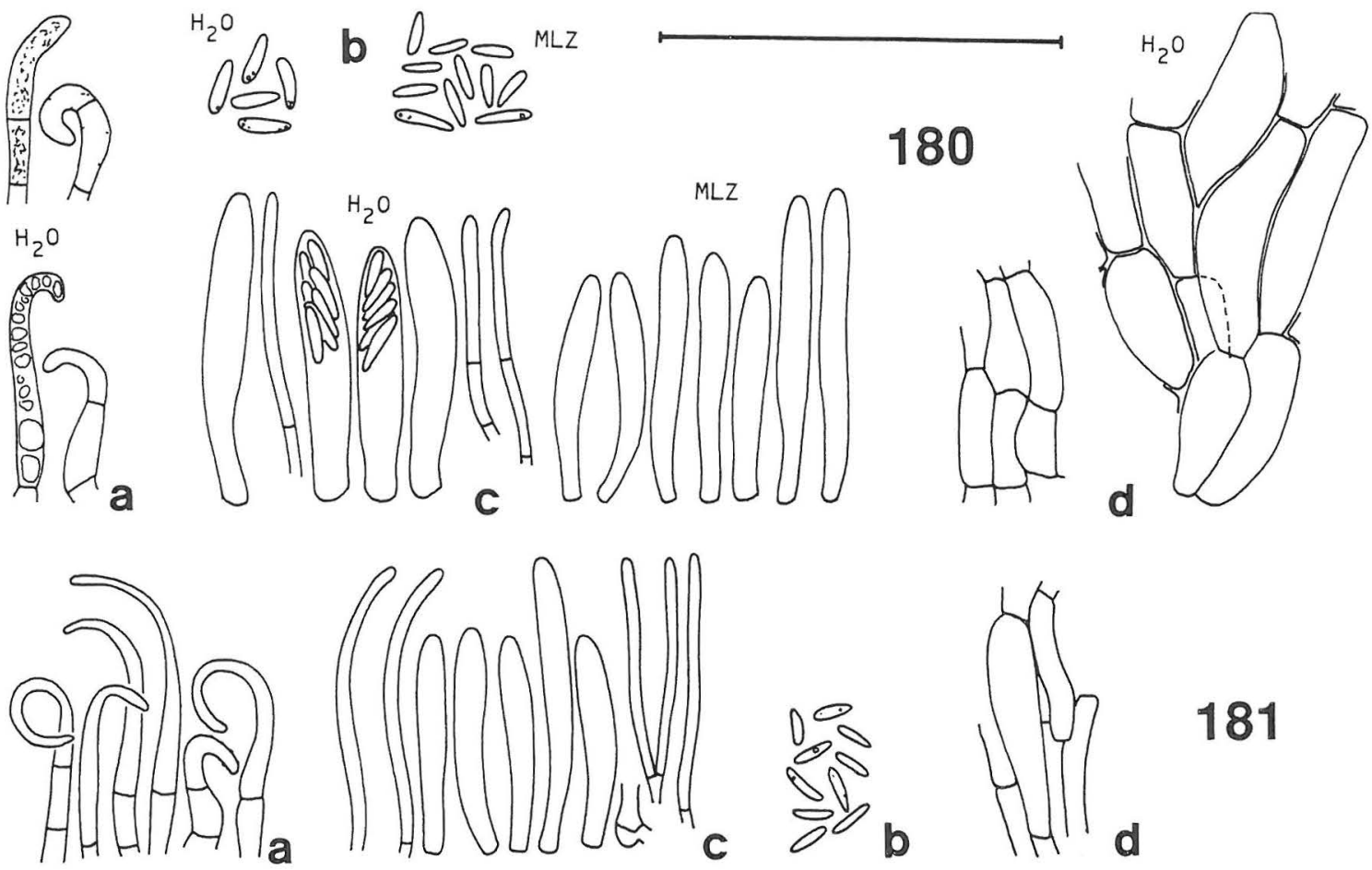

\section{1}
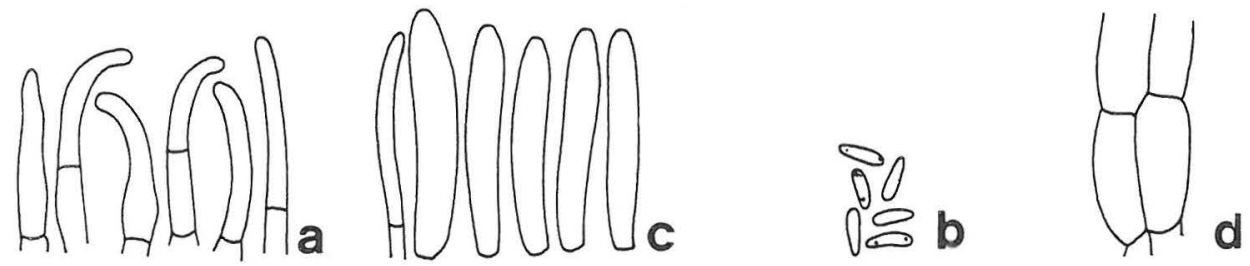

\section{2}

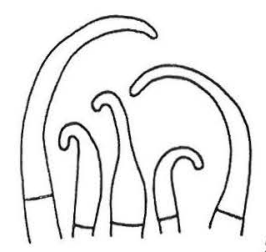

a
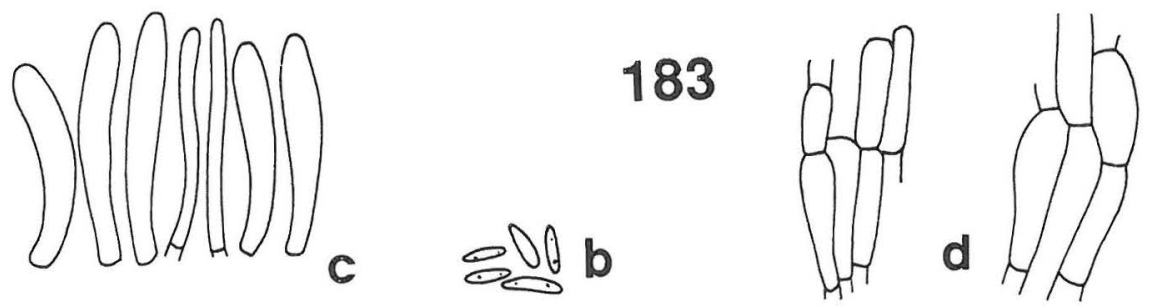

Figs. 180-183. Hamatocanthoscypha laricionis var. laricionis. a) hairs, b) spores, c) asci and paraphyses, d) excipulum. Scale $50 \mu \mathrm{m}$. - 180: SH 87/204. - 181: Fungi Exsiccati Suecici 2585. - 182: Lectotype of Hyaloscypha cuneata. - 183: Herb. Karsten 2693, syntype of Peziza acuum var. tenuissima.

Svr., Pilzflora Nordwestoberfrankens 7:42. 1983, nom. inval. (Art. 33.2). - Hamatocanthoscypha cuneata (Velen.) Svr., Ceská Mykol. 39:207. 1985. — Lectotype (Svrček 1985: 207): [Czechoslovakia,] Bohemia, Mnichovice, in conis piceis, 25.9.1925 Velenovsky (PRM, 149328, examined).

Uncinia laricionis Velen. var. lanensis Velen., Monogr. Discom. Bohem. 295; P1. 16:45. 1934. - Holotype (the only specimen mentioned): [Czechoslovakia, Lany,] ad conum piceae exc., 27.VIII.1929 Velenovský (PRM 150913, examined). Also cited as Uncinia lanensis by Velenovsky in the legend for figure 45.

Hyaloscypha lycopodii Velen., Monogr. Discom. Bohem.: 282; PI. 15:55. 1934. - Calycellina lycopodii (Velen.) Svr., Ceská Mykol. 39:211. 1985. — Holotype (the only specimen 
mentioned): [Czechoslovakia,] Bohemia, Mnichovice, Zbuzany, [Lycopodium clavatum, 14.XI.1929 Velenovský (PRM 151162, examined).

?Hyaloscypha curvipila Grelet, Rev. Mycol. 16:92, Fig. 35. 1951. - Holotype (the only specimen mentioned): [France,] Vienne. Savigne, in conis abietis excelsae, 13.IX.1932 (not seen, not located in PC).

Hyaloscypha juniperi E. Müller, Sydowia 21:149. 1968. - Holotype (original designation): Switzerland, Valesia. Aletschwald prope Brigua, Moränenweg, 20.IX.1965 Müller (ZT, not seen; isotype in UPS studied from a slide).

Apothecia gregarious, superficial, up to $550 \mu \mathrm{m}$ in diam when fresh, typically $50-300 \mu \mathrm{m}$ in diam when dry, cupulate throughout development, rarely undulate-crenulate when large, varying between the populations from clearly stipitate, with stipe up to $130 \mu \mathrm{m}$ long and $50 \mu \mathrm{m}$ wide, to more rarely sessile on a gradually narrowing base. Disc shallow to plane when fresh, bordered by a thin but dense zone of marginal hairs which rise only slightly above the hymenium; margin fleshy and incurved when dry, often obscuring part of the hymenium. Hair cover dense, minute, snow-white. Colour pure white when fresh, dry apothecia ranging from pure white to Straw (K79) to Apricot (L65) or orange brownish (M57), rarely with reddish tinge (L47, M47); colours modified by the overall hair cover.

Ectal excipulum of textura prismatica, cells on middle flanks prismatic but typically inflated and/or elongated, 9.4-24.0(-31.0) x 3.7-8.2(-8.9) $\mu \mathrm{m}, \overline{\mathrm{x}}=$ $14.7 \times 5.4 \mu \mathrm{m}(\mathrm{n}=60), \bar{Q}=2.7$, becoming smaller towards margin, on stipe narrowly prismatic-textura porrecta. Inner excipulum of narrower textura prismatica, cells ca. 9-14 × 2-4 $\mu \mathrm{m}$. Walls in ectal parts thin to $0.6 \mu \mathrm{m}$ thick in $80 \%$ of the populations, 0.8 $1.2 \mu \mathrm{m}$ thick in others, becoming thinner inwards, hyaline, partially $\mathrm{CB}+$ in $87 \%$ of the populations, $\mathrm{CB}-$ in others, $\mathrm{MLZ}-;$; fragmentarily $\mathrm{BF}+, \mathrm{TB}+$, CR-, CRB-, NR-, LUG-.

Hairs $10-28(-46) \times 2.0-3.6 \mu \mathrm{m}$, varying between the populations from almost cylindrical to narrowly

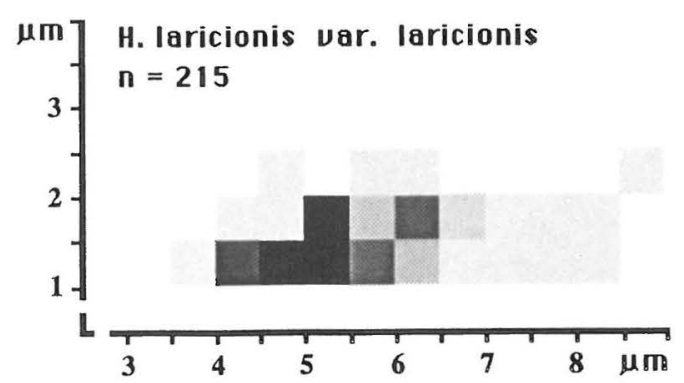

Fig. 184. Percentage sporogram of Hamatocanthoscypha laricionis var. laricionis. conical, uncinate, in a few populations only hairs with apically undulating to straight hairs predominate, these always mixed with uncinate hairs, aseptate to basally two-septate, typically without pigment, in five populations containing pigment, inconspicuously yellowish when fresh in water, deep red in LUG, dissolved in CB, MLZ, CR. Apex blunt, ranging between populations from scarcely to strongly tapering (to $0.6 \mu \mathrm{m}$ ), apex very rarely minutely solidified, without an apical globule in MLZ. Wall thin, hyaline, dull, never locally prominently thickened (except for the apex), negative in all reagents, smooth to minutely and loosely roughened when fresh in water, such variation occurring within the same apothecium, typically smooth in CB and MLZ, rarely with a few, prominent warts in $C B$, MLZ; warts typically not deforming in $\mathrm{CB}, \mathrm{MLZ}$, in one population clearly deforming in MLZ.

Asci 23-36(-41) x 4.0-5.1(-6.3) $\mu \mathrm{m}, \overline{\mathrm{x}}=28.5 \times$ $4.6 \mu \mathrm{m}(\mathrm{n}=55), \bar{Q}=6.1$ when fresh in water; 19 $35(-39) \times 3.4-5.4(-5.8) \mu \mathrm{m}, \overline{\mathrm{x}}=28 \times 4.5 \mu \mathrm{m}(\mathrm{n}=$ $44), \mathrm{Q}=6.3$ in MLZ; 20-33(-37) $\times$ 3.0-4.4(4.8) $\mu \mathrm{m}, \overline{\mathrm{x}}=25.5 \times 3.8 \mu \mathrm{m}, \bar{Q}=6.8$ in $C B$, eightspored, cylindrical to cylindrical-clavate with a slightly conical apex. Apical pore MLZ+ when fresh in water, when dry MLZ++ in $78 \%$ of the populations, MLZ+ in others, LUG+ (blue). Wall in mature asci apically thin to $0.4 \mu \mathrm{m}$ thick (in CB, MLZ), negative in all reagents. Contents MLZ-, LUG-. Asci arising from croziers.

Spores $4.8-7.0(-7.5) \times 1-2(-2.3) \mu \mathrm{m}, \overline{\mathrm{x}}=6.0 \times$ $1.8 \mu \mathrm{m}(\mathrm{n}=50), \overline{\mathrm{Q}}=3.4$ when fresh in water; 4.1$7.2(-8.0) \times 1.0-1.8(-2.3) \mu \mathrm{m}, \overline{\mathrm{x}}=5.5 \times 1.5 \mu \mathrm{m}(\mathrm{n}$ $=215) \mathrm{Q}=4.1$ in $\mathrm{CB}, \mathrm{MLZ}$; cuneiform-subfusiform, rarely oblong-elliptic, aseptate. Wall hyaline, not seen to turn brownish in aged spores. Guttulae minute, light to dark when fresh in water, similar to often lacking in dry material.

Paraphyses cylindrical to slightly widened at the apex, typically unpigmented, in five populations with inconspicuously yellowish pigment similar to that inside the hairs, first dichotomous branches occurring below the level of the asci. Terminal cells 18-31 $x$ $1.2-2.0 \mu \mathrm{m}, \overline{\mathrm{x}}=23.7 \times 1.6 \mu \mathrm{m}(\mathrm{n}=40)$, the widened apices $1.8-2.3 \mu \mathrm{m}$ wide.

Cultural characters (10 populations, 28 strains). Radial growth slow on MA, $10.9 \mathrm{~mm} /$ month $(\mathrm{n}=$ 89,7 popul., 19 strains). Mats with creamy white to Cream (K75) to Beige (L91) basic colour, frequently with blackish grey (T92, S92) or dark brown (P70, R71) zones or sectors. Aerial mycelium lacking to scanty and velvety, rarely abundant and densely interwoven, cottony; always greyish white or light grey 
(L92). Margin indistinct, thin, submerged. Hyphal strands frequently present, at the inoculum erect and entangled, elsewhere running in the agar, extending to $1 \mathrm{~cm}$ from the inoculum, concolorous with the mat or dark brown (R75). Formation of pigmented sectors or zones frequent, no colour change in surrounding agar but after prolonged ( 2 years) storage occasionally producing bright greenish yellow (L85) colour on agar surface, colour due to amorphous exudate. Yeast-like growth frequent after spore germination or at a later stage, conglomerations formed in and on the agar, these grey to light yellowish to orange.

Spores germinating readily on wood agars, growth moderate on PA, QA, somewhat slower on $\mathrm{BA}$, retarded on LA, yeast-like growth observed on all wood agars, producing a brown phialidic anamorph on MA, BA, PA, QA after ca. 9 months, when abundant the hyaline conidia forming $2 \times$ $2 \mathrm{~mm}$, snow-white areas on agar surface. Apothecial primordia produced in one strain on MA, these not developed further, juvenile apothecia (no mature asci) developed in one strain on MA.

The colouring in culture is variable, in some strains the basic light colours prevail in all replicates whereas others may show marked heterogeneity in the abundance and distribution of dark pigments. In addition to the anamorph, recognition in culture is facilitated by the general scarcity of aerial mycelium, the strong hyphal strands, which are often present, and the light basic colour, which is always present to some extent. Yeast-like areas in the mycelium often have a yellowish or orange tinge, which assists in recognition.

Distribution and phenology. Although the present material is fragmented, it seems that $H$. laricionis var laricionis has a wide, more or less continuous geographical range. It has mostly been collected in $\mathrm{Eu}-$ rope, but stray collections are known from the Yukon, Washington State, the Caucasus and Japan. As noted by Holm and Holm (1977), it fruits relatively

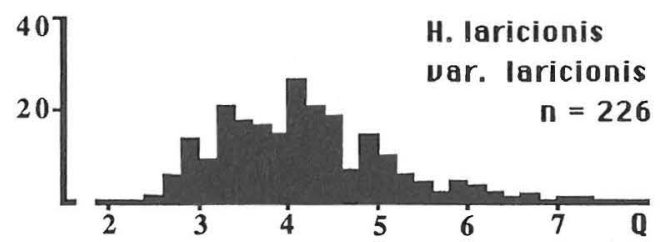

Fig. 185. The distribution of spore quotient Q in Hamatocanthoscypha laricionis var. laricionis. late. In the present material the peak is reached between September and November, the collections made during the summer months being very few.

Ecology. Hamatocanthoscypha laricionis var. laricionis grows mostly on conifers. It is found more rarely on Lycopodium and has once even been collected even on leaves of Rhododendron. It inhabits small wood fragments amongst litter. Fallen needles, cones and chips of bark are the domain of var. laricionis. When attacking a fallen branch, the apothecia are seen on the smallest twigs, avoiding the more bulky parts. The occurrence on Lycopodium was mentioned by Holm and Holm (1981) as "accidental". Strong invasion by var. laricionis of heterogeneous litter may result in occasional occurrence on such a relatively long-lived and somewhat woody substrate as Lycopodium. Likewise, some hardwood-inhabiting polypores (e.g. Lenzites betulinus (L.: Fr.) Fr. and Trametes hirsuta (Wulf.: Fr.) Pilát) exceptionally occupy fallen coniferous branches and fruit on them, if the secondary substrate has long remained in contact with the original piece of hardwood (Niemelä, pers. comm.). The scarcity of apothecia on Lycopodium seems to point to a similar case. The apothecia on Rhododendron were also exceptionally small, and the occurrence might be similarly "accidental".

Fallen cones are the substrate for $H$. laricionis. In all, 19 collections originate from cones: Picea (11), Pinus (3), Abies (3), Cryptomeria (1), Larix (1). The rest of the material originates from the following substrates: Lycopodium (6), Picea twigs (5), Picea needles (4), Pinus needles (3), Juniperus needles (1), Picea bark (1), Pinus bark (1), Rhododendron leaves (1). As seen from the above list, the species differs ecologically from the closely related $H$. ocellata and the other two varieties, which prefer a substrate on which var. laricionis does not occur or seldom fruits.

Discussion. Hamatocanthoscypha laricionis var. laricionis greatly resembles var. dryopteridis, var. minutissima, $H$. ocellata and $H$. uncinata, and is the commonest of the five. $H$. ocellata differs in being corticolous and in the clearly different shape and biguttulate nature of the spores. Holm and Holm (1977), who recognized dark-pigmented specimens on juniper as a variety of $H$. laricionis, had difficulty in delimiting the two taxa. As discussed under $H$. uncinata var. phaeotricha, the differences from the present species lie in the type of excipulum and the spores, rather than in the pigmentation of the hairs and excipulum. On the whole, spore size and shape separate var. laricionis fairly clearly from these two 
taxa. In the present taxon the greatest morphological variation is found in the hairs. In most populations they are strongly uncinate and taper slightly towards the blunt apex. The rest of the collections have more tapering or less clearly uncinate hairs. Unbroken gradation occurs, however, between the populations. As in $H$. uncinata and $H$. ocellata, the hairs in some populations may have scattered, prominent warts. As variation from smooth to warted hairs exists within the individual apothecia as well, it can be considered acceptable within $H$. laricionis. In the collection from Washington these warts deformed in MLZ, giving the hairs a strikingly different appearance. All the other morphological characters are relatively stable.

Interesting chemical variation was observed when one population from Picea cones was noted to have yellowish pigment inside the hairs. During a closer scrutiny MLZ was allowed to replace water in a mount, which immediately gave rise to countless red to blue globules around the apothecium. The phenomenon was later seen in five additional collections, all from cones of Picea and consisting of both fresh and dry material. Ten further populations from other substrates were studied in respect to this feature, but the reaction was lacking. These populations did not show the yellowish pigment either. Apparently Melzer's reagent concentrates the yellow pigment into globules and stains it. This pigment rapidly disappears in MLZ or CB, but stains and persists in LUG, in which the formation of coloured globules is lacking. These chemical variants agree in other respects with the material from other substrates.

Dennis (1956) believed that the present taxon belonged to Hyaloscypha, and it was transferred to the genus by Nannfeldt in the Fungi Exsiccati Suecici in 1958. Of the many names used for the taxon, Karsten's var. tenuissima is the oldest (Karsten 1869), but has not been employed at the specific level. Possible synonymy with Hyaloscypha lycopodii Velen. was suggested by Holm and Holm (1981), but rejected by Svrček (1985), who transferred Velenovský's species to Calycellina because of its hair shape. This character does not, however, warrant separation from $H$. laricionis on coniferous substrates, if the total variation is considered. Even in the material from Lycopodium there is wide variation from straight to clearly uncinate hairs. Such variation also characterizes the type collection of $H$. lycopodii. Nor do the collections from Lycopodium have the yellow pigment of Calycellina. Holm and Holm (1981) gave no taxonomic status to the collections on Lycopodium. In this case the ecology plays a smaller role than in, e.g., material from ferns or bark, recognized at species or varietal level, the reason being that occasional and accidental occurrence on Lycopodium does not result in changes in morphology (see discussion under section "Ecology").

The lectotype of Hyaloscypha cuneata was selected by Svrček (1985), who also transferred it to $\mathrm{Ha}$ matocanthoscypha. No reason was given for recognition at specific level, but apparently the only slightly uncinate hairs were decisive. I found that the hairs, and all the other diagnostic characters of the lectotype, fall within range of variation of $H$. laricionis. The hair characteristics of the type differ markedly from the description and illustration by Velenovsky (1934). This discrepancy requires a re-examination of the remaining syntypes. It may be that Velenovský was exaggerating when he described and illustrated the hairs as acute. The other characters in the diagnosis match those of $H$. laricionis.

The present taxon comes very close to $P$ silocistella conincola (Velen.) Svr. in its morphology, ecology and chemical properties. The neotype of that species differs mainly in its marginal hairs, which are mostly straight. They are intermixed, however, with slightly uncinate hairs, just as in Svrček's (1979) illustration of the neotype. Such variation is present in some populations of $H$. laricionis. Svrcek reported that the pore did not stain in MLZ, but I found a strong MLZ+ reaction. The neotype of $P$. conincola could well represent an extreme of the present taxon. Synonymization should, however, await confirmation by further material.

Exsiccatae studied: Lundell, S. \& Nannfeldt, J., Fungi Exsiccati Suecici 2584 (S), 2585 (UPS).

Material studied: Austria. Niederösterreich: Rekawinkl, 1915 Keissler (W). Canada. Yukon: Kluane Lake, 1987 SH 87/173* (TUR). Czechoslovakia. Bohemia: Karlstejn, 1986 Svrcek \& SH 86/51* (TUR). Lany, 1929 JV (PRM, type of Uncinia laricionis var. lanensis). Mnichovice, 1925 (type of Hyaloscypha cuneata), 1927 (type of Uncinia laricionis var. laricionis), 1929 (type of Hyaloscypha lycopodii) JV (PRM). Denmark. Sjalland: Sorø Sønderskov, 1978 Korf et al. (CUP). Finland. Varsinais-Suomi: Kaarina, 1986 SH 86/171* (TUR). Laitila, 1987 SH 27/211* (TUR). Lemu, 1987 SH 87/204* (TUR). Parainen, 1985 SH 85/154 (TUR). Turku, 1988 SH 88/102 (TUR). Vehmaa, 1987 SH 87/207* (TUR). Satakunta: Punkalaidun, 1984, 1985 SH 84/259, 85/126* (TUR). Uusimaa: Tenhola, 1986 SH 86/151* (TUR). Etelä-Häme: Korpilahti, 1986 SH 86/82 (TUR). Lammi, 1985 SH 85/109, 85/116 (TUR). Tammela, 1868 PAK 2693, 2694 (type of Peziza acuum var. tenuissima), 2695 (H). Somero, 1986 SH 86/24* (TUR). Pohjois-Savo: Kuopio, 1985 SH 85/99*, 85/100 (TUR). Siilinjärvi, 1985 SH 85/78 (TUR). Great Britain. Herefordshire: Gt Doward, 1975 Graddon (IMI). Warwickshire: 1971 Clark 2160 (TAA). Suffolk: Dunwich Forest, 1977 Ellis \& Ellis (IMI). Japan. Yamagata Pref.: Shirabutakayu, 1958 Shimizu \& Korf 1179 (CUP). Kushiro Prov.: Akan Nat. Park, 1963 Korf et al. 2498 (CUP). 
Sweden. Dalarna: Folkărna, 1980 Holm \& Holm 2235f (UPS). Garpenberg, 1974 Holm \& Holm 351d (UPS). St. Kopparberg-Sundbom, 1974 Holm \& Holm 326h (UPS). Uppland: Alsike, 1974 Holm \& Holm 392b (UPS). Dalby, 1979, 1980 Holm \& Holm 1893j, 2272d (UPS). Switzerland. Valesia: Aletschwald, 1965 Müller (UPS, isotype of Hyaloscypha juniperi). Thurgau: Wängi, 1974 Matheis 600 (Herb. Matheis). U.S.A. Washington: Gray's Harbor Co., Westport, 1979 Haines 3405 (NYS). U.S.S.R. Estonian S.S.R.: Distr. Kohtla-Järve, Boroni, 1967 AR (TAA, two collections). Distr. Tartu, Elva, 1966 AR (TAA). Karachayevo-Cherkess: Teberda, 1968 AR (TAA).
30. Hamatocanthoscypha laricionis var. dryopteridis

Hamatocanthoscypha laricionis (Velen.) Svr. var. dryopteridis (Bøhler) Huhtinen, comb. et stat. nov. - Hyaloscypha dryopteridis Bøhler, Norw. J. Bot. 21:89, Fig. 6. 1974. - Holotype (original designation): Norway, Akershus, Baerum, Isidalen, NM 7946, on dead parts of Dryopteris filix-mas, 16.X.1969 Bøhler 333/69 (O, examined).

Apothecia $30-180 \mu \mathrm{m}$ in diam when dry, stipitate on a $20-30 \mu \mathrm{m}$ wide, $30-60 \mu \mathrm{m}$ long stipe, more
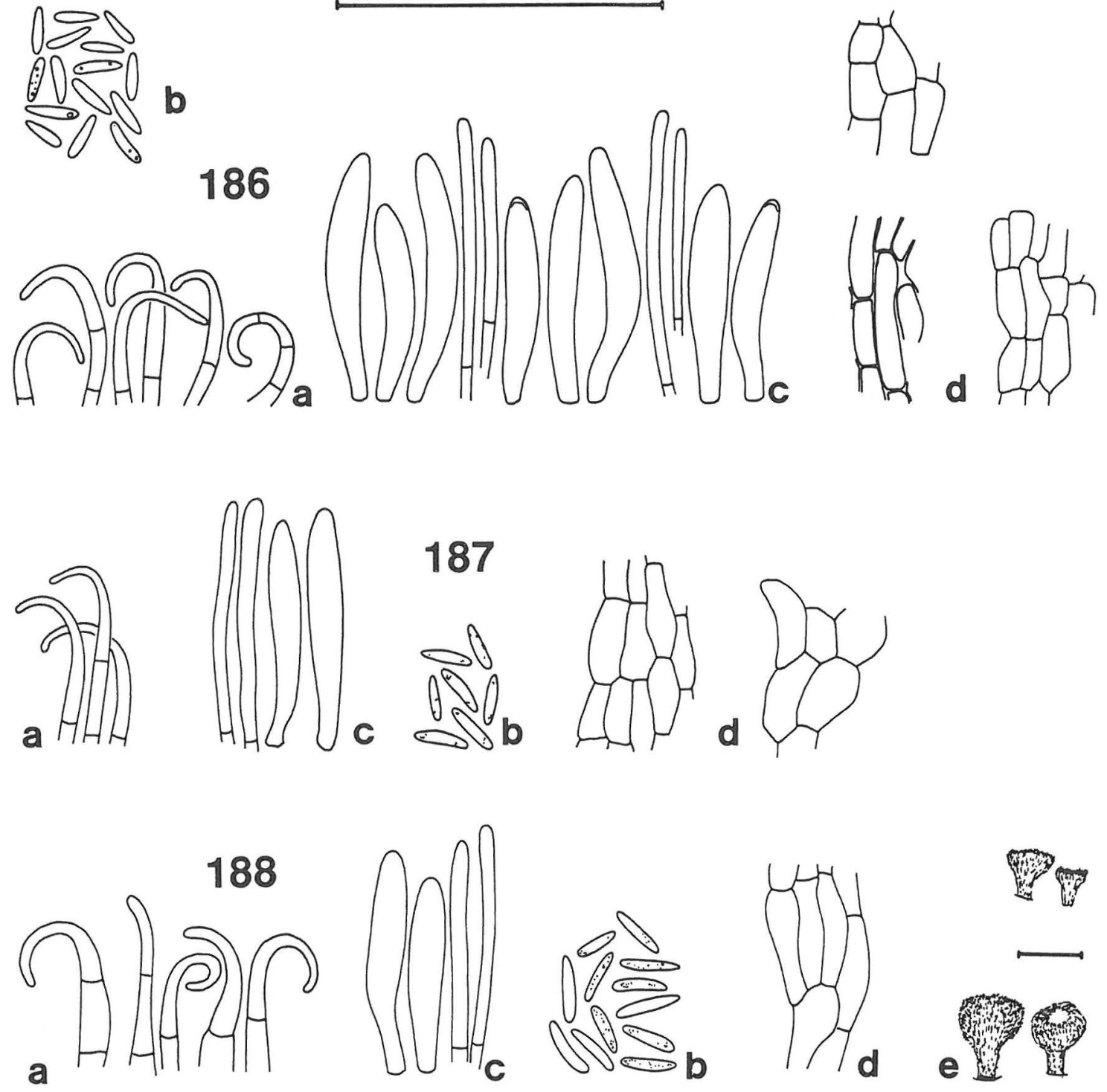
rarely sessile on a tapering base. Margin prominent, incurved, obscuring part of the hymenium when dry. Hair cover dense, minute, snow-white. Colour whitish to Cream (K75), disc concolorous to faint yellowish (K85) when dry.

Ectal excipulum of textura prismatica, cells on middle flanks prismatic but typically somewhat inflated and/or elongated, 7.8-17.0(-21.0) x 3.2-7.8(8.8) $\mu \mathrm{m}, \overline{\mathrm{x}}=11.4 \times 4.9 \mu \mathrm{m}(\mathrm{n}=50), \bar{Q}=2.3$, not notably changing size or shape towards margin, at the base narrowly prismatic. Inner excipulum of narrower textura prismatica to textura porrecta. Walls in ectal parts thin to $0.4 \mu \mathrm{m}$ thick, hyaline, totally or towards base CB+, MLZ-; CR-, LUG-, MLZ-.

Hairs 15-28 x 2-3 $\mu \mathrm{m}$, cylindrical-tapering, uncinate to strongly recurved, aseptate to basally one- to two-septate. Apex blunt, tapering to $1.2-1.6 \mu \mathrm{m}$, never solidified, without an apical globule in MLZ, not bearing resin. Wall thin, not locally prominently thickened, hyaline, smooth in CB, MLZ, smooth to frequently minutely encrusted in $\mathrm{CR}$ and water, $\mathrm{CB}-$, MLZ-; CR-, LUG-.

Asci $27-41(-42) \times 4.2-6.0(-6.2) \mu \mathrm{m}, \overline{\mathrm{x}}=33.5 \times$ $5.0 \mu \mathrm{m}(\mathrm{n}=26), \bar{Q}=6.8$ in $C B$; eight-spored, cyl-

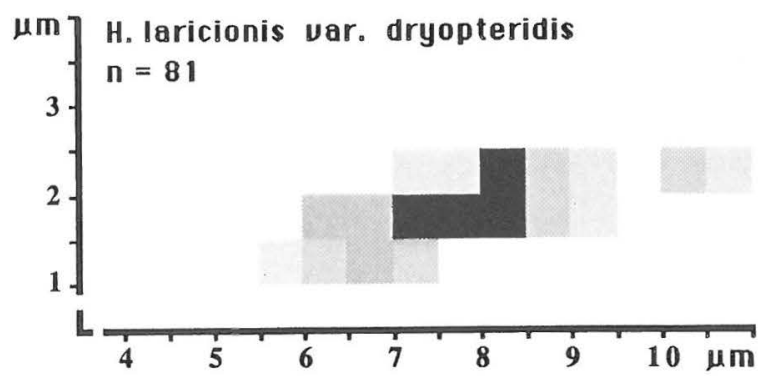

Fig. 189. Percentage sporogram of Hamatocanthoscypha laricionis var. dryopteridis.

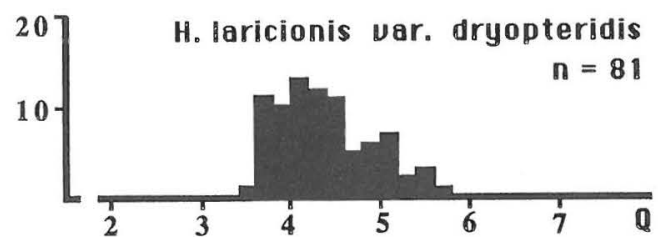

Fig. 190. The distribution of spore quotient Q in Hamatocanthoscypha laricionis var. dryopteridis. indrical-clavate with a slightly conical apex. Apical pore MLZ+, LUG+ (blue). Wall in mature asci apically $0.4-1.0 \mu \mathrm{m}$ thick (in CB, MLZ), negative in all reagents. Contents $\mathrm{MLZ+}$ (orange), frequently LUG+. Asci arising from croziers.

Spores $6.2-9.2(-10.7) \times 1.8-2.2 \mu \mathrm{m}, \overline{\mathrm{x}}=7.7 \times$ $1.8 \mu \mathrm{m}(\mathrm{n}=81), \bar{Q}=4.3$ in $\mathrm{CB}$ and MLZ; cuneiform-subfusiform, rarely oblong-ellipsoid, typically aseptate, once seen to become one-septate after discharge. Guttulae minute, light in CB and MLZ.

Paraphyses cylindrical to slightly widened at the apex, first dichotomous branches typically occurring below the level of the ascal bases. Terminal cells 28$47 \times 1.0-2.3 \mu \mathrm{m}, \overline{\mathrm{x}}=35.8 \times 1.8 \mu \mathrm{m}(\mathrm{n}=14)$, the widened apices $1.8-2.8 \mu \mathrm{m}$ wide.

\section{Cultural characters. Not known.}

Distribution and phenology. The variety is known only from one small area in southern Norway. The collections have been made in May, August and October (cf. Bøhler 1974).

Ecology. The scanty material suggests that var. dryopteridis is restricted to fern remains. All the collections confirmed by Bøhler and in the present study originate from Dryopteris. The two collections with juvenile apothecia on Polystichum braunii which were discussed by Bøhler (1974) could not be included with certainty in the present variety and further collections are needed.

Discussion. Hamatocanthoscypha laricionis var. dryopteridis differs from the type variety in its larger spores and asci. The reason for treating it as a variety of $H$. laricionis is the overall similarity in morphology. The spores have the cuneiform shape characteristic of the type variety. The degree of ecological isolation is hard to assess, since the type variety ranges from conifers to Lycopodium and even leaves of Rhododendron. On the other hand, the difference in spore size is not caused by specialization to coniferous bark, as in H. ocellata. The material of var. laricionis from Lycopodium and Rhododendron suggests that when it occurs on less woody substrates, the ascal and spore characteristics remain unchanged. Specialization to ferns and the slight morphological differences, justify separation at varietal level only. In all the studied collections the critical morphological characters are very constant. The greatest variation

Figs. 186-188 (Left). Hamatocanthoscypha laricionis var. dryopteridis. a) hairs, b) spores, c) asci and paraphyses, d) excipulum, e) dry apothecia. Scale $50 \mu \mathrm{m}$, for apothecia $100 \mu \mathrm{m}$. — 186: Holotype. — 187: Bøhler 360/69. — 188: Bøhler 346/69. 
occurs in the shape and size of the excipular cells, which can range from regularly prismatic to elongated or inflated within the same apothecium.

When describing Hyaloscypha dryopteridis Bøhler (1974) claimed that the paraphyses were unusually scanty or even lacking. I found the paraphyses of the same material as conspicuous and abundant as in any species of the genus.

Material studied: Norway. Akershus: Bæerum, 1969, 1971 Bøhler 333/69 (type), 346/69, 360/69, 387/69, 10/71 (O).

\section{Hamatocanthoscypha laricionis var. minutissima}

Hamatocanthoscypha laricionis (Velen.) Svr. var. minutissima (Velen.) Huhtinen, comb. et stat. nov. - Uncinia minutissima Velen., Monogr. Discom. Bohem.: 295; Pl. 16:45, 46. 1934. - Lectotype (indicated on the label by Svrcek, formally designated here): [Czechoslovakia,] Mnichovice, mala Žihev mlyn, in acutus Pini laricionis , XI.1929 Velenovský (PRM 151168, examined).

Hyaloscypha minima Velen., Monogr. Discom. Bohem.: 278; Pl. 15:52. 1934. - Holotype (the only specimen mentioned): [Czechoslovakia,] Mnichovice, u Brožka, Pinus, VI.1928 Velenovský (PRM 151184, examined).

Apothecia $30-100 \mu \mathrm{m}$ in diam when dry, stipitate on a $20-30 \mu \mathrm{m}$ wide, $20-50 \mu \mathrm{m}$ long stipe. Hair cover dense, minute, white. Colour faint yellowish.

Ectal excipulum of textura prismatica, cells on middle flanks prismatic to elongated, 7.6-17.0($21.0) \times 3.3-6.2(-7.5) \mu \mathrm{m}, \overline{\mathbf{x}}=12.3 \times 4.4 \mu \mathrm{m}(\mathrm{n}=$
35), $\bar{Q}=2.8$, becoming smaller towards the margin. Walls in ectal parts thin to $0.8 \mu \mathrm{m}$ thick, hyaline, fragmentarily but clearly $\mathrm{CB}+, \mathrm{MLZ}-$ in one collection, with a few blackish violet nodules in the other.

Hairs 8-25 x 1.8-2.7(-3.6) $\mu \mathrm{m}$, cylindrical-tapering, uncinate to strongly recurved, aseptate to basally one-septate. Apex blunt, tapering to 1.2$1.6 \mu \mathrm{m}$, not solidified, without an apical globule in MLZ, not bearing resin. Wall thin, never locally prominently thickened, hyaline, smooth, CB-, MLZ-.

Asci $15.8-24.3 \times 2.6-3.3 \mu \mathrm{m}, \overline{\mathrm{x}}=20.6 \times 2.9 \mu \mathrm{m}$ $(\mathrm{n}=14), \bar{Q}=7.5$ in CB. Apical pore MLZ+. Contents MLZ-. Asci arising from simple septa.

Spores $3.3-5.2 \times 1.0-1.6 \mu \mathrm{m}, \overline{\mathrm{x}}=4.4 \times 1.3 \mu \mathrm{m}$ ( $\mathrm{n}=15), \bar{Q}=3.5$ in $\mathrm{CB}$, cuneiform, rarely oblongelliptic, aseptate. Guttulae lacking in CB.

Paraphyses cylindrical, terminal cells $13-31 \times$ $1.2-2.0 \mu \mathrm{m}, \overline{\mathrm{x}}=20.1 \times 1.5 \mu \mathrm{m}(\mathrm{n}=8)$.

\section{Cultural characters. Not known.}

Distribution and phenology. Known only from two specimens from Velenovskýs collecting grounds in central Bohemia. Found in June and in November.

Ecology. As discussed below, the taxon is possibly restricted to pine needles.

Discussion. This variety was treated as synonymous with Hamatocanthoscypha laricionis by both
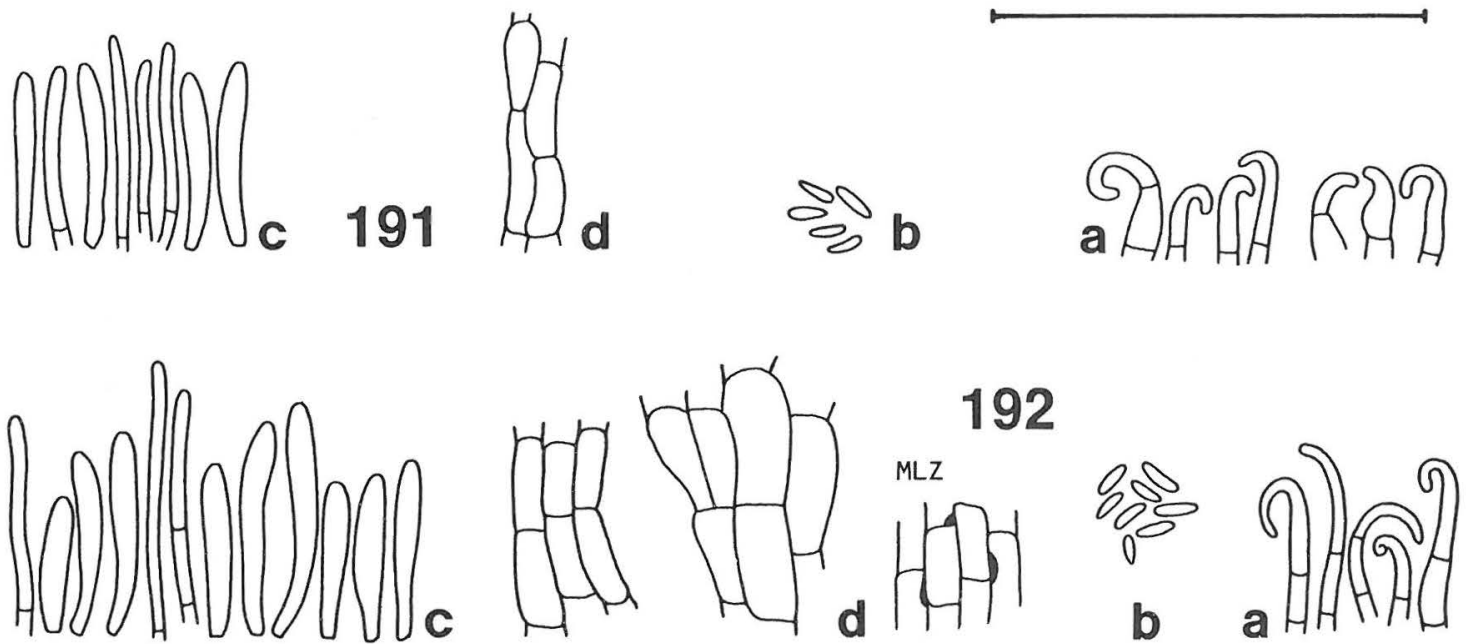

Figs. 191-192. Hamatocanthoscypha laricionis var. minutissima. a) hairs, b) spores, c) asci and paraphyses, d) excipulum. Scale $50 \mu \mathrm{m}$. - 191: Holotype of Hyaloscypha minima. - 192: Holotype of Uncinia minutissima. 
Holm and Holm (1977) and Svrček (1985), who all failed to see the difference from $H$. laricionis in the ascal bases and in the ascal and spore size. The type collection of Uncinia minutissima showed an additional difference in the excipulum, which has a few scattered nodules that are deep amyloid in MLZ. These have not been observed in the larger material of var. laricionis. As is the case in Hyaloscypha aureliella, this chemical feature varies between the populations in the present taxon.

It is noteworthy that these two collections from pine needles were the only ones among the over fifty studied collections of $H$. laricionis in which the asci arise from simple septa. Var. laricionis mainly occurs on other substrates and the untypical ecology is coupled with asci and spores which are also untypical of that taxon. The spore shape agrees with the general type in $H$. laricionis and as the ecological isolation is incomplete, the present material is recognized at varietal level rather than kept as a distinct species.

Although I have not seen the whole syntypic material of Uncinia minutissima, the lectotypification suggested by Svrček on the label is validated here. The exceptionally small asci and spores were mentioned in the original diagnosis.
Material studied: Czechoslovakia. Bohemia: Mnichovice, 1928 (type of Hyaloscypha minima), 1929 (type of Hyaloscypha minutissima) JV (PRM).

\section{Hamatocanthoscypha melanobasis}

Hamatocanthoscypha melanobasis (Arendh. \& R. Sharma) Huhtinen, comb. nov. - Debaryoscyphus melanobasis Arendh. \& R. Sharma, Mycotaxon 25:134; Pl. 6:1-6. 1986. - Holotype (original designation): Bhutan, Thimphu, Nawephu, on angiosperm bark, 26.IX.1980 Sharma (PAN 17590, not seen; isotype in Herb. Arendholz examined).

Apothecia scattered to gregarious, superficial, up to $200 \mu \mathrm{m}$ in diam when dry, cupulate throughout development, stipitate, with up to $200 \mu \mathrm{m}$ long and $100 \mu \mathrm{m}$ wide stipe. Margin fleshy and slightly incurved when dry. Hair cover dense, minute, whitish. Colour Straw (K79) to more brownish (M80) when dry, stipe base frequently dark brown to black.

Ectal excipulum of textura prismatica, cells on middle flanks prismatic but typically inflated and/or elongated, $11.5-18.0 \times 3.7-7.2 \mu \mathrm{m}, \overline{\mathrm{x}}=14.8 \times$ $4.7 \mu \mathrm{m}(\mathrm{n}=25), \bar{Q}=3.1$, becoming textura por-
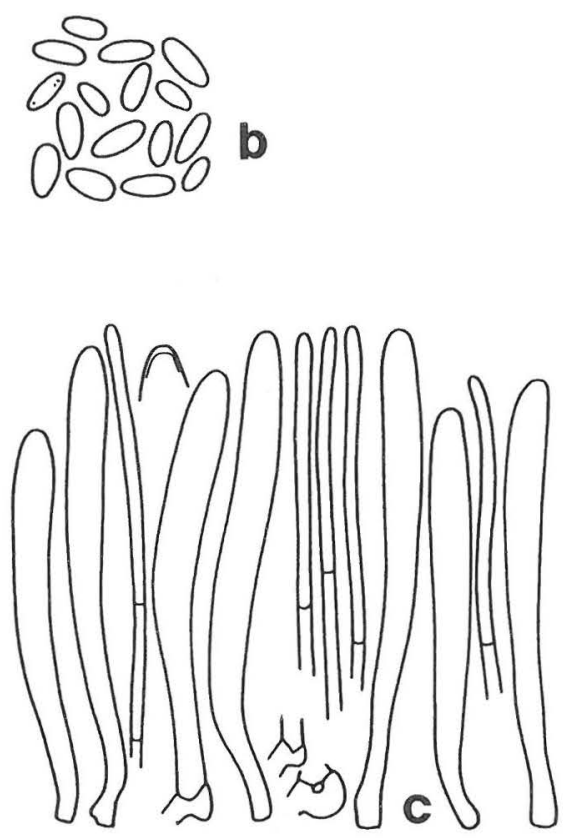

Fig. 193. Hamatocanthoscypha melanobasis, isotype. Scale $50 \mu \mathrm{m}$, for apothecia $100 \mu \mathrm{m}$.
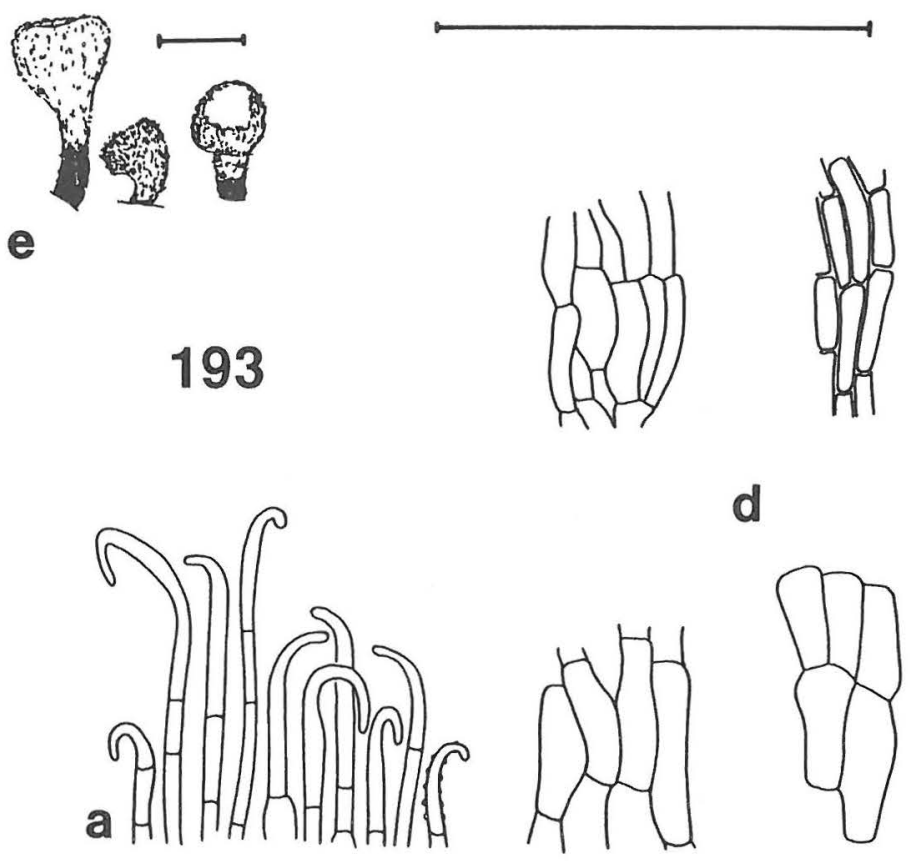
a) hairs, b) spores, c) asci and paraphyses, d) excipulum, e) dry apothecia. 
recta close to the margin, on stipe narrowly prismatic-textura porrecta, cells up to $30 \mu \mathrm{m}$ long. Inner excipulum of narrower textura prismatica-textura porrecta. Walls in ectal parts $0.4-0.6 \mu \mathrm{m}$ thick, clearly brown at stipe base, becoming lighter upwards, close to the margin hyaline to fragmentarily brownish, CB-, MLZ- to MLZ+ (blue) after $\mathrm{KOH}$ pretreatment.

Hairs $15-40 \times 1.8-2.0 \mu \mathrm{m}$, almost cylindrical to narrowly conical, uncinate, aseptate to basally twoseptate but merging gradually into the excipulum at the margin, not bearing resin or containing pigment. Apex blunt, tapering to $0.8-1.3 \mu \mathrm{m}$, not solidified, without an apical globule in MLZ. Wall thin, hyaline to brown, dull, never locally thickened, CB-, MLZ-, smooth, more rarely with a few prominent, hyaline to brown warts, which do not deform in CB, MLZ.

Asci $46-57 \times 4.4-5.5 \mu \mathrm{m}, \overline{\mathrm{x}}=51.7 \times 4.9 \mu \mathrm{m}(\mathrm{n}=$ $20), Q=10.5$ in lactic acid, eight-spored, cylindrical to cylindrical-clavate, with an elongated base, apex slightly conical. Apical pore MLZ- even after $\mathrm{KOH}$ pretreatment. Wall in mature asci apically 0.2 $0.5 \mu \mathrm{m}$ thick, negative in CB, MLZ. Contents MLZ-. Asci arising from croziers.

Spores $4.5-6.9(-7.5) \times 2.2-3.2(-3.4) \mu \mathrm{m}, \overline{\mathrm{x}}=$ $5.5 \times 2.6 \mu \mathrm{m}(\mathrm{n}=63), \mathrm{Q}=2.1 \mathrm{in}$ lactic acid, elliptic to broadly elliptic to broadly subfusoid, aseptate. Wall hyaline, not seen to become brownish in aged spores. Guttulae typically lacking in MLZ and lactic acid.

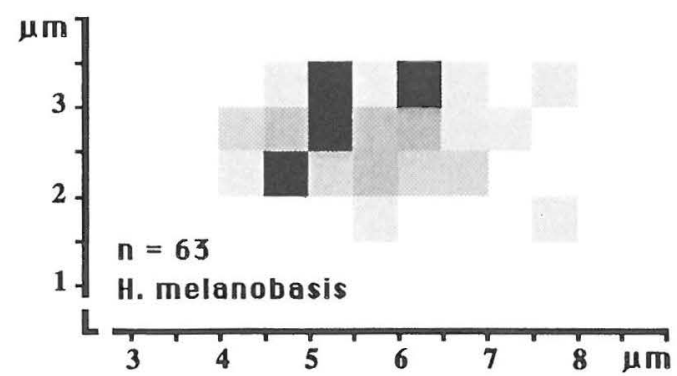

Fig. 194. Percentage sporogram of Hamatocanthoscypha melanobasis.

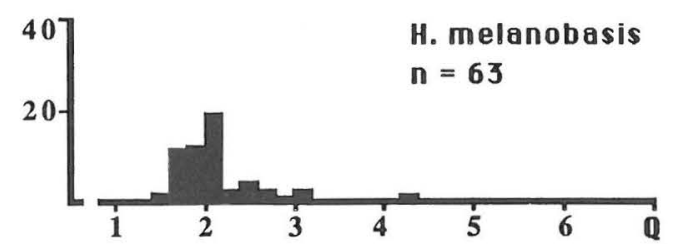

Fig. 195. The distribution of spore quotient Q in Hamatocanthoscypha melanobasis.
Paraphyses cylindrical, unpigmented, first dichotomous branches occurring below the level of the asci. Terminal cells $27-36 \times 1.4-1.8 \mu \mathrm{m}, \overline{\mathrm{x}}=31.9 \mathrm{x}$ $1.6 \mu \mathrm{m}(\mathrm{n}=10)$.

\section{Cultural characters. Not known.}

Distribution and phenology. The species is known only from the type locality in Bhutan.

Ecology. The type specimen originates from angiosperm bark. Thus, the ecology deviates from that of the bulk of the genus.

Discussion. The narrow and MLZ-asci, the yellow colour of fresh apothecia and the broad spores differentiate $H$. melanobasis from all other species of the genus. The asci are distinguished by a mean $\mathrm{Q}$ value of 10.5 , which is the extreme for the three studied genera. $H$. obsoleta has similar, although smaller, spores, but the asci and strongly uncinate hairs separate these taxa. H. melanobasis was described in the monotypic Debaryoscyphus in Leotiaceae (Sharma et al. 1986), the new genus being erected on account of the narrowness of the excipular cells. However, the excipulum of $H$. melanobasis, with elongated or inflated prismatic cells, does not deviate greatly from that of most of the species of Hamatocanthoscypha. Moreover, Debaryoscyphus shows similarities in the hairs, stipitate apothecia, lack of a dextrinoid reaction, tendency to brown pigmentation, occurrence on a non-bulky substrate, and presence of small, often subfusoid spores. To my mind, the genus should be treated as synonymous with Hamatocanthoscypha.

In one $\mathrm{KOH}$-pretreated apothecium, an interesting chemical reaction was noted in the excipulum. When studied in mass, it showed a very light blue tinge in MLZ. The colouring is too faint to be seen when the apothecium is squashed. This character was also found in $H$. obsoleta, but its taxonomic value will remain uncertain until more material is discovered.

Material studied: Bhutan. Nawephu, 1980 Sharma (Herb. Arendholz, isotype).

\section{Hamatocanthoscypha obsoleta, sp. nov.}

Apothecia cupulata, stipitata, minuter pilosa; specimina exsiccata pallide ochracea vel rubiginosa, pili nivei. Excipulum externum textura prismatica, cellulis 8-11 $\times 2.5-3.5 \mu \mathrm{m}$ prope marginem, cellulis deorsum 8-16 x 3.4-7.0 $\mu \mathrm{m}$, aliquantum crassotunicatis vel crassotunicatis, hyalinis, in solutione Mel- 

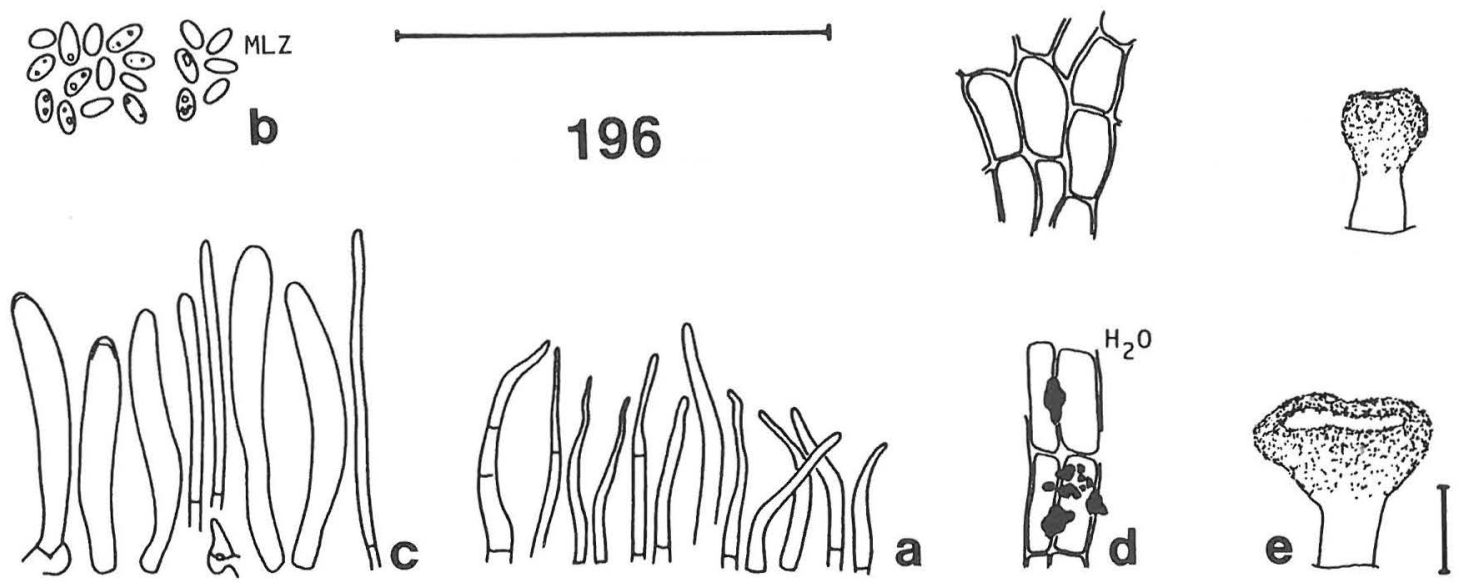

Fig. 196. Hamatocanthoscypha obsoleta, holotype. a) hairs, b) spores, c) asci and paraphyses, d) excipulum, e) dry apothecia. Scale $50 \mu \mathrm{m}$, for apothecia $100 \mu \mathrm{m}$.

zeri non coloratis, post solutionem kalii amyloideis; excipulum in toto crystallis hyalinis vel pallide brunneis obtectus. Pili 15-25 x 1-2 $\mu \mathrm{m}$, anguste conici vel fere cylindrati, leves, tenuiter tunicati, aseptati vel basaliter uniseptati, in solutione Melzeri non colorati. Asci $28-45 \times 3.2-4.4 \mu \mathrm{m}$, amyloidei, in basi uncinati, crystallis brunneis. Sporae 3.2-4.3-2.0 $2.6 \mu \mathrm{m}$, late ellipsoideae vel subglobosae, non septatae, minuter guttulatae. Paraphyses cylindraceae, 1.2-1.8 $\mu \mathrm{m}$ latae, cellulis terminalibus 20-35 $\mu \mathrm{m}$ longis.

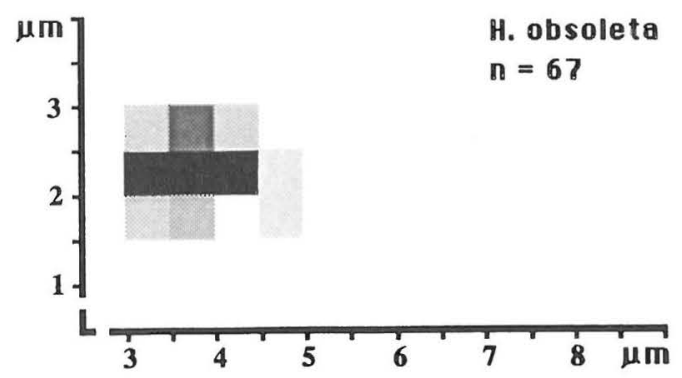

Fig. 197. Percentage sporogram of Hamatocanthoscypha obsoleta.

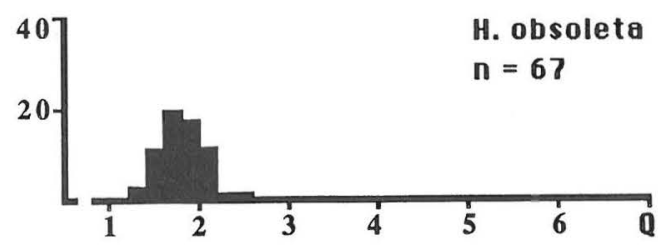

Fig. 198. The distribution of spore quotient $\mathrm{Q}$ in Hamatocanthoscypha obsoleta.
Holotype: Cuba. Nimanima, on rotten logs, Wright 367 (W, as Fungi Cubensis Wrightiani 686)

Apothecia gregarious, superficial, up to $500 \mu \mathrm{m}$ in diam when dry, cupulate, stipitate. Stipe stout, short to $100 \mu \mathrm{m}$ long, up to $70 \mu \mathrm{m}$ wide. Margin prominent, incurved, obscuring part of the hymenium when dry. Hair cover sparse, inconspicuous, snowwhite; stipe naked. Colour light yellowish brown to reddish brown (N35) when dry.

Ectal excipulum of textura prismatica, cells on middle flanks prismatic, $8-16 \times 3.4-7.0 \mu \mathrm{m}, \overline{\mathrm{x}}=12$ $\times 5.1 \mu \mathrm{m}(\mathrm{n}=20), \bar{Q}=2.8$, becoming more elongate towards margin, ca. 8-11 x 2.5-3.5 $\mu \mathrm{m}$. Walls in ectal parts 0.6-1.2 $\mu \mathrm{m}$ thick, hyaline, MLZ-, after $\mathrm{KOH}$ pretreatment locally $\mathrm{MLZ}+$ (blue) $\mathrm{BF}+$, fragmentarily $\mathrm{CR}+, \mathrm{CRB}+, \mathrm{NR}+, \mathrm{CB}-, \mathrm{LUG}-$. Excipulum covered by abundant, hyaline to brownish crystals, dissolving in $\mathrm{KOH}, \mathrm{MLZ}$; partly dissolved in $\mathrm{CB}$, persistent in other mountants.

Hairs $15-25 \times 1-2 \mu \mathrm{m}$, narrowly conical to almost cylindrical, straight to bent, aseptate to basally oneseptate. Apex tapering to $0.5-1.0 \mu \mathrm{m}$, not solidified, without an apical globule in MLZ. Wall thin, never locally thickened, hyaline, dull, smooth, MLZ-.

Asci $28-45 \times 3.2-4.4 \mu \mathrm{m}, \overline{\mathrm{x}}=32.9 \times 4.0 \mu \mathrm{m}(\mathrm{n}=$ 15), $\bar{Q}=8.3$ in CB, eight-spored, cylindrical-clavate to almost cylindrical, with slightly conical apex. Apical pore MLZ+, IKI+ (blue). Wall in mature asci apically $0.5-0.8 \mu \mathrm{m}$ thick (in CB). Contents LUG+, MLZ-, many asci with medium brown, minutely crystalline basal inclusions, persistent in water and CB. Asci arising from croziers. 
Spores 3.2-4.3(-4.7) $\times 2.0-2.6(-2.8) \mu \mathrm{m}, \overline{\mathrm{x}}=$ $3.7 \times 2.3 \mu \mathrm{m}(\mathrm{n}=67), \bar{Q}=1.7$ in $\mathrm{CB}$, broadly elliptic to subglobose, aseptate, wall slightly thickened in $\mathrm{CB}, 0.5 \mu \mathrm{m}$ thick in water-based reagents, hyaline, pale brownish in aged discharged spores. Guttulae lacking or with one small, light guttula at each end.

Paraphyses cylindrical to slightly widened at the apex, unpigmented, terminal cells $20-35 \times 1.2$ $1.8 \mu \mathrm{m}(\mathrm{n}=5)$.

\section{Cultural characters. Not known.}

Distribution and phenology. Known only from the type locality in Cuba. The collection is undated.

Ecology. The only available collection was apparently made from the outer bark of rotten logs.

Discussion. Hamatocanthoscypha obsoleta is easily recognized by the small roundish spores and the excipulum bearing a crystalline encrustation. It could possibly also be treated as a stray species of Hyaloscypha, but the number of alien characters would be greater in that genus. The stoutly stipitate apothecia, small spores, somewhat elongated excipula and lack of dextrinoid reactions all speak for placement in Hamatocanthoscypha. The massive crystalline exudates on the excipulum and the brown crystals inside the asci are, of course, unique characters in this genus. The faint amyloid reaction, in scattered areas of the excipulum after $\mathrm{KOH}$ pretreatment is a similarity to $H$. melanobasis.

The hairs vary within the same apothecium, ranging from almost cylindrical to regularly tapering. They are not as wide or as clearly curved as in $H$. laricionis, but fall within the range of variation of the genus. For instance, Hamatocanthoscypha ocellata has similar hairs. The spores are more roundish than the basic cuneiform-subfusoid type of Hamatocanthoscypha, but are closer to it than to the majority of Hyaloscyphas. Although small, the spores were relatively thick-walled, which was best observed in water-based stains.

Material studied: Cuba. Nimanima, Wright 367 (K; W, type).

\section{Hamatocanthoscypha ocellata, sp. nov.}

Apothecia cupulata, stipitata, pilosa; specimina exsiccata straminea vel aurantiobrunnea, pili nivei. Excipulum externum textura prismatica, cellulis 11-18(-26) $\times$ 3.4-5.6(-6.2) $\mu \mathrm{m}$. tenuiter tunicatis, hyalinis vel brunneis, in solutione Melzeri non coloratis. Pili 18-39 $\times 2-3(-3.8) \mu m$, anguste conici, uncinati vel in apice undulati, raro recti, leves vel conspicue tuberculati, tenuiter tunicati, in apice raro solidi, aseptati vel basaliter septati, hyalini vel brunnei, in solutione Melzeri non colorati. Asci 28-39 × 3.4-4.5 $\mu \mathrm{m}$, octospori, amyloidei, in basi uncinati. Sporae 4-12 × 1.8-3.0 $\mu \mathrm{m}$, ellipsoideae, raro cuneatae, non septatae, biguttulatae. Paraphyses cylindraceae, 1.4-2.3 um latae, 19-40 $\mu \mathrm{m}$ longis, raro minuter incrustatae.

Holotype: Japan. Kushiro Prov.: Akan National Park, wood along road between Akan-cho and Mt. Meakan, on Picea jezoensis branch, 28.IX.1963 Kamei, Yokoyama, Yamaguchi \& Korf (CUP-JA 2527).

Apothecia sparse to densely gregarious, superficial, up to $500 \mu \mathrm{m}$ in diam when fresh, 50-200(400) $\mu \mathrm{m}$ in diam when dry, cupulate to clavate when juvenile, cupulate when mature, stipitate on a 20 $40 \mu \mathrm{m}$ wide, $40-120 \mu \mathrm{m}$ long stipe. Disc shallow when fresh, bordered by a thin zone of marginal hairs which rise only slightly above the hymenium; margin prominent and incurved when dry, obscuring part of the hymenium. Hair cover dense, prominent, white. Colour pure white when fresh, brownish orange (M65) to Straw (K79) when dry, modified by the overall hair cover.

Ectal excipulum of textura prismatica, cells on middle flanks prismatic but typically inflated and/or elongated, 11-18(-26) x 3.4-5.6(-6.2) $\mu \mathrm{m}, \overline{\mathrm{x}}=$ $14.4 \times 4.9 \mu \mathrm{m}(\mathrm{n}=40), \bar{Q}=3.1$, becoming elongated and narrower towards margin, at the base narrowly prismatic. Inner excipulum of narrower and more elongated cells. Walls in ectal parts thin, at the base up to $0.6 \mu \mathrm{m}$ thick, CB-, CR-, MLZ-, LUG-, hyaline to clearly brown, such variation occurring even within the same apothecium, pigment distributed evenly in the walls.

Hairs $18-39 \times 2-3(-3.8) \mu \mathrm{m}$, narrowly conical, uncinate to apically undulating, more rarely straight, aseptate to basally two-septate, merging gradually into the excipulum, juvenile hairs and hairs on lower flanks often nearly cylindrical. Apex narrow, more rarely blunt, tapering to $0.5-1.0 \mu \mathrm{m}$, infrequently minutely solidified, without an apical globule in MLZ. Wall thin, never locally prominently thickened (except for the apex), CB-, CR-, MLZ-, LUG-, varying from hyaline to clearly brown and from smooth to prominently warted, such variation occurring within the same apothecium; warts brown when occurring on brown-walled hairs, otherwise hyaline, not deforming in any mountants. Solidifications $\mathrm{CR}+, \mathrm{CB}-, \mathrm{MLZ}-$.

Asci $28-39(-45) \times 3.4-4.5(-5.8) \mu \mathrm{m}, \overline{\mathrm{x}}=33.4 \times$ $4.1 \mu \mathrm{m}(\mathrm{n}=97), \bar{Q}=8.2$ in $C B$; eight-spored, cylindrical-clavate with a slightly conical apex. Apical pore MLZ+ when fresh in water, MLZ+ to MLZ++ when dry, LUG+ (blue). Wall in mature asci apically 

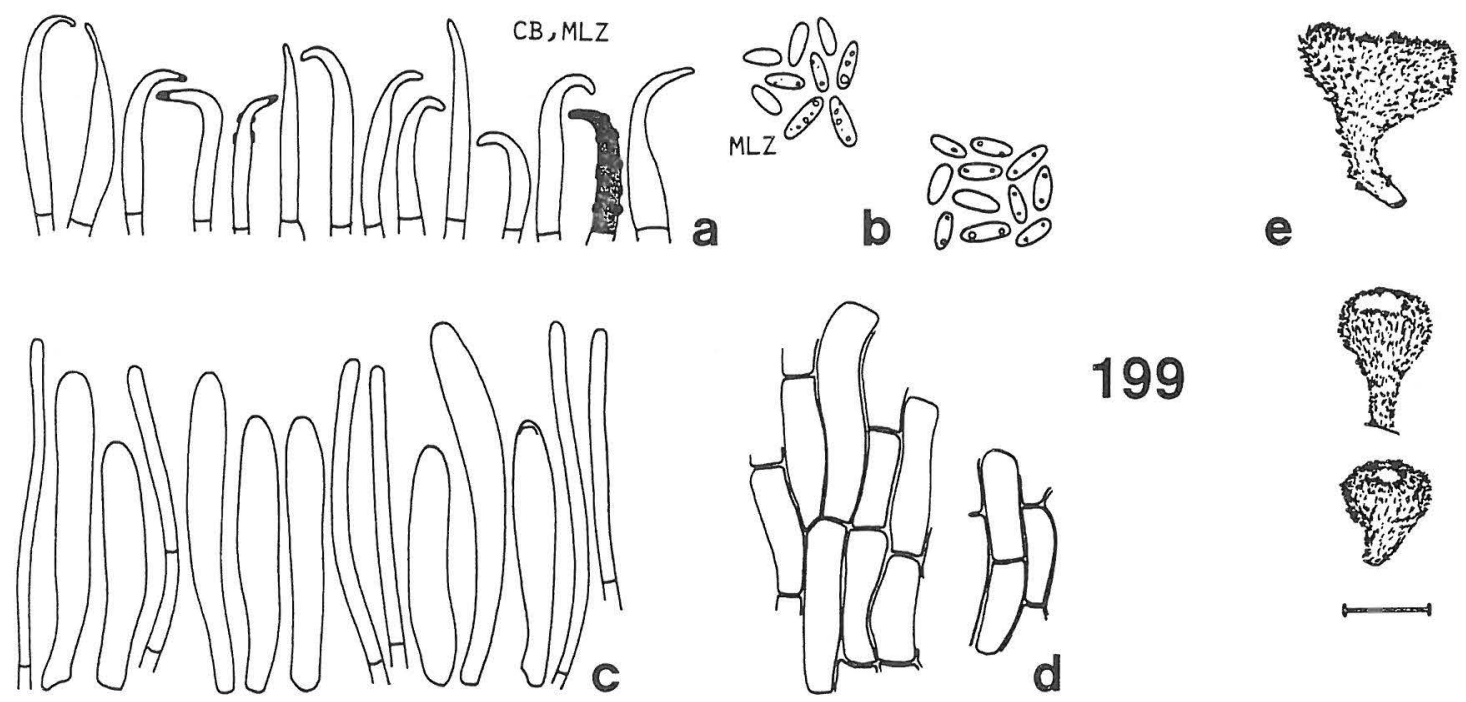

199
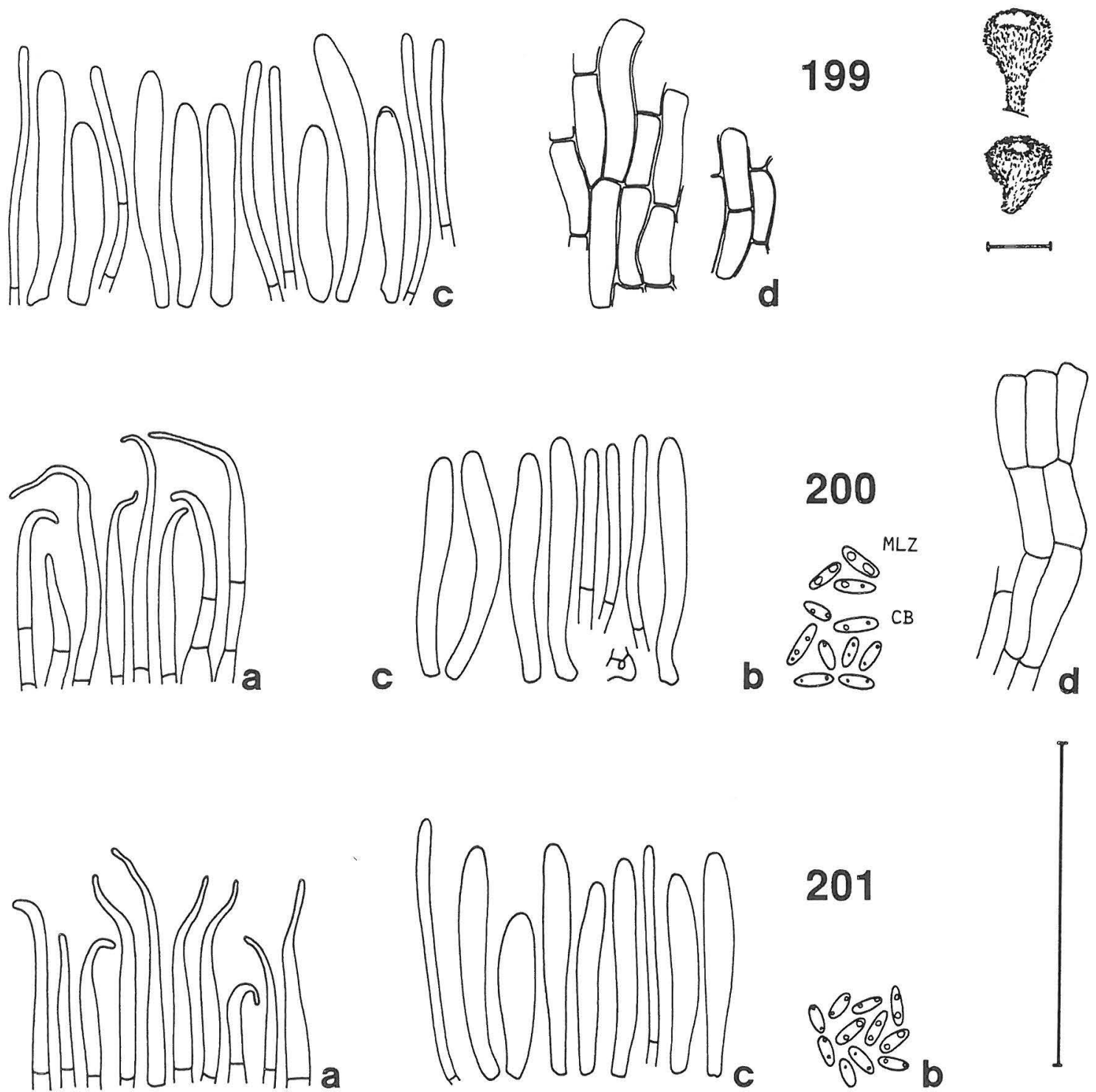

\section{1}

Figs. 199-201. Hamatocanthoscypha ocellata. a) hairs, b) spores, c) asci and paraphyses, d) excipulum, e) dry apothecia. Scale $50 \mu \mathrm{m}$, for apothecia $100 \mu \mathrm{m}$. - 199: Holotype. - 200: SH 84/262. - 201: SH 84/256. 
0.4-0.8 $\mu \mathrm{m}$ thick (in CB, MLZ), negative in all reagents, at the base with or without prominent $\mathrm{CR}++$ thickenings. Contents MLZ-, faintly orange in LUG. Asci arising from croziers.

Spores $5.6-12.0 \times 2.2-3.0 \mu \mathrm{m}, \overline{\mathrm{x}}=7.1 \times 2.5 \mu \mathrm{m}$ $(\mathrm{n}=23), \bar{Q}=3.0$ when fresh in water; $4.0-6.3(-$ $7.0) \times 1.8-2.4(-2.7) \mu \mathrm{m}, \overline{\mathrm{x}}=5.2 \times 2.1 \mu \mathrm{m}(\mathrm{n}=$ 163), $\bar{Q}=2.5$ in CB and MLZ; elliptic, often with somewhat tapered apices, rarely subfusiform-cuneiform, aseptate. Wall hyaline, not seen to become brownish in aged spores. Guttulae prominent, typically with one light guttula at each end when fresh in water, similar in CB, MLZ and CR, more rarely lacking, in one population guttulae typically refractive in $\mathrm{CR}$.

Paraphyses cylindrical, unpigmented, smooth or bearing a few small warts when fresh in water, smooth in CB and MLZ, smooth or often with velum-like, apical remnants in CR, first dichotomous branches occurring below the level of the asci. Terminal cells $19-40 \times 1.4-2.3 \mu \mathrm{m}, \overline{\mathrm{x}}=26.6 \times 1.7 \mu \mathrm{m}$ $(\mathrm{n}=30)$.

\section{Cultural characters. Not known.}

Distribution and phenology. In addition to the type locality in Japan, the species is known from four collections made in a small area in SW Finland. The type material was collected in late September and the Finnish collections were all made in mid-October after the first frosts. In Finland $H$. ocellata fruits to-

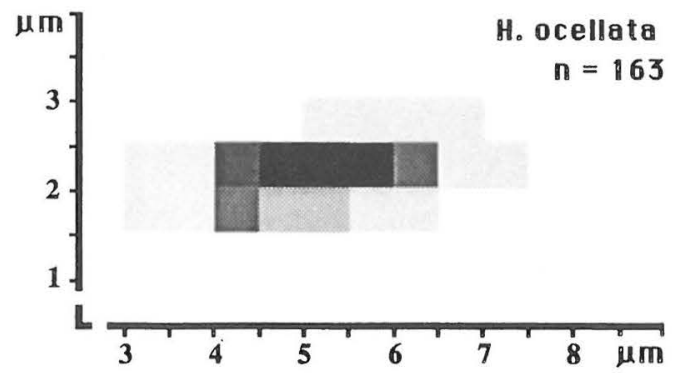

Fig. 202. Percentage sporogram of Hamatocanthoscypha ocellata.

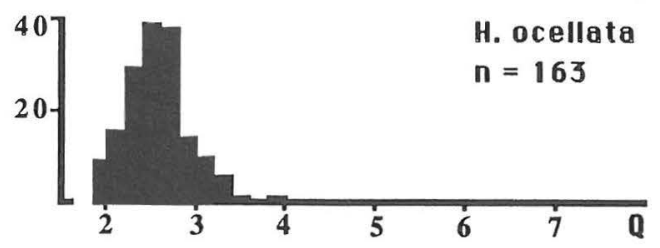

Fig. 203. The distribution of spore quotient $\mathrm{Q}$ in Hamatocanthoscypha ocellata. gether with Gorgoniceps viridula Huhtinen \& Iturriaga (1987).

Ecology. All the collections originate from fallen pieces of outer bark of conifers. In the Finnish material apothecia were found on pieces of Pinus bark next to the ground or on the underside of corticated twigs. As the type collection also occupies a corticated branch, it seems that $H$. ocellata has specialized to coniferous bark as a substrate. The closely related $H$. laricionis seems to have a different ecological preference. Outer bark is a difficult substrate to occupy for nearly all species of Hamatocanthoscypha and Hyaloscypha and $H$. ocellata is the only known species that appears to favour this substrate.

Discussion. The relatively broad and ellipsoid, biguttulate spores distinguish the present species from all other members of the genus. The hairs, with their narrowly conical shape and tapered apices, also differ from those of the closely related Hamatocanthoscyphas. Brown pigment in the hairs and excipular walls, prominent warts and sparsely encrusted paraphyses are further distinguishing characters.

The amount of brown pigment varies greatly between the apothecia of a single population. The range extends from hyaline apothecia to apothecia in which random areas, especially towards the base, are deeply pigmented. This pigmentation does not affect the overall colouring of the apothecia. Variable pigmentation is also seen in $H$. uncinata var. phaeotricha, in which the occurrence of warts on the hairs is related to the pigment distribution. In the present species the warts may be hyaline and may also occur on hyaline hairs.

The material is fairly homogeneous. The only notable differences between the type collection and the others are the refractive (in CR) bodies in the spores of the type and its lack of CR++ ascus bases. In spite of the great geographical distance between the collections, the substrate is the same and it seems that the present species is stable in all critical morphological and chemical characteristics.

As in $H$. obsoleta the hair shape of $H$. ocellata is closer to that prevailing in Hyaloscypha, than to the more cylindrical hairs of Hamatocanthoscypha. However, many characters support the present placement, for example the elongated/inflated excipular cells, stipitate apothecia, uncinate hairs, small spores, lack of dextrinoid reactions and presence of brown pigment in the excipulum and hairs.

Material studied: Finland. Satakunta: Punkalaidun, 1984 SH 84/256, 84/258, 84/262, 84/263 (TUR). Japan. Kushiro Prov.: Akan Nat. Park, 1963 Korf et al. 2527 (CUP, type). 


\section{Hamatocanthoscypha uncinata var. uncinata}

Hamatocanthoscypha uncinata (Phill.) Huhtinen, comb. nov. - Peziza uncinata Phill., Grevillea 5:117. 1877. - Dasyscypha uncinata (Phill.) Sacc., Syll. Fung. 8:456. 1889. Unguiculella uncinata (Phill.) Dennis, Kew Bull. 17:372. 1963. - Unciniella uncinata (Phill.) K. Holm \& L. Holm, Symb. Bot. Upsal. 21:19. 1977. - Lectotype (selected here): [U.S.A.,] California, Blue Canyon, on pine leaves, Dr. Harkness 3514 (NY, Ellis collection; examined).

Apothecia scattered to gregarious, superficial, $100-400 \mu \mathrm{m}$ in diam when dry, cupulate throughout development, sessile on a tapering base to shortly stipitate on a $60-70 \mu \mathrm{m}$ wide stipe. Disc shallow when dry, bordered by a copious margin, which is clearly incurved when dry, obscuring part of the hymenium. Hair cover dense but inconspicuous, blackish. Colour blackish, at the margin occasionally whitish.

Ectal excipulum of textura prismatica to textura angularis, such variation occurring mostly between the populations, cells on middle flanks, regularly prismatic to isodiametric, $4.9-9.2 \times 3.0-5.8(-$ 9.1) $\mu \mathrm{m}, \overline{\mathrm{x}}=6.6 \times 4.1 \mu \mathrm{m}(\mathrm{n}=40), \bar{Q}=1.6$, not notably changing in shape or size towards the margin, at the base small and roundish. Inner excipulum of narrower textura prismatica to textura porrecta. Walls in ectal parts $0.6-0.8 \mu \mathrm{m}$ thick, deep brown, pigment distributed evenly in the walls, inner excipulum lighter to hyaline, pigment often encrusted; walls MLZ-, CB-.

Hairs $20-42 \times 2.8-3.2 \mu \mathrm{m}$, cylindrical-tapering, uncinate, very rarely straight, aseptate to basally oneseptate, merging into the excipulum gradually. Apex tapering to $1.5-2.2 \mu \mathrm{m}$, not solidified, not exuding an apical globule in MLZ. Wall thin to clearly thickened due to concentration of pigment in the outer curve of the hairs, such variation occurring within the populations, clearly brown, becoming hyaline at the margin close to the hymenium, pigment distributed evenly in the walls or forming low, amoeboid warts.

Asci $30-45(-46) \times 3.8-4.8(-5.0) \mu \mathrm{m}, \overline{\mathrm{x}}=35.3 \times$ $4.1 \mu \mathrm{m}(\mathrm{n}=35), \bar{Q}=8.6$ in CB ; eight-spored, cylindrical-clavate with a slightly conical apex. Apical pore $\mathrm{MLZ}+$ when dry. Wall in mature asci 0.5 $0.8 \mu \mathrm{m}$ thick (in CB, MLZ). Contents MLZ-. Asci arising from croziers.

Spores $5.0-8.4(-9.8) \times 1.3-2.0(-2.5) \mu \mathrm{m}, \overline{\mathrm{x}}=$ $6.6 \times 1.8 \mu \mathrm{m}(\mathrm{n}=111), \bar{Q}=4.0$ in CB and MLZ; cuneiform-subfusiform, rarely oblong-elliptic, aseptate. Wall hyaline, not seen to become brownish in aged spores. Guttulae typically lacking in $\mathrm{CB}$ and MLZ.
Paraphyses cylindrical to slightly widened at the apex, unpigmented, first dichotomous branches occurring typically below the level of the ascal bases. Terminal cells $17-37 \times 1-2 \mu \mathrm{m}, \overline{\mathrm{x}}=27.8 \times 1.5 \mu \mathrm{m}$ $(n=20)$, the widened apices $1.8-2.3 \mu \mathrm{m}$ wide.

\section{Cultural characters. Not known.}

Distribution and phenology. In addition to the type collection from California, the taxon is known only from central Norway and northern Sweden. The Nordic collections were made at the end of August.

Ecology. The taxon has so far been collected from needles of Juniperus and Pinus. The original material includes a piece of outer bark, which in the original diagnosis (Phillips 1877) was said to be from Quercus. A coniferous host would have been more probable, but this could not be confirmed.

Discussion. The present taxon is distinguished from all the other members of the genus by the heavy pigmentation and the peculiar one-sided thickening of the hairs. The latter character differentiates it clearly from var. phaeotricha. The inclusion of a species with a brown excipulum in Hamatocanthoscypha is based on the widely varying pigmentation in var. phaeotricha, in which totally hyaline to blackish apothecia can occur within a single population. Such a variable character has little value concerning the generic delimitation of Hamatocanthoscypha.

The pigmentation also varies greatly in the present variety, as noted by Holm and Holm (1977). In the original material the pigment occurs on the hairs as low, amoeboid warts, but in the Nordic collections it is evenly distributed. The microtopography of the pigment makes the hairs look totally different, but both types show the one-sided thickening taxon. The other diagnostic characters are stable in the Nordic and Californian collections.

As noted by Holm and Holm (1977), the species is out of place in Unguiculella, the genus suggested by Dennis (1963). The Holms combined Peziza uncinata with Unciniella K. Holm \& L. Holm, which, however, was published five months later than Hamatocanthoscypha.

Material studied: Norway. S $\phi r$-Trøndelag: Oppdal, 1973 Holm \& Holm 92e (UPS). Sweden. Torne Lappmark: Kårsavagge, 1959 Santesson 13511d (UPS). U.S.A. California: Blue Canyon, 1876 Harkness (NY, lectotype and syntype). 

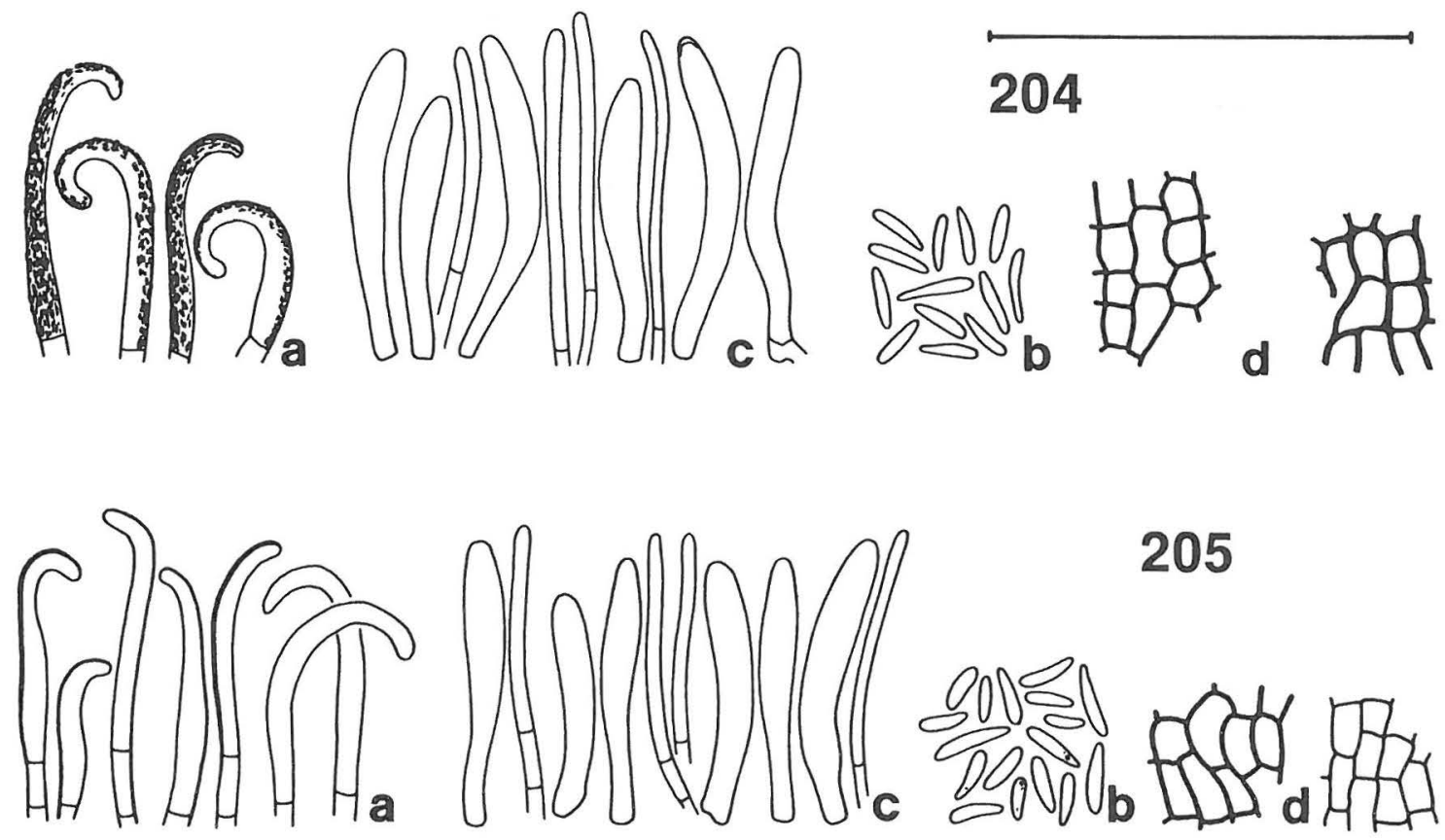

Figs. 204-205. Hamatocanthoscypha uncinata var. uncinata. a) hairs, b) spores, c) asci and paraphyses, d) excipulum. Scale $50 \mu \mathrm{m}$. - 204: Lectotype. - 205: Santesson 13511d.

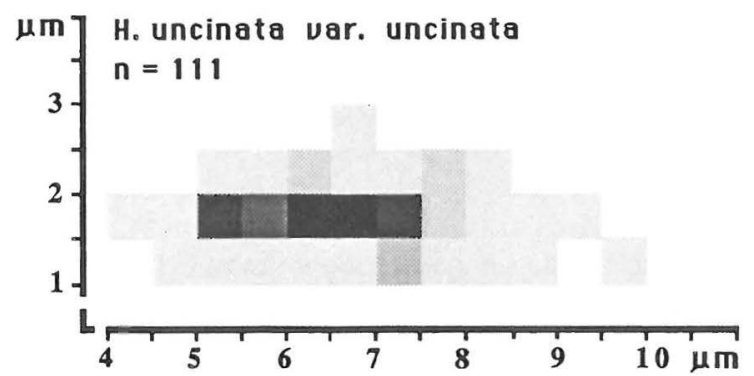

Fig. 206. Percentage sporogram of Hamatocanthoscypha uncinata var. uncinata.

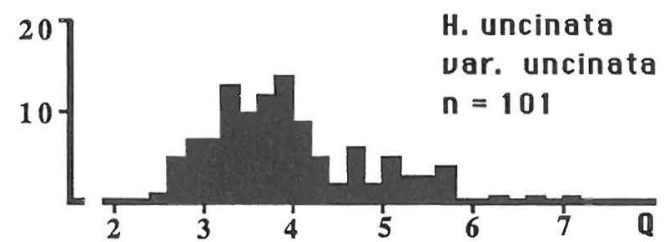

Fig. 207. The distribution of spore quotient $\mathrm{Q}$ in Hamatocanthoscypha uncinata var. uncinata.
36. Hamatocanthoscypha uncinata var. phaeotricha

Hamatocanthoscypha uncinata (Phill.) Huhtinen var. phaeotricha (K. Holm \& L. Holm) Huhtinen, comb. nov. - Unciniella laricionis (Velen.) K. Holm \& L. Holm var. phaeotricha K. Holm \& L. Holm., Symb. Bot. Upsal. 21:18; Figs. 3j, 12d, 12e. 1977. - Holotype (original designation): Norway, Sør-Trøndelag, Oppdal, Kongsvoll, W slope of S Kuntshø, near the river Blesebeck, on the upper border of the birchwood, alt. ca. $1000 \mathrm{~m}, 22$.VIII.1973 K. Holm \& L. Holm 86b (UPS, examined).

Apothecia scattered to gregarious, $50-220 \mu \mathrm{m}$ in diam when dry, cupulate throughout development, rarely undulate-crenulate when large, stipitate on a $30-50 \mu \mathrm{m}$ wide, $20-80 \mu \mathrm{m}$ long stipe, more rarely sessile on a somewhat tapering base. Disc shallow to plane when dry, bordered by a copious margin, which is clearly incurved when dry, obscuring part of the hymenium. Hair cover dense, varying between the populations from blackish brown to white. Colour varying within and between the populations from blackish to greyish brown (N70) to brownish (M77) to greyish (L92) to Cream (K75), the darker colours modified by a whitish hair cover, stipe often darker than the flanks, disc often lighter than the flanks. 

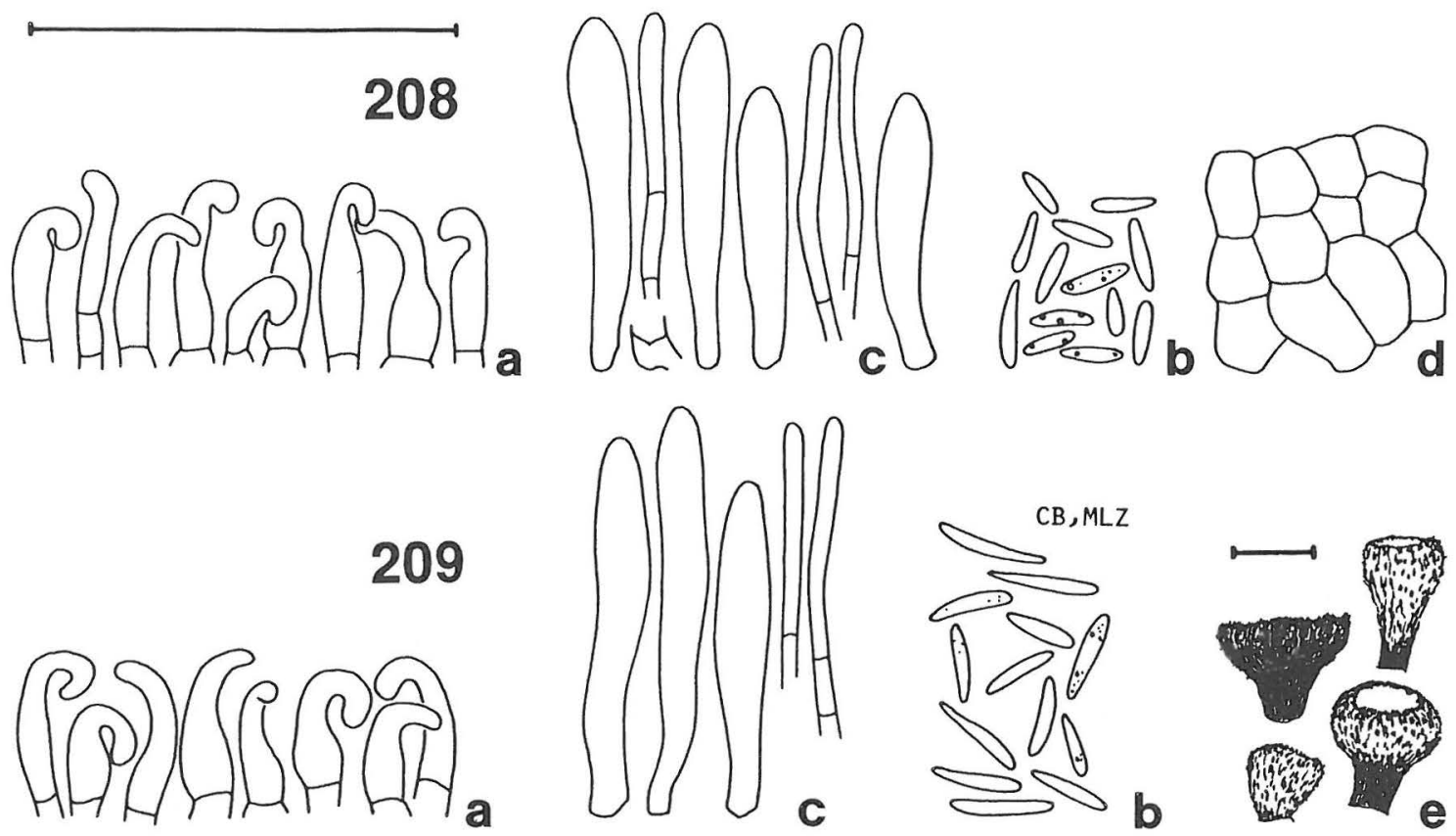

Figs. 208-209. Hamatocanthoscypha uncinata var. phaeotricha. a) hairs, b) spores, c) asci and paraphyses, d) excipulum, e) dry apothecia. Scale $50 \mu \mathrm{m}$, for apothecia $100 \mu \mathrm{m}$. — 208: Holotype. - 209: 1978 Andersson.

Ectal excipulum of textura prismatica, cells on middle flanks varying within the populations from isodiametric to regularly prismatic, more rarely clearly elongated, 4.3-13.5(-21.0) x 3.6-7.0(-8.2) $\mu \mathrm{m}$, $\overline{\mathrm{x}}=8.8 \times 5.0 \mu \mathrm{m}(\mathrm{n}=100), \bar{Q}=1.8$, rarely becoming smaller towards margin, at the base more roundish. Inner excipulum of narrower textura prismatica. Walls in ectal parts $0.4-1.0 \mu \mathrm{m}$ thick, deep to faintly brown to hyaline, such variation occurring within and between the populations, pigment distributed evenly in the walls, inner excipulum lighter to hyaline, pigmentoften encrusted; walls CB-, CR-, LUG-, $\mathrm{MLZ}-$, pigmented areas $\mathrm{BF}+, \mathrm{CRB}+, \mathrm{NR}+, \mathrm{TB}+$.

Hairs varying between the populations from 15$25 \times 2.2-3.2 \mu \mathrm{m}$ to $18-23 \times 3.0-5.8 \mu \mathrm{m}$, cylindricaltapering to lageniform, uncinate, aseptate to basally one-septate, merging gradually into the excipulum. Apex tapering to $1.8-2.0 \mu \mathrm{m}$ in narrow-haired populations, to $2.2-3.0 \mu \mathrm{m}$ in others, not solidified, without an apical globule in MLZ. Wall thin, never locally prominently thickened, clearly brown to hyaline, such variation may occur even within the same apothecium, smooth or sparsely warted when pig- ment occurrs as encrustations. Pigment $\mathrm{BF}+\mathrm{CRB}+$, $\mathrm{NR}+, \mathrm{TB}+, \mathrm{CB}-, \mathrm{CR}-$, LUG-, MLZ-

Asci $26-41 \times 4.2-5.0(-5.7) \mu \mathrm{m}, \overline{\mathrm{x}}=30.5 \times$ $4.7 \mu \mathrm{m}(\mathrm{n}=14), \bar{Q}=6.4$ in MLZ; $29-41 \times 4.3-$ $6.6(-6.8) \mu \mathrm{m}, \overline{\mathrm{x}}=33.7 \times 5.3 \mu \mathrm{m}(\mathrm{n}=26), \bar{Q}=6.4$ in CB. Apical pore MLZ+ when dry, LUG+ (blue). Wall in mature asci apically $0.4-1.0 \mu \mathrm{m}$ thick (in CB, MLZ). Contents MLZ-, frequently LUG+. Asci arising from croziers.

Spores 5.4-11.7(-13.6) $\times 1.2-2.6(-2.8) \mu \mathrm{m}, \overline{\mathrm{x}}=$ $8.1 \times 1.9 \mu \mathrm{m}(\mathrm{n}=150), \bar{Q}=4.3$ in CB and $\mathrm{MLZ}$; cuneiform-subfusiform, rarely oblong-elliptic, aseptate, less than $1 \%$ of the discharged spores one-septate. Guttulae lacking to minute in $\mathrm{CB}$, inconspicuous in MLZ, when present scattered and light.

Paraphyses cylindrical to slightly widened at the apex, unpigmented, first dichotomous branches typically occurring below the level of the ascal bases. Terminal cells $19-35 \times 1.5-2.3 \mu \mathrm{m}, \overline{\mathrm{x}}=24.7 \times$ $1.8 \mu \mathrm{m}(\mathrm{n}=35)$, the widened apices $1.8-2.8 \mu \mathrm{m}$ wide, mean septal intervals $7.2 \mu \mathrm{m}(\mathrm{n}=25)$.

Cultural characters. Not known. 


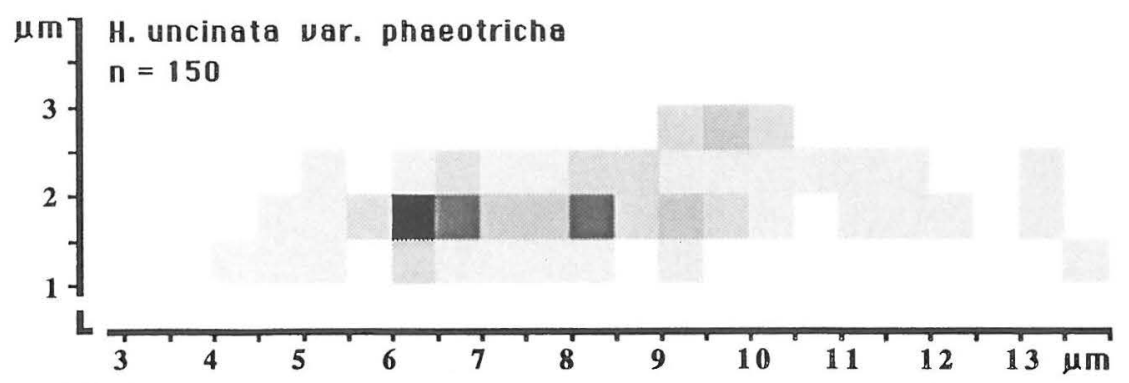

Fig. 210. Percentage sporogram in Hamatocanthoscypha uncinata var. phaeotricha.

Distribution and phenology. Outside Norway and Sweden, the variety is known from a single American collection. The few collections suggest a more or less continuous fruiting season, lasting from the early springtime occurrence of snow-free patches to the end of the growing season.

Ecology. The present material originates predominantly from juniper needles. The collection from the U.S.A. was made from needles of Abies. Holm and Holm (1977) suggested that this taxon is likely to be a true juniper fungus. Although not strictly so, var. phaeotricha may be ecologically less diversified than $H$. laricionis. The ecological ranges in these two taxa are only partially overlapping.

Discussion. The variety is related to the many species close to $H$. laricionis. $H$. uncinata var. uncinata differs mainly in its hairs, which have a unique one-sided thickening and are relatively long. Both characters are lacking in the present material. The hairs in var. phaeotricha vary between the populations from the "normal" nearly cylindrical and uncinate type to a rarer lageniform apically very broad type. The latter hair type does not occur in the other members of Hamatocanthoscypha. The apothecia of var, uncinata are always deeply pigmented and lack the variation found in var. phaeotricha. The hymenial characters are similar, but the excipular cells of the

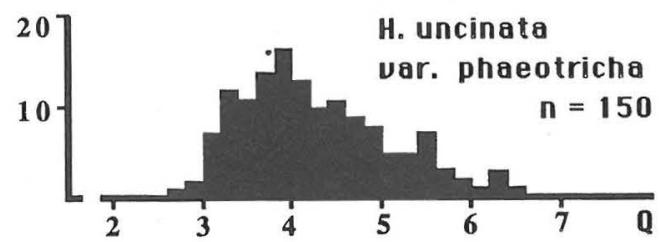

Fig. 211. The distribution of spore quotient Q in Hamatocanthoscypha uncinata var. phaeotricha. type variety are somewhat more isodiametric and darker than in var. phaeotricha. As discussed below, the pigmentation is variable in both these taxa with predominantly brown hairs and excipulum. They are treated as varieties rather than species, because the one-sided hair wall thickenings and other distinguishing characters are linked with the pattern of pigmentation, and the ecology and distribution overlap.

The variability in pigmentation in var. phaeotricha led Holm and Holm (1977) to treat it as a variety of Hamatocanthoscypha laricionis (as Unciniella laricionis). They pointed to intermediary forms with lighter apothecia and both brown and white hairs. They also believed that var. phaeotricha and var. laricionis can co-occur on the same substrate. Similar problems were encountered in the present study when scattered unpigmented apothecia were observed among swarms of brownish to blackish apothecia of var. phaeotricha in rich collections from juniper needles. The colour differences are due to variation in both hair and excipular pigmentation. The former may even vary in a single apothecium, the lower and marginal hairs being hyaline, or the variation can be seen at the margin, where the outermost hairs are pigmented and those closer to the hymenium are hyaline. In some populations the excipula show unbroken gradation from totally hyaline to basally or totally pigmented. In non-variable populations the hairs and excipula are always clearly pigmented. This variability does not, however, cause difficulties in delimitation from the light-coloured $H$. laricionis, which has a different type of excipulum and somewhat smaller spores.

Although the elongated excipular cells typical of Hamatocanthoscypha occur in some of the populations, these are all characterized by more prismatic to more isodiametric cells. This excipular structure connects the two brown-pigmented varieties and separates them clearly from $H$. laricionis. 
Material studied: Norway. Sфr-Trфndelag: Kongsvoll, 1973 Holm \& Holm 86b (UPS, type), 102a. Sweden. Östergötland: Östra Husby, 1968 Holm \& Holm 6a-68 (UPS). Uppland: Bălinge, 1974 Holm \& Holm 376c, 380b (UPS). Börje, Hässelby, 1974 Holm \& Holm 387k, 397e (UPS). Dalby, 1975 Holm \& Holm 442c (UPS); Upsala-Năs, 1973 Holm \& Holm 31d. Härjedalen: Tănnäs, 1967 Holm \& Holm 11b-67, 17f-67 (UPS). Torne Lappmark: Jukkasjärvi, 1978 Andersson (UPS). U.S.A. Washington: Yakima Co., E of Mt. Rainier Nat. Park, 1968 Denison \& Trappe (NYS 1007).

\section{Hamatocanthoscypha uncipila}

Hamatocanthoscypha uncipila (Le Gal) Huhtinen, comb. nov. - Hyaloscypha uncipila Le Gal., Bull. Soc. Mycol. France. 70:214, Fig. 12. 1954. - Uncinia uncipila (Le Gal) Raitv., Scripta Mycol. 1:74. 1970. — Lectotype (selected here): Fig. 12, Bull. Soc. Mycol. France 70:215. 1954.

Hamatocanthoscypha sulphureocitrina Svr., Česká Mykol. 37:65. 1983. - Holotype (original designation): [Czechoslovakia,] Bohemia meridionalis, Třeboň, inter locum "Prátr" dictum et piscinam Dubový rybnik, 20.XI.1968 Křǐ̌ \& Svrcek (PRM 829228, not examined).

Chrysothallus spiralis Velen. sensu Dennis, Kew Bull. 30:352, Fig. 3c. 1975.

Pezizella viridiflavescens Rehm in sensu Raitviir, Fol. Crypt. Est. 9:1. 1977.

Apothecia gregarious, superficial, $50-250 \mu \mathrm{m}$ in diam when dry, cupulate throughout development, rarely undulate-crenulate when large, clearly and stoutly stipitate, with stipe up to $120 \mu \mathrm{m}$ high and $90 \mu \mathrm{m}$ wide. Margin fleshy and incurved when dry, clearly to almost totally obscuring the hymenium. Hair cover dense, minute, white to sulphur-yellow. Colour sulphur-yellow when fresh, such apothecia rarely intermixed with totally hyaline apothecia, stipe typically hyaline to basally brownish, dry apothecia similar to Apricot (L65), colours modified or not by the hair cover.

Ectal excipulum of textura prismatica, cells on middle flanks prismatic but often inflated and/or elongated, varying between the populations from ca $8-15 \times 2-4 \mu \mathrm{m}$ to ca. $10-25 \times 4-9 \mu \mathrm{m}, \overline{\mathrm{x}}=12.7 \times$ $4.4 \mu \mathrm{m}$ ( $\mathrm{n}=70$, incl. both types), $\mathrm{Q}=2.9$, in largecelled type becoming smaller and regularly prismatic towards margin, on stipe narrowly prismatic. Inner excipulum of narrower textura prismatica. Walls in ectal parts $0.4-0.8 \mu \mathrm{m}$ thick, becoming thinner inwards, hyaline, at the base occasionally brownish, negative in all reagents.

Hairs $10-35 \times 2.0-3.8 \mu \mathrm{m}$, almost cylindrical to narrowly conical, uncinate, infrequently intermixed with straight to slightly bent hairs, aseptate to basally two-septate, especially at margin containing a resi- nous, evenly distributed, light yellowish pigment when fresh in water, in dry material pigment inconspicuous, not observed in $\mathrm{CB}, \mathrm{MLZ}, \mathrm{CR} ; \mathrm{BF}+$, $\mathrm{CRB}+, \mathrm{NR}+, \mathrm{TB}+, \mathrm{LUG}-$. Apex blunt, tapering to 1-2 $\mu \mathrm{m}$, not solidified, without an apical globule in MLZ. Wall thin, hyaline, dull, never locally prominently thickened, negative in all reagents, smooth when fresh in water, smooth in dry material, more rarely with a few, prominent warts; warts deforming or not in $\mathrm{CB}, \mathrm{MLZ}$.

Asci $34-59 \times 5.3-7.3 \mu \mathrm{m}, \overline{\mathrm{x}}=45.6 \times 6.3 \mu \mathrm{m}(\mathrm{n}=$ 11), $\bar{Q}=7.2$ when fresh in water; $28-47(-48) \times 4.4$ 5.8(-6.0) $\mu \mathrm{m}, \overline{\mathrm{x}}=37.6 \times 5.1 \mu \mathrm{m}(\mathrm{n}=35), \bar{Q}=7.3$ in MLZ; $23-39(-41) \times 3.2-5.8(-6.0) \mu \mathrm{m}, \overline{\mathrm{x}}=31.6 \times$ $4.2 \mu \mathrm{m}(\mathrm{n}=18), \bar{Q}=7.7$ in CB; eight-spored, cylindrical to cylindrical-clavate with a slightly conical apex. Apical pore MLZ- and LUG- in $63 \%$ of the populations, MLZ+ to MLZ++ and LUG+ (blue) in others. Wall in mature asci apically $0.5-1.0 \mu \mathrm{m}$ thick (in $\mathrm{CB}, \mathrm{MLZ}$ ), negative in all reagents, at the base with prominent $\mathrm{CR}++$ thickenings. Contents MLZ-, rarely $\mathrm{MLZ}+$ (orange), typically LUG+. Asci arising from croziers.

Spores $5.0-10.3(-11.5) \times 2-3 \mu \mathrm{m}, \overline{\mathrm{x}}=7.2 \times$ $2.6 \mu \mathrm{m}(\mathrm{n}=27), \bar{Q}=2.8$ when fresh in water; 4.8 $9.0(-10.3) \times 1.4-2.7(-3.0) \mu \mathrm{m}, \overline{\mathrm{x}}=6.7 \times 2.1 \mu \mathrm{m}(\mathrm{n}$ =126), $\bar{Q}=3.3$ in CB and MLZ; elliptic to oblongelliptic to subfusoid, more rarely cuneiform, aseptate in $80 \%$ of the populations, up to $32 \%$ of the discharged spores one-septate in others, often septate while still inside the asci, septum MLZ-, wall not seen to become brownish in aged spores. Guttulae inconspicuous to lacking when fresh in water, only tiny, dark bodies present, similar to often lacking in dry material.

Paraphyses cylindrical to slightly widened at the apex, typically containing yellowish substance similar to that inside the hairs, first dichotomous branches occurring below the level of the asci. Terminal cells $13-39 \times 1.0-2.6 \mu \mathrm{m}, \overline{\mathrm{x}}=28.1 \times 1.8 \mu \mathrm{m}(\mathrm{n}=30)$, the widened apices $1.8-2.5 \mu \mathrm{m}$ wide.

Cultural characters (3 populations, 11 strains). Radial growth moderate on MA, $15.1 \mathrm{~mm} /$ month (n $=47,2$ popul., 8 strains). Mats black, at the margin dark reddish brown (T13) or olivaceous (R91), extreme margin light reddish brown (N27) or whitish for 2-4 mm. Aerial mycelium lacking or rarely formed on a sector or on the inoculum, snow-white or grey (N92). Margin indistinct, even, submerged. Hyphal strands frequent, running in the agar. Zonation lacking, sector formation rare, in two populations not changing the colour of surrounding agar, in one population agar changed to reddish brown 

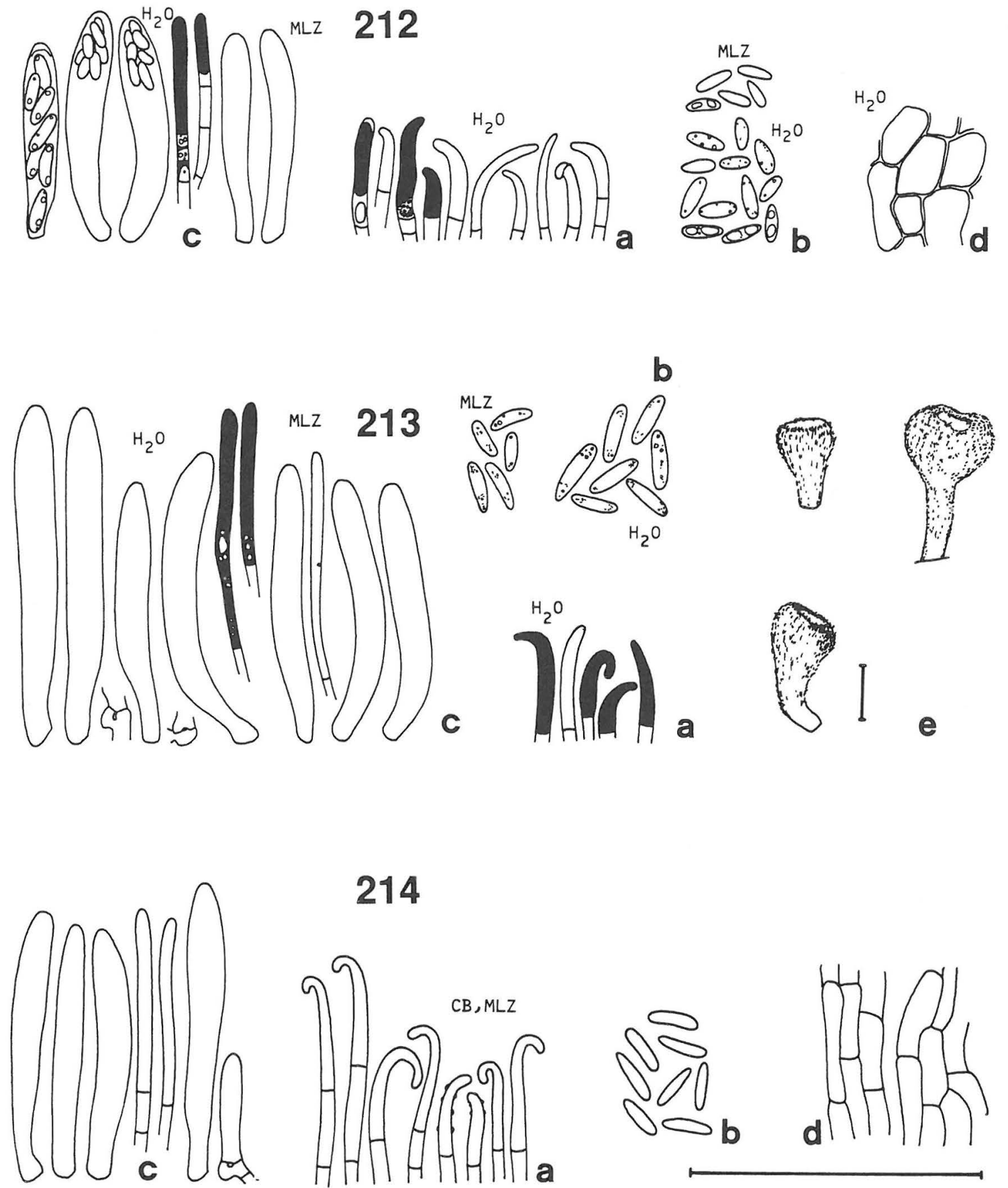

214

Figs. 212-214. Hamatocanthoscypha uncipila. a) hairs, b) spores, c) asci and paraphyses, d) excipulum, e) dry apothecia. Scale $50 \mu \mathrm{m}$, for apothecia $100 \mu \mathrm{m}$. - 212: SH 87/179. - 213: SH 86/134. — 214: 1973 Reid. 
(M20). Yeast-like growth frequent in all strains, forming gregarious conglomerations, these black to Cinnamon (N69). Context soft at surface, odour not specific.

Growth moderate on BA, PA, QA, LA. Producing a brown phialidic anamorph (1 single-spore strain) on PA, QA, LA. Apothecia not produced in single spore or multispore trials.

The dark basic colour with reddish brown or olivaceous tones at margin, lack of aerial mycelium and frequent yeast-like growth assist in distinguishing $H$. uncipila in culture. A brown, phialidic anamorph, with close resemblance to that linked to $H$. laricionis, characterized wood agar and leaf agar trials of one strain. The difference lay in the conidia, which were produced in chains in the present species. The same anamorph was not observed on MA and since no other strain was tested on wood agars, the connection reported here requires to be confirmed.

Distribution and phenology. Although rarely collected, the species occupies a geographically wide area. In addition to the material from Finland, Estonia, Czechoslovakia and Great Britain, stray collections extend the range into Svalbard, Japan and Canada. The type collection comes from Switzerland (Le Gal 1954). The collections were made between August and November.

Ecology. No clear trend can be seen within the scanty material. $H$. uncipila is apparently capable of exploiting a wide range of hosts. Three collections originate from Populus, in one of them apothecia occur on both wood and bark, in others on decorticated wood. The type was collected on cone scales of $P i$ nus and Abies and one further collection originates from cone scales of Picea. The species has possibly been introduced into the middle arctic Longyearbyen area in Svalbard, since it was found on coniferous

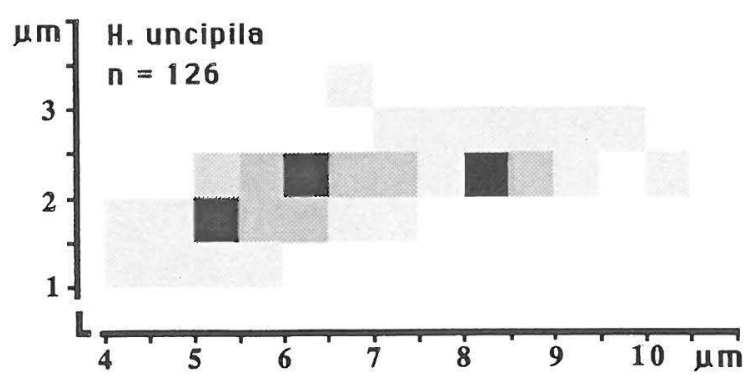

Fig. 215. Percentage sporogram of Hamatocanthoscypha uncipila. construction timber. Indigenous occurrence on driftwood, in the face of severe drought on the seashore, is highly improbable. The rest of the material originates from: Salix (1), Quercus (1), Betula (1), softwood (1). The type of Hamatocanthoscypha sulphureocitrina was collected on Salix (Svrcek 1983).

Discussion. The bright sulphur yellow colour distinguishes $H$. uncipila from other species of the genus. Although a yellowish pigment was observed in five populations of $\boldsymbol{H}$. laricionis, their general appearance remained white. However, in one population of $H$. uncipila studied in fresh condition, bright yellow apothecia grew together with totally white ones. A similar observation was made by Karsten in his collection from Betula. On closer scrutiny, these white apothecia were found to contain the same pigment inside the hairs and paraphyses, but in much smaller amounts, which did not affect the general colouring. Similar variation occurs in some populations of $P$. lachnobrachya. In such cases $H$. uncipila is distinguished from $H$. laricionis by the spore shape and size, and the stouter apothecia. In two collections the yellow colour had turned to Apricot upon drying.

The ascal apices show marked inconsistency in their reaction in MLZ. This occurrence of positive and negative $M L Z$ reactions is not coupled with any other morphological or chemical character studied. Hence, it is best accepted as a character varying between the populations. The size of the excipular cells and spore septation vary in the present species, as in fairly many species of Hyaloscypha. As in $\mathrm{H}$. laricionis, some hairs in an apothecium show occasional warts. In spite of this variation, $H$. uncipila is a taxonomically sound, easily recognizable species. The populations are linked by the rare type of pigmentation, ascus and spore characteristics, and hair characteristics.

The present material fits remarkably well with Le Gal's (1954) original diagnosis of Hyaloscypha uncipila. Unfortunately, no material could be located in Paris. The name should be reclaimed by neotypification with material originating from the original substrate of $H$. uncipila, preferably coupled with single spore isolates. The Code, however, only allows a lectotypification based on the microscopical plate of the original diagnosis (Arts. 7.4, 9.3).

The synonymy of $H$. sulphureocitrina is deduced solely from the diagnosis, because the type material was not available for study, but the similarity is so convincing that taxonomic confusion is not probable. Dennis (1975) treated the species under Chrysothallus spiralis, but Velenovsky's concept of that species represents a totally different taxon (see under Hyalo- 
scypha albohyalina). Hyaloscypha uncipila was transferred to the illegitimate Uncinia Velen. by Raitviir (1970a), who neglected the yellow pigmentation stressed in Le Gal's diagnosis and regarded the apothecia as similar to those of $H$. laricionis. Hence, Raitviir (1977b) later proposed combination with another species, Pezizella viridiflavescens Rehm. Study of a syntypic collection in $\mathrm{S}$ and the illustration by Nannfeldt (1932) indicate that this is a different species.

Although yellow, the pigment in $H$. uncipila differs from that characterizing the genus Phialina, which turns golden in $\mathrm{MLZ}$ and persists in CB and MLZ mounts. The distribution is also different, being diffuse in $H$. uncipila and vacuolate in Phialina. The diffuse nature of the pigment is easily demonstrated from dried apothecia, which in mass show a bright yellow colouring under the microscope but which after tapping lack any yellow tinge. Consequently, the pigment is not seen in CB or MLZ mounts. In the material from Japan, the pigment was clear in a water mount of dry apothecia but dissolved rapidly after MLZ was added. All in all, it seems that the yellow pigment inside the hairs and paraphyses of $H$. uncipila does not offer a strong link with the genus Phialina, or with those species of Hyaloscypha with yellowish, extraneous resin on the hairs.

Material studied: Canada. Alberta: Edmonton, 1987 SH 87/179* (TUR). Finland. Varsinais-Suomi: Turku, 1986 SH 86/134* (TUR). Etelä-Häme: Lempäälä, 1986 Læssøe (C). Tammela, 1870 PAK 2686 (H). Japan. Nagasaki Pref.: Unzen Nat. Park, 1957 Korf et al. 305 (CUP). Norway. Svalbard: Longyearbyen, 1988 SH 88/65* (TUR). Great Britain. Bedfordshire: Heath and Reach, 1973 Reid (K). U.S.S.R. Estonian S.S.R.: Distr. Rakvere, Vinni, 1970 AR (TAA). Heinassaar, 1979 AR (TAA).

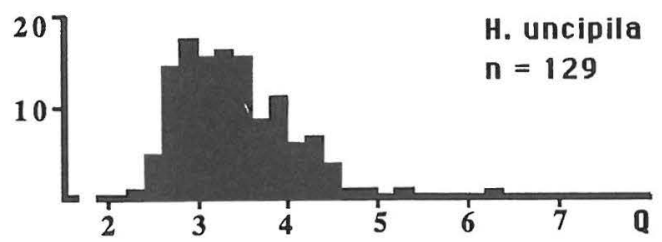

Fig. 216. The distribution of spore quotient $\mathrm{Q}$ in Hamatocanthoscypha uncipila. 


\section{PHIALINA}

Phialina Hőhnel, Mitt. Bot. Inst. Techn. Hochsch. Wien 3:61, 72. 1926. - Lectotype (unintentionally selected by Nannfeldt 1932: 272): Peziza deparcula P. Karst., Not. Săllsk. Fauna Flora Fenn. Förh. 10:191. 1869.

Setoscypha Velen., Monogr. Discom. Bohem.: 271. 1934. - Holotype (the only binomial mentioned): Setoscypha clavispora Velen., Monogr. Discom. Bohem.: 271. 1934.

Phialoscypha Raitv., Fol. Crypt. Est. 8:1. 1977. - Holotype (original designation): Peziza lachnobrachya Desm., Ann. Sci. Nat. 16:322. 1851.

Apothecia gregarious, more rarely confluent, superficial, $50-800 \mu \mathrm{m}$ in diam when fresh, 50$500 \mu \mathrm{m}$ in diam when dry, cupulate to scutellate to seemingly pulvinate, broadly to narrowly sessile to shortly stipitate. Disc shallow to plano-convex, in larger taxa often prominently convex after the margin becomes appressed to the substrate, disc spongy to even, margin consisting of a thin to prominent layer of hairs, not incurved when fresh, incurved or straight when dry. Hair cover sparse to dense, prominent to minute. Colour white to yellowish to vivid or sulphur yellow when fresh, Dry apothecia whitish to yellowish to vivid yellow or reddish brown, more rarely brownish or greyish. Ectal excipulum of textura prismatica, rarely of textura angularis, cells lying parallel to the receptacle surface, on middle flanks with mean length ranging between 6.9 and $14.5 \mu \mathrm{m}$, mean width between 4.1 and $8.3 \mu \mathrm{m}$, mean $Q$ value 1.8 , becoming narrower inwards, more roundish towards the base, towards margin similar or becoming smaller or narrower. Inner ex- cipulum lacking or poorly developed, showing weak delimitation from the thin subhymenium. Walls in ectal parts thin, $2.5 \mu \mathrm{m}$ thick, hyaline or yellowish to basally brownish, in one species faint to medium brown close to the margin MLZ-. Hairs narrowly conical to slightly lageniform, often with a filiform apex, apically flexuose-curved-cincinnate, more rarely straight, aseptate when short, multiseptate when longer. Apex tapering to a fine point, rarely septate, thin-walled, rarely solidified, without an apical globule in MLZ. Wall thin to somewhat thickened, hyaline, smooth or minutely warted at the apex, MLZ-. Warts stable to deforming in $\mathrm{CB}$ and MLZ. Hairs rarely with traces of resinous exudates but frequently with bright yellow pigment, which is not dissolved in CB or MLZ. Asci cylindrical-clavate, eight-spored or four-spored, mean length ranging between ca. 26 and $55 \mu \mathrm{m}$, mean width between 4.7 and $7.0 \mu \mathrm{m}$, pore MLZ+, asci arising from croziers. Spores elliptic to fusoid, mean length ranging between 6.1 and $15.9 \mu \mathrm{m}$, mean width between 1.8 and $3.2 \mu \mathrm{m}$, aseptate to septate, septum MLZ-, aguttulate to clearly guttulate. Paraphyses cylindrical to clavate, not protruding clearly above the level of the asci, frequently containing similar pigment to that seen inside the hairs, dichotomously branched, rarely with numerous branches close to apex, often closely septate, mean width of lower parts ranging between 2.0 and $2.6 \mu \mathrm{m}$. Most species inhabiting leaves, more rarely herbaceous stems or ferns, only exceptionally on woody substrates.

\section{Key to species of Phialina}

The spore measurements were made on material mounted in CB or MLZ.

1 Asci predominantly eight-spored

- Asci predominantly four-spored

2 Growing on Filipendula, excipulum prismatic to seemingly hyphoid, walls clearly thickened, dry apothecia clearly brown to reddish 45 P. ulmariae

- Growing on leaves or ferns, excipulum prismatic, walls thin-walled to slightly thickened, apothecia whitish to yellowish when dry

3 Growing on leaves, exceptionally other litter, spores fusoid to oblong-elliptic, apothecia yellow to whitish

- Restricted to ferns, spores fusoid, apothecia bright yellow

4 Spores narrowly fusoid, $1.3-2.0 \mu \mathrm{m}$ wide, densely guttulate ... 40 P. lachnobrachya 38 P. flaveola

- Spores frequently $>10 \mu \mathrm{m}$ long

6 Hairs rigid, conical, multiseptate $100-250 \mu \mathrm{m}$ long, excipulum hyaline $41 P$. lachnobrachyoides Hairs flexuose, uncinate, simple to apically branched, aseptate, $15-34 \mu \mathrm{m}$ long, excipulum brownish 
7 Hairs apically strongly curved or cincinnate, spores oblong-elliptic, minutely guttulate, frequently $>7 \mu \mathrm{m}$ long, mean spore length $6.8-7.7 \mu \mathrm{m}$ 39 P. foliicola

- Hairs straight to slightly flexuose, spores elliptic, clearly guttulate, rarely $>7 \mu \mathrm{m}$ long, mean spore length ca. $6 \mu \mathrm{m}$ $43 P$. pusilla

\section{Phialina flaveola}

Phialina flaveola (Cooke) Raitv., Fol. Crypt. Est. 9:1. 1977. - Peziza flaveola Cooke, Grevillea 1:131. 1873. - Mollisia flaveola (Cooke) Phill., Man. Brit. Discomyc.: 192. 1887. Pezizella flaveola (Cooke) Sacc., Syll. Fung. 8:288. 1889. Urceolella flaveola (Cooke) Boud., Hist. classific. discom. Europe: 128. 1907. - Dasyscypha flaveola (Cooke) Höhnel, Mitt. Bot. Inst. Techn. Hochsch. Wien 3:76. 1926. - Hyaloscypha flaveola (Cooke) Nannf., Trans. Brit. Mycol. Soc. 23: 252. 1939. - Type: not designated, syntype suitable for lectotypification (cited by Dennis 1949): King's Lynn, Norfolk, Sept. 1872 (K, Herb. Cooke; not seen).

?Calloria chrysostigma (Fr.) Phill. sensu Phill., Man. Brit. Discomyc.: 328. 1887.

Pezizella chrysostigma (Fr.) Sacc. sensu Rehm, Rabenh. Krypt.-Fl. ed. 2, 1(3): 682. 1892.

Apothecia scattered to gregarious, superficial, 50$300 \mu \mathrm{m}$ in diam when dry, cupulate throughout development, margin in larger apothecia not appressed to the substrate, narrowly sessile on an ill-defined base. Disc shallow, not spongy or pitted when mature, bordered by a thin zone of marginal hairs, which rise only slightly above the hymenium; margin only slightly incurved when dry. Hair cover dense, light yellowish, entangled. Colour vivid yellow to sulphur yellow when fresh, more rarely ochraceous; dry apothecia similar, rarely more brownish (L79).

Ectal excipulum of textura prismatica, cells on middle flanks regularly prismatic to somewhat isodiametric, 6.6-14.5(-22) x 3.8-7.7(-10.6) $\mu \mathrm{m}, \overline{\mathrm{x}}=$ $10.4 \times 5.6 \mu \mathrm{m}(\mathrm{n}=80), \overline{\mathrm{Q}}=1.9$, becoming smaller towards margin. At the base composed of brown textura angularis to textura epidermoidea. Inner excipulum of similar texture. Walls in ectal parts thin to $0.4 \mu \mathrm{m}$ thick, up to $1.0 \mu \mathrm{m}$ thick in three populations, in one population such variation dependent on age, hyaline, brown at the base, $\mathrm{CB}-, \mathrm{CR}_{-}$, more rarely $\mathrm{CB}+, \mathrm{CR}+$, negative in other reagents.

Hairs $16-53 \times 2-4 \mu \mathrm{m}$, narrowly conical to slightly lageniform, flexuose to cincinnate, typically one- to two-septate, the basal parts frequently with bright yellow pigment. Apex tapering to $0.5-1.0 \mu \mathrm{m}$, seldom slightly widened, not solidified, without an apical globule in MLZ. Wall thin and dull, never locally prominently thickened, hyaline, smooth in 55\% of the collections, in others with scattered, minute, apical warts, warts deforming or not (in CB, MLZ), wall negative in all reagents. Pigment amorphous, persistent in all reagents, $\mathrm{BF}+$ (red), $\mathrm{CB}+$ (blue), $\mathrm{CRB}+$ (blue), LUG+ (reddish brown), NR+ (red), $\mathrm{TB}+$ (green), in CR forming "hollow" bodies with a light centre and reddish margins.

Asci $26-44 \times 5.3-6.8(-7.5) \mu \mathrm{m}, \overline{\mathrm{x}}=35.2 \times$ $6.1 \mu \mathrm{m}(\mathrm{n}=22), \bar{Q}=5.8$ in MLZ; $27-41(-44) \times$ 4.6-6.6(-7.8) $\mu \mathrm{m}, \overline{\mathrm{x}}=33.9 \times 5.5 \mu \mathrm{m}(\mathrm{n}=51), \bar{Q}=$ 6.4 in $\mathrm{CB}$; four-spored, cylindrical-clavate with a slightly conical apex. Apical pore MLZ++, LUG+ (blue). Wall in mature asci apically $0.8-1.2 \mu \mathrm{m}$ thick (in CB, MLZ), wall negative in all reagents. Contents MLZ-, infrequently LUG+ (faintly reddish). Asci arising from croziers.

Spores 11.8-17.8(-20.2) x 1.8-3.5(-3.8) $\mu \mathrm{m}, \overline{\mathbf{x}}$ $=14.7 \times 2.5 \mu \mathrm{m}(\mathrm{n}=128), \bar{Q}=6.2$ in CB and MLZ; fusoid or broadly fusoid to oblong-elliptic (rarely), straight to slightly curved, rarely apically beaked, aseptate in ca. $80 \%$ of the populations, often oneseptate in others, rarely while still inside the ascus, septum MLZ-. Wall hyaline, not seen to turn brownish in aged spores. Guttulae inconspicuous to lacking in MLZ, abundant and tiny to few and large in $\mathrm{CB}$ (dry material).

Paraphyses cylindrical to clavate, frequently containing bright yellow, amorphous pigment similar to that inside the hairs, first dichotomous branches occurring typically at or below the level of the ascal bases. Terminal cells $12-37 \times 1.6-3.0 \mu \mathrm{m}, \overline{\mathrm{x}}=20.6$ $\times 2.2 \mu \mathrm{m}(\mathrm{n}=50)$, clavate apices $2.3-4.2 \mu \mathrm{m}$ wide, closely septate below, mean septal intervals $9.8 \mu \mathrm{m}$ $(\mathrm{n}=30)$.

\section{Cultural characters. Not known.}

Distribution and phenology. P. flaveola seems to be a European taxon, known from the Nordic countries to Corsica. Easternmost records are represented by two collections from the Caucasus. Collected between June and November, without any obvious peak. Evidently fruiting somewhat later than $P$. ulmariae.

Ecology. The species has been collected almost solely from dead stems and leaves of Pteridium aquilinum. The single exception originates from Sweden (Holm \& Holm 411, UPS), a collection made from leaves of Dryopteris dilatata. 

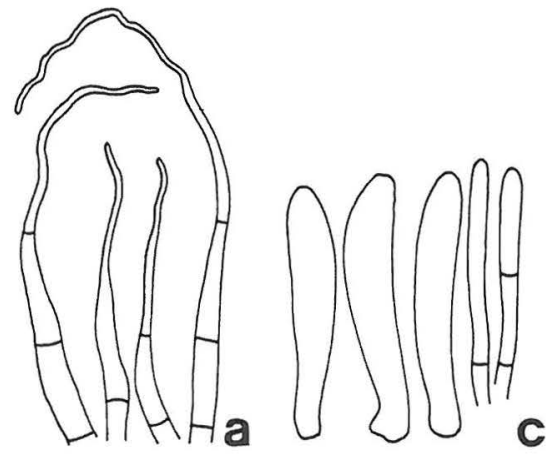

217
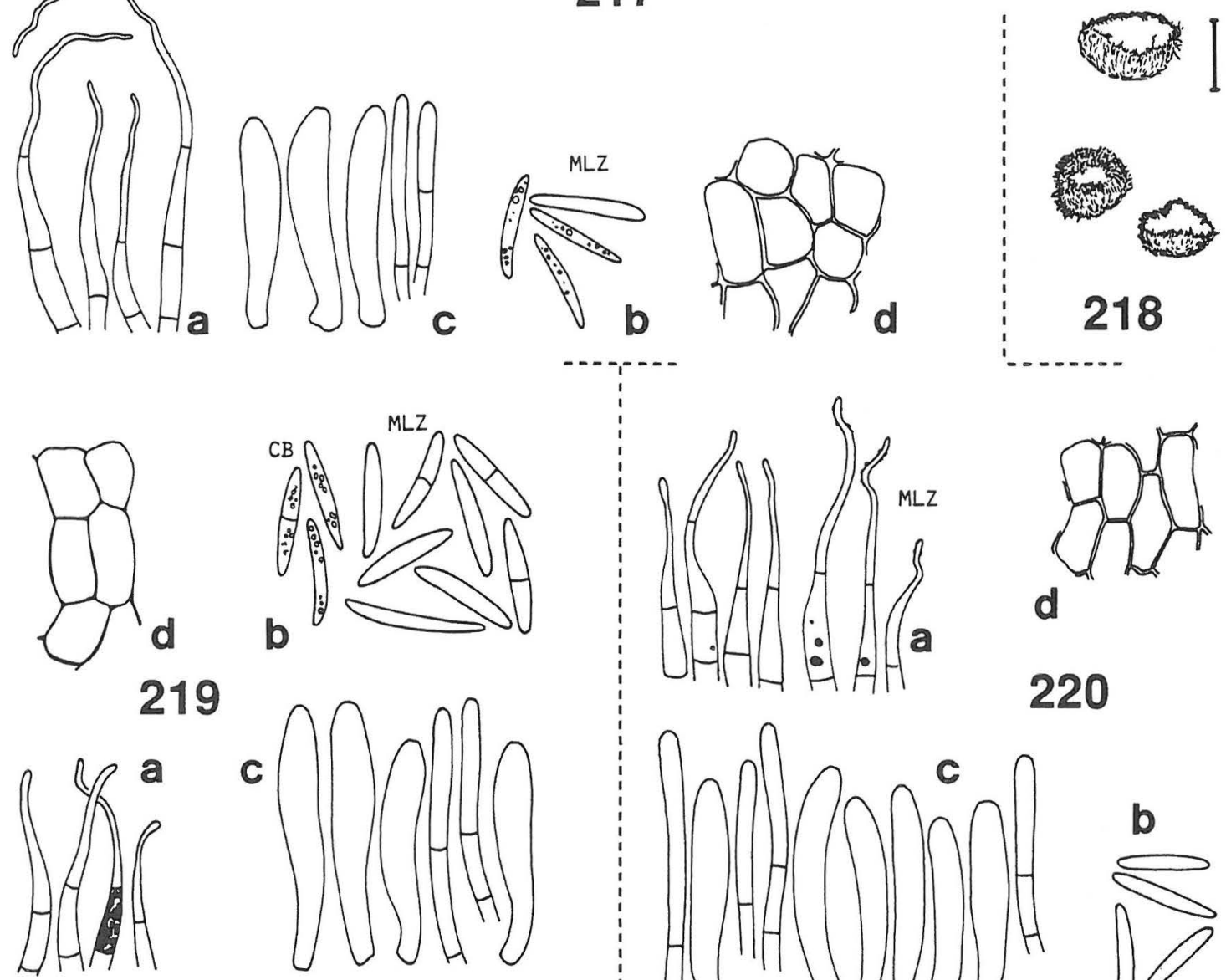

220
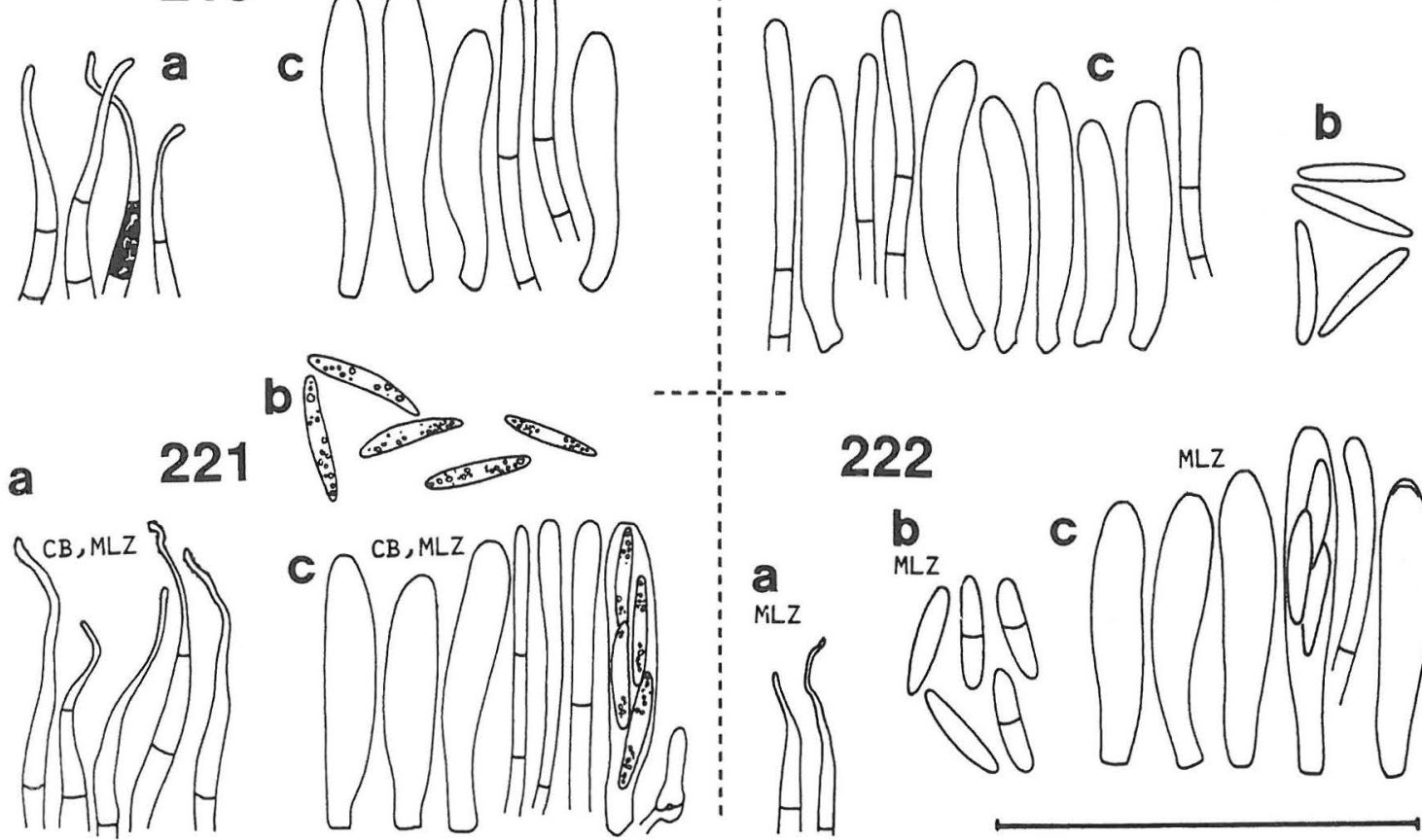

Figs. 217-222. Phialina flaveola. a) hairs, b) spores, c) asci and paraphyses, d) excipulum. Scale $50 \mu \mathrm{m}$, for apothecia $100 \mu \mathrm{m} .-$ 217: Kryptogamae exsiccatae 1439. - 218: Dry apothecia from various collections. - 219: Demoulin \& Korf 72-94. - 220: 1978 Clark. - 221: 1963 Dennis. - 222: Korf \& Demoulin 72-92. 
Discussion. The species is recognized by the substrate, yellow apothecia, four-spored asci and fusoid spores. It is closely related to $P$. lachnobrachya and $P$. ulmariae. Although there is considerable overall similarity between the three, they seem to be genetically isolated. In addition to the differences discussed below, this conclusion is supported by the unlikelihood that a certain genome would result in specialization on three widely different substrates only, i.e. leaves, Pteridium and Filipendula. As the data on the ecology are scanty, however, it is not possible to decide whether the three taxa should be recognized as varieties, or as different species. The second alternative, which does not disturb the existing classification, is chosen here.

Morphologically, $P$. flaveola is distinguished from $P$. lachnobrachya by divergent trends rather than clear differences. The variation in spore shape is smaller in $P$. flaveola, the excipulum is composed of less isodiametric cells and the excipular walls tend to be slightly thicker. The present species also lacks long-haired populations. The shape of dry apothecia is cupulate, in contrast to the often pulvinate shape in $P$. lachnobrachya, due to the margins becoming appressed to the substrate. The latter species has larger apothecia with often spongy discs, and greater variation in the apothecial colour.

$P$. ulmariae was treated as a synonym of $P$. flaveola by Raitviir (1970a). The morphological differences between the two taxa are, indeed, slight but as noted previously, it is unlikely that a single species would specialize on Filipendula and Pteridium, while avoiding numerous other types of herbaceous stems. Some fairly clear-cut differences also exist. The excipulum of $P$. ulmariae is massively thickened, to the extent of resembling textura oblita and this structure may well be responsible for the characteristic brown to reddish brown colouring in its dry apothecia. Its apothecia are clearly larger than those of $P$. flaveola and there is a difference in the fruiting periods.

$P$. flaveola lacks pronounced variation, the populations being especially uniform in ascal and spore characteristics. The difference between short-haired and longer-haired populations is not marked. The populations vary most widely in excipular structure. This variation parallels that frequently observed in Hyaloscypha, i.e. there are small-celled and largecelled populations and the same applies to excipular wall thickness.

I have not studied all the syntypes of Peziza flaveola and refrain from lectotypifying the species. Dennis (1949) studied and illustrated Plowright's collection in Herb. Cooke made in September, which would be a logical choice if sufficiently abundant.

Exsiccatae studied: Cooke, M., Fungi Brit. exs. 550 (IMI, K). - Kryptogamae exsiccatae 1439 (S, from Austria). Phillips, W., Elvellacei britannici 129 (K). - Rabenhorst, G., Fungi Europaei 2210 (CUP, K, M, PAD). - Rehm, H., Ascomyceten 564 (S, from Switzerland).

Material studied: Belgium. Liège (BR). Finland. Pohjois-Savo: Vehmersalmi, 1985 SH 85/89 (TUR). France. Corsica: Foret d'Aitone, 1972 Demoulin \& Korf 72-92, 72-94 (CUP). German Democratic Republic. Sachsen: Pulsnitz, 1882 Staritz (S, Herb. Rehm). Great Britain. Mon-

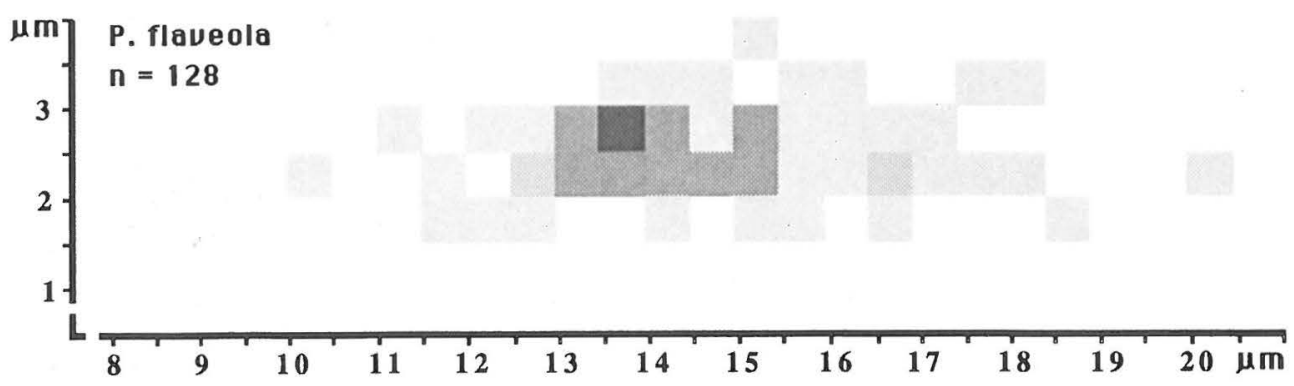

Fig. 223. Percentage sporogram of Phialina flaveola.

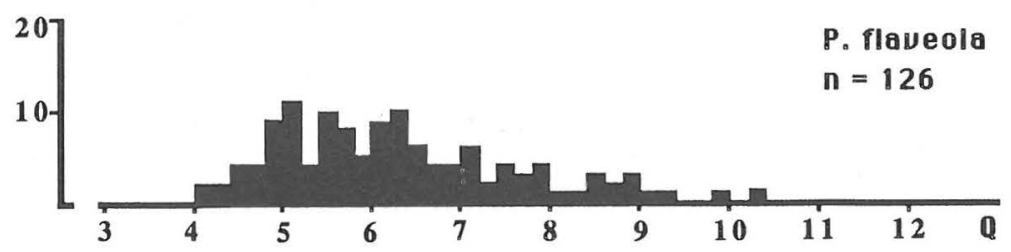

Fig. 224. The distribution of spore quotient $\mathrm{Q}$ in Phialina flaveola. 
mouthshire: Not localizable, 1964 Graddon 1678 (K). Herefordshire: Gorsley, 1976 Graddon 2923 (IMI). Warwickshire: Dumbles Wood, 1972 Clark (K). Hertfordshire: Linton Wood, 1976 Graddon (K). Argyll: Mull, 1978 Clark (E). W. Ross: Kinlochleven, 1963 Dennis (K). Norway. Akershus: Ringiåsen, 1971 Bøhler 13/71 (O). Sweden. Dalarna: Sundborn, 1974 Holm \& Holm 411 (UPS). Uppland: Dalby, 1976 Holm \& Holm 931d (UPS). U.S.S.R. Georgian S.S.R.: Lagodekhi Nat. Res., 1967 AR (TAA, two collections).

\section{Phialina foliicola}

Phialina foliicola (Graddon) Huhtinen, comb. nov. - Uncinia foliicola Graddon, Trans. Brit. Mycol. Soc. 69:263, Fig. 14. 1977. - Holotype (original designation): [Great Britain,] Warwickshire, Bannams Wood, ad folia marchida Alni glutinosae, X.1970 Clark (K, not seen).

Hyaloscypha himalayensis Arendh. \& R. Sharma, Mycotaxon 25:127; PI. 3:1-7 and P1. 4:1-6. 1986. - Holotype (original designation): [Bhutan,] near Chimakothi, Bunakha, 29.VII.1981 Sharma (PAN 17694, not seen; isotype in Herb. Arendholz examined).

Apothecia gregarious, superficial, 50-100(150) $\mu \mathrm{m}$ in diam when dry, cupulate, centrally sessile to indistinctly stipitate, almost naked to minutely hairy. Disc plane when dry, not spongy or pitted when mature, bordered by an inconspicuous, plane margin, which is rarely slightly incurved. Hair cover sparse to dense, inconspicuous, somewhat whitish at the margin. Colour somewhat brownish (L77) in dry material.

Ectal excipulum of textura angularis to textura prismatica, cells on middle flanks angular and isodiametric to prismatic, $4.4-10.0 \times 2.5-6.8$ (7.5) $\mu \mathrm{m}, \overline{\mathrm{x}}=6.9 \times 4.9 \mu \mathrm{m}(\mathrm{n}=30), \bar{Q}=1.4$, becoming clearly smaller towards margin and base. Inner excipulum composed of similar cells. Walls in ectal parts $0.4 \mu \mathrm{m}$ thick, hyaline, basally moderately brown, fragmentarily CB+, MLZ-.

Hairs 8-19 x 1.5-2.5 $\mu \mathrm{m}$, narrowly conical, apically strongly curved to cincinnate, more rarely straight to flexuose, aseptate, with bright yellow pigment. Apex tapering to $0.5-0.8 \mu \mathrm{m}$, not solidified, without an apical globule in MLZ. Wall thin and dull, never locally prominently thickened, hyaline, smooth, CB-, MLZ-. Pigment amorphous, yellow, $\mathrm{MLZ}+$ (golden), $\mathrm{CB}+$.

Asci $26-34 \times 4.8-6.0 \mu \mathrm{m}, \overline{\mathrm{x}}=29.7 \times 5.5 \mu \mathrm{m}(\mathrm{n}=$ 18), $\bar{Q}=5.4$ in MLZ; $19-31 \times 4.3-6.2 \mu \mathrm{m}, \overline{\mathrm{x}}=25.6$ x $5.3 \mu \mathrm{m}(\mathrm{n}=58), \bar{Q}=4.8$ in CB; eight-spored, clavate to cylindrical-clavate with a slightly conical apex. Apical pore MLZ+. Wall in mature asci apically $0.5-0.8 \mu \mathrm{m}$ thick. Contents MLZ-. Asci arising from croziers.

Spores $6.0-8.3(-8.7) \times 1.6-2.2 \mu \mathrm{m}, \overline{\mathbf{x}}=7.3 \times$ $2.0 \mu \mathrm{m}(\mathrm{n}=80), \bar{Q}=3.8$ in $\mathrm{CB}$ and MLZ; oblong- elliptic, more rarely elliptic, aseptate. Wall hyaline, not seen to become brownish in aged spores. Guttulae inconspicuous to lacking in CB and $M L Z$.

Paraphyses cylindrical to slightly clavate, frequently containing bright yellow, amorphous pigment similar to that inside the hairs. Terminal cells 6$20 \times 1.6-2.3 \mu \mathrm{m}, \overline{\mathrm{x}}=12.5 \times 2.0 \mu \mathrm{m}(\mathrm{n}=11)$, closely septate below, mean septal intervals $6.8 \mu \mathrm{m}$ $(n=14)$.

\section{Cultural characters. Not known.}

Distribution and phenology. The type of Hyaloscypha himalayensis originates from Bhutan. Describing the species, Sharma et al. (1986) reported one additional collection from the Indian subcontinent. The species has been described and reported from Great Britain under the name Uncinia foliicola (Graddon 1977, Clark 1980, Ellis \& Ellis 1985). The range is further extended by a collection from Colombia. The collections were made between June and October.

Ecology. P. foliicola fruits mainly on leaves and has been reported to grow on leaves of $A$ lnus, $B e$ tula, Rubus, and Salix (Ellis \& Ellis 1985). The material of $H$. himalayensis also originates from leaves (Sharma et al. 1986), whereas the Colombian collection was made on a fern rachis.

Discussion. Phialina foliicola is closely related to $P$. pusilla, but has differently shaped hairs, somewhat narrower spores, wider asci and more angular and basally brownish excipulum. The excipulum is coloured beyond the extreme base, which in many species of the genus shows dark brown tissue.

The variation between the three studied specimens is fairly small. The hairs in the type of $H$. himalayensis are more dwarfish than those of the others. As these collections are alike in other characters, such variation can be accepted in the species. The gelatinous sheath surrounding the spores, which was reported by Sharma et al. (1986), was observed by me only once (in $\mathrm{CR}$ ), which may be due to the deteriorating effect of $\mathrm{CB}$ and MLZ.

In the Colombian material, the apical pores of the asci were very faintly $M L Z+$ and the characteristic positive reaction was observed only after $\mathrm{KOH}$ pretreatment. The amount of pigment in this particular specimen was small and there was no bright golden $M L Z$ reaction. The scantiness of the specimens does not allow closer scrutiny of the total variation in this respect, but the overall similarity and stability in many characters allow inclusion of the collection in $P$. foliicola. 

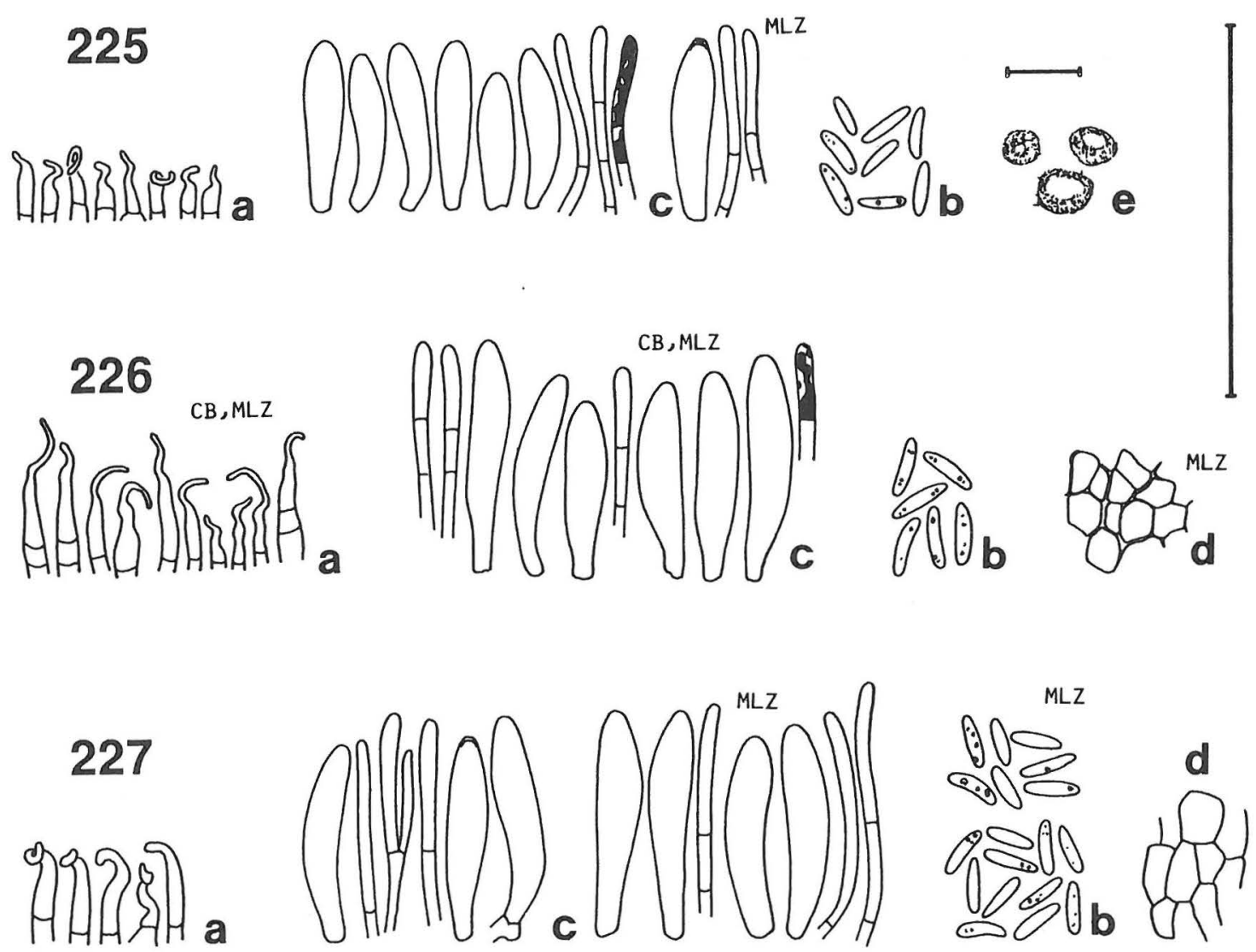

Figs. 225-227. Phialina foliicola. a) hairs, b) spores, c) asci and paraphyses, d) excipulum, e) dry apothecia. Scale $50 \mu \mathrm{m}$, for apothecia $100 \mu \mathrm{m}$. - 225: Isotype of Hyaloscypha himalayensis. - 226: 1978 Ellis \& Ellis. - 227: Dumont et al. Co-232.
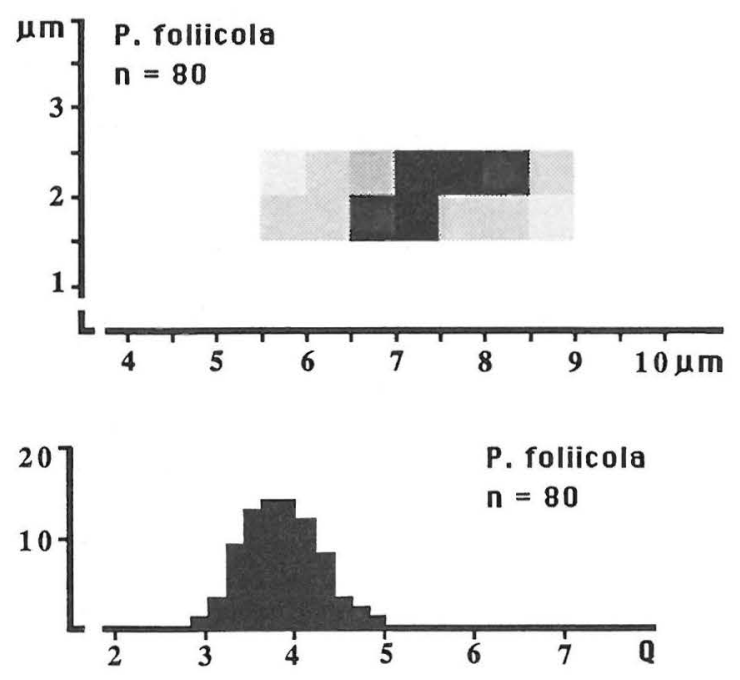

Although the type of Uncinia foliicola was not studied, the possibility of taxonomic confusion is slight. The original diagnosis by Graddon (1977) matches the present material very well, the only critical feature not mentioned being the nature of the ascal bases in the type collection.

Material studied: Bhutan. Bunakha, near Chimakothi, 1981 Sharma (Herb. Arendholz, isotype of Hyaloscypha himalayensis). Colombia. Dpto. Cundinamarca: Boyaca, 1974 Dumont e tal. Co-232 (NY). Great Britain. Suffolk: Norman Gwatkin Res., 1978 Ellis \& Ellis (IMI).

Fig. 228 (Left above). Percentage sporogram of Phialina foliicola.

Fig. 229 (Left below). The distribution of spore quotient $\mathrm{Q}$ in Phialina foliicola. 


\section{Phialina lachnobrachya}

Phialina lachnobrachya (Desm.) Raitv., Scripta Mycol. 1:27. 1970. - Peziza lachnobrachya Desm., Ann. Sci. Nat. 16:322. 1851. - Trichopeziza lachnobrachya (Desm.) Sacc., Syll. Fung. 8:418. 1889. - Pezizella lachnobrachya (Desm.) Höhnel, Sitzungsber. Akad. Wiss. Wien, Math. Nat. Cl. 115: 1285. 1906. - Urceolella lachnobrachya (Desm.) Boud., Hist. classific. discom. Europe: 130. 1907. - Hyaloscypha lachnobrachya (Desm.) Nannf., Nova Acta Soc. Sci. Upsal. Ser. 4., 8:273. 1932. - Phialoscypha lachnobrachya (Desm.) Raitv., Fol. Crypt. Est. 8:2. 1977. - Calycellina lachnobrachya (Desm.) Baral in Baral \& Krieglsteiner, Beih. Z. Mykol. 6:52. 1985. - Setoscypha lachnobrachya (Desm.) Svr., Ceská Mykol. 41:196. 1987. - Lectotype (selected here): [France,] sur les deux faces des feuilles seches des Acer pseudo-platanus et platanoides, en automne (as Pl. crypt. France, ed. 2: 1603; K; examined).

Peziza araneocincta Phill., Gard. Chron. 14:308, Fig. 57. 1880. - Lachnella araneocincta (Phill.) Phill., Man. Brit. Discomyc.: 271. 1887. - Trichopeziza araneocincta (Phill.) Sacc., Syll. Fung. 8:417. 1889. - Dasyscypha araneocincta (Phill.) Massee, Brit. fung.-fl.: 337. 1895. - Hyaloscypha lachnobrachya (Desm.) Nannf. var. araneocincta (Phill.) Dennis, Mycol. Pap. 32:73. 1949. - Lectotype (selected here): [Great Britain,] Leigh Down, Bristol, Bucknall (K, examined).

Pezizella punctiformis (Grev.) Rehm f. minor Rehm ex Starb., Bih. Svensk Vet.-Akad. Handl. 21:31; Pl. 1:20. 1895, nom. inval. in synon. - Pezizella minor Starb., Bih. Svensk Vet.-Akad. Handl. 21:31. 1895. - Hyaloscypha minor (Starb.) Boud., Hist. classific. discom. Europe: 127. 1907. Eupezizella minor (Starb.) Höhnel, Mitt. Bot. Inst. Techn. Hochsch. Wien 3:79. 1926. - Lectotype (selected here): Suecia, Uplandia, Uppsala, in foliis Aceris platanoidis, VIII.1893 Starbäck (S, Ex Herb. Rehm; examined). Out of the three fragments of the original collection (in S) the selected one bears Rehm's annotations.

Pezizella punctiformis (Grev) Rehm var. minor Rehm, Bih. Svensk Vet.-Akad. Handl. 21:31. 1895, nom. inval. err. cit. in synon.

Pezizella tetraspora Feltg., Rec. Mem. Trav. Soc. Bot. Luxemb. 16:54. 1903. - Lachnella tetraspora (Feltg.) Höhnel, Sitzungsber. Akad. Wiss. Wien, Math. Nat. Cl. 115: 1284. 1906. - Mollisiella tetraspora (Feltg.) Boud., Hist. classific. discom. Europe: 142. 1907. - Dasypezis tetraspora (Feltg.) Höhnel, Mitt. Bot. Inst. Techn. Hochsch. Wien 3:79. 1926. - Holotype (the only specimen mentioned): Luxemburg, Scheidhof, an făulen Salix pentandra-blättern, XI.1902 Feltgen (F 8641, Herb. Höhnel; examined).

Pezizella jaapii Rehm in Jaap, Verh. Bot. Ver. Prov. Brandenb. 49:9. 1907. - Lectotype (selected here): [German Democratic Republic, Kreis Parchim,] Triglitz in der Prignits, auf faulenden Birken- und Pappelnblättern, 2.X.1904 Jaap (S, Ex Herb. Rehm; examined). The specimen bears Rehm's annotations.

Setoscypha clavispora Velen., Monogr. Discom. Bohem.: 271; P1. 17:65-69. 1934. - Holotype (the only specimen mentioned): [Czechoslovakia,] Bohemia, Mnichovice, Huba¿kov, ad. fol. Quercus, 31.X.1930 Velenovský (PRM 153018, examined).

?Hyaloscypha oligospora Velen., Monogr. Discom. Bohem.: 283; Pl. 14:38. 1934. - Lectotype (Svrček 1985: 213): [Czechoslovakia,] Bohemia, Mnichovice, Hubackov, [on fallen leaves of Quercus sp. lying in a swamp,] 30.IX.1929 Vele- novsky (PRM 151172, not seen; synonymy indicated by Svrček 1985).

?Hyaloscypha lutea Raschle, Nova Hedwigia 30:655, Fig. 1. 1978. - Holotype (original designation): Switzerland, Canton VS, Aletschwald Nature Reserve, 10.IX.1970 Müller \& Dennis (ZT, not seen).

Hyaloscypha betularum Svr., Česká Mykol. 36:146; P1. 1:3. 1982. - Holotype (original designation): [Czechoslovakia,] Bohemoslovakia, Nemíž prope Vlasim, area tuta "Na ostrově", 23.IX.1981 Svrcek (PRM, not seen). The synonymy is based on a study of a paratype, see discussion in the text.

Apothecia gregarious, superficial, up to $800 \mu \mathrm{m}$ in diam when fresh, typically $100-400 \mu \mathrm{m}$ in diam when dry, cupulate when juvenile, cupulate to scutellate when mature, when fresh margin in larger apothecia typically appressed to the substrate and the apothecia seemingly pulvinate, narrowly sessile on an ill-defined base or on a 30-50 $\mu \mathrm{m}$ wide short stipe. Disc plane to convex when fresh, often spongy and pitted when mature, bordered by a thin zone of marginal hairs, which are appressed to the substrate or rise above the hymenium; margin typically inconspicuous when dry, only slightly incurved. Hair cover sparse, whitish. Colour vivid yellow to yellow to white when fresh, such variation occurring partly within the same population, the light yellowish discs occasionally showing whitish areas; dry apothecia very pale yellowish to ochraceous to yellow (K85) to vivid yellow, such variation also occurring within the same population, more rarely colour slightly more brownish (K89) to Beige (L91).

Ectal excipulum of textura prismatica, cells on middle flanks varying between the populations from somewhat isodiametric to regularly prismatic (more rare), 7.4-13.0(-21.0) x 4.8-9.0(-10.0) $\mu \mathrm{m}, \overline{\mathrm{x}}=$ $9.9 \times 6.3 \mu \mathrm{m}(\mathrm{n}=90), \bar{Q}=1.6$, becoming smaller towards margin, at the base composed of brown textura epidermoidea. Inner excipulum composed of slightly narrower cells. Walls in ectal parts thin to $0.4 \mu \mathrm{m}$ thick in $96 \%$ of the populations, $0.8-1.0 \mu \mathrm{m}$ thick in others, hyaline, brown at the base, $\mathrm{CB}$-in ca. $80 \%$ of the populations, $\mathrm{CB}+$ in others, negative in other reagents.

Hairs $18-64(-130) \times 2.5-4.0 \mu \mathrm{m}$, narrowly conical to slightly lageniform, often with a long filiform apex, flexuose to cincinnate, more rarely straight, one- to multiseptate, the basal parts frequently with bright yellow pigment. Apex tapering to $0.4-1.0 \mu \mathrm{m}$, very rarely inconspicuously solidified, without an apical globule in MLZ. Wall thin and dull, in a few populations somewhat thickened and refractive, never locally prominently thickened, hyaline, smooth in $75 \%$ of the populations (in CB and MLZ), occasionally bearing scattered, minute, apical warts in others, warts deforming or not in $\mathrm{CB}$ and $\mathrm{MLZ}$, wall nega- 

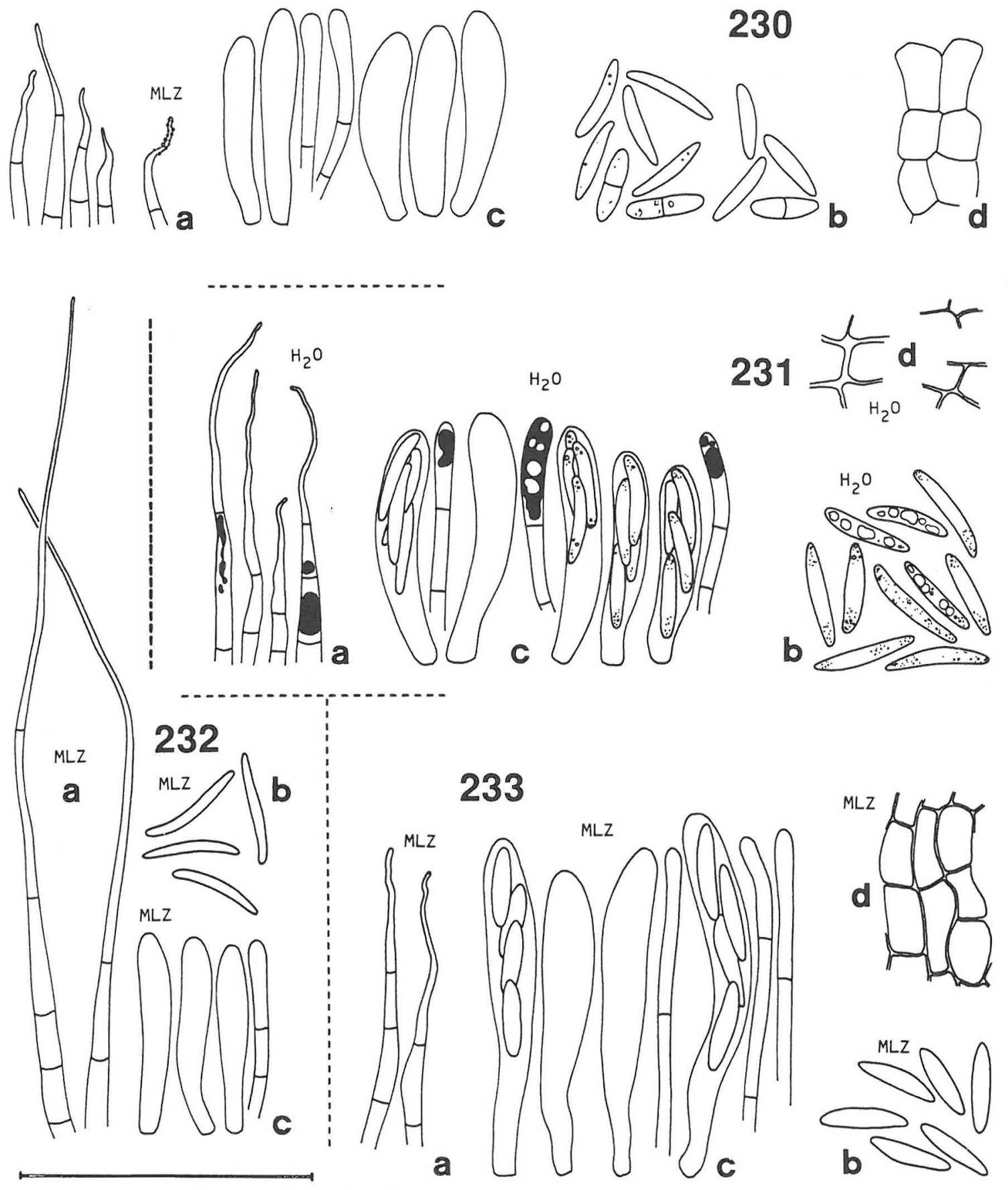

Figs. 230-235. Phialina lachnobrachya. a) hairs, b) spores, c) asci and paraphyses, d) excipulum, e) dry apothecia. Scale $50 \mu \mathrm{m}$, for apothecia $100 \mu \mathrm{m}$. - 230: Isolectotype of Pezizella punctiformis f. minor. - 231: SH 87/195. - 232: Matheis 980. - 233: Matheis 970. — 234: SH 84/180. — 235: SH 84/176. 

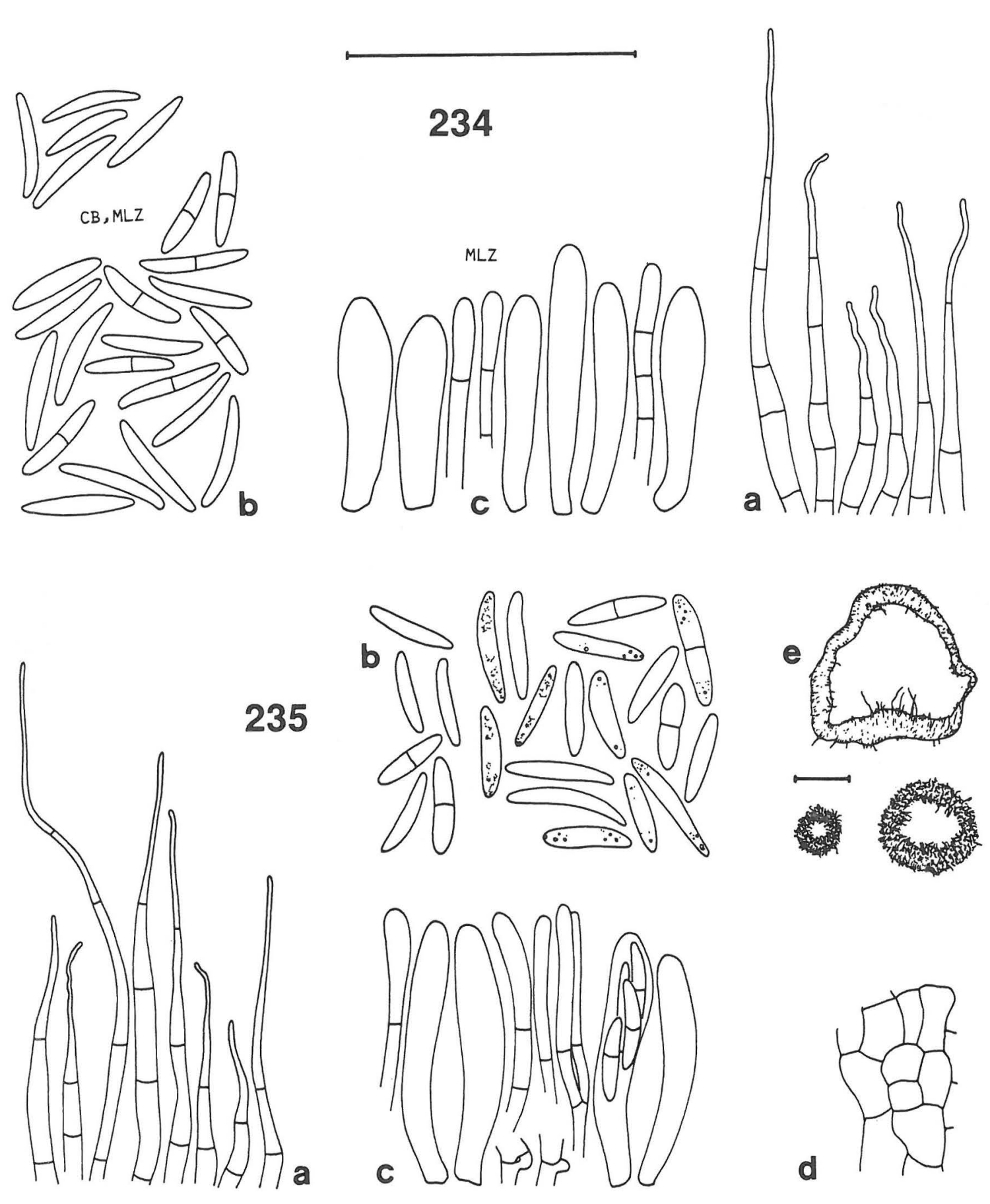
tive in all reagents. Pigment amorphous, persistent in all reagents, $\mathrm{BF}+(\mathrm{red}), \mathrm{CB}+$ (blue) $\mathrm{CRB}+$ (blue), LUG+ (reddish brown, R16), MLZ+ (golden), NR+ (red), TB+ (green), in CR forming "hollow" bodies with a light centre and reddish margins.

Asci $28-52(-63) \times 5-8(-9.7) \mu \mathrm{m}, \overline{\mathrm{x}}=37.3 \times$ $6.3 \mu \mathrm{m}(\mathrm{n}=84), \overline{\mathrm{Q}}=5.9$ in MLZ; $26-44(-53) \times$ $4.0-7.4(-8.4) \mu \mathrm{m}, \overline{\mathrm{x}}=33.8 \times 5.7 \mu \mathrm{m}(\mathrm{n}=112), \bar{Q}$ $=6.0$ in $\mathrm{CB}$; four-spored (in two populations rarely six-spored, in one population once eight-spored), cylindrical-clavate with a slightly conical apex. Apical pore MLZ++ when fresh and dry, LUG+ (blue). Wall in mature asci apically $0.8-1.3 \mu \mathrm{m}$ thick (in $\mathrm{CB}, \mathrm{MLZ}$ ), negative in all reagents. Contents MLZ-, very rarely MLZ+ (orange), rarely LUG+. Asci arising from croziers.

Spores 11.0-18.8(-22.2) x 2.0-3.5(-4.0) $\mu \mathrm{m}, \overline{\mathrm{x}}$ $=15.0 \times 2.7 \mu \mathrm{m}(\mathrm{n}=425), \bar{Q}=5.9$ in CB and MLZ; mostly fusoid to narrowly fusoid when in asci, after being discharged similar or becoming more broadly fusoid to oblong-elliptic, straight to slightly curved to apically beaked (especially in asci), aseptate in $77 \%$ of the populations, frequently one-septate after being discharged in others, rarely while still inside the asci. Wall hyaline, not seen to become brownish in aged spores. Guttulae prominent and light to lacking when fresh in water, typically inconspicuous to frequently lacking in dry material.

Paraphyses cylindrical to clavate, frequently containing bright yellow, amorphous pigment similar to that inside the hairs, first dichotomous branches occurring typically at or below the level of the ascal bases. Terminal cells 8-34 x (1.3-)2.0-3.7 $\mu \mathrm{m}, \overline{\mathrm{x}}=$ $17.7 \times 2.5 \mu \mathrm{m}(\mathrm{n}=50)$, clavate apices $2.3-4.0 \mu \mathrm{m}$ wide, closely septate below, mean septal intervals $9.2 \mu \mathrm{m}(\mathrm{n}=50)$.

Cultural characters (1 population, 3 strains). Radial growth slow on MA, $10.3 \mathrm{~mm} / \mathrm{month}(\mathrm{n}=10,1$ popul., 2 strains). Mats snow-white to Cream (K75) to Corn Silk (K80), colouring uniform or with dark brown (R75), blackish or greyish black (S92) central areas. Aerial mycelium lacking and surface doughlike or restricted to $5 \mathrm{~mm}$ from the inoculum, low, cottony, white. The central area often folded or strongly convoluted. Margin indistinct, minutely fimbriate, submerged. Hyphal strands lacking, zonation and sector formation occasional, showing rhythmic growth in unicolorous mat or with an indistinct reddish (P17) zone. No colour change in surrounding agar, no yeast-like growth. Context somewhat tough at surface, odour not distinct.

Growth moderate on LA, QA, retarded on BA, PA. On MA producing a dematiaceous anamorph in abundance after 9 months (single spore and multispore trials) but conidia not borne. No apothecia formed in single-spore or multispore trials.

The abundant, black conidiophores densely covering the central areas, or the convoluted appearance together with the light colouring and dough-like surface characterize $P$. lachnobrachya in culture. As the species is apparently rare in Finland, only one population was obtained for isolation, the results thus being preliminary. Production of apothecia in culture was reported by Müller (1977), but his description suggests that part of the material belonged to some other species, as eight-spored asci were mentioned. The reports of apothecial production in culture under the names $H$. lachnobrachya var. araneocincta (Müller 1977) and H. lutea (Raschle 1978) represent $P$. lachnobrachya as circumscribed here. In both cases no anamorph was produced in culture.

Distribution and phenology. The species is widely distributed over most of Europe, and reaches Italy (Raschle 1978) and Algeria in the Mediterranean area. Collections from North America, the Caucasus, Soviet Far East and Japan indicate an extensive

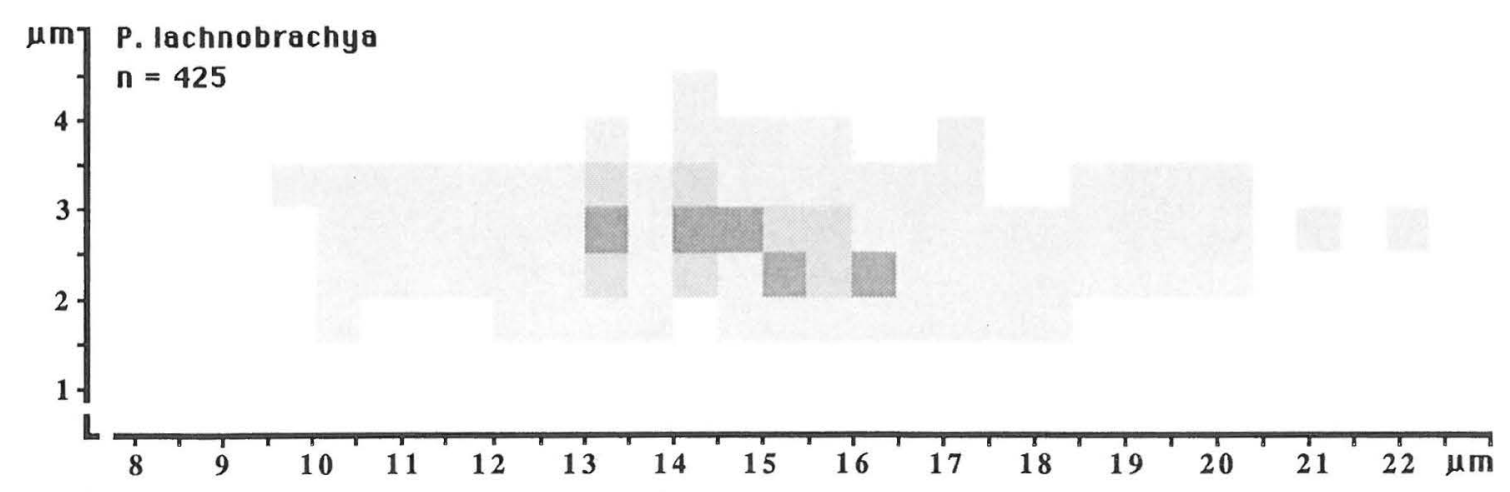

Fig. 236. Percentage sporogram of Phialina lachnobrachya. 
range. Many abundant collections from Canada were made from the timberline area. In Finland $P$. lachnobrachya seems, surprisingly, to be a rare species at least in southern parts of the country.

Phialina lachnobrachya fruits mainly in the autumn, between August and November, and has a clear peak in Europe in September-October. All the collections dating from June have been made in the mountains of Tadzhikistan, and the only collection made in spring originates from Algeria.

Ecology. Phialina lachnobrachya is a foliicolous species, only exceptionally found on other substrates. I know two such collections from capsules of Aesculus. The species has a wide variety of foliar substrates. Three collections have been made from petioles of Acer (2) and Fraxinus (1) the rest from leaves of: Betula (21), Acer (16), Alnus (7), Quercus (4), Salix (2), Rhododendron (2), Corylus (1), unidentified (3). The material of $H$. lutea (not studied), originates from leaves of Rhododendron (Raschle 1978). Arendholz (1979) reported the species from leaves of Acer, Betula, Tilia and Quercus. One collection from leaves of Andromeda polifolia was reported by Eriksson (1970).

Discussion. Phialina lachnobrachya is distinguished from other foliicolous species of the genus by the four-spored asci. The other two closely related foliicolous species, $P$. lachnobrachyoides and $P$. setiigera, have eight-spored asci. $P$. lachnobrachya has few populations in which the spores match those of $P$. lachnobrachyoides in narrowness and guttulation: the spore size showed notable overlap in only five populations studied by me. The spores of $P$. setiigera differ from the bulk of $P$. lachnobrachya in their predominantly elliptic shape. In characters other than the spores and asci, the three species are closely similar.

The variability in the present species is exceptionally wide when compared with that occurring in Hyaloscypha, but clearly parallels the variation seen in the two closely related species of Phialina. The variation may be linked with the foliar substrate, which may require more genotypic variability than more stable, bulky, ligneous substrates. Leaf litter is degraded much more rapidly than a fallen trunk. Leafy substrates are subject to recurrent desiccation and other major changes in the immediate environment; temperature fluctuates more sharply than below a partly buried moss-carpeted trunk. Thus evolutionary history may be responsible for the variability noted here. It may be noted that the species of $\mathrm{Hya}$ loscypha showing the widest variation is $H$. herbarum, which inhabits ligneous substrates ranging from large trunks to small fragments of wood amongst the litter and frequently occupies herbaceous litter. It seems that the variation in a species should be judged in relation to its ecological range. As discussed below, I do not believe that the large variation in the present material indicates the existence of a species aggregate.

In $P$. lachnobrachya the populations show an unbroken gradation of characteristics, in which the extremes are far apart. The variation is too gradual, however, to establish discontinuities and separate taxa with stable characters. Variation is seen in three major characters: size of asci, size and shape of spores, and hair length, the last-mentioned being as variable as in Hyaloscypha: the hairs may show a many-fold difference in length between the populations. This feature is independent of the other morphological characters, and the few long-haired populations merely represent the extreme of a smooth gradation, so that subdivision is unnecessary. This conclusion partly agrees with the proposal made by Dennis (1949) that only the rank of variety be given to Peziza araneocincta.

At first sight, the differences between the populations in spore size and shape are rather great. For instance, comparison of the mostly oblong-elliptic spores of the type of Pezizella tetraspora with the narrowly fusiform spores of some collections might suggest the existence of different taxa. Populations close to Hyaloscypha lutea represent another extreme. But the same variation is seen in a smaller scale during growth of the apothecium in many populations. When still in the asci, the spores are

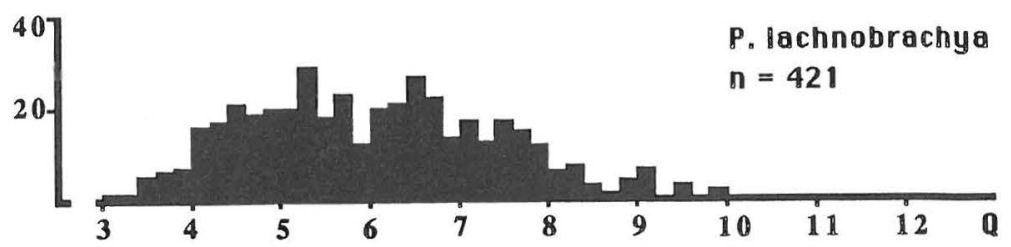

Fig. 237. The distribution of spore quotient $\mathrm{Q}$ in Phialina lachnobrachya. 
fusiform, but they become broader after being discharged. The ontogenic variation does not, however, explain the whole scale of variation. There are populations in which the spores are more or less elliptic from the very first and populations in which the spores remain narrowly fusoid after discharge. Amongst the discharged spores in some populations, however, the variation ranges from narrowly fusoid to oblong-elliptic and the two "spore types" are definitely linked. To some extent, spore width is connected with ascal size, especially with width of the asci. My study of 63 populations indicates that fairly large morphological variation in the spores and asci needs to be accepted. The rare populations with permanently elliptic spores may perhaps reveal a diverging trend, but are linked with the bulk of the material by populations in which the mature spores are identical but the developing spores fusoid.

The beaked spore apex, which was highly exaggerated in Velenovsky's (1934) illustration of Setoscypha clavispora, is also a variable character. It is seen best in the uppermost spores of an ascus and is apparently caused by lack of space or pressure in the ascus pushing the spores towards the apical cone. After discharge the character often disappears as the width increases. The typical excipulum is thin-walled and only a few populations with clearly thickened walls are known. Such interpopulational variation is a common feature in Hyaloscypha. In one collection from Finland the wall thickness was noted to vary within the same population.

The variation in apothecial colouring has caused confusion. Both white and yellow populations (when fresh) are known and I observed this variation within two Canadian populations. Although the apothecia are whitish to the naked eye, they always contain the typical yellow pigment of Phialina species. The presence of this pigment is of taxonomic importance, not its abundance. The confusion is largely due to the fact that Desmazières (1851) in the original diagnosis and in the widely distributed exsiccatum stressed the white colour of the apothecia and discussed other white species as possible sources of confusion. The assumption that Desmazières was describing a constantly white foliicolous species led Raschle (1978) and Svrček (1982) to treat yellow populations as new taxa. However, the original material of Peziza lachnobrachya shows the typical yellow pigment inside the paraphyses and hair bases. The number of spores per ascus has also caused taxonomic confusion. Müller (1977) claimed that $P$. lachnobrachya could be distinguished from var. araneocincta by its eightspored asci. He also stated that the diagnostic characters in both taxa were constant in culture. Whether he cultured $P$. lachobrachyoides or a deviating population of $P$. lachnobrachya cannot be ascertained.

Stability is seen in many characters. The asci are almost invariably four-spored. Only two populations had asci with six or more spores, but such asci were very rare. In all populations the asci arise from croziers, and Arendholz's (1979) observation to the contrary appears to be erroneous. The apical plug always turns blue in MLZ. The hair shape is relatively constant, the main source of variation being the cincinnate/flexuose versus straight nature of the apex. Septation, smoothness, thin walls and lack of solidifications are characteristic. In one quarter of the populations some of the hairs may show minute apical roughness. In most apothecia the typical isodiametric excipular type is dominant or occurs together with textura prismatica.

The synonymy of $H$. betularum with the present species is based on the paratype in Velenovsky's herbarium (PRM 152562), which was discussed by Svrček (1982). He also confirmed that one of the studied CUP collections from Japan was $H$. betularum. When transferring Peziza lachnobrachya to Phialina, Raitviir (1970a) reported that the asci were four- and eight-spored. This was possibly due to failure to recognize the eight-spored collections, later described as a separate species in Phialoscypha. The genus Phialoscypha was based on the reported lack of septa in the hairs and the reported solidity of the long filiform hair apices. Both these characters are lacking, however, in the original material. Recently Svrček (1987d) brought Setoscypha into daylight. This monotypic genus is based on $P$. lachnobrachya (Svrček 1985). This decision to separate a foliicolous genus with a special type of hair is here supported in a modified sense.

Exsiccatae studied: Desmazières, M., Plantes cryptogames de France 1603 (K, type of Peziza lachnobrachya) and Pl. crypt. France 2003 (K, P). - Phillips, W., Elvellacei britannici 169 (E, G, K). - Lundell, S. \& Nannfeldt, J., Fungi Exsiccati Suecici $2583(\mathrm{~K}, \mathrm{~S})$. - Jaap, O., Fungi sel. exs. 130 (E, K, P, TUR). — Rehm, H., Ascomyceten 1160 (F, S) and $1162(\mathrm{~F})$.

Material studied: Algeria. Alger, 1915 Maire 3133 (MPU). Austria. Tirol: Silvretta-Stausee, 1985 Matheis 967 (TUR). Belgium. Liège: Beaufays, Mouton 37 (BR). Not localized, Mouton (BR). Canada. Northwest Territories: Inuvik, 1984 SH 84/162, 84/176, 84/178a, 84/180, 84/185a, 84/210 (TUR). Czechoslovakia. Bohemia: Mnichovice, 1930 (type of Setoscypha clavispora), 1931 (paratype of Hyaloscypha betularum) JV (PRM). Federal Republic of Germany. Winterberg, 1892 Wagner (S). Finland. Satakunta: Vammala, 1987 SH 87/195* (TUR). Koillismaa: Kuusamo, 1978 Korf (CUP, two collections). France. Parc de Libiscy, 1850 Roberge (P). German Democratic Republic. Berlin area: Lichterfelde, 1893 Sydow (S, Herb. Rehm). 
Zehlemdorf, 1893 Sydow (S, Herb. Rehm). Parchim: Triglitz, 1904 (type of Pezizella jaapii), 1905, 1912 Jaap (S, Herb. Rehm). Potsdam: Freshdorfer Moor, 1969 Benkert (BHU, K). Great Britain. Somerset: Bristol, Bucknall (K, type of Peziza araneocincta). Yorkshire: Rokeby, 1904 (K, Herb. Crossland). Not localizable, 1949 Bramley (K) Pickering, 1972 Bramley K72/12b (K). Not localized, Bramley (K). Westerness: Caisteal nan Con, 1977 Dennis (K). Japan. Gumma Pref.: Lake Marunuma, 1957 Korf 136 (CUP). Tochigi Pref.: Nikko, 1963 Imai \& Korf 2731 (CUP). Kushiro Prov.: Akan Nat. Park, 1963 Korf et al. 2492 (CUP). Luxemburg. Sheidhof, 1902 Feltgen (F, type of Pezizella tetraspora). Norway. Hordaland: Bergen, 1987 Olsen (TUR, two collections). Sweden. Uppland: Dalby, 1978 Korf (CUP). Uppsala, 1893 Starbäck (S, Herb. Rehm, type of Pezizella punctiformis $\mathrm{f}$. minor). Switzerland. Bern: Le Fuet, 1985 Matheis 980 (TUR). Graubünden: S. Bernardino Pass, 1985 Matheis 970 (Herb. Matheis). Thurgau: Münchwilen, 1972 Matheis 199 (K). Güttinger Wald, 1985 Matheis 995 (TUR). U.S.A. Washington: Baker Lake, 1941 Mains 0207 (MICH). U.S.S.R. Estonian S.S.R.: Distr. Kohtla-Järve, 1967 AR (TAA). Distr. Paide, Laupa, 1976 Kullman (TAA). Distr. Pärnu, 1976 Kullman (TAA). Distr. Rakvere, Saka, 1964 AR (TAA). Kăsmu, 1964 AR (TAA). Distr. Voru, Haanja, 1976 Kullman (TAA). Karachayevo-Cherkess: Teberda, 1968 AR (TAA, three collections). Tadzhikistan: Hissar Mts., 1982 Kullman (TAA). Ljuli-Harvi, 1979 Kullman, AR (TAA). Tsehil-Dara, 1979 Kullman (TAA).

\section{Phialina lachnobrachyoides}

Phialina lachnobrachyoides (Raitv.) Huhtinen, comb. nov. Phialoscypha lachnobrachyoides Raitv., Fol. Crypt. Est. 8:2. 1977. - Holotype (original designation): U.S.S.R., Khabarovsk Region, Dolmi, Tilia amurense, 17.VIII.1966 Remm (TAA 44315, examined).

Apothecia gregarious, superficial, $100-500 \mu \mathrm{m}$ in diam when dry, cupulate when juvenile, cupulate to scutellate when mature, in larger apothecia margin occasionally appressed to the substrate, narrowly sessile on an ill-defined stipe to shortly and stoutly stipitate. Disc convex to plane when dry, spongy and pitted when aged, bordered by a thin zone of marginal hairs, which are appressed to the substrate or rise above the hymenium, margin inconspicuous to slightly incurved when dry. Hair cover sparse, whitish. Colour varying between the populations from very faint ochraceous or ochraceous to vivid luteous to more brownish (L77) when dry.

Ectal excipulum of textura prismatica, cells on middle flanks regularly prismatic to almost isodiametric, 6.2-12.7(-14.8) x 4.0-7.0(-8.0) $\mu \mathrm{m}, \overline{\mathrm{x}}=$ $9.8 \times 5.3 \mu \mathrm{m}(\mathrm{n}=60), \overline{\mathrm{Q}}=1.8$, not notably changing size or shape towards margin, at the base com- posed of brown textura epidermoidea. Inner excipulum composed of narrower cells. Walls in ectal parts thin to $0.4 \mu \mathrm{m}$ thick, $0.8-2.0 \mu \mathrm{m}$ in one population, hyaline, brown at the base, negative in all reagents.

Hairs $18-120 \times 2.5-3.5 \mu \mathrm{m}$ narrowly conical, often with a long filiform apex, flexuose to cincinnate, more rarely straight, one- to multiseptate, the basal parts frequently with bright yellow pigment. Apex tapering to $0.4-1.0 \mu \mathrm{m}$, not solidified, not exuding an apical globule in MLZ. Wall thin and dull, never locally thickened, hyaline, smooth in most populations, in others frequently with warts deforming in MLZ, wall negative in all reagents. Pigment amorphous, persistent in all reagents, $\mathrm{BF}+$ (red), $\mathrm{CB}+$ (blue), $\mathrm{CRB}+$ (violet), LUG+ (red), $\mathrm{MLZ}+$ (golden), NR+ (red), $\mathrm{TB}+$ (violet).

Asci $32-53(-55) \times 4.8-7.4(-7.8) \mu \mathrm{m}, \overline{\mathrm{x}}=43.1 \times$ $6.0 \mu \mathrm{m}(\mathrm{n}=43), \bar{Q}=7.2$ in MLZ; $29-43 \times 4.2$ $5.6 \mu \mathrm{m}, \overline{\mathrm{x}}=38.3 \times 5.1 \mu \mathrm{m}(\mathrm{n}=20), \overline{\mathrm{Q}}=7.6$ in $\mathrm{CB}$; eight-spored, cylindrical-clavate with a slightly conical apex. Apical pore MLZ++ when dry, LUG+ (blue). Wall in mature asci apically $0.6-1.0 \mu \mathrm{m}$ thick (in $\mathrm{CB}, \mathrm{MLZ}$ ), negative in all reagents. Contents MLZ-, inconspicuously LUG+ at the extreme apex and base. Asci arising from croziers.

Spores $11.4-20.0(-21.8) \mu \mathrm{m}, \overline{\mathrm{x}}=15.9 \times 1.8 \mu \mathrm{m}$ ( $\mathrm{n}=93$ ), $\overline{\mathrm{Q}}=8.8$ in $\mathrm{CB}$ and MLZ; narrowly fusoid, straight to curved to apically beaked, aseptate (only one septate spore seen). Wall hyaline, not seen to become brownish in aged spores. Guttulae prominent and light, filling the spores, rarely lacking or inconspicuous in dry material.

Paraphyses cylindrical to clavate, frequently containing bright yellow, amorphous pigment similar to that inside the hairs, first dichotomous branches occurring at or below the level of the ascal bases. Terminal cells $11-31 \times 1.8-2.8 \mu \mathrm{m}, \overline{\mathrm{x}}=23.0 \times 2.2 \mu \mathrm{m}$ $(\mathrm{n}=30)$, apices $2.4-3.6 \mu \mathrm{m}$ wide, closely septate below, mean septal intervals $11.4 \mu \mathrm{m}(\mathrm{n}=20)$.

\section{Cultural characters. Not known.}

Distribution and phenology. The species is apparently restricted to the Soviet Far East and Japan. It is sympatric with the widely distributed Phialina lachnobrachya. The few collections have been made in July and August.

Ecology. Phialina lachnobrachyoides is foliicolous, known from the following substrates: Tilia (3), Aesculus (1), Betula (1), Carpinus (1), ? Fagus (1). In the mixed collections by Nazarova, which were cited by Raitviir (1977), the present species occurs on leaves of Tilia only. 

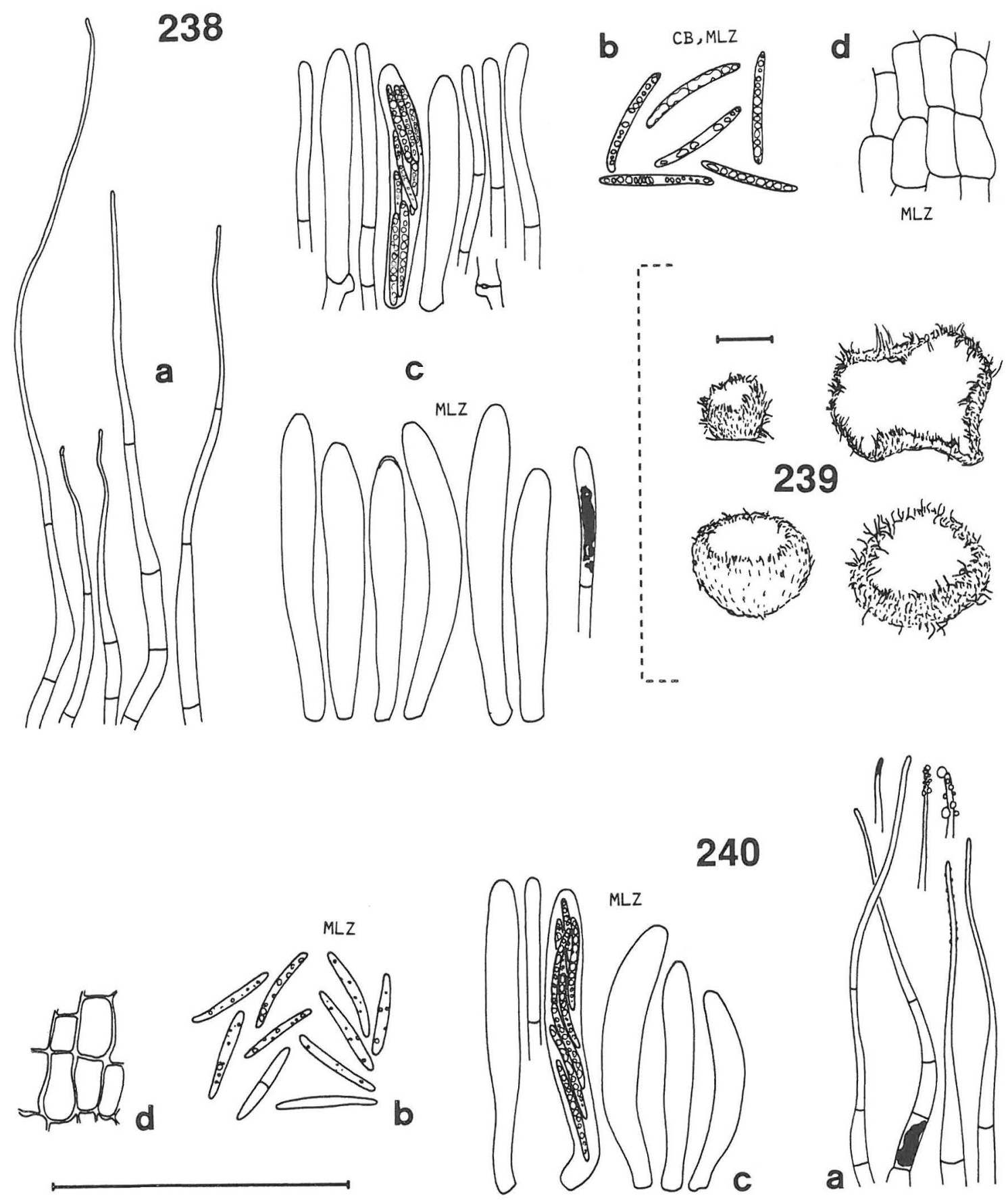

Figs. 238-242. Phialina lachnobrachyoides. a) hairs, b) spores, c) asci and paraphyses, d) excipulum. Scale $50 \mu \mathrm{m}$, for apothecia $100 \mu \mathrm{m}$. - 238: Holotype. — 239: Dry apothecia from various collections. — 240: Dolmi, 1966 Remm. — 241: 1961 Nazarova. — 242: Shimizu \& Korf 1231. 

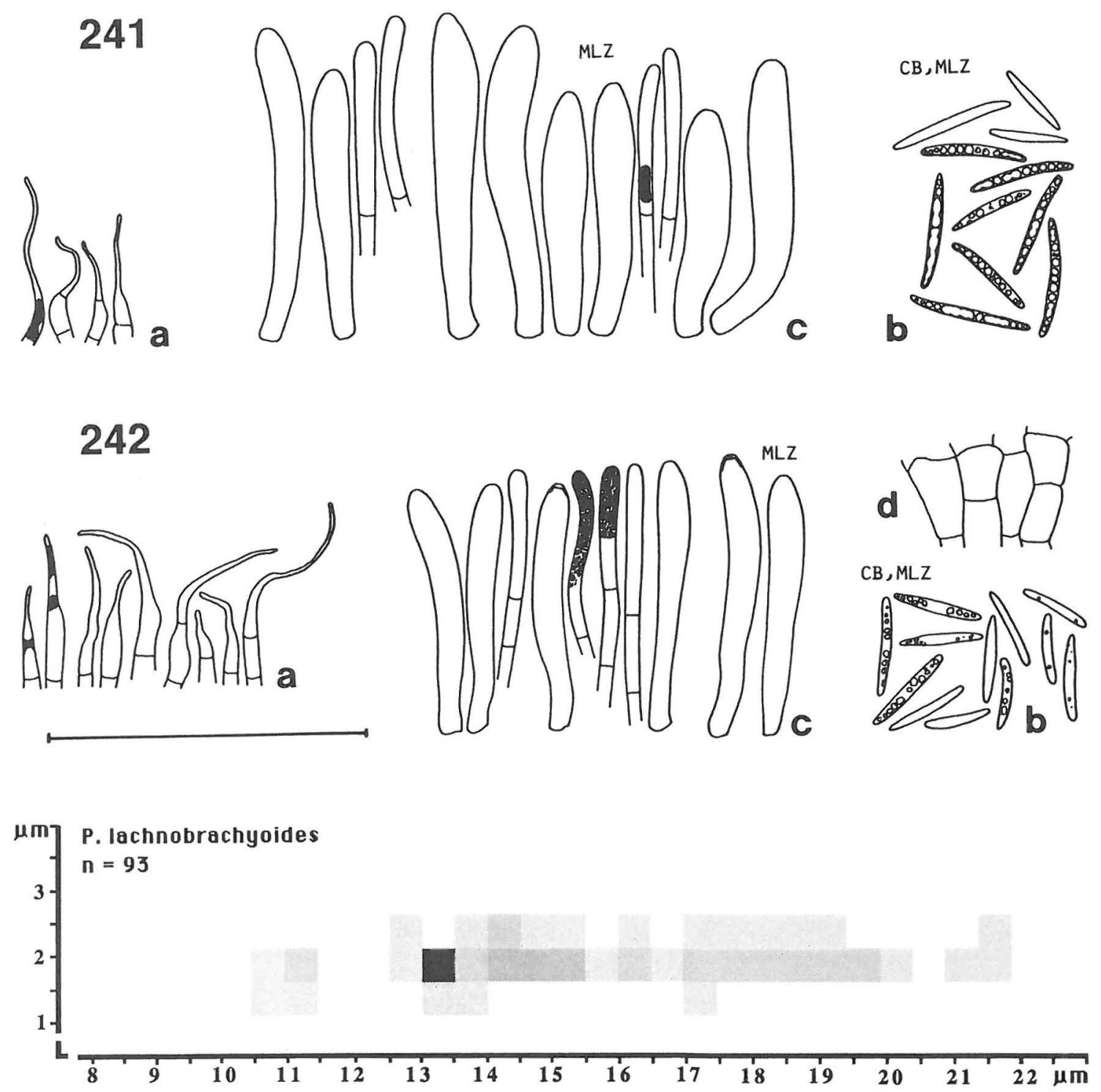

Fig. 243. Percentage sporogram of Phialina lachnobrachyoides.

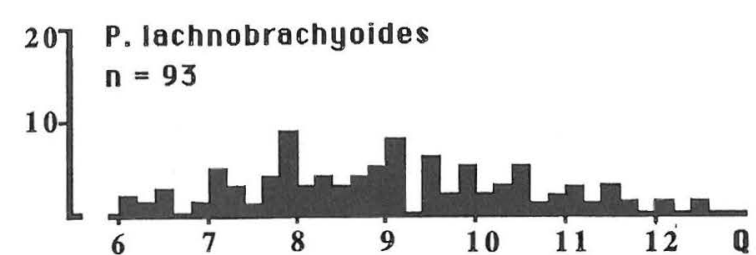

Fig. 244. The distribution of spore quotient $Q$ in Phialina lachnobrachyoides.
Discussion. The eight-spored asci and narrowly fusoid, guttulate spores facilitate recognition of the species under the microscope. The spores of $P$. lachnobrachya are seldom equally narrow and fusoid, and they are borne in four-spored asci and lack conspicuous guttulae. Most populations of $P$. lachnobrachya have clearly wider spores than those of the seven known collections of $P$. lachnobrachyoides. In 
other characters the two species are almost alike and no divergent trends are evident in $P$. lachnobrachyoides. $P$. setiigera is distinguished from the present species by the elliptic spores.

The variability in $P$. lachnobrachyoides parallels that in $P$. lachnobrachya, and the similar variational patterns suggest a close relationship. There are shorthaired and long-haired populations, and the excipular walls may be thin or clearly thickened. The variation in ascal size is much smaller than in $P$. lachnobrachya, and a notable difference is seen in the stability of the spore size and shape. One Japanese collection (Korf 1304) differs from the rest of the material in having more cincinnate and curved hairs; the type has long and relatively straight hairs. But in all the other characters Korf's specimen matches $\boldsymbol{P}$. lachnobrachyoides and the deviating hairs must be accepted as part of the variation of the species.

In addition to the uniform spores, the populations all have eight-spored asci, croziers, MLZ++ ascal plugs, typical $P$ hialina pigment, similar apothecia and similar paraphyses, and are alike in basic hair characters, such as wall thickness and septation.

Material studied: Japan. Akita Pref.: Towada Lake, 1959 Korf \& Korf 1304 (CUP). Yamagata Pref.: Shirabutakayu, 1958 Shimizu \& Korf 1231 (CUP). U.S.S.R. Khabarovsk Region: Dolmi, 1966 Remm (TAA, type and another collection). Unlocalized, 1966 Remm (TAA). Primorsk Region: Subutinski Nat. Res., 1961, 1962 Nazarova (TAA).

\section{Phialina pseudopuberula}

Phialina pseudopuberula (Graddon) Raitv., Fol. Crypt. Est. 9:1. 1977. - Hyaloscypha pseudopuberula Graddon, Trans. Brit. Mycol. Soc. 58:151, Fig. 4. 1972. - Holotype (original designation): [Great Britain,] Herefordshire, Llangrove, XI.1965 Graddon 1718 (K, not seen; isotype in E examined).

Phialina plowrightii Arendh., Morphologisch-Taxonomische Untersuchungen an blattbewohnenden Ascomyceten aus der Ordnung der Helotiales: 53; Pl. 9:1. 1979. - Holotype (original designation, Arendholz 1979: 54): [Great Britain,] St. Clement, Terrington, Plowright (K; as Phillips, W, Elvell. Brit. 88 in Herb. Broome; examined).

Uncinia pallidipilosa Arendh., Morphologisch-Taxonomische Untersuchungen an blattbewohnenden Ascomyceten aus der Ordnung der Helotiales: 57; Pl. 7:5 and Pl. 8:1. 1979. Holotype (original designation): [Federal Republic of Germany,] Schleswig-Holstein, Ahrensburg, U-Bahn Bahndamm, in foliis marcidiis Querci, 29.X.1977 Schmidt (Herb. Schmidt, not seen; paratypes in BR examined).

Hamatocanthoscypha tenuipila Graddon, Trans. Brit. Mycol. Soc. 87:329, Fig. 4. 1986. - Holotype (original designation): [Great Britain,] Herefordshire, Ross-on-Wye, IX.1984 Graddon 4198 (Herb. Graddon, not seen; isotype in TUR examined).
Hyaloscypha uncinata Arendh. \& R. Sharma, Mycotaxon 25:124; Pl. 2:1-4 and Pl. 3:5, 7. 1986. - Holotype (original designation): [India,] Bomdila, West Kameng, Arunachal Pradesh, 23.VIII.1981 Sharma 17833 (PAN, not seen; isotype in Herb. Arendholz examined).

Hamatocanthoscypha acericola Svr., Ceská Mykol. 41:21; Pl. 2:2. 1987. - Holotype (original designation): [Czechoslovakia, Bohemia,] Radotín, VIII.1924 Velenovský (PRM 148463, examined).

Apothecia gregarious, superficial, mostly 100 $300(-530) \mu \mathrm{m}$ in diam when dry, cupulate, broadly sessile to narrowly sessile on an ill-defined base or on a 30-50 $\mu \mathrm{m}$ wide, up to $60 \mu \mathrm{m}$ high stipe. Disc convex to plane when dry, not spongy or pitted when mature, bordered by a thin zone of marginal hairs which rise only slightly above the hymenium; margin only slightly incurved when dry. Hair cover dense, white. Colour in disc greyish to greyish brown (M91), to Cream (K75) to ochraceous (L60) when dry, flanks light brownish (M75) close to the margin, brownish (N75) below, blackish at the base, rarely whitish; these colours modified by the overall hair cover.

Ectal excipulum of textura prismatica, cells on middle flanks regularly prismatic to somewhat isodiametric, 7.8-13.2(-16.4) x 3.2-8.5(-11.3) $\mu \mathrm{m}, \overline{\mathbf{x}}$ $=9.5 \times 5.4 \mu \mathrm{m}(\mathrm{n}=110), \bar{Q}=1.8$, becoming smaller towards margin, at the base more roundish. Inner excipulum composed of slightly narrower cells. Walls in ectal parts uniformly $0.4-1.5 \mu \mathrm{m}$ thick, rarely side walls thickened and apical walls thin, brown to light brown, similar or hyaline towards the margin, clearly pigmented parts $\mathrm{CB}+, \mathrm{CRB}+, \mathrm{NR}+$, $\mathrm{TB}+, \mathrm{MLZ}-$; the more hyaline parts negative in all reagents.

Hairs $15-34 \times 2-4 \mu \mathrm{m}$, narrowly conical to conical to slightly lageniform, flexuose to uncinate, rarely straight, simple to apically branched, aseptate, typically containing bright yellow pigment, more rarely exuding yellowish resin. Apex tapering to 0.5$1.5 \mu \mathrm{m}$, irregularly minutely to prominently solidified, without an apical globule in MLZ. Wall thin and dull, never locally prominently thickened (except for apex), smooth, hyaline, rarely brownish, negative in all reagents. Pigment inside the hairs amorphous, persistent in all reagents, $\mathrm{CB}+$ (blue), LUG+ (reddish brown), MLZ+ (golden).

Asci $51-88 \times 6.4-9.7 \mu \mathrm{m}, \overline{\mathrm{x}}=66 \times 8.6 \mu \mathrm{m}(\mathrm{n}=$ 10), $\bar{Q}=7.7$ in MLZ; 43-68 $\times 5.6-8.0(-8.8) \mu \mathrm{m}, \overline{\mathrm{x}}$ $=56.2 \times 7.0 \mu \mathrm{m}(\mathrm{n}=54), Q=8.1$ in CB; eight spored, in two populations often also four-spored, cylindrical-clavate with a slightly conical apex. Apical pore $\mathrm{MLZ}++$ when dry, LUG+ (blue). Wall in mature asci apically $0.8-1.2 \mu \mathrm{m}$ thick (in $\mathrm{CB}, \mathrm{MLZ}$ ), negative in all reagents. Contents MLZ-, rarely LUG+. Asci arising from croziers. 
Spores $9.1-14.1 \times 2.0-4.1(-4.4) \mu \mathrm{m}, \overline{\mathrm{x}}=11.3 \times$ $3.2 \mu \mathrm{m}(\mathrm{n}=171), \bar{Q}=3.8$ in CB and MLZ, elliptic to oblong-elliptic to subfusoid, aseptate, in $29 \%$ of the populations one-septate, with or without an apical cilium in $60 \%$ of the populations, cilia totally lacking in others. Wall hyaline, rarely seen to become brownish in aged spores. Guttulae prominent to lacking in dry material, such variation occurring mostly between the populations.

Paraphyses cylindrical to apically somewhat widened, frequently containing bright yellow, amorphous pigment similar to that inside the hairs, first dichotomous branches and anastomoses typically occurring below the level of the ascal bases. Terminal cells $14-29(-64) \times 1.8-3.5 \mu \mathrm{m}, \overline{\mathrm{x}}=23.1 \times 2.5 \mu \mathrm{m}$ ( $=53$ ), subclavate apices $2.0-3.8 \mu \mathrm{m}$ wide, often closely septate below, mean septal intervals $11.6 \mu \mathrm{m}$ $(\mathrm{n}=55)$.

\section{Cultural characters. Not known.}

Distribution and phenology. The material originates mainly from central and western Europe with only two more remote collections, from the Caucasus and India. $P$. pseudopuberula appears to be an autumnal species: most collections were made between August and November.

Ecology. P. pseudopuberula is a foliicolous species. Most of the material originates from leaves of Quercus (8 collections), but there are single collections from Acer, Betula, Castanea and Fagus. Furthermore, the paratype of Uncinia pallidipilosa consists of leaves of both Quercus and Castanea. The type collection of Hyaloscypha uncinata was collected from dead angiosperm leaves (Sharma et al. 1986).

Discussion. The many distinctive features enable a rapid recognition. The hairs in the apothecia may be simple, but often have typical bifurcate to branched, or undulating-cincinnate apices. Another striking feature lies in the spores, which in many populations often have apical cilia. These have escaped notice in earlier studies (e.g. Dennis 1949, Graddon 1972, Arendholz 1979, Graddon 1986), apparently because they are inconspicuous under low magnification. The asci arise from croziers and have MLZ++ apical plugs. A further character assisting recognition is the colour of the the excipulum, which is always more or less clearly brown under the microscope. The colour is incorporated in the excipular walls. In some of the populations, the hairs may also be pigmented. Due to its character combination, $P$. pseudopuberula can hardly be confused with any other foliicolous species.

Although easy to recognize, the species is hard to place in any of the existing genera. The basic hair shape is that of a Hyaloscypha or Phialina. However, the yellow pigment excludes close relationship with Hyaloscypha, which was suggested by Graddon (1972) in the first generic placement. Phialina was proposed by Arendholz (1979) for Phialina plowrightii, on account of the Hyaloscypha-like hairs and the thick excipular walls. This placement, emphasizing the link with Peziza ulmariae, is supported here. The uncinate nature of the hairs was stressed when the species was described anew by Graddon (1986) and placed in Hamatocanthoscypha. In the type specimen uncinate hairs predominate amongst the scattered forked ones, and in general appearance the specimen does, indeed, resemble a Hamatocanthoscypha. This genus was also proposed by Svrček (1987a) for H. acericola, but it is predominantly lignicolous, is characterized by stipitate apothecia, elongated excipular cells and small cuneiform spores, and its species rarely possess yellow pigment. In contrast to the depiction by Graddon (1986), I found that the hairs in the type specimen of $H$. tenuipila were aseptate.

Placement in Phialina is supported by the typical yellow pigment, the ecology, the broad paraphyses, the MLZ++ apical pores and the basic shape of the hairs and spores. As in the other shorter-haired species of the genus, the hairs tend to be aseptate in the exceeding part. The present species does, however, have characters giving it in a rather isolated status within the genus. Examples in Hymenoscyphus and Arachnopeziza (White 1944, Huhtinen 1985b, 1987d) diminish the taxonomic value of spore cilia at the generic level. Svrcek (1977a) used prominent hair branching as a generic character in Dendrotrichoscypha. My study of the type specimen of $D$. acanthopila revealed, however, that the genus is based on a species of Mollisina, which is the only genus in the Hyaloscyphaceae in which a larger natural unit is delimited by branched hairs. This character must be accepted at least in Hyalopeziza and Urceolella, which have predominantly simple hairs. Possibly the most alien element for $P$ hialina is the brown pigment of the excipular walls, and rarely also the hair walls, which typically results in a faint to medium brown colour under the microscope. In many cases a brown excipulum coincides with other major characters delimiting natural genera, but most of these genera possess a deep brown excipulum whereas, more faint pigmentation is relatively rare. It is interesting to note that in Hamatocanthoscypha uncinata var. phaeo- 

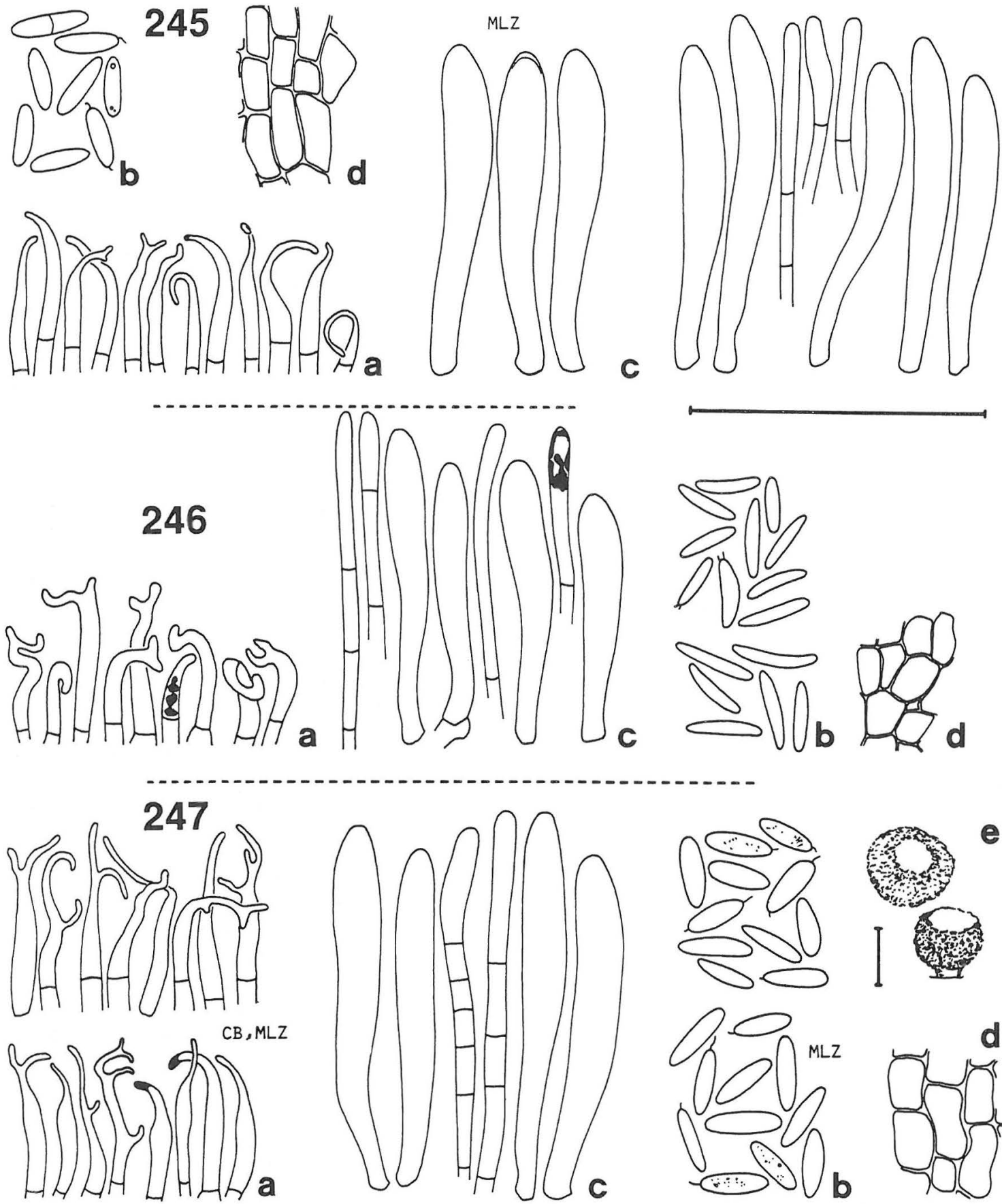

Figs. 245-251. Phialina pseudopuberula. a) hairs, b) spores, c) asci and paraphyses, d) excipulum, e) dry apothecia. Scale 50 $\mu$ m, for apothecia $100 \mu \mathrm{m}$. - 245: Isotype of Hamatocanthoscypha tenuipila. - 246: Holotype of Hamatocanthoscypha acericola. 247: Isotype of Hyaloscypha pseudopuberula. - 248: Isotype of Hyaloscypha uncinata. - 249: SH 85/48. - 250: Mouton 461, paratype of Uncinia pallidipilosa. - 251: Herb. Mouton, paratype of Uncinia pallidipilosa. 


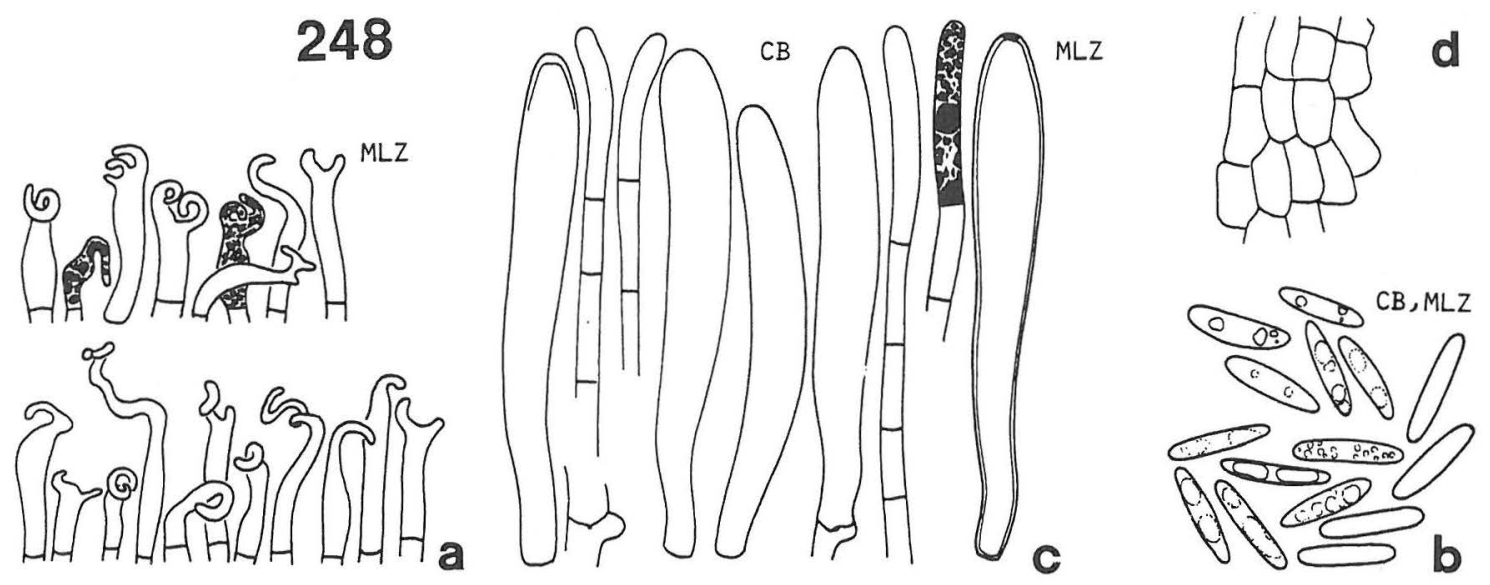

249
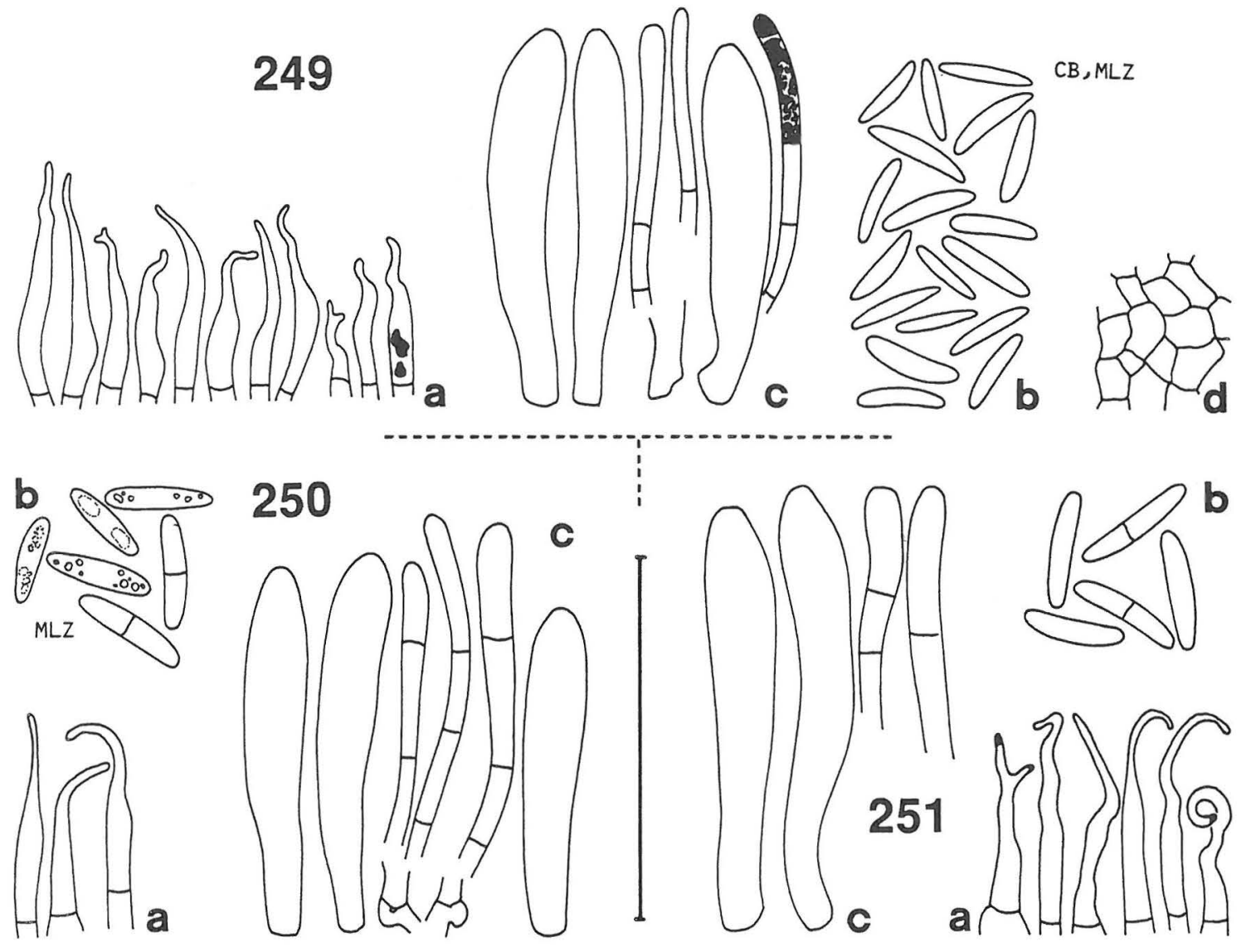
tricha the amount of brown pigment varies greatly within a single population. There is little evidence of the taxonomic value of a somewhat brownish versus hyaline excipulum, and as the pigmentation is variable in the present species and in var. phaeotricha, I shall refrain from erecting a new genus for Hyaloscypha pseudopuberula and accept this variation in Phialina.

The hair and spore characteristics vary most between the populations. The proportion of branched hairs varies greatly within an apothecium and the populations also differ in this respect. In some the branched hairs dominate whereas in others they are rare. In one population they were not seen at all. The spores vary as in $P$. lachnobrachya, but the apical cilia are distinctive when present. The apical solidifications are as variable as in Hyaloscypha. Spores with apical cilia are present in many populations, but in variable proportions, and sometimes cilia were not observed at all. In two populations some of the asci become four-spored after abortion of four of the primary spores. Such asci occur amongst the normal, eight-spored ones. Spore shape varies mostly between the populations, from elliptic to oblong-elliptic; a subfusoid type prevails in populations also having clearly wider, oblong-elliptic spores. Similar intrapopulational variation was often seen in $P$. lachnobrachya. These spores do, although rarely, show the typical cilia. The presence versus absence of a spore septum parallels the variation observed in the larger-spored Hyaloscyphas. Although wide variation was accepted within spore characteristics, the unbroken gradation and the similarity in other critical features favour the present delimitation. The variational pattern and magnitude in $P$. pseudopuberula matches that in another widespread foliicolous Phialina: $P$. lachnobrachya.

The synonymy of Uncinia pallidipilosa is based on four paratype collections in BR, which were cited by Arendholz (1979). These collections by Mouton bear scanty collection data but all originate from the same site. They are possibly fragments of a single, larger collection. The leaves bear many species but the original diagnosis can only mean the present taxon. My measurements of the spores gave a notably different size range than that reported by Arendholz (1979). Unfortunately the holotype (in herb. Schmidt) was not forwarded for study, so it remains to be seen whether this collection bears smaller spores (9-12 x 3-4.5 $\mu \mathrm{m}$ : Arendholz 1979).

The isotype of Hyaloscypha uncinata lacks the brownish colours characterizing many other collections. The apothecia are predominantly yellowish with flanks appearing white due to the dense fur of hairs. As already noted under $P$. lachnobrachya, the amount of yellow in the disc is due to the abundance of yellow pigment inside the paraphyses and the total scale of variation is from bright yellow to white. The

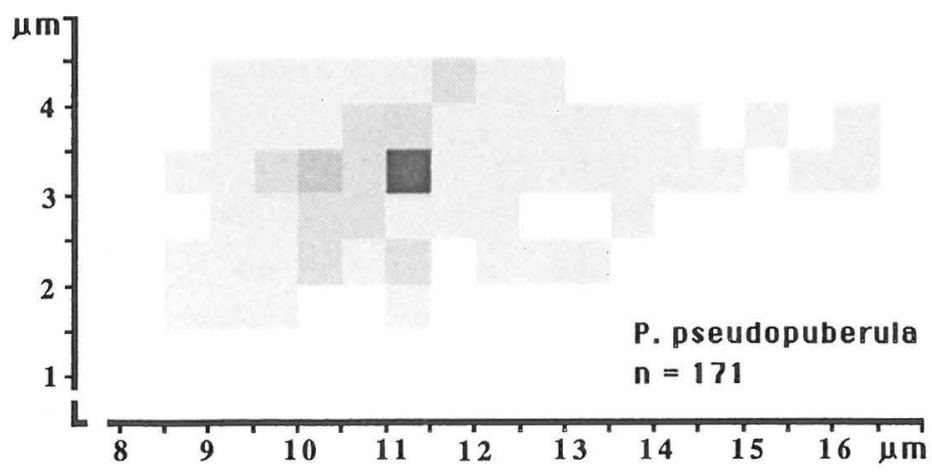

Fig. 252. Percentage sporogram of Phialina pseudopuberula.

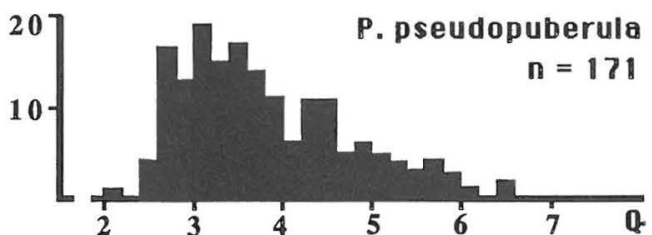

Fig. 253. The distribution of spore quotient $\mathrm{Q}$ in Phialina pseudopuberula. presence of the pigment in the paraphyses is of taxonomic value, not its abundance. Similarly, variation in the abundance of pigment accounts for the colour variation in $P$. pseudopuberula; when pigment is scanty the overall colour of the disc is brownish or greyish, due to the influence of the coloured excipulum. In the type of $H$. uncinata the brownish tone in the excipular walls is faint, being apparent only under 
the microscope, and it does not affect the overall colouring. In other populations a yellow disc occurred, often in combination with a clearly brown excipulum.

In the type collection of Hamatocanthoscypha acericola the hairs often bear resinous matter which is quite similar to the pigment inside the hairs. In MLZ such conglomerations vary from golden to somewhat reddish and these were mentioned in the original diagnosis (Svřek 1987a).

Exsiccatae studied: Cooke, M., Fungi Brit. exs. 574 (E). Phillips, W., Elvell. Brit. 88 (K, Herb. Broome, type of Phialina plowrightii; UPS).

Material studied: Belgium. Liege: Beaufays, Mouton (BR, paratypes of Uncinia pallidipilosa, four collections). Czechoslovakia. Bohemia: Radotín, 1924 JV (PRM, type of Hamatocanthoscypha acericola). Great Britain. Devon: Slapton Ley, 1981 Clark (IMI). Suffolk: Walberswick Nat. Res., 1978 Ellis \& Ellis (IMI). Herefordshire: Llangrove, 1965 Graddon 1718 (E, isotype of Hyaloscypha pseudopuberula). Ross-on-Wye, 1984 Graddon 4198 (TUR, isotype of Hamatocanthoscypha tenuipila). Worcestershire: Nutnells Wood, 1978 Clark (IMI). India. West Kameng: Arunachal Pradesh, 1981 Sharma (Herb. Arendholz, isotype of Hyaloscypha uncinata). Netherlands. Wageningen, 1950 Gremmen (CUP). Norway. Oppland: Kongsvoll, 1985 SH 85/48 (TUR). U.S.S.R. Georgian S.S.R.: Lagodekhi Nat. Res., 1967 AR (TAA).

\section{Phialina pusilla}

Phialina pusilla Huhtinen, nom. nov. (Art. 72 Note 1). Hyaloscypha pusilla Arendh. \& R. Sharma, Mycotaxon 17: 498; PI. 9:1-6 and Pl. 10:4. 1983, nom. illeg. (Art. 64.1, non Hyaloscypha pusilla (Oeder: Fr.) Boud.). - Holotype (original designation): [India,] W-Bengal, Batasi, $2250 \mathrm{~m}$, on dead fallen grass leaves, 9.IX.1979 Sharma (PAN 17257, not seen; isotype in herb. Arendholz examined).

Apothecia gregarious to confluent, superficial, $50-180 \mu \mathrm{m}$ in diam when dry, cupulate, narrowly sessile to very inconspicuously stipitate, naked to minutely hairy. Disc shallow to plane when dry, not spongy or pitted when mature, bordered by an inconspicuous margin, which is plane to slightly incurved when dry, occasionally undulate-crenulate. Hair cover sparse, inconspicuous, somewhat whitish at the margin. Colour ochraceous (L79) to reddish brown (N35, P27) in dry material.

Ectal excipulum of textura prismatica, cells on middle flanks fairly isodiametric to prismatic, 4.0 $10.8 \times 3.0-6.2 \mu \mathrm{m}, \overline{\mathrm{x}}=7.1 \times 4.1 \mu \mathrm{m}(\mathrm{n}=25), \bar{Q}=$ 1.7 , becoming regularly prismatic towards margin. Walls in ectal parts thin to $0.4 \mu \mathrm{m}$ thick, yellowish, $\mathrm{CB}+, \mathrm{CR}-, \mathrm{MLZ}$.

Hairs 9-24 x 2.2-4.0 $\mathrm{m}$, narrowly conical, straight to somewhat flexuose, rarely branched, aseptate, frequently with bright yellow pigment. Apex tapering to $0.5-1.0 \mu \mathrm{m}$, not solidified, without an apical globule in MLZ. Wall thin and dull, never locally prominently thickened, hyaline, smooth in $\mathrm{CB}$ and $\mathrm{MLZ}$; $\mathrm{CB}-$, CR-, MLZ-. Pigment amorphous, yellow, $\mathrm{CB}+, \mathrm{MLZ}+$ (golden).

Asci $31-34 \times 3.8-5.0 \mu \mathrm{m}, \overline{\mathrm{x}}=33.7 \times 4.7 \mu \mathrm{m}(\mathrm{n}=$ 14), $\bar{Q}=7.2$ in MLZ and CB, eight-spored, cylindrical-clavate with a slightly conical apex. Apical pore MLZ++. Wall in mature asci apically 1.0 $1.4 \mu \mathrm{m}$ thick, in MLZ side walls often prominently thickened. Contents MLZ-. Asci arising from croziers.

Spores $4.8-7.4 \times 1.8-2.7 \mu \mathrm{m}, \overline{\mathrm{x}}=6.1 \times 2.2 \mu \mathrm{m}$ ( $\mathrm{n}=20$ ), $\bar{Q}=2.8$ in CB and MLZ; elliptic, aseptate. Wall hyaline, not seen to become brownish in aged spores. Guttulae large, usually two in each spore, rather inconspicuous in CB and MLZ.

Paraphyses cylindrical, frequently containing bright yellow, amorphous pigment similar to that inside the hairs. Terminal cells $10-25(-38) \times(1.5-) 2$ $3 \mu \mathrm{m}, \overline{\mathrm{x}}=23.5 \times 2.3 \mu \mathrm{m}(\mathrm{n}=11)$, closely septate below, mean septal intervals $9.1 \mu \mathrm{m}(\mathrm{n}=13)$.

\section{Cultural characters. Not known.}

Distribution and phenology. Known only from the type locality in India. The type specimen was collected in September. leaves.

Ecology. The type originates from dead grass

Discussion. The yellow pigment in the hairs and paraphyses, the broadness of the paraphyses, the somewhat isodiametric excipulum, the hair shape, and the herbicolous habit place the present species in Phialina rather than Hyaloscypha, which was the genus originally chosen by Arendholz and Sharma (1983). The closely related $P$. foliicola has similar spores and asci, but differs in the typically curved to coiled hairs. The predominantly oblong-elliptic spore shape of $P$. foliicola, and the difference in spore size also assist in distinguishing the two taxa. The asci of $P$. foliicola are slightly wider than those of the present species. In the type collection the hairs were rarely dichotomously branched, which is a feature reminiscent of $P$. pseudopuberula. However, the two taxa differ in the ascus and spore sizes.

The scanty isotype material did not allow a thorough study of the present species. However, the ascal walls that often appear clearly thickened in $\mathrm{MLZ}$ seem to be characteristic. Another interesting feature was found inside the spores, which (especially in 


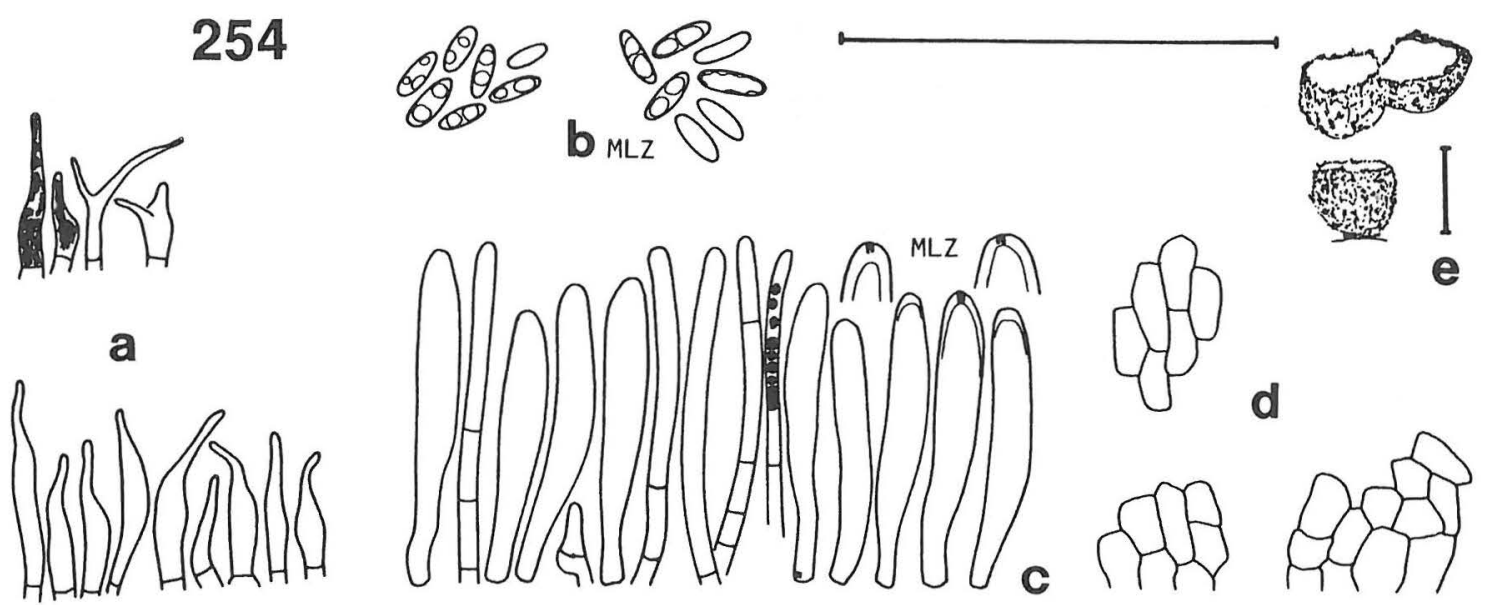

Fig. 254. Phialina pusilla, isotype. a) hairs, b) spores, c) asci and paraphyses, d) excipulum, e) dry apothecia. Scale $50 \mu \mathrm{m}$, for apothecia $100 \mu \mathrm{m}$.

CR) show one or two unstained, refractive, hyaline, solid bodies. Whether these are the indistinct guttulae seen in CB and MLZ remains to be confirmed.

In the original diagnosis, Arendholz and Sharma (1983) reported that the asci are MLZ-, which is in partial contrast to my observations on the same material. They also gave a smaller width for the spores and paraphyses.

Material studied: India. West Bengal: Batasi, 1979 Sharma (Herb. Arendholz, isotype).

\section{Phialina setiigera, sp. nov.}

Apothecia cupulata vel margine appressa, cupulata, breviter stipitata, pilosa, alba vel luteola vel pallide sulphurea; specimina exsiccata similes sed non alba. Excipulum externum textura prismatica, cellulis prismaticis vel plus minusve isodiametris, 10.8-21.0 × 5.8-10.5(-14.8) $\mu \mathrm{m}$, parietibus tenuibus, hyalinis, in solutione Melzeri non coloratis. Pili 100-250 x 4.0-6.5 $\mu \mathrm{m}$, anguste conici, leves, aliquantum crassotunicati, multiseptati, in solutionee Melzeri non colorati, pigmento aureo. Asci 40-65 × 5.0-7.4 $\mathrm{mm}$, octospori, amyloidei, in basi uncinati. Sporae 9.0-11.8 $\times 2.7-3.5 \mu \mathrm{m}$, late fusoideae vel ellipsoideae vel naviculatae, non septatae, non guttulatae. Paraphyses cylindraceae vel in apice minuter dilatatae, pigmento aureo, dense septatae, 2.0-3.4 $\mathrm{m}$ latae, cellulis terminalibus 13-18 $\mu \mathrm{m}$ longis.

Holotype: Canada. Yukon: Kluane Lake, Outpost Mtn., alt. ca. $910 \mathrm{~m}$, a steep ravine with a small creek, on leaves of
Alnus crispa, very abundant, 19.IX.1987 Huhtinen 87/133 (TUR, isotypi in CUP, TAA, K, cultures in CBS and ATCC).

Apothecia gregarious, superficial, typically 100 $500 \mu \mathrm{m}$ in diam when dry, cupulate throughout development, margin in larger apothecia rarely appressed to the substrate, shortly stipitate on a ca. $50 \mu \mathrm{m}$ wide and up to $80 \mu \mathrm{m}$ high stipe. Disc often convex when fresh, plane and rarely convex when dry, often spongy and pitted when mature, bordered by a thin and sparse zone of marginal hairs, which rise above the hymenium, margin often appressed to the substrate when fresh, inconspicuous when dry, plane to only slightly incurved. Hair cover sparse, whitish. Colour white to pale ochraceous to pale sulphur yellow when fresh; dry apotecia ochraceous to pale sulphur yellow.

Ectal excipulum of textura prismatica, cells on middle flanks prismatic to somewhat isodiametric, $10.8-21.0 \times 5.8-10.5(-14.8) \mu \mathrm{m}, \overline{\mathrm{x}}=14.5 \times$ $8.3 \mu \mathrm{m}(\mathrm{n}=30), \bar{Q}=1.7$, becoming smaller towards margin, at the base roundish and small. Inner excipulum composed of narrower textura prismatica, cells ca. $15-23 \times 3.2-6.8 \mu \mathrm{m}$. Walls in ectal parts thin to $0.4 \mu \mathrm{m}$ thick, hyaline, $\mathrm{CB}+, \mathrm{CR}+, \mathrm{BF}-$, CRB-, LUG-, MLZ-, NR-, TB-.

Hairs 100-250 x 4.0-6.5 $\mu \mathrm{m}$, narrowly conical, straight, multiseptate, septal intervals $6-14 \mu \mathrm{m}$ in the lower $1 / 3$, with bright yellow pigment. Apex tapering to $1.0-1.5 \mu \mathrm{m}$ in mature hairs, blunt and 2-4 $\mu \mathrm{m}$ wide in juvenile hairs, solidified, without an apical 


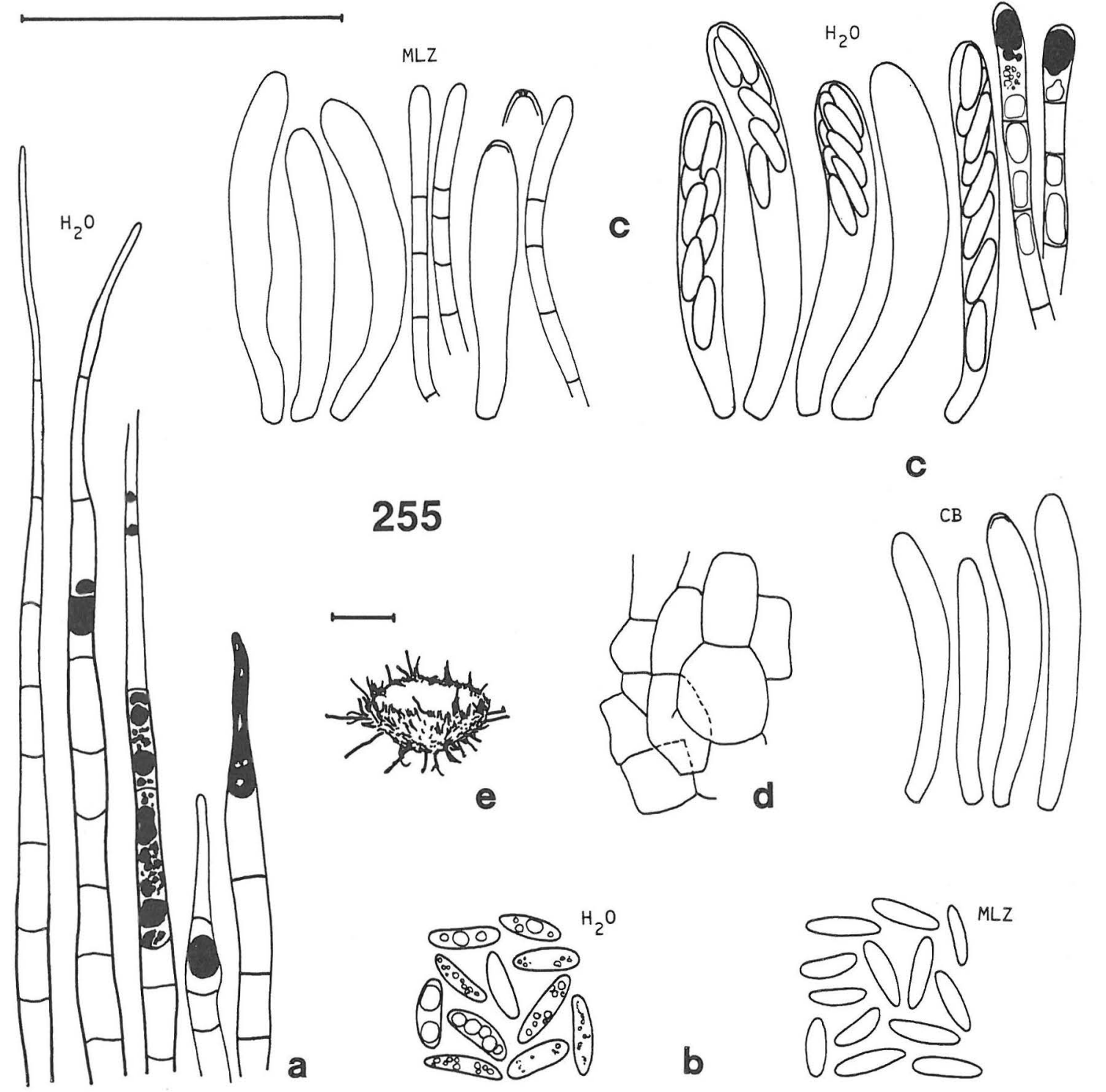

Fig. 255. Phialina setiigera, SH 87/154. a) hairs, b) spores, c) asci and paraphyses, d) excipulum, e) dry apothecia. Scale $50 \mu \mathrm{m}$, for apothecia $100 \mu \mathrm{m}$.

globule in MLZ. Wall basally $0.6 \mu \mathrm{m}$ thick, $0.4 \mu \mathrm{m}$ in medial parts, becoming thinner towards apex, refractive, rarely locally prominently thickened, hyaline, smooth, negative in all reagents. Pigment amorphous, persistent in all reagents, $\mathrm{BF}+$ (red), $\mathrm{CB}+$ (blue), CRB+ (blue), LUG+ (reddish brown),
MLZ+ (golden), NR+ (red), TB+ (greenish), in CR forming "hollow" bodies with a light centre and reddish margins.

Asci $50-64 \times 6.8-9.4 \mu \mathrm{m}, \overline{\mathrm{x}}=57.4 \times 7.8 \mu \mathrm{m}(\mathrm{n}=$ 5), $\bar{Q}=7.4$ when fresh in water; $45-65 \times 5.8-7.4(-$ 7.8) $\mu \mathrm{m}, \overline{\mathrm{x}}=56.2 \times 6.6 \mu \mathrm{m}(\mathrm{n}=59), \bar{Q}=8.4 \mathrm{in}$ 
MLZ; $40-61 \times 5.0-6.2 \mu \mathrm{m}, \overline{\mathrm{x}}=49.0 \times 5.9 \mu \mathrm{m}(\mathrm{n}=$ 13), $\bar{Q}=8.5$ in CB; eight-spored, cylindrical-clavate with a slightly conical apex. Apical pore MLZ++ when fresh cand dry, LUG+ (blackish violet). Wall in mature asci apically $0.8-1.0 \mu \mathrm{m}$ thick (in $\mathrm{CB}$, $M L Z)$, negative in all reagents. Contents MLZ-, infrequently LUG+. Asci arising from croziers.

Spores $9.8-13.3 \times 2.8-4.0 \mu \mathrm{m}, \overline{\mathrm{x}}=11.1 \times$ $3.3 \mu \mathrm{m}(\mathrm{n}=14), \bar{Q}=3.4$ when fresh in water; 9.0 $11.8 \times 2.7-3.5 \mu \mathrm{m}, \overline{\mathrm{x}}=10.6 \times 3.1 \mu \mathrm{m}(\mathrm{n}=90), \bar{Q}=$ 3.5 in CB and MLZ; broadly fusoid to elliptic with slightly tapered apices, occasionally naviculate, aseptate. Wall hyaline, not seen to become brownish in aged spores. Guttulae inconspicuous to lacking when fresh in water (mature spores), lacking in dry material (MLZ).

Paraphyses cylindrical to clavate, frequently containing bright yellow, amorphous pigment similar to that inside the hairs, anastomosing below the level of the ascal bases. Terminal cells $13-18 \times 2-3 \mu \mathrm{m}, \overline{\mathrm{x}}=$ $15.8 \times 2.6 \mu \mathrm{m}(\mathrm{n}=15)$, clavate apices $2.8-3.4 \mu \mathrm{m}$ wide, closely septate below, mean septal intervals $10.0 \mu \mathrm{m}(\mathrm{n}=30)$.

Cultural characters ( 2 population, 7 strains). Radial growth slow on MA, $10.7 \mathrm{~mm} /$ month $(\mathrm{n}=10,2$ popul., 4 strains). Mats with black basic colour, margin black to Cinnamon (N69) to dark brown (T71). Aerial mycelium snow-white to grey (N92),

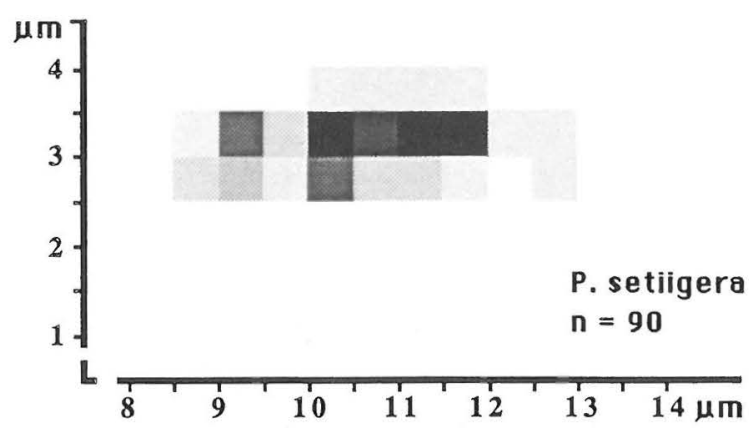

Fig. 256. Percentage sporogram of Phialina setiigera.

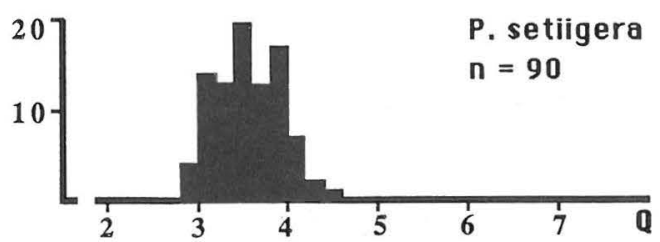

Fig. 257. The distribution of spore quotient $Q$ in Phialina setiigera. cottony, extending to $2-4 \mathrm{~mm}$ from the margin. Margin distinct, minutely uneven, submerged. Hyphal strands lacking, zonation lacking, sector formation lacking, no colour change in surrounding agar, no yeast-like growth. Context soft at surface, odour not distinct.

Spores germinating readily on wood agars, growth moderate to retarded on BA, PA, QA, LA. Producing mature apothecia in abundance after 10 months (single-spore strains) on MA, apothecia scattered on LA, exceptional on BA, apothecial primordia produced on PA, these not developed further. The 7 strains isolated from a cultivated apothecium all identical. Anamorph not produced in single-spore or multispore trials.

The black basic colour, covered by a densely cottony, white to grey aerial mycelium characterizes $P$. setiigera in culture. Apothecial production was exceptionally rich on MA, the agar surface in the storage tubes being occasionally totally filled with caespitose apothecia.

Distribution and phenology. The species is so far known only from two areas in western Canada, close to the timberline. The collections were all made in September.

Ecology. All the collections were made in dense thickets of Alnus crispa. In two exceptionally rich collections $P$. setiigera occurs together with less abundant $P$. lachnobrachya. $P$. setiigera was found among the type material of Hyalopeziza digitipila from Inuvik (N.W.T.). Alnus crispa is apparently a favourable habitat for foliicolous Hyaloscyphaceae. At Inuvik $P$. lachnobrachya and $H$. digitipila were clearly dominant on the thick leaf carpets under Alnus crispa. At Kluane Lake (Yukon) similar mass occurrences of apothecia were observed, but the dominant was $P$. setiigera.

Discussion. The species is easily recognized by the long, stiff, densely multiseptate hairs and the broad spores. The variation between the three populations is small. As in P. lachnobrachya, the apothecia may vary within one population from snow-white to clearly yellowish. Under the microscope such apothecia are identical and their hairs and paraphyses contain the typical Phialina pigment. The hairs match those in the long-haired populations of $P$. lachnobrachya. The hairs of $P$. lachnobrachya are only rarely thickened, but thickening almost always occurs in $P$. setiigera.

Many characters appear constant in the few collections studied. The spore and ascus sizes seem to 
vary within narrow limits. All the populations have long and somewhat stiff hairs, croziers, MLZ++ apical pores, $\mathrm{CB}+$ excipulum and spores lacking guttulae and septa.

Material studied: Canada. Northwest Territories: Inuvik, 1984 SH 84/185 (TUR, mixed in type of Hyalopeziza digitipila). Yukon: Kluane Lake, 1987 SH 87/133* (type), 87/154* (TUR).

\section{Phialina ulmariae}

Phialina ulmariae (Lasch in Rabenh.) Dennis, Brit. cup fungi and their allies: 102. 1960. - Peziza ulmariae Lasch in Rabenh., Flora 36:201. 1853. - Trichopeziza ulmariae (Lasch in Rabenh.) Lambotte, Fl. mycol. belge: 524. 1880. - Urceola ulmariae (Lasch in Rabenh.) Quélet, Enchir. fungorum: 322. 1886. - Mollisia atrata (Pers.: Fr.) Karst. "form" ulmariae (Lasch in Rabenh.) Phill., Man. Brit. Discomyc.: 182. 1887. - Mollisia ulmariae (Lasch in Rabenh.) Rehm, Rabenh. Krypt.Fl. ed. 2, 1(3): 531. 1892. - Pyrenopeziza ulmariae (Lasch in Rabenh.) Boud., Hist. classific. discom. Europe: 129. 1907. - Hyaloscypha ulmariae (Lasch in Rabenh.) Nannf. ex Gremmen, Fungus 26:36. 1956, nom. inval. (Art. 33.2). - Calycellina ulmariae (Lasch in Rabenh.) Korf, Mycotaxon 14:2. 1982. - Type material (the exsiccatum mentioned, not specified further): [Poland,] Driesen [= Drezdenko] (as Rabenhorst's "Klotzschii herbarium vivum mycologicum sistens fungorum per totam germaniamn crescentium collectionem perfectam 1723").

Peziza deparcula P. Karst., Not. Sällsk. Fauna Flora Fenn. Förh. 10:191. 1869. - Helotium deparculum (P. Karst.) P. Karst., Not. Săllsk. Fauna Flora Fenn. Förh. 11:240. 1870. Pseudohelotium deparculum (P. Karst.) Sacc., Syll. Fung. 8: 293. 1889. - Pezizella deparcula (P. Karst.) Rehm, Rabenh. Krypt.-Fl. ed., 2., 1(3): 673. 1892. - Belonidium deparculum (P. Karst.) Massee, Brit. fung.-fl.: 497. 1895. - Urceolella deparcula (P. Karst.) Boud., Hist. classific. discom. Europe: 128. 1907. - Phialina deparcula (P. Karst.) Höhnel, Mitt. Bot. Inst. Techn. Hochsch. Wien 3:61. 1926. - Hyaloscypha deparcula (P. Karst.) Nannf. ex Dennis, Mycol. Pap. 32:74. 1949. - Holotype (the only existing specimen, the diagnosis extracted from this specimen): [Finland,] Tavastia australis, Tammela, Mustiala, ad Spiraeam, 6.VII.1866 Karsten (H, Herb. Karsten 2182, examined).

Phialoscypha spiraeicola Raitv., Fol. Crypt. Est. 8:3. 1977 ("spiraeaicola"). - Holotype (original designation): [U.S.S.R.,] Khabarovsk Region, Bitschevaya, Spiraea sp., 11.VIII.1966 Remm (TAA 44323, examined).

?Urceolella ulmariae Boud., Hist. classific. discom. Europe: 129. 1907. - Pseudohelotium ulmariae (Boud.) Sacc. \& Trott., Syll. Fung. 12:662. 1913. - Type: not seen.

Apothecia gregarious to confluent, superficial, typically $150-500 \mu \mathrm{m}$ in diam when dry, cupulate throughout development, varying within the same population from broadly sessile to sessile on an illdefined stipe to shortly and stoutly stipitate. Disc shallow to plano-convex when fresh, not spongy and pitted when mature, bordered by an inconspicuous margin when fresh; when dry disc often clearly concave, margin fleshy and prominently incurved when dry, occasionally undulate-crenulate. Hair cover minute, dense at the margin, dense to sparse below, whitish. Colour deep ochraceous (L60) to more orange (M57) to Apricot (L65) when fresh; dry apothecia typically dark brown (R29) or Chestnut (R27) to somewhat more reddish (S20), in some populations orange brown (N59, P59), rarely light yellowish brown (L77).

Ectal excipulum varying between the populations from modified textura prismatica with thin apical and massively thickened side walls to textura prismatica, cells on middle flanks varying correspondingly from narrowly prismatic or seemingly hyphoid, 9-18 $\times 3$ $6(-6.4) \mu \mathrm{m}, \overline{\mathrm{x}}=11.7 \times 4.6 \mu \mathrm{m}(\mathrm{n}=30), \bar{Q}=2.5$ to regularly prismatic or somewhat isodiametric, $8-17 \times$ (3.5-) 4.0-9.2 $\mu \mathrm{m}, \overline{\mathrm{x}}=11.1 \times 5.8 \mu \mathrm{m}(\mathrm{n}=30), \overline{\mathrm{Q}}=$ 1.9 , becoming smaller towards margin, at the base more roundish or excipulum composed of brown textura epidermoidea. Inner excipulum composed of narrower cells. Walls in ectal parts variably thickened, in ca. $70 \%$ of the populations walls uniformly 1-2 $\mu \mathrm{m}$ thick, in ca. $20 \%$ side walls $1.0-2.6 \mu \mathrm{m}$ thick and apical walls thin, in ca. $10 \%$ walls 0.4 $1.0 \mu \mathrm{m}$ thick, hyaline, hyaline to brown at the base, negative in all reagents.

Hairs $10-44 \times 2-4 \mu \mathrm{m}$ narrowly conical, flexuose/ cincinnate to curved, more rarely straight, often oneor two-septate, the basal parts frequently with bright yellow pigment. Apex tapering to $0.4-1.0 \mu \mathrm{m}$, not solidified, without an apical globule in MLZ. Wall thin and dull, never locally prominently thickened, hyaline, smooth in $89 \%$ of the populations, occasionally bearing scattered, minute, apical warts in others, warts deforming or not in CB and MLZ, wall negative in all reagents. Pigment amorphous, persistent in all reagents, $\mathrm{BF}+$ (red), $\mathrm{CB}+$ (blue), $\mathrm{CRB}+$ (blue), LUG+ (reddish brown), MLZ+ (golden), $\mathrm{NR}+$ (red), TB+ (green), in CR forming "hollow" bodies with a light centre and reddish margins.

Asci $23-39(-40) \times 4.6-5.8(-6.8) \mu \mathrm{m}, \overline{\mathrm{x}}=29.9 \times$ $5.3 \mu \mathrm{m}(\mathrm{n}=25), \overline{\mathrm{Q}}=5.6$ in MLZ; $26-41(-43) \times$ 4.5-6.3 $(-7.2) \mu \mathrm{m}, \overline{\mathrm{x}}=33.8 \times 5.2 \mu \mathrm{m}(\mathrm{n}=82), \overline{\mathrm{Q}}=$ 6.3 in $\mathrm{CB}$; four-spored, in one population rarely sixspored, cylindrical-clavate with a slightly conical apex. Apical pore MLZ++ when dry, LUG+ (blue). Wall in mature asci apically $0.8-1.3 \mu \mathrm{m}$ thick (in CB, MLZ), negative in all reagents. Contents MLZ-, rarely LUG + , at the base and apex yellowish pigment also often present, staining reactions similar to those of hair pigment. Asci arising from croziers.

Spores $11.0-16.3(-19.8) \times 1.5-2.6(-3.2) \mu \mathrm{m}, \overline{\mathbf{x}}$ $=14.0 \times 2.1 \mu \mathrm{m}(\mathrm{n}=177), \bar{Q}=7.1$ in CB and MLZ; 

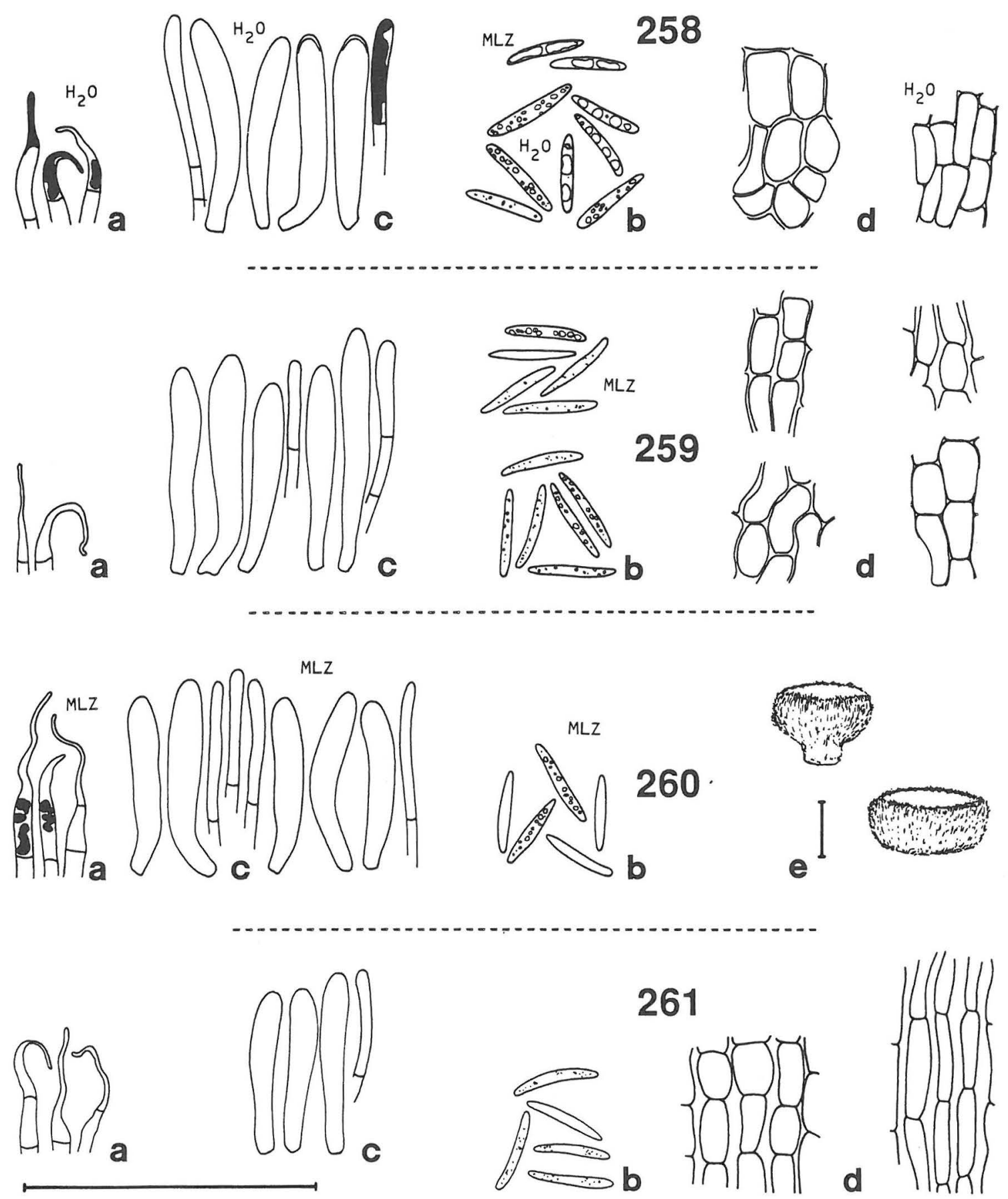

261

Figs. 258-261. Phialina ulmariae. a) hairs, b) spores, c) asci and paraphyses, d) excipulum, e) dry apothecia. Scale $50 \mu \mathrm{m}$, for apothecia $100 \mu \mathrm{m}$. - 258: SH 87/82. - 259: 1900 Crossland. - 260: Holotype of Peziza deparcula. — 261: 1887 Sydow. 
fusoid, rarely oblong-elliptic, straight to slightly curved, rarely apically beaked, aseptate. Wall hyaline, not seen to become brownish in aged spores. Guttulae prominent and light when fresh and dry.

$P$ araphyses cylindrical to clavate, frequently containing bright yellow, amorphous pigment similar to that inside the hairs, first dichotomous branches occurring typically at or below the level of the ascal bases. Terminal cells $15-30 \times 1.5-3.0 \mu \mathrm{m}, \overline{\mathrm{x}}=21.1$ $\times 2.3 \mu \mathrm{m} \quad(n=65)$, clavate apices $2-4 \mu \mathrm{m}$ wide, closely septate below, mean septal intervals $7.2 \mu \mathrm{m}$ $(\mathrm{n}=50)$.

\section{Cultural characters. Not known.}

Distribution and phenology. The species is widely distributed in Europe and the U.S.S.R. In its phenology it differs clearly from the closely related $P$. lachnobrachya and $P$. flaveola. Most populations have been collected in mid-summer (Fig. 17).

Ecology. All but two collections have been made from dead stems of Filipendula ulmaria. The exceptions originate from leaves of Spiraea and Salix.

Discussion. The species is recognized by the substrate, the yellow apothecia, which turn dark reddish brown or brownish upon drying, the narrowly fusoid and clearly guttulate spores, and the clearly to massively thickened excipular walls. The closest relative, $P$.flaveola, lacks such an excipulum, retains its yel- low colour upon drying and is smaller. The difference in phenology is also noteworthy. As discussed under $P$. flaveola, $P$. ulmariae and $P$. lachnobrachya are distinguished by divergent trends rather than by clear-cut differences.

Taxonomically, the most significant variation occurs in the excipular structure. The studied populations show a gradation from textura prismatica with somewhat thickened walls, through textura prismatica incrassata to tissue resembling textura oblita. Similar gradation was recently discussed in Arachnopeziza (Huhtinen 1987d). An excipular type similar to that in Fig. 261 occurred in a taxon definitely belonging to Arachnopeziza. I suggested that it had developed from an originally hyphoid excipulum by thickening of the side walls only, the septa remaining thin, or that it was the result of irregular thickenings in an originally prismatic structure. The evidence provided by $P$. ulmariae suggests that this rare excipular type is prismatic in origin. In some populations the walls become equally thick in all directions, while in others the upper and lower walls remain thin. The reason for this difference is obscure, but the unbroken gradation indicates that this character does not exclude Peziza ulmariae from close relationship to $P$. lachnobrachya and $P$. flaveola. In other respects the variation between the populations is small.

A similar excipular structure was discussed by Arendholz (1979) in the treatment of Bertault's (1977) new section Phialina in Hyaloscypha. Arend-

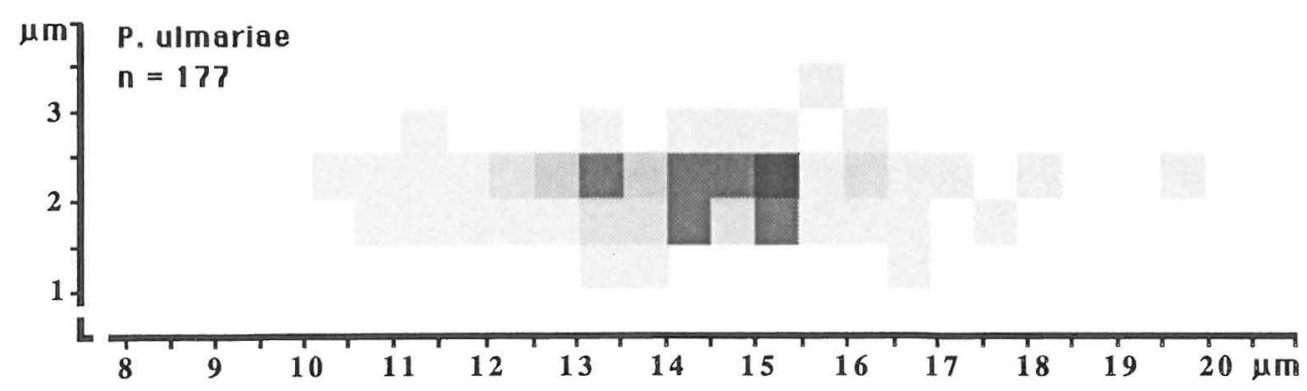

Fig. 262. Percentage sporogram of Phialina ulmariae.

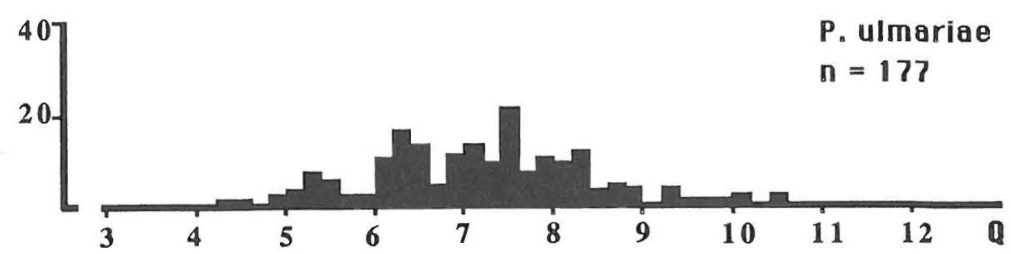

Fig. 263. The distribution of spore quotient $Q$ in Phialina ulmariae. 
holz pointed out the difference between textura oblita, with thick walls and thin lumina, and the structure he called textura prismatica incrassata, which is characterized by prismatic cells and, consequently, wide lumina. However, my generic distinction between Hyaloscypha and Phialina sensu Arendholz is based mainly on other characters than those stressed by him.

Unlike the other material, two collections originating from the Khabarovsk region in the U.S.S.R. have been made from leaves, one from Spiraea (type of Phialoscypha spiraeicola) and the other from Salix. Superficially, they both resemble $P$. lachnobrachya when dry, because of their yellow colouring. However, the prismatic excipulum has strongly thickened walls, frequently up to $2 \mu \mathrm{m}$ thick, which is a highly anomalous character in $P$. lachnobrachya. The small asci and the narrowly fusoid and clearly guttulate spores also differentiate them from that species, and link the collections with P. ulmariae. Apart from the colour of dried apothecia and the ecology, these two collections clearly come within the variational range of $P$. ulmariae, and Phialoscypha spiraeicola is here put in its synonymy. It is, of course, curious that the strict ecological distinction of the well-studied European material, is obscured in the Far East.

The eight-spored Urceolella ulmariae Boud. was treated as a synonym of present species by Korf (1982), who made no comment on the number of spores in the asci. When describing the species, Boudier (1907) specifically pointed out this difference from $U$. deparcula (P. Karst.) Boud. No type material of the species exists in PC and apparently Boudier's taxon has remained uncollected ever since. Gremmen's (1956) observation on the variable spore number in asci of $P$. ulmariae suggests that Boudier's species could be synonymous. Eight-spored asci were not, however, encountered in the present material.

Exsiccatae studied: Jaap. O., Fungi sel. exs. 359 (M, P). Krieger, K., Fungi saxonici $2125(\mathrm{H}, \mathrm{K}, \mathrm{M}, \mathrm{PAD}, \mathrm{S})$. Rehm, H., Ascomyceten 905 (K, M, NY, S). - Sydow, P., Mycotheca Marchica 1369 (K, S).

Material studied: Belgium. Liège, Mouton (BR). Czechoslovakia. Bohemia: Mnichovice, 1986 Svrčk \& SH 86/33 (TUR). German Democratic Republic. Berlin: Wilmersdorf, 1887 Sydow (S, Herb. Rehm). Parchim: Triglitz, 1904 Jaap (S, Herb. Rehm). Ober-Lausitz: Bachlau, 1908 Feurich (S, Herb. Rehm). Finland. Uusimaa: Kirkkonummi, 1987 SH 87/82 (TUR). Etelä-Häme: Tammela, 1866 PAK (H, type of Peziza deparcula). France. Quevilly, Letendre (H). Great Britain. Devon: Slapton Ley, 1981 Clark (IMI). Herefordshire?: Not localizable, 1972 Bower (K). Norfolk: Kings Lynn, 1979 Moore (K). Whetafen, 1947 Dennis (K).
Lincolnshire: Tumby, 1908 Hawley (K, Herb. Crossland). Yorkshire: Halifax, 1900 Crossland (S, Herb. Rehm). Hornsea, 1900 (NY, Herb. Massee). Perthshire: Not localized (K, Herb. Rea). Ebudes: Isle of Mull, 1968 Dennis (K). Isle of Tiree, 1973 Dennis (K). Isle of Skye, 1983 Dennis (K). Not localizable, 1949 Ellis \& Ellis (IMI). Ireland. Dublin, 1950 Hassell (K). Norway. Akershus: Drøbak, 1893 Starbăck (S). Nordland: Træna, 1988 Olsen (TUR). Sweden. Gotland: Morby, 1913 Vestergren (S). U.S.S.R. Estonian S.S.R.: Distr. Harju, Riisipere, 1965 AR (TAA). Distr. Hiiumaa, Hanikatsi Islet, 1972 Kullman (TAA) Distr. Tartu, Elva, 1965 AR (TAA, two collections). Latvian S.S.R.: Jelgava, 1965 AR (TAA). Kalnciems, 1966 AR (TAA). Lithuanian S.S.R.: Kaunas, 1966 AR (TAA). Khabarovsk Region: Korfovsk, 1966 Remm (TAA). Bitchevaya, 1966 Remm (TAA, type of Phialoscypha spiraeicola). Sakhalin Region: Homutovo, 1970 Kullman \& AR (TAA). 


\section{EXCLUDED, DUBIOUS AND IMPERFECTLY KNOWN TAXA}

1. Hyaloscypha acerina Velen., Monogr. Discom. Bohem.: 277; P1. 14:18. 1934, nomen dubium. - Holotype (the only specimen mentioned): [Czechoslovakia,] Bohemia, Radotín prope Pragam, in valle "Radotinske údolí" dicto, VIII.1924 Velenovský (PRM 148463, not seen).

According to Svrcek (1985), the type specimen has no apothecia matching the protologue. A new species, Hamatocanthoscypha acericola, was recently described by Svrcek (1987a), on the basis of one of the fungi in the type specimen. Svrcek's species is here treated as a synonym of Phialina pseudopuberula.

2. Uncinia acerina Velen., Monogr. Discom. Bohem.: 294; Pl. 15:32. 1934. - Holotype (the only specimen mentioned): [Czechoslovakia,] Radotín, in foliis aceris campestris, 30.VIII.1924 Velenovský (PRM 148617, examined).

A study of the abundant holotype showed that Velenovsky's illustration and description match the specimen fairly well. Placement in Calycellina is suggested by the cylindrical and mostly aseptate hairs, the yellow pigment tuming golden in MLZ, the foliicolous habit, the elliptic-fusoid spores and the gross morphology of the apothecia (cf. Lowen \& Dumont 1984). Except for the frequently uncinate hairs, the specimen agrees well with $C$. populina (Fuckel) Höhnel.

3. Hyaloscypha acuminatula Svr., Česká Mykol. 32: 202. 1978. - Holotype (original designation): [Czechoslovakia,] Bohemoslovakia, Slovakia septentrionalis, montes Vysoké Tatry, ad ripam lacus "Trojrohé pleso" dicti apud casam alpinam "Kežmarská chata", alt. 1614 m., 11.VIII.1956 Kubicka \& Svrčk (PRM, not seen).

The type specimen was not available for study, but Dr. Svrcek kindly supplied me with the original annotations and drawings of the type. The conical and acuminate hairs, the voluminous asci and especially the long and narrow spores suggest conspecificity with the polyphagous Hyaloscypha herbarum, but formal synonymy will not be proposed until the type can be studied.

4. Hyaloscypha acuum (Alb. \& Schw.: Fr.) Velen., Monogr. Discom. Bohem.: 278. 1934. - Peziza acuum Alb. \& Schwein., Consp. fung. Lusat: 330. 1805. - Type: not designated.

Judging from Velenovsky's illustration, his material fits the present concept of this dwarfish species. The rough cylindrical-clavate hairs exclude it from Hyaloscypha. The choice of genus has wavered between Dasyscyphus, Cistella and Clavidisculum Kirschst. (Dennis 1949, Svrcek 1962 and 1987d, Matheis 1977, Raitviir 1970a). Further synonymy is given by, e.g., Carpenter (1981).

5. Hyaloscypha albolutea (Pers.) Velen. Monogr. Discom. Bohem.: 272. 1934. - Peziza sulphurea var. albolutea Pers., Syn. method. fung.: 649. 1801. - Type: not seen.

Under Peziza sulphurea B P. albolutea Persoon (1801) referred to plate 8 in his earlier work (Persoon 1800), where $P$. sulphurea is illustrated as a lignicolous, apparently hyalo- scyphaceous fungus. Connection with the genera studied here is, however, unlikely. The material examined by Velenovsky partly belonged to $H$. aureliella (one collection studied). Svrcek (1985) also reported other species of Hyaloscypha from the material.

6. Uncinia antirrhini Velen., Monogr. Discom. Bohem.: 412. 1934, nomen dubium. - Holotype (the only specimen mentioned): [Czechoslovakia, Bohemia,] Mnichovice, Vresiny, Antirrhinum orontium, VII.1934. Velenovský (PRM 153252, examined).

The holotype convolute was empty.

7. Hyaloscypha asperipila Svr., Česká Mykol. 31:9; PI. 1:3. 1977. - Holotype (original designation): [Czechoslovakia,] Bohemoslovakia, Bohemia meridionalis, Třebon̆, in alneto paludoso "U Jindr̊̊" dicto, 10.XI.1956 Kubicka (PRM 799375, not seen).

After studying a paratype (in BHU), I could conclude that $H$. asperipila is a synonym of Hyaloscypha carpinacea Velen. and $H$. salicina Velen. The golden yellow pigment, broad paraphyses, elliptic-fusoid spores, foliicolous habit and cylindrical hairs exclude it from Hyaloscypha. The species is closely related to Calycellina, but the prominent warts on the hairs make it uncertain whether it belongs to that genus (Huhtinen 1990).

8. Hyaloscypha barbata (Velen.) Svr., Česká Mykol. 39:216. 1985. - Hyaloscypha quercina Velen. var. barbata Velen., Monogr. Discom. Bohem.: 276. 1934. - Lectotype (Svřek 1985: 216): [Czechoslovakia,] Bohemia, Jevany, Fagus, XI.1923 Velenovsky (PRM 149740, examined).

This variety was recently transferred to the genus Protounguicularia, and was shown to offer an earlier name for $P$. brevicapitata Raitv. \& Galán (Huhtinen 1987a).

9. Hyaloscypha betulina Velen., Monogr. Discom. Bohem.: 276; PI. 14:29. 1934. - Lectotype (Svrcek, 1985: 206): [Czechoslovakia, Bohemia,] Mnichovice, Betula alba, 23.IV.1928 Velenovsky (PRM 147639, examined).

Type material fits the original diagnosis and illustrations well. As already concluded by Svrcek (1985), H. betulina belongs to Cistella. The clavate hairs were covered with prominent, long spines.

10. Hyaloscypha byssacea (P. Karst.) Boud., Hist. classific. discom. Europe: 127. 1907. - Peziza byssacea P. Karst., Fungi Fenniae Exsiccati 741. 1868. - Type: not designated.

The exsiccatus in $\mathrm{S}$ contained a helotiaceous fungus related to Crocicreas Fr.

11. Uncinia callunae Velen., Novitates mycol. noviss.: 140. 1947, nomen dubium. - Holotype (the only specimen mentioned): [Czechoslovakia,] ad caules putridos Callunae loco insolato, merid. prope Mirošovice maio 1942, data from Velenovský (1947), examined.

The type convolute is empty. 
12. Hyaloscypha candidata (Cooke) Boud., Hist. classific. discom. Europe: 127. 1907. - Peziza candidata Cooke, Grevillea 1:130.1873. - Type not designated, original material in NY studied.

Both the original material and Massee's plate marked "drawn from type" indicate that $P$. candidata belongs to Arachnopeziza.

13. Hyaloscypha caricicola (Sacc.) Velen., Monogr. Discom. Bohem.: 280. 1934. - Dasyscypha caricicola Sacc., Syll. Fung. 8:450. 1889. - Type: not seen.

The identity of Dasyscypha caricicola is unknown. The material examined by Velenovsky was shown to belong to Psilachnum lateritioalbum (P. Karst.) Höhnel by Svrček (1985).

14. Hyaloscypha carnosa (Rodway) Spooner, Biblioth. Mycol. 116:423. 1987. - Helotium carnosum Rodway, Pap. Proceedings Royal Soc. Tasmania: 155. (1920) 1921. Holotype (the only specimen mentioned): [Australia,] Tasmania, Cascades, Hobart, VIII.1919 Rodway (HO 32529, examined).

When transferring the species to Hyaloscypha, Spooner (1987) stressed the presence of scanty, more or less tapered hairs. Studying the holotype specimen, I found that there were numerous clavate hyphal ends and the specialized hairs were more or less cylindrical. They were also multiseptate in the protruding part. The prevailing hair type clearly excludes $H$. carnosum from Hyaloscypha. Psilocistella might be the most suitable genus for the species.

15. Hyaloscypha carnosula Velen., Monogr. Discom. Bohem.: 281; P1. 14:43. 1934. - Lectotype (Svrček 1985: 206): [Czechoslovakia,] Bohemia, Kosoř, Carex, VI.1927 Velenovský (PRM 149665, examined). (1985).

The species was recently placed in Discocistella by Svrček

16. Hyaloscypha carpinacea Velen., Monogr. Discom. Bohem.: 273; PI. 14:2. 1934. - Lectotype (Svrček, 1985: 206): [Czechoslovakia,] Bohemia, Mnichovice, Mirosovice, in foliis carpineis, 16.IX.1929 Velenovský (PRM 151167, examined).

The name is discussed under the conspecific $H$. asperipila. Hyaloscypha salicina Velen. is a further synonym.

17. Hyaloscypha cejpii Velen., Monogr. Discom. Bohem.: 277; Pl. 14:16. 1934. - Lectotype (Svrček, 1978: 204): [Czechoslovakia, Bohemia,] Lysá n[ad] L[abem], Salix cinerea, 24.V.1924 Velenovsky (PRM 148693, examined).

This species has been placed in Psilocistella and Microscypha by Svrček $(1978,1985)$. In Finland, too, the species is found on the underside of leaves of Salix caprea. The roundish excipular cells with brownish walls point to Microscypha rather than to Psilocistella (Svrček 1985).

18. Hyaloscypha ceracella (Fr.) Boud., Hist. classific. discom. Europe: 126. 1907, nomen dubium. - Peziza ceracella Fr., Syst. Mycol. 2:114. 1823. - Type: not designated.
No original material of this species is preserved in $\mathbf{S}$ or UPS. Saccardo's (1875) interpretation of the species was erroneous, according to Mycotheca Veneta 1507 in RO. His material had naked apothecia, whereas Fries described short coloured hairs in $P$. ceracella.

19. Hyaloscypha ciliata (Fuckel) Migula, Thome's Kryptogamen-Flora 3(3,2): 1092. 1913, lapsus calami pro Hyalopeziza ciliata Fuckel.

20. Hyaloscypha ciliifera (P. Karst.) Boud., Hist. classific. discom. Europe: 127. 1907. - Peziza ciliifera P. Karst., Fungi Fenniae Exs. 546. 1866. - Type: not designated.

Karsten's exsiccatum (in $\mathrm{H}$ ) contains a helotiaceous species. The short marginal hairs mentioned by Karsten (1869) were not found. Korf (1971) placed the species in Pezoloma Clem.

21. Hyaloscypha cladii Nag Raj \& Kendrick, Monograph of Chalara and allied genera: 183, Fig. 61b. 1975. - Holotype (original designation): [Great Britain,] Dorset, Wareham, Sugar Hill, 26.V.1961 (IMI 89626b, not seen).

An isotype (IMI 89626f) was studied. The species is the holotype of Phaeoscypha Spooner and the differences from Hyaloscypha were discussed by Kirk and Spooner (1984). Hyaloscypha cladii-marisci Svr. and Uncinia scintillans Graddon are synonyms (see below).

22. Hyaloscypha cladii-marisci Svr., Česká Mykol. 40:209; P1. 2:4. 1986. - Holotype (original designation): [Czechoslovakia,] Bohemia centralis, in prato uliginoso "Polabská cemava" dicto (area tuta) prope pagum Mělnická Vrutice, 18.IX.1985 Pivnicková (PRM, not seen).

The type specimen was not available for study, but Dr. Svrcek kindly supplied me with his original annotations and drawings. These, together with the protologue, clearly indicate that $H$. cladii-marisci is a synonym of Phaeoscypha cladii $(\mathrm{Nag}$ Raj \& Kendrick) Spooner (see above).

23. Hyaloscypha clavuliformis (P. Karst.) Boud., Hist. classific. discom. Bohem.: 127. 1907. - Peziza clavuliformis P. Karst., Fungi Fenniae Exs. 742. 1868. - Type: not designated.

Karsten's (1871) description of Pezicula clavuliformis ( $\mathrm{P}$. Karst.) P. Karst. suggests that the species is not hyaloscyphaceous.

24. Hyaloscypha contorta Velen., Monogr. Discom. Bohem.: 285. 1934. - Holotype (the only specimen mentioned): [Czechoslovakia,] Bohemia, Mencice near Mnichovice, on wood of fallen trunk of Sarothamnus scoparius, 2.VIII.1926 Velenovský (PRM 151186, not examined), data from Svrcek (1985).

The scanty type specimen was not studied. Svr飞ek (1985) listed the species as a synonym of Unguiculella falcipila Höhnel. Velenovsky (1934) did not cite any illustration, but Svrček referred to one (Plate 14:19) with the legend $H$. sarothamni. Apparently Velenovsky had confused drawings of species on Sarothamnus (see discussion under $H$. herbarum). 
25. Hyaloscypha cookei (Pass.) Boud., Hist. classific. discom. Europe: 127. 1907. - Peziza cookei Pass., Nuovo Giorn. Bot. Ital. 3:258. 1875. - Type: not seen.

No material of $P$. cooke $i$ was studied by me, but Dennis (1963), who had seen it, placed the species in Urceolella. Rashle (1977) suggested that it might be placed in Unguicularia.

26. Hyaloscypha cretacea Velen., Monogr. Discom. Bohem.: 278; P1. 14:32. 1934. - Lectotype (Svrcek, 1985: 207): [Czechoslovakia,] Bohemia, Vysehorovice, in foliis Artemisiae vulgaris, 17.VI.1923 Velenovsky (PRM 149289, examined).

Hyaloscypha cretacea was recently transferred to Dendrotrichoscypha Svr. by Svrcek (1985), but this taxon belongs to Mollisina Höhnel (Huhtinen 1987c). The type species of Dendrotrichoscypha is also a typical species of Mollisina (holotype in PRM examined).

27. Hyaloscypha cretacea Velen. var. lupuli Velen., Monogr. Discom. Bohem.: 279: PI. 14:31. (as Hyaloscypha lupuli). 1934. - Lectotype (Svřcek 1985: 207): [Czechoslovakia,] Bohemia, Radotín, Humulus lupulus, V.1924 Velenovský (PRM 148834, examined).

The type specimen contains a species which is closely related to Cistella grevillei (Berk.) Raschle, having long, almost cylindrical hairs, which bear cyanophilic spines. Svræek (1985) recognized it as an independent species in Cistella.

28. Hyaloscypha cylindrospora Velen., Monogr. Discom. Bohem.: 276; PI. 14:17. 1934. - Lectotype (Svřek 1985: 207): [Czechoslovakia,] Bohemia, Kostelec n[ad] Č[ernymi] Lesy, on a dead leaf of Betula sp., 3.V.1927 Velenovský (PRM 150873, not examined), data from Svrček (1985).

The species was recently synonymized with Merostictis betulicola (Fuckel) Svr. (Svrčk 1985).

29. Hyaloscypha cylindrospora Velen. var. alba Velen., Monogr. Discom. Bohem.: 276. 1934, nomen dubium. - Holotype (the only specimen mentioned): [Czechoslovakia, Bohemia,] Mnichovice, ad folia betulina, IX.1933 Velenovský, data from Velenovský (1934).

Type material not preserved in PRM (Svrčk 1985). The description is too scanty for recognition.

30. Uncinia daedaleae Velen., Novitates mycol.: 194. 1939, nomen dubium. - Holotype (the only specimen mentioned): [Czechoslovakia, Bohemia,] Mnichovice, Daedalea quercina, VI.1939 Velenovský (PRM 153198, examined).

The type convolute was empty. The description is barely adequate for recognition.

31. Hyaloscypha dematiicola (Berk. \& Broome) Nannf., Trans. Brit. Mycol. Soc. 20:205. 1936. - Peziza dematiicola Berk. \& Broome, Ann. Mag. Nat. Hist. 15:459; Pl. 15:20. 1865. - Lectotype (Huhtinen 1987d: 19): [Great Britain,] 31.III. 1863 Broome (K, examined).
Peziza dematiicola is the type species of Dematioscypha and was recently treated by me (Huhtinen 1987d) in a paper in which Svřek's (1977c) generic segregation was supported.

32. Hyaloscypha dennisii Bertault, Kew Bull. 31:705, Fig. 1. 1977. - Holotype (original designation): [Morocco,] Khemiss-de-Sahel, Larache, leaves of Arbutus unedo, 28.I.1959 Malençon (MPU, examined).

Hyaloscypha dennisii was based on a sclerotiniaceous fungus, whose hairs resemble those of Hyaloscypha, but are borne in a totally different manner than in the Hyaloscyphaceae. The outer excipulum has a thin discontinuous outer layer composed of hyphae running parallel to the surface and the hairs arise from these hyphae.

33. Hyaloscypha dentata (Pers.) Boud., Bull. Soc. Mycol. France 1:118. 1885. - Peziza dentata Pers., Icon. descr. fung. 1:5. 1798. - Type: not designated, apparently no original material preserved.

This is the type species of Cistella and was the other species listed under Hyaloscypha in connection with the diagnosis.

34. Hyaloscypha dentata (Pers.) Boud. var. straminea (Fuckel) Boud., Hist. classific. discom. Europe: 127. 1907. Niptera dentata (Pers.) Fuckel f. straminea Fuckel, Jahrb. Nassauischen Vereins Naturk. 25-26:335. 1871-1872. Type: not seen.

I do not know the identity of this taxon.

35. Hyaloscypha epicalamia (Fuckel) Boud., comb. inval. err. cit. in synon.

This combination was listed under the synonymy of Crocicreas stramineum (Berk. \& Broome) Carpenter by Carpenter (1981). He referred to Boudier (1907: 127), where Fuckel's epithet was, however, combined with Urceolella on page 129.

36. Hyaloscypha epilobii Velen., Monogr. Discom. Bohem.: 275; PI. 14:11. 1934. - Holotype (the only specimen mentioned): [Czechoslovakia,] Bohemia, Mnichovice, Kunice (silva), Epilobium angustif., 15.X.1928 Velenovský (PRM 151076, examined).

This is a typical species of Mollisina, as suggested earlier by me (1987c). Svrček (1985) proposed placing it in Dendrotrichoscypha, the type of which also belongs to Mollisina.

37. Uncinia epilobii Velen., Novitates mycol. noviss.: 138. 1947, nomen dubium. - Holotype (the only specimen mentioned): [Czechoslovakia, Bohemia,] Strancice, Epilobium angustifoloium, 23.VII.1941 Velenovsky (PRM 154106, examined).

The holotype convolute was empty. The original diagnosis refers fairly clearly to a species of Mollisina.

38. Hyaloscypha eriophori (Quél.) Velen., Monogr. Discom. Bohem.: 279. 1934. - Erinella eriophori Quél., Champ. Jura et Vosges, Suppl. 10:12; Pl. 11:1. 1880. (cita- 
tion combined from Saccardo (1889) and Dennis (1949)). Type: not seen.

The identity of Quélet's species was not studied. The material examined by Velenovsky belonged at least partly to Cistella (according to PRM 150171). Recently, Svrcek (1985) reported conspecificity with Discocistella glyceriae (Velen.) Svr.

39. Hyaloscypha farinacea (Cooke \& Massee) Boud., Hist. classific. discom. Europe: 127. 1907. - Pseudohelotium farinaceum Cooke \& Massee, Grevillea 21:71. 1893. Syntype: [Great Britain,] Carlisle, on bark of willow (K, examined).

The specimen bears a drawing and determination by Dr. M.A. Sherwood, stating that it contains an immature species of Tympanis Tode.

40. Uncinia galiorum Velen., Monogr. Discom. Bohem.: 296; ?P1. 14:20 (sub Hyaloscypha galiorum). 1934. - Holotype (the only specimen mentioned): [Czechoslovakia, Bohemia,] Strančice, Galium mollugo, VII.1931 Velenovský (PRM 154059, examined).

The holotype specimen represents Unguiculella eurotioides. The name Hyaloscypha galiorum was not accepted by Velenovsky because it was cited by him as a herbarium name under $H$. herbarum. The apparent error in citation of the generic name in the legend for Plate 14 is discussed under $H$. herbarum.

41. Hyaloscypha glyceriae Velen., Monogr. Discom, Bohem.: 279. 1934. - Lectotype (Svrčk 1979: 198): [Czechoslovakia,] Bohemia, Chocerady, Glyceria spectabilis, 13.VI.1925 Velenovský (PRM 149513, examined).

As stated by Svrcek (1985), H. glyceriae seems to be conspecific with $H$. eriophori. The marginal hairs are broadly clavate and densely covered with cyanophilic spines. The spores are more frequently septate than in the type of $H$. eriophori.

42. Hyaloscypha graminum Velen., Monogr. Discom. Bohem.: 280; P1. 14: 6. 1934. - Lectotype (Svrček, 1985: 208): [Czechoslovakia,] Bohemia, Mnichovice, Myšlín, Holcus lanatus, Glyceria plicata, 20.VII.1927 Velenovsky (PRM 150257, examined).

Svrcek (1985) placed this species in Psilachnum Höhnel. I found that the paraphyses were cylindrical, but the specimen was not studied in detail. Hyaloscypha iridina Velen. var. nivea Velen. is conspecific, as already noted by Svrček (1985).

43. Hyaloscypha hamulata (Feltg.) Boud., Hist. classific. discom. Europe: 126. 1907. - Pezizella hamulata Feltg., Vorst. Pilz-Fl. Luxembourg Nachtr. 3:51. 1903. - Type: not designated.

As discussed under "Generic relationships", the typification of Pezizella hamulata needs to be clarified. But the affinity to Unguiculella is evident even from the badly preserved slide in the type specimen.

44. Hyaloscypha hederae Velen., Monogr. Discom. Bohem.: 272; Pl. 14:24. 1934. - Lectotype (Svrček, 1985:
209): [Czechoslovakia,] Bohemia, Mnichovice, Hedera, X.1927 Velenovsky (PRM 151058, examined).

I found the lectotype convolute empty. Svrčk (1985) indicated synonymy with $H$. perpusilla.

45. Hyaloscypha hellebori Velen., Monogr. Discom. Bohem.: 275; Pl. 14:12. 1934, nomen dubium.

No specimen is preserved in PRM (Svřek 1985).

46. Hyaloscypha herbicola (Svr.) Korf, Mycotaxon 7: 485. 1978. - Polydesmia herbicola Svr., Ceská Mykol. 21: 149, Fig. 4. 1967. - Holotype (original designation): [Czechoslovakia,] Slovakia, montes Belanské Tatry, in valle "Kotlina Siedmich pramefov" supra Tatranská Kotlina, ad caulem iac. senecionis nemorensis, 26.VII.1958 Svr飞ek (PRM 666708 , examined from slides preserved in CUP).

Unfortunately, the type specimen in PRM was not available for study. My observations are based on slides made by Dr. Korf. The excipulum is clearly brown and the hairs taper to an acute point. The paraphyses occasionally protrude ca. $15 \mu \mathrm{m}$ above the level of the ascal apices and are hair-like in appearance. These characters clearly exclude the species from Hyaloscypha and suggest placement in Unguiculella.

47. Hyaloscypha hungarica (Rehm in Winter) Velen., Monogr. Discom. Bohem.: 274. 1934. - Pezizella hungarica Rehm in Winter, Flora 30:526. 1872. - Type: not seen.

Raschle (1978) listed Pezizella hungarica in the synonymy of Cistella grevillei. The material examined by Velenovsky was recently shown to belong to Albotricha Raitv. (Svrček 1987a).

48. Hyaloscypha hyalina (Pers.: Fr.) Boud., Icon. mycol. liste prelim.: 4. 1904, nomen confusum. - Peziza hyalina Pers., Syn. meth. fung.: 655. 1801. - Type: no original material left.

Thanks to the kindness of Dr. Machiel Noordeloos, I was able to solve the problem of the description of Peziza hyalina. In his 1801 compilation Persoon referred to an earlier description of $P$. hyalina in his Tent. disp. meth. Fung. from 1797. The same publication had appeared even earlier, in 1794, in Neues Mag. Bot. But Persoon's citation referred to Peziza corticalis, which bears the same diagnosis as $P$. hyalina (Persoon 1794, 1801). Consequently, the year 1801 stands as the starting point for the epithet hyalina.

Hyaloscypha hyalina has caused confusion in the genus in numerous connections. Dennis (1949) commented on the typification and pointed out that Fries, when sanctioning the name, also cited Nees's (1817) treatment. In this treatment the substrate was stated to be Quercus, which is the sole character with diagnostic value. The treatment by Albertini and Schweinitz (1805: 326) contained no diagnostic features, nor did that by Fries (1823). As regards the possible neotypification of $P$. hyalina, one would have to depend solely on the ecology, which is hardly desirable. As no type material exists and as the name has been used for a large number of taxa in Hyaloscypha, it is best discarded. 
49. Hyaloscypha hyperici Velen., Novitates mycol.: 193. 1939. - Holotype (the only specimen mentioned): [Czechoslovakia,] Bohemia, Mnichovice, collis Budíkov, Hypericum perforatum, 31.X.1938 Velenovský (PRM 151096, examined).

The species bore no prominent hairs. It was stated to be closely related to Mollisiella chlorinella (Ces.) Svr. by Svrcek (1985).

50. Hyaloscypha hypericicola Svr., Česká Mykol. 32: 202. 1978. - Holotype (original designation): [Czechoslovakia,] Bohemoslovakia, Slovakia septentrionalis, montes Belanské Tatry, Tatranská kotlina, ad viam versus locum "Červená hlína" dictum, alt. ca. 1500 m, 18.VI.1959 Kubička \& Svř̌ek (PRM, not seen).

Unfortunately, the type was not available for study and the original diagnosis and annotations provided by Dr. Svrcek do not clarify the status of $H$. hypericicola. The identity of this species should be determined when material is located in PRM.

51. Hyaloscypha incrustata Velen., Monogr. Discom. Bohem.: 273; Pl. 14:1. 1934.

No material is preserved in PRM (Svrcek 1985), but the original diagnosis seems to be characteristic enough to allow neotypification.

52. Uncinia invisibilis Velen., Monogr. Discom. Bohem.: 294; Pl. 15:31. 1934, nomen dubium. - Holotype (the only specimen mentioned): [Czechoslovakia, Bohemia,] Mnichovice, Prunus domestica, VIII.1926 Velenovsky (PRM 147953, examined).

I could not detect the fungus to which Velenovsky's original diagnosis refers. Only $H$. fuckelii var. alniseda is present in the specimen.

53. Hyaloscypha iridina Velen., Monogr. Discom. Bohem.: 275; Pl. 14: 26. 1934. - Lectotype (Svrčk 1985: 210): [Czechoslovakia,] Bohemia, Mnichovice-Božkov, "Jezérko", Eriophorum angustifolium", 12.VIII.1929 Velenovsky (PRM 149478, not seen).

Svrček (1985) indicated relationship to Hyaloscypha leuconica and judging from the protologue and Svrček's description, the species may be another synonym of $H$. leuconica.

54. Hyaloscypha iridina Velen. var. nivea Velen., Monogr. Discom. Bohem.: 275. 1934. - Holotype (the only specimen mentioned): [Czechoslovakia,] Bohemia, Mnichovice, "Jburany", Holcus, 30.V.1933 Velenovský (PRM 151221, examined).

Svrčk (1985) observed that this is a synonym of $H$. graminum, which he placed in Psilachnum in the same study.

55. Hyaloscypha lachnoides Velen., Monogr. Discom. Bohem.: 281; Pl. 14:42. 1934. - Holotype (the only specimen mentioned): [Czechoslovakia,] Bohemia, Mnichovice, Hrusice-Ondřejov, Juncus communis, 1.VIII.1927 Velenovský (PRM 147795, examined from a microscopic slide).

Svrček (1985) reported synonymy with Discocistella fugiens (Buckn.) Svr.
56. Hyaloscypha lacustris Velen., Novitates mycol. noviss.: 138. 1947. - Holotype (the only specimen mentioned): [Czechoslovakia,] Bohemia, Mnichovice, lacus Božkov, 2.VII.1942 Velenovský (PRM 151160, examined).

This is a mollisiaceous species, as already noted by Svrcek (1985).

57. Uncinia laricina Velen., Novitates mycol. noviss.: 139. 1947, nomen dubium. - Holotype (the only specimen mentioned): [Czechoslovakia, Bohemia,] Mnichovice, Larix europ., 12.VI.1941 Velenovsky (PRM 153249, examined).

The type convolute was empty and the meagre description is of limited diagnostic value.

58. Hyaloscypha leucella (P. Karst.) Boud., Hist. classific. discom. Europe: 126. 1907. - Peziza leucella P. Karst., Fungi Fenniae Exs. 651. 1867. - Type: not seen.

I have not seen original material of Peziza leucella. Original collections have been studied by Dennis (1956), Raschle (1978) and Carpenter (1981). Their observations indicated that the species does not belong to Hyaloscypha.

59. Hyaloscypha lignatilis Velen., Monogr. Discom. Bohem.: 278; PI. 15:53. 1934. - Holotype (the only specimen mentioned): [Czechoslovakia,] Bohemia, Mnichovice, ad truncum pini silvestris, 6.VII.1929 Velenovsky (PRM 151135, examined).

The species was transferred to Psilocistella by Svrček (1977c). It clearly belongs to that genus, but may be conspecific with $P$. obsoleta (Velen.) Svr. (Huhtinen 1990).

60. Hyaloscypha lonchitidis Velen., Monogr. Discom. Bohem.: 286; Pl. 15:45. 1934. - Lectotype (Svrček 1985: 211): [Czechoslovakia,] Slovakia, Choð, Aspidium longhitidis, VIII.1925 Velenovský (PRM 150902, examined).

Velenovsky (1934) cited only one collection, which Svrcek (1985) selected as a lectotype. Svrcek also cited a syntype, which was not cited in the protologue. The species was shown to be conspecific with Incrupila aspidii (Lib.) Raitv.

61. Uncinia longispora Velen., Monogr. Discom. Bohem.: 295. 1934. - Holotype (the only specimen mentioned): [Czechoslovakia, Bohemia,] Strančice, St. Anna, Sambucus racemosa, 5.V.1934 Velenovsky (PRM 154105, examined).

I found the holotype convolute empty, but the diagnosis is characteristic enough to allow neotypification.

62. Hyaloscypha lunispora Velen., Monogr. Discom. Bohem.: 277; Pl. 14:14. 1934. - Holotype (the only specimen mentioned): [Czechoslovakia, Bohemia,] Mnichovice, Hubackov, Populus tremula, 23.IX.1929 Velenovsky (PRM 151126, examined).

This seems to be a species of Calycellina, as noted by Svrčk (1985).

63. Hyaloscypha luteola Ahmad, Biol. Soc. Pakistan Monogr. 7:198. 1978. — Lectotype (selected here): [Pakistan,] 
Changa Manga, on dead branches of Prosopis juliflora, 24.II.1962 Ahmad 15392 (BPI, examined).

Describing the species, Ahmad (1978) cited two "holotype" specimens. Lectotypification with one of these syntypes (Art. 7.7) is thus needed. The species has clearly brown excipulum and hairs resembling those of Protounguicularia. Thus it obviously cannot belong to Hyaloscypha, but the proper genus for Ahmad's species remains to be found.

64. Hyaloscypha luzulae Velen., Monogr. Discom. Bohem.: 281; Pl. 14:41. 1934. - Holotype (the only specimen mentioned): [Czechoslovakia,] Bohemia, Myšlín near Mnichovice, on a dead leaf of Luzula campestris, 26.VIII.1924 Velenovský (PRM 150170, not examined).

Svrcek (1985) transferred this species to Calycellina.

65. Hyaloscypha martialis Velen., Monogr. Discom. Bohem.: 272. 1934. - Holotype (the only specimen mentioned): [Czechoslovakia,] Bohemia, Mnichovice, Hubačkov, ad cupulam quercinam, 6.III.1934 Velenovský (PRM 151250, examined).

This species was recently transferred to Psilachnum by Svrček (1985). Some paraphyses resemble the somewhat tapering hairs of that genus but cylindrical paraphyses are commoner. The stipitate apothecia, lack of dextrinoid reactions, the excipulum and the slightly cuneiform spores suggest placement in Hamatocanthoscypha, but Svrcek's choice is supported here, because the paraphyses do not taper in Hamatocanthoscypha. More collections are needed to clarify the prevailing type of paraphyses in this species.

66. Hyaloscypha martii Velen., Monogr. Discom. Bohem.: 281. 1934. - Holotype (the only specimen mentioned): [Czechoslovakia, Bohemia,] Mnichovice, Luzula albida, 15.III.1934 Velenovský (PRM 151261, examined).

The hyphoid excipulum excludes relationship to Hyaloscypha (Svřek 1985). The apothecia in the specimen were all immature.

67. Hyaloscypha microcarpa (Fuckel) Boud., Hist. classific discom. Europe: 127. 1907. - Niptera microcarpa Fuckel, Jahrb. Nassauisches Vereins Naturk. 25-26:334. 1871-1872. - Type: not seen.

I have not studied material of Niptera microcarpa and its relationships cannot be deduced from the original diagnosis.

68. Hyaloscypha microscopica Velen., Monogr. Discom. Bohem.: 280; P1. 14:5. 1934. - Lectotype (Svrček 1985: 212): [Czechoslovakia,] Bohemia, Krkonoše, Eriophorum, VIII.1927 Cejp (PRM 150830, seen).

I did not prepare a mount of the scanty material. The species was recently transferred to Discocistella by Svrček (1985).

69. Hyaloscypha microspis (P. Karst.) Velen., Monogr. Discom. Bohem.: 212. 1934. - Peziza microspis P. Karst., Fungi Fenniae Exs. 647, 1867. - Type: not seen.

I have not studied Karsten's original material, which was reported to be a species of Mollisina by Dennis (1956). The material examined by Velenovsky belongs to Discocistella fugiens (Svrček 1962).

70. Hyaloscypha microstoma (Wallr.) Boud., Hist. classific discom. Europe: 126. 1907. - Peziza microstoma Wallr., Fl. Crypt. Germ. 2:495. 1833. - Type: not seen.

I have not located original material of $P$. microstoma, nor seen a modern treatment of the species. Judging from the short descriptions given by Saccardo (1889) and Rehm (1896), the name could be based on a species of, e.g., Unguicularia. The urceolate shape of the apothecia, in particular, could be diagnostic of the glassy-haired hyaloscyphaceae.

71. Hyaloscypha millepunctata (Lib.) Boud., Hist. classific. discom. Europe: 126. 1907. - Peziza millepunctata Lib., Pl. Crypt. Ard. 2:128. 1832. - Type: not seen.

Peziza millepunctata was transferred to Unguicularia by Dennis (1949) and is a well-known species of the genus (Raschle 1977).

72. Hyaloscypha milliaria Velen., Monogr. Discom. Bohem.: 276. 1934. - Lectotype (SvrCek 1985: 212): [Czechoslovakia,] Bohemia, Karlštejn, on rotten wood of Ulmus sp., X.1926 Velenovský (PRM 148709, not seen).

Svrček (1985) synonymized this name with Cistella dentata. Another collection of $H$. milliaria in Velenovsky's herbarium also contained apothecia of a typical Cistella (PRM 148635).

73. Uncinia minima Velen., Monogr. Discom. Bohem.: 294. 1934. - Holotype (the only specimen mentioned): [Czechoslovakia, Bohemia,] Mnichovice, Carpinus, IX.1933 Velenovsky (PRM 153195, examined).

This is a typical species of the genus Mollisina.

74. Hyaloscypha minutella Boud., Icon. mycol. expl.pl. 1:18. 1905. - No specimens preserved.

Plate 522 in Boudier's "Icones Mycologicae", which appeared in 1910, is a fairly detailed illustration of a hyaline, lignicolous fungus much resembling species of Hyaloscypha. The spore size fits $H$. vitreola and $H$. herbarum fairly well. The former species was given as a possible synonym by Raitviir (1970a). Boudier, however, illustrated the margin as composed of cylindrical hairs forming small, irregular teeth. This feature suggests that neither of the above species was in his possession. Until a better match with the plate is collected, $H$. minutella must remain a mystery.

75. Hyaloscypha mirabilis Velen., Monogr. Discom. Bohem.: 283; P1. 14:37. 1934. - Holotype (the only specimen mentioned): [Czechoslovakia,] Bohemia, Karlštejn, on fallen leaves of Acer pseudoplatanus, 16.X.1926 Velenovsky (PRM 150890, not seen).

The type convolute was found to be empty by Svrček (1985). He suggested synonymy with Hyalopeziza ciliata Fuckel, but another interpretation of Hyaloscypha mirabilis was given by Kirk (1984). 
76. Hyaloscypha mollisiaeformis Velen., Monogr. Discom. Bohem.: 276. 1934. - Holotype (the only specimen mentioned): [Czechoslovakia,] Bohemia, Mnichovice, the hill "Plecháx", on bark of a decaying trunk of Populus tremula, IX.1933 Velenovsky (PRM 151239, not examined).

Svrčk (1985) synonymized the present species with $\mathrm{Mol}$ lisia sublividula (Nyl.) P. Karst. sensu Sacc.

77. Phialina montana Raitv., Fol. Crypt. Est. 23:5, Fig. 2. 1984. - Holotype (original designation): U.S.S.R., Tadzhikistan, Montes Pamiro-Alaici, in pratis subalpinis jugi Petri I in vicinis pagi Tavildara, in caulibus putridis plantae dicotyledorum, 21.VI.1982 Raitviir (data from the diagnosis) (TAA 94921, examined).

Although the excipulum and hairs are basally brownish, Raitviir's species is a typical Calycellina with herbicolous habit, persistent yellow pigment inside the hairs and paraphyses, oblong-fusoid spores and cylindrical marginal hairs. Hence, a new combination is proposed: Calycellina montana (Raitv.) Huhtinen comb. nov. - Basionym: Phialina montana Raitv., Fol. Crypt. Est. 23:5. 1984.

78. Hyaloscypha myriadea Boud., Hist. classific discom. Europe: 127. 1907. - Pezizella myriadea Mouton, Compt. Rend. Bull. Soc. Royal Bot. Belg. 36:17. 1897 (citation from Saccardo, Syll. Fung. 14). - Isolectotype (indicated in herb. by Arendholz): [Belgium,] 18.XII.1880 Mouton? 54 (BR, examined).

This is a species of Calycellina, which superficially resembles Phialina lachnobrachya, also abundant in the specimen. The cylindrical hairs and paraphyses contain the characteristic persistent, yellow pigment. The small spores and asci arising from simple septa separate the specimen from the well-known foliicolous members of the genus. The combination Phialina parenchymatosa (Velen.) Graddon was based on conspecific material examined by Graddon. A new combination is proposed: Calycellina myriadea (Mouton) Huhtinen, comb. nov. - Basionym: Pezizella myriadea Mouton, Compt. rend. Bull. Soc. Royal Bot. Belg. 36:17. 1897.

79. Hyaloscypha nana (Sacc.) Boud., Hist. classific discom. Europe: 127. 1907. - Helotium nanum Sacc., Fungi Veneti Novi Vel Critici: 36. 1875. - Type: not designated, original material preserved.

I have studied a collection in PAD referred to as "presumed holotype" by Arendholz. It contained only a few juvenile apothecia, which were totally naked and are thus not hyaloscyphaceous. Carpenter (1981) referred the species to Allophylaria.

80. Uncinia nardi Velen., Monogr. Discom. Bohem.: 296; Pl. 16:40, 41. 1934. - Holotype (the only specimen mentioned): [Czechoslovakia, Bohemia,] Mnichovice, BožkovMirošovice, Nardus, V.1931 Velenovský (PRM 153192, examined).

Only a small fragment of the scanty material was studied. The marginal hairs were cylindrical-clavate, but the uncinate habit and septation depicted by Velenovsky seem to be fictitious.
81. Hyaloscypha nympharum Velen., Monogr. Discom. Bohem.: 281; Pl. 14: 44. 1934. - Lectotype (Svrcek 1985: 213): [Czechoslovakia,] Bohemia, Vyžlovka pr. Jevany, Iris pseudacorus, 28.V.1927 Velenovsky (PRM 149817, examined).

Svrček (1985) transferred the species to Psilocistella. I found that the marginal hairs were short, blunt to cylindricalclavate hyphal projections. The excipulum was composed of rounded cells with prominent intercellular spaces. Hence, placement in the Dermateaceae seems more natural than in Psilocistella, which has textura prismatica in the excipulum.

82. Phialina obscura Raitv., Fol. Crypt. Est. 23:6, Fig. 3. 1984. - Holotype (original designation): U.S.S.R., regio Primorsk, distr. Ternei, reservatum Sichote-Alinicum, Maisa, Ulmus sp., ad foliis putresc., 13.IX.1976 Murdvee (TAA 111272 , examined).

The proper genus for this intriguing discomycete is not clear to me. It cannot be kept in Phialina used in the present sense because of the cylindrical hairs. The excipular walls are clearly brown and minutely encrusted with the pigment. When dry, the apothecia have a nearly black disc, which contrasts with the deep red colouring of the margin. The character combination would be anomalous in Calycellina.

83. Hyaloscypha ochracea Velen., Monogr. Discom. Bohem.: 279. 1934. - Holotype (the only specimen mentioned): [Czechoslovakia,] Slovakia, Tatra, Vysoké Tatry, Tatranská Lomnica, alt. ca. $1800 \mathrm{~m}$, Adenostyles, VII.1924 Pilát (PRM 148800, examined).

This species was synonymized with Discocistella micacea (Pers.: Fr.) Svr. by Svrcek (1962). The cylindrical-clavate hairs bear cyanophilic spines and the species is closely related to Cistella grevillei.

84. Hyaloscypha oenotherae Velen., Monogr. Discom. Bohem.: 279; P1. 15:48. 1934. - Holotype (the only specimen mentioned): [Czechoslovakia,] Bohemia, Mnichovice, Oenothera, 24.VIII.1924 Velenovsky (PRM 147655, examined).

This is a typical species of Mollisina. Svræek (1985) referred the material to the genus Denrotrichoscypha.

85. Hyaloscypha olivacea Velen., Monogr. Discom. Bohem.: 282; P1. 14:28. 1934. - Holotype (the only specimen mentioned): [Czechoslovakia,] Bohemia, Jimy, silva Vidrholec, Quercus, 30.V.1925 Velenovsky (PRM 154061, examined).

The species was recently reduced to a variety of Dematioscypha richonis (Boud.) Huhtinen (Huhtinen 1987d).

86. Hyaloscypha opalina (Quél.) Boud., Hist. classific discom. Europe: 126. 1907. - Peziza opalina Quél., Grevillea 8:117; Pl. 131:7. 1880. - Type: not designated.

The smoothness and waxy appearance of the apothecia, together with the lilac colouring suggest affinity to Hyalinia Boud. and Orbilia Fr. 
87. Hyaloscypha paludosa Velen., Monogr. Discom. Bohem.: Pl. 14:25. 1934, lapsus calami pro Eriopeziza paludosa Velen.

The notes on the holotype collection suggest that $H$. paludosa was a manuscript name only. Apparently Velenovský changed his mind at a relatively late stage, because the other species of Eriopeziza were depicted in Plates 6 and 8. Although the generic name was changed in the text, it was left in the legend, with the unintentional result of two different names. These should not be treated as alternative names, in view of the apparent mistake in the legend for Plate 14.

88. Hyaloscypha paludosa Dennis, Kew Bull. 16:325. 1962. - Holotype (original designation): [Great Britain,] Derbyshire, Ashop Clough, N slope of Kinder Scout, [alt. $550 \mathrm{~m}] 1800 \mathrm{ft}$, 9.VII.1960 Palmer 8 (K, examined).

When describing the species, Dennis (1962) ignored the frequent septa in the hairs. These are blunt and resemble the hairs in subg. Eupezizella. The septation is here considered to exclude Dennis's species from Hyaloscypha. The morphological characters show a close match with the two species which were originally described in Protounguicularia and later transferred to Arachnopeziza (Raitviir \& Galán 1986, Huhtinen 1987d). The few collections of the present species (from Czechoslovakia, DDR, Finland) are all herbicolous and the merging of herbicolous species of similar appearance in Arachnopeziza needs to be examined.

89. Hyaloscypha pani Velen., Monogr. Discom. Bohem.: 285. 1934. - Holotype (the only specimen mentioned): [Czechoslovakia,] Bohemia, Jevany, on dead leaves of Lycopodium clavatum, 10.X.1925 Velenovský (PRM 149707, not seen).

This species was recently transferred to Hyalopeziza by Holm and Holm (1981). Their conclusions were based on study of a slide made from the type collection. Holm and Holm suggested that $H$. pani may be a true lycopod fungus.

90. Hyaloscypha parasitica (Ell. \& Everh.) Sherwood, Mycotaxon 5:222. 1977. - Stictis parasitica Ell. \& Everh., J. Mycol. 4:54. 1888 (cited from Sherwood 1977). - Holotype: [U.S.A.,] New Jersey, on Diatrype, Ellis (NY, not seen).

A study of the isotype specimen in CUP revealed that Stictis parasitica is a synonym of Dematioscypha dematiicola.

91. Hyaloscypha parenchymatosa Velen., Monogr. Discom. Bohem.: 277; P1. 14:13. 1934. - Lectotype (Svrček 1985: 214): [Czechoslovakia,] Bohemia, Mnichovice, "Hubackov", on a dead leaf of Quercus sp., 27.X.1931 Velenovský (PRM 151144, not seen).

According to Svrcek (1985) the lectotype convolute is empty and rest of the original material does not match the protologue. The material examined by Graddon (1979), who proposed a new combination in Phialina, is Pezizella myriadea. Judging from Velenovsky's text and illustration, $H$. parenchymatosa is conspecific with Graddon's and Mouton's material.

92. Hyaloscypha parenchymatosa Velen., Novitates mycol. noviss.: 137. 1947, homon. illeg. (Art. 64.1). -
Holotype (the only specimen mentioned): [Czechoslovakia,] Bohemia, Mnichovice, Božkov, in prato uliginoso, 10.VII.1941 Velenovský (PRM 151093, examined).

The type specimen is Psilachnum acutum (Velen.) Svr. as already noted by Svr飞ek (1985).

93. Hyaloscypha parvula (De Not.) Boud., Hist. classific discom. Europe: 127. 1907. - Peziza parvula De Not., Microm. Italici 1:58; P1. 2:1-5. 1839. - Type: not seen.

I have not traced the original material of $P$. parvula. The plate provided with the diagnosis excludes relationship with Hyaloscyphaceae. The apothecia were depicted as smooth. Boudier's transfer to Hyaloscypha caused a change in the name Hyaloscypha parvula Arendh. \& R. Sharma (see under $H$. occulta).

94. Hyaloscypha pellucens (Ces.) Boud., Hist. classific discom. Europe: 127. 1907. - Peziza pellucens Ces., Flora Bot. Gesellsch. Regensb. 35:396. 1852; Rabenhorst's "Klotschii Herb. viv. mycol. 1620." - Type: not designated.

The exsiccatum in RO contained a species of Cistella with cyanophilic spines on the cylindrical-clavate hairs. In spore size it closely matches Cistella caricis (Raitv.) Raitv., for which Cesati's species apparently offers an older name.

95. Hyaloscypha pellucida Velen., Monogr. Discom. Bohem.: 283; Pl. 14:39. 1934, nomen dubium. - Holotype (the only specimen mentioned): [Czechoslovakia,] Bohemia, Vsesimy near Mnichovice, on a fallen leaf of Fagus silvatica, 3.X.1931 Velenovský (PRM 151220, not seen).

According to Svrcek (1985), the species might be Hyalopeziza ciliata. The hairs were, however, described as relatively short.

96. Hyaloscypha phyllophila (Desm.) Boud., Hist. classific discom. Europe: 127. 1907. - Peziza phyllophila Desm., Ann. Sci. Nat. Bot. 17:96. 1842. - Type: not seen.

I have not studied Desmazières's original material. Carpenter (1981) studied a syntype and stated that the species belongs to Hymenoscyphus S.F. Gray. Boudier (1907) erroneously cited Karsten as the author of $P$. phyllophila.

97. Uncinia pilosa Velen., Novitates mycol.noviss.: 140. 1947 , nomen dubium. - Holotype (the only specimen mentioned): [Czechoslovakia,] Mnichovice, ad truncum sectum Fraxini, X.1942 Velenovský (data from Velenovsky) (PRM, seen).

The holotype convolute was empty. The description is too vague to allow taxonomic conclusions.

98. Hyaloscypha priapi Velen. var. obsoleta Velen., Monogr. Discom. Bohem.: 272. 1934. - Holotype (the only specimen mentioned): [Czechoslovakia, Bohemia centralis, Jevany, ad lignum putridum Fagi sylvaticae, 10.X.1925 Velenovský (PRM 150906, examined).

This variety was transferred to Psilocistella as $P$. obsoleta by Svrček (1977c) and was made the type species of the genus. It may be conspecific with $H$. lignatilis. 
99. Hyaloscypha priapi Velen. var. vernalis Velen., Monogr. Discom. Bohem.: 272. 1934. - Lectotype (Svrček, 1977c: 198): [Czechoslovakia, Bohemia,] Mnichovice, malá Hrušov, 19.V.1941 Velenovský (PRM 151115, examined).

This variety was recently transferred to Psilocistella as $P$. vernalis by Svrcek (1985). The lectotype shows the typical hairs of the genus.

100. Hyaloscypha pteridina Velen., Monogr. Discom. Bohem.: 282. 1934. - Lectotype (Svrčk 1985: 215): [Czechoslovakia, Bohemia,] Mnichovice, Hrusice, 12.VIII.1927 Velenovsky (PRM 151075, examined).

This is Micropodia chrysostigma, as suggested by Svrček (1985).

101. Phialina puberula (Lasch in Rabenh.) Höhnel, Mitt. Bot. Inst. Techn. Hochsch. Wien 3:72. 1926. - Hyaloscypha puberula (Lasch in Rabenh.) Nannf. ex Dennis, Mycol. Pap. 32:73. 1949. - Peziza puberula Lasch in Rabenh., Flora 34:566. 1851. - Type: not seen.

This species was recently synonymized with Calycellina punctata (Fr.) Lowen \& Dumont (1984).

102. Hyaloscypha punctiformis (Grev.) Boud., Hist classific discom. Europe: 126. 1907. - Peziza punctiformis Grev., Scot. crypt. fl., Edinburgh 2:63. 1823 (from Lowen \& Dumont 1984). - Neotype (Lowen \& Dumont 1984: 1008): [Great Britain,] Argyll, Glen Mane Garden, on fallen oak leaves, 6.IX.1963 Henderson (E, examined).

This species was recently neotypified and synonymized with Calycellina punctata (Fr.) Lowen \& Dumont (1984).

103. Hyaloscypha punctiformis (Grev.) Boud. var. robustella (Sacc.) Boud., Hist. classific discom. Europe: 126. 1907. - Pseudohelotium punctiforme (Grev.) Sacc. var. robustellum Sacc., Michelia I: 427. 1878. - Type: not seen.

The identity of this variety was not studied.

104. Hyaloscypha punctoidea (P. Karst.) Boud., Hist. classific discom. Europe: 126. 1907. - Helotium punctoideum P. Karst., Not. Sällsk. Fauna Flora Fenn. Förh. 11:215. 1870. - Type: Dennis (1956) cited a collection dated 2.X.1868 as "typus". Karsten's diagnosis referred to two collections, so apparently Dennis's citation should be taken as a lectotypification. Lectotype (in $\mathrm{H}$ ) not studied.

Dennis (1956) studied and depicted the species concluding that it belongs to Pezizella.

105. Hyaloscypha pusilla (Oeder: Fr.) Boud., Hist. classific discom. Europe: 127. 1907, nomen dubium. - Helvella pusilla Oeder: Fr., Flora Danica; P1. 779:3. 1782. (data from Carpenter 1981). - Type: not seen.

I have not located material of Helvella pusilla. Carpenter (1981) remarked that the illustration was undiagnostic.

106. Hyaloscypha pygmaea (Mouton) Boud., Hist. classific discom. Europe: 127. 1907. - Trichopeziza pygmaea
Mouton, Bull. Soc. Royal Bot. Belg. 36:19. 1897. - Holotype (the only specimen mentioned): [Belgium,] Beaufays on leaves of Carpinus, Mouton 473 (BR, examined).

This species was recently transferred to Hyalopeziza (Huhtinen 1987c).

107. Hyaloscypha quercina Velen. var. barbata Velen., Monogr. Discom. Bohem.: 276. 1934. - Hyaloscypha barbata (Velen.) Svr., Česká Mykol. 39:216. 1985. — Lectotype (Svrčk 1985: 216): [Czechoslovakia,] Bohemia, Jevany, Fagus, XI.1923 Velenovsky (PRM 149740, examined).

This epithet was recently shown to offer an earlier name for Protounguicularia brevicapitata Raitv. \& Galán, the type species of Protounguicularia (Raitviir \& Galan 1986, Huhtinen 1987a).

108. Hyaloscypha quercina Velen. var. resinacea Dennis, Kew Bull. 30:353, Fig. 4b. 1975. - Holotype (original designation): [Great Britain,] Warwickshire, Tamworth-inArden, on rotten wood of Sorbus aucuparia, I.1974 Clark (Graddon 2338) (K, examined).

This variety was recently transferred, as a form, to Protounguicularia barbata (Velen.) Huhtinen (Huhtinen 1987a).

109. Hyaloscypha radiostriata (Feltg.) Boud., Hist. classific discom. Europe: 126. 1907. - Pezizella radiostriata Feltg., Rec. Mem. Trav. Soc. Bot. Luxemb. 16:52. 1903. Type: not seen.

I have not traced original material of Feltgen's species. It was stated to belong to Cistella by Höhnel (1906).

110. Hyaloscypha reticulata Vacek, Česká Mykol. 2:119; Figs. 1-4. 1948. - Lectotype (selected unintentionally by Svræek 1986): [Czechoslovakia,] Bohemia, Hořelice, ad ramulum arborum frondosum (sub terram), 31.VIII.1947 Vacek (PRM 685970, examined).

This unique species was recently placed in a new, monotypic genus Calyptellopsis Svr. by Svrček (1986). Svrček cited PRM 685970 as holotype, although Vacek reported two collections and did not indicate the type. Hence, Svrček actually lectotypified the species (for a similar case, see Unguiculella in "Generic relationships").

111. Hyaloscypha richonis (Boud.) Dennis, Mycol. Pap. 32:66. 1949. - Urceolella richonis Boud., Bull. Soc. Mycol. France 4:82. 1888. - Holotype (the only specimen mentioned): [France,] St. Amand-sur-Fion, XII.1885 Richon (PC, examined).

The species was recently transferred to Dematioscypha (Huhtinen 1987d).

112. Phialina rosae Raitv., Eesti NSV Tead. Akad. Toim., Biol. 18:68, Fig. 3. 1969. - Holotype (original designation): [U.S.S.R.,] Kirghizia, Tian-Shan occidentalis, Montes Tshatkal, reservatum Sary-Tshelek, apud lacum SaryTshelek, ad foliis emortuis Rosae sp., 5.VIII.1967 Raitviir (TAA 44603, examined).

This species may best be accommodated in Calycellina. 
113. Hyaloscypha salicina Velen., Monogr. Discom. Bohem.: 284. 1934. - Lectotype (Svrcek 1985: 216): [Czechoslovakia, Bohemia,] Radotín, IX.1925 Velenovský (PRM 150903, examined).

This species is conspecific with $H$. carpinacea, as already suggested by Svrcek (1985). Hyaloscypha asperipila is a further synonym (see discussion under that epithet).

114. Uncinia salicina Velen., Novitates mycol. noviss.: 139. 1947, nomen dubium. - Holotype (the only specimen mentioned): [Czechoslovakia, Bohemia,] Mnichovice, Hubackov, Salix amygdalina, 4.VI.1940 Velenovsky (PRM 153248 , examined).

The type specimen contained a few apothecial fragments, which did not permit any taxonomic decisions.

115. Uncinia sarothamni Velen., Monogr. Discom. Bohem.: 296; ?P1. 14:19. 1934. - Holotype (the only specimen mentioned): [Czechoslovakia, Bohemia,] Mnichovice, VII.1926 Velenovsky (PRM 149400, examined).

This species is synonymous with Unguiculella eurotioides (P. Karst.) Nannf. The confusion in the citation of illustrations between the taxa from Sarothamnus is discussed under $H$. herbarum and $H$. contorta.

116. Uncinia scintillans Graddon, Trans. Brit. Mycol. Soc. 63:481, Fig. 5. 1974. - Hyaloscypha scintillans (Graddon) Dennis, British Ascomycetes: 178. 1981. - Holotype (original designation): [Great Britain,] Warwickshire, Snitterfield Bushes, on Carex pendula, 7.XII.1972 Clark (Graddon 2250) ( $K$, examined). pha.

The material is Hyaloscypha cladii, the type of Phaeoscy-

117. Hyaloscypha senilis Velen., Monogr. Discom. Bohem.: 282; Pl. 14:27. 1934. - Holotype (the only specimen mentioned): [Czechoslovakia,] Bohemia, Liblice, prope Vsetaty, VII.1925 Fechtner (PRM 148384, examined).

I could not find any true hairs on the mounted apothecia. The other characters point to the synonymy with $H$. perpusilla proposed by Svrcek (1985). The asci were devoid of croziers, however, and $H$. senilis may be a further synonym of var. alniseda. Not having found the hairs depicted by Velenovsky, I will refrain from proposing the synonymy. The substrate was hardwood but its structure did not agree with the original report (in truncis quercinis).

118. Hyaloscypha sesleriae Velen., Monogr. Discom. Bohem.: 280; Pl. 14:7. 1934, nomen dubium. - Holotype (the only specimen mentioned): [Czechoslovakia,] Bohemia, Radotín, on a dead leaf of Sesleria coerulea, VIII.1924 Velenovský (PRM 149239, not seen).

Svrcek (1985) studied the type specimen and found only immature, mollisioid apothecia.

119. Hyaloscypha straminea (Berk. \& Broome) Boud., Hist. classific discom. Europe: 127. 1907. - Peziza stramineum Berk. \& Broome, Ann. Mag. Nat. Hist. 7:182. 1851. -
Lectotype (Carpenter 1981: 178): [Great Britain,] Fotheringay (K, not seen).

This taxon was shown to be a good species in Crocicreas by Carpenter (1981).

120. Uncinia subcorticalis Velen., Monogr. Discom. Bohem.: 295. 1934. - Holotype (the only specimen mentioned): [Czechoslovakia, Bohemia,] Mnichovice, collis Plecháč, in cortice Piceae exs., X.1928 Velenovský (PRM 151131, examined).

The specimen had cuneiform spores and elongated excipulum, which suggest close affinity to Hamatocanthoscypha. The hairs varied from straight to uncinate and were typically brown and sparsely warted. As some species in Hamatocanthoscypha exhibit these features, Uncinia subcorticalis may well belong to the genus. The scanty type material did not, however, allow closer scrutiny.

121. Hyaloscypha subliciformis (P. Karst.) Boud., Hist. classific discom. Europe: 127. 1907. - Peziza subliciformis P. Karst., Not. Sällsk. Fauna Flora Fenn. Förh. 10: 205. 1869. - Holotype: [Finland, Tavastia australis,] Mustiala, ad Art., 31.X.1867 Karsten (H, Herb. Karsten 884; not seen).

The epithet appeared in the index of Karsten's 1869 compilation with a footnote in which he briefly mentioned the emendation of $P$. sublicoides. Apparently the index was written somewhat later than the main study, where only Peziza sublicoides was cited. This name was not, however, validly published (Art. 34.1a) Dennis (1956) accepted the species in Allophylaria.

122. Hyaloscypha substipitata Velen., Novitates mycol. noviss. 137. 1947. - Holotype (the only specimen mentioned): [Czechoslovakia,] Bohemia, Myslín near Mnichovice, on a dead culm and leaf of Phalaris arundinacea, 30.VII.1941 Velenovský (PRM 151103, not seen).

The species was synonymized with Psilachnum acutum by Svrčk (1985).

123. Hyaloscypha subtilis Velen., Monogr. Discom. Bohem.: 273; Pl. 14:33. 1934. - Holotype (the only specimen mentioned): [Czechoslovakia,] Bohemia, Mnichovice, on dead leaves of Pyrus communis, 7.X.1931 Velenovsky (PRM 151222, not seen).

Svrčk (1985) found the convolute empty. He suggested that the species was a synonym of Hyalopeziza ciliata.

124. Hyaloscypha subtilis Velen. var. drupacea Velen., Monogr. Discom. Bohem.: 273. 1934. - Holotype (the only specimen mentioned): [Czechoslovakia] Bohemia, Mnichovice, Menčice, Prunus spinosa, IX.1933 Velenovský (PRM 151267, examined).

This is a synonym of Hyalopeziza pygmaea (Mouton) Huhtinen (Huhtinen 1987c).

125. Hyaloscypha sulfurina Dennis, Kew Bull. 14:439, Fig. 12. 1960. - Holotype (original designation): Venezuela, 
Sierra de Santo Domingo, Edo. Merida, on Espeletia, 25.VII.1958 Dennis 2437 (K, examined).

The long, tapering, multiseptate hairs and the typically two- to three-septate spores clearly exclude the species from Hyaloscypha. The hair walls are hyaline and bear abundant amorphous resin, which is greenish or olivaceous in $\mathrm{KOH}$. Due to the overall abundance of resinous exudates the presence of a similar sap inside the hairs could not be verified. The hairs are thin-walled and bear no apical crystal masses. Although the paraphyses are filiform, the species is best placed in Trichopeziza Fuckel (cf. Raitviir 1987). A new combination is proposed: Trichopeziza sulfurina (Dennis) Huhtinen, comb. nov. - Basionym: Hyaloscypha sulfurina Dennis, Kew Bull. 14:439, Fig. 12. 1960.

126. Hyaloscypha sulphurea Velen., Monogr. Discom. Bohem.: 273; P1. 14:3. 1934, nom. inval. (Art. 34.1a). Incrupila sulphurea Svr., Čská Mykol. 39:218. 1985. Holotype (cited as neotype when published): [Czechoslovakia,] Bohemia centralis, Vrane $\mathrm{n}$ [ad] Vlt[avou], ad folium putridissimum Carpini betuli in strato alto foliorum deiectorum in fauce silvatica uda, rarissime, 31.V.1953 Svrček (PRM, not seen).

This species was not accepted by Velenovsky (1934), which is evident from the synonymy given under Hyaloscypha albolutea (Pers.) Velen. var. albolutea and from the index, where the present species is also listed under $H$. albolutea. The similarity between the illustrations also deserves attention. Apparently Velenovsky changed his mind about the validity of $H$. sulphurea at a relatively late stage and only indicated the synonymy, failing to remove the description. A neotypification was proposed for the species by Svrček (1985) and also a new combination with Incrupila. A direct reference to a Latin diagnosis was given, fulfilling the establishment of Incrupila sulphurea Svr.

127. Hyaloscypha sulphureopilosa Svr., Ceská Mykol. 40:211; P1. 3:2. 1986. - Holotype (original designation): [Czechoslovakia,] Bohemia centralis, Kladno, in monte Kožova Hora (alt. $446 \mathrm{~m}$ ), ad squamas strobilorum deiectorum $P i$ ceae abietis in palude ad marginem stagni silvatici, 23.X.1983 Svrčk (PRM, not seen).

The type specimen was not available for study but Dr. Svrček kindly provided me with the original annotations and drawings of the holotype. From all the available evidence, it seems that $H$. sulphureopilosa is merely based on a marginal population of Hamatocanthoscypha uncinata. The hairs were stated to be often curved (Svrček 1986). A similar collection from Picea cones is preserved in TUR and the apothecia in this collection show variation from straight to uncinate hairs, and from broad to narrow hairs. Definite conclusions must, however, await more material from spruce cones.

128. Hyaloscypha tenuispora Velen., Monogr. Discom. Bohem.: 285; P1, 14:35. 1934. - Holotype (the only specimen mentioned): [Czechoslovakia, Bohemia,] Mnichovice, Carex, 18.XI.1925 Velenovský (PRM 149481, examined).

The species was synonymized with Calycellina luzulae (Velen.) Svr. by Svř̌ek (1985).
129. Hyaloscypha terrestris Velen., Monogr. Discom. Bohem.: 275; Pl. 12:14. 1934, nomen dubium. - Holotype (the only specimen mentioned): [Czechoslovakia,] Bohemia, Mnichovice, "Boukalova stran", on loamy ground with a thin covering of moss, 11.VIII.1931 Velenovský (PRM 151214, not seen). (1985).

The type convolute was found to be empty by Svrček

130. Hyaloscypha tumidula (Rob. ex Desm.) Grelet, Rev. Mycol. 16:95. 1951. - Peziza tumidula Rob. ex Desm., Ann. Sci. Nat. 16:325. 1851. - Type: not seen.

I have not studied original material of $P$. tumidula. Arendholz (1979) treated the species under Mollisia and specifically mentioned the lack of hairs.

131. Uncinia truncorum Velen., Monogr. Discom. Bohem.: 294; Pl. 15:33-35. 1934. - Holotype (the only specimen mentioned): [Czechoslovakia, Bohemia,] Všenory, 20.V.1926 Cejp (PRM 149198, examined).

The brownish excipulum with angular structure and the cylindrical-clavate hyphal projections at the margin exclude the species from Hamatocanthoscypha. The type material is scanty and the identity remained unsolved.

132. Hyaloscypha typhacea Velen., Monogr. Discom. Bohem.: 285; Pl. 14:36. 1934, nomen dubium. - Holotype (the only specimen mentioned): [Czechoslovakia,] Bohemia, Neratovice, "Čeminovsko", on dead rhizome of Typha sp., 15.IX.1926 Velenovsky (PRM 149120, not seen).

The holotype convolute was found to be empty by Svrčk (1985).

133. Hyaloscypha ulmacea Velen., Monogr. Discom. Bohem.: 277. 1934, nomen dubium. - Holotype (the only specimen mentioned): [Czechoslovakia, Bohemia,] Karlstejn, infra "Vodopady", Ulmus, 16.X.1926 Velenovský (PRM 150911, seen). (1985).

The type convolute was empty, as already noted by Svrček

134. Hyaloscypha umbrina Velen., Monogr., Discom. Bohem.: 277; Pl. 14:15. 1934. - Holotype (the only specimen mentioned): [Czechoslovakia,] Bohemia, Praha-Slivenec, on fallen leaves of Alnus glutinosa, VI.1925 Vélenovský (PRM 150874, not seen).

Svrček (1985) synonymized the species with Mollisia rabenhorstii (Auersw.) Rehm.

135. Uncinia uncinata Velen., Monogr. Discom. Bohem.: 295; Pl. 15:36, 37. 1934. - Holotype (the only specimen mentioned): [Czechoslovakia, Bohemia,] Mnichovice, VII.1926 Velenovský (PRM 149449, examined).

No apothecia matching the protologue were found in the type specimen. The description and illustration by Velenovsky suggest that the species is close to, e.g., Hamatocanthoscypha 
laricionis. A newer collection on Hypericum could answer the question whether $H$. laricionis is capable of fruiting on this substrate.

136. Phialina viridiflavescens (Rehm) Raitv., Fol. Crypt. Est. 9:1. 1977. - Pezizella viridiflavescens Rehm., Rabenh. Krypt.-Fl. ed. 2, 1(3): 658. 1892. - Syntype: [German Democratic Republic,] Berlin, Bellevuegarten, an abgefallenen Rinde, IX.1887 Sydow (S, Sydow's Mycoth. Marchica 1746; examined).

The material examined by Raitviir, on which the combination with Phialina was based, was Hamatocanthoscypha uncipila. The original material had already been depicted by Nannfeldt (1932). The curious clavate terminal cells with a clear apical thickening are a major difference from other mollisioid taxa and leave the species without a suitable genus.

137. Hyaloscypha zalewskii Descals \& Webster, Trans. Brit. Mycol. Soc. 67:528, Figs. 1, 2. 1976. - Type: original material lost.

The type collection is not preserved in EXR (Dr. Webster, in litt.) or in K (Dr. Spooner, in litt.). The only material preserved in IMI was a dried culture bearing a single, minute, juvenile apothecium. The structure of the hairs suggests affinity to $H$. albohyalina and $H$. herbarum. The few spores seen were notably smaller than those depicted by Descals \& Webster (1976). The elongated shape of the spores and especially the ecology suggest that $H$. zalewskii is a synonym of $H$. herbarum. I was not, however, able to obtain the conidial state of $H$. herbarum. Descals and Webster (1976) reported that production of Clathrosphaerina took place within six weeks in all their strains. Unfortunately, the exact identity of $H$. zalewskii must remain uncertain until further cultural work is carried out. Synonymization with $H$. herbarum, having overlapping ecology, seems probable.

\section{ACKNOWLEDGEMENTS}

This study was supported financially for four and a half years by the Academy of Finland and I am deeply grateful for the opportunity to devote my time to this work. Likewise, the Finnish Mycological Society is thanked for all help in connection with its publication. The staff in the Herbarium of the University of Turku deserve special thanks for their cooperation. I also owe a debt of gratitude to the Department of Electron Microscopy and the Zoology Department in the University of Turku for technical assistance. The curators of numerous herbaria are thanked for arranging loans of the material and especially for their patience in waiting for it to be returned! All my colleagues abroad and in Finland who helped me with discussions, collections or in other ways are warmly thanked.
I am most grateful to my friend Dr. Tuomo Niemelă, who kindly undertook the labour of editing the monograph and accompanied me on many trips to the mycological "terra incognita" of the Canadian timberline. Likewise, I would like to thank Dr. Pekka Isoviita for unstinting help with the nomenclatorial aspects of the study, and for other comments on the paper. Mrs. Anna Damström, M.A., bravely completed the laborious task of revising the English text. Mr. Krister Karttunen, M.Sc. is thanked for revising the Latin descriptions.

Finally, I take this opportunity to thank Prof. emer. Paavo Kallio who, by offering me the opportunity to travel to the Canadian North and to Svalbard during the preliminary stage of my studies, initiated my interest in mycology on a geographically wider scale.

\section{References}

Abdullah, S.K. \& Webster, J. 1983: The aero-aquatic genus Pseudaegerita. - Trans. Brit. Mycol. Soc. 80:247254.

Abdullah, S.K., Horie, Y. \& Udagawa, S. 1986: New or interesting aero-aquatic conidial fungi from Japan. - Nova Hedwigia 43:507-513.

Ahmad, S. 1978: Ascomycetes of Pakistan 1. - Biol. Soc. Pakistan Monogr. 7.

Albertini, J.B. \& Schweinitz, L.D. 1805: Conspectus fungorum in Lusatiae superioris agro niskiensi crescentium e methodo Persooniana. - 376 pp., 12 tables. Leipzig.

Arendholz, W.-R. 1979: Morphologisch-taxonomische Untersuchungen an blattbewohnenden Ascomyceten aus der Ordnung Helotiales. - 115 pp., 16 tab. PhD Thesis, Univ. Hamburg.

Arendholz, W.-R. \& Sharma, R. 1983: Some new or interesting Helotiales from the eastern Himalayas. - Mycotaxon 17:473-512.

Atchley, W.R., Gaskins, C.T. \& Anderson, D. 1976: Statistical properties of ratios 1. Empirical results. - Syst. Zool. 25:137-148.

Baral, H.O. 1984: Taxonomische und ökologische Studien über die Koniferen bewohnenden europäischen Arten der Gattung Lachnellula Karsten. - Beitrăge Kenntnis Pilze Mitteleuropas 1:143-156.

- 1987a: Der Apikalapparat der Helotiales. Eine lichtmikroskopische Studie über Arten mit Amyloidring. - Z. Mykol. 53:119-136.

- 1987b: Lugol's solution/IKI versus Melzer's reagent: hemiamyloidity, a universal feature of the ascus wall. - Mycotaxon 24:399-450.

Baral, H.O. \& Krieglsteiner, G.J. 1985: Bausteine zu einer Askomyzeten-Flora der Bundesrepublik Deutschland: in Süddeutchland gefundene inoperculate Diskomyzeten mit taxonomischen, ökologischen und chorologischen Hinweisen. - Beih. Z. Mykol. 6:1-226.

Bertault, R. 1977: Hyaloscypha dennisii sp. nov. - Kew Bull. 31:705-708.

Berthet, P. 1964: Essai biotaxinomique sur les Discomycètes. - 157 pp. PhD Thesis, Univ. Lyon.

Bøhler, H.C. 1974: Taxonomical studies on some Norwegian Helotiales (Ascomycetes) on fern remains. Norw. J. Bot. 21:79-100. 
Boidin, J. 1971: Nuclear behaviour in the mycelium and the evolution of the basidiomycetes. - In: Petersen, R.H. (ed.), Evolution in the higher basidiomycetes: 129-148. Knoxville.

Boudier, E. 1885: Nouvelle classification naturelle des Discomycètes charnus. - Bull. Soc. Mycol. France 1:91120.

- 1907: Histoire et classification des Discomycètes d'Europe. - 221 pp. Paris.

- 1905-1910: Icones Mycologicae. - 600 tab. Paris.

- 1909-1910: Icones Mycologicae 3. - 178 tab. Paris.

- 1911: Icones Mycologicae 4. Texte descriptif. - Paris.

Breitenbach, J. \& Kränzlin, F. 1981: Pilze der Schweiz 1. Ascomyceten. - 313 pp. Luzern.

Cailleux, A. 1981: Code des couleurs des sols. Boubée.

Cain, R.F. 1948: Myriogonium, a new genus among simplified ascomycetes. - Mycologia 40:158-167.

Cannon, P.F., Hawksworth, D.L. \& SherwoodPike, M.A. 1985: The British Ascomycotina. An annotated checklist. - 302 pp. Huddersfield.

Carpenter, S.E. 1981: Monograph of Crocicreas (Ascomycetes, Helotiales, Leotiaceae). - Mem. New York Bot. Gard. 33:1-290.

Cash, K. \& Stevenson, J.A. 1961: Proposal to conserve the generic name Cistella Quélet (Hyaloscyphaceae) against Cistella Blume (Orchidaceae). - Taxon 10:244.

Chadefaud, M. 1943: Sur les divers types d'elements dangeardiens des ascomycetes et sur la formation des asques chez la pezize Pustularia catinus. - Rev. Sci: 77-80.

Clark, M.C. 1980: A fungus-flora of Warwickshire. - 272 pp. London.

Clements, F.E. \& Shear, C.L. 1931: The genera of fungi. - 496 pp., 58 tab. New York.

Cooke, R.C. \& Rayner, A.D.M. 1984: Ecology of saprotrophic fungi. - $415 \mathrm{pp}$. London \& New York.

Davis, P.H. \& Heywood, V.H. 1963: Principles of angiosperm taxonomy. - $558 \mathrm{pp}$. Edinburgh \& London.

Dennis, R.W.G. 1949: A revision of the British Hyaloscyphaceae, with notes on related European species. - Mycol. Pap. 32:1-97.

- 1950: New fungi. - Kew Bull. 1950: 170.

- 1953: Hyaloscypha quercina Vel. in England. - Kew Bull. 1953: 295.

- 1955a: An overlooked species of Unguiculella. - Kew Bull. 1955: 136.

- 1955b: Fungi from Sierra Leone: Pezizales and Helotiales. - Kew Bull. 1955: 363-368.

- 1955c: Ascomycetes from Tristan da Cunha. - Results Norweg. Exped. Tristan da Cunha 36:1-10.

- 1956: A revision of the British Helotiaceae in the Herbarium of the Royal Botanic Gardens, Kew, with notes on related European species. - Mycol. Pap. 62:1-216.

- 1960: Fungi venezuelani: 3. - Kew Bull. 14:418-458.

- 1962: New or interesting British Helotiales. - Kew Bull. 16:317-327.

- 1963: A redisposition of some fungi ascribed to the Hyaloscyphaceae. - Kew Bull. 17:319-379.

- 1971: New or interesting British microfungi. - Kew Bull. 25:335-374.

- 1975: New or interesting British microfungi. - Kew Bull. 30:345-365.

- 1981: British Ascomycetes. - 585 + 40 pp., 44 tab. Vaduz.

Descals, E. \& Webster, J. 1976: Hyaloscypha: perfect state of Clathrosphaerina zalewskii. - Trans. Brit. Mycol. Soc. 67:525-528.

Desmazières, M. 1851: Sur les plantes cryptogames, récemment découvertes en France. - Ann. Sci. Nat. 16: 296-330.

Döbbeler, P. 1984: Symbiosen zwischen Gallertalgen und Gallertpilzen der Gattung Epigloea (Ascomycetes). Beih. Nova Hedwigia 79:203-239.

Ellis, M.B. 1971: Dematiaceous Hyphomycetes. - 608 pp. London \& Reading.

Ellis, M.B. \& Ellis, J.P. 1985: Microfungi on land plants. An identification handbook. -818 pp. London \& Sydney.

Emmons, C.W. 1932: The development of the ascocarp in two species of Thielavia. - Bull. Torrey Club 59:415420.

Eriksson, B. 1970: On Ascomycetes on Diapensiales and Ericales in Fennoscandia. - Symb. Bot. Upsal. 19:1-71, 8 tab.

Eriksson, O. \& Hawksworth, D.L. 1986: Outline of the Ascomycetes - 1986. - Systema Ascom. 5:185324.

Fisher, P.J. 1978: Survival of aero-aquatic hyphomycetes on land. - Trans. Brit. Mycol. Soc. 71:419-423.

Fries, E.M. 1823: Systema mycologicum 2. - 621 pp. Lundae.

Fuckel, L. 1869-1870: Symbolae mycologicae. - Jahrb. Nassauischen Ver. Naturk. 23-24:1-459, 6 tab.

Furtado, J.S. 1966: Significance of the clamp-connection in the Basidiomycetes. - Persoonia 4:125-144.

le Gal, M. 1939: Florule mycologique des Boils de la Grange et de l'Etoile. Discomycetes 2. - Rev. Mycol. 4:25-63.

- 1954: Étude critique sur les discomycètes récoltés en Suisse au cours de la session de 1953. - Bull. Soc. Mycol. France 70:187-218.

Gillet, C.-C. 1879: Champignons de France. Les Discomycètes. - $230 \mathrm{pp}$. Alencon.

Graddon, W.D. 1972: Some new discomycete species 2. - Trans. Brit. Mycol. Soc. 58:147-159.

- 1974: Some new discomycete species. - Trans. Brit Mycol. Soc. 63:475-485.

- 1977: Some new discomycete species. - Trans. Brit. Mycol. Soc. 69:255-273.

- 1986: Some new discomycete species 7. - Trans. Brit. Mycol. Soc. 87:328-333.

Greis, H. 1938: Die Entstehung der Basidiomycetenschnallen aus den Ascomycetenhaken. - Jahrb. Wiss. Bot. 86:81-106.

Grelet, L.-J. 1951: Les Discomycètes de France d'après la classification de Boudier. - Rev. Mycol. 16:80-100.

Gremmen, J. 1956: Taxonomic notes on mollisiaceous fungi 4. Fungus 26:32-37.

Greuter, W., Burdet, H.M., Chaloner, W.G., Demoulin, V., Grolle, R., Hawksworth, D.L., Nicolson, D.H., Silva, P.C., Stafleu, F.A., Voss, E.G. \& McNeill, J. 1988: International code of botanical nomenclature. - Regnum Vegetabile 118:1328.

Grosser, D. 1977: Die Holzer Mitteleuropas. - 208 pp. Heidelberg \& New York.

Häffner, J. 1987: Die Gattung Helvella. - Beih. Z. Mykol. 7:1-165.

Haines, J.H. 1974: Notes on the genus Trichopezizella with descriptions of new taxa. - Mycologia 66:213-241. 
- 1980: Studies in the Hyaloscyphaceae 1. Some species of Dasyscyphus on tropical ferns. - Mycotaxon 11: 189-216.

Haines, J.H. \& McKnight, K.H. 1977: Notes on two American Hyaloscyphaceae on aspen. - Mycotaxon 5: 423-431.

Haines, J.H. \& Dumont, K.P. 1984: Studies in the Hyaloscyphaceae 3. The long-spored, lignicolous species of Lachnum. - Mycotaxon 19:1-39.

Harmaja, H. 1977: A note on Helvella solitaria (syn. H. queletii) and $H$. confusa n. sp. - Karstenia 17:40-44.

Hein, B. 1977: Revision der Gattung Laetinaevia Nannf. (Ascomycetes) und Neuordnung der Naevioideae. - Beih. Wildenowia 9:1-136.

- 1981: Zum Wert von Paraphysenauflagerungen für die Taxonomie des Hysteropezizella-Komplexes (Dermateaceae, Mollisioideae). - Nova Hedwigia 34:449-466.

Hennebert, G.L. \& Bellemère, A. 1979: Les formes conidiennes des Discomycetes. Essai taxonomique. Rev. Mycol. 43:259-315.

von Höhnel, F. 1902: Fragmente zur Mykologie 1. Sitzungsber. Akad. Wiss. Wien, Math.-Nat. Cl. 111:1-70.

- 1903: Mykologische Fragmente. — Ann. Mycol. 1:391414.

- 1906a: Revision von 292 der von J. Feltgen aufgestellten Ascomycetenformen auf Grund der Originalexemplare. Sitzungsber. Akad. Wiss. Wien, Math.-Nat. Cl. 115:1139.

- 1906b: Fragmente zur Mykologie 2. - Sitzungsber. Akad. Wiss. Wien, Math.-Nat. Cl. 115:1-47.

- 1909: Fragmente zur Mykologie 6. - Sitzungsber. Akad. Wiss. Wien, Math.-Nat. Cl. 118:1-178, tab. 1.

- 1918: Fragmente zur Mykologie 22. - Sitzungsber. Akad. Wiss. Wien, Math.-Nat. Cl. 127:1-86.

- 1926a: Über die Gattung Pezizella Fuckel. - Mitt. Bot. Inst. Techn. Hochsch. Wien 3:54-80.

- 1926b: Über die Gattung Pezizella Fuckel. - Mitt. Bot. Inst. Techn. Hochsch. Wien 3:94-108.

Holm, K. \& Holm, L. 1977: Nordic junipericolous ascomycetes. - Symb. Bot. Upsal. 21:1-70.

- 1981: Ascomycetes on Nordic Lycopods. - Karstenia 21:57-72.

Holmgren, P.K., Keuken, W. \& Schofield, E.K. 1981: Index Herbariorum 1. The herbaria of the world. - Regnum Vegetabile 106:1-452.

Hora, F.B. 1960: New check list of British agarics and boleti 4. Validations, new species and critical notes. Trans. Brit. Mycol. Soc. 43:440-459.

Hughes, S.J. 1958: Revisiones hyphomycetum aliquot cum appendice de nominibus rejiciendis. - Canad. J. Bot. 36:727-836.

Huhtinen, S. 1984: Additions to the ascomycetous fiora of the Canadian North. - Karstenia 24:1-11.

- 1985a: Finnish records of discomycetes: Unguicularia equiseti sp. nov. and Albotricha laetior. - Karstenia 25: $17-20$.

- 1985b: Mycoflora of Poste-de-la-Baleine, northern Québec. Ascomycetes. - Naturaliste Canad. 112:473-524.

- 1987a: The genus Protounguicularia in Europe. - Beiträge Kenntnis Pilze Mitteleuropas 3:457-463.

- 1987b: New Svalbard fungi. - In: Laursen, G.A. \& Ammirati, J.F. (eds.), Arctic and alpine mycology 2:123-151. New York.

- 1987c: Three new species, and the histochemical delimita- tion of genera in the glassy-haired Hyaloscyphaceae. Mycotaxon 29:267-283.

- 1987d: Taxonomic studies in the genera Protounguicularia, Arachnopeziza and Dematioscypha. - Mycotaxon 30:928.

- 1988: Five glassy-haired Hyaloscyphaceae. - Karstenia 27:8-14.

- 1990: Some hyaloscyphaceous species new to North America, collected from the Canadian timberline. Arctic and alpine mycology 3 . In press.

Huhtinen, S. \& Niemelä, T. 1985: Mycoflora of Postede-la-Baleine, northern Québec. Introduction. - Naturaliste Canad. 112:437-444.

Huhtinen, S. \& Cannon, P.F. 1987: Proposal to conserve Hyaloscypha Boudier with $\mathrm{H}$. vitreola ( $\mathrm{P}$. Karsten) Boudier as the conserved type (Fungi). - Taxon 36:649651.

Huhtinen, S. \& Iturriaga, T. 1987: Finnish records of discomycetes: Gorgoniceps viridula sp.nov., and its cultural characters. - Mycotaxon 29:189-194.

Jones, J.P. 1981: Nuclear behaviour in Hypocreales: Calonectria crotalariae. - Mycologia 73:923-930

Jones, J.P. \& McGawley, E.C. 1984: Karyology and ascus formation in Cordyceps capitata. - Canad. J. Bot. 62:2146-2149.

Jones, J.P. \& Clay, K. 1987: Ascus and crozier development in the Balansiae. - Canad. J. Bot. 65:1027-1030.

Julien, J.B. 1958: Cytological studies of Venturia inaequalis. - Canad. J. Bot. 36:607-613.

Karsten, P.A. 1869: Monographia Pezizarum fennicarum. - Not. Sällsk. Fauna Flora Fenn. Förh. 10:99-206.

- 1871: Mycologia Fennica. — Bidr. Känned. Finlands Natur Folk 19:1-250.

- 1887: Symbolae ad Mycologiam Fennicam 19. - Medd. Soc. Fauna Flora Fenn. 14:85-94.

Kirk, P.M. 1984: New or interesting microfungi 13. Ascomycetes on Laurus nobilis leaf litter. - Mycotaxon 19: 307-322.

Kirk, P.M. \& Spooner, B.M. 1984: An account of the fungi of Arran, Gigha and Kintyre. - Kew Bull. 38:503597.

Kohn, L.M. 1979: A monographic revision of the genus Sclerotinia. - Mycotaxon 9:365-444.

Kohn, L.M. \& Korf, R.P. 1975: Variation in ascomycete iodine reactions: $\mathrm{KOH}$ pretreatment explored. - Mycotaxon 3:165-172.

Korf, R.P. 1951: A monograph of the Arachnopezizeae. Lloydia 14:129-180.

- 1971: Some new Discomycete names. - Phytologia 21:201-207.

- 1973: Discomycetes and Tuberales. - In: Ainsworth, G.C., Sparrow, F.K. \& Sussman, A.S. (eds.), The Fungi. An advanced treatise 4A: 249-319. New York \& London.

- 1978: Revisionary studies in the Arachnopezizoideae: A monograph of the Polydesmieae. - Mycotaxon 7:457492.

- 1982: New combinations and a new name for discomycetes illustrated by Boudier in the Icones Mycologicae. Mycotaxon 14:1-2.

Korf, R.P. \& Dumont, K.P. 1968: The case of Lambertella brunneola: an object lesson in taxonomy of the higher fungi. - J. Elisha Mitchell Sci. Soc. 84:242-247.

Korf, R.P. \& Kohn, L.M. 1980: Revisionary studies in the Hyaloscyphaceae 1. On genera with "glassy" hairs. 
- Mycotaxon 10:503-512.

Korf, R.P. \& Zhuang, W.-Y. 1984: The ellipsoid-spored species of Pulvinula (Pezizales). - Mycotaxon 20:607616.

- 1985: Some new species and new records of discomycetes in China. - Mycotaxon 22:483-514.

Küppers, H. 1981: DuMont's Farben-Atlas. - 163 pp.

Köln.
Kuyper, T.W. 1985: Clitocybe metachroa and the problem of the variable species. - Agarica 6:11-27.

- 1988: Specific and infraspecific delimitation. - In: Bas, K., Kuyper, T.W., Noordeloos, M.E. \& Vellinga, E.C. (eds.), Flora Agaricina Neerlandica 1:30-37. Rotterdam \& Brookfield.

Lagarde, J. 1906: Contribution a l'etude des Discomycetes charnus. - Ann. Mycol. 4:125-256.

Lowen, R. \& Dumont, K.P. 1984: Taxonomy and nomenclature in the genus Calycellina (Hyaloscyphaceae). - Mycologia 76:1003-1023.

Luard, E.J. 1983: Two problems encountered in preparation of fungi grown at low osmotic potential for microscopy. - Trans. Brit. Mycol. Soc. 80:529-533.

Massee, G. 1895: British Fungus-Flora 4. - 522 pp. London \& New York.

Matheis, W. 1977: Cistella amenticola sp. nov. nebst Bemerkungen über einige andere Cistella-Arten. - Friesia 11:85-93.

Migula, W. 1913: Kryptogamen-Flora von Deutschland, Deutsch-Österreich und der Schweiz 3, Pilze 3. - In: Thome, O.W. (ed.), Flora von Deutschland, Österreich und der Schweiz: 685-1404. Gera.

Müller, E. 1967: Neufunde von Hyaloscyphaceae aus den Alpen. - Sydowia 21:143-153.

- 1977: Zur Pilzflora des Aletschwaldreservats (Kt. Wallis, Schweiz). - Beitr. Kryptogamenflora Schweiz 15:1-126.

Nag Raj, T.R. \& Kendrick, B. 1975: A monograph of Chalara and allied genera. - 200 pp. Waterloo.

Nannfeldt, J.A. 1932: Studien über die Morphologie und Systematik der nicht-lichenisierten inoperculaten Discomyceten. - Nov. Acta Soc Sci. Upsal. Ser. 4, 8:1-368, 20 tab.

- 1936: Notes on type specimens of British inoperculate discomycetes (First part, notes 1-50). - Trans. Brit. Mycol. Soc. 20:191-206.

- 1939: Notes on type specimens of British inoperculate discomycetes (Second part, notes 51-100). — Trans. Brit. Mycol. Soc. 23:239-252.

- 1976: Iodine reactions in ascus plugs and their taxonomic significance. - Trans. Brit.Mycol. Soc. 67:283-287.

Nees von Esenbeck, C.G. 1817: Das System der Pilze und Schwămme. - $334 \mathrm{pp}$. Würzburg.

Nylander, W. 1869: Observationes circa Pezizas Fenniae. - Not. Sällsk. Fauna Flora Fenn. Förh. 10:1-97.

Parmasto, E. \& Parmasto, I. 1987: Variation of basidiospores in the Hymenomycetes and its significance to their taxonomy. - Bibliotheca Mycol. 115:1-168.

Persoon, C.H. 1794: Tentamen dispositionis methodicae fungorum. - Neues Mag. Bot. 1:81-128.

- 1798: Icones et descriptiones fungorum minus cognitorum 1. - Lipsiae.

- 1800: Icones et descriptiones fungorum minus cognitorum 2. - Lipsiae.

- 1801: Synopsis methodica fungorum. - 708 pp. Gottingae.
Pfister, D.H. 1976: A synopsis of the genus Pulvinula. Occ. Pap. Farlow Herb. Harvard Univ. 9:1-19.

Phillips, W. 1877: Fungi of California and the Sierra Nevada Mountains. - Grevillea 5:113-118.

- 1887: A manual of the British Discomycetes. - 462 pp. London.

Quélet, L. 1886: Enchiridion fungorum. - $352 \mathrm{pp}$. Lutetiae.

Raitviir, A. 1970a: Synopsis of the Hyaloscyphaceae. Scripta Mycol. 1:1-115.

- 1970b: On the dimorphism of the ascospores in the Hyaloscyphaceae. - Eesti NSV Tead. Akad. Toim., Biol. 19:382-384.

- 1977a: A new genus of the Hyaloscyphaceae. - Fol. Crypt. Est. 8:1-8.

- 1977b: New taxa of the Hyaloscyphaceae. - Fol. Crypt. Est. 9:1-8.

- 1977c: The genus Dasyscyphella. - Eesti NSV Tead. Akad. Toim., Biol. 26:29-35.

- 1985: The arcto-alpine species of the Hyaloscyphaceae. Agarica 6:137-146.

- 1986: The problem of genus in discomycetes. - Scripta Mycol. 15:149-158.

- 1987: Notes on the taxonomy and nomenclature of Belonidium, Trichopeziza and Lachnum (Hyaloscyphaceae) in the light of homologous series concept. - Eesti NSV Tead. Akad. Toim., Biol. 36:313-318.

Raitviir, A. \& Galán, R. 1986: A new genus of the Hyaloscyphaceae. - Int. J. Mycol. Lichenol. 2:221-234.

Raschle, P. 1977: Taxonomische Untersuchungen an Ascomyceten aus der Familie der Hyaloscyphaceae Nannfeldt. - Sydowia 29:170-236.

- 1978: Neufunde und Neukombinationen von Hyaloscyphaceae Nannfeldt, (Helotiales). — Nova Hedwigia 30:653672.

Rehm, H. 1896: Die Pilze Deutschlands, Oesterreichs und der Schweiz 3. Ascomyceten: Hysteriaceen und Discomyceten. - In: Rabenhorst,L. (ed.), Kryptogamen-Flora von Deutschland, Oesterreich und der Schweiz: 1-1275. Leipzig.

Rifai, M.A. 1968: The Australasian Pezizales in the Herbarium of the Royal Botanic Gardens Kew. - Verh. Kon. Nederl. Akad. Wetensch., Afd. Natuurk. 2, 57:1-295.

Rogers, J.D. 1972: Hypoxylon cohaerens: cytology of the ascus. - Mycopathol. Mycol. Appl. 48:161-165.

Ryvarden, L. 1976: The Polyporaceae of North Europe 1. - 214 pp. Oslo.

Saccardo, P.A. 1875: Fungi veneti novi vel critici 4. Atti Soc. Ven.-Trent. Sc. Nat. 4:1-41.

- 1889: Sylloge fungorum omnium hucusque cognitorum 8. - 1143 pp. Patavii.

- 1913: Sylloge fungorum omnium hucusque cognitorum 22. - 1612 pp. Patavii.

Sanders, P.F. \& Webster, J. 1978: Survival of aquatic hyphomycetes in terrestrial situations. - Trans. Brit. Mycol. Soc. 71:231-237.

Savile, D.B.O. 1955: A phylogeny of the Basidiomycetes. - Canad. J. Bot. 33:60-104.

Schumacher, T. \& Sivertsen, S. 1987: Sarcoleotia globosa (Sommerf.: Fr.) Korf, taxonomy, ecology and distribution. - In: Laursen, G.A., Ammirati, J.F. \& Redhead, S.A. (eds.), Arctic and alpine mycology 2:163-176.

Schroeter, J. 1908: Die Pilze Schlesiens. - In: Cohn, F. (ed.), Kryptogamen-Flora von Schlesien 3 (2): 1-597. 
Seaver, FJ. 1961: The North-American cup-fungi (Inoperculates). -428 pp. New York.

Sharma, M.P. 1986: Indian Hyaloscyphaceae. - Nova Hedwigia 43:381-422.

Sharma, M.P., Arendholz, W.-R. \& Thind, K.S. 1986: Some more observations on eastern Himalayan Helotiales. - Mycotaxon 25:119-140.

Sherwood, M.A. 1974: A new Hyaloscypha from New York. - Phytologia 28:15-16.

- 1977: The Ostropalean fungi. - Mycotaxon 5:1-277.

Spooner, B.M. 1987: Helotiales of Australasia: Geoglossaceae, Orbiliaceae, Sclerotiniaceae, Hyaloscyphaceae. Biblioth. Mycologica 116:1-711.

Spooner, B.M. \& Dennis, R.W.G. 1985: New or interesting Ascomycetes from the Highlands and Islands. - Sydowia 38:294-316.

Starbäck, K. 1895: Discomyceten-Studien. - Bih. Svensk Vet.-Akad. Handl. 21:1-42, 2 tab.

Stevenson, J. 1879: The fungi of Scotland. - $443 \mathrm{pp}$. Edinburgh \& London.

Svrð̌k, M. 1962: O rodu Discocistella gen. nov. a některých jeho druzich. De genere Discocistella gen. nov. familiae Hyaloscyphacearum. - Česká Mykol. 16:9-13.

- 1967: Species novae Discomycetum (Helotiales) e montibus Belanské Tatry, Slovakiae. Nové druhy diskomyceti̊ (Helotiales) z Belanských Tater na Slovensku. — Ceská Mykol. 21:146-150.

- 1976: New or less known Discomycetes 3. Nové nebo méněznámé diskomycety 3. - Česká Mykol. 30:8-16.

- 1977a: New or less known Discomycetes 4. Nové nebo méněznámé diskomycety 4. - Česká Mykol. 31:8-14.

- 1977b: New or less known Discomycetes 5. Nové nebo méněznámé diskomycety 5. — Česká Mykol. 31:133-138.

- 1977c: New or less known Discomycetes 6. Nové nebo méněznámé diskomycety 6. — Česká Mykol. 31:193-200.

- 1978: New or less known Discomycetes 9. Nové nebo méněznámé diskomycety 9. — Ceská Mykol. 32:202-204.

- 1979: New or less known Discomycetes 10. Nové nebo méněznámé diskomycety 10. — Ceská Mykol. 33:193206.

- 1982: New or less known Discomycetes 11. Nové nebo méněznámé diskomycety 11. - Česká Mykol. 36:146153.

- 1983: New or less known Discomycetes 12. Nové nebo méněznámé diskomycety 12. - Ceská Mykol. 37:65-71.

- 1984: A taxonomic revision of inoperculate discomycetes described by J. Velenovsky in the genus Helotium, preserved in National Museum, Prague. - Acta Mus. Nat. Pragae 15B: 129-215.

- 1985: Notes on the genus Hyaloscypha (Helotiales). Poznámky o rodu Hyaloscypha (Helotiales). — Česká Mykol. 39:205-219.

- 1986: New or less known Discomycetes 14. Nove nebo menězname diskomycety 14. - Ceská Mykol. 40:203217.

- 1987a: New or less known Discomycetes 15. Nove nebo menězname diskomycety 15 . - Ceská Mykol. 41:16-24.

- 1987b: Über zwei neue Discomycetengattungen (Helotiales). - Sydowia 39:219-223.

- 1987c: New or less known Discomycetes 16. Nove nebo menězname diskomycety 16. - Česká Mykol. 41:88-96.

- 1987d: Evropské rody diskomycetů celedi Hyaloscyphaceae (Helotiales). The European genera of the family
Hyaloscyphaceae (Helotiales). — Česká Mykol. 41:193206.

- 1988: New or less known Discomycetes. 17. Nove nebo menězname diskomycety. 17. — Ceská Mykol. 42:76-80.

Sydow, H. \& Sydow, P. 1919: Mykologische Mitteilungen. - Ann. Mycol. 17:3-47.

Tibell, L. 1978: The genus Microcalicium. - Bot. Notiser 131:229-246.

- 1987: Australasian Caliciales. - Symb. Bot. Upsal. 27:1280.

Weber, N.S. 1972: The genus Helvella in Michigan. Mich. Bot. 11:147-201.

Velenovsky, J. 1934: Monographia Discomycetum Bohemiae. - 436 pp., 30 tab. Pragae.

- 1939: Novitates mycologicae. - 208 pp. Pragae.

- 1947: Novitates mycologicae novissimae. - 168 pp., 2 tab. Pragae.

White, W.L. 1943: Studies in the genus Helotium 3. History and diagnosis of certain European and North American foliicolous species. - Farlowia 1:135-170.

- 1944: Studies in the genus Helotium 4. Some miscellaneous species. - Farlowia 1:599-617.

Zhuang, W.-Y. 1988: A monograph of the genus Unguiculariopsis (Leotiaceae, Encoelioideae). - Mycotaxon 32:183.

Zhuang, W.-Y. \& Korf, R.P. 1986: A monograph of the genus Aleurina Massee (= Jafneadelphus Rifai). Mycotaxon 26:361-400.

Accepted for publication

on 6 February 1989 


\section{INDEX}

\section{Index to species, varieties and forms}

acanthopila 221

acericola $220-222,225,233$

acerina $137,138,233$

acerum 50

aciculare 53

acuminatula 233

acutum 240, 242

acuum 52, 183, 184, 233

aggregata 54

alba 235

albohyalina $62-65,67,69,73,74,76,79,80,84,93,103,107$, $111,122,126,132,136,141,154,166,175,179,204,244$ albolutea $109,233,243$

alniseda $56,65,69,76,79,80,84,131,132,134,141,142,150$, $151,162,167,170,179,237,242$

amyloideopilosa $72,109,112$

anderssoni 109

antirrhini 233

araneocincta $62,211,214,216$

asperipila $233,234,242$

aspidii 237

atomaria 52,109

aureliella $47,52,56,58-61,64-66,69,72-74,76,78-80,82,84$, $90,91,97,103,107,115,117,118,133,157,171,172,191$, 233

auricolor 52

australis 129

barbata $51,61,115,233,241$

betularum 211,216

betulicola 235

betulina 233

brdensis 50

brevicapitata 233,241

britannica $64-66,76,79,83,90,112,113,117,118$

bulbopilosa $65,69,76,79,84,150,151,158$

byssacea 233

callunae 233

calycina 150

candida $49,52,53,66,90,91,115,118$

candidata 234

capitata 60

capreolaria 129,133

caricicola 234

caricis 240

carnosa 234

carnosula 234

carpinacea $233,234,242$

cejpii 234

ceracella 234

cerberi $58,137,138,142$

chlorinella 237

chrysostigma 206,241

ciliata $47,50,234,238,240,242$

ciliifera 234

cincinnata $62,147,151,154$

cladii $53,234,242$

cladii-marisci 234

clavispora $205,211,216$

clavuliformis 234

conincola 54,187 contorta 234,242

cookei 235

copiosa 109

copiosus 129

corticalis 236

cretacea 235

cuneata $183,184,187$

cupularum 137, 138, 142

curvipila 185

cylindrospora 235

daedaleae $58,60-63,65,73,74,76,77,79,81,82,84,97,120$, $132,133,141,235$

degenerans 151,155

dematiicola 48, 52, 235, 240

dennisii 48,235

dentata $47,51,52,235,238$

deparcula 48, 49, 205, 229, 230, 232

diabolica 58,124

digitipila 228

drupacea 242

dryopteridis $78,186,188$

epicalamia 235

epilobii 235

epiporia $58,62,73,74,81,84,126$

eriobasis 112

eriophori 235,236

eurotioides 142, 236, 242

faginea 163

falcata 109

falcipila $54,55,234$

farinacea 236

flaveola $50,77,78,206,231$

foliicola $61,74,209,225$

fuckelii $56,58,61-63,65-67,69,73,74,76,77,79,80,83,84$, $97,103,104,112,122,123,129,133,134,136,141,154,155$, $158,166,167,169,175,178$

fugiens $52,237,238$

fuscostipitata 51

galiorum $141,142,236$

glyceriae 236

graminum 236, 237

granulosella 52,109

grevillei $64,235,236,239$

grisella 53

hamata 151

hamulata $54,55,236$

hederae 236

helicotricha 74, 182

hellebori 236

herbarum $58,63,64,67,73,74,76,77,79,82,84,97,103,133$, $136,137,150,167,169,215,233,234,236,238,242,244$

herbicola 236

himalayensis 209,210

hirta 50

hungarica 236

hyalina $47,48,52,99,109,112,120,123,124,129,133,151,155$, $176,179,236$

hyperici 237

hypericicola 237

imberbe 72

incerta 176

incrustata 237

intacta $64,67,74,79-84,103,141,142,166,179$

invisibilis 237 
iridina 236,237

jaapii 211

juniperi 185

lachnobrachya $48-50,53,54,62,67,74,78,84,203,205,208$, $211,217,219,220,224,228,231,239$

lachnobrachyoides $68,74,77,78,215-217$

lachnoides 237

lacustris 237

lagenipilus 163

lanensis 184

laricina $129,155,237$

laricionis $49,54,57-59,71,74,75,77,78,81,83,84,111,181$, $183,184,194,196,198,200,203,204,244$

lateritioalbum 234

latispora $58,64,71,145$

lectissima 93,94

leucella 237

leuconica $53,58,61,62,65-67,69,73,74,76,79,80,84,111$, $132,147,154,155,158,175,178,237$

lignatilis $163,237,240$

lignicola 99, 100, 101

ligniseda 111

lonchitidis 237

longevestita 151

longispora 237

lunispora 237

lupuli 235

lutea 211,215

luteola 237

luteopallida 120

luzulae 238,243

lycopodii 184,187

mali 129

martialis 238

martii 238

maxima 176

melanobasis $64,181,191,194$

mercurialis 66

micacea 239

microcarpa 238

microscopica 84, 238

microspis 238

microstoma 238

millepunctata 84,238

milliaria 238

minima 190,238

minor 211,212

minutella 238

minutissima 126, 186, 190

mirabilis 238

mollisiaeformis 239

montana 239

myriadea 239, 240

nana 239

nardi 239

nectrioidea 151

nipponica $64,90,118,155,175$

nivea 236,237

nympharum 239

obscura 109, 239

obsoleta $58,163,192,196,237,240$

occulta 74,158

ocellata $74,186,187,194,196$

ochracea 239 oenotherae 239

oligospora 211

olivacea 52,239

opalina 239

pallidipilosa $220,222,224$

paludicola $136,167-169$

paludosa 48,240

pani 240

parasitica $54,90,99,240$

parenchymatosa 239,240

parvispora 172

parvula 158,240

pellucens 240

pellucida 240

perpusilla $129,133,136,169,179,236,242$

pezizellaeformis 93

phaeotricha 186, 196-198, 221, 222

phyllophila 240

pilosa 240

pinaceus 109

plowrightii $50,220,221$

populina 233

priapi $65,69,71,79,84,103,160,165,240,241$

protonematosa 93,94

pseudopuberula $50,67,70,71,77,137,220,224,225,233$

pteridina 241

puberula $48,49,51,241$

punctata 241

punctiformis $52,211,212,241$

punctoidea 241

pusilla $209,225,241$

pygmaea 241,242

quercicola $63-65,67,69,73,74,76,79,82,84,103,123,144$, $160,162,163$

quercina $115,163,166,233,241$

quercus $73,120,123,124,133$

rabenhorstii 243

radiostriata 241

rehmii 55

resinacea 61,241

resinifera 109,113

reticulata 60,241

richonis $52,61,239,241$

robustella 241

rosae 241

roseoguttata $79,84,115,116,118$

rubi-fruticosi 137

ruborum 137

salicina $233,234,242$

sarothamni $64,98,137,138,141,142,234,242$

scintillans 234,242

scutula 66

secalina $73,133,136,167$

senilis 242

sesleriae 242

setiigera $57,59,82,84,215,220,226$

setosa 151,152

spinosae 129,176

spiraeicola $229,231,232$

spiralis $65,69,76,79,84,97,99,107,117,126,136,145,150$, $162,201,203$

stereicola 52

stevensonii $108,109,171$

straminea 235,242 
stramineum 235

strobilicola $67,73,78,90,170$

subcorticalis 242

subliciformis 242

sublicoides 242

sublividula 239

substipitata 242

subtilis 50,242

sulfurina 242

sulphurea 233, 243

sulphureocitrina 201, 203

sulphureopilosa 243

sydowii 109

tapesioides 113,115

tenuipila $220,221,222$

tenuispora 243

tenuissimum 183, 184, 187

terrestris 243

tetraspora 211,215

tigillaris 70, 72, 103-105, 157

tiliae 129

trapezispora 120,121

truncorum 243

tumidula 243

typhacea 243

ulmacea 243

ulmariae $50,60,68,74,77,78,206,208,221,229$

umbrina 243

uncinata $55,62,72,186,187,196,197,200,220-222,224,243$

uncipila $64,71,74,81,83,84,104,201,244$

usitata $64,65,173$

velenovskii 109,112

vernalis $162,163,166,241$

viridiflavescens $201,204,244$

viridula 196

vitreola $47,48,58,61,62,65,66,69,73,74,76,79,80,84,90$, $91,97,103,132,133,136,141,147,150,157,160,165,175$, 176, 179,238

zalewskii 244

\section{Index to genera}

Albotricha 62, 236

Allophylaria 47, 50, 239, 242

Amicodisca 50

Arachnopeziza 60, 71, 73, 91, 112, 157, 221, 231, 234, 240

Arachnoscypha 47

Betulina 50, 51, 71

Bisporella 50

Calycellina 48-52, 71, 74, 187, 233, 237, 238, 239, 241

Calyptellopsis 60,241

Chalara 53

Cheiromycella $79,82,83,89,111,120$

Chrysothallus 51, 104, 203

Ciliolarina 111

Cistella $47-49,51,52,64,73,163,233,235,236,240,241$

Cistellina 52

Clathrosphaerina 79,244

Clavidisculum 52, 233

Crocicreas 50, 233, 242

Cudoniella 72

Dasyscypha 54
Dasyscyphella $60,71,73$

Dasyscyphus 60, 233

Debaryoscyphus 49,181, 192

Dematioscypha $46,51,52,71,235,241$

Dendrotrichoscypha 221, 235, 239

Dennisiodiscus 58, 142

Discocistella 52, 234, 238

Eriopeziza 240

Eupezizella 49, 52, 53, 62, 72, 73, 90,91, 115, 120, 157, 172, 240

Fuscoscypha 53

Gorgoniceps 196

Haplographium 52

Helotium 48, 66, 72

Helvella 66

Hyalinia 239

Hyalopeziza 47, 48, 53, 55, 73, 90, 151, 158, 221, 241

Hymenoscyphus $66,67,72,240$

Incrupila 48, 51, 62, 243

Lachnellula $66,111,150$

Lachnum 53, 61, 62, 71, 73

Laetiporus 86

Lenzites 186

Microcalicium 66

Microscypha 48, 53, 234

Mollisia 47, 55, 73, 243

Mollisina 47, 49, 60, 71, 221, 235, 238, 239

Mycena 72

Orbilia 239

Perrotia 73

Pezizella 47, 52, 54, 91, 204, 206, 241

Pezoloma 234

Phaeohelotium 72

Phaeoscypha 53, 234, 242

Phialophora 79, 83, 131

Phialoscypha 50, 53, 205

Protounguicularia 51, 61, 71, 72, 91, 115, 233, 238, 240, 241

Pseudaegerita 79, 83, 89, 97, 102-104

Pseudohelotium 133

Psilachnum 236-238

Psilocistella 53,54,71,73,162, 163, 166, 187,234,237, 239-241

Pyrenopeziza 66

Scutoscypha 51,71

Setoscypha $49,53,54,205,216$

Trametes 186

Trichopeziza 243

Truncicola 54, 90

Tympanis 236

Uncinia 49, 142, 181, 204, 209

Unciniella 49, 181, 197, 200

Unguicularia $54,72,73,235,238$

Unguiculariopsis 54,55

Unguiculella 48, 49, 54, 55, 142, 158, 197, 236, 241, 242

Urceolella 61, 221, 232, 235

Zoellneria 50

\section{Index to substrates}

Abies 111, 132, 183, 186, 200, 203

Acer $137,141,178,211,215,221,233,238$

Adenostyles 239

Aesculus 74, 102, 215, 217

Alnus 93, 97, 102, 132, 134, 136, 141, 150, 154, 176, 178, 209, 
$215,226,228,243$

Amylocystis 126, 128

Andromeda 215

Antirrhinum 233

Arbutus 235

Artemisia 235, 242

Aspidium 237

Betula $82,86,97,102,118,132,136,141,144,150,154,160$, $162,166,176,178,203,209,215,217,221,233,235$

Calluna 150, 154, 233

Carex 234, 242, 243

Castanea 102,221

Carpinus 93, 103, 109, 129, 132, 133, 144, 217, 234, 238, 241, 243

Corylus 93, 97, 99, 103, 132, 141, 154, 166

Crataegus 97, 103, 141, 166

Cryptomeria 97,186

Daedalea $120,122,235$

Diatrype 240

Dryopteris 188, 189, 206

Epilobium 73, 141, 235

Eriophorum 168, 169, 237, 238

Espeletia 175,243

Fagus $97,102,132,136,141,150,154,160,162,166,176,178$, $217,221,233,240,241$

Filipendula 57, 74, 208, 231

Fraxinus $103,132,215,240$

Galium 236

Glyceria 236

Hedera 236

Holcus 236, 237

Humulus 235

Hymenochaete 122,132

Hypericum 237

Iris 239

Juncus $132,169,237$

Juniperus $109,111,186,197$

Larix $97,102,103,109,111,115,129,132,150,151,154,186$, 237

Libertia $132,133,167$

Lonicera 136

Luzula 238

Lycopodium 74, 185-187, 189, 240

Malus 129, 132

Melampyrum 141

Molinia 132, 169

Nardus 239

Nothofagus 103

Oenothera 239

Peniophora 132

Phalaris 242

Phellinus 132

Phragmites 137, 141

Phylica 129,132

Picea $86,102,111,115,126,132,136,151,153,154,184,186$, $187,194,203,242,243$

Pinus $82,86,97,105,109,111,141,150,151,154,155,170-172$, $186,190,196,197,203,237$

Polystichum 189

Populus $86,97,103,109,132,136,141,154,166,178,203,211$, 237,239

Postia 128

Potentilla 169

Prosopis 238
Prunus $97,103,129,132,136,166,176,178,237,242$

Pseudotsuga 111

Pteridium 74, 206, 208

Pyrus 242

Quercus 73, 81, 82, 86, 93, 97, 98, 102, 115, 120, 122-124, 129, $132,133,136,137,141,149,150,154,162,163,165,166$, $178,197,203,211,215,220,221,236,238-242$

Rhododendron $186,189,215$

Rosa $141,145,147,241$

Rubus 102, 132, 137, 141, 150, 209

Rumex 141

Salix 73, 86, 93, 97, 99, 129, 132, 136, 144, 151, 154, 203, 209, $211,215,231,234,236,242$

Sambucus 103, 237

Sarothamnus 137, 141, 234, 242

Sasa 102

Secale 167

Senecio 236

Sesleria 242

Sorbus $61,136,144,154,241$

Spiraea 229, 231

Symphoricarpus 136, 141, 154

Taxus 111, 150

Tilia 97, 129, 132, 215, 217

Typha $132,169,243$

Ulex 103

Ulmus 178, 238, 239, 243

Vaccinium 154

Verbascum 137, 141

Viburnum 132 